\title{
Abstract of 44th National Conference of Association of Clinical Biochemists of India (ACBICON 2017)
}

Association of Clinical Biochemists of India-2017

\section{Awadhesh Saran Memorial Oration}

Towards patient-centred health care---Biochemist's responsibilities

\section{Sucheta P Dandekar}

Professor \& Head, Department of Biochemistry, In Charge Central Clinical Biochemical Laboratory Seth G.S. MedicalCollege, Mumbai

$\mathrm{T}$

here is a constant challenge to better health needs behind.

Right from harmonization of laboratories to expansion of technology, many trends shall affect how we Biochemists work and think as we try and amalgamate the fast progressing technology with our day to day work.

Thus, the trends in laboratory technology and the effects on quality asects to better patient care shall be discussed in detail.

\section{G. P. Talwar Memorial Oration}

How to Imagine the Future of Laboratory Medicine

\section{Prof. Maurizio Ferrari}

Maurizio Ferrari, MD

Professor of Clinical Pathology, Vita-Salute San

Raffaele University, Milan, ItalyIFCC President

ttempting to predict the future or being dogmatic $A$ about what may or what may not transpire in the future is a risky business, and history provides numerous examples of predictions and assumptions that have proved to be wrong.

Laboratory medicine exists within the broader framework of medicine and healthcare. Therefore, changes and advances in these areas will impact the future direction of laboratory medicine.

We may predict an increasing numbers of patients with chronic diseases, a development of more personalised medicine and preventative medicine, a rising patient and clinical expectations and a shift to increasingly supporting more frail patients in the community.

Moreover laboratory testing will be influenced by many factors such as healthcare reforms, cost containment strategies, consolidation of laboratories and in vitro diagnostic testing, as well as the impact of new technologies and tests on the existing laboratory organization. Laboratory presents many concrete opportunities in the near future to deliver better and more 
efficient care. They are underpinned by rapid technological change like:-efficiency can be improved and at a time of great financial pressure, it must be,there is considerable scope to improve the effectiveness of existing services to provide an increasingly useful language and standards for testing and reporting, working together with other diagnostic disciplines,improving the fields of molecular testing and genomics. -developing point of care testing carried out at the bedside or nearby to patients and biosensors to have applications in health care, -smartphones and the ease of entering the market for apps will expand a parallel consumer market with implications for traditional services. A bright future for laboratory medicine is in front of us.

\section{K. L. Gupta Memorial Oration}

\section{Identification of Novel Biomarker of Human Urinary Bladder Cancer Using High-Density Oligonucleotide Microarray}

M. L. B. Bhatt ${ }^{1}$, P.K. Singh ${ }^{1,2}$, A. K. Srivastava ${ }^{1}$, S.K. Rath ${ }^{3}$, M.M. Goel ${ }^{4}$, D. Dalela ${ }^{5}$.

1. Department of Radiotherapy, King George's Medical University, Lucknow, Uttar Pradesh 1,2. Pankaj Kumar Singh: Department of Biochemistry, All India Institute of Medical Science, Bhopal, Madhya Pradesh,

3. Division of Toxicology, CSIR-Central Drug Research Institute, Lucknow, Uttar Pradesh, 4. Department of Pathology, King George's Medical University, Lucknow, Uttar Pradesh, 5. Department of Urology, King George's Medical University, Lucknow, Uttar Pradesh, India.

INTRODUCTION: Urothelial bladder cancer (UBC) is a frequently occurring malignancy of the urinary tract.Treatment of UBC remains a challenge despite advances in surgical, radiotherapy and chemotherapeutic options. Gene expression profiling is an innovative way to identify relevant differentially expressed candidate genes to be used as molecular bio-markers and novel therapeutic targets.

MATERIALAND METHODS: Tissue specimens were obtained from 7 patients with pathologically confirmed non-muscle-invasive $(n=2)$ and invasive $(n=5)$ UBC. Total RNA was extracted from the bladder tumor tissues using TRIzol reagent. Human Bladder Total RNA was used as a universal reference for Affymetrix high-density oligonucleotide microarray analysis. The expression data for each sample was generated on Affymetrix Human Gene 1.1 ST arrays. Subsequently, we validated the expression profile of five differentially expressed cancertestis (CT) genes (ZNF165, CEP55, PBK/TOPK, TTK and D40) using reverse transcriptase quantitative PCR and immunohistochemistry in another bladder tumour cohort $(n=76)$ and $(n=65)$.

RESULTS:We got 1105 differentially expressed genes which included 380 up-regulated genes and 725 downregulated genes respectively. We observed mRNA overexpression of all five CT genes in testis and UBC patients. CT genes mRNA expression were detected in range of $43 \%-59 \%$ of bladder tumors. Relative mean fold mRNA expression of all five CT genes were found to be significantly $(\mathrm{p}<0.01)$ higher in muscle-invasive bladder cancer as compared to non-muscle-invasive bladder cancer patients. Moreover, Immunohistochemical expression of CT genes was detected in range of 56\%$70 \%$ of bladder tumors.

CONCLUSION:We found that all the five CT genes were expressed in a large fraction of bladder tumors and consequently, this may pave the way for successful immunotherapy with high efficiency. The CT genes identified in our study, could serve as a valuable target for development of anticancer agents or cancer peptide vaccines for UBC.

KEY WORDS : Urinary Bladder Cancer; Oligonucleotide microarray; Gene expression; Cancer-testis; Peptide vaccine 
Dr. T. N. Pattabhiraman Memorial Oration

Task Force on Indian Normative Clinical Laboratory Parameters

\section{T Malati}

Director, Krebs Biochemicals \& Industries Limited, Hyderabad Formerly Senior Professor\& Head, Department of Biochemistry, Nizams Institute of Medical Sciences, Hyderabad 500008, Hyderabad, Telangana

$\mathrm{O}$ ver the years it has been observed that that diagnostic and pathological investigations/tests done in Indian Laboratories for diagnosis and prognosis of all diseases and syndromes use the reference values provided by Western literature such as WHO or other healthcare institutions of the world.

In view of multi ethnicity of Indian population, a consensus emerged from the association the Medically Aware and Responsible Citizens of Hyderabad (MARCH) regarding the need to set up Indian standards for biochemical parameters.

It has been decided to set up a task force on Qualitycontrol of various laboratory parametersSetting up Indian Normative Values. The proposed project was named "INCLAP" the Indian Normative Clinical Laboratory Parameter.

The presentation will not only highlight the course of events followed from the time of inception of this much needed project but also inspire ACBI to play a role in setting up Indian Laboratory standards.

\section{Seth GS Medical College \& KEM Hospital Oration}

\section{Alcoholic Liver Disease: Pathogenesis, Biochemical Markers and Treatment}

\section{Subir Kumar Das}

Department of Biochemistry, College of Medicine \& JNM Hosptal, WBUHS, Kalyani, Nadia 741235, WB
A lcoholic beverages, and the problems they engender, have been familiar in human societies since the beginning of recorded history. Alcohol is no longer viewed as a threat to all people, but rather to a small subclass of "alcoholics" or, in today's technical terms, people who are "alcohol-dependent". Ethanol is primarily metabolized in the liver by the multiple isoenzymes of alcohol dehydrogenase (ADH) and microsomal ethanoloxidizing system (MEOS) and by other ethanolmetabolizing enzymes such as catalase. About $90 \%$ to $95 \%$ of ethanol is eliminated by oxidation. The rest is excreted by the kidneys, the lungs and the skin. Oxidative stress is a well recognized key step in the pathogenesis of ethanol-associated liver injury. Oxidative stress and associated cellular injury promote inflammation. TNF$\alpha$ and its inducible cytokines interleukin (IL)-6, are associated with alcoholic fibrosis.TGF- $\beta$, one of the most powerful profibrogenic mediators, plays a major role in the development of liver cirrhosis and regulates ECM gene expression and matrix degradation. It is believed to be a potent activator of hepatic stellate cells resulting in ECM synthesis and correlates well with the presence of fibrosis in patients with ALD. Among a variety of blood tests used to aid the diagnosis of alcohol consumption and related disorders, laboratory tests are particularly useful in settings where cooperativeness is suspected or when a history is not available. Antioxidants could have beneficial effects in reducing the incidence of ethanol-induced changes in lipid, proteins and nucleic acids. Preventive measures were more effective than curative treatment. Glutathione replacement would seem to be an obviouschoice in view of the extensive evidence implicating glutathionedepletion in the pathophysiology of alcohol-inducedliver dysfunction. Indeed, cytokinedirected therapy has been shown to prevent liver injury in a rat model of ALD, autologous bone marrow cell infusion (ABMI) therapy is a candidatemodality in the treatment of decompensated liver cirrhosis. However, abstinence is the foundation of therapy for an alcohol problem. 


\section{Plenary}

\section{Baicalein, A Natural Flavonoid, Activate of Nrf2/ Autophagy Axis to Suppresses the Expression of Il-6 In Human OA Chondrocytes}

\section{Tariq M. Haqqi}

\section{Department of Anatomy \& Neurobiology, Northeast Ohio Medical University, Rootstown, Ohio, USA}

BACKGROUND: Inflammation is an important component of osteoarthritis (OA) pathogenesis. IL-6 is implicated in OA pathogenesis as it suppresses anabolic factors and upregulate the expression of catabolic proteins.Here, we used an in vitro model of inflammation in OA to investigate the potential of Baicalein, a natural flavonoid found in root extract of Scutellaria baicalensis, to suppress the expression of IL-6 and determined the molecular mechanism by investigating the role of Nrf2 and autophagy activation in human OA chondrocytes.

METHODS: Primary human OA chondrocytes were prepared by enzymatic digestion of deidentified and discarded cartilage from donors with OA who underwent total knee arthroplasty. Autophagy activation was investigated by immunoblotting for LC3-I and LC3-II, ATG5and autophagic flux. Expression and activation of Nrf2 was determined by immunoblotting and by a luciferase reporter assay, respectively. mRNA and protein expression of Nrf2 regulated genes HO-1, NQO1, SOD2 was studied by qPCR and immunoblotting, respectively. Total protein levels and activation of ERK1/2 and its upstream and downstream signaling molecules was assayed by immunoblotting.For molecular docking studies using theGlide tool in Schrödinger Maestro suite, the crystal structure of Keap1 protein in complex with a small chemical compoundK67 (PDB CODE: 4ZY3) was extracted from the Protein Data Bank and used as docking structure template.

RESULTS: OA chondrocytes showed high levels of IL6 expression upon stimulation with IL-1 $\beta$. However, pretreatment of OA chondrocytes with Baicalein, in a dose dependent manner, abolished the IL- $1 \beta$-induced upregulation of IL-6 expression.Baicalein induced macro-autophagy in $\mathrm{OA}$ chondrocytes in vitro as indicated by significantly $(\mathrm{p}<0.05)$ increased expression of LC3II, ATG5, ATG3, ATG12, ATG16L1 and Beclin1.Using LC3 turn over assay, we determined that Baicalein treatment enhanced autophagy flux demonstrating the induction of active autophagyin human OA chondrocytes. We next examined the signaling pathways involved in the activation of autophagy and results showed that Baicalein induced autophagy in OA chondrocytes through activation of mTOR signaling via AMPK/TSC2/mTOR/P70S6K axis. Additionally, Baicalein treated OA chondrocytesalso showed enhanced activity of Nrf2/ARE as revealed by Nrf2/AREluciferase reporter assay.In silico molecular docking studies indicated that Baicaleinactivates Nrf2 by disrupting the Keap-1/Nrf-2 interaction by blocking the Nrf-2binding site in the Keap-1 protein.Additionally, OA chondrocytes treated with Baicalein, and in a dose dependent manner,showed enhanced expression, both at mRNA and protein levels, of Nrf2 target genes HO-1, NQO1, and SOD2. We next determined the molecular events involved in Baicalein mediated activation of Nrf2 and it was found that treatmentof OA chondrocytes with Baicalein activated MEK1/2-ERK1/2-ELK-1 signaling in a time dependent manner. Inhibition of ERK1/2 activation or inhibition of autophagy using small molecules or siRNA mediated depletion of target genes expression abolished the protective effects of Baicalein in OA chondrocytes under pathological conditions.

CONCLUSION: The present study indicates that Baicaleinexert the anti-inflammatory effect, at least in part, by activating Nrf2/autophagy axis through activation of MEK1/2-ERK1/2-ELK1 and AMPK/TSC2/ mTOR/P70S6 Kpath-waysin human OA chondrocytes under pathological conditions. This property indicates that Baicaleincould be developed as an effective adjunct therapyfor the suppression of OA pathogenesis. 


\section{Plenary}

Clinical Biochemistry at the Summit of Mt. Everest: Correct Interpretation of Arterial Blood Gases and Acid-Base Status.

Gustavo Zubieta-Calleja \& Natalia Zubieta De Urioste

High Altitude Pulmonary and Pathology Institute IPPA, La Paz,Bolivia zubieta@altitudeclinic.com

$\mathrm{E}$ nvironmental factors play a crucial role in the establishment of normal biochemical ranges and should be fully comprehended in order to avoid misinterpretation that could only imperil health. Following an expedition that measured blood gases near the summit of Mt. Everest at $8400 \mathrm{~m}$, the calculation of acid-base balance using the sea level Van Slyke equation has misled scientists. Changes in arterial blood gases $(\mathrm{O} 2$ and $\mathrm{CO} 2$ ) and in acidity or alkalinity (changes in $\mathrm{H}+$ ion concentration) are discussed in terms of base excess (BE) and base deficit (BD). The mathematical relations between them are expressed by the well-known Van Slyke equation. In his quest for a precise measurement of the factor causing variation, Siggaard-Andersen changed these terms to "titratable hydrogen ion in extracellular fluid" (with an inverse sign). We later renamed it as "titratable hydrogen ion difference [from the normal]" (THID). The normal for any altitude is zero. That is the perfect homeostatic environment for cells from the acid-base point of view. As the altitude increases, the barometric pressure decreases exponentially and so do the arterial oxygen partial pressure $(\mathrm{PaO} 2)$ andthe arterial carbon dioxide partial pressure $(\mathrm{PaCO} 2)$. Hence the acid-base values also change accordingly, inducing changes in the mountaineer's blood chemistry (CO2, bicarbonates, $\mathrm{pH})$. Likewise with time at high altitude, hemoglobin increases as an adaptive process and acts as an additional buffer. Consequently, under hypoxic conditions at the very high (extreme) altitudes of Mt Everest ( $\mathrm{PaO} 2: 28 \mathrm{mmHg}$ ), using the Van Slyke equation for sea level conditions without corrections is invalid and, hence, inappropriate. TheCauldwell expedition that sampled blood gases at $8400 \mathrm{~m}$, calculated a BD of $-6.9 \mathrm{mM}$. We have previously created the correction factors for the Van
Slyke equation for different altitudes. Upon applying them at the summit of Mt. Everest, we found that the true THID of the four subjects was $0.7 \mathrm{mM}$. One can appreciate that the optimal acid-base balance (close to zero) is fundamental for the conquest of such extreme altitude. All cells and biochemical reactions in the organism, require an optimal $\mathrm{pH}$ and acid-base balance for optimal function, transcendental for survival. The remarkable adaptation to chronic hypoxia leads unequivocally to survival even at the extreme hypoxic levels of the summit of Mt. Everest, supporting the concept that man can adapt to life even on the summit of Mt. Everest as proposed by the late Prof. Dr. GustavoZubieta-Castillo (Sr), over 14 years ago.

\section{Plenary}

\section{Tropical Fruits as Functional Foods for Metabolic Syndrome}

\section{Lindsay Brown}

\section{School of Health and Wellbeing, University of Southern Queensland, Toowoomba, Australia}

unctional foods provide health benefits in chronic inflammatory bowel disease, in addition to nutrition. The metabolic syndrome includes central obesity, insulin resistance, elevated blood pressure, impaired glucose tolerance, non-alcoholic fatty liver disease and dyslipidaemia; these signs are due to chronic low-grade inflammation combined with oxidative stress. We have shown that a diet high in fructose and saturated/trans fats induces these cardiovascular, liver and metabolic signs in rats. We have shown that components of foods can reverse all these symptoms in diet-induced obese, hypertensive and insulin-resistant rats. In particular, tocotrienols from palm oil, dietary fibre from tropical seaweeds, anthocyanins from tropic Australian native fruits such as Davidson's plums, and polyphenols from Garcinia fruits such as achacha lowered blood pressure, prevented inflammatory cell infiltration into the heart, liver and fat pads, improved plasma lipid profiles and decreased plasma inflammatory biomarkers. All these interventions, especially tropical fruits and seaweeds, 
could be produced commercially, sustainably and costeffectively in many tropical countries, with the aim of reducing the incidence of metabolic syndrome, and decreasing the risk of costly cardiovascular and metabolic disorders.

\section{Plenary}

\section{Angiogenic Growth Factors in the Heart: An Endogenous Route for Cardiac Preservation}

\section{Hari S. Sharma, PhD, DSc}

Institute for Cardiovascular Research, VUM, University Medical Center, Amsterdam, The Netherlands

$\mathrm{P}$ atients with total coronary artery occlusions are known to have more developed collateral circulation compared to patients with stenosis and consequently, we hypothesised, that they may represent the ideal human model to examine endogenous, local growth factor production in vivo.Vascular endothelial growth factor (VEGF) is a heparin-binding homo-dimeric peptide mitogen with target cell specificity to vascular endothelial cells and it promotes growth of blood vessels i.e. angiogenesis in vivo. Using molecular cloning, we have shown earlier that VEGF exists at least as four different species of 121, 165, 189, and 206 amino acids due to alternative splicing of mRNA. We have elucidated the expression and regulation of VEGF in the heart of various species. Using human specific oligonucleotide primers in RT-PCR, we have cloned and characterized porcine heart derived cDNA molecules encoding VEGF121 as well as VEGF165. We have examined the expression pattern and cellular distribution of VEGF in the porcine heart during ischemic ventricular dysfunction achieved by two cycles of short coronary occlusions and followed by reperfusion. Myocardial expression of VEGF mRNAs ( 1.7 and $3.9 \mathrm{~kb}$ ) was increased by 2 fold in the reperfused myocardium. Immunoreactive VEGF was localized mainly in cytoplasm of myocytes in a diffused pattern as well as in smooth muscle layers of the coronary blood vessels. We have shown that the expression of VEGF (both at mRNA and protein level) is up-regulated by adenosine in microvascular endothelial cells in vitro and that tumor necrosis factor- $\beta$ (TNF- $\beta$ ), a potent cytokine mainly secreted by macrophages in the ischemic myocardium is involved in upregulating the VEGF expression in cultured cardiomyocytes. Furthermore, endogenous VEGF and bFGF concentrations were prospectively examined in the coronary circulation and periphery of 14 male patients (seven with mean diameter stenosis $83 \%$, mean age 63, and a further seven with total coronary artery occlusion, mean age 64 undergoing elective left coronary system intervention. VEGF concentrations were significantly higher in the coronary sinus samples of patients with occlusions compared to those with stenoses (400.2 (176.7) v103.3 (19.8) pg/ml, $\mathrm{p}=0.026)$. In contrast, no significant differences between local and systemic concentrations of bFGF for either the whole cohort of patients or those with occlusions were observed. Taking together, our results clearly show that the cardiac myocytes are the major source of VEGF in the heart, of which the expression can be regulated by a number of factors. Increased local VEGF but not bFGF production in the diseased coronary circulation in patients with total coronary occlusions suggest a potential paracrine role for this growth factor in pathophysiologic collateral formation and supports its use as a therapeutic tool for angiogenesis, particularly in patients with inoperable coronary heart disease.

\section{Plenary}

\section{Advances in Cardiac Biomarkers in Health \& Disease}

\section{Virendra K Misra}

MBBS; MD; PGDC(UK); FAHA; FAPSC; FISC; FGHA

Sr. Cardiologist, Sheikh Khalifa Medical City,. Abu Dhabi, UAE

Wational Academy of Clinical Biochemistry (NACB) in 1999, establishing analytical and clinical recommendations for use of cardiac markers in coronary artery diseases. 
Since then the objective is to suggest the specific cardiac markers, its role, its appropriate use to establish the diagnosis in different clinical scenario. The role of cardiac troponins as diagnostic biomarkers of myocardial injury in acute coronary syndrome (ACS) is well established. More than a decade journey from 1stgeneration assays, now 5th-generation high-sensitivity cardiac troponin (hs-cTn) assays as changed the diagnostic capability. During acute ischemia (STEMI or NONSTEMI) Troponin is released from the cytosolic pool of the myocytes. Its subsequent release is prolonged with degradation of actin and myosin filaments. Isoforms of the protein, $\mathrm{T}$ and $\mathrm{I}$, are specific to myocardium. The CK-MB isoform ofcreatine kinase is expressed in heart muscle. It resides in the cytosol and facilitates movement of high energy phosphates into and out of mitochondria.

Lactate dehydrogenase (LDH) catalyses the conversion of pyruvate to lactate. LDH-1 isozyme is normally found in the heart muscle and LDH-2 is found predominantly in blood serum. A high LDH-1 level to LDH-2 suggest MI. But after wide availability of High sensitive troponin, usefulness of LDH is questionable specially due to its nonspecific nature.Myoglobin is less used again a non specific enzyme. Myoglobin is the primary oxygencarrying pigment of muscle tissue. It is high when muscle tissue is damaged.

Other less used biomarkers are hs-CRP, which is used to predict to determine risk of future heart attacks in people who have already have stablished CAD. NT-proBNP or $\mathrm{BNP}$ are useful in diagnosis and for prognosis of congestive cardiac failure.

\section{Plenary}

Improvement in the Quality Management System As Per Iso 15189 and Sustanance for the Continuation of Accreditation

\section{Thuppil Venkatesh}

Lead assessor NABL PAO DAC Nepal accreditation board AERSSC and international faculty lead assessor and Professor emeritus SJMC Bangalore Principal assessor NABH Principal Adviser QCI

Contact.venkatesh.huppil@gmIl.com
$\mathrm{M}$ edical testing laboratories comprising of majority of testing activities in Clinical Biochemistry which include clinical chemical pathology, immunology, serogy, molecular Biology, genetics and endocrinology across the globe is the major portion of the evidence based medical services when compared to anatomical pathology and clinical microbiology. Hence Clinical diagnostic laboratory services are highly advanced in the area of Clinical biochemistry all over the world. Any laboratory under the broad title pathological laboratory is truly a clinical chemistry laboratory. The quality management system (QMS) for attaining total quality in good laboratory practices is totally an objective process in Clinical chemistry section which depends on the state of art technology and a good quality control mechanism. However subjectivity is always a component and is noticed in anatomical pathology and clinical microbiology which has bias and high uncertainties.

QMS as management commitment and passion with appropriate documented policies for the procurement of high quality desources is the basic need as per ISO 15189 international standard. Other standards such as safety and POCT standards are also part of GLP. In addition speaker will be highlighting the importance of other seven resources and seven quality determinants during the talk. The importance of specific quality dequirementa of resources to be handled by competent persons is stressed during the discussion. Importance of documented procedures and maintenance of appropriatetechnicL and quality records will be highlighted to establish the evidence for QMS.

In order to sustain the accreditation status the improvement of quality and competence and the key lerformance indicators to minimiE risk to the end user will be discussed in detail. 


\section{IL- 1}

\section{My Journey with Human Cord Blood Stem Cells in Making Models for Developmental Neurotoxicity}

\author{
AB Pant \\ Developmental Toxicology Laboratory, System \\ Toxicology and Health Risk assessment Group \\ CSIR-Indian Institute of Toxicology Research \\ (CSIR-IITR), Vishvigyan Bhawan, \\ 31 MG Marg, Lucknow, \\ Uttar Pradesh-226001, India \\ Email:abpant@iitr.res.in; abpant@ rediffmail.com
}

$\mathrm{T}$ he developmental neurotoxicity (DNT) due to environmental chemicals, drugs and NCEs calls for effective therapeutic measures. Due to ethical constraints, elaborate experimental protocols and inter species differences associated with animal-based DNT models, the need for their appropriate substitute became necessary. Hence, we utilize the pluripotency potential of human umbilical cord blood stem cells (hCBSCs) to convert them into neural subtypes and subsequently as the tool to evaluate the DNT. Our studies provide deeper insights into the complex processes involved in neuronal development, injury and repair and demonstrate the mapping of cellular and sub-cellular events of differentiation of hCBSCs into morphological and functional neuronal cells. We discovered that how the master regulator signalling molecules/ cascades are critical to convert hCBSCs into functional neurons and what exactly happens when things go wrong during the intricate process of neuronal development. We also uncovered novel links between the xenobiotic metabolizing capabilities and their regulators in hCBSCs derived neuronal cells all through the differentiation. We further attempted to create functional three-dimensional (3D) neuronal niche organoid using cells combined with scaffolds that facilitate cell growth, organization and differentiation. The results are promising for the development of 3D network niche of proliferating and non-proliferating stem cells, progenitor cells, differentiating neuronal and fully differentiated cells in hydrogel scaffolds initiated by hanging drop cultures. The hydrogel scaffolds were found to be capable of affirming the culture and axonal outgrowth and migration of multilayered cells. The ultrastructural studies confirm the neurite outgrowth and synaptic conjunctions in 3D cultures. The data generated thus far not only proposes a much sought after framework for screening the newly developed drug candidate molecules for their safety assessment for developmental neurotoxicity, but as well to evaluate the neuroprotective potential in developing brain in a high throughput fashion.

KEYWORDS:Stem cells, developmental neurotoxicity, 3D neural organoid culture, Organophosphate pesticides

\section{IL- 2}

Identification and Functional Characterization of Novel Circrna and Mirna Molecules Employing C. Elegans Model: Implications for Age Associated Neurodegenerative Diseases

\section{Aamir Nazir}

Senior Scientist, Division of Toxicology and Experimental Medicine, CSIR-Central Drug Research Institute, Lucknow.

$\mathrm{T}^{\mathrm{h}}$ The exciting world of research with RNAs, has to its credit some breakthrough findings that havesignificantly affected multiple strategies of disease research and treatments. circRNAs are a class of noncoding RNAs, which are produced by scrambling of exons at the time of splicing. These molecules are known to act as miRNA sponges thereby modulating the repression function carried out by miRNAs; thus a single molecule affects hundreds of its target genes. Considering the multi-factorial nature of Parkinson's disease, we hypothesize presence of a commoncircRNA/microRNA trigger for the multiple manifestations of the ailment. We endeavored to study these interesting molecules employing transgenic C. elegans strain expressing 'human' alpha-synucleinalong-with YFP reporter gene. Using the approach of RNAseR-exonuclease treatment of total RNA, followed by amplification using divergent primers, we validated the presence of two very well expressing circRNAs in C. elegans. We further went on to validate the sequence of identified circRNA4 and carried out functional genomics studies with its 
synthesizing gene zip-2. Employing RNAi of zip-2 we studied the associated endpoints of alpha-synuclein aggregation in transgenic $\mathrm{C}$. elegans model followed by whole transcriptome analysis employing NextGen sequencing on IlluminaNextSeq 500 platform, which revealed a number of interesting targets that are affected vis-à-vis circRNA4 down-regulation. We further carried out global microRNA profiling of all known and novel microRNA molecules of C. elegans, using NextGen sequencing. Highly conserved microRNA molecule, let7 , known to regulate important functions of development and viability, was studied via creation of let-7 loss-offunction modeltowards studying the downstream effects on known associated molecules. Employing Timmons and Fire feeding vector L4440, we cloned let-7miRNA and quantifiedlet-7 levels. We further went on to study the whole transcriptome profile of PD model of C. elegans under let-7 silenced condition. Our studies identify circzip- 2 as an interesting target and reveal novel set of genes that are affected under let-7 silenced condition in the PD model.

\section{IL- 3}

\section{A Novel Histone Deacetylase Inhibitor as the Specific Posttranslational Modifier for Possible Epigenetic Therapeutics in Leukaemia}

Javeed Ahmad Bhat ${ }^{1}$, Neena Capalash ${ }^{2}$, Abid Hamid*

1. Cancer Pharmacology Division, CSIR-Indian Institute of Integrative Medicine, Jammu-180001, E-mail: ahdar@iiim.ac.in;

2. Department of Biotechnology, Panjab University, Chandigarh-160014, India

$\mathrm{T}_{\mathrm{s}}$ he balance in acetylation levels of histones is essential for cellular physiology and aberrations in these acetylation levelshave been observed in diseased states like cancer. During carcinogenesis, global hypoacetylation of histone $\mathrm{H} 4$ is evident and interestingly, loss of acetylation of histone $\mathrm{H} 4$ andhypoacetylation of histone $\mathrm{H} 3$ has emerged as a hallmark of malignant transformations. Increasing acetylation level of histones by histone deacetylase
(HDAC) inhibition promotes cell death, differentiation and growth arrest of cancer cells. In cancer cells, an imbalance exists between histone acetylase(HAT) and HDACactivities and current research focuses actively on seeking competitive HDAC inhibitors (HDACi) for chemotherapeutic intervention.During the last decade, four HDAC inhibitors have been approved by FDA as drugs against various cancers. However, in spite of clinical advantages like high specificity towards cancer cells and less drug resistance, the toxicity associated with HDACi is mainly due to their pan-HDAC inhibition activity and may be addressed by designing the class and isoform specific inhibitors. We rationally designed and synthesized a library of novel HDACi molecules and discovered one potential molecule targeting Class I HDAC's and evaluated its mechanism of action on chromatin state at the specific histone residues with characteristic modifications during leukemogenesis. From findings ofinvitro studies, we analysed the effect of this novel inhibitor on different in vivomurine models of leukemiaandour findings indicate that,this class I specific inhibitor have significant potency in checking leukemia.

\section{IL 4}

\section{Low Protein Diet And Vitamin D Supplements In Chronic Kidney Disease (Ckd) Patients}

\author{
Afrozul Haq \\ Professor\& Head, Department of Food Technology, \\ School of Interdisciplinary Sciences, Jamia Hamdard \\ (Hamdard University), New Delhi-110062, India. \\ Email: haq2000@gmail.com
}

fter the age of 40, kidney filtration begins to fall by
aging of the kidneys, many conditions such as damage
of the kidneys are more common in older people. These
patients may also suffer with diabetes, high blood
pressure and cardiovascular diseases CVD).Chronic
kidney disease (CKD) increases the risk of heart attack
and stroke, and in some cases can progress to kidney
failure requiring dialysis or transplantation. The
appearance of micro-albuminemia and/ or low eGFR is 
an indicator to start low protein diet to prevent complications and to improve the quality of life of CKD patients.

Patients with CKD have a high prevalence of both 25(OH)D and 1,25(OH)2D deficiency1. Nutritional vitamin D supplements can replete diminished 25(OH)D substrate in stages 1 to 5 of CKD2. A single monthly 50,000 IU ergocalciferol capsule safely repletes almost all vitamin D-deficient dialysis patients within 6 months3.The ability to convert 25(OH)D to its active form $1,25(\mathrm{OH}) 2 \mathrm{D}$ in the kidney is lost as renal function declines4,5. Active vitamin D therapy is given to correct $1,25(\mathrm{OH}) 2 \mathrm{D}$ deficiency in stages 3 to 5 of CKD2. Serum Para Thyroid Hormone (PTH) level decreaseswhileserum $1,25(\mathrm{OH}) 2 \mathrm{D}$ level increases, reduced proteinuria, improvingendothelial cardiovascular markersand decrease of inflammatory markers.

Low protein diet preserves nutritional status in patients with chronic renal failure. Protein restriction is indicated as soon as the eGFR becomes lower than $60 \mathrm{ml} / \mathrm{min} /$ $1.73 \mathrm{~m} 2$ body surface. The newly developed low-protein Indica rice is expected to help many CKD patients in China and Southeast Asia6. Our goal for hemodialysis patients should be a healthy lifestyle that can be maintained in the long run, rather than a short-term "diet".Use Vitamin D supplementation to CKD patients as they are generally deficient. Low serum $25(\mathrm{OH}) \mathrm{D}$ level is reported in approximately $90 \%$ of CKD and dialysis patients25(OH)D is the fuel for endocrine renal and cellular 1,25(OH)2D synthesis. Low vitamin D status leads to SHPT. 25(OH)D deficiency \& insufficiency are associated with progression of renal disease, morbidity and mortality in CKD and dialysis patients.

\section{IL- 5}

\section{Can We Predict Fracture Healing Outcome Early? -Evaluation of CYR61 A Noval Marker.}

\author{
Ajai Singh', Abbas Ali Mahdi ${ }^{2}$ \\ 1. Department of Orthopaedic Surgery and \\ 2. Biochemistry, King George's Medical University, \\ Lucknow, Uttar Pradesh, India.
}

INTRODUCTION: Angiogenesis is a prerequisite for fracture repair, whereas insufficient blood supply is likely to result in delayed/non-union. As CYR61 is the key regulator of angiogenesis process, we hypothesised that CYR61 might play a vital role in fracture healing.

METHODS: Total 107 adult patients with simple, fresh traumatic diaphyseal fractures of both bones of the leg managed conservatively were included in the study. For biochemical analysis, blood sample $(3 \mathrm{ml})$ was collected at 04 th, 07 th, 10th, 15th\& 20th days of post-fracture follow-up. CYR61 mRNA and protein expression were measured by qRT-PCR and western blotting assay respectively. Clinico-radiological follow-up was done at $06^{\text {th }}, 10^{\text {th }}, 16^{\text {th }}, 20^{\text {th }} \& 24^{\text {th }}$ of post-fracture weeks using RUST scoring to analyze fracture healing progression and their final outcomes, which were further correlated with the quantified CYR61 expressions.

RESULTS: As per clinico-radiological status at the 24th week, patients were divided into two groups: Group I (normal healing $\mathrm{n}=91$ ) and Group II (impaired healing $\mathrm{n}=16)$. The mean age of the patients were $32.08 \pm 9.33$ (85 Male/06 female) in group I and 33.2 \pm 9.03 (13 Male/ 3 female) in group-II, showed insignificant difference. Mean RUST score at $24^{\text {th }}$ week in Group-I $(11.08 \pm 0.86)$ and Group-II $(5.87 \pm 0.59)$ were statically significant. Expressions of CYR61 mRNA as well as protein gradually up-regulated and were higher at all follow up intervals in Group-I compared to Group-II. Moderate correlation was found between the CYR61 expression levels with the fracture healing progression.

CONCLUSION : CYR61 may predict the healing outcomes of simple diaphysealtibial fractures. 


\section{IL- 6}

\section{Regulation of Signalling Cascade by CD44 Variants Display Promising Therapeutic Potential In Urothelial Carcinoma of Bladder}

Alpana Sharma, Vivek Anand

Department of Biochemistry, All India Institute of Medical Sciences, New Delhi.

INTRODUCTION: Urothelial carcinoma of bladder (UBC) is the 9th most common cancer and 13th most common cause of cancer death worldwide. The prognosis of this malignancy is still debatable. Interactions between tumor cells and the extracellular matrix (ECM) are found to be associated with promotion of cell motility, adhesion, migration and metastasis. Hyaluronic acid (HA) is a nonsulfated glycosaminoglycan component of ECM that provides tissue homeostasis and maintains ECM architecture. HA is able to transmit signals originating from ECM into the cell, changes its metabolism, and linked to the promotion of cell migration and metastasis. HA mediated signalling pathway molecule includes, Hyaluronic acid synthase (HAS) which synthesizes HA, and CD44 cell surface HA-receptor that mediates downstream effect of HA. HA synthesis is inhibited by 4-Methylumbelliferone (4-MU). In UBC there is dearth of literature that shows HA and its downstream signalling effect comprehending CD44 variants.

MATERIALAND METHODS: Study cohort included 50 UBC patients with low and high grades of muscle invasive bladder cancer undergoing radical cystectomy and 50 healthy subjects for blood samples only. ELISA, Q-PCR, Immunohistochemistry and Immunoblotting were done for HA-family members. Variant analysis of CD44 was performed in patients and HT1376 cells. Effect of CD44 silencing on downstream signaling was observed. Combinatorial treatment of 4-MU (4Methylumbelliferone) with Cisplatin and Doxorubicin on chemosensitivity was also observed.

RESULTS: This study demonstrated higher expression of HA, HAS2, and CD44 in Indian UBC patients. ROC curve analysis of serum ELISA showed HA and CD44 as the potential diagnostic marker for UBC. Splice variant analysis of CD44 demonstrated that isoforms containing
CD44v3 and CD44v6 exons may have distinct role in bladder cancer progression. Downregulation of CD44v3 by shRNA has substantive effect on cell cycle and downstream signaling. Furthermore, we observed that 4-MU in combination with low dose of Cisplatin and Doxorubicin increases chemosensitivity of bladder cancer cells, promote their apoptosis, and also reduces expression various downstream signalling molecules.

CONCLUSION: Our study describes crucial role of these key molecules HA, HAS and CD44 in bladder cancer. Silencing CD44 expression reveals their vital role in intracellular signalling in bladder cancer progression. Higher expression of these molecules in UBC patients with cut-off value of higher sensitivity and specificity explains their importance as a potential biomarker. Also, we observed significance of 4-MU in enhancing the chemosensitivity, indicating their clinical and translational importance which can be exploited in designing better therapeutics for management of Urothelial carcinoma of bladder in future.

KEY WORDS: Urothelial carcinoma of bladder (UBC), Hyaluronic Acid (HA), CD44-Variant, 4Methylumbelliferone (4-MU).

\section{IL- 7}

\section{Respiratory Circadian Rhythm:Clinical Utility In Bronchial Asthma}

\section{Anand Srivastava}

\section{Department of Respiratory Medicine, King George's}

Medical University, UP, Lucknow

$\mathrm{T}$ here are more than 100 acute and chronic common and rare human diseases, conditions, and syndromes which are characterised by diurnal and $24 \mathrm{~h}$ patterns of circadian rhythm.Bronchial asthma, which is characterized by recurring symptoms, chronic inflammation of airway, reversible airflow limitation, and bronchial hyperresponsiveness, is on such condition. There is diurnal variability of either late evening/ overnight or early morning symptoms which significantly compromises nocturnal sleep, daytime productivity, and overall quality of life, and thus knowledge of variable 
clinical presentation is of great relevance to patient management. Lung function of asthmatic patients frequently worsens between midnight and early morning. Thus peak flow meter should be used as an objective tool in determining an individual patient's day to night changes in lung function. As we continue to learn more about circadian changes, better approaches to treating the disease with the same medications will emerge.

\section{IL- 8}

\section{Lean Management and its Challenges}

\section{Arun Raizada}

Senior Consultant and HOD, Biochemistry, Medanta-The Medicity, Gurgaon

BACKGROUND: Continual improvement as is an indispensable part of Clinical Lab's Quality Management Policy so introducing newer technologies and concept are evident. In this context to address improvement of service quality \& total Clini Lab process environment introducing Lean Managementas a powerful tool to eliminate waste i.e. non value added activity in the process is requirement of contemporary Lab Medicine in a tertiary care hospital.In a tertiary care hospital's clinical laboratory, lean enables lab to streamline their operations and deliver quality and timely result to the patients in challenging need.

METHODS: The concept of Lean 5S are sort, set in order, shine, standardize \& sustain which are used to eliminate waste, unnecessary inventory, operator motion, sample transport time etc. The lean concept was introduced with Total Laboratory Automation (TLA); analyzers were placed for chemistry \& immunoassay parameters in a manner to implement lean distance travelled by operators, technologists. Secondly manual sample ID verification steps were removed to work faster on the lean time because these were done manually by technologist before the samples were registered \& centrifuged at the sample receiving area. With the help of lean implementation urgent samples were sorted out easily into urgent racks and in turn improve urgent samples TAT.
RESULTS: Implementation of lean exhales shortening the distance travelled by operators, technologists on a mark of lean distance. Now 162 feet traveled instead of 1162 feet travelled earlier lean model incorporation. Benefits for the elimination of verification steps were to save time approximately $5 \mathrm{hrs}$ per technologist. Urgent samples TAT were improved upto $72 \%$, prior it was only $50 \%$.

CONCLUSION: Increasing efficiency and saving time with these successfully applied aspects it requires staff training and motivation to implement lean principles which is still a challenge and development area. Another development area is to implement lean from the sample collection department to transport and storage of samples after processing and may be done with coming time. With these challenges lean is a powerful tool for identifying and eliminating non value added activities, increasing productivity \& speed of the process.

\section{IL- 9}

\section{Novel Therapeutic Statergies to Treat Temporal Lobe Epilepsy-Induced Cognitive Deficits}

\section{BS Shankaranarayana Rao}

Department of Neurophysiology, National Institute of Mental Health and Neuro Sciences (NIMHANS) Bengaluru - 560 029, India. bssrao.nimhans@gmail.com

$\mathrm{T}$ emporal lobe epilepsy (TLE) is a chronic neurological disorder constituting about $60 \%$ of adult epilepsies and most often refractory to medication. Spontaneous recurrent seizures (SRS) are the hallmark of TLE and are often associated with neurodegeneration and cognitive impairments. The mechanisms of generation of SRS, epileptogenesis and associated cognitive deficits are yet to be understood. Accordingly, we have used an animal model of TLE to assess the seizure characteristics, neurodegeneration, spatial learning, memory and depressive-like behaviour. We have also evaluated the effect of levetiracetam (LEV) treatment, exposure to enriched environment (EE) and the combination of EE and LEV for 14 days on TLE- 
induced deficits. Epileptic rats were video-monitored using phenotyper to behaviourally characterise seizure frequency and duration followed by analysis of EEG and field potentials from the hippocampus, somatosensory and parietal cortices. TLE was associated with increased SRS, altered EEG coherence, hippocampal neurodegeneration, gliosis and cognitive deficits. Interestingly, EE, LEV and combination therapy reduced SRS in TLE rats. Interestingly, the combination therapy prevented the anhedonia, spatial learning and memory deficits in TLE. Taken together, the study demonstrates that TLE causes behavioural alterations, cognitive deficits and aberrant brain activity in addition to the progressive increase of seizures. EE and LEV interventions independently ameliorated most of the alterations induced by TLE, whereas combination therapy of EE and LEV was more successful in complete restoration. Inclusion of positive stimuli of EE along with the pharmacological treatment would help in potentiating the effect of AED and curbing the side-effects produced by chronic antiepileptic therapy. Hence, treatment strategies could be designed with non-pharmacological interventions that utilize components of EE combined with AED. The results of present study might help to develop newer strategies by combining pharmacological and non-pharmacological approaches to treat drug resistant epilepsies and associated comorbid conditions including depression and spatial learning deficits. Our findings might help to better understand the neural basis of brain damage and susceptibility to various neurological and psychiatric disorders.

KEY WORDS: Temporal lobe epilepsy, neurodegeneration, gliosis, spatial learning and memory deficits, depressive-like behaviour, seizure episodes, levetiracetam, enriched environment, epileptogenesis, spontaneous recurrent seizures, local field potentials and electrocorticography.

ACKNOWLEDGEMENTS: Acknowledge the financial support from the CSIR, DST, DBT, SERB and NIMHANS.

\section{IL- 10}

\section{Digitoxigenin Augments 2-Dg Induced Toxicity In Cancer Cells By Targeting Hypoxia-Induced Factor 1- $\alpha$-Mediated Signaling.}

Akanchha Shukla,Garima Dhawan, Romila Singh, JyotikaRajawat, Manohar Singh, Nidhi Shukla, Arun Kumar Jajoriya, Divya Mishra and D.P Mishra*

\section{Cell Death Research Laboratory, Endocrinology \\ Division, CSIR-Central Drug Research Institute, Lucknow, INDIA \\ * Corresponding author email: dpm@cdri.res.in}

A ccelerated glycolysis ensures ATP levels meet the demands of highly proliferating cancer cells. Cancer cells significantly differ from the normal cells in producing as much as $50 \%$ of the cellular ATP by anaerobic glycolysis. This differential metabolic profile is recently perceived as a target of novel therapeutic strategies. However, current therapeutic agents targeting glycolytic enzymes lack specificity, have off target effects and limited clinical potential. The glycolytic inhibitor, 2-Deoxy Glucose (2-DG) has been used as a therapeutic agent targeting hexokinase2 (HK2) enzyme in cancer. However, 2-DG treatment is known to induce prosurvival Akt /HIF1 $\alpha$ signaling. Enhanced therapeutic efficacy has been observed with the combination of phosphatidylinositol-3-kinase/Akt inhibitors in 2-DGbased chemotherapy/radiotherapy. Cardiac glycosides induce potent anticancer activities by modulation of $\mathrm{Src}$ kinase signaling, induction of TRAIL, TNF $\alpha, \mathrm{NF} \alpha \mathrm{B}$ induced cell death, Topoisomerase II and HIF1 $\alpha$ inhibition. Therefore, we studied the effects of a cardiac glycoside Digitoxigenin (DGX) in an effort to counter 2 -DG induced pro-survival HIF $1 \alpha$ signaling in Glioblastoma cells. Digitoxigenin enhanced cytotoxicity of 2-DG in a dose dependent manner showing synergistic effects. Combined treatment with subtoxic doses of 2DG $(1 \mathrm{mM})$ and DGX $(0.5 \mu \mathrm{M})$ significantly reduced colony formation, invasion and migration in U87MG and LN229 glioblastoma cells. Protein profiling studies showed significant reduction of 2-DG induced HIF1 $\alpha$, HK2 and other pro-survival protein expression. Thus, these results collectively suggest that the combined 
treatment of 2-DG and DGX inhibits proliferation, invasion and migration by targeting pro-survival HIF1? signaling in glioblastoma cells. In conclusion, this study revealed that in glioblastoma cells digitoxigenin augments 2-DG induced toxicity by targeting HIF1 $\alpha$.

\section{IL- 11}

\section{Point-of-Care Diagnostics: Current State-of- Affairs, Limitations And Future}

D Asthana ${ }^{1}$, R. Valiathan ${ }^{1}$, L. Friedman ${ }^{1}$ and

M. Rathore ${ }^{2}$

\section{University of Miami - Miller School of Medicine, Miami, \\ 2. University of Florida, Jacksonville, Florida, USA}

$\mathrm{P}$ oint-of-care testing is becoming a crucial component of the healthcare delivery both in and out of clinical settings. Minute Clinics, Urgent Care or Rapid care centers are now a common site in many pharmacies and shopping malls. Online and mobile diagnostic applications are allowing the patient/consumer to take their health into their own hands. Presenter will examine point of care testing; current state of affairs, how it's being used in the minute clinics, in pharmacies, and by the consumer. In addition, discussion will cover limitations of current point-of-care tests, devices and barriers to implementations for wider utilization. Presentation will also address technological advances and future of the field in general.

\section{IL 12}

\section{Fusing Elements of other Teaching Strategies for Lecturing Beyond Conventions and to Foster Student's Creativity}

\section{Dinesh Puri}

University College of Medical Sciences, New Delhi

$\mathrm{W}^{\mathrm{c}}$ e have arrived an era wherein accelerated information input and expanding knowledge base has created an avalanche of new information. The students are digital-natives, who have grown up with technology, intimately familiar with and accustomed to finding information online. In this era more creative thinking, less rote memorization of facts and, more importantly, a little enjoyment during teaching-learning sessions is likely to succeed more. The current education system however, creates several obstacles to meaningful learning and creation of open space for creative expression. Preparing students for exams (87.3\%), vast syllabus $(86.4 \%)$, inadequate teaching staff and other teaching-resources $(80.3 \%)$ and conflicting curriculum demands $(76.4 \%)$ are major hindrance to scope for nurturing creativity. Divergent thinking forms important part of creativity and problem solving, which are effectively squander in these pressure situations to which our students are persistently exposed.

This lends credence to statement of Sir Ken Robinson, an international advisor on education "All students have tremendous talents but we squander them ruthlessly". , and the question is what can be done in the current situation.

Teaching in Medical colleges continues to be didactic, lecture based and teacher-centric, which is not highly rated by medical educationists. Most important aspect of lecturing is stimulation of student to become active learner.This talk will focus on various factors that "Rob" the lecturer of chance of oratory, and the student of little enjoyment. A flipped classroom approach, fusing elements of various innovative methods that emphasize avoidance of zealotry with respect to pedagogical approach. Merits of deviation from strict adherence to specific criteria and traditional lecturing will be discussed. Emphasis will be on basic tenet of constuctivism that students are active learners and must construct knowledge for themselves. Role of teacher is to facilitate learners in coherent way of thinking as a procedural guide. Role of student-interaction, dramatization, role play and humor will be highlighted, based on research but more importantly on personal experience of the author. 


\section{IL-13}

\section{Introduction to Competency Based Education}

\section{Farzana Mahdi}

Professor, Department of Medical Education, Era's Lucknow Medical College, Lucknow, Era University, Lucknow

A competency is an observable ability of a health professional, integrating multiple components such as knowledge, skills, values and attitudes.

A set goal can be achieved by constructing competencies which would have specific learning objectives.

Competencies should be constructed in all the three domains of knowledge, skills and attitude as well as at different levels of Miller's pyramid.

Competency based curricula helps students achieve their goal. These are the details that shall be discussed in the symposium on medical education.

\section{IL- 14}

Deciphering The Role of COQ10 Deficiency In Fibromyalgia Syndrome: Implications on the Amelioration of its Symptoms by Ubiquinol-10 Supplementation

Farzana Mahdi, Ghizal Fatima

Era's Medical College and Hospital, Lucknow, India

Zibromyalgia Syndrome (FMS) is now recognized as a major health problem. The pathophysiologic mechanisms of FMS are difficult to identify and current drug therapies have demonstrated limited effectiveness. Recently a study reported coenzyme Q10 (CoQ10) deficiency, mitochondrial dysfunction, and oxidative stress from patients with FMS. We therefore plan out this first study on supplementation of Ubiquinol-10 in Indian patients with FMS. For this we measured plasma levels of CoQ10 in patients of FMS and in healthy control subjects $(n=20)$, suggesting the presence of decreased levels of antioxidant in this disease. However, plasma level of CoQ10 was significantly decreased in FMS relative to healthy controls, suggesting that FMS is associated with coenzyme Q10 deficiency and increased oxidative stress. Moreover, plasma level of oxidative stress parameters was significantly higher in FMS patients than in controls, suggesting increased tissue oxidative damage in FMS. Moreover, to compensate for the loss of quality of life (QOL),next, we examined the effect of ubiquinol-10 supplementation (200 mg/day for 30days) in FMS patients. This resulted in an increase in coenzyme Q10 levels and a decrease in oxidative stress parameters. Suggesting that ubiquinol-10 supplementation improved the QOL and symptoms severity among FMS patients.

KEYWORDS:Fibromyalgia Syndrome, CoQ10, ubiquinol-10

\section{IL-15}

\section{Interpretation of Iron Status}

\section{Helen Martin}

SA Pathology

$I^{r}$ ron is an essential nutrient for almost all living cells, however too little or too much iron can cause significant health problems. Iron deficiency, caused mainly by dietary deficiency or blood loss is common world-wide and the World Health Organisation estimates that more than a billion people, including $40-50 \%$ of all children are iron deficient. Iron overload is also common although the causes are more varied. Iron status is assessed by a combination of biochemical and haematological markers that traditionally include iron, transferrin, transferrin saturation, ferritin, haemoglobin, mean corpuscular volume and mean corpuscular haemoglobin. While some patterns of these tests, such as those in classical iron deficiency or primary iron overload of long standing, are easy to interpret, many are difficult. This is particularly true when iron deficiency co-exists with significant inflammatory illness; in such settings additional tests such as soluble transferrin receptor or hepcidin, markers of inflammation such as C-reactive protein or erythrocyte sedimentation rate, or 
haemoglobin response to a clinical trial of iron therapy may be helpful.

\section{IL- 16}

Changing Paradigms of Chemotherapy: Utilizing Metabolically Elevated Copper For Selective Non Enzymatic Dna Damage and Consequent Cell Death.

Imrana Naseem, Asim Rizvi, Sandesh Chibber, Atif Zafar, Saman Khan

Department of Biochemistry, Faculty of Life Sciences, The Aligarh Muslim University, Aligarh.

Malignancies are characterised by a systemic metabolic elevation of copper, both in the serum and within the malignant tissue. The cause of this copper elevation is not known, however cellular copper elevation is one of the several metabolic characteristics which distinguish normal cells from malignant cells. Some research groups have implicated copper elevation as a cause /effect phenomenon for anigiogenesis and metastasis. Interestingly, copper elevation has been shown for almost all malignancies, irrespective of the tissue of origin.

Our laboratory for the past several years has been demonstrating that metabolic copper elevation can be utilised for inducing selective cell death in cancer cells, by a Fenton like, non enzymatic, reactive oxygen species mediated, DNA damaging reaction, which in turn causes selective cell death while sparing normal cells.

The talk will explore this idea theoretically, while building a rational experimental framework to explore this reaction. The talk will include our work, ranging from demonstration of this reaction in vitro, for standard chemotherapeutic drugs, methotrexate and 5 F Uracil. An alternative but similar reaction shown by Vitamin D, and its demonstration in vitro, in vivo, in silico and ex vivo followed by animal studies on DEN induced hepatocellular carcinoma. More recent studies on the plant derived molecule coumesterol and its reaction with copper will be explored, along with its demonstration in DMBA induced breast carcinogenesis, and our preliminary attempts at synthesising coumarin based, selective copper chelators will be discussed.
IL-17

Antimicrobial peptides: Role of defensins as antimycobacterial agents against active and latent bacilli.

Richa Sharma1 ${ }^{1}$ Uma Nahar Saikia ${ }^{2}$, Sadhna Sharma1, Indu Verma ${ }^{1}$

1. Department of Biochemistry, Post Graduate Institute of Medical Education and Research, Chandigarh 160012, India,

2. Department of Histopathology, Post Graduate Institute of Medical Education and Research, Chandigarh 160012, India.

$\mathrm{O}$ ne third latently infected population together with emergence of multidrug and extensively drug resistant Mycobacterium tuberculosis (M. tb) point towards the urgent need for alternative avenues besides the current chemotherapy. Human antimicrobial peptides (AMPs), the first line of defense against invading microbes, acting either by direct antimicrobial activity or by stimulating the immune system, can be promising targets against TB. In the present work, the potentially active human AMPs and their biologically active motifs were screened out and identified by in silico approaches. On the basis of in silico analysis, HBD-1, having highest antimicrobial score by using AntiBp2, CAMP and AMPA web servers, was selected for further analysis. Scanning of HBD-1 by in silico tools led to identification of Pep B (ACPIFTKIQGTCYRG) as active motif having antimicrobial and immunomodulatory potential respectively. Another defensin, HNP-1, which has already been reported as a potential antimycobacterial agent from our lab was also screened for its antimicrobial motif that resulted into identification of a sequence Pep H (RRYGTCIYQGRLWAF). Activity of HBD-1,Pep-B and Pep-H was determined against actively growing $\mathrm{M}$. tb in vitro, inside monocyte derived macrophages (MDMs) and dormant bacilli in in vitro potassium deficiency and human PBMC granuloma models using colony forming unit enumeration. HBD-1 ,Pep B and Pep $\mathrm{H}$ not only displayed bactericidal activity against in vitro growing $M$. tb but also inhibited intracellular mycobacterial growth at concentrations lower than in vitro MICs asindicated by significant $(\mathrm{p}<0.001)$ reduction 
(more than $1 \mathrm{log}$ ) in bacillary load in infected MDMs as compared to control infected cells. Also combinations of (HNP-1 + HBD-1) and (Pep H + Pep B) showed significant $(p<0.001)$ activity in restricting the mycobacterial growth in MDMs at $1 / 4$ th and $1 / 8$ th of their individual effective concentrations. Further, antimicrobial activity of AMPs and their motifs against intracellular bacilli was supported by significant increase $(\mathrm{p}<0.001)$ in IFN ? and NO release by infected MDMs. Although at higher concentrations, HBD-1, Pep-B, HNP1 and Pep $\mathrm{H}$ led to decrease in dormant mycobacterial load both in vitro as well as inside human granuloma model.The antimycobacterial activity of both AMPs and their motifs was also significantly higher $(\mathrm{p}<0.01$; $\mathrm{p}<0.001$ ) than rifampicin and isoniazid against latent TB models. Thus, the present study indicates that human AMPs and their motifs are effective antimicrobial players against both actively growing and dormant mycobacteria.

\section{IL- 18}

\section{High Sensitivity Troponin I: What We Should Know?}

\section{Jaganathan Sickan}

Associate Medical Director, Medical \& Scientific Affairs, Abbott Diagnostics

$\mathrm{C}$ ardiac troponins are the preferred biomarkers for diagnosing Acute Myocardial Infarction(AMI). In patients with Acute Coronary Syndrome, increased levels of cTns are associated with increased risk of death and re-admissions, and identify patients who benefit from early, intensified treatment. In patients with stable Coronary Artery Disease(CAD), cTn measured by a high sensitive assay is detectable in the large majority of patients. Interestingly, even levels below the 99th percentile of a healthy reference population provide prognostic information, and predict risk of mortality and development of Heart Failure, and in AMI.

It is important to understand that the term "high sensitivity "reflects the assay's characteristics and does notrefer to a difference in the form of cardiac troponin being measured. Several names have been used in the literature forthese assays, including "high-performance," "highly sensitive," "high-sensitive," "ultrasensitive," "novelhighly sensitive," "sensitive," and "high sensitivity." The term "high-sensitivity" be uniformlyused for publication in Clinical Chemistry and throughout the scientific literature. This term, however, begs the question.

How does one define a High sense assay? In a scorecard concept, an assay was proposed to be hs if it met 2 basic criteria.

1 First, the total imprecision (CV) at the 99th percentile value should be $<10 \%$.

1 Second, measurable concentrations below the 99th percentile should be attainable with an assay at a concentration value above the assay's limit of detection for at least 50\% (and ideally_95\%) of healthy individuals to attain the highest level of scorecard designation.

The Major inequalities exist between men and women in the treatment and outcome of acute coronary syndromes, with an increase in both early and late deaths in women. Importantly, women with suspected acute coronary syndrome are less likely to have a diagnosis of myocardial infarction, an observation previously attributed to atypical symptoms and less reliable findings on electrocardiography. The use of high sensitivity troponin I assay hasidentified potentially important differences between the sexes, with the 99th centile (upper reference limit) being twofold higher in men than in women.A recent study done by A shah et al published

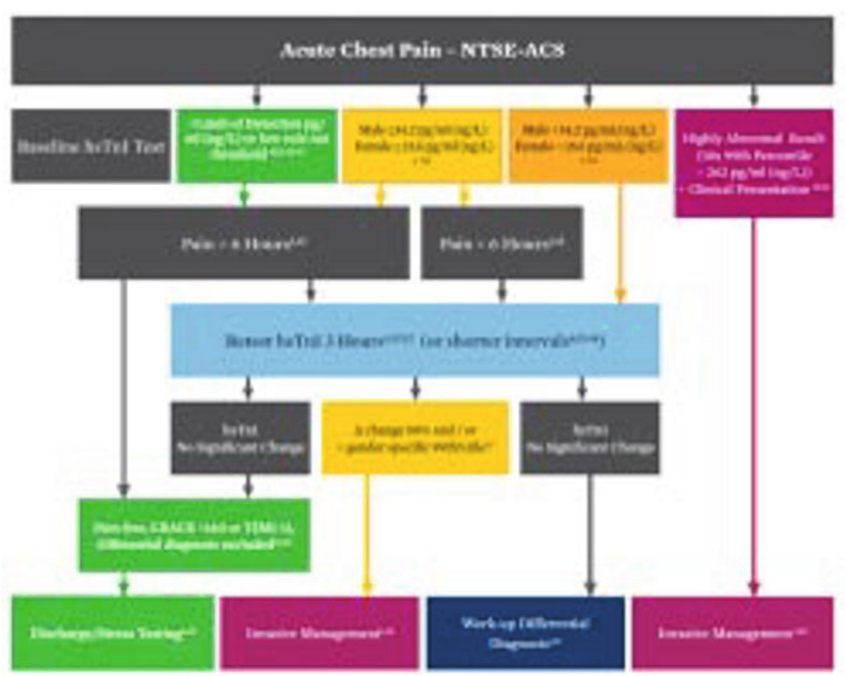


in BMJ, improves the diagnosis of ACS in women, when high sensitive troponin I (hsTnI) was used compared to the contemporary troponin.

Use of high sensitive troponin assays improve the diagnosis of myocardial infarction, and use of these assays has been associated with reductions in recurrent myocardial infarction and death.

One of the major advances of high sensitivity assays over contemporary assays is that troponin concentrations can be quantified in healthiest people.

The Abbott ARCHITECT STAT High Sensitive Troponin-I (hsTnI) algorithm has beendeveloped using previous recommendations and clinical research data relating to performance. One must keep in mind that there should always be clinical assessment of any suspected AcuteCoronary Syndrome (ACS) patient as well as pretest probability determination. Additionally, thisalgorithm is designed to provide a template for guidance and should be evaluated and adjustedfor your site as appropriate. The performance criteria for the different cutoffs in the algorithmare depicted below:

1 LoD (Limit of Detection) - $100 \%$ NPV(negative predictive value).

1 99th percentile or URL (upper referencelimit) suspected ACS patients may fall belowthese cutoffs as early presenters or slow risingtroponin concentrations.

l $50 \%$ delta - $94 \%$ PPV (positive predictivevalue).

$110 \times 99$ th percentile (abnormally hightroponin value $262 \mathrm{pg} / \mathrm{mL}$ ) at presentation andclinical presentation (ST-segment depression, deep T wave inversions without $\mathrm{Q}$ waves,continued chest pain, hemodynamic instability,etc.) may be ruled in as appropriate with $98 \%$ specificity.

\section{IL-19}

\section{Medical Error and Disclosure in Quality Care and Patient Safety- : An Overview}

\section{Jawahar (Jay) Kalra}

Department of Pathology and Laboratory Medicine, University of Saskatchewan and Royal University Hospital, Saskatoon, Saskatchewan, Canada.

$\mathrm{A}$ dverse events are inevitable due to the complexity of the healthcare process. Medical errors have gained significance due to their increased prevalence which is detrimental to the safety of patients.Failure to inform the patient of adverse events caused by a medical error compromises the autonomy of the patient. It also jeopardizes the opportunity to improve the quality in health care. Disclosure of an adverse event is an important element in managing the consequences of a medical error. Physicians should seek to disclose medical errors to patients and their families on both ethical and pragmatic grounds. By following an open disclosure policy, patient's autonomy can be preserved and malpractice claims can be reduced effectively. The complexities of medical error disclosure to patients present ideal opportunities for medical educators to probe how learners are balancing the ethical complexities involved in error disclosure. The designing of an error disclosure policy requires integration of various aspects including bioethics, physician-patient communication, quality of care, and team-based care delivery. There are many policies and procedures proposed by the national and international regulators to have consistent and standard medical error disclosure and reporting policies. The majority of provinces in Canada have adopted some form of a disclosure policy while others are in the process of developing such policies. These Canadian provincial initiatives, though similar in content, remain isolated because of their non-mandatory nature and absence of federal or provincial laws on disclosure. We suggest a uniform policy centered on addressing errors in a nonpunitive manner and respecting the patient's right to an honest disclosure should be a standard of care.

KEYWORDS: Adverse events, Medical Error, patient Safety, medical error disclosure 


\section{IL- 20}

\section{LEAD As Poison - It's Action Mechanism}

Krishan Kumar Sharma ${ }^{1 *}$, KshemaThakur $^{1}$, Chaffy Sachdeva ${ }^{1}$

1. Department of Biochemistry, Dr. Yashwant Singh Parmar Government Medical College, Nahan-173001, Distt. Sirmaur, Himachal Pradesh-India.

*Corresponding and presenting author: Department of Biochemistry, Dr. Yashwant Singh Parmar Government Medical College, Nahan-173001, Distt. Sirmaur, Himachal Pradesh-India.

Email:drkksharma8@gmail.com

Mobile: 94189-27288.

$\mathrm{L}^{\mathrm{e}}$ ead is widely distributed in the environment. Also its wide industrial and household applicability is dependent on itsphysico-chemical properties, low cost and a visibly easy workability.Lead from various sources such as food, water, soil and airfinds its way to the inside of the human body. Besides this, adults also face the occupational exposure to lead which also accounts to the 'take home lead' with their clothes.It was documented that in case of adults, $20-70 \%$ of the ingested lead enters the blood while almost $100 \%$ of the inhaled lead finds its way into the bloodstream.Because it is nonbiodegradable and has a slow rate of elimination, it tends to accumulate in body and this chronic or acute accumulation of even small quantities of lead leads to the condition termed as lead poisoning (plumbism). Plumbism is a hazardous occupational and environmental disease that affects millions of adults and children worldwide.

Lead presents its toxicity by two ways: inorganic lead and organic lead. Inorganic lead is defined as lead oxides, metallic lead, and lead salts. The organic lead compounds include the tetra-alkyl lead compounds mainly tetraethyl lead and tetramethyl lead which have been incorporated into gasoline as anti-knock additives.Based on the BLL, the different effects of lead on individuals have been observed. Despite the interesting fact that no normal physiological role for lead could be found, lead toxicology causes numerous serious pathophysiological manifestations in the patients.Oxidative stress understandably the major mechanism of lead poisoning is caused when generation of free radicals is more than ability of the system to readily detoxify the body from them, as their accumulation results in cellular damage.Lead exposure leads to hematological changes that result due to the poisoning of three enzymes of heme biosynthesis pathway: ?-aminolevulinic acid dehydratase(ALAD), ferrochelatase, and ?aminolevulinic acid synthetase. Also, RBCs become more fragile as their membrane is damaged because lead disrupts the maintenance of the cell membrane.Most sensitive organ to lead exposure is brain. Neurons may be directly or indirectly harmed byaccumulation of heme precursors, such as ALA, due to influence of lead on ALAD. Lead also hinders the development of neurotransmitters and organisation of ion channels.Bones are the primary site for the deposition of lead in our body. Lead is thought to be deposited in two segments in the bones. Cortex, the site for deposition of the nonexchangeable pool is present deep in the bone and the exchangeable pool is found at the bone surface. Lead from exchangeable pool is actively re-absorbed and may get easy access to plasma. After this mobilization, lead from the non-exchangeable pool is shifted to the surface.In women, lead toxicity leads to miscarriage, pregnancy hypertension, infertility, premature membrane rupture, premature delivery and pre-eclampsia. Increased BLL are associated with delayed puberty in girls.Lead interaction with human chorionic gonadotropin (HCG) indicated that lead acetate changed the secondary structure of HCG by loosening and destruction of the HCG skeleton and by increasing the hydrophobicity around Tyr residues, which results in decreased bioactivities of HCG, suggesting a direct interactions of lead with sex hormones and suggesting a possible mechanism of lead induced reproductive toxicity at molecular level. Metabolic changes during pregnancy mobilise the lead from bones into the blood, thus increasing the toxic effects of lead.Lead toxicity leads to histopathological changes in the renal proximal tubular epithelium and results in interstitial nephritis, which is usually related to high blood pressure.Lead is projected to alter the permeability of blood vessels and collagen synthesis.In order to justify the carcinogen status of organic lead compounds, it must be noted that these compounds are metabolized in the body into a number 
of metabolites including inorganic lead (Group 2A carcinogen) prior to excretion in the urine.ALAD gene polymorphismsare known to influence the BLL and thus directly affect the susceptibility of individualstowards lead poisoning. Lead stronglyinhibits ALAD enzyme stoichiometrically and at the molecular level, displacing a zinc ion at the metal binding siteproduces inhibition through a change in the enzyme's quaternarystructure.

A foresight by Ronald Lane mentionedthat 'There isonly one way to prevent lead poisoning and one way only and that is to make the process safe.The importance of education of the workerhimself must be stressed.'Its treatments though are available but prevention remains most important as the negative neurotoxicological effects manifested in the growing children, pregnant women and foetus cannot be reversed. We need to cover a long distance in order to make the environment safe for our health and for the coming generations.

\section{IL- 21}

\section{Nutritional Modulation of Gene Expression and Noncommunicable Diseases}

\section{Kailash Bhattacharyya}

Professor and Head, Department of Biochemistry, Gauhati Medical College and Hospital, Guwahati- 781032

$\mathrm{N}^{\text {on }}$ on-communicable diseases like Coronary artery disease (CAD), Hypertension, Diabetes Mellitus and Cancer can develop because of interaction of genetic and environmental factors, particularly when countries become more affluent because of changes in life style including decreased physical activity and dietary transition. All the factors related with changes in lifestyle modulate gene expression. Diet and lifestyle factors are closely related to heritability of the variant phenotypes. These are dependent on various nutrients and environmental factors for their expression.

Studies have revealed that specific nutrients interact with the genetic codes possessed by all nucleated cells. Diet and lifestyle also influence epigenetic changes. These epigenetic changes result in changes of the gene expression through transcription and translation. An interaction between the nutrients and nuclear receptors stimulate signaling pathway which results in modulation of epigenetic changes and gene expression.

Thus it has been established that an increased susceptibility to Coronary artery diseases, Diabetes, Central Obesity, Hyperinsulinemia and Lipoprotein(a) excess in young age group of Indians show a genetic predisposition to these problems because of gene and environment.

Antioxidant vitamins, Coenzyme Q10, n-3 fatty acids have a beneficial influence on phenotype expression. Saturated fat and Sugars may have hazardous effects on phenotype expression. Epigenetic modifications induced by diet are associated with every step of growth and development and thereby it is related to noncommunicable diseases like tumorogenesis, CAD, Diabetes and Hypertension etc. Diet induced epigenetic modification is an effect which manifests itself through gene expression.

KEYWORDS: Dietary Transition, Epigenetic Changes, Gene Expression, Lipoprotein (a)

\section{IL- 22}

\section{Generation and Characterization of Anti-Hiv-1 Chuman Recombinant Monoclonals}

\section{Kalpana Luthra, Professor}

Department of Biochemistry, AIIMS, New Delhi

$\mathrm{H}^{\mathrm{v}-1}$ IV-1 subtype $\mathrm{C}$ is responsible for more than $50 \%$ of the infections globally and more than $90 \%$ infections in India. Till date, there is no effective vaccine against HIV-1. Recent animal studies and human Phase I trials showed promising results of the protective effect of anti-HIV-1 broadly neutralizing antibodies (bnAbs). Majority of the bnAbs targeting HIV-1 have been isolated from non-subtype $\mathrm{C}$ infected donors. Recombinant antibody technology enables generation of a large repertoire of monoclonals with diverse specificities. Mapping the epitope specificities of bnAbs provides useful information for vaccine design. Interaction 
between CD4 binding-site (CD4bs) on the HIV-1 envelope glycoprotein and CD4 receptor on the host immune cells is the primary event leading to HIV-1 infection. The CD4bs is a highly conserved region, comprised of a conformational epitope and is a potential target of bnAbs such as VRC01 that is presently under human clinical trials. Recombinant single chain antibody fragments (scFvs) can access masked epitopes due to their small size and have shown the potential to inhibit viral replication and neutralize a broad range of viruses. We characterized plasma samples of antiretroviral naïve HIV-1 infected donors for the presence of bnAbs. Pooled peripheral blood mononuclear cells (PBMCs) from select donors whose plasma antibodies exhibited cross neutralizing activity were used to construct a phage recombinant single chain variable fragment $(\mathrm{scFv})$ library with high diversity. The library was panned and screened by phage ELISA usingtrimeric recombinant proteins to identify viral envelope specific clones. Three scFvmonoclonals D11,C11 and 1F6 selected from the library cross neutralized subtypes A, B and C viruses at concentrationsranging from $0.09 \alpha \mathrm{g} / \mathrm{mL}$ to $100 \alpha \mathrm{g} / \mathrm{mL}$. The D11 and 1F6 scFvs competed with mAbs b12 and VRC01 demonstrating CD4bs specificity, while C11 demonstrated N332 specificity. This is the first study toidentify cross neutralizing scFvmonoclonals with CD4bs and N332 glycan specificities from India.Cross neutralizing anti-HIV-1 human scFvmonoclonals can be potential candidates for passiveimmunotherapy and for guiding immunogen design.

\section{IL- 23}

\section{Inborn Errors of Metabolism; Five-year experience in a tertiary care hospital}

\section{Dr. D.M. Vasudevan, MD, FRCPath, FACBI}

Amrita Institute of Medical Sciences, Elamakkara P.O., Cochin, Kerala

$\mathrm{M}$ ore than 500 inborn errors of metabolism (IEM) are described. While individually rare, the incidence for inborn errors of metabolism, collectively, is about 1 in 1,000 newborns. India with 28 million births every year; the magnitude of the problem is very high.
Undetected cases of metabolic disorders lead to permanent mental retardation. About $1 / 3$ rd of pediatric mental retardation cases may be attributed to the inability to detect a metabolic disorder in early childhood.

Metabolic pathophysiology of IEM includes: accumulation of substrate or precursors, redirection of substrate into alternative pathways, deficiency of products or subsequent products, and secondary effects by any of the above onunrelated pathways. Inborn errors of metabolism includes any of the biomolecule metabolism defect, such as carbohydrate, amino acid, lipid, trace metal disorders, peroxisomal Disorders and lysosomal storage disorders.

Poor feeding, vomiting, diarrhoea, dehydration, temperature instability, reduced heart beat, involuntary movements, irritability, seizures, abnormal muscular tone, skin rashes, loss of or no growth of hair, developmental delay, altered facial features are common symptoms of IMDs. In children of school going age loss of hearing, learning disorders, aggressiveness, seizures and mental retardation are the symptoms.

New born screening programs have started in 1953 for PKU. In India, no comprehensive data is available. Hospital based mass newborn screening program has been introduced only very few hospitals in India. It should be a mandatory screening of all newborns. Therefore, clinicians, parents, hospital staff, general public are need awareness of the need for screening program.

Cheap, Easy to do, Reliable and quick screening tests are offered to general population of patients as well as healthy subjects. Screening of all newborn babies can be done in all small labs. The screening test will define "at risk" population, but do not give definitive answer. If suspicious, send samples to tertiary care laboratories. Advanced techniques including thin layer chromatography, HPLC, GC/MS, and Tandem mass spectrometry - are extremely sensitive and wiill give a final diagnosis. Further definite diagnosis is done either by DNA probe analysis or by specific enzyme assays.

During a 5-year period, we have analaysed a total of more than 10,000 samples. The most commonly detected inborn errors, in lowering frequency are: congenital adrenal hyperplasia, hyperhomocystenemia, organic acidurias, pheochromocytomas, porphyrias, maple syrup 
urine disease, maple syrup urine disease. An algorithm has been developed for IEM screening. A rapid screening test kit, which can screen 35 most common IEM has been developed.

It should be remembered that Inborn errors are rare; but if undetected, mental retardation is the consequence.

\section{IL- 24}

\section{Fatty Acid Synthase: A New Therapeutic Target For Pulmonary Hypertension}

\section{Kashif Hanif}

\section{Division of Pharmacology, CSIR-Central Drug Research Institute}

$\mathrm{P}$ ulmonary hypertension (PH), a disorder prevalent in chronic obstructive pulmonary diseases patient and in army personnel and natives at high altitude, is caused by severepulmonary vasoconstriction resulting in right ventricle (RV) hypertrophy which leads to heart failure. We have found that inhibition of Fatty acid Synthase (FAS), which plays an important role in de novo fatty acid synthesis, was protective as it reversed pulmonary vascular remodelling and reduced right ventricle pressure associated with pulmonary hypertension. Further, there was increased FAS expression and activity and increased level of palmitate, product of FAS catalyzed reaction, in hypoxic cardiomyocytes and RV of Monocrotaline treated pulmonary hypertensive rats. In our study, we observed the elevated level of cellular ROS and oxidative stress markers, increased pro-inflammatory cytokine, TNF- $\alpha$, and decreased anti-inflammatory cytokine, IL10 , along withincreased expression of p65 subunit of $\mathrm{NF} \alpha \mathrm{B}$ in hypoxic cardiomyocytes and RV of Monocrotaline-treated rats. FAS inhibition either by siRNA or C75 (2 mg/kg weekly, i.p.), a pharmacological FAS inhibitor, decreased FAS activity and expression, oxidative stress and inflammation. In our study, we found the loss of mitochondrial membrane potential, reduced levels of NAD and ATP in hypoxic cardiomyocytes and RV of MCT-treated rats. Inhibition of FAS attenuated the mitochondrial dysfunction as evident by restoration of mitochondrial membrane potential and increased ATP and NAD level in hypoxic cardiomyocytes and RV of
MCT-treated rats. We also found that markers (LC3BII and p62) of autophagy, which plays a role in hypertrophic remodelling of ventricles, were also reversed by FAS inhibition. In conclusion, this study shows that de novo fatty acid synthesis gets modulated in pulmonary hypertension and inhibition of FAS is protective in pulmonary hypertension.

\section{IL- 25}

Nexrutine Inhibits Azoxymethane Induced Colonic Aberrant Crypt Formation In Rat Colon And Induced Apoptotic Cell Death In Colon Adenocarcinoma Cells

Kausar Mahmood Ansari ${ }^{1}$ and Shamshad Alam ${ }^{1}$

1. Environmental Carcinogenesis Laboratory, Food, Drug, and Chemical Toxicology Group CSIR-Indian Institute of Toxicology Research (CSIR-IITR), Vishvigyan Bhawan, 31, Mahatma Gandhi Marg, Lucknow 226001, Uttar Pradesh, India.

$\mathrm{C}$ olon cancer is the third most common cause of death in the worldwide. Therefore, new preventive strategies are warranted for preventing colon cancer. Nexrutine (NX), an herbal extract from Phellodendron amurense, has been shown to have an anti-inflammatory, anti-microbial and anti-cancer activity for various tissuespecific cancers, but its chemopreventive efficacy has not been evaluated for colon cancer. Here, we explored the mechanism of chemopreventive/chemotherapeutic efficacy of NX against colon cancer. It was observed that dietary exposure of NX significantly reduced the number of azoxymethane (AOM)-induced aberrant crypt foci $(\mathrm{ACF})(* \mathrm{p}<0.005)$ in rats. In addition, significant inhibition of AOM-induced cell proliferation and reduced expression in inflammatory markers COX-2, iNOS as well as proliferative markers PCNA and cyclin D1 were also seen. Along with these, NX exposure significantly enhanced the apoptosis in the colon of AOM treated rats. Furthermore, in vitro studies, NX $(2.5,5,10 \mu \mathrm{g} / \mathrm{ml}, 48 \mathrm{~h})$ decreased the cell survival and colony formation while induced G0/G1 cell cycle arrest and apoptosis in colon adenocarcinoma cells COLO205 and HCT-15. However, NX had the minimal cytotoxic effect on IEC-6 normal 
rat intestinal cells, suggesting its high therapeutic index. NX treatment also modulates the level of Bax and Bcl-2 proteins along with cytochrome c release, enhanced cleavage of poly (adenosine diphosphate-ribose) polymerase as well as the catalytic activity of caspases 3 and 9 in both COLO205 and HCT-15 cells. Based on these in vivo and in vitro findings, we suggest that NX could be a useful candidate agent for colon cancer chemoprevention and treatment.

KEY WORDS: Chemoprevention, ACF, Nexrutine, Apoptosis, Colon Cancer

\section{IL- 26}

\section{Molecular Diagnostics In Metabolic Disorder: A Clinician's Approach}

\section{Kausik Mandal}

Department of Medical Genetics, C Block; Sanjay

Gandhi Post-Graduate Institute of Medical Sciences;

Raibereli Road. Lucknow -226014. India.

Email: mandal.kausik@gmail.com;

kausik@sgpgi.ac.in

$\mathrm{M}$ etabolic disorders are essentially single gene disorders. In such disorders, there is a defect in the normal metabolic process in the body, in most occasions arising from a defective enzyme, encoded by a mutated gene. Most such disorders are inherited as autosomal recessive manner, where both the copies of a gene (alleles) get mutated, producing a very low level of enzyme, resulting in manifestation of the disease. Some of these are X-linked disorders where mainly males manifest the disease.

For molecular diagnosis of such disorders, Sanger sequencing of the causative genes are done to look for small scale mutations. When genes are large or there are more than one gene giving rise to the same phenotype and metabolic derangement, molecular diagnosis becomes challenging. In such situations, next-generation sequencing technique (NGS) is advocated to look for sequence variations. We can explore sequence variations of the whole genome by NGS. Exome sequencing, based on NGS technique is a very useful technique and widely used these days. Through exome sequencing, we tend to see all the coding regions (exons) of the known genes of the genome, thereby reducing the cost and time of analysis. NGS is a very powerful technique; but it is important to note that there are limitations as well. NGS cannot detect large deletions and duplications and various complex rearrangements. For deletions and duplications (copy number variations/ CNVs) inside genes, MLPA (Multiplex Ligation-Dependent probe Amplification) technique is used. Cytogenetic microarray (CMA) though can scan the whole genome for CNVs, it cannot detect CNVs inside genes. With platforms using SNP array, CMA can be used for finding homozygous regions in genome to search for genes responsible for autosomal recessive disorders in consanguineous families.

We will discuss the usefulness of the various molecular techniques with case scenarios.

\section{IL- 27}

\section{A Successful Crosstalk Between Glycation and Oxidation Can Generate Aggressive Immunogenic Neoepitopes On Self Proteins: A Likely Event In The Development of Systemic Lupus Erythematosus}

\section{Khursheed Alam}

Department of Biochemistry, Faculty of Medicine, Aligarh Muslim University, Aligarh, India

*Email: kalam786@rediffmail.com

The glycation (caused by reducing sugars, dicarbonyls 1 etc) and oxidationare episodes of normal physiology. There is an inbuilt mechanism to tackle them; namelythe glyoxalase system-I and an array of antioxidants. In this study, $1 \mathrm{mg} / \mathrm{ml}$ of whole histone (Sigma) was mixed with $40 \mathrm{mM}$ deoxyribose and placed at $37 \mathrm{oC}$ for 9 days to generate complete AGEs. The whole-histone-AGEs was then treated with hydroxyl radical for $3 \mathrm{~h}$ generated from Fenton's system, and the biophysical changes in oxidized-whole-histone-AGEs (OWHA) were studied by an array of techniques namely- UV, fluorescence, CD and FTIR spectroscopy. Furthermore, NBT colorimetric assay was used for Amadori adducts which went on decreasing with AGEs formation. Free ?-amino groups 
and arginine were assayed to demonstrate their participation in glycation cascade. Oxidation and aggregation were studied as free thiols, protein carbonyls, and thioflavin $\mathrm{T}$ and Congo red binding. The OWHA was injected into rabbits and induced antibodies were characterized. The binding profile, specificity and crossreactivity of naturally occurring autoantibodiesin SLE serahave been studied against OWHA. The OWHA showed hyperchromicity at $276 \mathrm{~nm}$, decrease of tyrosine fluorescence accompanied by increase in pentosidine, AGE-specific and ANS fluorescence, shift in amide bands I and II, increase in melting temperature, compared to native controls. Autoantibodies in SLE sera showed strong binding with GWHA.Highly immunogenic nature of OWHAand presence of good amount of autoantibodies against OWHAin SLE sera points out its likely role in the induction and/or progression of systemic lupus erythematosus, at least in a sub-group of patients.

\section{IL- 28}

\section{Modulation of the Complement System In Inflammatory Diseases and Its Therapeutic Control}

\section{Lalit M. Srivastava}

Department of Biochemistry and Lab. Medicine,
Sir Ganga Ram Kolmet Hospital, New Delhi.

$\mathrm{T}$ he Complement system provides a rapid and efficient means to protect the host from invasive microorganisms. Due to its diverse biological andfunctional activities, Complement is the major mediator of inflammation, a natural response to the host tissue through any injury. Evidences areforthcoming to show that Complement significantly contributes to the regulation of Immune response. Furthermore, the effector functions arising from complement activation carry the potential for harming the host by directly or indirectly mediating inflammatory tissue damage or destruction.

Inappropriate or excessive activation of the Complement system can lead to harmful, potentially life-threatening consequences due to severe anddrastic inflammatory destruction. Genetic complement deficiencies and/ or complement depletion have been shown to be beneficial in reducingtissue and organ injury due to severe complement dependent inflammation.

Considerable clinical and experimental evidence alongwith the work done in our laboratory implicate the role of Complement and ComplementReceptors ( CR1 ; MCP; DAF and CD 59 ) in the pathogenesis of various inflammatory diseases viz. Immune Complex and Autoimmune disorders, as also organ failure as a consequence to sepsis and multiple trauma. We have found that the value of CR1/E in patients with SLE was significantlylower than their consanguineous relatives and healthy subjects. CR1/E was found to be stable in consecutive samples in controls. In patients thenumbers varied between low and high during the course of treatment. Our results led to suggest that low levels of the CR1 on erythrocytes in SLEpatients are acquired during the course of the disease.

Recently considerable advances have been made towards the utility of measurements of complement proteins $(\mathrm{C} 1$, $\mathrm{C} 2, \mathrm{C} 3, \mathrm{C} 4, \mathrm{C} 5$ and their breakdown products) and complement receptors for the diagnosis and assessment of disease severity and response to therapy as well as of prognosticvalue in early recognition of patients at risk to develop various forms of nephropathy, multiple organ failure after trauma or with graft rejection followingrenal transplantation. Hence the work progress on complement system, its receptors alongwith complement derived breakdown products (viz. C3a, C5a)or protein-protein complexes provide a comprehensive insight into the activation state of the system and have led to provide strategies towards a novel therapeutic approach and control of the complement system for many inflammatory diseases. 


\section{IL- 29}

\section{Mechanistic Role of Nutraceuticals In Proliferation And Apoptosis of Cancer Cell Lines}

M. Arshad, Asif Jafri, M. Serajuddin

Molecular Endocrinology Lab, Department of

Zoology,University of Lucknow, Lucknow-226007

Email:arshadm123@rediffmail.com

$\mathrm{N}^{4}$ utraceuticals are the natural bioactive compounds including broad diversity of structures and functionalities, provide an excellent pool of molecules for the prevention and treatment of many diseases including cancer without side effect.Nutraceuticals are thesubstances that extracted from food sources with additional health benefits along with the basic nutritional values. They are basically categorised into a three key terms- nutrients, herbals, dietary supplements and dietary fibers on the basis oftheir natural source and chemical composition. Since last two decades the extensive studies on various cancer cells showed potent anti-proliferative and apoptosis efficacy of these nutraceuticals. Some of the promising nutraceuticals that have shown excellent resultsareNaringenin, Piperine, Curcumin, Euplatin, Rutin, Caffeic acid, Quercetin, Apigenin,Silibinin, Norcantharidin, Saffron, Parthenolide, Fiestin, Lupeol etc. Theypossess anticancer properties against skin, breast, bone, prostate, cervix, oral, lung, ovarian, and leukemia cancer cells. The anticancer efficacy of these promising compoundsare due to modulation of the cell viability, induction of intrinsic and extrinsic ROS production, nuclear fragmentation/ condensation, depolarisation of mitochondrial membrane potential (MMP), cell cycle check-points, caspases induction and expression of various oncogenic genes. These findings provide a mechanistic role and mode of action of these potent compounds on proliferation and apoptosis of various cancer cells. Understanding the effects of various nutraceuticals on cancer cells may enhance their usagesin the prevention and treatment of various cancers without adverse effect.Further studies are needed to validate cancer preventive and curative efficacy of potent nutraceuticals at clinical level.

KEYWORDS: Nutraceuticals, Cancer cells, Proliferation, Apoptosis
IL- 30

\section{Potential Role of Lycopene In Targeting Proprotein Convertase Subtilisin/Kexin Type-9 to Combat Hypercholesterolemia}

Sahir Sultan Alvi ${ }^{1}$, Irfan A. Ansari ${ }^{1}$, M. Salman Khan ${ }^{1 *}$

1. Clinical Biochemistry \& Natural Product Research Lab., Department of Biosciences, Integral University, Lucknow-226026, India

Droprotein convertase subtilisin/kexin type 9 (PCSK9) is a serine protease of the proprotien convertase (PC) family that has profound effects on plasma low density lipoprotein cholesterol (LDL-C) levels, the major risk factor for coronary heart disease (CHD), through its ability to mediate LDL receptor (LDL-R) protein degradation and reduced recycling to the surface of hepatocytes. Thus, the current study was premeditated not only to evaluate the role of lycopene in targeting the inhibition of PCSK-9 via modulation of genes involved in cholesterol homeostasis in HFD rats but also to examine a correlation between HFD induced inflammatory cascades and subsequent regulation of PCSK-9 expression. Besides the effect of lycopene on hepatic PCSK-9 gene expression, PPI studies for PCSK9-Lycopene complex and EGF-A of LDL-R were also performed via molecular informatics approach to assess the dual mode of action of lycopene in LDL-R recycling and increased removal of circulatory LDL-C.We for the first time deciphered that lycopene treatment significantly down-regulates the expression of hepatic PCSK-9 and HMGR, whereas, hepatic LDL-R expression was significantly up-regulated. Furthermore, lycopene ameliorated inflammation stimulated expression of PCSK-9 via suppressing the expression of inflammatory markers. The results from our molecular informatics studies confirmed that lycopene, while occupying the active site of PCSK-9 crystal structure, reduces the affinity of PCSK-9 to complex with EGF-A of LDL-R, whereas, atorvastatin makes PCSK-9-EGF-A complex formation more feasible than both of PCSK-9-EGF-A alone and Lycopene-PCSK-9-EGF-A complex. Based on above results, it can be concluded that lycopene exhibits potent hypolipidemic activities via molecular 
mechanisms that are either identical (HMGR inhibition) or distinct from that of statins (down-regulation of PCSK9 mRNA synthesis). To the best of our knowledge, this is the first report that lycopene has this specific biological property. Being a natural, safer and cost effective agent, lycopene could be used as a complete regulator of cholesterol homeostasis and ASCVD.

\section{IL-31}

Epithelial-To-Mesenchymal Transition: Its Diagnostic And Prognostic Significance In Human Urothelial Carcinoma of Bladder.

\section{Minal Garg \\ Department of Biochemistry, University of Lucknow, Lucknow-226007}

INTRODUCTION: Epithelial-to-mesenchymal transition (EMT) is a key feature in the pathogenesis of tumors of epithelial origin including urothelial carcinoma of bladder (UCB). It is marked by the phenotypic transition of epithelial cells (non-motile) to mesenchymal cells (motile and invasive) and confers tumor cells an increased resistance to anoikis/ apoptosis. EMT biomarkers may help to elucidate biologic features to identify patients at high risk for recurrence, metastasis and progression and therefore, possess prognostic significance.

MATERIAL AND METHODS: The present work has been undertaken to examine the quantitative expression of EMT markers including epithelial, mesenchymal and EMT-activating transcription factors (EMT-ATFs) in sixty five human tumor tissues [non muscle invasive bladder cancer (NMIBC) and muscle invasive bladder cancer (MIBC)]by real time-quantitative polymerase chain reaction (RT-qPCR)and immunohistochemistry (IHC). Expression results were correlated with clinicopathological variables for their clinical relevance.

RESULTS:Statistical relevance was observed between focal loss of membranous expression of E-cadherinand novel expression of mesenchymal markers $[\mathrm{N}$-cadherin and Vimentin]. Kaplan Meier along with log rank statistics was used to determine the significant influence of novel expression of mesenchymal markers on short progression free survival (PFS) $(\mathrm{p}=0.048)$. Abnormal expression of EMT-ATFs [Snail, Slug, Zeb and Twist] positively correlates with histological grade and pathological stage of tumor.

CONCLUSIONS: Molecular validation of EMT marker proteins in bladder cancer as evident by correlation of expression with clinicopathological features may prove to be a sensitive and effective diagnostic and prognostic tool. It might help in improving early detection of recurrence in low grade NMIBC nevertheless, further studies are required in more number of clinical samples.

KEY WORDS: Biomarkers; Clinicopathological variables; Clinical relevance; Epithelial-to-mesenchymal transition (EMT); Non-muscle invasive and muscle invasive bladder cancer

\section{IL- 32}

Glycoxidation of Human Low Density Lipoprotein: Role In Diabetes, Periodontitis And Coronary Artery Disease

Moinuddin, MinhalAbidi, Md Masum Rizwee

Department of Biochemistry, Faculty of Medicine, J.N. Medical College, AMU, Aligarh-202002, U.P, India.

INTRODUCTION:Advanced glycation end products (AGEs) are synthesized via the non-enzymatic glycation and oxidation (glycoxidation) of proteins, lipids and nucleic acids. AGEs have got a role in diabetes, periodontitis (PD) and Coronary artery disease (CAD); the three have known co-morbidity. Glycation alters the structural and functional properties of the proteins. Glycation of low density lipoprotein (LDL) with MGO leads to the formation of AGEs and generation of reactive oxygen species (ROS).It plays a role in the enhanced unregulated uptake of lipids by cells in wall of artery in diabetic patients. Enhanced inflammatory processes in DM could be a contributing factor to CAD and PD.

METHODOLOGY:LDL was glycated with MGO in varying concentrations / time and the structural changes were analyzed by various biophysical and biochemical 
techniques. Antibodies against MGO-LDL were raised in experimental animals and characterized by direct binding and competitive-inhibition ELISA. Anti-glycated LDL autoantibodies in sera of type 2 DM, PD and CAD patients were evaluated by ELISA.

RESULTS: Modified LDL exhibited hyperchromicity, increase in fluorescence intensity, shift in amide I and amide II bands in FT-IR, loss of alpha helix in CD, aggregation in ThT assay SEM and TEM. Modified LDL was found to be highly immunogenic as compared to native LDL. High titre of auto-antibodies against MGOLDL in type 2 DM, PD and CAD patients was also found.

CONCLUSION:Glycation leads to structural distresses in LDL that can alter the physiological behavior. Experimental induction of antibodies against MGO-LDL and presence of auto-antibodies against MGO-LDL in type $2 \mathrm{DM}, \mathrm{PD}$ and CAD patients' sera point towards the generation neo-epitopes on LDL upon glycoxidative damage. The MGO-LDL has been projected as one of the links in the DM-PD-CAD co-morbid triangle.

KEYWORDS: Diabetes Mellitus, Periodontitis, Low Density Lipoprotein, Antibodies, Glycoxidation.

\section{IL- 33}

\section{Genetic Variants And Expression of Cox2 And Enos In Type 2 Diabetes Mellitus}

Monisha Banerjee*, Sushma Verma, Atar Singh Kushwah and Neena Srivastavaa

Molecular \& Human Genetics Laboratory, Department of Zoology, University of Lucknow, Lucknow-226007; aDepartment of Physiology, King George's Medical University, Lucknow-226003 *Email: monishabanerjee30@gmail.com; mhglucknow@yahoo.com

BACKGROUND: High glucose concentrations alter the nitric oxide $\left(\mathrm{NO}^{*}\right)$ bioavailability resulting in accumulation of reactive oxygen species (ROS), leading to endothelial dysfunction.Type 2 Diabetes Mellitus (T2DM) patients have a reduced ability to generate Nitric oxide (NO*), whose synthesis is catalyzed by nitric oxide synthase (NOS). Endothelial NOS (eNOS) is activated by insulin-signaling pathways. Severalvasodilator and constrictor molecules involved in the pathogenesis of macrovascular diseases are synthesized by cyclooxygenase (COX)enzyme. COX2 is the inducible form associated with inflammation and is also the target of non-steroidal anti-inflammatory drugs (NSAIDs), commonly used in diabetic treatment. The aim of present study was to understand the correlation, if any, between COX2 and eNOS gene variants and their expression in T2DM cases as compared to normal healthy controls.

MATERIAL AND METHODS: Blood samples from 472 subjects (221 each of controls and 251 T2DM cases) were collected after ethical approval and individual written consent. All subjects were genotyped by polymerase chain reaction-restriction length polymorphism (PCR-RFLP) using specific primers and restriction enzymes. Genotypic, allelic, carriage rate frequencies were calculated and expression study was done by real-time polymerase chain reaction (RT-PCR) using SYBR Green assay and confirmed by western blot analysis.

RESULTS: The COX2 gene polymorphism, $-765 \mathrm{G} / \mathrm{C}$ showed correlation with $\mathrm{COX} 2$ expression. The ' $\mathrm{GC}+\mathrm{CC}$ genotype individuals with T2DM showed a significantly higher COX2 expression when compared to ' $\mathrm{GC}+\mathrm{CC}$ ' controls $(\mathrm{P}<0.05)$. Genotyping analysis in the present study also showed ' $\mathrm{C}^{* 1}$ as a significant risk allele $(\mathrm{P}=0.022)$. No correlation of eNOS $-786 \mathrm{~T} / \mathrm{Cgene}$ variant was found with its expression.

CONCLUSION:The present study will be able to describe the individual and combined effects of different gene variants on T2DM susceptibility inthe study population.Expression studieswill provide leadsto discover target genes for antidiabetic therapy.

KEYWORDS: Type 2 diabetes mellitus, genetic variants, expression, $\mathrm{COX} 2$, eNOS 


\section{IL- 34}

\section{Should Cystatin C Be used by itself or with other Markers to Identify and Stage the Patients with Chronic Kidney Disease}

\section{Mridula Mahajan}

$C$ hronic kidney disease (CKD) being asymptomatic often remains undiagnosed or is diagnosed at an advanced stage. Specific symptoms of CKD usually develop at advanced stage or may be detected by chance following routine blood and urine examination. The disease is often associated with old age, diabetes, hypertention and cardiovascular disease etc. It describes abnormal kidney function and/ or structure. Urine and blood tests are used to detect and monitor kidney disease. Currently the key markers being used are abnormal urinary albumin $(>30 \mathrm{mg} / \mathrm{g}$ creatinine) and a persistant reduction in estimated glomerular filteration rate ( $\mathrm{e}$ GFR $<60 \mathrm{ml} / \mathrm{min} / 1.73 \mathrm{~m} 2$ ) for three months or more irrespective of clinical diagnosis . Another way of assessing kidney functions has been by using a combination of e GFR and albumin:creatinine ratio (ACR) . Increased ACR and decreased eGFR have been shown to be associated with increased risk of CKD. Accordingly patients with CKD have been staged from Stage 1 to Stage 5. Another important aspect is that kidney functions tend to decline with age and such people with age related decline in kidney functions may not progress to kidney failure. Under such circumstances staging of CKD may not work as the prognosis for a 75 year old patient with eGFR of $55 \mathrm{ml} / \mathrm{min} / 1.73 \mathrm{~m} 2$ may be different than that for a 45 year old patient with same e GFR. In addition eGFR may be too narrow a basis for assessing the risk of progression of CKD. Such findings potentiated the need for fairly comparable biomarkers for early detection of less severe cases of CKD.

Serum creatinine concentration has been found to vary with muscle mass depending upon age,sex,race and level of physical fitness. So serum creatinine levels may vary in individuals with same level of kidney function. Such findings potentiated the need for fairly stable novel biomarker for early diagnosis and staging of CKD.
Recently cystatin c level has been established as fairly comparable tool for estimating GFR. Cystatin C is made by all nucleated cells and not just by muscle as a result the blood levels of cystatin c are fairly similar between individuals who have same GFR. Cystatin c has also been identified as an inflammatory marker.

\section{IL- 35}

\section{Ashwagandha, The Traditional Indian Medicinal Plants In Modern Medicine Perspectives}

\section{Neelam S Sangwan}

$\mathrm{A}$ shwagandha (Withania somnifera) is one of the most reputed Indian medicinal plants and is used as a major ingredient in more than 200 traditional herbal preparations. Ashwagandha is credited with important pharmacological activities including anti stress, neuroprotection, reversal of Alzheimer's disease, immuno modulation, osteo protective, cardiovascular etc. Recent decade has witnessed tremendous upsurge in the knowledge and information on biochemical and phytochemical aspects of plant. Under NMITLI program, we have advanced the knowledge on various aspects of phytochemicals in Ashwagandha including its phytochemistry, molecular biology, genomics and pharmacological investigations. The major phytomolecules of Ashwagandha are withanolides, the C28, ergostane molecules with lactone ring. Our studies revealed that significant variations exists in phytomolecular levels in herbal preparations. Also, the molecules forms the basis for pharmacological actions. The efforts in this directions revealed distinct activities of Ashwagandha associated with osteo health, immunomo-dulation, anti stroke and other activities. Different withanolides thus may be utilised for development of specific formulation for specific health problems. Various development towards the enhancement of molecules in plants for specific and extract specific activities will be discussed in the meeting. 


\section{IL-36}

\section{Metabolic Reprogramming Targeting Alters the Proliferative Behavior of Hepatocellular Cancer}

Nissar Ahmad Wani' ${ }^{1}$, Bo Zhang ${ }^{2}$, Kun-yu Teng ${ }^{3}, 5$, Juan M. Barajas ${ }^{3}, 5$, Tasneem Motiwala ${ }^{4}$, Peng $\mathrm{Hu}^{3},{ }^{5}$, Lianbo $\mathrm{Yu}^{4}$, Rafael Brüschweiler ${ }^{2},{ }^{6},{ }^{7}$ Kalpana Ghoshal ${ }^{3},{ }^{5}$ and Samson T. Jacob1,

1. Department of Biotechnology, Central University of Kashmir, Srinagar,

2. Department of Chemistry \& Biochemistry,

3. Department of Pathology,

4. Department of Biomedical Informatics,

5. Comprehensive Cancer Center,

6. Department of Biological Chemistry and Pharmacology,

7. Campus Chemical Instrument Center (CCIC) $N M R$, The Ohio State University, Columbus, $\mathrm{OH}$

$\mathrm{H}$ epatocellular carcinoma (HCC) represents approximately $90 \%$ of all cases of primary liver cancer.Sorafenib, the only approved targeted therapy, prolongs median survival by ?3 months. Therefore, there is anurgent need to identify new therapeutic strategies to treat this deadly disease. Glycolysis is substantially elevated inmany tumors including sorafenib resistant, prompted us to screen several drugs with anti-glycolytic activity. Wedemonstrate that the combination of glycolytic inhibitor 2-deoxy-D-glucose (2DG) and sorafenib drastically reducestumorigenesis of sorafenib sensitive and resistant cells. However, other drug combination does not showany synergistic effect. Since only the combination showed promising results. We extended our study to test naturalcompound zerumbone; a monocyclic sesquiterpene. We observed that this compound reduced proliferation andclonogenic survival of HCC cells by altering metabolic reprograming, arrested cells at $\mathrm{G} 2 / \mathrm{M}$ phase of cellcycle and by inducing apoptosis. To uncover the underlying molecular mechanisms, first, we performed profiling ofphosphokinases that showed significant inhibition in activation of PI3K/AKT/mTOR and STAT3 in HCC cells treatedwith zerumbone, pathways associated with worst patient survival. Gene expression profiling using microarray and analysis of microarray data using Gene
Set Enrichment Analysis (GSEA) and Ingenuity Pathway Analysis (IPA) revealed that zerumbone treatment resulted in significant deregulation of genes regulating apoptotic, cell cycle and metabolism. Indeed, tracing glucose metabolic pathways by growing HCC cells with $13 \mathrm{C}$-glucose and measuring extracellular and intracellular metabolites by 2D-NMR showed a reduction in glucose consumption and reduced lactate production, suggesting glycolytic inhibition. Additionally, zerumbone impeded shunting of glucose-6-phosphate through the pentose-phosphate-pathway, thereby forcing tumor cells to undergo cell cycle arrest and apoptosis. So, combined inhibition of glycolysis and mTOR signaling disrupted metabolicre-programming and thereby cell cycle and apoptosis in HCC and inhibited their growth. Thesefindings reveal novel therapeutic strategies to realize the potential benefit from targeting the Warburg effect.

\section{IL- 37}

Cellular-Xenobiotic Interaction In A Tissue Specific Pattern Leads To Diversification In Mode of Cell Death Response

\author{
Pradeep Kumar Sharma ${ }^{1}$, Deepika Arora ${ }^{1,2}$
}

1. Environmental Carcinogenesis Lab, Food Drugs and Chemical Toxicology Group, CSIR-Indian Institute of Toxicology Research, Vishvigyan Bhawan, 31, Mahatma Gandhi Marg, Lucknow-226001, Uttar Pradesh, India.

2. Present Address: Department of Biosciences and Bioengineering, IIT-Guwahati, Assam India

INTRODUCTION:Understanding the consequence of human cellular interaction with environmental contaminants such as pesticides is a challenging task. The occurrence of different cell death modalities in cells exposed to the same toxicant render mitigation strategies less effective. The molecular basis of such heterogeneous response remains poorly understood.

MATERIALAND METHODS:Here, we have explored the molecular basis of heterogeneity in human cell line of different tissue origins(such as liver, breast, brain, ovarian, kidney, and pancreases) when exposed to 
deltamethrin (DLM; a common pesticide) which is widely used for various pest management programs.

RESULTS:Our data demonstrate that DLM exposure resulted in induction of different mode of cell deaths in different human cell lines. Under similar treatment conditions, DLM was able to induced apoptosis (HEK and Hela), necrosis (MCF-7, Panc-1 and SHSY5Y) and necroptosis (HepG2). Interestingly, the DLM induced apoptotic cell death was differentially regulated in HEK (caspase-independent) and Hela (caspase 8-dependent) cells. Most interestingly, DLM exposure resulted in RIPK3-dependent necroptosis in HepG2 cells. Of note, PARP1 activation, loss of MMP, mitochondrial ROS and mitochondria fragmentationwere the common events in all the cell types. Pharmacological inhibition of PARP1 rescued DLM induced cell death significantly in all the cell types which suggested that it could play central role in diverse modes of cell death induced by DLM.

CONCLUSION:Tissue specificity may direct the cellular response against any xenobiotic substance and also account for heterogeneous modes of cell death in different tissues upon a toxicant exposure.

KEYWORDS:Heterogeneity, Xenobiotic, Deltamethrin, Apoptosis, Necroptosis.

\section{IL 38}

\section{Critical Thinking :- Building Good Judgement}

\section{Dr. Praveen Sharma}

\section{HOD \& Prof., Deptt of Biochemistry, AIIMS Jodhpur, India, Member IFCC-CCLM, Chair APFCB Communication Committee}

$\mathrm{C}$ ompetent reasoning and problem solving is not unique to health care. Lives depend on competent clinical reasoning. Thus it is a moral imperative for health care providers to strive to monitor and improve their clinical reasoning and care related judgments. Thus, focus on assessing competence in reasoning and problemsolving is also becoming a standard in the workplace.Excellence in professional judgment is the result of the sound use of critical thinking skills and the reliable and strong disposition to use those critical thinking skills.At every level from novice to expert, clinical judgment regarding diagnosis, treatment, and ongoing evaluation of patient outcomes is a fundamentally complex reasoning process which is applied to problems characterized by a multiplicity of potentially varying parameters. This consumes cognitive resources including time to think as it relies upon core critical thinkingskills and habits of mind.The discussion below outlines what explores the challenge we face as researchers and educators to facilitate improvements in clinical reasoning for ourselves, our students and our peers. Thus as a researcher and mentors efforts are needed to improve clinical reasoning skills and to be demonstrated in a variety ofhealth science disciplines and laboratory medicine.

\section{IL- 39}

\section{Endothelial Dysfunction \& Oxidative Stress In Type 2 Diabetes Mellitus}

\section{R. Dhananjayan}

Consultant \& Head, Dept. of Biochemistry, Apollo Speciality Hospitals, Vanagaram, Chennai - 600095 , India

INTRODUCTION: Endothelial dysfunction (ED) is an imbalance in the production of vasodilator factors and when this balance is disrupted, it predisposes the vasculature towards prothrombotic and pro-atherogenic effects.It is focussed as a potential contributor to the pathogenesis of vascular disease in diabetes mellitus (DM). Type 2DM (T2DM) is a disorder of insulin secretion and insulin resistance, a condition in which cells fail to use insulin properly, sometimes combined with an absolute insulin deficiency.Under physiological conditions, there is a balanced release of endothelialderived relaxing and contracting factors, but this delicate balance is altered in DM and atherosclerosis, thereby contributing to further progression of vascular and endorgan damage.DM produces disturbances of lipid profile, especially an increased susceptibility to lipid peroxidation, which is responsible for increased incidence of atherosclerosis. An enhanced oxidative stress has been observed in increased free radical 
production, lipid peroxidation and diminished antioxidant status.Studies indicate that risk is detectable in childhood, though these disorders may not emerge until adulthood.

\section{ENDOTHELIAL DYSFUNCTION \& OXIDATIVE}

STRESS: Hyperglycemia, dyslipidemia, increased intimal medial thickening (IMT) may lead to ED subsequently leading to atherosclerosis. Measuring lipids, markers of endothelial function, such as IMT, nitrite (NO) and high sensitive C Reactive Protein (hsCRP) in patients with T2DM and in affected families may be useful in early prediction of ED and risk of Coronary Artery Disease (CAD).Increased oxidative stress due to hyperhomocysteinemia and oxidative stress inducing factors may lead to reduced bioavailability of NO. Increased oxidative stress and reduced NO levels may cause altered IMT and ED in patients with T2DM.

We have conducted a study aimed to assess the markers of ED in patients with the family history of T2DM from South Indian population. A total of 450 subjects were included in the study comprising Group I of T2DM, Group II of age and sex matched healthy controls, Group III of children of T2DM patients and Group IV of children of healthy controls. The blood samples of $60 \mathrm{~T} 2 \mathrm{DM}$ cases and 60 controls were randomly taken for the estimation of homocysteine (HCY) and oxidative stress markers.Results indicated that IMT was significantly higher in T2DM patients, compared with control subjects with no family history of DM. The fasting plasma glucose (FPG), glycated haemoglobin (HbA1c), serum Total Cholesterol, Triacylglycerol,LDL-Cholesterol, ApolipoproteinB (Apo B) and hsCRP levels were significantly increased, whereas HDL-Cholesterol and NO levels were significantly decreased in T2DM patients. However, there were no differences in FPG, HbA1c, serum lipids, Apo A1, Apo B, hsCRP and IMT in children of T2DM patients and healthy controls. The serum NO andplasma glutathione (GSH) levels were significantly decreased in T2DM patients than healthy controls. The plasma MDA and HCY were significantly increased in T2DM patients than healthy controls. There was a significant positive correlation with $\mathrm{HCY}$ and MDA; significant negative correlation with $\mathrm{NO}$ and GSH with IMT was observed.
CONCLUSION: According to our study, the IMT was significantly higher in the T2DM patients and further demonstrated that it increases with age and family history. The increased levels of lipids, hsCRP, IMT and decreased NO levels might contribute to the risk of ED in patients with T2DM. No differences in FPG, HbA1c, serum lipids, Apo A1, Apo B, hsCRP and IMT in children of T2DM patients and healthy controls, thus appear that children of T2DM patients may not be at risk for DM and ED in the very young age.Elevated HCY, MDA and decreased GSH and NO were observed in patients with T2DM. Further, it was also observed that increased HCY and MDA were found to be positively correlated with IMT, whereas NO and GSH were negatively correlated with IMT. These findings clearly indicate that T2DM patients are highly susceptible to ED and might increase the risk for CAD.

\section{IL- 40}

\section{Advanced Glycation End Products (Ages) As Biomarkers In Diabetes And Its Complications}

\section{RT Erasmus}

\author{
Stellenbosch University and National Health \\ Laboratory Services(NHLS), Tygerberg Hospital, \\ Cape Town, South Africa
}

dvanced Glycation End products (AGEs) are a
heterogeneous group of compounds derived from
non-enzymatic glycation of proteins, lipids and nuclear
acids through complex and sequential reactions known
as the Maillardreaction.AGEs may form endogenously,
and glycation of molecules may negatively affect their
function. Theseversatile molecules can be a factor in
aging and in the development or worsening of many
degenerative diseases, such as diabetes, atherosclerosis,
chronic kidney disease, and Alzheimer's disease. A high
consumption of AGEs through the diet has been directly
linked to all of these chronic diseases.Modern diets are
linked to high levels of AGEs. Certain foods, such as
animal foods high in fat and protein, are more susceptible
to AGEs formation during cooking. Several animal and
human studies have shown that eating a low-AGE diet
results in lower levels of AGEs in blood and tissues by 
up to 53\%, plus a lower risk of heart and kidney disease, as well as increased insulin sensitivity. This is because AGEs adversely affect the body's cells, promoting oxidative stress and inflammation.High levels of inflammation over a long period of time can cause damage to every organ in the body. Decreased clearance of serum AGEs may further increase tissue AGEs accumulation. Evaluation of AGEs and their accumulation has been difficult because their structure is complex and heterogeneous. Non-invasive skin autofluorescence (SAF) measurement serves as a proxy for tissue accumulation of AGEs. Diabetes, hypertension and obesity all showed strong association with SAF, particularly when gender differences were taken into account. Further research using large number of samples will clarify the association between AGE's and their relationship with chronic diseases in different racial groups.

\section{IL- 41}

\section{Drug Repurposing In Alzheimer's Disease: Focus on Adult Neurogenesis}

\section{Rajnish Kumar Chaturvedi ${ }^{1,2}$}

Developmental Toxicology Laboratory, Systems Toxicology \& Health Risk Assessment Group

1. CSIR-Indian Institute of Toxicology Research(CSIR-IITR) Vishvigyan Bhawan, 31

Mahatma Gandhi Marg, Lucknow-226001 Uttar Pradesh, India

2. Academy of Scientific and Innovative Research(AcSIR) CSIR-IITR Campus Lucknow

lzheimer's disease (AD) is a progressive
neurodegenerative disorder that impairs learning,
memory and cognitive judgment of an individual and is
accompanied by mood swings, disorientation and finally
delirium. The complex pathology of the AD is
characterized by loss of neurogenesis, synaptic damage
accompanied by substantial neuronal loss. Therefore, the
most important eventin neuroregeneration and
neuroprotection in AD is the selection of drugs that can
act on multiple targets involved in the degenerative
process and can induce neurorestorative/neuroprotective activities to produce fully functional neurons that can restore brain functions. For that reason neurogenesis is an attractive research avenue for therapy in $\mathrm{AD}$ since it is easily modifiable. Ethosuximide (ETH), is an anticonvulsant drug used for the treatment of epileptic seizures. However, the effects of ETH on endogenous neurogenesis and associated cellular and molecular mechanism(s) remain unexplored. Therefore, we studied the effects of ETH on rat multipotent neural stem cell (NSC) proliferation, differentiation adult hippocampal neurogenesis by generating amyloid beta (A $\beta 1-42)$ toxin-induced rat model of AD-like phenotypes. ETH potently induced NSC proliferation and neuronal differentiation in the hippocampus-derived NSC in vitro. Moreover, ETH enhanced NSC proliferation and differentiation in the rat model of the $\mathrm{AD}$ by activating the PI3K$\cdot$ Akt and Wnt $\beta$-catenin transduction pathways leading to behavioural recovery in a rat model of AD. However, inhibition of the PI3K·Akt and Wnt. $\beta$-catenin pathways effectively blocked the mitogenic and neurogenic effects of ETH. In addition to this, in silico molecular target prediction docking studies suggest that ETH interacts with Akt, Dkk-1, and GSK$3 \beta$. This collectively indicates that ETH augments structural plasticity in rat hippocampus by stimulating endogenous neurogenesis via PI3K·Akt and Wnt· $\beta$ catenin signalling pathways to reverse the behavioral changes associated with AD.

\section{IL- 42}

\section{General Aspects of Competencies In Biochemistry}

\section{Rohini Bhadre}

Professor, Dept of Biochemistry, K. Somaiya Medical College \& Hospital, Everard Nagar, Sion, Mumbai 40022. Maharashtra, India.

rbbhadre@gmail.com

$\mathrm{C}$ ompetency based medical education is Gennext. This talk will stress on how the competencies can be framed in the different domains of Biochemistry citing examples. Including the teaching learning and the assessment methods. 
It will also emphasize on how the Biochemistry departments in Medical Institution will have to move from the traditional methods of teaching to competency based medical education.

\section{IL- 43}

\section{Association Of Fructosamine And Hba1c In Newly Diagnosed Type 2 Diabetes Mellituspatients}

Roshan Alam ${ }^{1 *}$, Saba Khan ${ }^{1}$, Mohammad Mustufa Khan $^{1}$

1. Department of Biochemistry, IIMS\&R, Integral University, Lucknow

INTRODUCTION: Uncontrolled blood sugar is the main culprit for high levels of morbidity and mortality in diabetic patients worldwide. The blood glycated haemoglobin (HbAlc) and fructosamine provides a good picture of average plasma glucose for the previous 2-3 months and 2-3 weeks, respectively.

AIM: To associate the HbA1c and Fructosamine in newly diagnosed Type 2 diabetes mellitus (T2DM) patients to find an alternative marker and better predictor for early development of diabetic complications.

MATERIAL AND METHODS: Total 70 subjects (35 T2DM and 35 age and gender matched healthy controls) were enrolled from rural and sub-urban population of North India.Biochemical investigations were performed by commercial kits using Chemistry Autoanalyzer.P $<0.05$ was considered statistically significant.

RESULT: The mean of fasting blood sugar (FBS), postprandial blood sugar (PPBS), HbA1c and fructosamine have significantly increased in T2DM patients compared to healthy controls $(\mathrm{p}<0.0001)$. FBS, PPBS, HbA1c and Fructosamine have significant positive correlation in T2DM patients $(\mathrm{p}<0.01)$. Fructosamine has significant positive correlation with FBS, PPBS and HbA1c $(\mathrm{p}<0.01$, $\mathrm{p}<0.01, \mathrm{p}<0.01$, respectively).A linear regression was performed to find the predictor of Fructosamine. Model showed HbA1c as the only strong predictor $(\mathrm{p}=0.002)$. In addition, a linear regression was performed to find the predictor of HbA1c. Model showed FBS and Fructosamine as the strong predictors $(\mathrm{p}=0.000, \mathrm{p}=0.002$, respectively).

CONCLUSION:Fructosamine has significant positive correlation with FBS, PPBS and HbA1c in T2DM patients. HbA1c has been observed as strong predictor of fructosamine. However, FBS and fructosamine have seen as strong predictors of HbA1c. Fructosamine may act as a potential biomarker for the diagnosis of diabetes and its complications in Asian population.

KEYWORDS: Glycated haemoglobin, Fructosamine, Type 2 diabetes mellitus, Body mass index

\section{IL- 44}

\section{A Prooxidant Mechanism of Cancer Chemopreventive Properties of Plant Polyphenols}

SM Hadi, Mohd Farhan

Department of Biochemistry, Faculty of Life Sciences, Aligarh Muslim University, Aligarh-202002, India

INTRODUCTION:In the last couple of decades there has been some interest in alternative mechanisms of apoptosis induction which do not involve caspases. This is particularly of interest in relation to cancer cells. We have hypothesized that mobilization of endogenous copper ions by plant polyphenols such as EGCG and consequent oxidative degradation of cellular DNA could be an important mechanism of their anticancer properties.

OBJECTIVES:Over the years we have validated our hypothesis to a considerable degree. We further confirm the hypothesis byusing analogues of EGCG to identify the structural features of tea catechins important for mobilizing endogenous copper and breakage of cellular DNA in cancer cells.

METHODOLOGY:Comet assay to study DNA breakage, MTT assay for cell proliferation and Histone/ DNA Elisa for apoptosis induction was used to examine the catechin mediated oxidative breakage of cellular DNA in various cancer cell lines. 
RESULTS \& CONCLUSION:Catechins have been shown to inhibit cell proliferation and induce apoptosis in different cancer cell lines and that such cell death is prevented to a significant extent by copper chelator neocuproine. Further, normal breast epithelial cells (MCF-10A), cultured in a medium supplemented with copper (MCF-10A-Cu), become sensitized to EGCG induced growth inhibition. Copper transporters Ctr1 and ATP7A are found to have an increased expression in MCF-10A-Cu cells and EGCG inhibits the expression of both the copper transporters in such cells. Moreover, silencing of copper transporter $\mathrm{Ctr} 1$ by siRNA reduces the sensitivity of MCF-10A-Cu cells to EGCG. We conclude that the position and the number of hydroxyl groups in various catechins determine their capacity to mobilize endogenous copper and degrade cellular DNA.

KEYWORDS: Green tea catechins, Cancer cells, Hydroxyl groups

\section{IL- 45}

\section{Micro RNA's: Their Potential As Minimally Invasive Cancer Biomarkers}

\section{Sabhiya Majid}

Professor, Department of Biochemistry, Government Medical College, Srinagar

$\mathrm{m}$ iRNAs, a family of small, endogenous, and evolutionarily conserved non-coding ribonucleic acids have been involved in regulation of several essential, cellular, and functional processes. They are known to play key roles in all types of cancer and function as oncogenes or tumour-suppressors. The development of clinically validated biomarkers for cancer remains a top priority in field of cancer molecular biology. MicroRNAs have many characteristics of an ideal biomarker,notably their inherent stability and resilience. Recent blood-based miRNA profiling studies, reporting their presence in serum and plasma, revealed that circulating miRNAs have potential power to be examined as novel non invasive prognostic and diagnostic biomarkers of cancers.
IL- 46

\section{Nanomedicines for the Treatment of Tuberculous Meningitis}

\section{Sadhna Sharma}

Department of Biochemistry, Postgraduate Institute of Medical Education and Research, Chandigarh-160012

Tuberculous meningitis (TBM) is the most common form of central nervous system tuberculosis and has very high morbidity and mortality. Empiric treatment includes at least four first-line drugs, mainly isoniazid, rifampin, pyrazinamide, and ethambutol or streptomycin. Adjunctive treatment with corticosteroids has been shown to improve mortality however treatment regimen has number of drawbacks, which include longer treatment schedule of almost 18-24 months, associated toxicity and limited ability to cross the blood brain barrier. It necessitates the development of improved formulations of these drugs that can specifically target the desired sites. Nanomedicine based strategies have been successfully employed to address these shortcomings of conventional therapy.Double coated PBCA nanoparticles can be a promising vehicle to deliver anti tubercular drugs to brain and for drug targeting to the brain, our work suggest possibility of using nanoparticle formulation through oral route which is more convenient and patient friendly mode of drug delivery.Pharmacokinetic analysis showed sustained release of drugs in the plasma and brain for up to six days. Successful generation of murine model was confirmed by increased and stable bacillary loads in mice as well as histopathology of mouse tissues showing meningitis in brain, granuloma in lungs and tuberculous pathology in spleen. Drug loaded nanoparticles were found to be more effective, 4 or 8 doses of nanoencapsulated formulation were equi-efficacious to 32 or 64 doses of conventional therapy. 


\section{IL- 47}

\section{Histone Deacetylase Inhibitors and Their Potential Importance In Better Management of Gastric Cancer}

\section{Sanjay Gupta}

Principal Investigator, Epigenetics and Chromatin Biology Group, Gupta Laboratory, Advanced Centre for Treatment, Research \& Education in Cancer, Tata Memorial Centre, Kharghar,

Navi Mumbai 410210, MH, India;

sgupta@actrec.gov.in

$\mathrm{A}$ cetylation of histones is an essential Post Translation Modification (PTM), which influences overall chromatin architecture and thus controls critical processes such as replication, transcription. Two enzymes known as Histone acetyl transferases (HATs) and Histone deacetylases (HDACs) govern the dynamics of acetylation on chromatin. Largely owing to the reversible nature of histone PTMs, their modifying enzymes are considered to be important targets for therapeutic intervention. This led to the development and investigation of HDAC inhibitors (HDACi) as anticancer "epi-drugs".

A recent finding from our group on a study undertaken in human gastric cancer patient samples demonstrates an increase in the levels of HDAC activity in gastric cancer. This is coupled with with global histone deacetylation and chromatin condensation. We hypothesize that due to increased HDAC activity, the compact chromatin organization decreases the binding of chemotherapeutic drug to the DNA and affects the efficacy of the drug. Thus, inhibition of HDAC activity by using HDACi could potentially alter global chromatin organization and enable more binding of the chemo-drug to chromatin, which may lead to enhanced cell death.

Also, our group has recently developed and demonstrated the importance of liquid biopsy (serum) based monitoring of histone PTMs, HATs and HDACs. This novel approach provides the first evidence that liquid biopsy is a valuable predictive tool for monitoring disease progression. Importantly, with the advent of epi-drugs, liquid biopsy based 'real time' monitoring will be useful for subgrouping of the patients for epi-drug treatment, predicting response to therapy, early relapse and prognosis. In the presentation, we will discuss how histone modifications and their modifiers are potentially involved in cancer diagnosis, prognosis and their utility in terms of better disease management.

\section{IL- 48}

\section{Targeting Cellular Redox Regulators For Development of Anti-Cancer, Anti-Inflammatory and Radio-Protective Drugs}

\section{Santosh Kumar Sandur}

\section{Radiation Biology \& Health Sciences Division, Bhabha Atomic Research Centre, Trombay, Mumbai-400094}

$\mathrm{C}$ ellular redox status is determined by a balance between levels of ROS inducers and ROS scavengers. The regulation of redox homeostasis is vital for maintaining cellular health and normal functions. The cells have intrinsic as well as extrinsic inducers of ROS including mitochondrial ETC, metabolic defects, ER stress, oncogenes, hypoxia, xenobiotics and ionizing radiation. The scavengers of ROS in the cells include a battery of antioxidant genes controlled by NRF-2 and small molecules reduced glutathione, NADPH anddietary antioxidants. Modulation of cellular redox is a promising anti-cancer strategy. Thioredoxin and thioredoxin reductase system regulated by NRF-2 also play a vital role in maintenance of cellular redox homeostasis. We have used in-silico docking and high content screening to identify novel modulators of NRF-2 and TRX/TRXR system which disrupt cellular redox homeostasis. Docking studies revealed that baicalein (5,6,7 trihydroxy flavone) isolated from dried roots of Scutellariabaicalensis (Arjuna) could bind to the active site of thioredoxin reductase. Baicalein treatment led to significant reduction in the activity of thioredoxin reductase and nuclear levels of thioredoxin-1 thereby increasing ASK1 levels in lymphoma cells. We established a causal role of cellular redox modulation in potent anti-cancer efficacy of baicalein using CRISPRCas9 based knock-out of ASK1 and over-expression of 
thioredoxin-1. Besides showing anti-tumor efficacy, baicalein exhibited anti-inflammatory action via inhibition of NF-KB transactivation through modulation of TRX without inhibiting the DNA binding efficacy of $\mathrm{NF}-\mathrm{kB}$. Baicalein also activated Nrf-2 via inhibition of MKP3 and activation of ERK. The activation of NRF-2 was associated with increased abundance of hematopoietic stem cells and mitigation of ionizing radiation induced toxicity in vivo. We have established a causal role of NRF-2 using chemical inhibitors and knockout mice. Taking clues from the efficacy studies and its mechanism of action, we are preparing nanoformulations for increasing the therapeutic potential of this candidate drug.

\section{IL- 49}

\section{Malabsorption Function Tests In Various Diseases}

\section{SatyaVati Rana}

Department of Super Specialty Gastroenterology, Post Graduate Institute of Medical Education \& Research, Sector 12, Chandigarh, India

$\mathrm{M}$ alabsorption is a condition caused by the decreased ability to digest and/or absorb nutrients from food. It can be seen in various diseases. Various biochemical tests are available that are used to diagnose maldigestion and malabsorption like lactose hydrogen breath test, Urinary D- Xylose test, Fecal Pancreatic Elastase measurement. Lactose Hydrogen breath test is a noninvasive and inexpensive technique which is used to measure lactose intolerance/ malabsorption. Urinary Dxylose test is used to estimate the change in integrity of small intestine due to various diseases. Fecal pancreatic elastase is measured to establish the exocrine function of the pancreas in diseases like Chronic Pancreatitis. The importance of these malabsorption tests in different diseases such as Celiac disease, Type 2 Diabetes Mellitus etc will be presented and discussed.

\section{IL- 50}

\section{Exploring Semen Protein Signatures For Understanding Male Fertility/Infertility In Acute Lymphoblastic Leukemia (All) Survivors}

\author{
$\underline{\text { Savita Yadav }}{ }^{* 1}$, Paras Jain ${ }^{1}$, Sarman $\operatorname{Singh}^{2}$ \& \\ Sameer Bakhshi ${ }^{3}$ \\ 1. Department of Biophysics, All India Institute of \\ Medical Sciences, New Delhi-110029 \\ 2. Department of Laboratory Medicine, AIIMS, \\ New Delhi-110029 \\ 3. Department of Lab Oncology,IRCH,AIIMS, \\ New Delhi- 110029
}

INTRODUCTION: ALL patients have a high chance of survival as a result of new treatment protocols that combines chemotherapy and radiation therapy. Infertility is one of the major consequences of these treatments. Sperm count and motility are done in routine, but they provide only a brief idea about fertility status. This study was undertaken since no proteomic based study for fertility status of treated cancer survivors was currently available which can detect or predict differential proteomics of cancer survivors. Aim of this study was to focus on differentially expressed proteins in semen of ALL survivors with normal persons of same age group.

MATERIAL AND METHOD: The major spotlight of this study is to explore the differential proteomics of human semen to find differentially expressed proteins with normal persons of same age group. Then identification of differentially expressed proteins in ALL survivors by MALDI-TOF/MS followed by validation of differentially expressed proteins in cancer survivors by western blotting and Immunoassay (ELISA).

RESULTS: Total 50 samples were used in this study out of them 30 samples were found to be having normal semen parameters. DIGE experiments were done to know the differentially expressed proteins in patients having normal semen parameter when compared with normal controls, 24 differentially expressed spots were identified, then these differentially expressed spots subjected to MALDI/TOFF experiment 8 differentially expressed proteins were identified, out of them 3 proteins were selected on the basis of their properties, mascot 
score and fold change and confirmed by Western blotting and then validated by ELISA in large number of samples.

CONCLUSION: Overall the differential expression proteomic study of semen of cancer survivors is needed to answer the role of semen proteins in maintaining the fertility of such individuals. The change in expression of these proteins in cancer survivorsmay provide the information about the adverse effects of cancer treatment on fertility; it will help in designing some cure related to fertility, which may further help in improving fertility in ALL survivor men. These proteins can be used in developing strategy for ALL survivors and aid those patients in future to father their own child. ALL patients may be advised for stem cell (spermatogonia) preservation before start of treatment thereby helps to overcome mental stress and social stigma related to infertility.

KEY WORDS: Semen, Differential expression, Protein signatures, Acute Lymphoblastic Leukemia, Male fertility

\section{IL- 51}

\section{Anemias and Variant Windows - A Challenge For Interpretation of Glycated Haemoglobin}

\author{
Bhargava S, Mahato K, Chugh P, Manocha A, Kankra
} M, Singla P, Sharma A, Gupta V, Jose S.

Sir Ganga Ram Hospital, New Delhi

INTRODUCTION: The process of glycation is nonenzymatic and irreversible, attaching a sugar residue to the amino terminal of proteins. Glycatedhaemoglobin (HbA1c) is the major glycated protein measured in the laboratory for purpose of clinical evaluation and management. The two major variants that affect this process are the blood glucose concentration and the life span of the red blood cells (RBC). Therefore, indirectly the haemoglobin also affects the process of glycation and the recommendation is to interpret $\mathrm{HbA} 1 \mathrm{c}$ with caution in presence of anemia. Many studies have evaluated the correlation between average blood glucose and $\mathrm{HbA} 1 \mathrm{c}$, but there are very few studies that have assessed the alteration in $\mathrm{HbA} 1 \mathrm{c}$ with haemoglobin levels; there are no guidelines to help the clinician in interpretation in the presence of anemia. Hence, this study was conducted to evaluate the correlation between $\mathrm{Hb}$ and $\mathrm{HbA} 1 \mathrm{c}$ when there is anemia. Also, the manufacturers' claim that a haemoglobin variant below $60 \%$ does not affect the HbA1c measurement was evaluated.

MATERIALS AND METHODS: Consecutive subjects were enrolled over a period of one month. All patients with co-morbidities were excluded. The enrolled 1211 subjects were divided into four groups on basis of their haemoglobin level. Group 1 included male subjects with $\mathrm{Hb} \leq 13.0 \mathrm{~g} / \mathrm{dl}$, group 2 included female subjects with $\mathrm{Hb} \leq 12.0 \mathrm{~g} / \mathrm{dl}$, group 3 included male and female subjects with $\mathrm{Hb} \leq 10.0 \mathrm{~g} / \mathrm{dl}$ and group 4 included all subjects with $\mathrm{Hb} \leq 8.0 \mathrm{~g} / \mathrm{dl}$. HbA1c was measured by HPLC on the BioRad Variant Turbo. Out of these 1211 subjects 45 had a variant window of $<60 \%$. These 45 subjects were further subdivided into two groups-one (group A) with a variant window between $30 \%$ and $60 \%$, and the other (group B) with a variant window of less than $30 \%$. The correlation between their random blood glucose and glycated haemoglobin was derived. The first 40 subjects were selected with random blood glucose varying from $98 \mathrm{mg} / \mathrm{dL}$ to $350 \mathrm{mg} / \mathrm{dL}$ and their fructosamine and albumin levels were estimated. Taking a cut-off of RBG as $160 \mathrm{mg} / \mathrm{dL}$ (the upper limit of the biological reference interval), serum fructosamine cut off value was also estimated.

RESULTS: As haemoglobin decreases, the correlation (r) between random blood glucose and glycated haemoglobin decreases, but the significance remains the same ( $\mathrm{p}<0.001)$, till the haemoglobin reaches $8.0 \mathrm{gm} / \mathrm{dl}$. Below this haemoglobin, the significance decreases significantly ( $\mathrm{p}=0.022)$, even though it is still significant. The correlation of glycated haemoglobin with random blood glucose was significant $(\mathrm{p}=0.017)$ in patients with variant window $<60 \%$, but this significance was markedly increased $(\mathrm{p}<0.001)$ in those with a variant of less than $30 \%$. To derive a cut-off for fructosamine corresponding to a high blood glucose (>160 mg/dl), a receiver operating curve analysis was done, which revealed a cutoff of $340.5 \mu \mathrm{mol} / \mathrm{L}$.

Conclusion: Glycated haemoglobin should be interpreted 
with great caution when haemoglobin is $<8.0 \mathrm{gm} / \mathrm{dl}$ and when the variant window is $>30 \%$. The cutoff for normal fructosamine may be much higher in our population as indicated by our results of 40 subjects; hence, a multicentricstudy may be needed to establish the BRI for our Indian population.

\section{IL- 52}

\section{Growth Differentiation Factor-15 As A Stress Responsive Cytokine In Changing of Lifestyle Diseases: An Expression of Metabolic Syndrome}

\section{S Prakash}

Department of Laboratory Medicine, AIIMS, New Delhi-110029

$\mathrm{M}$ etabolic syndrome is a major global public health challenge due to increased consumption of fatty foods, calorie rich food and inadequate physical activity that altered the levels of glucose, HbA1C, glucagon, lipids or methyl glyoxal and subsequently releases a novel cytokines i.e. growth differential factor -15 (GDF15). Lifestyle modification with the diet and exercise adjustment is the core therapeutic approach for NASH. GDF-15 has pivotal role and their expression has been linked with oxidative stress, protein glycation, and inflammation changes in various diseases state conditions.Non alcoholic fatty liver disease has become a common cause for the chronic liver disease and recently it has been observed that diet, genetics and lifestyle contribute to the onset and progression of NASH which may progress to cirrhosis and hepatocellular carcinoma. Blood samples were collected from the OPD of Gastroenterology, and department of Medicine AIIMS, New Delhi. Each of them was screened for serology and subsequently patients were sub-grouped for NASH $(\mathrm{n}=42)$, HBV $(\mathrm{n}=56), \mathrm{HCV}(48)$ and non insulin dependent diabetes mellitus $(n=44)$. Forty five healthy subjects were also included and screened for anti HCV, HBsAg and HBeAg in the study. Clinical, biochemical, haematological investigations were done for all subjects. Evidence suggest that various biomarkers such as adiponectin, leptin, Insulin Resistance, BMI, proinflammatory and anti-inflammatory cytokine,oxidants stress index, micronutrient antioxidants, genetic polymorphism such as SNPs and GDF-15 are associated in metabolic abnormalities. GDF15 levels were significantly higher in NASH subjects than in controls $(\mathrm{P}=.014)$. Among NAFLD patients, the highest quartile of GDF15 levels was significantly associated with a risk of higher viral loads even after adjustment for age, gender, body mass index, hypertension, diabetes, aspartate aminotransferase, platelet, albumin, insulin resistance and low HDL but not with NASH risk. Multivariate analysis may provide the significant information with the association between age, genders, waist circumference, hypertension, insulin resistance. GDF-15 levels have some hidden relation to changes in body weight, waist hip ratio, BMI and HOMA IR. Our finding suggests that high circulating GDF-15 levels may serve as a novel biomarker of NAFLD hence there is a need to develop anti-fibrotic micronutrient antioxidant that can improve the quality of life.

\section{IL- 53}

Cathepsin L And B As Potential Markers For Liver Fibrosis: Insights From Patients And Experimental Models.

\section{Shyam S. Chauhan}

Department of Biochemistry, All India Institute of Medical Sciences, Ansari Nagar, New Delhi-110029

INTRODUCTION: Lysosomal cysteine proteases cathepsin L (CTSL) and B (CTSB) play crucial role in extracellular matrix (ECM) degradation and tissue remodeling, which is a prominent feature of fibrogenesis. The aim of this study was to determine the role and clinical significance of these cathepsins in liver fibrosis.

MATERIALS AND METHODS: Hepatic histological CTSL and CTSB expression were assessed in experimental models of liver fibrosis, patients with liver cirrhosis, chronic viral hepatitis, and controls by realtime PCR and immunohistochemistry. Plasma levels of CTSL and CTSB were analyzed in 51 liver cirrhosis patients (Child-Pugh stages A, B and C) and 15 controls.

RESULTS: Significantly enhanced CTSL mRNA 
$(\mathrm{P}=0.02)$ and protein $(\mathrm{P}=0.01)$ levels were observed in the liver of carbon tetrachloride-treated mice compared with controls. Similarly, hepatic CTSL and CTSB mRNA levels $(\mathrm{P}=0.02)$ were markedly increased in Abcb4-/(ATP-binding cassette transporter knockout) mice compared with wild-type littermates. Elevated levels of CTSL and CTSB were also found in the liver $(\mathrm{P}=0.001)$ and plasma $(\mathrm{P}<0.0001)$ of patients with hepatic cirrhosis compared with healthy controls. Furthermore, CTSL and CTSB levels correlated well with the hepatic collagen $(\mathrm{r}=0.5, \mathrm{P}=0.007 ; \mathrm{r}=0.64, \mathrm{P}=0.0001)$. CTSL and CTSB levels increased with the Child-Pugh stage of liver cirrhosis and correlated with total bilirubin content ( $r=0.4 / 0.2 ; \mathrm{P}$ ?0.05). CTSL, CTSB, and their combination had a high diagnostic accuracy (area under the curve: $0.91,0.89$ and 0.96 , respectively) for distinguishing patients from controls.

CONCLUSION: Our data demonstrate the over expression of CTSL and CTSB in patients and experimental mouse models, suggesting their potential as diagnostic biomarkers for chronic liver diseases.

KEY WORDS: Proteases, Fibrosis, extracellular matrix, Immunohistochemistry, Real time PCR

\section{IL- 54}

\section{Deciphering The Role of Circadian Rhythm In Fibromyalgia Syndrome Patients}

\section{Siddharth K. Das ${ }^{1}$, Ghizal Fatima ${ }^{2}$}

1. Head, Department of Rheumatology, KGMU, Lucknow,

2. Assistant Professor, Department of Molecular Medicine, ELMCH, Lucknow.

INTRODUCTION: Fibromyalgia syndrome (FMS) patients have disturbed sleep patterns which may lead to altered circadian rhythm in hormones and cytokines.Therefore, aim of this study was to appraise the circadian rhythm disturbances in hormonal profiles (Melatonin, Serotonin and Cortisol) and cytokines (IL$6 \&$ TNF-alpha) and to correlate it with BMI and FIQR in women with FMS.
METHODS: Hormonal profiles and cytokines were measured, by ELISA, every 6 hours during $24 \mathrm{hr}$ period in 50 female patients satisfying ACR criteria for FMS, and 50 healthy females without FMS. FMS was assessed by FIQR. BMI was calculated by a standard formula of weight in kilograms/height in meter square.

RESULTS: A significantly higher cortisol levels were observed in the evening $(\mathrm{p}<0.05)$ and midnight $(\mathrm{p}<0.01)$ in patients groups. Significantly higher melatonin levels were observed in morning $(\mathrm{p}<0.01)$, and midnight hours $(p<0.01)$ in patients group. Serotonin levels were lower among patients throughout the day. Serum TNF-? levels were substantially increased in patients but showed no circadian variation. In contrast, no difference in the levels of IL-6 was found. Melatonin morning levels were positively correlated with FIQR among patients group.Mid-night level of cortisol was found negatively correlated with FIQR in patients group. FIQR was found negatively correlated with serotonin in morning and midnight among patients group.

CONCLUSION: There is an irregularity in circadian rhythm of serum cortisol and melatonin in FMS patients. BMI and FIQR were found associated with the disturbed levels of cortisol, melatonin and serotonin in FMS patients. However, no changes in cytokines were observed.

KEY WORDS: Hormonal profiles, Cytokines, Fibromyalgia syndrome

\section{IL- 55}

\section{Formulating Competencies In Biochemistry}

\section{Sucheta Dandekar}

Professor and Head, Department of Biochemistry, Seth G. S. Medical College and KEM Hospital, Acharya Donde Marg, Parel, Mumbai 400012. Maharashtra, India

$\mathrm{I}^{\mathrm{n}}$ n order to support the current trends of events towards competency based education, this talk shall elaborate on the development of learning objectives, competencies, teaching learning methods, assessment strategies, 
clubbed to the different degrees of expertise, from novice to expert.

One particular topic will be chosen from the undergraduate curriculum and the audience shall be walked through the detailing of the competencies.

It is hoped that the deliberations that follow shall help the formulation of a complete set of competencies and prepare us towards a new era in Biochemistry.

\section{IL- 56}

\section{Lifestyle Disorders}

Sudhir Mehrotra, Pushkar Singh Rawat

Department of Biochemistry, University of

Lucknow-226 007 U.P.

$\mathrm{L}$ ifestyle disorders are the health disorders due to careless and vegetative living or the diseases which are linked to the bad or sluggish way of life and wrong eating habits.

The main risk factors of these lifestyle disorders are alcohol, drugs, junk food, adverse nutrition, lack of physical activity, stress etc. Lifestyle disorders include heart diseases, diabetes type 2, respiratory diseases, cancer, mental health issues, obesity etc. These disorders are causing about $50 \%$ mortality worldwide. India is second largest capital of the lifestyle disorders following China.

Prevention is the only remedy that aims to reduce the rate of people suffering from lifestyle disorders. They can be prevented by living a balanced and stress free life. A balanced and stress free lifestyle can be achieved by adopting healthy nutritional diet, regular physical and mental exercise, refraining from smoking and alcohol drinking, limiting the use of cellular phones etc.

The government should also undertake the task of educating people about the health hazards linked to the sort of lifestyle people, intentionally or unintentionally undertake. Many efforts have been made to aware people of the hazards related to smoking and drinking, but the current scenario of mortality by cancer, lung, liver and heart damage clearly leaves much to be done.

Not only parents, but the educational institutions should also promote healthy living by holding counselling sessions to persuade the young generation to use less mobiles and spend time with the nature, giving stress on physical activities. The lecture would cover various aspects of causes, consequences and remedial measures for the abatement of various common lifestyle disorders in India such as cardiovascular diseases, cancer, type II diabetes mellitus, obesity related disorders which are leading causes of health related issues along with mortality.

\section{IL- 57}

\section{Phytochemicals: A Possible Herbal Drug For The Treatment Of Post Prandial Hyperglycemia}

\section{Dr. Suman Bala Sharma}

Director-Professor, Deptt of Biochemistry, University College of Medical Sciences, (University of Delhi) Dilshad Garden, Delhi- 110095

$\mathrm{D}$ iabetes is a group of metabolic diseases characterized by hyperglycemia resulting from defects in insulin secretion, insulin action, or both.Currently, there is renewed interest in plant-based medicines and functional foods modulating physiological effects in the prevention and cure of diabetes and obesity. The plant kingdom is a wide field to search for natural effective oral anti-hyperglycemic agents that have minimum side effects. More than 1200 plant species have been recorded to be used empirically worldwide for their alleged anti-hyperglycemic activity.Recently, considerable research has been focused on postprandial hyperglycemia apart from fasting glycemic control because it doubles the risk of death from cardiovascular disease. Therefore, phytochemicals (natural compounds)showing improvement in post-prandial hyperglycemia via alpha-glucosidase and alpha-amylase inhibition from plant sources offer an attractive strategy for the control of type 2 diabetes. Guanidium compounds have been reported to possess anti-diabetic activities from last 3 decades. Metformin, a biguanide, is a widely used 
drug against diabetes and was derived from medicinal plant. This study highlights the novel anti-diabetic compound isolated and purified from aqueous extract of germinated Glycine max seeds. The compound contains guanidine groups and has been designated as Glynide (patent filed) which was found to be beneficial in controlling both fasting and post prandial hyperglycemia by showing salutary effects on glycemic index and inhibition of alpha-glucosidase and alphaamylase.Postprandial hyperglycemia is characterized by hyperglycemic spikes that induce oxidative stresswhich increases the risk of atherosclerosis in diabetics. Glynide also showed significant antioxidant activity as well as potential in correcting dyslipidemia in STZ+NAD induced diabetic rats.

Conclusion: Hence, Glynide can be a single anti-diabetic compound which can be used for controlling both fasting as well as post prandial hyperglycemia and also reduces the risk of atherosclerosis.

\section{IL- 58}

\section{HCMV mIR U170-3p: Its Biological Functions on Human Antiviral Mechanisms}

\section{Sunil Babu Gosipatala}

Department of Biotechnology, Babasaheb Bhimrao Ambedkar University, Lucknow- 226025

$\mathrm{H}^{\mathrm{s}}$ uman cytomegalovirus (HCMV) is a ubiquitous pathogen causing significant morbidity and mortality in immunocompromised individuals and neonates. It exhibits seroprevelance of $\sim 60 \%$ in the United States and as high as $100 \%$ in developing countries of lower socioeconomic environments. HCMV is a DNA virus, belongs to ?- Herpesviridae family having genome of size $230-240 \mathrm{~Kb}$. Upon primary infection it establishes lifelong latency in its host, i.e., Humans. HCMV evolved with humans and exhibits various strategies to counter the human antiviral mechanisms for its survival in the human. The discovery of viral miRNAs and their spectacular biological roles, attracted us to investigate the role of HCMV miRNAs on human antiviral mechanisms. HCMV reported to encode 26 mature miRNAs from 16 precursor miRNAs, reported to exhibit immune evasive roles. By using various bioinformatic tools, we attempted to speculate the HCMV miRNAs role on apoptosis, autophagy etc. Our in silico results show HCMV miRNAs target the apoptosis and autophagy, HCMV miR, UL-70-3p \& UL148D target the apoptotic pathway. They target the mRNAs of extrinsic/intrinsic and ER-induced apoptotic pathways. In this paper, we discuss our in silico results, how HCMV uses its miRNA machinery in overcoming the antiviral mechanisms in benefitting its survival in the host.

KEY WORDS: Antiviral mechanisms, Apoptosis, autophagy, Human Cytomegalovirus (HCMV), Viral MiRNAs, UTR

\section{IL- 59}

\section{Molecular Medicine: Opportunities and Challenges}

\section{Swasti Tiwari}

Additional Professor \& Head, Department of Molecular Medicine \& Biotechnology, Sanjay Gandhi Postgraduate Institute of Medical Sciences (SGPGIMS), Lucknow

$\mathrm{M}$ olecular medicine holds great promise to improve patient care by increasing the precision and predictability of diagnostic procedures, and by individualizing and targeting therapeutic approaches. Recent technological advancements and biological discoveries have given a tremendous boost to the development of molecular medicine-based approaches. These developments have unprecedented possibilities to impact the fundamentals and practice of medicine, however, certain challenges needs to be overcome before that could happen. We, in the department of Molecular Medicine \& Biotechnology department at Sanjay Gandhi PGI, are using some of these approaches with the ambition to predict development of human diseases in a non-invasive/ less-invasive manner. My talk will highlight findings from some of these studies. In addition, recent advancements in Molecular Medicine, and associated challenges in their clinical implementation will also be discussed. 
IL- 60

\section{Strategies For Construction of Bacterial Vaccine Vectors For Mucosal Immunity}

\section{Thungapathra M, Anit Kaur}

Department of Biochemistry, Postgraduate Institute of Medical Education and Reserarch, Chandigarh, India.

$\mathrm{D}$ iarrhea is the second biggest killer of children globally, with more than 800,000 under-fives dying every year according to Unicef. A quarter of these deaths occur in India. Vaccines can effectively control the morbidity and mortality due to diarrheal diseases. Availability of multivalent vaccines for common diarrhoeal diseases is highly desirable. The prerequisite for developing multivalent vaccines is the availability of a safe vector. Genetically constructed live attenuated bacterial vectors may be derived from pathogenic bacteria or the commensal bacteria. This presentation describes the construction of a Vibrio cholera chassis which can be made to deliver the antigens of common mucosal pathogens. The implications of using such vaccine vectors on the mucosal immunity will be discussed

\section{IL- 61}

Utility of Serum Il-6, Il-8 Levels and Apache Ii Score For The Prediction of Clinical Outcome In Patients With Acute Respiratory Distress Syndrome

Deme Swaroopa $^{1}$, Kakarla Bhaskar ${ }^{2}$, Vijay Kumar Kutala ${ }^{3}$

Departments of

1. General Medicine,

2. Respiratory Medicine and

3. Clinical Pharmacology and Therapeutics, Nizam's Institute of Medical Sciences (NIMS), Hyderabad-500082, India

BACKGROUND AND AIM: Studies on potential biomarkers in experimental models of acute lung injury (ALI), and clinical samples from patients with ALI have provided evidence to the pathophysiology of the mechanisms of lung injury and predictor of clinical outcome.Because of the high mortality and substantial variability in outcomes in patients with ARDS, identification of biomarkers like cytokines is important to determine prognosis and guide clinical decisionmaking.

MATERIALS AND METHODS: In this study, we have included 30 patients admitted to ICU diagnosed with ARDS and serum samples were collected on day 1 and 7 and were analyzed for serum IL- 6 and IL- 8 by ELISA method and APACHE II scoring was done on day 1.

RESULTS: The mortality in the patients observed with ARDS was $34 \%$. APACHE II score was significantly higher in non-survivors as compared to survivors. There were no significant differences in gender, biochemical and hematological parameters among the survivors and non-survivors. Serum IL-6 and IL-8 levels on day 1 were significantly higher in all the ARDS patients as compared to healthy controls and these levels were returned to near normal basal levels on day 7. The serum IL-6 and IL-8 levels measured on day 7 were of survivors. As compared to survivors, the IL-6 and IL-8 levels were significantly higher in non-survivors measured on day 1 . Spearman's rank correlation analysis indicated a significant positive correlation of APACHE II with IL-8. By using APACHE II score, IL-6 and IL-8, the ROC curve was plotted and provided predicable accuracy of mortality (outcome) was $94 \%$.

CONCLUSION: The present study highlighted the importance of measuring the cytokines like IL-6 and IL8 in patients with ARDS in predicting the clinical outcome. 


\section{IL- 62}

\section{Iem and Nbs Awareness}

\section{Vikas Bhatia,}

Voice for no voice people, Founder Member MERD India (parent support group)

$\mathrm{I}_{\mathrm{r}}^{\mathrm{n}}$ India, the health care priorities are still limited to reducing the Infant Mortality Rate and meeting basic infrastructure requirements. The Government of India is yet to have a mandatory screening program for newborn babies.

Every Newborn has a right to lead a healthy and normal life and the first step towards that is to get a Newborn Screening (NBS) Test. As the Newborn cannot speak for itself it is the responsibility of healthcare providers, the parents, the society and the government to make NBS available for every Newborn.

Medical fraternity must be aware that besides communicable diseases there are almost lessthan equal number of newborns who are suffering because of-noncommunicable diseaseslike treatableIEMs.

Treatable IEMs are those set of disorders which if detected \& properly treated before the onset of symptoms can help a Newborn lead a very normal and healthy life. The only way to detect them is by routinely performing screening tests on all newborns (normal or otherwise). Apart from infectious diseases, IEMs could be the largest group of disorders, completely hidden from public and government eye.If we look around, may be many family could have lost or affected children in inexplicable circumstances, probably due to IEMs.

In center where-NBS is available and when it come positive, then confirmation of the diagnosis is required. here very less centers there with such facility so experts with their available connections send the samples aboard, by the time the results are available, mostly many children would died, so we should be set up reference labs so that confirmation of diagnosis will be at the earliest and many children can be saved.

IL- 63

\section{Time Waits for Nobody: Possible Impact of Disrupted Life-Style on Human Health}

Vinod Kumar

IndoUS Center for Biological Timing, Department of Zoology, University of Delhi, Delhi 110 007, India;

daptation to the surrounding environment is of
crucial importance to life. Biological systems regulate their internal environments as well as their relationships to the outside world. The regulatory mechanism usually involves negative feedback, which always generates oscillations since there is bound to be delay in the feedback loop. The period of these rhythms can be from minutes to several months. In all vertebrates, the circadian $($ circa $=$ about; dian $=$ day $)$ organization, showing rhythmicity of about a day, is driven by a central oscillation generating system. There are also circadian oscillators in the peripheral tissues. Long livedspecies also appear controlling the time and duration of the different life-history stages in the year using another clock system, called the circannual (circa = about; anuum = year) system. Modern human lifestyle with extended hours of wakefulness and associated extended frequent feeding and exposure to light significantly and chronically perturbs the circadian system, profoundly enhances risk factor for diabetes, obesity cancer and early aging. Also, humans like many vertebrates show robust seasonal rhythms in their health and wellbeing; the birth month has been suggested to have the effects that might persist throughout life. Human lifestyles under artificial conditions of 'eternal summer' provide the most extreme example for disconnects from the natural season, and this in the long-run may make humans vulnerable to increased morbidity and mortality.In this lecture, I intend to emphasize the need for a better understanding of the daily and seasonal biology against the backdrop of rapid changes in the climate and lifestyles and other anthropogenic impacts in the surroundings. Interestingly, the lecture is to arouse enthusiasm among medical scientist, since for most part my lab continues to work in the area of basic science research using diurnal birds as the model system. Like humans, birds are diurnal, have invaded almost all walks 
of the ecosystems, and hence offer an excellent experimental system to understand the mechanism of temporal adaptation without pre-disposition to the metabolic syndrome and early agingas in humans. For example, migratory birds show hyperphagia, body fattening and gain weight up to $200 \%$, and exhibit concurrent gonadal growth under long days. This isspontaneously followed by decrease in food intake, body fat store with total loss of gained body mass, and gonadal regression. However, surprisingly, there is no report of any metabolic diseases including diabetes or early aging in these birds.

\section{IL 64}

\section{Circulatory miRNA:Novel Tool for Diagnostic and Therapeutic Biomarker for Cardiovascular Disease}

Wahid Ali, Mabood Khan, Mohd Tasleem

Post Graduate Department of Pathology, King George's Medical University, Lucknow

$\mathrm{C}$ ardiovascular diseases (CVDs) have now become the leading cause of mortality in India. 30 million heart patients are residing in India, where 14 million are in urban areas and 16 million in rural areas. More than $80 \%$ of sudden cardiac deaths in the world are caused by atherosclerotic coronary artery disease (CAD), and the remaining $20 \%$ of cases are caused by other diseases including congenital heart disease, cardiomyopathies, aortic valve disease, left ventricular hypertrophy and other cardiac disorders. One of the major challenges in cardiovascular disease is the identification of reliable biomarkers in serum/plasma. In the past few years, the crucial role of different micro-RNAs (miRNAs) in the cardiovascular system has been widely recognized. Recently, it was discovered that extracellular miRNAs circulate in the bloodstream and that such circulating miRNAs are remarkably stable. This extracellular miRNA stability is due to packaged with lipid vesicles or by being associated with protein or lipoprotein complexes. Although the precise cellular release mechanisms of miRNAs remain largely unknown. The identification of stable circulating miRNAs challenges a number of concepts and launches a new generation of potential biomarkers. Various circulatory miRNAs like miR-1, miR133, miR122, miR-126, miR-129 and miR155 are known to be stable blood based biomarkers for cardiovascular disease. These findings have raised the possibility that circulatory miRNAs may play a role as diagnostic and therapeutic biomarkers in cardiovascular disease.

KEYWORDS: CVD, CAD, miR133, miR122, miR155, Biomarker, Serum, Plasma.

\section{IL 65}

\section{Sperm DNA Damage, Protamines and Oxidative Stress In Infertile Men}

\section{Dr. Z.G. Badade}

Dept. of Biochemistry, MGM Medical College, Navi Mumbai-410209

$I^{n}$ mpairment of spermatogenesis and defective sperm function is the most prevalent cause of male infertility. Recent reports indicate that increasing male infertility could be due to genomic abnormalities. The etiology of sperm DNA damage is multi-factorial and compromised due to nuclear defects, protamine deficiency and oxidative stress. In present study, we aim to assess sperm DNA integrity, protamine-1/protamine-2 ratio, oxidative stress markers and semen characteristics in infertile and fertile men.

The study includes fertile controls and infertile men which are subgrouped into four groups based on result of semen analysis as normospermic infertile, oligospermic, asthenospermicand oligoasthenospermic men. Sperm DNA integrity in terms of DNA fragmentation Index (DFI), P1/P2 ratio, TOS, TAC and OSI are assessed in fertile and subgroups of infertile men.

DNA fragmentation Index is significantly elevated and $\mathrm{P} 1 / \mathrm{P} 2$ ratio is significantly high in subgroups of infertile patients as compared to control. TOS, OSI are found to be significantly increased whereas TAC levels are significantly decreased in all subgroups of infertile patients as compared to control $(\mathrm{p}<0.001)$. We observed relatively increase linear trend of DNA damage, P1/P2 
ratio and OSI from normospermic, oligospermic, asthenospermic to oligoasthenospermic infertile men.

Male infertility irrespective of normal and abnormal semen parameters may result due to seminal oxidative stress induced DNA damage or alerted protamine ratio. Therefore, evaluation of DFI, protamine ratio and OSI can be used for diagnosis, prognosis of male infertility in addition to routine semen parameters in case of infertility with unknown origin, which can be an additional measure to infertility evaluation to decide the treatment strategies.

\section{IL 66}

\section{Regulation of Monocyte NADPH Oxidase: Role of Pattern Recognition Receptors}

\section{Deena H. Elsori}

Activation of the NADPH oxidase enzyme complex results in the production of the oxygen free radical, superoxide anion (O2.-). Superoxide anion is critical for host defense against fungal and bacterial pathogens and efficient immune responses; however, uncontrolled monocyte-derived O2.- may contribute to chronic inflammation and tissue injury. We have previously identified several pathways that regulate the activity of NADPH oxidase in human monocytes; however, the receptor(s) responsible for the activation of NADPH oxidase in primary human monocytes have not yet been determined. This study shows that pattern recognition receptors, namely Dectin-1 and Complement Receptor 3 , are essential for regulating NADPH oxidase activity in Zymosan-activated human monocytes. We show that TLR2 and TLR4 are not required for NADPH oxidase activation by Zymosan. In addition, this study focuses on Dectin-1 downstream signaling and complex formation with intracellular signaling proteins. Our findings in human monocytes are supportive of the prior recognized role of Src and Syk tyrosine kinases in regulating Dectin-1-mediated ROS production in murine macrophages. Our data also shows that Src and Syk are tyrosine phosphorylated in Zymosan- treated cells and that they both regulate each other's activity. Furthermore, we focused on Dectin-1 complex formation with intracellular signaling proteins including Syk, Src and $\mathrm{PKC} \delta$, protein kinases that regulates NADPH oxidase activity in human monocytes. This is the first study to show the involvement of PKC $\delta$ in Dectin-1 signaling. We found that the activity of PKC $\delta$ is required for its own complex formation with Dectin-1 as well as SykDectin-1 interaction. In contrast, Src and Syk inhibitors had no effect on PKC $\delta$ association with Dectin-1. Blocking the activity of Src inhibited phospho-Syk/ Dectin-1 complex formation which supports the role of Src in regulating Syk tyrosine phosphorylation/ activation. Our data confirms that Dectin-1, a pattern recognition receptor, is a key player in the regulation of NADPH oxidase in Zymosan-activated human monocytes and we introduce PKC? as a novel player in Dectin-1 signaling. To expand on this project, the study also includes data reporting an endogenous pathophysiological protein, osteopontin, as a novel ligand for Dectin-1. We show here that osteopontin induces the oxidative burst in monocytes through Dectin-1. We also show data supporting binding of osteopontin to Dectin-1.

Taken together, our study provides new insights into Dectin-1 ligands and downstream signaling in primary human monocytes and highlights novel signaling pathways utilized in these important cells. Our findings are relevant for understanding the regulation of NADPH oxidase in the innate immune response and in chronic inflammatory diseases. 


\section{IL 67}

\section{Drug Metabolizing Genes as Biomarkers for Predicting Tobacco Induced Cancers}

Devendra Parmar, Vinay Yadav, Feza Hasan, Sudhir Singha, Madan Lal Brahma Bhatta, Rahat Hadib and Tridev Katiyar

Developmental Toxicology Division, CSIR-Indian Institute of Toxicology Research, M.G.Marg, Lucknow;

a. Department of Radiotherapy, King George's Medical University, Lucknow;

b. Department of Radiation Oncology, Dr. R.M.L Institute of Medical Sciences, Lucknow

$\mathrm{T}$ obacco induced cancers including head and neck squamous cell carcinoma (HNSCC) and lung cancer are among the most common malignancies that possibly involves a combination of exposure to the carcinogens as well as inheritance of genetic differences in the enzymes catalyzing their metabolism and detoxification. Our studies have shown that variant genotypes of drug metabolizing enzymes such as cytochrome P450s (CYPs) and glutathione S-transferases (GSTs) modify the risk to tobacco induced squamous cell carcinoma of head and neck (HNSCC) and lung. Prevalence of certain CYP haplotypes (haplotypes of CYP1A1, 1B1 and 2E1) and combinations of genotypes of CYPs and GSTs in cases demonstrated the importance of gene-gene interactions in determining the risk to these malignancies. Likewise, several fold increase in the risk in tobacco users (both in the form of chewing or smoking) have indicated the importance of interaction of environmental risk factors in determining the risk to the malignancy of head \& neck or lungs. To determine if the susceptible genotype changes are associated with phenotypes of increased resistance or susceptibility, studies were initiated to identify the expression and regulation of PAHmetabolizing CYP1A1 and 1B1 isoenzymes in freshly prepared rat peripheral blood lymphocytes (PBL) isolated from the control and cases suffering from lung cancer. RT-PCR studies demonstrated significantly higher mRNA expression of polycyclic aromatic hydrocarbon (PAH)-responsive CYP1A1 isoenzyme in the freshly prepared PBL isolated from lung cancer cases. This increase in the mRNA expression was found to be associated with an increase in the catalytic activity of CYP1A1 in freshly prepared PBL. Interestingly, the variant genotypes of CYP1A1 in the controls were found to be associated with much higher increase in the expression of blood lymphocyte CYP1A1 when compared to those with wild type genotypes. Further, the cases with variant genotypes of CYP1A1 showed much higher increase in the mRNA expression of CYP1A1 and its associated activity in the freshly prepared PBL when compared to the controls. These studies have led us to conclude that CYPs could not only be used as a tool to predict susceptibility to environment induced malignancies but, importantly, integrating expression and functional data with genetic deficiencies may allow more precise identification of biomarkers that may help to identify true risks of exposure and diseases.

\section{IL 68}

Epigenetic Mechanisms Regulate Placental Genes In Normal and Pathological Pregnancies: Search For Novel Fetal Dna Epigenetic Marker

Rahat $\mathrm{B}^{1}$, Hamid $\mathrm{A}^{2}$, Bagga $\mathrm{R}^{1}$, Kaur Jyotdeep ${ }^{\mathbf{1}^{*}}$

1. Postgraduate Institute of Medical Education and Research, Chandigarh -160012, India;

2. Indian Institute of Integrative Medicine, Jammu 180001, India

$\mathrm{P}$ acental development involves a complex structural and physiological reorganization during pregnancy, directed towards the establishment of a continuous nutrient supply to the developing fetus and proper maintenance of pregnancy. Placentation, thus, resembles tumourigenesis in many aspects like rapid cell division, migration and invasion, overlapping gene expression profiles and escape from immune detection and is hence referred to as pseudomalignant tissue. Regulation of placental development involves a highly complex differential global gene expression profile varying with gestational age.The importance of these intrinsic molecular controls become increasingly evident in abnormal pregnancies that are characterized by aberrant trophoblast proliferation/invasion like preeclampsia and hydatidiform mole. However, the molecular and 
epigenetic mechanisms involved in the regulation of various malignancy and invasion related genes during the advancement of gestational period and their aberrations in pregnancy related disorders have largely been unknown. Therefore in this study, we sought to examine the epigenetic regulation of various molecular events during normal pregnancy and their dysregulation in pregnancy related disorders. Based on the previous knowledge regarding the presence of fetal DNA in maternal plasma, we further extended. our study to search for fetal epigenetic markers in maternal blood, exploiting the differential methylation pattern of genes in maternal blood and placenta.

Pregnant women were enrolled in five groups, based on the three trimesters in normal pregnancy and two pregnancy related disorder groups viz, preeclampsia and hydatidiform. Placental villi sample and maternal blood was obtained from each pregnant woman. Search of fetal epigenetic marker in maternal plasma, based on the difference in methylation between maternal DNA and placental villi revealed a series of potential fetal epigenetic markers.We have also identified a few epigenetic fetal DNA markers that were specifically detected in pathological pregnancies viz, C-MYC and VEGF, hypermethylated form of which acted as marker for preeclamptic pregnancy and both preeclamptic and molar pregnancy respectively, while SNRPN, which was identified as hypomethylated pattern in maternal plasma acted as fetal epigenetic marker for molar pregnancy.

\section{IL 69}

\section{Inorganic Nanoparticles - An Effective Drug Delivery System Against Cancer}

\section{Mohd Sajid Khan}

Nanomedicine \& Nanobiotechnology Lab, Department of Biosciences, Integral University, Lucknow.

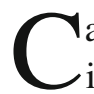
ancer is most common and prevalent disease which is responsible for millions of death per annum. Numerous drugs with several delivery systems have been emerged but none have provided satisfactory result. In the same direction nanotechnology has shown a substantial solidarity against cancer. Metal and metal oxide nanoparticle with encapsulated anticancer proteins or biomolecules, bioconjugated with highly potent single or multiple drugs (who have severe side effects) have been found highly effective against cancer at very much low concentration. In the given investigations the enzymatic gold nanoparticles have been synthesized by using a natural anticancer agent bromelain (a cysteine protease) as a reducing as well as a capping agent. Eventually, these NPs were used to bioconjugate with cisplatin alone, cisplatin along with doxorubicin, and cisplatin in combination with doxorubicin and Methotrexate. These combos were found highly effective against osteosarcoma at much lower concentration with minimum side effects due to the synergistic effect of bromelain with these bioconjugated drugs. Further, glucose encapsulated Cadmium Oxide nanoparticles (GCdO NPs) have been synthesized chemically by using glucose as a capping agent. The inhibitory role of GCdO NPs against Human Cervical cancer cell line-HeLa and Human osteosarcoma cell line- Saos- 2 by preventing early and lateglycation was also explored. These nanoparticles were found effective antiglycating agents and they were found to be highly effective against AGEs intervention. Also, 2D \& 3D conformational changes in capping protein during the enzyme mediated synthesis of gold nanoparticles was also studied by using characterization techniques fluorescence and circular dichroism spectroscopy.

KEY WORDS: Nanoparticles, Bromelain, Cisplatin, Doxorubicin, Methotrexare.

\section{IL 70}

\section{An In-Vivo And In-Vitro Approach To Establish A Link Between Ages And Lung Cancer}

Saheem Ahmad ${ }^{* 1,2}$, Hamda Khan ${ }^{1,2}$

1. Department of Biosciences, Integral University, Lucknow

1. IIRC-1, Laboratory of glycation biology and metabolic disorders, Integral University, Lucknow.

INTRODUCTION- The cellular response of advanced glycation end products (AGEs) is mainly mediated 
through the receptor for AGEs (RAGE). AGE-RAGE axis is believed to activate theintracellular signaling cascade which is accountable for the inflammatory reactions. Thisin turnmay further impact the initiation of various forms of tumours or cancers. In general, the association between hyperglycemia and the initiation of cancers has been the subject of investigation for many years. However, the association is not fully understood. The aim of this study was to establish a link between in vivo glycation model and cancer animal model. MethodsThis is the first study, which reported the detection of AGEs biomarkers in the cancer inin vivoanimal model. Furthermore, the data has been also reported for the comparative study between both in vivo glycation and cancer animal model. To find out the association of cancer and AGEs, we have also prepared in vitro glycation model system through A549 lung cancer cell line.

Results- In the present study the cell imaging, reactive oxygen species (ROS) measurement, spectrofluorometric analysis and ELISA, experiments showed that AGEs were found to be increased which might result in oxidative stress generation and cell proliferation in A549 cells. The glycation and oxidative markers in A-549 lung cancer cells were found to be detected and it implies that both the glycation and oxidation has detrimental effect on perturbed cells. Un-modified sample (control) had negligible effect on the A-549 cancer cells as expected.

CONCLUSION- This study would pave the way for the researchers to further probe for the establishment of AGEs and cancer.

KEYWORDS: Advanced glycation end product, Glycoxidation process, protein oxidation, AGEs biomarkers, ELISA.

\section{IL 71}

\section{Sensitization of Cancer Cells To Chemotherapeutic Agents By A Diterpenoid: Evidence For The Role of NF-k Band Lncrna Signaling}

Nikee Awasthee, Vipin Rai, Sumit S. Verma, Shruti Mishra, Subash C. Gupta

Laboratory for Translational Cancer Research, Department of Biochemistry, Institute of Science, Banaras Hindu University, Varanasi-221005, Uttar Pradesh, India

$\mathrm{T}$ he development of resistance mechanism by cancer cells to the existing drugs is a major roadblock to the cancer therapy. Therefore, agents that can sensitize the cancer cells to the existing drugs are needed.Accumulating evidence over the past several years has indicated that Mother Nature has been a "gold mine" for drug discovery. Here, we provide evidence that bharangin, a diterpenoid isolated from Premnaherbacea exhibit activities against multiple cancer types including breast cancer. Bharangin sensitizes cancer cells to doxorubicin and gemcitabine. The pro-inflammatory cytokine induced NF- B activation in cancer cells that was suppressed by bharangin. The diterpenoid also suppressed phosphorylation and degradation of I B, I B? kinase activation, p65 nuclear translocation and p65 phosphorylation all of which are required for NF- B activation. Immunoprecipitation and molecular docking studies indicated that bharangin can directly inhibit binding of p65 to DNA. The diterpenoid also suppressed the expression of proteins involved in tumor cell survival, proliferation, invasion and angiogenesis. Interestingly, the genetic deletion of p65 abolished the effects of bharangin. The diterpenoid also induced expression of tumor suppressor IncRNAs (MEG-3, MHRT, NEAT, GAS-5), while down-regulating oncogenic H19 expression. Furthermore, an abundance in the p65 expression was observed in breast tumor tissues. The pro-inflammatory transcription factor, NF-?B is known to be regulated by H19 IncRNA. Overall, the suppression of H19 expression and NF-?B activation by bharangin may contribute to its anti-cancer activities. 


\section{IL 72}

\section{Epigenetic Regulation of Cripto1, An Oncofetal Protein.}

Arnab Pal, Reetu Thakur, Roshan Verma, Jaimanti Bakshi, Amanjit Bal.

Post Graduate Institute of Medical Education and Research (PGIMER), Chandigarh, India, 160012

INTRODUCTION: Cripto1 (CR1), a member of the EGF-CFC protein family which differentially expresses during early embryogenesis, is involved in the regulation of different signaling pathways during fetal development. CR-1 is also an oncogenic growth factor involved in tumorigenesis through influencing cell proliferation, survival, migration and invasion. Over expression of CR1 is detected early embryonic life and in various cancers but how the expression of CR1 in cancer tissue is regulated is not clearly known. There are reports that CR1 auto-regulates its own expression but it does not explain the differential expression of CR1 in different phases of life. We proposed that CR1 is regulated by epigenetic regulations viz promoter DNA Methylation and histone modifications, like other oncofetal proteins. In this study, we analyzed the CR1 expression and concurrent $\mathrm{CR} 1$ promoter Methylation pattern in tumor tissue collected from OSCC patients as well as in immortalized cancer cell lines.

MATERIAL AND METHODS: Two cell lines Embryonal Carcinoma cell line (Ntera2) and Oral Squamous cell carcinoma (Cal27) and fiftybiopsy proven OSCC cases were included in the current study.CR1 mRNA expression and concurrent $\mathrm{CR} 1$ promoter methylation at CpGisland $\sim 0.5-0.8-\mathrm{kb}$ upstream to the Transcription start site (TSS)was analyzed by RT-PCR and bisulfite sequencing respectively.Expression of CR1 protein both soluble formand membrane bound form were also checked by ELISA and Immunohistochemistry respectively.

RESULTS: Ntera2 (Embryonal carcinoma cell line) shows significantly high CR1 mRNA and proteinexpression whereas Cal27 (Oral squamous cell carcinoma) shows very less expression of CR1. CR1 promoter methylation pattern analysis shows that all the
CpGsites at the $\mathrm{CpG}$ island are significantly hypomethylated in Ntera2 cell line and hyper methylated in Cal27 cells. Methylation pattern and expression analysis reveals that $\mathrm{CpG}$ island present upstream to the TSS site might have a role in the regulation of CR1 expression.

In case of OSCC patients $30 \%$ of cases shows high expression of CR1 mRNA.Methylation pattern analysis shows that there is a site specific patchy change in DNA Methylation, which may be explained by the presence of heterogonous cell population in tumor tissue, also evident by the IHC pattern.

CONCLUSION: Promoter DNA Methylation regulates the expression of CR1 in tumor cell lines.

KEY WORDS: Oncofetal Protein, Promoter DNA Methylation, OSCC, Ntera2, Cal27.

\section{IL 73}

Sparaoxonase-1, Lecithin Cholesterol Acyl Transferase, Apolipoprotein A-I And Hdl Cholesterolin Alcoholic Liver Cirrhosis

\section{Shashikant Nikam \\ Professor and Head, Department of Biochemistry, Belgaum Institute of Medical Sciences, Belagavi-590001, Karnataka.}

lcoholic liver cirrhosisand chronic liver disease are
the 10th leading cause of death for men. Alcoholic liver cirrhosis is the most common complication of ethanol abuse. Alcoholic fatty liver progresses to alcoholic hepatitis, cirrhosis and liver failure. Lipoproteins are synthesized by the liver and secreted into the circulation. Alcoholic liver cirrhosis causes alteration in lipoprotein metabolism producing liver steatosis and necrosis. ApolipoproteinA-I, the most abundant protein of HDL and acts as cofactor for lecithin cholesterol acyl transferase (LCAT).Paraoxonase-1 (PON-1) is an enzyme synthesized in liver and has an esterase activity towards lipid peroxides and circulates in plasma bound to High-Density Lipoproteins cholesterol (HDL-C). Considering these facts thestudy was planned to find out the role of PON-1, LCAT, 
ApolipoproteinA-I and HDL in relation to risk of atherosclerosis in alcoholic liver cirrhosis patients.

Several studies has been shown thatthe serum PON-1 activity, LCAT activity, Apolipoprotein A-I and levels of HDL cholesterol,were significantly reducedin patients with alcoholic liver cirrhosis. The study conclude that the decrease in PON-1, LCAT, Apolipoprotein A-I and HDL cholesterol in alcoholic liver cirrhosis may contribute to high risk of atherosclerosis in alcoholic liver cirrhosis patients.

KEYWORDS: Alcoholic liver cirrhosis, Paraoxonase1, Lecithin cholesterol acyl transferase, Apolipoprotein A-I, HDL

\section{IL- 74}

\section{Vitamin D: Molecular Actions Across A Variety of Biological Systems}

\section{HA Morris}

School of Pharmacy and Medical Sciences, University of South Australia,Chemical Pathology, SA Pathology, Adelaide, South Australia howard.morris@unisa.edu.au

$\mathrm{T}$ The well characterised endocrine pathway of vitamin D metabolism and its activities are solely responsible for vitamin $\mathrm{D}$ regulation of plasma calcium and phosphate homeostasis under control of serum 1,25dihydroxyvitamin $\mathrm{D}$, the biologically active metabolite of vitamin D. This pathway protects against the metabolic bone disease of rickets in children or osteomalacia in adults. The critical level for serum 25-hydroxyvitamin $\mathrm{D}$ to maintain adequate serum 1,25-dihydroxyvitamin D is $20 \mathrm{nmol} / \mathrm{L}(8 \mathrm{ng} / \mathrm{ml})$ and is synthesised by the kidney.In contrast adequate serum 25-hydroxyvitamin D protects against osteoporosis and reduces risk of fracture at a higher level becausethis activity depends on 1,25dihydroxyvitamin Dbeing synthesized bybone cells. This protective effect against fracture is only observed in combination with adequate dietary calcium intake. Similar metabolism of vitamin D protects against cancer death and infectious disease. Metabolism of 25hydroxyvitamin $\mathrm{D}$ to 1,25 -dihydroxyvitamin $\mathrm{D}$ by macrophages has been well described to enhance killing of microorganisms. The critical level for serum 25hydroxyvitamin $\mathrm{D}$ for metabolism by non-renal cells is 50 to $75 \mathrm{nmol} / \mathrm{L}$ ( 20 to $30 \mathrm{ng} / \mathrm{ml}$ ). Such autocrine actions of 1,25-dihydroxyvitamin $\mathrm{D}$ have now been demonstrated in skin, prostate, breast and colonic tissues, in these latter tissues to protect against cancer. In these tissues activities of vitamin D include reduction of cell proliferation and stimulation of cell maturation, activities which reduce the risk of cancer.The critical level of serum 25-hydroxyvitamin D for optimal health of these tissues is dependent on the level of the enzyme which converts 25-hydroxyvitamin D to 1,25-dihydroxyvitamin D (CYP27B1) and therefore may vary for different tissues. There is general international consensus that screening for vitamin D status is of limited value but a number of high risk groups warrant evaluation of serum 25 hydroxyvitamin D levels. These include patients with chronic kidney disease, osteoporosis or fragility fractures and elderly individuals who are institutionalized.

\section{IL- 75}

\section{Potential risk Predictors For Cardiovascular diseases}

\section{Tomris OZBEN}

IFCC Executive Board, Treasurer Board of Directors, IFCC Foundation for Emerging Nations (FEN)

Akdeniz University Medical Faculty

Department of Clinical Biochemistry 07058

Antalya Turkey

n association exists between increased incidence of cardiovascular diseases (CVDs) and chronicdiseases such as end-stage chronic renal disease (CRD), diabetes mellitus (DM) and obstructive sleep apnea syndrome (OSAS). The high systemic oxidative stress in $\mathrm{CRD}$ and DM is considered as a major pathogenic mechanism leading to CVDs as demonstrated in ourstudies and publications. OSAS is a condition characterized by-cessation of respiration due to bstruction of the upper air-way during sleep. Its prevalence is approximately $4 \%$ in males and $2 \%$ in females in the adult population. Persistent system 
icinflammation in OSAS plays a crucial role in the pathogenesis of CVDs such as systemic arterial hypertension, coronary artery diseases, and heart failure. In OSAS, each episode of air way obstruction is followed by a decreased arterial $\mathrm{O} 2$ saturation. Intermittent hypoxia and hypoxia/reoxygen at ion sequencesin OSAS are believed to be the major reasons to increase cardiovascular risk by enhanced sympathetic activation and oxidative stress. There is limited and conflicting information in the literature investigating risk predictors for CVDsin OSAS patients. In order to identify potential risk biomarkers for CVDs in OSAS, we measured plasma soluble tumor necrosis factor receptor-1 (sTNF-R1), myeloperoxidase (MPO)as markers of persistent systemic inflammation; total antioxidant capacity (TAC), total oxidativ estatus (TOS), ischaemia-modified albumin (IMA) and advanced oxidation protein products (AOPP) as markers of oxidative stress and copept in as a new marker of CVDs. Increased systemic inflammation as indicated by elevated plasma MPO activity and sTNF$\mathrm{R} 1$ levels in the OSAS patients might contributeto-the higher incidence of CVDs. High systemicoxi-dativestress as indicatedbyincreased TOS and decreased TAC levels is reflected by increased AOPP with out causing an increase in IMA. In contrast to high copept in levels in CVDs, our finding of low copept in levels in the OSAS patients might be due to low secretion of antidiuretichormone. Therefore, we consider plasma MPO activity, sTNF-R1, TOS, TAC and AOPP levels in the OSAS patients as potential risk predictors for CVDs.

KEYWORDS: cardiovasculardiseases; copeptin; myeloperoxidase; tumornecrosisfactor receptor-1; ischaemia-modifiedalbumin; advancedoxidation protein products.

\section{IL- 76}

\section{Emerging Trends in Quality Management}

\section{Bernard Gouget}

Counselor for Public Health FHF;

Chair-Human Health Care Committee-COFRAC;

IFCC Chair-Nominations Committee;

General Secretary of the International Francophone

Federation of Clinical biology and

Lab Medicine ( FIFBCML

$\mathrm{L}$ aboratory services are currently at thecentre of attention regarding quality due to their wide ranging impact on patient care.Numerous global initiatives all around the world have focused on medical laboratory harmonization, standardization and accreditation. The accreditation is reinforcing confidence as official recognition of competences and is offering a common framework of reference.Though accreditation has many advantages and advocated largely, it is still challenging for the laboratories to fulfill all requirements and the criteria of accrediting bodies. So identifying the different challenges faced by the laboratories during the process of accreditation is important for proper planning and action to resolve those goals. The reason to start the process of accreditation is interest in improvement of quality of lab services with better documentation of processes and responsibilities or interest of management.

The accreditation improves the processes in the laboratories, increasing the quality in all areas in labs reduction of errors in the pre-analytical processes, facilitation of accurate and rapid diagnosis, participation in acceleration and efficiency of treatment, facilitation of personalized medicine development, continuous improvement. Achieving and maintaining accreditation is still challenging for many countries due to; trained staff turnover, training and mentorship inadequacy, low staff satisfaction, quality and quantity of instruments, top management support, and sometimes poor quality laboratory facility.Facing the Lab medicine challenges and having in mind the medicalization of the profession, there is a strong need to have a coordinated strategy and to adopt a more proactive approach to respond to the changing of the international laboratory medicine landscape.Concerning quality, the strategic plans of IFCC 
include focusing of accreditation of medical labs based on ISO standards and cooperation with International, Regional Accreditation and National accreditation bodies. Nevertheless, theaccreditation laboratory standards have to be developed in compliance with international standards but with a focus on priorities and available resources in the country. The standards provide direction and a framework to laboratories on appropriate country-specific aspects of laboratory quality to achieve their goals for accreditation. However, cooperation and input from many stakeholders are also required. As in other resource-constrained countries, a successful accreditation program depends on knowledge and attitudes of personnel on laboratory quality; human and financial resources for personnel, equipment, and other infrastructure; and most important, a commitment from leaders. Sometimes with a flexible and feasible national program, laboratory quality systems can be gradually improved of the whole laboratory management system and laboratories that demonstrated outstanding performance in the quality management process will be strongly encouraged to enroll in an established ISO 15189 accreditation scheme. Thisapproach for laboratory accreditation is certainly affordable, sustainable, effective, and scalable.

\section{IL- 77}

\section{Circulatingfree Tumour DNA (ctDNA) And Circulatingtumourcells (CTC) As Novel Biomarkers; Liquid Biopsy}

\section{Tomris Ozben}

IFCC Executive Board, Treasurer Board of Directors, IFCC Foundation for Emerging Nations (FEN) Akdeniz University Medical Faculty Department of Clinical Biochemistry 07058 Antalya Turkey

The genomic profiles of cancer patients are highly variable which dramatically influence the development of the disease and the efficacy of potential treatments. Personalized health care employs molecular diagnostics to test each patient's genomic variants as a guide for the best-treatment. Analysis of circulating tumour DNA (ctDNA) and circulating tumor cells (CTC) has created a revolution in molecular diagnostics. The clinical implications of ctDNA and CTC are a recent and rapidly developing-research topic. Intensive research is on going to investigate ctDNA and CTC as diagnostic and prognostic biomarkers in cancer. Several studies have shown the potential role of ctDNA and CTC slevelsshed by tumours in to the plasma of cancer patients in the assessment of different malignanciesas a prognostic and predictive tool for early diagnosis and early intervention, clinical management and follow-up of cancer patients identifying specific genomic alterations to guide therapeutic selection, monitoring therapeutic responses, and detecting recurrence. Recent technological advances make it possible to use blood and plasma as a "liquid biopsy". Blood is a readily obtained, repeatable clinical sample; whereas serial tumour biopsy is often challenging, more expensive and not without risk. Instead of extensive imaging and invasive tissue biopsies, liquid biopsies can be used for screening of tumours that are not yet visible on imaging and before symptoms arise and to guide cancer treatment to detect diease progression or treatment resistance. I will present an overview of the exciting developments in this area, as well as looking forward to developments in future.

KEYWORDS: liquid biopsy; circulating free tumour DNA; circulating tumour cells

\section{IL- 78}

\section{Standardization and Harmonization In Laboratory Medicine}

\section{$\underline{\text { H A Morris }}$}

School of Pharmacy and Medical Sciences, University of South Australia and Chemical Pathology, SA Pathology Adelaide SA 5000 Australia

$\mathrm{T}$ he practice of medicine continues to be subject to intense pressures to improve performance and decrease costs. In laboratory medicine various pressures drive this process: (1) Globalization of laboratory practices with standardization and harmonization across national borders, (2) Global acceptance of evidencebased medicine as a foundation for best practice setting 
common critical clinical decision limits; (3)High investment costs and rapid development of new technologies requiring international demonstration of cost effectiveness before acceptance by stakeholders funding health care services; (4) Re-evaluation of reimbursement from activity- to patient outcome-based criteria. The profession has vigorously responded particularly through the IFCCand national professional societies establishing alliances with international metrology and regulatory agencies as well as the In Vitro Diagnostics Industry. The establishment of the Joint Committee for Traceability in Laboratory Medicine (JCTLM) is an important achievement. This movement has harnessed significant resources to markedly develop the theory of knowledge in this area and demonstrated major progress to providing comparability with many clinical laboratory assays including cholesterol, PSA, plasma protein and haemoglobin A1c.Currently most clinical laboratory analytes are not suitable for assay standardization strategies. A second approach is the International Consortium for Harmonization in Laboratory Medicine formed to develop an infrastructure to coordinate international harmonization activities. The encouragement and support for these developments including the provision of resources remain current issues for our profession.

\section{IL- 79}

\section{Towards The Cybersecurity Paradigm of Lab Medicine And E-Health}

\section{Bernard Gouget}

Counselor for Public Health FHF;

Chair-Human Health Care Committee-COFRAC;

IFCC Chair-Nominations Committee;

General Secretary of the International Francophone

Federation of Clinical biology

and Lab Medicine ( FIFBCML)

$\mathrm{T}$ he vast opportunity for digital technologies?to transform the way healthcare is delivered has been increasingly recognized around the world. Citizens continue to take a more co-creative role in decisions about their own healthcare, and new technologies can enable and facilitate this emergent trend. eHealth encompasses a diverse range of technologies, including broadband connectivity, software, digital networking, big data, mobile connectivity, smart infrastructure and even articial intelligence, which support the delivery and improvements in health and medical care for individuals and communities. Its potential to improve service delivery for patients is just as broad, ranging from smarter, more cost?effective models of care, remote monitoring, improved access particularly in rural areas, rapid data analysis, which is helping shift the traditional treatment paradigm of illness to a collaborative, proactive management of wellness. The healthcare systems and Laboratory Medicine are focused on as critical infrastructures that ought to be protected from all types of fears, including cyber security threats and attacks. Despite continual progress in the systemic risk management of cyber domain, it is clear that anticipation and prevention of all possible types of attack and malfunction are not achievable for current or future cyber infrastructures. The medical devices and lab instruments may be highly vulnerable, especially if timely security updates is not provided or if the devices are not updated by the medical facility or the patient with the device. In many cases the existence of the security threat has not been assessed or recognized. The presentation will focus on the investigation of the cyber security paradigm, adaptive systems in a healthcare critical information infrastructure.

\section{IL 80}

\section{Leadership \& Team Development: Success Together}

\section{Dr. Pradeep K Dabla}

Chair, IFCC-TFYS

Associate Professor, Department of Biochemistry, G.B.Pant Institute of Postgraduate Medical Education \& Research (GIPMER), GNCTD, Delhi-110002

$\mathrm{T}$ eam is described simply as "a group of people who are working through collective endeavour toward a common goal". Effective team working is an essential ingredient for any organisational success. Successful 
teams can help transform an organisation, increase outputs and deliver on organisational objectives. It is crucial in supporting how we blend the invaluable skills, capabilities, knowledge, experience and diversity of staff to develop a new culture and vision with leadership and foremost participation at is heart. This topic covers range of attributes and real time model for successful teamwork in context of IFCC-TFYS.

International Federation of Clinical Chemistry \& Laboratory Medicine (IFCC) recognized the need for a support group to help young scientists for promoting the essential contribution of laboratory medicine at the centre of healthcare. Thus to address these challenges, Task Force - Young Scientists (TFYS) was constituted in 2010. The aim of TF-YS is to ensure that young scientists make a significant and growing contribution to the activities of IFCC and other National programmes. IFCC-TFYS able to cross the barrier and created a strong young scientists support group involving more than 30 global IFCC member countries covering global regions. We use modern information technology \& social media to establish networks and facilitating the communication, 24/7 using Facebook, Twitter, Linked In and others. We partnered with other National and International societies to deliver educational workshops, trainings, mentorship programmes, webinars to learn perspectives \& principles of Laboratory Management \& Leadership namely "IFCCTFYS Survey", "IFCC-TFYS Mentorship Programme", "Research Booklet", "Young Scientists Awards \& Grants", Webinars and Lab-Surfing. TFYS is working with commitment to help the new generation facing challenges in the field of laboratory medicine.Thus, there are different crucial elements of team work such as composition of teams, working together; motivation and the leadership within teams that contribute to develop a quality results successfully.

\section{IL 81}

\section{Glycogen phosphorylase BB: A more sensitive and specific marker for early diagnosis of acute myocardial infarction}

Dr. Neelima Singh, Vedika Rathore, Roshan Kumar Mahat
Department of Biochemistry, GR Medical College, Gwalior

\section{ABSTRACT:}

Cardiac markers are used to evaluate functions of heart. However, there are no satisfactory cardiac biomarkers for the diagnosis of acute myocardial infarction (AMI) within 4 hour of onset of chest pain. Among novel cardiac markers, glycogen phosphorylase BB (GPBB) is of particular interest as it is increased in the early hours after AMI. The present study was conducted with the objective to find out the sensitivity and specificity of GPBB over other cardiac markers i.e. myoglobin and CKMB in patients of AMI within 4 hour after the onset of chest pain. The study includes 300 AMI patients and 100 normal healthy individuals as controls. In all the cases and controls, serum GPBB and myoglobin concentrations were measured by ELISA where as CKMB was measured by diagnostic kit supplied by ERBA. The sensitivity and specificity of glycogen phosphorylase $\mathrm{BB}$ (GPBB) were greater than CKMB and myoglobin in patients of AMI within 4 hour after the onset of chest pain. Hence, glycogen phosphorylase BB (GPBB) can be used as additional biomarker for the early diagnosis of AMI.

KEYWORDS: Glycogen Phosphorylase BB, Acute Myocardial Infarction, CKMB, Myoglobin

\section{IL 82}

\section{Dose and time dependent effect of fluoride in rats}

AVakdevi Validandi, Srinivas Dheeravath and Arjun L. Khandare*

$\mathrm{T}$ The present study was conducted to evaluate the effect of fluoride $(\mathrm{F})$ in Wistar NIN rats in a dose and time dependent manner. Male adult 36 Wistar NIN rats were taken from the National Centre for Laboratory Animal Sciences, National Institute of Nutrition, Hyderabad, India and were randomly divided into six groups (Group I: control, group II: Fluoride $5 \mathrm{mg} / \mathrm{L}$, Group III: Fluoride 10 mg/L, Group IV: Fluoride 15 mg/ L, Group V: Fluoride $50 \mathrm{mg} / \mathrm{L}$ and Group VI: Fluoride $100 \mathrm{mg} / \mathrm{L}$ ). Group I served as control and Group II to VI 
were administered respective $\mathrm{F}$ concentrations in their drinking water. Animals from each group were examined after 30, 60 and 90 days for $F$ toxicity changes. It was observed that $\mathrm{F}$ induce dose and duration dependent diet intake, body weight gain, signs of dental fluorosis, urinary, serum, bone and teeth fluoride levels along with histopathological changes in liver and kidney tissue. Results of the study revealed that there was a significant decrease in diet and water intake in the Group VI compared to Group I, II, III. The significantly increased exposure of $\mathrm{F}$ through water in group $\mathrm{V}$ and VI as compared to other groups has shown alterations in their food conversion ratio, body weight gain, and significant increase in the urinary and serum fluoride levels. Increased fluoride dose dependent development in dental fluorosis was observed. Significant increase in bone and teeth fluoride levels in the Group V and VI compared to Group I, II, and III was observed. Histopathology of kidney showed focal fibrosis in medulla with few dilated tubules in the kidney of $100 \mathrm{ppm}$ fluoride group compared to control, 5, 10, 15 and $50 \mathrm{ppm}$ fluoride groups. Whereas histopathology of liver showed microvacuolation in $33-66 \%$ liver in 50 ppm fluoride group compared to control, 5, 10, 15 ppm fluoride groups. Microvacuolation in $>90 \%$ liver was observed in 100 ppm fluoride group compared to control, 5, 10, 15 and $50 \mathrm{ppm}$ fluoride groups. However, there was no significant differences between the body organ ratio of brain, kidney, heart, testes and liver among all the groups. In conclusion the dose dependent effect of $\mathrm{F}$ starts at $15 \mathrm{mg} / \mathrm{L}$ at third month and the effect of $50 \mathrm{mg} / \mathrm{L}$ starts from second month onwards.

\section{IL 83}

\section{Effect of Alpha -Tocopherol on Antitubercular Drugs Induced Hepatotoxicity}

\section{Rajiv Nehra \& Shashank Tyagi}

Department of Biochemistry, LLRM Medical College, Meerut

BACKGROUND: Mycobacterium, the causative organism of tuberculosis, is notorious for its ability to develop resistance with mono therapy. To prevent emergence of resistance, combination of antitubercular drugs is given for months to years that can lead to side effects. Hepatotoxicity is one of the commonest sideeffect with antitubercular drugs. This study was aimed to explore the hepatoprotective potential of ?-tocopherol against experimentally induced hepatotoxicity in albino rabbits.

METHODS: This experimental study was carried out on 30 rabbits of either sex. They were divided into three groups comprising 10 animals each. Hepatotoxicity is induced experimentally in rabbits following a standard protocol. Group I received normal saline (10 ml/kg bw). Rabbits in group II were treated with first line Antitubercular drugs Isoniazid( $5 \mathrm{mg} / \mathrm{kg} \mathrm{bw}$ ), Rifampicin (20 mg/kg bw) and Pyrazinamide (25 mg/kg bw) concurrently. Group III received ? Tocopherol $200 \mathrm{mg} /$ kg bw along with group II drugs. All drugs were administered by oral route for 90 days. On last day of experiment blood samples were taken to investigate the plasma levels of alanine aminotransferase (ALT), alkaline phosphatase (ALP) and serum total bilirubin.

RESULTS: Serum levels of ALT were found to be markedly elevated upon oral administration of anti tubercular drugs for 90 days. A statistically significant reduction in ALT levels was noticed when ?-Tocopherol was given in doses of $200 \mathrm{mg} / \mathrm{kg}$ bw along with Ant tubercular drugs for same duration. Similar results were obtained with serum ALP \& serum total bilirubin.

CONCLUSIONS: ?-tocopherol (200 mg/kg bw, oral) was found to have hepatoprotective effect against antitubercular drugs induced hepatotoxicity in albino rabbits.

KEYWORDS: Antitubercular drugs, Hepatotoxicity, ?tocopherol, Antioxidents 


\section{IL 84}

\section{Arsenic Induced Cognitive Deficits Associated with Alterations in NMDA Receptor Induced Post-synaptic Signalling in Hippocampus: Protective Role of Curcumin}

Pranay Srivastava, Yogesh K. Dhuriya, Aditya B. Pant and Vinay K. Khanna

Developmental Toxicology Laboratory, System Toxicology and Health Risk Assessment Group, CSIR - Indian Institute of Toxicology Research, 31 Mahatma Gandhi Marg, Lucknow - 226 001, India

$\mathrm{P}$ rotective role of curcumin in arsenic induced cognitive deficits involving NMDA receptor dysfunctions has been investigated. Treatment with curcumin ameliorated arsenic induced alterations in NMDA receptors, its receptor subunits and synaptic proteins - pCaMKII $\alpha$, PSD-95 and SynGAP both in vivo in rat hippocampus and in vitro in primary hippocampal cultures. Curcumin was found to protect arsenic induced changes in hippocampus by modulating decreased levels of BDNF, pAkt, $p$ Erk $1 / 2$, pGSK3 $\beta$ and pCREB. Treatment of hippocampal cultures with pharmacological inhibitors for $\operatorname{Erk}^{1} \frac{1}{2}$, GSK3 $\beta$ and Akt individually was found to inhibit levels of CREB and proteins associated with $\mathrm{PI} 3 \mathrm{~K} / \mathrm{Akt} / \mathrm{GSK} 3 \beta$ pathway. Arsenic induced learning and memory deficits in rats assessed by water maze and Y-maze were found to be improved on treatment with curcumin. The findings are interesting and exhibit that curcumin exercises its neuroprotective effect through PI3K/Akt pathway which could affect NMDA receptor and downstream signalling through $\operatorname{TrK} \alpha$ and BDNF in arsenic induced cognitive deficits in hippocampus.

\section{IL 85}

\section{Rationalized approach using Nano-medicines: A preclinical study of $\mathrm{pH}$ triggered intracellular delivery of Doxorubicin for effective tumor regression}

\section{Dr. P.R. Mishra}

Pharmaceutics Division, Preclinical South PCS 002/ 011, CSIR-Central Drug Research Institute B.S. 10/1, Sector-10, Jankipuram Extension, Sitapur Road, Lucknow-226031, India

$\mathrm{N}$ ano-medicine has shown great promise in enhancing the outcome of chemotherapy due to their improved pharmacokinetic and bio-distribution profiles which leads to improved accumulation of drug at tumor site especially through enhance permeation retention (EPR) effect. However it results in poor cellular internalization and insufficient intracellular drug release hampers the efficacy.

We have focused on the development of $\mathrm{pH}$ responsive nanoparticle that not only trigger the release of drug at acidic $\mathrm{pH}$ but also facilitate the process of endosomal escape by generation of cationic charge. This cationic charge absorb the protons of acidic environment by means of specific chemical groups and then cause swelling/or rupture of the endo/lysosome. Moreover these charge reversal particle also propose a solution to the dilemma of surface charge of particles suitable for systemic administration and tumor targeting.

We have developed small molecular weight vitamin modified $\mathrm{pH}$ sensitive charge reversal nanoparticles (NP) for efficient intracellular delivery of Doxorubicin (DOX). Confocal microscopy illustrated that NP treatment kept higher DOX accumulation inside the cells than conventional $\mathrm{pH}$ insensitive lipid nanoparticles. The cationic charge of nanoparticles subsequently facilitated the endosomal escape and promoted the nuclear accumulation of DOX. Furthermore, in vitro cytotoxicity, apoptosis, cell cycle arrest and mitochondrial membrane depolarization studies supported the enhanced efficacy of NP in comparison to free DOX and non-modified. Intravenous pharmacokinetics and bio-distribution 
investigations indicated that $\mathrm{pH}$ sensitive nanoparticles can significantly prolong the blood circulation time of DOX in biological system and increase the drug accumulation to tumor site. Consequent to this NP also exhibited much enhanced therapeutic efficacy and lower toxicity in tumor-bearing rats compared to free DOX. The reduction in toxicity was confirmed by histological and survival analysis. In conclusion, these results suggest that the VitB6 modified charge reversal nanoparticles can be a novel platform for the successful delivery of anticancer drugs. 


\section{FP- 1}

\section{Laboratory and Point of Care Testing A Real Time Solution}

\section{Aditi Gupta \\ Consultant, Uniworld Garden, Gurgaon 122001}

BACKGROUND: Many medical laboratory tests can now be done near the patient (point-of-care testing, POCT), ranging from basic blood glucose measurement to complex coagulation testing. The Indian market for POCT systems has grown rapidly in the last ten years. The Indian market is now almost 1500 crores corresponding to about a third of the total market for in vitro diagnostic testing.

Switching from conventional laboratory testing to POCT shortens the time to decision-making about further testing or treatment, as delays are no longer caused by specimen transport and preparation, and the test results are rapidly available at the point of care. Better medical outcomes and lower costs may benefit the patient.

METHOD: In our 1250 bedded hospital, array of POCT devices are present including 139 Glucometers, 6 Arterial Blood Gas machine, 3 Cardiac POCT, 3 Drug screening, 5 Glycosylated Hemogloin, POCT for Urea and Creatinine. The quality control of these different POCT devices is maintained periodically (as per guidelines by NABL -National accreditation board for calibration and testing laboratories and CAP College of American pathologists) so as to ensure the correct results are delivered to the Clinician and patient for better medical outcome.

RESULTS: The available methods and equipment enable persons not specially trained in laboratory medicine to perform high-quality laboratory testing at the point of care, under certain conditions. The benefits of POCT includes rapid decision making,reducing operating times, reduce high dependency,post operative care time,reduced emergency room time and reduce number of hospital bedsrequired.

CONCLUSION: POCT for certain applications is a useful complement to conventional laboratory testing.POCT has been facilitated by the increasing miniaturization of laboratory instruments and procedures. The key advantages of POCT are that it dispenses with sample transport to the laboratory and sample preparation.

The future utilization of POCT will depend not only on technical advances, but also on developments in costs and reimbursement. Before POCT is introduced in a hospital or outpatient practice, a cost-benefit analysis should be performed, because the introduction is costly and requires a certain amount of organizational work especially for quality management. The potential medical and economic benefits should be assessed individually in each case.

\section{FP- 2}

\section{Sonic Hedgehog-Gli1 Cell Signalling Pathway Nexus for the Progression of Brain Tumoriogenesis}

\section{Mehdi H. Shahi}

Interdisciplinary Brain Research Centre (IBRC), JNMC, Faculty of Medicine, A.M.MU, Aligarh, U.P.,

$\mathrm{D}$ rug resistance is very common phenomena to understand for the effective therapy of the most common brain tumour glioma. Mitogenic cell signalling pathway Sonic-hedgehog Gli1 (Shh-Gli1) has substantial contribution in the tumoriogenesis. Although, mitogenic pathway Shh roles in brain tumour drug resistance is not thoroughly investigated. Interestingly, we found that ShhGli1 signalling pathway regulates the expression of novel stem cell marker, BMI1 (B-Cell specific MoloneyMurine Leukemia Virus), in brain tumour. Noteworthy, we also demonstrated high expression pattern of Multidrug resistance protein in brain tumour. MRP expression was decreased by BMI1 siRNA and Shh cell signalling specific inhibitor Gli antagonist 61 (GANT61). Intriguingly, GANT61 inhibited brain tumour in vitro cell colony compared to temozolomide, common drug for brain tumour treatment. Moreover, a synergic effect of GANT61 and temozolomide significantly decreased the LD50 of temozolomide in the cell colony experiments. Therefore, our results suggest that there is 
a potential network of Shh-Gli1-BMI1 cell signalling to regulate MRP and to promote chemoresistance in glioma.

KEY WORDS: BMI; MRP; Shh; Glioma; Chemoresistance; GLI1

\section{FP- 3}

\section{Quantification of DTT Metrics In Various Fiber Bundle In Patients With Frontal Lobe Injury and Its Correlation Withneuropsychological Tests}

Raj. Kumar' ${ }^{1}$ Mazhar Husain² R. K. Gupta ${ }^{3}$, and R. K.S. Rathore ${ }^{4}$

1. Dept.of Biochemistry BRD Medical College, Gorakhpur, Uttar Pradesh, India,

2. Neurosurgery, Sahara Hospital, Lucknow, Uttar Pradesh, India,

3. Radiodiagnosis, Fortis Hospital Gurgaon.

4. Mathematics and Statistics, Indian Institute of Technology, Kanpur, Uttar Pradesh, India,

INTRODUCTION: Traumatic brain injury (TBI) results from an outside force traumatically injure the brain secondary to falls, vehicle accidents and violence. It is known to affect cognitive, physical and psychological skills. Frontal lobes play an essential role in attention, long term memory and executive functions. Most of the quantitative DTI studies are based on region of interest (ROI) analysis and may not represent the true extent of Diffuse Axonal Injury(DAI) in these patients. Diffusion tensor tractography (DTT) helps to demonstrate structural abnormality of the whole fiber tracts and is proposed for DAI quantification in patients with frontal lobe injury to assess DAI in various fiber bundles and to look for correlation of these fiber bundles measures with various neuropsychological tests (NPT).

MATERIALS AND METHODS: Human subjects: Conventional MRI and DTI was performed within 7 days and after 6 months of injury in 21 TBI patients (15 male; age range from 15-45 years) showing unilateral/or bilateral frontal lobe injury.

IMAGING PROTOCOL: T2, T1, T2-FLAIR, T2* GRE and DTI data was acquired on 1.5 T GE unit.
NP TESTS: NPT were performed in controls and patients at 6 months following injury. NPT included number connection tests (NCT A and B), figure connection tests (FCT A and B) and performance subset of modified Wechsler Adult Intelligence Scale (WAIS$\mathrm{P}$, modified for Indian population.

STATISTICAL ANALYSIS: Multiple comparisons using Bonferroni, Post Hoc tests were performed.

RESULTS: We found reduced fractional anisotropy (FA) and increased mean diffusivity (MD) values in all WM tracts in TBI patients compared to controls, NPT scores were found to be significantly impaired in follow-up patients compare to controls and some of these tests showed significant correlation with DTI indices with different WM tracts.

CONCLUSION: White Matter tracts which show significant difference on DTT were also correlated with those NPT which are associated with main function of frontal lobe such as memory, attention, visual and motor function. It appears more realistic methods for DAI quantification in TBI patients and provides information about structural integrity and connectivity of whole fiber tracts.

KEY WORDS: DTI, TBI, WM Tract, DAI,NPT 
FP- 4

Id1 Ablation Enhances Hematopoietic Stem Cell Self-Renewal and Suppresses Hematopoietic Ageing by Reducing Proliferative Stress

Satyendra K. Singh ${ }^{1,4}$, Stephen Gadomski ${ }^{1}$, Shweta Singh ${ }^{1}$, Lei Sun ${ }^{1}$, Alexander Pfannenstein ${ }^{1}$, Serguei Kozlov ${ }^{2}$, Lino Tessarolloㄹ, Kimberly D. Klarmann ${ }^{1,3}$ and Jonathan R. Keller ${ }^{1,3}$

1. Mouse Cancer Genetics Program, Center for Cancer Research, NCI. Frederick, MD 21702, USA.

2. Center for Advanced Preclinical Research, and

3. Basic Science Program, Leidos Biomedical Research Inc. Frederick National Laboratory for Cancer Research, Frederick, MD 21702, USA.

4. Department of Stem Cell and Cell Culture, Center for Advanced Research, King George's Medical University, Lucknow, India, 226003

$\mathrm{D}$ efining mechanisms that maintain tissue stem cells during homeostasis and stress is important in tissue regeneration and repair, cancer and ageing. Inhibitor of DNA binding (Id1-4) proteins are helix-loop-helix (HLH) transcription factors. Id proteins are often deregulated in human cancers, where they contribute to tumor growth, invasiveness and metastasis, and have been linked to the self-renewal of cancer stem cells (CSCs). Thus, a complete understanding of the function of Id genes in normal adult stem cells and CSCs could lead to the development of novel therapies. Id1 is induced in hematopoietic stem cells (HSCs) by pro-inflammatory cytokines that promote HSC proliferation and differentiation in vitro and in vivo, suggesting that Id1 may function during stress hematopoiesis. Id1-/- HSCs show increased self-renewal in serial bone marrow transplantation (BMT) assays, which is correlated with decreased proliferation, mitochondrial biogenesis, and ROS production. As a result, Id1-/- HSCs harbor less DNA damage, show increased quiescence and reduced aging. Mechanistically, we found cytokines and other pro-inflammatory stimuli present in the hematopoietic microenvironment after ?-irradiation induced Id1 expression. Id1-/- HSCs fail to properly respond to cytokine-induced proliferation, suggesting that HSC exhaustion is mediated, in part, by chronic proliferative stress. Id1-/- HSCs are protected from other chronic proliferative stresses including chronic genotoxic and inflammatory stress, and ageing. Thus, targeting Id1 inhibition may be therapeutically useful to improve HSC survival and function during BMT, chronic stress and ageing.

KEYWORDS: Stem cells; Hematopoiesis; Self-renewal; Quiescence; Proliferative stress; Ageing.

\section{FP- 5}

\section{Syndrome X, C-Reactive Protein And Proteinuria In Newly Diagnosed Patients of Type 2 Diabetes Mellitus- A Cross-Sectional Study}

Sangeeta Singh, Basant Joshi, Seema Gupta, Ashok Kumar

Government Medical College, Haldwani, Uttarakhand

INTRODUCTION:-Diabetes Mellitus (DM) is the commonest metabolic abnormality in the world. Type 2 diabetes, the commonest form of diabetes constitutes nearly $90 \%$ of diabetic population in any country. The Syndrome X consists of a constellation of metabolic abnormalities that confer increased risk of cardiovascular disease and diabetes mellitus.Inflammation, as assessed by $\mathrm{C}$-reactive protein (CRP) may be an important precursor of the syndrome $X$ and type 2 diabetes.Syndrome $\mathrm{X}$ is a known risk factor for proteinuria in the general population.

MATERIALS AND METHODS:-100 patients of newly diagnosed type 2 diabetes mellitus in the age group of 25-60 years were included in the study. Patients with Gestational diabetes mellitus were excluded from the study.

RESULTS:- Of the 100 patients studied, 58 patients has syndrome $\mathrm{X}$ according to the International Diabetes Federation (IDF) criteria. Among the studied subjects 33 males had increased WHR $>0.90$ (Normal <0.90), whereas all the females had WHR $>0.85$ (Normal $<0.85$ ). Mean waist circumference in males with MS was $96.26 \pm 1.01$ as compared to those without syndrome $\mathrm{X}$ 
(85.6 \pm 50.57$)$ and mean waist circumference in females with MS was $(90.3 \pm 40.68)$ as compared to those without syndrome $X(75.63 \pm 0.25)$. Mean serum hs-CRP levels in patients with syndrome $X$ was $(6.35 \pm 1.07)$ as compared to those without syndrome $X(7.09 \pm 1.30)$. Mean Proteinuria levels in patients with metabolic syndrome was $(549.34 \pm 68.80)$ as compared to those without syndrome $X(456.74 \pm 33.04)$. The mean Proteinuria level was markedly raised in patients of metabolic syndrome as compared to those without syndrome $\mathrm{X}$. This difference was found to be markedly significant $(\mathrm{p}<0.01)$.

CONCLUSION:-In conclusion, considering that Syndrome $\mathrm{X}$ is a modifiable risk factor, early detection of syndrome $\mathrm{X}$ would be a cost-effective strategy to decrease the prevalence of proteinuria and chronic kidney disease in the general population.

KEY WORDS:-Diabetes Mellitus, Syndrome X, C Reactive Protein and Proteinuria

\section{FP- 6}

\section{Genetic Association of ADIPOQ Gene Variants (RS266729 and RS17846866) With Circulatory Adiponectin Levels and Type 2 Diabetes In North Indians}

Mohammad Mustufa Khan*, Sangeeta Singh*, Israr Ahmad*, Satyendra Kumar Sonkar**, Gyanendra Kumar Sonkar*

*Department of Biochemistry,

** Department of Medicine, King George's Medical University, UP, Lucknow

A diponectin gene (ADIPOQ) variants and its transcript have associated with Type 2 diabetes mellitus (T2DM) in different ethnicity. We aimed to find the association of ADIPOQ gene variants (rs266729 and rs17846866) with its circulatory adiponectin levels in T2DM and normal healthy control (NHC) groups. A total of 300 subjects (150 T2DM and $150 \mathrm{NHC}$ ) aged between 25-75 years were recruited in the study. Various anthropometric and clinical parameters were investigated. Circulatory adiponectin levels were also evaluated. Polymerase chain reaction-restriction fragment length polymorphism (PCR-RFLP) method was used for genotyping. Mean levels of anthropometric and biochemical parameters were significantly raised in T2DM than NHC. While, the levels of circulatory adiponectin was found significantly lower in T2DM as compared to NHC ( $\mathrm{p}=0.02)$. Genotyping analysis of rs 17846866 of ADIPOQ gene variant shown that there were no significant association of TT versus TG genotype $(\mathrm{p}=0.13)$ as well as TT versus GG genotype $(\mathrm{p}=0.11)$ with T2DM patients and NHC. However, T and G allele frequencies of the rs 17846866 had significant association with T2DM cases as compared to NHC $(\mathrm{p}=0.02)$. There was significant impact of rs17846866 on circulatory adiponectin level, PPBS, HbA1c and serum creatinine $(\mathrm{p}<0.001,0.04,0.03$ and $<0.001$, respectively). Circulatory adiponectin level was significantly lower in GG genotype as compared to TG \& GG genotypes $(\mathrm{p}<0.001)$. Haplotype frequency analysis in T2DM and NHC of ADIPOQ gene; variants $-11377 \mathrm{C} / \mathrm{G}$ and $+10211 \mathrm{~T} / \mathrm{G}$ showed that $\mathrm{C}$ allele of -11377 was negatively correlated with $\mathrm{T}$ allele of +10211 , i.e. $\mathrm{CT}$ was protective haplotype while CG haplotype increased the risk for T2DM.

\section{FP- 7}

Vitamin D Mediated Selective Cell Death of Malignant Cells: A 'Possible' Non Enzymatic, Copper Induced, ROS Mediated Pathway For Dna Breakage

Asim Rizvi, Imrana Naseem

Department of Biochemistry, Faculty of Life Sciences, Aligarh Muslim University, Aligarh.

Copper is elevated in malignancies. This systemic metabolic feature of cancer is common to almost all known malignancies and to malignancies across tissue of origin and species. Using in vitro, ex vivo, and in vivo models we show that Vitamin D in malignant cells causes copper induced, reactive oxygen species mediated DNA breakage selectively in malignant cells. Using computational methods, we show how the Vitamin D receptor (VDR) serves as a chaperone molecule to 
mediate this Fenton like reaction between water soluble DNA and fat soluble Vitamin D within the aqueous nuclear milieu of malignant cells.

The bigger idea of utilising characteristic metabolic features of malignancies to develop selective and targeted therapeutic strategies will be discussed at some length.

\section{FP- 8}

\section{Effect of Bortezomib In Mice Sperm}

Kamla Kant Shukla ${ }^{\mathrm{a}, \mathrm{b}}$, Praveen, Sharmaa Mayung Geol Pang ${ }^{\mathrm{b}}$

a. Department of Biochemistry, All India Institute of Medical Sciences, Jodhpur, 342005 Rajasthan

b. Department of Science \& Technology, Chung-Ang University, Anseong, Gyeonggi-do, South Korea.

INTRODUCTION: Physiologically normal spermatozoa are essential for successful fertilization of thefemale gamete both in vitro and in vivo, as well as for normal embryonic development. Theseprocesses require that the spermatozoa possess a specific set of proteins. Since maturespermatozoa are silent during both transcriptional and translational events and are thereforeincapable of protein synthesis, the presence or absence of specific antagonist molecules is anideal mechanism by which overall sperm function may be maximized or minimized. Therefore,we investigated an antagonistic approach to inhibit fertility parameters in the mouse model. Bcl-2 (B-cell lymphoma 2), Cytochrome C, caspase and procaspase 3 proteins was evaluated by qPCR and western blot analysis which were correlated with fertility rates of individual mice spermatozoa by in-vitro fertilization (IVF) procedures.

MATERIAL AND METHODS: ICR mice of age (8-12) weeks was used to prepare the mouse sperm suspension. The spermatozoa were collected accordingly based on the methods described previously (Tayama et al., 2006). The spermatozoa were incubated to disperse for $12 \mathrm{~min}$ in presence of $5 \% \mathrm{CO} 2$ at $37^{\circ} \mathrm{C}$ in incubator. Then the sperm suspension was incubated for further 90 min at same incubation condition in air for capacitation in BM. The BSA media was additionally supplemented with 1,10 , and $100 \mu \mathrm{M}$ of bortezomib in separate falcon tube.

RESULT:The present study showed that bortezomib decreased the sperm motility and viability alongwith decreased gene expression of $\mathrm{Bcl}-2$, caspase and procaspase 3 on a dose- dependent manner. However, cytochrome c expression was found significantly increased in the treatment groups. Furthermore, we also found a decreased cleavage rate and embryonic development in all different treatment groups. However, higher doses showed a negative effect of treatment compared with control groups. These above findings demonstrate that bortezomib dependent phosphorylation of p53 either is attributable to its ability to induce apoptosis, or activate pathways that are stimulated in response to cell death in sperm cells.

CONCLUSION: The interdisciplinary approach in the current study of the bortezomib is found to be a negative strategy which could be toxic and inhibit fertility rates as well as overall sperm physiology and embryo development in mice.

KEY WORDS: Male infertility, IVF, mice, gene expression,bortezomib.

\section{FP- 9}

\section{Human Papilloma Virus Oncoproteins Blocks Ecdysoneless (ECD) A "Bypass" for the Recruitment of Clients of R2TP Complex.}

Riyaz A Mir ${ }^{1}$, Ying Zhang ${ }^{2}$, Hamid Band ${ }^{3}$, Vimla Band ${ }^{3}$

\section{Dept. of Biochemistry All India Institute of Medical Sciences New Delhi India \\ 2. Abbott Molecular; Des Plaines, IL USA. \\ 3. University of Nebraska Medical Center Omaha Nebraska USA.}

INTRODUCTION: -HPV is associated with more than $95 \%$ of invasive cervical cancers.High risk types such as HPV type 16 and type 18 are among most common types found in cervical cancers and are the main factors implicated in cervical carcinogenesis. HPV encodes two proteins E6 and E7 which together promote cellular 
proliferation, prolong cell-cycle progression, and prevent apoptosis. HPV Virus maintains these cellular processes by abrogating the function of two key tumor suppressors retinoblastoma protein (pRB) and p53 by E7 and E6 respectively. One of the least studies area is the folding, assembly and remodeling of these two tumor suppressors. Recently a newly discovered multi molecular Co-chaperone complex called R2TP has come in to play. $\mathrm{R} 2 \mathrm{TP}$ complex is considered as master regulator of cell growth and survival. It is involved in the assembly/ remodeling/activation of large protein or protein-RNA complexes. R2TP complex is composed of four proteins: RUVBL1, RUVBL2, RPAP31 and PIH1D1. Notably PIH1D1 component of R2TP complex binds to phosphorylated proteins, these proteins in turn brings clients to R2TP -HSP90 complex for assembly and remodeling. The phosphorylation site recognized by PIH1D1 component of R2TP complex is phosphorylated by evolutionary conserved Enzyme kinase CK2 on a motif DSDD/E. We recently found human Ecdysoneless (ECD) contains this motif and interacts with R2TP complex.Interestingly Human ECD was discovered during the interactome analysis of E6 proteins from high risk HPV 16 and 18. ECD has been shown to interact with tumor suppressors retinoblastoma protein $(\mathrm{pRB})$ and P53.Based on our preliminary data and published studies we hypothesize that HPV E6 protein may prevent recruitment and remodeling of tumor suppressor $\mathrm{pRB}$ and P53 by blocking ECDs binding to PIH1D1 component of R2TP complex.

MATERIALAND METHODS: Recombinant proteins were expressed in either BL21 competent cells, or Sf9 insect cells and purified by affinity chromatography. The interaction between ECD and E6 was tested by immunoprecipitation, GST pull down and Histag pull down assay followed by western blotting. Effect of E6 on ECDs phosphorylation was tested by invitro kinase assay.Recruitment of pRB to PIH1D1 component of R2TP complex was tested by immobilizing GSTP1H1D1 on GST beads, pRB was expressed in HEK293 cells lysates collected prom these cells were either used directly (or ECD was Immunodepeleted) for incubation with GSTPIH1D1. Role of R2TP complex and effect of E6 on pRB activation was tested by arresting E6 expressing and control epithelial cells in $\mathrm{G} 2 / \mathrm{M}$ phase of cell cycle by nocodazole treatment followed by cell cycle analysis. R2TP complex components were knocked down by siRNA.

RESULTS: Here in this study we found HPV E6 interacts with the ECD in the same region where it interacts with PIH1D1 component of R2TP complex. Up on further biochemical analysis we found E6 interaction with ECD prevents latter's phosphorylation by CK2. This phosphorylation is prerequisite for PIH1D1 interaction. Up on further exploration lysate prepared from the cells transfected with HPV E6, PIH1D1 component of R2TP complex was unable to pull down ECD from these lysates. Based on the previous studies that $\mathrm{pRB}$ interacts with ECD we suspect ECD may act as a bridge between R2TP complex and pRB and HPV E6 might block this bridge. To test this hypothesis, we employed various biochemical techniques, intriguingly ECD turned out be bridge between ECD and pRB and HPV E6 is blocking this bridge by directly interacting with ECD. To elucidate the biological significance of $\mathrm{pRB}$ recruitment to R2TP complex, knockdown of ECD and PIH1D1 component of R2TP complex component leads to inactivation of $\mathrm{pRB}$ and thus increase the proliferation of these cells. The same phenotype was observed upon transfection of HPV E6 in cells containing wild type pRB.

CONCLUSION: In this study we have discovered a novel mechanism of retinoblastoma ( $\mathrm{pRB}$ ) inactivation by human papilloma virus. This study will pave the way for the future therapeutic strategy and in the management of HPV induced malignancies.

KEY WORDS: HPVE6, PRB, R2TP COMPLEX, ECDYSONELESS, 


\section{FP- 10}

\section{Understanding The Genetics of Genes Involved In Autoimmunity \& Innate Immunity and their Association with Non-Segmental Vitiligo in a South Indian Tamil Cohort}

\section{Medha Rajappa}

Additional Professor of Biochemistry, Jawaharlal Institute of Postgraduate Medical Education and Research, Puducherry, India.

INTRODUCTION: Non-segmental vitiligo (NSV) is an autoimmune disease of the skin in which melanocytes are destroyed by antigen-specific $\mathrm{T}$ cells, resulting in patchy depigmentation. Altered genotype of more than 100 genes in vitiligo has recently been reported worldwide in various populations. While adaptive immunity and its mediators such as cytokines plays a clear role in disease progression, initiating factors are largely unknown.Mediators of innate immunity such as toll like receptors (TLR) and NACHT Leucine rich repeats PYD containing protein (NLRP) have been shown to play a pivotal role in the disease pathogenesis. Hence we undertook to investigate the possible influence of tumor necrosis factor (TNF)-?(rs1799964, rs1800630, rs1799724, rs1800629, rs361525), TLR-4(rs4986790) and NLRP1(rs2670660) gene polymorphisms on vitiligo riskin South Indian Tamils.

MATERIALS AND METHODS: This was acase control study involving 264 vitiligo patients and 264 age, gender and ethnicity matched controls. Amplified refractory mutation specific (ARMS)-PCR and TaqMan 5' allele discrimination assay was employed for genotyping of the genetic variants. Plasma protein levels were measured by ELISA.

RESULTS: We observed that the patients with the risk allele A of TNF- $\alpha$ (rs1800629) and haplotypes: AGCTC andAGCCAconferred an increased susceptibility to vitiligo. There was no significant association between other TNF- $\alpha$ SNPs and disease risk. Genotype-phenotype analysis showed that there was an association of TNF- $\alpha$ SNP rs361525 with the female gender. A significant increase in TNF-? level was found in NSV patients, compared to controls and the higher levels of TNF- $\alpha$ were found in the minor variant genotype AA, when compared with that of heterozygous $\mathrm{AG}$ and major variant GG genotypes of rs1800629. GG genotype of NLRP1(rs2670660)was found to strongly associated with $\mathrm{NSV}$, than controls $(\mathrm{p}<0.0001)$ and plasma NLRP1 was higher in NSV, compared to controls $(\mathrm{p}<0.0001)$. None of the genotypes of TLR-4(rs4986790) was associated with NSV ( $(p>0.05)$ and plasma TLR-4 was not different in NS V compared to controls.

CONCLUSION: Our results suggest that polymorphisms in TNF- $\alpha$ (rs1800629) and NLRP1(rs2670660) genesare genetic factors for susceptibility to NSV in South Indian Tamil ethnicity.

KEY WORDS: Autoimmune, innate immunity, gene polymorphisms, association, south Indians.

\section{FP- 11}

\section{Immunological Studies on DNA Modified with Quinacrine}

Safia Habib, K S Asha and Asif Ali

Department of Biochemistry, Jawaharlal Nehru

Medical College, Faculty of Medicine, Aligarh Muslim University, Aligarh, Uttar Pradesh, India.

INTRODUCTION: Quinacrine is a heterocyclic threering compound, available in the market under various labels like atabrine, mepacrine etc. This drug has therapeutic potential for its antiprotozoal, antibacterial activity, adjuvant cancer therapy and rheumatoid arthritis. It is a FDA approved drug for malaria, giardiasis and tapeworm infection. DNA is one of the biological targets for quinacrine since it interacts with double-stranded DNA by intercalation; groove binding and covalent binding.

AIMS AND OBJECTIVES: The study involves modification of DNA with quinacrine and characterization of the modified DNA through various physicochemical techniques. The modified DNA has been used as an antigen to raise antibodies in female rabbits. The induced antibodies have been characterised by ELISA for their antigen binding specificity. The 
induced antibodies have been also assessed for binding to DNA isolated from hepatocellular carcinoma patients.

MATERIALS AND METHODS: Calf thymus DNA,S1 nuclease, Quinacrine, anti-rabbit and anti-human IgG alkaline phosphatase conjugates, Protein-A Agarose affinity column, Freund 's complete and incomplete adjuvants, etc were purchased from Sigma-Aldrich, USA.

DNA modified in presence of quinacrine was characterised by UV-vis and CD spectroscopy.Thermal denaturation studies,Dynamic light scattering (DLS),FTIR, Agarose gel electrophoresis, SDS-PAGE, Scanning and Transmission Electron Microscopy.

RESULTS: Compared to native DNA,quinacrine modified DNA exhibited hypochromicity which increasedwith increasing concentration of quinacrine. Similarlya decrease in ellipticity from $10 \mathrm{mdeg}$ to $4 \mathrm{mdeg}$ at $275 \mathrm{~nm}$ was observed in modified DNA. The change in the size of DNA was observed by an increase in the hydrodynamic radius of DNA from $615 \mathrm{~nm}$ for native to about $712 \mathrm{~nm}$ for the modified DNA. Significant structural changes were observed in the modified DNA when compared to the native form. Antigenicity of the modified DNA was confirmed by high titre antibodies $(>1: 6400)$ raised in New Zealand white rabbits.

CONCLUSION:The structural modifications in the quinacrine modified DNA would have implications in the normal function of DNA. Antibodies against modified DNA may be used as a probe for diagnosis of hepatocellular carcinoma.

KEY WORDS: DNA, quinacrine, IgG, ELISA, hepatocellular carcinoma.

\section{FP- 12}

\section{A Study To Assess and Correlate Serum Levels of Visfatin With Insulin Resistance Among Patients with Pre-Diabetes And Diabetes at a Tertiary Care Hospital In Uttarakhand}

Manisha Naithani, Anissa Atif Mirza, Sarama Saha, Debadyuti Sahu

All India Institute of Medical Sciences, Rishikesh.

INTRODUCTION: Adipocytokines like Visfatin may play a role in the pathogenesis of insulin resistance. This study seems imperative considering an increase in prevalence of diabetes in Uttarakhand. It would serve to clarify the role of Visfatin and to correlate it with insulin resistance in Pre Diabetics and Diabetics would enhance our knowledge of this novel marker.

MATERIAL AND METHOD: It was a clinic based cross sectional study. Serum levels of Visfatin were assessed in patients having Pre Diabetes and Diabetes and attending OPD of AIIMS, Rishikesh. Healthy, age and sex matched controls were randomly selected from the people working in AIIMS, Rishikesh. Visfatin levels were measured along with the BMI, blood pressure, lipids, glucose, insulin and hsCRP levels, and HOMAIR indexes.One-way ANOVA and linear regression analysis were carried out using SPSS 23.

RESULTS AND OBSERVATIONS: Serum Visfatin levels were not significantly different in diabetic $(\mathrm{n}=49,14.2282 \pm 5.9 \mathrm{ng} / \mathrm{ml})$ subjects compared with pre diabetic subjects $(n=21,15.0109 \pm 4.4 \mathrm{ng} / \mathrm{ml})$. However, serum Visfatin showed a significant association with Fasting blood glucose levels even after adjusting for age, sex andbody mass index (BMI). Levels werealso not correlated to BMI and waist hip ratio in the present study. We could not find a significant correlation between serum Visfatin and insulin resistance.

CONCLUSION: In the present study serum Visfatin levels were notcorrelated with BMI and waist hip ratio and a significant negative correlation was found with glucose levels.

KEYWORDS: Visfatin, insulin resistance, homeostasis model assessment of insulin resistance. 
FP- 13

Frequency of ABO Blood Group and Rhesus Factor (D) In Patients of Type 2 Diabetes Mellitus Attending Tertiary Care Teaching Hospital of South India

\author{
Shrimanjunath Sankanagoudar ${ }^{1 *}$, \\ Shivanand $\mathrm{Mali}^{2}$, Sanjay Kambar ${ }^{3}$, \\ Sandip Chindi ${ }^{4}$
}

\section{1,2. Department of Biochemistry,}

3. Department of Community Medicine,

4. Department of Medicine, KLE University's, Jawaharlal Nehru Medical College (JNMC), Belagavi, Karnataka, India - 590010.

*Present Affiliation: Assistant Professor, Department of Biochemistry, All India Institute of Medical

Sciences (AIIMS), Jodhpur, India - 342005.

INTRODUCTION: Diabetes mellitus (DM) is a group of metabolic diseases characterized by hyperglycemia resulting from defects in insulin secretion, insulin action, or both. After discovery of ABO human blood group system by Landsteiner, many researchers had tries to make an attempt to correlate between $\mathrm{ABO}$ phenotype and disease susceptibility. Many reports have appeared in recent past suggesting an association between blood groups and DM but, published literature produces conflicting results with relevance to DM.

AIMS AND OBJECTIVES: To estimate prevalence of $\mathrm{ABO}$ and $\mathrm{Rh}$ (D) blood group type among diabetics attending tertiary care teaching hospital.

MATERIAL AND METHODS: With institutional ethical committee approval, we collected data of diabetic patients from medical record section for 12 months period from 1st Jan 2015 to 31st Dec 2015. Study design was descriptive and retrospective. Data of ABO and Rh (D) blood groups were represented in simple number and percentage distribution.

RESULTS: A total of 1,038 diabetic patient's medical records were collected during the study period for $\mathrm{ABO}$ grouping and Rh (D) typing. Out of these $59.34 \%$ were males. Rh (D) positive and negative blood groups were seen in $95.56 \%$ and $4.44 \%$ respectively. The frequency of blood groups O, A, B, AB was 32.36\%, 29.95\%, $29.28 \%$ and $8.37 \%$ respectively. The most prevalent blood group in both males and females was 'O' and least prevalent was 'AB' blood group.

CONCLUSION: Our study findings suggested DM was more frequently seen in individuals with blood group 'O' and these individuals should be closely monitored by the treating clinicians. The trend of observed prevalent percentage of blood groups for $\mathrm{DM}$ was $\mathrm{O}>\mathrm{A}$ ? $\mathrm{B}>\mathrm{AB}$.

KEY WORDS: ABO blood groups; Rh (D) typing; Diabetes; Tertiary care hospital; Retrospective.

\section{FP- 14}

\section{Evaluation of Risk Markers of CVD In Metabolic Syndrome}

Bharat Kumar Gupta ${ }^{1}$, Haren Baruah ${ }^{2 *}$

1. Professor, Dept. of Biochemistry, Subharti Medical College, Meerut,

2. Associate Professor, Dept. of Biochemistry, VCSG Government Institute of Medical Sciences \& Research, Srinagar, Uttarakhand.

INTRODUCTION: Metabolic syndrome is one of the major public health issues with $30-40 \%$ probability of developing type 2 Diabetes Mellitus and/or cardiovascular disease. On extensive search of literature we could hardly find any study which could find a strong correlation between basal C-peptide and insulin levels as a risk marker for CVD and whatever was available the workers have tried to establish that high C-peptide levels coexists with hyperinsulinemia in metabolic syndrome. So we tried to explore the levels of C-peptide and insulin as a risk marker of CVD in the patients of metabolic syndrome.

MATERIALAND METHODS: After obtaining ethical clearance, a total of 267 patients of metabolic syndrome were selected and enrolled for the present study. All the patients were subjected to complete general and systemic examination and findings noted. Waist circumference was also recorded in all of them. The special investigations like lipid profile, LFT, KFT, fasting blood 
glucose, C-peptide and Insulin levels were estimated in all the cases and findings recorded and statistically analysed.

RESULTS: Out of the total of 267 subjects, 15 (5.6\%), $12(4.5 \%)$ and $240(89.8 \%)$ had C- peptide level $<0.78$ (mean \pm SD $0.49 \pm 0.24), 0.78-1.89$ (mean \pm SD 1.35 \pm 0.45 ) and $>1.89$ (mean \pm SD $6.14 \pm 3.47$ ) respectively while in $3(1.1 \%), 198(74.1 \%)$ and $66(24.7 \%)$ subjects, the insulin levels were found to be in $<2$ (mean \pm SD $1.8 \pm 0.0), 2-25($ mean \pm SD 13.05 \pm 26.58$)$ and $>25$ (mean \pm SD 43.1 \pm 16.85$)$ respectively.

CONCLUSION: In our study 240 (89.8\%) subjects were having elevated C-peptide which is statistically significant $(\mathrm{p}<0.001)$, whereas only 66 (24.75) subjects had evaluated insulin level with no statistical significance. Therefore we came to a conclusion that fasting serum C-peptide levels is a better risk marker of CVD rather than fasting insulin levels in patients with Metabolic Syndrome.

KEY WORDS: Metabolic Syndrome, fasting blood glucose, fasting C-peptide, fasting Insulin and biomarker

\section{FP- 15}

\section{The Combination of Copeptin and High Sensitive Troponin I (Hs Trop I) I For Early Rule out of Acute Coronary Syndrome (ACS): An Observational Study}

Mamta Kankra, Anjali Manocha,

Parul Singla, Seema Bhargava

Department of Biochemistry, Sir Ganga Ram Hospital, New Delhi, India

INTRODUCTION : Copeptin is a marker of acute hemodynamic stress which is released from the pituitary within few minutes of occurrence of MI. Cardiac trop I is released into the blood stream within hours following MI or ischemic injury. High sensitive Trop I assay can detect elevated levels of C Tn I (above the $99^{\text {th }}$ percentile of an apparently healthy reference population) within 3 hours after the onset of chest pain. Rule-out of acute myocardial infarction (AMI) is a major challenge in the Emergency Department (ED). Around 10\% of all internal medicine emergency patients present to the ED with chest pain, but only $10 \%$ of these patients have an AMI as the underlying disease. Due to the potential hazards of overseeing an evolving MI, most patients are subjected to 6-12 hours observation in chest pain units (CPU) with a resultant excellent prognosis at high costs. We evaluated whether a protocol with combined testing of copeptin and hs trop I at admission in patients presenting with chest pain in less than or within six hours, low-tointermediate risk and suspected acute coronary syndrome (ACS) does not lead to a higher proportion of major adverse cardiac events than the current standard protocol.

MATERIAL AND METHODS: A total 120 subjects (aged $>18$ years) attending the ED with chest pain in less than or within six hours were enrolled in the study as per inclusion and exclusion criterion and after informed consent. Serum copeptin, hs trop I estimation of copeptin, and hs trop I was done along with the standard protocol followed in ED.Copeptin levels were estimated by immunoluminometric method andhs Trop Iwas estimated by chemiluminescent microparticle immunoassay.

RESULTS: Data was expressed as mean \pm SEM. Significance of association was determined by regression analysis, Student's t test and Spearman's correlation studies.ROC curves were generated to assess sensitivity and specificity throughout the concentration ranges of hs-TnI and copeptin, and to compare the ability of hsTnI alone and in combination with copeptin to diagnose ACS.A $p$ value of $<0.001$ was considered significant.

CONCLUSION: The overall diagnostic accuracy of hs Trop I alone was higher than copeptin alone. As copeptin is also secreted in non cardiac conditions triggered by stress reaction, such as sepsis, lower respiratory tract infections, or ischemic stroke. Combination of copeptin and hs Trop I improves diagnostic accuracy in patients attending the ED with chest pain within 6 hours of onset of chest pain.Hence, dual marker strategy i.e the combination of two in ED can be of help, when the diagnosis is questionable, especially in the time period of 3-6 hours after onset of chest pain.

KEY WORDS: Copeptin, ischemic stroke, hs Trop I 
FP- 16

Effect of Body Mass Index on the Association of Myocardial Work Stress, Uric Acid And Glycemic Status In Diabetes Mellitus In South Indian Population

Nivedita Nanda ${ }^{1}$, Sarvesh Ramanathan ${ }^{2}$, Sudhagar Mookkappan ${ }^{3}$.

1. Department of Biochemistry, Jawaharlal Institute of Postgraduate Medical Education and Research,

2. Pondicherry Institute of Medical Sciences,

3. Department of Medicine, Pondicherry Institute of Medical Sciences.

INTRODUCTION: Currently there is a need for simple yet affordable marker for monitoring cardiovascular (CV) risk especially for the poor section of the society. Uric acid (UA) is a routineparameter which has been linked to deterioration in glucose metabolismand $\mathrm{CV}$ morbidity separately. However, relationship of UA with glycemic status in subjects with different body mass index (BMI) has not been explored.

AIM: Therefore, the present study aims at evaluation of the metabolic and CV profiles in diabetes mellitus subjects with different BMI.

MATERIAL \& METHODS: The anthropometric, biochemical and noninvasive $\mathrm{CV}$ risk markers of myocardial work stress such as rate-pressure product (RPP) were assessed in diabetic subjectswith normal $(n=46)$ and high BMI $(n=80)$ compared with healthy age and gender-matched non-diabetic controls $(n=43)$.

RESULTS: High BMI diabetic subjects alone had increased diastolic blood pressure (DBP) $(\mathrm{P}<0.05)$, and RPP $(\mathrm{P}<0.05)$ compared to control. Mean fasting sugar (FSG)was highest $(\mathrm{P}<0.01)$ and $\mathrm{UA}(\mathrm{P}<0.05)$ was lowest in normal BMI DM patients. Our data shows that once DM sets in UA level starts rising with rise in FSG, DBP especially in high BMI DM patients. The myocardial work stress marker RPP was highest in high BMI DM patients and this was positively associated with increased UA level. The findings of the present study indicate the presence of increased myocardial work stressin DM with higher BMI also associated with a steady rise in UA level which is known antioxidant within physiological range.
Conclusion: DM subjects with higher BMI should monitor their $\mathrm{CV}$ profile more closely and this can be achieved by monitoring of simple, cheap and affordable markers such as UA and RPP even in a rural setting in developing countries.

KEYWORDS: Body mass index, Oxidative stress, Myocardialwork stress, Uric acid.

\section{FP- 17}

\section{Association of TNF-A 308 Gene Polymorphism In Brain Tumors: A Preliminary Study}

Manjulata Kumawat ${ }^{1}$, Dhara B. Dhaulakhandi ${ }^{2}$, Ishwar Singh ${ }^{3}$

Post Graduate Institute of Medial Sciences, Pt. B.D. Sharma, University of Health Sciences, Rohtak (Haryana), India 124001

TNF (Tumor Necrosis Factor) $\alpha$-gene encodes a multifunctional proinflammatory cytokine and an immunomediator located within MHC on chromosome $6 \mathrm{p} 21.3$. It is involved in the innate immune response- in regulation of a wide spectrum of biological processes as well as in several diseases. Here we have hypothesized if polymorphism of TNF- $\alpha 308$ gene could contribute to the pathogenesis of brain tumors.

INTRODUCTION: TNF alpha belongs to (TNF) superfamily. This cytokine is mainly secreted by macrophages. Being located in MHC region, TNF alpha genes present genetic linkage disequilibrium hence contain a large number of polymorphisms. Many single nucleotide polymorphisms (SNPs) in and around the TNF- $\alpha$ gene have been reported. An association between genotype and TNF-aproduction exists and it has been found that HLA-DR3, DR1, DR4, and DR7 are associated with higher TNF- $\alpha$ and HLA-DR2 and DR5 are associated with lower TNF- $\alpha$ responses. The role of TNF alpha in inflammation is well known. higher levels of TNF- $\alpha$ are related to the severity of inflammatory responses. It's not unlikely that TNF-alpha gene polymorphism could affect TNF- $\alpha$ production which in turn could lead to neuroinflammation in brain tumours. 
MATERIAL \& METHODS: DNA was extracted from tumor tissues which had been stored in appropriate stabilization buffer. We tested presence of TNF- $\alpha 308$ gene and its polymorphism in 13 clinical samples of Meningioma using PCR. The PCR reaction was optimized and performed as reported in the literature. Equal volumes of the PCR products from each sample were subjected to electrophoresis on a $1.5 \%$ agarose gel, stained with ethidium bromide and photographed.

RESULTS \& CONCLUSION: As expected we detected a PCR product of $863 \mathrm{bp}$ in the tumor samples tested and well matching to the results of the only study reporting TNF- $\alpha 308$ polymorphism in brain tumors. These results point to a potential relationship between TNF- $\alpha$ polymorphism and neuroinflamation. In this regard, biological and genetic regulation of TNF- $\alpha$ is important as trigger for TNF- $\alpha$ release could lead to greater TNF- $\alpha$ production resulting into severe inflammatory response. These preliminary results prompt to explore prognostic value of TNF- $\alpha$ genotyping with focus on developing anti-TNF- $\alpha$ therapy for treating brain tumors by targeting a specific TNF- $\alpha$ gene polymorph.

KEY WORDS: TNF- $\alpha$ 308, Brain Tumor, gene polymorphism, cytokines

\section{FP- 18 \\ Role of Vasodilator-Stimulated Phosphoprotein (VASP) In Osteosarcoma}

\author{
Kharb S, Halder M, Kundu Z* \\ Department of Biochemistry and Orthopedics*, \\ Pt. BDS PGIMS, Rohtak, Haryana
}

INTRODUCTION: Vasodilator-stimulated phosphoprotein (VASP) has been reported to play an important role in the process of cell migration and tumor metastasis. However, till date, no study has examined serum VASP levels in osteosarcoma.

AIMS AND OBJECTIVES: The present study was planned to analyse serum vasodilator-stimulated phosphoprotein (VASP) levels in osteosarcoma.
Materials and methods: Serum vasodilator-stimulated phosphoprotein (VASP) levels were studied in 50 subjects. They were categorised as patients with osteosarcoma (group I, $\mathrm{n}=25$ ), and musculo-skeletal pain (controls, group II, $\mathrm{n}=25$ ). Vasodilator-stimulated phosphoprotein levels were analysed in serum by doubleantibody sandwich enzyme-linked immunosorbent assay kits (Elabscience kit).

RESULTS AND CONCLUSION: Serum VASP levels were significantly decreased in osteosarcoma patients (group I) as compared to those with musculoskeletal pain (group II, $\mathrm{p}<0.05$ ). Serum VASP levels showed a positive correlation with serum calcium levels in both the osteosarcoma and control groups. Serum VASP levels showed a negative correlation with serum phosphorus levels in both osteosarcoma and control groups.Serum alkaline phosphate levels showed positive correlation with serum VASP levels in controls, which got inversed in osteosarcoma cases.Findings of the present study of negative correlation between VASP and ALP in osteosarcoma patients indicate that involved in the pathogenesis of osteosarcoma.

KEY WORDS: Osteosarcoma, VASP, alkaline phosphatase, calcium

\section{FP- 19}

\section{Role of Vitamin D and Its Associated Molecules In Innate Immunity In Pulmonary Tuberculosis As Compared To Household Contacts}

Sudhasini Panda ${ }^{1}$, Ambrish Tiwari ${ }^{1}$, Kalpana Luthra ${ }^{1}$, S.K. Sharma ${ }^{2}$, Archana Singh ${ }^{1}$

1. Department of Biochemistry, All India Institute of Medical Sciences, New Delhi-110029, India

2. Department of Medicine, All India Institute of Medical Sciences, New Delhi-110029, India.

INTRODUCTION: Vitamin D3 along with its nuclear receptor, vitamin $\mathrm{D}$ receptor (VDR) brings about immunomodulatory activities via antimicrobial peptide Cathelicidin, the latter has been found to be implicated in tuberculosis. Also the bioavailability of vitamin D depends upon vitamin D binding protein (VDBP). 
AIMS AND OBJECTIVES: We attempted to investigate the frequency of fok I VDR polymorphism along with vitamin D, VDR, VDBP and cathelicidin levels in tuberculosis patients and household contacts as compared to healthy controls and assess the implication of these findings in susceptibility to tuberculosis.

STUDY SUBJECTS AND METHODS: 60 active TB patients, 60 household contacts and 50 healthy controls were taken. Fok1 polymorphism was studied in all the groups by PCR-RFLP. VDR and VDBP mRNA levels were studied using real time PCR. The levels of serum VDR and cathelicidin were measured using ELISA. Vitamin D levels were measured in serum samples using CLIA.

RESULTS: No association was found between VDR polymorphism and susceptibility to tuberculosis (P $=0.12$ ). VDR mRNA levels were found to be lower in active TB group compared to household contacts and healthy controls with (p-0.0001). VDBP expression was lower in patients as compared to other two groups but the difference was not significant (p-0.19). The serum levels of Vitamin D and VDR were also found to be lower in active TB group as compared to healthy controls $(\mathrm{P}=$ $0.007 \& 0.001$ respectively). However, the expression of VDR and levels of vitamin D was significantly $(\mathrm{P}<$ $0.05)$ higher in household contacts compared to both active TB and healthy control groups. As expected, the level of cathelicidin was higher in patients as compared to other groups $(\mathrm{P}=0.01)$.

CONCLUSION: Higher level of cathelicidin was found in active TB group despite decreased expression of VDR and low vitamin D levels. This may be attributed owing to the trigger from both VDR and toll like receptors by M. Tuberculosis derived ligand. The higher frequency of wild type genotype, increased VDR expression along with increased vitamin D levels in household contacts compared to active TB group might be responsible for protection against the active disease. However, more sample size is required to validate the results.

KEY WORDS: Tuberculosis, cathelicidin, VDR polymorphism
FP- 20

\section{Study of Serum Levels of Gamma Glutamyl Transferase, Uric Acid and CRP In Patients With Chronic Obstructive Pulmonary Disease Patients In A Tertiary Care Hospital}

Mallika Barman, Barnali B Thakur

Gauhati Medical College, Guwahati, Assam

INTRODUCTION: COPD is a major and increasing public health problem and the 4th leading cause of death in the world. COPD is defined as a/preventable and treatable disease characterized by persistent airflow limitation that is not fully reversible. Risk factors for COPD are smoking, alpha1 anti-trypsin deficiency, recurrentbroncho pulmonary infections, air pollution,low socio economic status, low birth weight and history of severe childhood respiratory infections. GGT is a membrane bound enzyme and it initiates the turnover of glutathione which has important antioxidant propreties and levels of GGT has been shown to be upregulated in cases of oxidative stress.Uric acid is present in body fluids and known to have role asan endogenous free radical scavenger.CRP is an acute phase reactant and raises in inflammatory conditions.Uric acid and CRP are known to provide prognostic informationand thus enables to detect those at high risk.

MATERIALS AND METHODS: A cross sectional study was carried out for a period of 45 days (July-August 2017) and the patients were selected from the Dept of Pulmonary Medicine, GMCH and the study was conducted in the Dept of Biochemistry, GMCH.

The study group consists of patients with chronic pulmonary disease irrespective of duration and the control group of ageand sex matched healthy individuals. Both cases and controls were interviewed taking informed consent. Based on inclusion and exclusion criteria a minimum of 35 cases and controls age and sex matched were selected. Serum samples of patients were collected and analysed in central clinical laboratory in VITROS 5600 INTEGRATED SYSTEM ORTHO CLINICAL DIAGNOSTICS using the principle of chemi-illuminisence. 
RESULTS: GGT has been shown to be upregulated in oxidative stress.Activity of GGT as a possible marker of oxidative stress has been seen. Uric acid and CRP areknown to provide prognostic infornation and may help to detect patients at high risk of mortality thus facilitating identification of high risk COPD patients who can benefit from intensive management.

CONCLUSION: Studies have shown that serum GGT shows a significant elevation with acute exacerbsation of COPD. Studies have shown that CRP measurement provide incremental prognostic response and can be used in accurate detection of patients at high risk of mortality in COPD.

KEY WORDS: COPD, oxidative stress

\section{FP- 21}

Predictive Models Utilizing Plural Fluid Adenosine Deaminase In Diagnosing Tuberculous Plural Effusion (TPE) In An Area With A High Incidence of Tuberculosis And HIV.

S Girish, V Patil, A Joshi, N Ramraje

Grant Govt. Medical College, Mumbai, Maharashtra, India.

INTRODUCTION: Tuberculous Plural Effusion (TPE) is the most common form of the extra pulmonary tuberculosis in India.Prediction based on adenosine deaminase (ADA) is dependent on age as well as the tuberculosis incidence. As the determination of ADA is still not routinely used under National algorithm, the aim of this study was to evaluate the diagnostic usefulness of ADA along with other data from pleural fluid analysis using prediction models, in patients from populations with high prevalence of tuberculosis (TB) and HIV.

METHODS : Total 70 patients with pleural effusion (20 tuberculous, 21 infectious, 11 neoplastic, 15 miscellaneous, 3 idiopathic) were analysedretrospectively. Two predictive models; one included pleural fluid ADA values (model 1), and the other without ADA (model 2) were performed. The statistical modelling used analysis adjusted for the following covariates: gender, fever, chest pain, dyspnoea, cough, sputum, size and location of the effusion, accompanying pulmonary lesions, red and total nucleated cell counts, lymphocytes (\%), neutrophils (\%), plural fluid chemistry. The final regression tree was derived using the CART approach.

RESULTS: Model 1 selected two variables (ADA $>35$ $\mathrm{U} / \mathrm{L})$ and lymphocytes $(>31.5 \%)$ and correctly classified 68/70 effusions ( 1 false negative, 1 false positive). Model 2 (without ADA) selected five variables: lymphocytes (>31.5\%), CD4, plural fluid LDH, Alkaline phosphatase and plural fluid proteins correctly classifie4 and 59/70 effusions ( 8 false negatives, 3 false positives). The sensitivity [GD1] of models, 1 and 2 was $99.4 \%$ and $95.2 \%$, specificity $98.1 \%$ and $94.3 \%$ and accuracy $99 \%$ and $95 \%$.

CONCLUSIONS: In conclusion, in areas with a high prevalence of tuberculosis and HIV it is possible to safely predict TPE with either of the two models can be included in national diagnostic algorithm.

KEY WORDS: Predictive model, Plural fluid ADA, Tuberculous Plural effusion.

FP- 22

\section{Serum Magnesium Levels In Pre \& Post Liver Transplantation}

Pradeep Naik, Malikarjun M

Department of Clinical Biochemistry, Global hospitals, Hyderabad

INTRODUCTION: Serum magnesium $(\mathrm{Mg})$ exists in ionized $(62 \%)$, protein-bound $(33 \%)$ and anioncomplexed $(5 \%)$ forms. Total hypomagnesaemia invariably occurs during liver transplantation (LTx) mostly because of transfusion-related citrate toxicity and chelation of $\mathrm{Mg}$ during the an hepatic phase, Patients with end-stage liver disease (ESLD) are prone to hypomagnesaemia as a result of malnutrition, malabsorption, diarrhea, secondary hyperaldosteronismand diuretic treatment.

MATERIAL \& METHODS: This study evaluates the efficacy and safety of routine Mglevels in pre and post LTx to prevent hypomagnesaemia after LTx.We 
estimated GGT, ALP \& Mg levels in sera of 35pre LTxand postLTx patients. GGT, ALP activity and Magnesium levels were estimating till 10th day of post LTxin males ( $n=31$, females $(n=4)$ in IP \& 40 day in op. Total patients are divided in to three groupsbased on $\mathrm{Mg}$ levels in pre LTxHypomagnesaemia $(\mathrm{n}=12)$ normal $(\mathrm{n}=12)$ and Hypermagnesaemia $(\mathrm{n}=9)$. GGT, ALP activity andMg levels were estimated from 30 normal individuals and considered as a control group (Mgnormal range is1. 7-2.1mg/dL, Alp 30- 132 u/l GGT 7-62u/l).

RESULTS: Mg was supplemented in hypomagnesaemia patients and we observed no complications after post LTx. In one case of normal group we observed complications of ACR on 38 day of Post LTx due to developing of hypomagnesaemia who was not given $\mathrm{Mg}$ supplementation based on this observation we have started giving Mgsupplementation. After LTx $1.0 \mathrm{gm}$ of $\mathrm{Mg}$ was supplemented per day to patients having $\mathrm{Mg}$ normal levels $1.5-2.0 \mathrm{mg} / \mathrm{dl}$ in serum pre LTx even in normal group to overcome the complications of hypomagnesaemia. And $2.0 \mathrm{gm}$ of $\mathrm{Mg}$ was supplemented per day to patients had $\mathrm{Mg}$ levels below $1.5 \mathrm{mg} / \mathrm{dl}$ in serum pre LTx. The results showed lower prevalence of post-operative hypomagnesaemia in patients administered $\mathrm{Mg}$ supplementation post LTx the Mg has increasing trend up to 5th day of post LTx after that it reaches to normal range and maintain stable in both groups In hypermagnesaemia group no complications were observed after post LTx Severe hypermagnesaemia can exert an anti-calcium effect on the cardiovascular system, causing vasodilatation, which is deleterious during reperfusion that the reason we are not supplement $\mathrm{Mg}$ to the hypermagnesaemia pre LTx group.

CONCLUSION: Low levels of Mg post LT x may cause ACR maybe due to antibiotics and immunosuppressive drugs. Hence magnesium levels has to be maintain at normal level. $\mathrm{Mg}$ levels are also used to monitor dosage of immunosuppressive drugs.In response to the observation of significant pre LTxhypomagnesaemia in LTx patients, we recommend the practice of magnesium supplementation post LTx inhypomagnesium group and group with normal levels by monitoring Mg levels.

KEYWORDS: Hypomagnesaemia, preLTx, post LTx

\section{FP- 23}

\section{A Very Rare Case of Carbonate-Apatite Stone In 31 Years Old Male Patient}

Rajeev Gupta, Nitin Dayal, Anupama Arya, Dilip Kimar, Poonam Das

Institute of Laboratory Medicine, Max Super Specialty Hospital (West Block), 1-Press Encalve Road, Saket, New Delhi-110017, India

INTRODUCTION: Nephrolithiasis is a common condition, affecting 5 in 1000 persons. It is heterogeneous disorder, with stones developing from a wide variety of metabolic or environmental disturbances.

MATERIAL AND METHODS: The morphological studies were made with the whole calculus. Qualitative chemical analysis was done on pulverized calculus using clean pestle and mortar to obtain finely ground powder using kit provided by Biolabo Reagents, France. The results were confirmed by Fourier Transform Infrared Spectroscopy (FTIR) method.

RESULTS: Chemical Analysis results of Kidney stone showed mainly calcium Carbonate along with struvit (Magnesium Ammonium Phosphate) and FTIR method gave the composition of carbonate Apatite $100 \%$.

CONCLUSION: Magnesium Ammonium Phosphate (Struvite) forms stones at alkaline $\mathrm{pH}$, where the ammonium level is high. Struvite stones have been referred to infection stones and "triple Phosphate" stones. Carbonate ions were also commonly identified; they were assumed to be associated with calcium carbonate $\left(\mathrm{CaCo}_{3}\right)$. Calcite $\left(\mathrm{CaCo}_{3}\right)$ is extremely rare $(1 \mathrm{in} 20,000$ stones). Numerous clinical reports attest to the association of urea -splitting bacteria, struvite and carbonate (apatite) stones. All strains of Proteus and both strains of Staphylococcus split urea, and the stones formed were composed of struvite and Carbonate-apatite.

KEY WORDS: FTIR, Struvit, Apatite, Triple Phosphate stone 


\section{FP- 24}

\section{Relation of Catalytic Iron With The Cell Free Hemoglobin Resulted From The Dialysis Pump Injury of Rbcs In Patients on Maintenance Hemodialysis}

Mukhopadhyay B, Hegde U, Chakurkar V, Konnur A, Gang S, Rajapurkar M.

Muljibhai Patel Society for

Research in Nephro Urology,

Dr V Desai Marg, Nadiad-387001, Gujarat.

INTRODUCTION: Catalytic iron (CI) is the non transferrin bound iron involved in generation of toxic free radicals in body. Blood pump induced injury to RBC's may lead to increase in cell-free hemoglobin (fHb) after dialysis which is a potential source of CI. We examined the effect of dialysis session on generation of $\mathrm{fHb}$ and SCI in MHD patients.

MATERIALS AND METHODS: Eighty five MHD patients (63 males, 22 females) were included in this study. Heparin samples were collected pre and post dialysis ( $\mathrm{n}=522$ ) every 3 monthly, over 21 months period. Plasma were immediately separated and stored at $-700 \mathrm{C}$. The CI was measured by modified Bleomycin detectable iron assay. Plasma fHb levels were estimated by a highly sensitive ELISA kit (My Biosource, USA). C-Reactive protein (hsCRP) in the pre dialysis samples were also measured by Beckman AU480.

RESULTS: There were significant $(\mathrm{p}<0.0001)$ increase of mean post dialysis CI $(0.766 \pm 2.195 \mu \mathrm{mol} / \mathrm{l} \mathrm{Vs} 1.423$ $\pm 4.41 \mu \mathrm{mol} / \mathrm{l})$ and $\mathrm{fHb}(7.61 \pm+7.64 \mathrm{mg} / \mathrm{dl} \mathrm{Vs} 16.144 \pm$ $12.27 \mathrm{mg} / \mathrm{dl})$. The post dialysis change in $\mathrm{CI}$ in patients were significantly in correlation with the change in $\mathrm{fHB}$ (unpaired t test: two tailed $\mathrm{p}$ value $=0.0006$ ). The correlation in between the pre dialysis CI levels and hsCRP levels were highly significant ( $\mathrm{p}$ value $<0.0001$ ).

CONCLUSION: Our study shows thathemodialysis results in a significant increase in fHband CI. The dialysis pump induced fHb may thus be associated to increase oxidative stress and inflammation through the generation of $\mathrm{CI}$ in patients.
KEY WORDS:Iron, non transferring, MHD, cell-free hemoglobin

\section{FP- 25}

Interpretation of Clinical Biochemistry Results In the Context of Renal Dysfunction

Sudip Kumar Datta,Tapasyapreeti Mukhopadhyay, Sudhanshu Shekhar

All India Institute of Medical Sciences, New Delhi

INTRODUCTION: A lot of routinely used parameters in clinical biochemistry are known to be excreted through the kidneys. Hence, in patients with renal dysfunction some of these parameters may be falsely deranged without any pathological causes just as a fall-out of deranged excretory system.

MATERIALAND METHODS: We looked into several routine clinical biochemistry parameters in patients with different stages of kidney dysfunction without any other known or obvious underlying ailments in a crosssectional study design. Estimated glomerular filtration rate (eGFR) was calculated from serum creatinine levels using 4 parameter MDRD formula and was used for staging of chronic kidney dysfunction (CKD). The results were compared with 46 age-sex matched healthy controls and also with the normal reference ranges as quoted by the kit manufacturers. Appropriate statistical tools like t-test or ANOVA and Pearson's correlation tests were done for comparison.

RESULTS: Two parameters namely serum total amylase and acid phosphatasewere found to be significantly elevated in CKD patients without any obvious clinical causes. However, strong correlation with the eGFR was not observed.

CONCLUSION: In the setting of kidney dysfunction the interpretation of some routine clinical biochemistry parameters should be done with caution. 
KEY WORDS: Kidney dysfunction, CKD, interpretation

\section{FP- 26}

\section{Vitamin B12 Uptake In B12 Deficient and Replete Subjects}

\section{Dr. Sadanand S. Naik}

KEM Hospital, Pune, Deenanath Mangeshkar Hopsital \& Res Centre, Pune

$\mathrm{C}$ oba Sorb test was performed employing various doses $(1.5,3,6$, and $9 \mathrm{ug})$ and free forms $(\mathrm{OH}-\mathrm{B} 12$ and CN B12) in vitamin B12 deficient(plasma B12 <148 $\mathrm{pmol} / \mathrm{L}$ ) and sufficient participants(Plasma B12> 200 $\mathrm{pmol} / \mathrm{L})$.

We recruited 62 healthy individuals (32 males and 30 females) from the population with low cobalamin status (group A) and in the population with normal cobalamin status (group B), we recruited 45 healthy individuals (17 males and 28 females.

We have shown that oral intake of free $\mathrm{CN}-\mathrm{Cbl}$ induced a higher increase in circulating holoTC concentrations than free $\mathrm{HO}-\mathrm{Cbl}$, and that both individuals with a low and a normal cobalamin status have an absorptive capacity that is saturated above doses of $3-\mu \mathrm{g}$ three times a day. Further studies are needed to determine if $\mathrm{CN}-\mathrm{Cbl}$ or $\mathrm{HO}-\mathrm{Cbl}$ provides the

\section{IMPROVIT 2:}

Participants aged $>18$ years $(n=60)(27$ males and 33 females) were recruited from the Pune area in India, and the study was carried out at Deenanath Mangeshkar Hospital and Research Center, Pune, India, in the spring of 2016. Most of the participants were healthy staff at the hospital. Inclusion criterion was a plasma $\mathrm{Cbl}$ below $150 \mathrm{pmol} / \mathrm{L}$ measured two weeks prior to enrollment. The 60 participants were randomly divided into three groups (CN-group $(\mathrm{n}=20)$, HO-group $(\mathrm{n}=20)$, and placebo group $(\mathrm{n}=20)$ ) with an equal distribution of sex and age. All participants were asked to eat their usual diet throughout the study.
The participants received one daily dose of $3-\mu \mathrm{g} \mathrm{CN}$ B12 (CN-group), 3- $\mu$ g HO-B12 (HO-group), or no supplementation (placebo group) for eight weeksNo major differences were observed between males and females (data not shown).

In conclusion, we have demonstrated that eight weeks of daily oral treatment with $3-\mu \mathrm{g} \mathrm{CN}-\mathrm{B} 12$ or $3-\mu \mathrm{g} \mathrm{HO}-$ B12 causes an increase in total serum $\mathrm{Cbl}$ and holoTC, plus minor changes in the metabolic markers MMA and Hcy. Our findings show that the current RDA is insufficient for short-term restoration of an impaired B12 status. Future studies are requested to establish the dose of B12 needed in order to restore an impaired B12 status. A benefit of $\mathrm{HO}-\mathrm{B} 12$ versus $\mathrm{CN}-\mathrm{B} 12$ administration was suggested, but not definitively proven. Further studies should be directed to explore the dose and the form of B12 requested in order to restore and maintain an optimal B12 status in populations with low dietary intake of this vitamin.

Similarly, vitamin B12 deficient subjects were supplemented with $400 \mathrm{~mL}$ of milk containing $4 \mathrm{ug} / \mathrm{L}$ of B12 for 2 weeks and the results will be discussed

\section{FP- 27}

\section{A Marine Sponge Derived Natural Product, Arenosclerin E As An Inhibitor of Mycothiol-S- Conjugate Amidase}

Akansha Saxena, Amit Mani Tiwari, Sanjay Mishra*

Department of Biotechnology, IFTM University, Moradabad 244102, U.P., India.

INTRODUCTION: Marine sponges have potential sources for secondary metabolites and are considered as a drug treasure house.

MATERIAL AND METHODS: 3D model of Mycothiol-S-conjugate amidase (Mca) was determined by comparative homology modeling program MODELLER based on the crystal structure of 1-D-myoinositol 2-acetamido-2-deoxy-alpha-D-glucopyranoside deacetylase (MshB) from Mycobacteriumtuberculosis as a template. The computed model's energy was minimized and validated to obtain a stable model structure. Stable 
model was used for docking of nineteen bioactive compounds isolated from marine sponges against Mca using AutoDock 4.2. The docked complexes were validated and enumerated based on the AutoDock Scoring function to pick out the best marine inhibitors based onbinding energy.

RESULTS: From the entire marine compounds which were docked, we got best one (Arenosclerin E) of them with optimal binding energy $-13.11 \mathrm{kcal} / \mathrm{mol}$. Further the best-docked complex was analyzed through Python Molecular Viewer software for their interaction studies. The docked protein - inhibitor complex structure was optimized using molecular dynamics simulation for $5 \mathrm{ps}$ with the CHARMM-22 force field using NAMD incorporated in VMD 1.9.2 and then evaluating the stability of complex structure by calculating RMSD values.

CONCLUSION: Thus from the Complex scoring and binding ability its deciphered that this marine derived compound could be promising inhibitor for Mca as drug target yet pharmacological studies have to confirm it.

KEY WORDS : Mycothiol-S-conjugate amidase; Mycobacterium tuberculosis; Mca; NAMD; VMD

\section{FP- 28}

\section{Novel Biomarkers For Preeclampsia Screening And Diagnosis}

\section{Dr. Saswati Das}

\section{Consultant Biochemistry, Quest Diagnostics India}

Preeclampsia (PE) is an major cause of maternal and perinatal mortality and morbidity.Pre-eclampsia (PE) affects about $2 \%$ of pregnancies globally. A major challenge in modern obstetrics is early identification of pregnancies at high-risk of preterm $\mathrm{PE}$ and undertaking of the necessary measures to improve placentation and reduce the prevalence of the disease. Early PE (requiring delivery before 34 weeks) rather than late PE is associated with an increased risk of perinatal mortality and morbidity and both short-term and long-term maternal complications. Early identification of women at highrisk for PE could potentially improve pregnancy outcome as intensive maternal and fetal monitoring in such patients would lead to an earlier diagnosis of the clinical signs of the disease and the associated fetal growth restriction and avoid the development of serious complications through pharmacological interventions starting from the first trimester. The underlying mechanism for PE is thought to be impaired placentation, documented by the findings of abnormal blood flow in the uterine arteries and reduced maternal serum levels of placental products.PIGF (Placental Growth Factor) has been shown to be the most discriminating biochemical marker for pre-eclampsia, and early-onset pre-eclampsia in particular. The patient-specific risk of developing PE in the first trimester can be predicted by a combination of factors in the maternal history, prior or family history of PE, and the maternal blood pressure(MAP), uterine artery pulsatility index (UtPI), PAPP-A, PLGF. The antiangiogenic factor soluble fmslike tyrosine kinase 1 (sFlt1) and the sFlt-1:PlGF ratio has shown promise in clinical research as a biomarker for predicting and diagnosing preeclampsia in the second and third trimester.

\section{FP- 29}

\section{Clinical Symptoms and Management of Beta Thalassemia}

Nitu Nigam', Monica Agarwal², Prithvi Kumar Singh ${ }^{1}$, Syed Rizwan Hussain ${ }^{1}$

\section{Cytogenetics Unit, Department of Centre for advance research, KGMU, Lucknow \\ 2. Department of Obs \& Gyn, KGMU, Lucknow}

Thalassemia is a single gene disorder is characterised by reduction or absence of beta-globin chain. It is commomest in the Middle East, Southeast Asia, African and some Asian countries including India. Thalassemia has diverse clinical phenotypes. Thalassemia major patients present with severe anemia requiring regular blood transfusion for survival whereas Thalassemia trait is characterizedby mild hypochromic, microcytic anemia with elevated HbA2 levels. The prevalence of thalassemia in India 3.3\% and a high of $17 \%$ in certain communities. There are 30 million carriers and 
approximately 10000 children are born with the disease every year. Estimated cost for treatment is 1 lacs per child per year which is difficult to afford in developing countries. Management of thalassemics is not only traumatic to the family but also poses a tremendous socioeconomic burden on the country making.

Its control and prevention a cause of prime concern. Prenatal diagnosis, of thalassemia offers parents to make reproduction choices and therefore reduce the burden from the society.

KEYWORDS: Thalassemia, Beta-globin, Anemia, PrenatalDiagnosis, Blood transfusions

\section{FP- 30}

\section{MicroRNA: A New Potential Marker for Prostate Cancer}

Mohammad Waseem ${ }^{1,2}$, M. Serajuddin ${ }^{2}$, A A Mahdi ${ }^{1}$, S N Sankhwar ${ }^{1}$, DP Mishra ${ }^{3}$ MK Ahmad ${ }^{1}$

1. Department of Biochemistry, King George's Medical University, Lucknow, U. P. India

2. Department of Zoology, Lucknow University, Lucknow, U.P, India

3. Endocrinology division, CDRI, Lucknow, U.P. India

INTRODUCTION: Prostate cancer is maximally diagnosed cancer still it is the second leading cause of death in western world. So, there is urgent need of a new biomarker for diagnosis as well as therapy purpose. MicroRNAs (miRs) are noncoding RNA which regulate about $30 \%$ of human genes. Due to their stability, sensitivity and specificity in sample these miRs are current hot topic of research in the field of biomarker hunt.

MATERIAL AND METHODS: In the present study, we evaluated differentially expressed microRNA in benign prostatic hyperplasia (BPH), prostate cancer (PCa) on and control subjects by Microarray analysis. Most significantly up regulated microRNAs were validated through RT-PCR analysis.

RESULTS: Our result showed that miR-1827, miR-4510 and $\mathrm{miR}-130 \mathrm{~b}-3 \mathrm{p}$ were over expressed in prostate cancer as compared to control as well as BPH. MiR-1827, miR4510 was also found to be up regulated in high Gleason score and metastasis tissue. These miRs showed good specificity and sensitivity in receiver operating characteristic (ROC) analysis which may prove helpful in differentiating between prostate cancer and Control. High expression of miR-1827 correlated with the poor prognosis and low progression free survival in $\mathrm{PCa}$ subjects in comparison to low expression of miR-1827.

CONCLUSION: The putative microRNAs, miR-1827, miR-4510 and miR-130b-3p will be used as diagnostic marker and also for therapeutic purpose to overcome prostate cancer burden in coming scenario.

KEYWORD: Prostate cancer, BPH, Microarray, RTPCR, Diagnostic and Prognostic marker

\section{FB- 31}

MDR-1 Gene Polymorphisms In SteroidResponsive Versus Steroid-Resistant Nephrotic Syndrome In Children

Tabrez Jafar ${ }^{1}$, Narayan Prasad ${ }^{2}$, Abbas Ali Mahdi ${ }^{1}$,

1. Department of Molecular Medicine, Era University, Lucknow, India,

2. Department of Nephrology, Sanjay Gandhi Postgraduate Institute of Medical Sciences, Lucknow, India,

3. Department of Molecular Medicine, Era University, Lucknow, India

BACKGROUND:- The putative genetic regulation of multidrug resistance gene-1 (MDR-1) gene expression andP-glycoprotein function has not yet been clearly delineated in patients with nephrotic syndrome (NS). We undertook this study to examine the distribution of three most frequent MDR-1 exonic polymorphisms G3435C, G2677T/A and C1236T in patients with NS and control children to investigate their usefulness as markers of responsiveness of the disease to steroids.

METHODS:-Two hundred and sixteen children with 
NS and 216 healthy controls were genotyped for three exonicMDR-1 polymorphisms (G3435C, G2677T/A and $\mathrm{C} 1236 \mathrm{~T}$ ) by using the polymerase chain reactionrestriction fragment length polymorphism technique. The frequency distribution of genotypes/alleles was compared between patients with NS and controls and also between steroid-sensitive NS (SSNS) and steroidresistant NS (SRNS) patients.

RESULTS:-Of the total 216 cases of NS (median age of onset 5 years, 165 males), 137 had SSNS, and 79 had SRNS. Homozygous mutants of C3435T (TT versus CC, $\mathrm{P} 1 / 40.034$ ) and G2677T/A (TT 1 AA versus GG), P 1/4 0.030 ) were significantly higher in patients with NS compared to controls. The frequency distribution of homozygous mutant TT1AA compared to wild genotype GG was significantly higher in SRNS than SSNS patients (P 1/4 0.011) for G2677T/A, while the mutant genotypes for C3435T and C1236T were not differentbetween SRNS and SSNS patients. The combination-bearing mutant genotype either of C3435T or G2677T/A exhibited a significantly higher frequency of mutant genotypes distribution in SRNS patients. MDR-1 haplotypes did not differ significantly between SSNS and SRNS patients.

CONCLUSIONS:- Patients with NS carrying homozygous mutants of single nucleotide polymorphism (SNP) G2677T/A are prone to develop SRNS. The synergistic effect of mutant genotypes of SNPs G2677T/ $\mathrm{A}$ and $\mathrm{C} 3435 \mathrm{~T}$ in different combinations increase the risk of developing steroid resistance in patients with NS.

\section{FP- 32}

Follicle Stimulating Hormone and Testosterone Levels and Their Correlation with Sperm Concentration and Motility In Male Partners of Infertile Couples

Suruchi Mathur ${ }^{1}$, Kalbe Jawad ${ }^{1}$, Tariq Mahmood ${ }^{1}$, Seema Dayal ${ }^{2}$, Shikha Seth ${ }^{3}$

1. Department of Biochemistry,

2. Department of Pathology,

3. Department of Obstetrics \& Gynecology, Uttar Pradesh University of Medical Sciences, Saifai, Etawah, Uttar Pradesh

INTRODUCTION: Follicle Stimulating Hormone (FSH) and testosterone are the prime regulators of germ cell development. The successful and complete male germ cell development and function is dependent on the balanced interplay of these hormones along with others. This study was undertaken to assess and correlate the serum levels of FSH and testosterone along with the sperm concentration and sperm motility in males of infertile couples.

MATERIALS AND METHODS: In this prospective descriptive study, serum FSH and testosterone levels were estimated in 24 male partners of couples presenting with infertility at the out-patient clinic of a rural tertiary care medical college hospital. Microscopy of the semen to assess sperm concentration and motility according to the WHO criteria was done for all enrolled patients.

RESULTS: The serum FSH and testosterone levels were classified according to normal ranges. Seven (29.2\%) patients had low FSH levels and 17 (70.8\%) had normal FSH levels. Seven (29\%), 13 (54\%) and 4 (16.7\%) patients had low, normal and high levels of serum Testosterone. The sperm concentration, progressive and total motility was classified according to the WHO criteria. Fifteen $(62.5 \%)$ patients had normal semen microscopy findings. Three $(12.5 \%)$ patients had asthenozoospermia and $6(25 \%)$ had oligoasthenozoospermia. A significant correlation was found between FSH levels and sperm progressive motility $(r=0.406$, $\mathrm{p}=0.049$ ).A significant correlation was also found 
between the sperm concentration and sperm progressive motility $(r=0.432, p=0.035)$.

CONCLUSION: FSH levels directly correlate with sperm progressive motility. Sperm concentration also correlates significantly with sperm progressive motility. Larger studies may be required to validate this result.

KEY WORDS: Male infertility, Follicle Stimulating Hormone (FSH), Testosterone, Semen analysis

\section{FP- 33}

\section{Effect of Eight Weeks Oral Supplementation

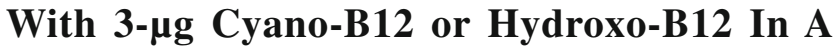 Vitamin B12 Deficient Population}

Eva Greibe ${ }^{1}$, Namita Mahalle ${ }^{2}$, Vijayshri Bhide ${ }^{2}$, Sergey Fedosov ${ }^{3}$, Christian W. Heegaard ${ }^{3}$, Sadanand $\mathrm{Naik}^{2}$, Ebba Nexo

\section{Department of Clinical Biochemistry and Institute} of Clinical Medicine, Aarhus University Hospital, Aarhus, Denmark;

2. Department of Pathology, Deenanath Mangeshkar Hospital and Research Center, Pune, India;

3. Department of Molecular Biology and Genetics, Aarhus University, Aarhus, Denmark.

Email:pnmahalle@gmail.com, cell no: 09689935996

PURPOSE: We compared the effect of eight weeks oral supplementation with cyano-B12 (currently used in vitamin pills) and hydroxo-B12 (predominant form in the diet) in a population with nutritional vitamin B12 deficiency.

METHODS: Fifty-one healthy Indian adults with baseline serum cobalamin $<150 \mathrm{pmol} / \mathrm{L}$ were supplemented for eight weeks with daily oral supplements of 3- $\mu$ g cyano-B12 $(n=15), 3-\mu$ g hydroxoB12 $(n=16)$, or placebo $(n=20)$. Blood at baseline and each following week was examined for total cobalamin, holotranscobalamin, methylmalonic acid, and homocysteine.

RESULTS: The study groups did not differ at baseline and were characterized by (median [range]) serum cobalamin (128 [68-191] pmol/L), holotranscobalamin (16 [6-41] pmol/L), methylmalonic acid (0.8 [0.3-1.7] $\mu \mathrm{mol} / \mathrm{L}) \&$ homocysteine $(17.9[8.5-100.9] \mu \mathrm{mol} / \mathrm{L})$. The group supplemented with cyano-B12 showed a higher increase in total serum cobalamin than the group treated with hydroxo-B12, while other biomarker changes were comparable in the two groups. After eight weeks of treatment, the biomarker values of the supplemented groups (pooled) significantly differed from the placebo group. Yet, the vitamin B12 status was still poor (cobalamin: 168 [87-302] pmol/L; holotranscobalamin: 19 [8-45] pmol/L; methylmalonic acid: 0.7 [0.2-1.7] $\mu \mathrm{mol} / \mathrm{L}$; homocysteine: 17.2 [2.6-96.8] $\mu \mathrm{mol} / \mathrm{L}$.

CONCLUSION: Eight weeks supplementation with 3- $\mu$ g cyano-B12 elevated serum cobalamin more than 3- $\mu \mathrm{g}$ hydroxo-B12, but all other biomarkers behaved alike in both groups. Supplementation with $3-\mu \mathrm{g}$ vitamin B12 did not rescue the low status in individuals with nutritional vitamin B12 deficiency.

\section{FP- 34}

\section{A Pilot Study of Insulin Resistance, Oxidative Stress and Chronic Inflammation In Opiate Dependent Males of Western Rajasthan}

Purvi Purohit ${ }^{1}$, Naresh Nebhinani ${ }^{2}$, Praveen Sharma ${ }^{1}$

1. Department of Biochemistry, All India Institute of Medical Sciences Jodhpur,

2. Department of Psychiatry, All India Institute of Medical Sciences Jodhpur

INTRODUCTION: Opium is the second-mostcommonly abused substance after tobacco. Ischemic heart disease is one of the most common cause of death in opiate addicts. Chronic use of opiates complicates the effects on cardiometabolic health.

MATERIALS AND METHODS: The study included 120 subjects (30 healthy controls, 60 opiate dependents with and without smoking dependence and 30 smokers) attending the de-addiction clinic at a tertiary care centre. Inclusion criteria was chronic opiate and/ smoking history for minimum one year, in male subjects under 50 years 
of age and no associated co-morbid conditions. The subjects were studied for metabolic syndrome (MS) components based on NCEP -ATP -III criteria and blood samples were analysed for fasting blood sugar (FBS), insulin, Homeostasis model assessment insulin resistance (HOMA-IR), lipid profile, Hs-CRP and total antioxidant capacity (TAC).

RESULTS: The study showed a significant difference across the four groups for waist to hip ratio $(\mathrm{F}=9.09, \mathrm{p}=0.00001)$, systolic blood pressure $(\mathrm{F}=3.64$, $\mathrm{p}=0.014)$, FBS $(\mathrm{F}=8.9, \mathrm{p}=0.00001)$ and $\mathrm{TAC}(\mathrm{F}=2.72, \mathrm{p}$ $=0.04)$ using one way ANOVA. However, there was nonsignificant difference for Hs CRP $(\mathrm{F}=2.20, \mathrm{p}=0.09)$ across the groups. The frequency of MS according to NCEP ATP-III criteria, was highest in the opiate + smoking group $(43.3 \%)$ followed by pure opiate dependents (26.7\%) and smoking group (14.81\%). Multiple regression analysis of opiate dependents showed that TAC, FBS, total cholesterol (TCH), triglycerides, low density lipoproteins (LDL) and Hs CRP ( $\mathrm{F}=4.80$, $\mathrm{p}<0.0001)$ independently predicted HOMA-IR. Further Pearson's correlation analysis showed significant association of HOMA-IR with FBS, TAC, TCH and LDL.

CONCLUSION: The study helps conclude that chronic opiate dependents are a unique group with significant low grade inflammation and oxidative stress that pushes for insulin resistance, even in the absence of comorbidities like diabetes or hypertension. The consumption of opiates with smoking further adds to this risk. Thus a routine monitoring of the cardio-metabolic profile in these subjects can prove to be useful.

KEYWORDS: Insulin resistance, Oxidative stress, Inflammation, Opiate addiction, Cardio-metabolic.

\section{FP- 35}

Association of ADIPOQ (+276 G/T) Gene Polymorphism And Oxidative Stress In Cardiovascular Disease of North Indian Women of Punjab

Jyot Amrita, ${ }^{*}{ }^{*}$ MridulaMahajan, ${ }^{2}$ AJS Bhanwer ${ }^{3}$

1. Department of Biochemistry, Sri Guru Ram Das Institute of Medical Sciences and Research, Amritsar, Punjab, India

2. Department of Biochemistry (Ex), Government Medical College, Amritsar, Punjab, India

3. Department of Human Genetics, Guru Nanak Dev University, Amritsar, Punjab, India

INTRODUCTION: Adiponectin gene plays an important role in inflammation and atherosclerosis. AdipoQ (+276G/T) gene polymorphism has been found to be associated with cardiovascular disease.

AIM: Our aim was to analyze the association of AdipoQ $(+276 \mathrm{G} / \mathrm{T})$ gene polymorphism and oxidative stress with cardiovascular disease in Punjabi women of North India.

MATERIAL AND METHODS: The present study included 400 women of whom 200 with CVD were considered as cases and 200 without CVD were considered as controls. They were screened for lipid profile, serum malondialdehyde (MDA), serum LDL carbonyl protein and serum superoxide dismutase (SOD). Genotyping was done by RFLP-PCR method.

RESULTS: Distribution of TT homozygotes with cardiovascular disease was significantly more $(\mathrm{p}<0.05)$ as compared to women without cardiovascular disease. $\mathrm{T}$ allele carriers of cases showed significant $(\mathrm{p}<0.05)$ higher frequency distribution as compared to controls. TT homozygotes conferred ? 9 to 11 fold high risk $(\mathrm{p}<0.05)$ towards CVD predisposition for MDA, LDL carbonyl protein and SOD.

CONCLUSION:In the present study significant association of AdipoQ $(+276 \mathrm{G} / \mathrm{T})$ gene polymorphism and oxidative stress with cardiovascular disease in Punjabi women of North India was observed.

KEY WORDS: AdipoQ gene, cardiovascular disease, gene polymorphism, malondialdehyde, 


\section{FP- 36}

\section{Cardiac Biomarkers: Present and Future}

\section{Mrinalini Patwardhan}

Dr Patwardhans Pathology Laboratory Ulhasnagar 3

INTRODUCTION-Acute coronary syndrome refers to a spectrum of clinical presentation ranging from those from ST- segment elevation myocardial infarction (STEMI) to Non ST- segment elevation myocardial infarction (Non STEMI) or unstable angina. This is one of the most-common serious illnesses in the industrialized world. Laboratory medicine now plays a crucial role in identifying risk factors, early events and conditions triggering plaque rupture in coronary ischemic disease. Progress in laboratory research has resulted from the discovery of new and more-promising biochemical markers of myocardial damage. Blood testing for biomarkers of myocardial injury plays an increasingly important role for the evaluation, diagnosis and managing triage of patients with chest pain.

AIM- Since it is crucial to identify the proper guide lines and new test parameters in secondary primary health sector in India this is small attempt to give brief about history, development in cardiac biomarkers merging biomarkers new guide lines and protocol

MATERIAL- The guidelines for the diagnosis of myocardial infarction (MI) have recently changed and prominently incorporate the results of cardiac marker testing in the clinical definition of MI. We review these updated guidelines for MI definition as it pertains to cardiac biomarker testing and further compare the differing biology and release kinetics of clinically relevant biomarkers. Finally, we define the contemporary use of cardiac biomarker testing for patients with chest pain, including appropriate integration of point-of-care testing into day-to-day clinical use. The measurement of multiple biomarkers and the use of point of care markers may accelerate current diagnostic protocols for the assessment of such patients. We also highlight the role of new cardiac markers as future cardiac biomarkers. Protocol for small lab.

RESULTS AND CONCLUSION- Guidelines of
European cardiology society and American college of Cardiology will be discussed. Further I will try to review current trends in our country and propose protocol with view of usefulness of biomarkers, cost effectiveness suitable for Indian scenario. With this the patient who is coming for angina pain in hospital will be followed properly with proper used of cardiac biomarkers.

KEY WORDS: Acute coronary syndrome, myocardial infarction, angina, ischemic

\section{FP- 37}

\section{Short Term Effectiveness of Scaling and Root Planing (SRP) on Some Biochemical Systemic Inflammatory Markers In Periodontitis Patients with and Without Diabetes Mellitus}

\author{
Abdul Samad Aziz ${ }^{1 *}$, Madhav Govind Kalekar ${ }^{1}$, \\ Adinath Narayan Suryakar ${ }^{2}$

\section{Department of Biochemistry, Grant Govt. Medical College, Mumbai,}

2. D. Y. PatilVidhyapeeth, Pune.

INTRODUCTION: Periodontitis and diabetes have mutual influence on each other. The hyperactive innate immune response and the resultant inflammation is a common factor associated with both the diseases, which probably have a synergistic effect when they co-exist in the host. The present study assesses the short term effectiveness of SRP on periodontal clinical parameters and some biochemical systemic inflammatory markers in periodontitis patients with and without type II diabetes mellitus.

MATERIALS AND METHOD: A total of 168 individuals with generalized chronic periodontitis (American Academy of Periodontitis 1999 criteria; Clinical Attachment Level (CAL)?3mm) were recruited and divided into the following groups based on their diabetes status (WHO 2006 criteria; Fasting Glucose? $7.0 \mathrm{mM} / \mathrm{L}=$ Diabetes $)$.

Group CP; $n=86$, mean age $=40.9 \pm 4.61, F$ Glucose $<6.05 \mathrm{mM} / \mathrm{L}$ 
Group CPDM; n=82, mean age $=47.7 \pm 7.03$, F Glucose $>7.0 \mathrm{mM} / \mathrm{L}$

The clinical parameters evaluated were Gingival Index (GI), Plaque Index (PI), Probing Depth (PD) and CAL. Biochemical systemic inflammatory markers estimated were Interleukins-6,-10, CRP besides F Glucose and HbA1c. SRP was performed on both the groups at baseline and a follow-up was done after 3 months.

RESULTS: The CPDM group showed significantly higher clinical damage and systemic levels of inflammatory markers. Post therapy improvements in study parameter's level were shown by both the groups. However, CPDM group's response to SRP therapy was lower than that of $\mathrm{CP}$ group.

CONCLUSION: The patients with periodontitis and diabetes showed higher inflammatory burden and relatively lower therapy response compared to nondiabetics. The lowered therapy response could be due to co-occurrence of diabetes with periodontitis thatmaycontribute to an additional oral and systemic inflammatory burden.

KEY WORDS: scaling, root planning, CPDM, HbA1c, SRP

\section{FP- 38}

\section{Quality Control -Does The Analyser Matter - Time To Rethink}

Kanchan Kulhari, Bhasker Mukherjee

Army Hospital ( $R \& R$ ), Base Hospital Delhi Cantt

INTRODUCTION:In order to maintain a strict control over the results generated in clinical chemistry laboratories, a number of procedures are followed to maintain the quality of results. The procedures followed in order to compare the performances between different laboratories are known as external quality assessment (EQA). Separate EQA programmes for different analysers are recommended as the methods and principles may be different for them.

OBJECTIVE \& AIM:To compare the performance of
EQA results from DIMENSION EXL 200 and VITROS 4600 analysers from two consecutive years and to analyse differences, if any.

MATERIAL \&METHODS:This clinical chemistry laboratory of this tertiary care hospital is enrolled in EQA with CMC Vellore. The lab has DIMENSION EXL 200 and VITROS 4600 analysers. The results from two different EQA cycles of 2016 (DIMENSION EXL 200) $\& 2017$ (VITROS 4600) are available for analysis and comparison. The variable index score (VIS) and standard deviation index (SDI) from these reports have been analysed.

Results:The data for 2016 for DIMENSION EXL 200 had 15 parameters in the EQA while VITROS 4600 had 20 parameters in the EQA list. There were some parameters that were not common and hence have not been compared. The overall VIS for the DIMENSION had 07 parameters $(46.7 \%)$ VIS $(<100)$ 'very good', 05 (33\%) VIS (100-150) 'good category', 02 (13\%) VIS (151-200) in 'satisfactory' and $01(07 \%)$ parameter had VIS (>200) 'not acceptable' category. The overall VIS for the VITROS 4600 had 15 parameters $(75 \%)$ with VIS (<100) 'very good', 04 (20\%) with VIS (100-150) 'good category', 01 (13\%) with' VIS (151-200) in 'satisfactory' category.

CONCLUSION:The VIS results from the VITROS 4600 as compared to DIMENSION EXL 200 showed marked improvement with $71.4 \%$ parameters undergoing a reduction in the VIS values while the SDI results were mostly similar

KEY WORDS: EQA, VIS, SDI, DIMENSION EXL 200 \& VITROS 4600. 


\section{FP 39}

\section{Understanding The Practices and Principles of Eqa Programme: CMC EQAS (Under The Ageis of Acbi)}

Pamela Christudoss, Geethanjlai Arulappan

Christian Medical College, Vellore

\begin{abstract}
AIM: To enable clinical laboratories to understand the importance of an EQA program in improving the quality of laboratory test results.
\end{abstract}

BACKGROUND: Quality in a clinical laboratory is defined as the degree of excellence and conformance to requirements leading to a reliable system. QC in a clinical laboratory is a statistical process used to monitor and evaluate the analytical process that produces patient results. The QC programme is primarily concerned with the identification of errors and the procedure to recognize and minimize them. The role of an effective QC system in these laboratories is to ensure that not only are medically significant errors detected, but no false rejections occur since retesting associated with these rejections attracts a high cost. An external quality monitoring scheme is a technique to challenge a laboratory's internal QC methods and procedures. It involves the principles of cooperation and coordination between the organizing laboratory and participants.

METHODOLOGY AND RESULTS: Some of the laboratories that have been participating continuously in all the CMC-EQAS programmes by analyzing QC sera every month of each cycle has resulted in a tremendous improvement in their performance. Over the years, the VIS and OMVIS of analytes of particular laboratories showed a decline, indicating overall improvement in performance. Their performance should certainly prove to be an encouragement to other laboratories to perform better in the EQAS.

This presentation will give you an overview of importance of scoring index, VIS, SDI, \% BIAS, OMVIS, L J CHARTS, examples of random errors and systematic errors of the participating laboratories and how recognizing them has helped the laboratory to further achieve good quality results.
BENEFITS OF EQA: Provides a basis for education and training of staff, an indicator of laboratory performance and systematic improvement of performance, allow poor performance to be addressed, provides a means for identifying and resolving problems and preventing their recurrence, enables the laboratory and clinicians to know that the tests results are reliable.

CONCLUSION: Participation in the EQAS has created awareness among laboratories to improve their performance. Deviant results have prompted reappraisal of analytical methods used. On the whole, CMC- EQAS has helped other participating laboratories minimize errors and improve the reliability of results.

KEY WORDS: EQA, QC, OMVIS, L J charts

\section{FP40}

Interleukin (IL)-33: Novel Inducer of Mast Cell Proliferation and New Therapeutic Target for Allergic Diseases

Rohit Saluja' ${ }^{1}$, Tomasz Hawro², Juergen Eberle², Martin K Church², Marcus Maurer ${ }^{2}$

Charite Universitatmedizin Berlin and All India

Institute of Medical Sciences AIIMS Bhopal, Bhopal, India 1. Charite Universitatmedizin Berlin, Germany, Berlin, Germany

INTRODUCTION: Interleukin-33 (IL-33), a member of the IL-1 cytokine family, is emerging as a new modulator of immune and inflammatory responses. Although IL-33 and its associated receptor ST2 are reportedly expressed in mast cells (MCs), the precise role of IL-33 in modulatingMC function has not been determined.

METHODS: For the present studies, we employed in vitro differentiated bone marrow derived mast cells (BMMCs) from C57BL/6 mice. BMMCs were treated for $24 \mathrm{~h}$ to $96 \mathrm{~h}$ with different concentrations of IL-33 and then checked for cell proliferation, degranulation, cytotoxicity, cell survival and apoptosis.

RESULTS: In the present studies, we explored IL-33 effects on MCs in vivo and in vitro. IL-33 increased the number of peritoneal and skin MCs in vivo. IL-33 also 
resulted in increased proliferation of MCs in vitro, as explored by WST assay. Cell cycle analysis further confirmed this result by showing increased G2 cell populations in MCs stimulated with IL-33. We found that IL-33-mediated MC proliferation requires ST2 and MyD88, is independent of Kit, and is mediated through a p38 MAPK-dependent pathway. IL-33 did not induce degranulation and was not cytotoxic for MCs.

CONCLUSION: we report here a novel role of IL-33 as an inducer of mast cell proliferation through ST2/ Myd88 and p38 MAPK-dependent pathway. This novel mechanism for increasing MC proliferation and numbers further defines the role of IL-33 in MC-dependent diseases including allergies and may help to develop novel approaches for the treatment of these disorders.

KEY WORDS: Interleukins, Allergy, Mast cell

\section{FP41}

Relationship Between Interleukin-17 and Testosterone with Disease Severity and Suicidal Behavior In Bipolar Disorder

\author{
H. Nandeesha, Neha Keshri, Shivanand Kattimani \\ Department of Biochemistry and Psychiatry, \\ JIPMER, Puducherry
}

BACKGROUND: The prevalence is bipolar disorder is increasing worldwide owing to its recurring nature and severity of episodes. Suicide is the most common complication and hormonal imbalance and inflammation plays a key role in it. The present study was undertaken as there are limited data about testosterone and interleukin-17 levels in bipolar disorders.

AIM: To assess testosterone, sex hormone binding globulin and Interleukin-17 levels and their association with disease severity and suicidal ideation in patients with bipolar disorder.

MATERIALS AND METHODS: 41 bipolar disorder cases and 41 age matched controls were enrolled in the study. Testosterone and Interleukin-17 were estimated in all the subjects.

RESULTS: Interleukin-17 was significantly increased and testosterone was significantly decreased in patients with bipolar disorder compared to controls. Also Interleukin-17 was inversely associated with testosterone in the cases. There was no association between testosterone and interleukin-17 with disease severity and suicidal behavior.

CONCLUSION: We conclude that testosterone is reduced and interleukin-17 is increased in bipolar disorder cases.

KEY WORDS: Interleukin 17, testosterone, bipolar disorder, inflammation

\section{FP42}

\section{Association Between AST, ALT and CK In Cases of Dengue Infection: A Prospective Case Control Study}

Pratibha Gavel ${ }^{1}$, Sujit Bharti

Heritage Institute of Medical Sciences ${ }^{l}$, Varanasi, Institute of Medical Sciences ${ }^{2}$, Banaras Hindu University, Varanasi

INTRODUCTION: Dengue is an endemic viral infection found in our country, known for its high morbidity and mortality. Existing biomarkers such as Alanine transaminase (ALT) andAspartate transaminase (AST) have been associated with severe dengue but evidences are not conclusive. Recently, Creatine kinase (CK) has emerged as a biomarker of dengue infection. These biomarkers depict the underlying pathophysiological processes in dengue.

AIM AND OBJECTIVE: We aimed to assess AST, ALT and $\mathrm{CK}$ in dengue infection and to assess the relationship amongst these biomarkers in dengue infection.

MATERIALAND METHODS: This was a prospective cohort study of confirmed dengue patients and healthy controls. Blood samples from patients coming to the hospital were screened for NS 1 antigen by lateral flow chromatography. The blood samples which were found positive for NS 1 antigen were confirmed for the presence of IgM antibody by ELISA. The IgM positive samples and controls were then tested for AST, ALT and CK. 
RESULTS: The biomarkers of muscle and liver involvement were found to be elevated. There was positive association between AST, ALT and CK.

CONCLUSION: The study concludes that CK is a good biomarker to assess the disease progression and severity. However,it is advised to use AST, ALT and CK in combination to assess the disease condition better.

KEY WORDS: Dengue, Creatine Kinase, Alanine Transaminase, Aspartate Transminase

\section{FP43}

\section{Association of Metabolic Syndrome and Oxidative DNA Damage In HIV/AIDS Patients}

Vinayak W. Patil, Kolgiri Vaishali H, Rajesh B. Rajekar

\section{Grant Medical College, and Sir J J Hospital Mumbai} 400008 Maharashtra

AIM: Metabolic syndrome (MS) is frequent in human immunodeficiency virus infection (HIV) and may be related to antiretroviral therapy (ART). The present study is aimed to determine association of metabolic syndrome with oxidative deoxynucleic acid (DNA) damage in HIV1 infected patients with different ART status.

METHODS: We used plasma levels of the oxidized base, 8-hydroxy-2-deoxyguanosine (8-OHdG), as our biomarker of oxidative DNA damage. $8-\mathrm{OHdG}$ was measured with 8-OHdG enzyme-linked immunosorbent assay (ELISA) kit. The biomarkers of MS were insulin resistance (IR), Cholesterol/HDL ratio, Waist circumference and Hypertension.

RESULTS: All subjects were randomly selected and grouped as HIV-negative (control group) ( $n=300)$, HIV

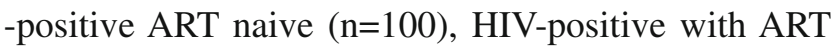
first line $(n=100)$ and HIV-positive with ART second line $(n=100)$. MS and oxidative DNA damage were significantly higher in HIV-positive patients with second line ART and first line ART than ART naive patients. In a logistic regression analysis, increased MS was positively associated with the increased DNA damage (OR: 29.68, 95\%:13.47, CI: 65.40) $\mathrm{P}=0.0000$.
CONCLUSIONS: In this case control study, we observed that ART plays a significant role in the development of MS and oxidative DNA damage in HIVpositive patients taking antiretroviral therapy. Awareness and knowledge of MS \& DNA damage in HIV/AIDS patients may prove helpful to clinicians to manage non AIDS diseases such as cardiovascular disease and cancer. Larger studies are warranted to determine exact role of ART in induction of MS and DNA damage.

\section{FP44}

\section{Comparative Analysis of Physico-Chemical Properties of Commonly Used Edible Oils In India}

Viyatprajna Acharya ${ }^{1}$,Jyotirmayee Bahinipati ${ }^{2}$, Govind Pratap Singh ${ }^{3}$, Karan Khandelwal ${ }^{4}$

1. Professor Biochemistry, IMS \& SUM Hospital, Bhubaneswar, Odisha;

2. Asst. Professor, KIMS, Bhubaneswar, Odisha;

3. Intern MBBS, KIMS, Bhubaneswar;

4. Final year MBBS, KIMS, Bhubaneswar.

INTRODUCTION: Role of dietary lipid is considered vital in metabolic syndrome, diabetes mellitus, CHD and cancer. PUFA-rich oils have been supplemented in Indian diets claiming to have beneficial effects on health due to their antioxidant property. But due to the laibility to be oxidised fast under heat, UV ray exposure and storage actually might be adding to the oxidant intake in the diet. Hence to estimate the oxidant product generation due to storage, temperature and UV exposure this study was taken up to analyse comparatively the physico-chemical properties between 4 major edible oils/ghee - Deshi ghee, palm oil, olive oil and rice bran oil.

MATERIAL AND METHODS: The 4 ghee/ oil samples were chosen and stored in the dark and physical properties like OD, smell, degree of unsaturation and chemical properties like quantity of primary oxidation products peroxide value (PV) and secondary oxidation products like p-Anisidine value (AV), Malondialdehyde (MDA) and TOTOX value were studied after exposing 
them to different storage conditions, duration of storage and temperatures every fortnightly for 2 months.

RESULTS: Rice bran oil showed high smoke point and better stability at higher temperature. Palm oil showed highest potential for oxidation whereas desi ghee showed the least and moreover, with storage it was totally free of oxidation products beyond 15 days. Olive oil was very much susceptible to oxidation under all types of exposure and duration of storage.

CONCLUSION: Rice bran oil and desi ghee can be chosen for deep frying. Palm oil should best be avoided for cooking. Olive oil may taken in the raw form but should be avoided in all modes of Indian cooking.

KEY WORDS: Peroxide value; Anisidine value; TBARS; TOTOX

\section{FP45}

\section{Role of Curccumin: An Insight Into Antioxidant Strategies Against Pesticide Toxicity}

\section{Tanzeel Ahmed}

Department of Biotechnology, School of Engineering and Technology, IFTM University, Lodhipur Rajput, Delhi Road (NH-24), Moradabad, Uttar Pradesh 244102, India.

Pesticides have become an integral part of ecosystem and their widespread use in public health programs and agriculture have resulted in severe environmental pollution leading to potential health hazards, the extent of which depends upon the amount of residue and its toxicity. The general population is subjected to low dose chronic pesticide exposure through food and environment. The residue analysis of human samples shows an increasing trend in pesticide levels in serum, adipose tissue, breast milk, urine and others. Implications on human health of pesticides residues following subchronic exposure have yet to be comprehensively answered.Exposure to pesticides may be associated with neurotoxicity, hepatotoxicity, immunotoxicity, genotoxicity and adverse reproductive outcomes, and their effects have been attributed to increased oxidative stress. Pesticides cause reactive oxygen species (ROS) generation in significant quantities, which has been suggested to result in oxidative stress and a signal for apoptosis. Findings from our studies have revealed a concomitant genotoxic and apoptotic effect of the pesticides in peripheral blood mononuclear cells. Since the long-term genotoxic effects of pesticides on humans cannot be ignored and it is therefore the need of the hour to search for protective measures to minimize their harmful effects. We havereported that PBMC undergoes dose-dependent apoptotic cell death following pesticide exposure and also highlights the effectiveness of cucumin in counteracting pesticide induced cytotoxicity. HSPs have emerged recently as an antiapoptotic molecules and functions to modulate the engagement or progression of apoptosis induced by pesticide. The inducible expression of HSPs could render the human PBMCs to withstand and recover from the toxic effects of subchronic pesticide exposure. Though the linkage between cellular events induced by stress and cell death is known, yet the molecular mechanism highlighting the specific role of heat shock protein (HSP) in pesticide-mediated cytotoxicity is not clear. To better understand the mechanism, we employ curcumin an HSP inducers, and also highlighting their attenuating effects against the apoptotic ability of these pesticides. We emphasizes that antioxidants protects cells efficiently, suggesting their possible therapeutic intervention as well as underscoring the mechanism of pesticide-induced toxicity at cellular level. Also, such studies will provide further insight into the molecular mechanisms behind pesticide poisoning and might suggest new diagnostic and therapeutic approaches for their toxicity and remedy. 


\section{FP46}

\section{Epigenetics: New Horizon for Diabetic Nephropathy Diagnosis and Prognosis}

Sangeeta Singh*, Satyendra Kumar Sonkar\#, Abbas

Ali Mahdi* Gyanendra Kumar Sonkar*

*Department of Biochemistry,

King George's Medical University, Lucknow

\#Department of Medicine, King George's Medical

University, Lucknow

Diabetic nephropathy (DN) is one of the most serious microvascular complications and remains the leading cause of end stage renal disease (ESRD). The persistent albuminuria has been considered to be central hallmark of DN. However, albuminuria has the capacity to indicate kidney damages for clinician, microalbuminuria is not a specific biomarker for prediction of $\mathrm{DN}$ prior to the onset of devastating complication. There are numerous cellular and molecular defects persisting prior to appearing clinical symptoms. Therefore, need to look for easy and accurate way to detect DN before its beginning. Moreover, better understanding of disease pathogenesis might be helpful to identify new biomarkers and novel mechanism underlying DN to improve existing therapeutic regime. It is now accepted that initiation and progression of $\mathrm{DN}$ are result of complex interaction between genetic and environmental factors that could be explained by epigenetic modifications. Environmental signals could alter the intracellular pathways by chromatin modifiers and regulate gene expression patterns leading to diabetes and its complications. However, our knowledge about the epigenetic mechanisms linking environmental factors and pathophysiology of diabetes and its associated complications is very limited. A hyperglycaemic environment in diabetic patients causes an imbalance in metabolic homeostasis that may alter gene expression. Recent epigenetic studies have focused on how gene transcription controls cell behavior in response to environmental changes. It will help to recognize novel approaches to inhibit, attenuate, or reverse the persistent deleterious consequences of diabetes in the kidney.

Diabetes profoundly impacts transcription programs in target cells through activation of multiple signaling pathways and key transcription factors leading to aberrant expression of pathological genes. Emerging evidence suggests that these factors associated with the pathophysiology of diabetic complications and metabolic memory might also be influenced by epigenetic mechanisms in chromatin such as DNA methylation.

\section{FP 47}

\section{Study on Liver Function In Pregnant Anemic Women Upon Iron and Folic Acid Therapy}

Amit Kumar Mani Tiwari ${ }^{1,2 *}$, Abbas Ali Mahdi1, Sanjay Mishra ${ }^{2}$

1. Department of Biochemistry, King George's Medical University, Lucknow, India

2. Department of Biotechnology, IFTM University, Moradabad, India

INTRODUCTION:Oral iron therapy is the most widely prescribed treatment for iron deficiency anemia. However, oral iron supplementation may also lead to various health problems. The recognition of these physiological variations is essential for the diagnosis of liver diseases during the course of pregnancy.Therefore, the objective of this study was to assess the variations in levels of routine LFTs before and after iron and folic acid treatment.

MATERIALS AND METHODS: Iron and folic acid supplementation was given to 500 anemic women ( mild $=200$, moderate $=200$, and severe $=100$ ) and 100 age matched non-anemic controls daily for 100 days. Blood index values and liver function parameters were estimated as per standard protocols.

RESULTS: Blood index values and liver function parameters were estimated as per standard protocols. Hemoglobin $(\mathrm{Hb})$ and total protein $(\mathrm{TP})$, iron $(\mathrm{Fe})$, albumin and alkaline phosphatase (ALP) levels were found significantly increased $(\mathrm{P}<0.001 ; \mathrm{p}<0.01 ; \mathrm{p}<0.05)$ in anemic and control subjects after treatment. Lipid peroxidation (LPx), aspartate transaminase (AST) and alanine transaminase (ALT) were increased in pre-treated groups and further increased after treatment. Moreover, gamma-glutamyl transpeptidase (GGT) was found to be 
decreased before even after iron and folic acid supplementation in all subjects.

CONCLUSION: Treatment with iron and folic acid although has good efficacy for $\mathrm{Hb}$ and body iron stores but for the cost of increasing the associated compartment of total bilirubin, AST, ALT and GGT levels. Authors suggested that liver function tests monitored at regular intervals during pregnancy.

KEY WORDS: Pregnancy, Iron deficiency anemia, Iron supplementation, liver function.

\section{FP 48}

\section{Association of Poor Glycemic Control and Hyperhomocysteinemia with Microalbuminuria}

Iyyapu Krishna Mohan, Siraj Ahmed Khan, Rachel Jacob, M. Vijaya Bhasker and K.S.S. Sai Baba

Department of Biochemistry, Nizam's Institute of Medical Sciences, Panjagutta, Hyderabad.

INTRODUCTION: Urinary microalbumin is a well documented biomarker for incipient diabetic kidney disease prompting aggressive management of diabetes to prevent risk for diabetic nephropathy. The rationale of the current study is to evaluate association of glycemic control and total plasma homocysteine with microalbuminuria.

MATERIALS AND METHODS:We have studied a total of 975 subjects, which includes 873 diabetic patients (514 men and 359 women) and 102 non-diabetic controls (57 men and 45 women). Immunoturbidimetric assay on Roche Coas c501analyzer was used to estimate urinary microalbumin levels and homocysteine levels by chemiluminescence method.

RESULTS: The mean age of these two groups were $61.5 \pm 23.1$ y vs. $59.1 \pm 10.4$ y, respectively $(p=0.29)$. The random blood glucose levels in these two groups were $149 \pm 77$ vs. $97 \pm 13 \mathrm{mg} / \mathrm{dl}(\mathrm{p}<0.0001)$. Urinary microalbumin levels were higher in diabetes group than non-diabetic group i.e. $79.0 \pm 185$ vs. $3.51 \pm 1.55 \mu \mathrm{g} / \mathrm{mg}$ creatinine $(\mathrm{p}<0.0001)$. Based on urinary microalbumin levels, the data was segregated into normal $(<30 \mu \mathrm{g} / \mathrm{mg}$ creatinine, $\mathrm{n}=653)$, microalbuminuria $(30-300 \mu \mathrm{g} / \mathrm{mg}$ creatinine, $n=268$ ) and clinical microalbuminuria $(>300 \mu \mathrm{g} / \mathrm{mg}$ creatinine, $\mathrm{n}=53$ ). Analysis of variance (ANOVA) revealed higher levels of fasting blood glucose (F: 39.76, p<0.0001), glycosylated hemoglobin (F: 64.31, $\mathrm{p}<0.0001)$ and total plasma homocysteine $(\mathrm{F}: 3.69$, $\mathrm{p}=0.04)$ in microalbuminuria and clinical microalbuminuria groups when compared to subjects with normal microalbumin levels. Age ( $\mathrm{F}: 2.24$, p=0.11) and gender $(2: 1.53, \mathrm{p}=0.22)$ have no influence on the urinary microalbumin.

CONCLUSION: Poor glycemic control and hyperhomocysteinemia are associated with microalbuminuria.

KEY WORDS: Poor glycemic control, Diabetic nephropathy, Hyperhomocyseinemia, Microalbuminuria.

\section{FP 49}

Comparison of Total Antioxidant Capacity In Term Small for Gestational Age (SGA) and Appropriate for Gestational Age (AGA) Newborns

Seema Lekhwani ${ }^{1}$, ND Vaswani ${ }^{2}$, Rajendra Dugesar ${ }^{2}$, Aparna Yadav ${ }^{3}$

1. Department of Biochemistry,

2. Department of Pediatrics,

3. Department of Microbiology, PGIMS, Rohtak, Haryana, India.

INTRODUCTION: When antioxidant defenses weaken, body cells and tissues are more prone to develop dysfunction/disease. The maintenance of an adequate antioxidant status is essential to protect body against destructive effects of free radicals. Total antioxidant capacity (TAC) is the measure of amount of free radicals scavenged by a test solutionto evaluate the antioxidant capacity of biological samples.

AIMS AND OBJECTIVES: This study was undertaken to estimate the total antioxidant capacity in term small for gestational age (SGA) newborns and compare it with term appropriate for gestational age (AGA) newborns. The study was conducted in the Department of 
Biochemistry in collaboration with the Departments of Pediatrics and Microbiology at Pt. B. D. Sharma PGIMS, Rohtak (Haryana).

MATERIALS AND METHODS: 82 newborns (Term AGA 46 and Term SGA 36) were enrolled for the study.Cord blood was procured and TAC of serum was determined by the TAC assay kit (Labor Diagnostika, Germany).

Results and conclusion: There was no significant difference in the total antioxidant capacity of theAGA and the SGA groups. There was significant difference in the mean birth weight of the AGA and the SGA groups. The ponderal index was significantly different between both the groups. Results show that weight for gestational age in term newborns does not significantly affect total antioxidant capacity.

KEY WORDS: SGA, AGA, TAC

\section{FP 50}

\section{Post-Mortem Biochemistry In Determining The Time of Death}

Rohini Karunakaran ${ }^{1 *}$, Surekha Bhat ${ }^{2}$, Srikumar P S ${ }^{3}$

1. Unit of Biochemistry, Faculty of Medicine, AIMST University, Bedong, Kedah, Malaysia

2. Department of Biochemistry, School of Medicine, St.Xavier University, Aruba

3. Unit of Psychiatry, Faculty of Medicine, AIMST University, Bedong, Kedah, Malaysia

INTRODUCTION: Post-mortem biochemistry is a promising branch of biochemistry in forensic medicine. Thus, post-mortem biochemistry, although not yet a commonly practiced investigatory tool, is emerging as an increasingly important one in modern medicine. Assessment of the cause and time of death is a vital ancillary procedure in post-mortem biochemistry.

AIM: The aim of this study is to summarize from available literature, the role of post-mortem blood investigations in determining the time of death.
METHODS: Previous research studies on post-mortem blood investigations and their correlation to time of death, was retrieved from PubMed. Excerpts were picked from these studies to summarize all the findings.

CONCLUSION: From previous studies it follows that blood pH, NADH, ammonia, hypoxanthine, triglyceride are good post-mortem blood markers for determining the time of death whereas lactate and uric acid fail to serve as good markers.

KEY WORDS: Post-mortem biochemistry, Time of death, Post-mortem blood

\section{FP 51}

Serum Human Epididymus Protein-4 (HE4) As A Biomarker In Ovarian Cancer and Its Comparison With CA-125 In Benign and Malignant Cases

Swarnima Singh ${ }^{1}$, Avinash Pandey ${ }^{2}$, Uday Kumar ${ }^{3}$, Anand $\mathrm{Saran}^{4}$, Rekha Kumari ${ }^{5}$, Juit Ram Keshari ${ }^{6}$

Department of Biochemistry ${ }^{1,3,4,5,6,}$

Regional Cancer Center ${ }^{2}$,

Indira Gandhi Institute of Medical Sciences, Patna ${ }^{1,2,3,4,5,6}$.

INTRODUCTION: Ovarian cancer is the third leading site of cancer among women in India.70\% of ovarian cancers are diagnosed with advanced disease leading to high fatality rate.Human epididymis protein 4 (HE4), also known as WAPfour disulphide core 2 (WFDC2),is a novel protein and is one of the more promising biomarkers for improving diagnosticperformance in ovarian cancer detection.

AIM:To evaluate a new tumor marker, HE4, in comparison with CA125 in diagnosis of ovarian cancer and benign gynecological diseases.

MATERIAL AND METHODS: Pre-operative serum HE4 and CA125 were determined in 146 patients with benign gynecologic diseases and 64 with ovarian cancers. HE4 and CA125 cutoffs were $140 \mathrm{pmol} / \mathrm{L}$ and $35 \mathrm{kU} / \mathrm{L}$, respectively. Sandwich Elisa, biotin-labelled antibody kit was used for HE4. 
RESULTS: HE4 showed a higher specificity than CA 125 in patients with benign gynecologicdiseases, with abnormal concentrations in $1.37 \%$ and $33.56 \%$ of the patients, respectively. Renalfailure was the most common cause of increased HE4 amongst benign cases. A significantly higher area under the ROC curve was obtained with HE4 than with CA 125 in the differential diagnosis of gynecologic diseases ( 0.874 vs 0.722 ). Both markers were clearly related to stage (HE4: $\mathrm{P}=0.004$; CA125: $\mathrm{P}=0.001$ ).) and histopathological type (HE4: $\mathrm{P}$ $=0.003$ and $\mathrm{CA} 125: \mathrm{P}=0.009$ in serous papillary ovarian cancer).

CONCLUSION: HE4 has significantly higher diagnostic specificity than CA 125 , and the combination of CA125 and HE4 improved the detection of ovarian cancer in all stages and histological types. HE4 use may be important in the differential diagnosis of ovarian cancer.

KEYWORDS : Human epididymis protein 4, HE4, Carbohydrate antigen125, Ovarian cancer

\section{FP 52}

\section{Advanced Glycation End Products-Mediated Renal Cell Dysfunction Andameliorating Effect of Ramipril And Losartan}

Pawan Kumar Kare ${ }^{1}{ }^{4}$, Rishila Ghosh ${ }^{1}$, Manushi Siddarth ${ }^{1}$, Basu Dev Banerjee ${ }^{1}$, Om Prakash Kalra ${ }^{3}$, Himanshu Madaan ${ }^{4}$, Ashok Kumar Tripathi ${ }^{1}$

1. Department of Biochemistry,

2. Department of Medicine, University College of Medical Sciences \& G.T.B. Hospital, Delhi, India.

4. Department of Biochemistry, Kalpana Chawla Govt. Medical College \&Hospital, Karnal, India.

INTRODUCTION:Hyperglycemia-mediated advanced glycation end products (AGEs) formation is one of the major factor leading to renal cell injury in diabetic patients. The interaction of AGEs with receptor for advanced glycation end products (RAGE) is believed to induce downstream signaling of various effectorsleading to renal fibrosis in diabetic nephropathy (DN). Angiotensin converting enzyme inhibitor (ramipril) and angiotensin II receptor blocker (losartan) are the antihypertensive drugs known for their reno-protective activity.

AIMS AND OBJECTIVES: The present study was designed to explore the effect of advanced glycation end products (AGEs) on renal cell dysfunction by assessingreactive oxygen species (ROS) production and mRNA expression of NADPH oxidase p47phox subunit, TGF $\beta 1$ and fibronectin in AGE-exposed renal cells along with effect of ramipril and losartan on these parameters.

MATERIAL AND METHODS: The cultured human renal proximal tubular epithelial cells (HK-2 cells)were treated with $200 \mu \mathrm{g} / \mathrm{ml}$ of artificially made AGEs using bovine serum albumin (AGE-BSA) and unmodified BSA for $24 \mathrm{~h}$. The HK-2 cells were also co-treated with drugs namely; ramipril $(5 \mu \mathrm{M})$ and losartan $(5 \mu \mathrm{M})$. ROS generation was assessed by using 2',7'dichlorodihydrofluorescein diacetate (H2DCFDA) method. NADPH oxidase $\mathrm{p} 47$ phox subunit, RAGE, TGF $\beta 1$ and fibronectin mRNA expression was assessed by using real-time PCR.

RESULTS: A significant increased $(\mathrm{p}<0.001)$ ROS production was observed in AGE-BSA-treated HK-2 cells as compared to control HK-2 cells treated with unmodified BSA. The mRNA expression of NADPH oxidase, RAGE, TGF $\beta 1$ and fibronectin in HK-2cells was found significantly increased $(\mathrm{p}<0.001)$ after $24 \mathrm{~h}$ treatment with AGE-BSA as compared to control. Ramipril and losartan significantly $(\mathrm{p}<0.05)$ scavenged theproduction of ROS and also significantly decreased the mRNA expression of NADPH oxidase p47phox subunit, RAGE, TGF $\beta 1$ and fibronectin.

CONCLUSIONS:AGEs induce renal cell damage and is one of the mechanisms leading to diabetic nephropathy.

KEYWORDS: Advanced glycation end products, Diabetic nephropathy, Angiotensin converting enzyme inhibitor and Angiotensin II receptor blocker 


\section{FP 53}

\section{Studies on Blood Lead Levels, Hemoglobin and Arterial Blood Pressure In Traffic Police In Dehradun Region.}

Rajeev S Kushwaha, Nirbhay Singh, Tariq Massod, Sumita Sharma, Swati Naithani, Anita Singh, Poonam Verma and R K Singh*.

Biochemistry and Physiology Department, SGRR Institute of Medical and Health Sciences, Shri Guru Ram Rai University, Dehradun-248001 (UK).

INTRODUCTION: Lead poisoning effect has been known since conducted in ancient times. Lead poisoning was founded largely as an occupational disease of adults Lead poisoning is now well documented and persists as a major public health problem throughout the world. It affects multiple systems and has role in the production of reactive oxygen species (ROS).

AIMS \& OBJECTIVE: This study was conducted to define blood lead levels, haemoglobin and arterial blood pressure in traffic police posted in different areas of Dehradun.

MATERIAL \& METHODS: Sixty-three subjects participated in this study. They were divided into two groups each comprising of 43 traffic policemen and 20 age-matched healthy controls. The blood samples were analyzed by Lead care II analyzer unit to determine the blood lead levels. Hemoglobin was measured by CBC coulter counter. A questionnaire was designed to assess the adverse health effects of the traffic policemen.

RESULTS \& CONCLUSION:Blood lead level was found to be $3.47 \pm 1.45$ in traffic police which was calculated to be statistically significant in comparison to the healthy controls with a mean of $2.15 \pm 1.16$ (mean $\pm \mathrm{SD}$ ). Blood pressure was recorded to be elevated in traffic police (both systolic and diastolic) in comparison to healthy counterparts whereas blood hemoglobin did not exhibit and significant change between the two groups. Blood lead levels were higher in traffic policebut within normal range as per CDC guidelines thanhealthy controls. It may be due to better environment conditions and lesser air pollution in Dehradun city.Hemoglobin was found to be normal whereas BP variation in traffic police could be due to their stressful duty hours.

KEY WORDS: Lead poisoning, ROS, hemoglobin

\section{FP 54}

Experimental Validation of Lipid Lowering and Antioxidant Potential of Tinosporacordifolia (M.): An Indigenous Medicinal Plant

Vishnu Kumar ${ }^{1}$, Farzana Mahdi ${ }^{1}$, Dharam Raj Singh ${ }^{2}$, Ranjana Singh, Abbas Ali Mahdi ${ }^{3}$ and Raj Kumar Singh ${ }^{4}$.

1. Department of Biochemistry, Era's Lucknow Medical College \& Hospital, Lucknow.

2. Kaya Chikitsha Vibhag, Rajkiya Ayurvedic Chikitsha Mahavidyalaya,

3. Department of Biochemistry, King George's Medical University, Lucknow,

4. Department of Biochemistry, T.S.M. Medical College, Lucknow

OBJECTIVE: To explore lipid lowering and antioxidant activities of Tinosporacordifolia (T. cordifoliaGiloy, Gurch, Gudici; Family Menispermaceae) in type 2 diabetic patients

MATERIAL AND METHODS: In this studyclinical trial had conducted on type 2 diabetic patients attending diabetes OPD, Kaya ChikitshaVibhag, Rajkiya Ayurvedic Chikitsha Mahavidyalaya, TouriyaGanj, Lucknow as per guide line of ethics. All biochemical assays had done by standard kit methods.

RESULTS: Oral administration of powdered stems of T. cordifoia $(50 \mathrm{mg} / \mathrm{kg}$ body weight, $\mathrm{p}$. o.) for 15 days in type 2 diabetic patients resulted significant decrease in the level of fasting blood sugar, total cholesterol, $\beta$, pre - $\beta$ lipoproteins and triglycerides. The decrease in lipids and apoprotein levels of $\beta$ lipoproteins was accompanied with stimulation of plasma lecithin cholesterol acyltransferase (LCAT). Lipid and apoprotein level of $\beta$ lipoproteins and antioxidant enzymes were recovered significantly. 
CONCLUSION: Results of this study lead to research and development of lipid lowering and anti oxidant drug from Tinosporacordifolia.

KEY WORDS: Tinosporacordifolia- $\beta$, Pre $\beta$ \& $\beta$ lipoproteins- Antioxidant enzymes - Lipoprotein profile - LCAT.

\section{FP 55}

\section{Prevalence of Metabolic Syndrome In Granite Workers of Khammam District}

Kalyan Kumar*, P.Srilakshmi**, K. Rambabu**

* Dept. Of Biochemistry, Shahjahanpur, Uttar Pradesh, India,

**Dept. Of Biochemistry, Mamata Medical College, Rotary Nagar, Khammam - 507 002,

Telangana State. India.

Email-ID: kakarlakalyan@gmail.com

BACKGROUND: The occurrence of the metabolic syndrome (MS) has significantly increased over the last few decades and has become a main health challenge worldwide. A very few studies are available on granite workers and industries very much localized in khammam. Hence this study has been taken up.

OBJECTIVES: Aim of our study is to assess the prevalence of MS and its components in granite workers.

MATERIALS \& METHODS: 210 workers of granite industries in and around Khammam, Telangana State of 20 - 50 age groups are included in this study. Blood pressures (BP), waist circumference (WC) were measured and blood was collected in fasting condition for the estimation of glucose and lipid profile. Incidence of metabolic syndrome was calculated based on updated National cholesterol education programme (NCEP) guidelines.

RESULTS: 69 subjects out of 210 were identified as having MS based on American Heart Association / Updated NCEP guidelines

CONCLUSION: MS should be identified and beneficial measures should be suggested early, so that the risk of hypertension, atherosclerosis, diabetes and the resultant morbidity and mortality can be delayed.

\section{FP 56}

\section{Academic Stress and Biochemical Parameters: A Correlational Study In Medical Students.}

\section{Trushna Shah}

SBKSMIRC, Sumandeep Vidyapeeth, Piparia, Waghodia, Vadodara, Gujarat.

INTRODUCTION : Stress is a reaction to change; it can be either positive or negative, and it affects both the body and the mind. Normally, stress stimulates the release of hormones such as adrenaline, increse heart rate, increasing blood sugar, cortisol and lipid prifile.Cortisol causes the mobilization of fats deposited in the adipose and other tissues, leading to an increased levels of lipids in the blood. Aim of our study is to see the relationship between academic stress and biochemical parameters in medical students.

Material and Method : Cross sectional study conducted in Department of Biochemistry, SBKS MIRC after Ethical clearance (number). Total 140 First year medical volunteered and gave consent were recruited.After recording of age, gender and demographic aspects stress was assessed with the Pre-validated Questionnaire Academic Stress Scale, PSS score, Blood glucose,Lipid profile and Cortisol.

RESULT : Mean PSS score in stressed and non-stressed group was $21.54 \pm 5.31$ and $10.47 \pm 1.55$ respectively and significantly higher in stressed students compare to non stressed students $(\mathrm{p}<0.001)$. Results shows $87.86 \%$ students were stressed and $12.14 \%$ students were non stressed.Cholesterol level was found to be elevated significantly in stressed when compared with non Stressed group $(\mathrm{p}<0.01)$ in study.Study does not show any significant difference in Salivary cortisol, Blood glucose, Triglyceride, HDL and LDL between Stressed and Non Stressed Students.

Conclusion: Academic stress is correlated with high cholesterol level which may further cause various other complications. Author suggest proper monitoring in large 
population and some stress relieving modalities for Medical students.

KEY WORD: Perceived stress scale, Academic Stress.

\section{FP 57}

\section{A Study to Assess Circulating Vitamin D Level and Its Association with Thyroid Disorder In Populations Attending Tertiary Care Hospital In Uttarakhand}

Sarama Saha, Anissa Atif Mirza, Manisha Naithani, Bela Goyal, Venkatapathy Kalia Moorthy

AIIMS Rshikesh, Department of Biochemistry

INTRODUCTION: VitaminD (vitD) deficiency is a global health problem. Synthesis of vit D in the skin following exposure to sunlight is the major source of vitamin $\mathrm{D}$ for humans. Moreover, vitD is a crucial component of innate immune system and plays a protective role against infection and other various disorders including thyroid.Uttarakhand has a geographic peculiarity (in latitudes north approx 310), cultural belief and isan endemic region to iodine deficiency.

AIMS AND OBJECTIVE: To measure the circulating vit $\mathrm{D}$ level to observe the trend of vitD level with respect to variations in season, age and sex and to investigate its association with thyroid profile among populations attending AIIMS Rishikesh.

MATERIAL AND METHOD:An observational studywas conducted in 1372 individuals.Data were collected from Biochemistry log book of AIIMS Rishikesh maintained from October 2015 to September 2016. The chemiluminescent immuno assay was carried out for measurement of parameters. One way ANOVA and Pearson's correlation coefficients were carried out with SPSS 23.

RESULTS: There was a significant $(<0.001)$ differencein vitD levels $(17.27 \pm 7.01 \mathrm{ng} / \mathrm{ml}$ vs. $29.60 \pm 16.51 \mathrm{ng} / \mathrm{ml}$ ) between winter and summer. There was no significant impact of gender on circulating vitamin $\mathrm{D}$ level. However younger age group ( $<20$ years) has higher vitD level than the older age group.
Furthermore, hypothyroid individuals have lower vitD level compared to euthyroid individuals $(17.39 \pm 10.72$ $\mathrm{ng} / \mathrm{ml}$ vs $22.72 \pm 12.76 \mathrm{ng} / \mathrm{ml} ; \mathrm{p}=0.125$. Pearson, $\mathrm{s}$ correlation coefficient showed a nonsignificant $(p=0.676)$ negative association between vitD and TSH level $(\mathrm{r}==$ 0.043 )

CONCLUSION: Present study suggests that vitD fluctuates seasonally in Uttarakhand and a nonsignificant negative association persists between vitD and TSH level.

KEYWORDS: Vitamin D, Thyroid profile, Uttarakhand, Hypothyroid, Euthyroid.

\section{FP 58}

\section{Association of Insulin Resistance (IR) With Viral Etiology and Serum TNF-A In Patients with Chronic Liver Disease}

Asif Iqbal, M. Irshad

\section{Clinical iochemistry Division, Department of Lab Medicine, All India Institute of Medical Sciences (AIIMS), New Delhi-110029, India}

INTRODUCTION: Insulin resistance (IR) is commonly observed in patients with chronic liver diseases in India and is increasingly associated with hepatitis virus infection. Several studies have shown that serum levels of TNF- $\alpha$ is significantly elevated in patients with chronic liver diseases, caused by both hepatitis B and C. However, the data are inconsistent due to high prevalence of hepatitis $\mathrm{C}$ viruses in many regions of the world. We therefore, studied the relationship between the IR, serum level of TNF- $\alpha$ and viral etiology of liver diseases.

MATERIALS AND METHODS:A total number of 79 patients including 35 patients (agerange 23-49 years) with chronic viral hepatitis $(\mathrm{CVH}), 33$ patients (age range 3458 years) with cirrhosis (CIR) and11 patients (age range 43-54) with hepatocellularcarcinoma (HCC) of both gender and in adult age groupwere included in this study plan.IR level was calculated using HOMA-IR formula. Bloodanalysis for insulin, C-peptide and TNF- $\alpha$ and hepatitis serology were done using EIAprocedure. Data 
were analyzed using appropriate statistical tests.

RESULTS: Although IR was higher than controls in all the disease groups, it was significantly high in $\mathrm{CVH}$ patients $(\mathrm{p}<0.01)$. Accordingly, C-peptide also showed a strong tendency to be elevated in $\mathrm{CVH}$ patients $(\mathrm{p}=0.06)$. However, analyzing IR and C-peptide levels across Hepatitis $\mathrm{B}$ and $\mathrm{C}$ viruses revealed that $\mathrm{HCV}$ and HBV had comparable effect in rising IR in CVH and $\mathrm{HCC}$ patients. In cirrhosis patients, however, $\mathrm{HCV}$ markedly increased IR levels while IR levels in HBV infected patients were comparable to controls $(\mathrm{p}<0.01)$. TNF- $\alpha$ was elevated in all the three disease groups when compared to controls. Further, TNF- $\alpha$ level was remarkably elevated in cirrhosis patients infected with $\mathrm{HCV}$ as compared to HBV $(\mathrm{p}<0.05)$ only. TNF- $\alpha$ level when stratified based on viral etiology in $\mathrm{CVH}$ and $\mathrm{HCC}$ patients were comparable to control patients.

CONCLUSION: HCV infection might play a role in inducing IR via increased TNF- $\alpha$ level in liver cirrhosis patients.

KEYWORDS: Chronic liver diseases, Insulin resistance, $\mathrm{C}$-peptide, Hepatitis viruses, TNF- $\alpha$

\section{FP 59}

\section{Oxidative Stress in Hepatic Manifestation of Dengue Virus Infection}

Abhishek Sharma, Nidhi Sharma

N.S.C.B. Medical College, Jabalpur, M.P, SHKM GMC Nalhar, Nuh, Haryana

OBJECTIVE: To study oxidative stress markers and antioxidant status in patients of hepatic manifestations of dengue fever.

INTRODUCTION: Manifestation of viral infection may be contributed by the alteration of oxidation/ reduction (Redox) status in virus infected patients and this alteration starts prior to symptoms of disease appear. Increased level of hepatic enzymes may be a useful marker of hepatic insult.

METHOD: We conducted a cross sectional hospital based study of 90 patients serologically confirmed to have dengue virus infection who have hepatic manifestations. Blood samples were collected on 4th day and onwards after the fever and analysed for liver function tests (LFT), prothombin time (PT) and thrombocytes and other haematological parameters. And to measure the oxidative stress blood was analysed for malondialdehyde (MDA), catalase (CAT), glutathione peroxidase(GPx ) and paraoxanase (PON).

RESULTS: Among the LFT parameters the level of Aspartate transaminase (AST) was significantly raised followed by alanine transaminase (ALT) and other parameters were either within normal limit or not significant. The level of MDA was significantly raised and level of GPx, CAT and PON was reduced. The significant correlation was found between ALT, AST and oxidative stress markers.

CONCLUSION: So we can conclude that in patients of dengue virus infection, raised liver enzyme (AST, ALT) may be associated with oxidative stress and AST can be used in a severity index and as prognostic marker of hepatic manifestations.

KEY WORDS- Oxidative stress, Antioxidants, Aminotransferase, Paraoxanase

\section{FP 60}

\section{Serum Angiotensin Converting Enzyme Activity In Kota Stone Workers Exposed To Silica Dust At Southern Rajasthan.}

Krishna Murari Lodha, A. K. Bhargava

Department of Biochemistry, Jhalawar Medical College, Jhalawar, Rajasthan

INTRODUCTION: Exposure to crystalline silica (silica dust) occurs in many industrialoperations worldwide. Kota stone mines in southern Rajasthan are common sites for exposure.Long term exposure of silica dust leads is non-curable progressive silicosis.Several biomarkers such as serum angiotensinconverting enzyme (ACE) activity have been examined to determine the extent of silicosis.

OBJECTIVES: Measurement of serum levels of 
Angiotensin converting enzyme in workers occupationally exposed to silica dust as biomarkers of severity of disease.

METHODOLOGY: A case-control study was carried out on 60 silica exposed workers and 60 non-exposed healthy controls. Serum levels of ACE were estimated by spectrophotometric method in both study groups.

RESULTS:The serum mean activity of ACE was higher in silica exposed workers $(48.82 \pm 6.74$ units/L) than in thehealthy controls $(23 \pm 6.14$ units/L). There was an association between the serum ACE level and the severity of silicosis. The results confirm that the serum ACE activity is elevated in silicosis and suggest that the elevation is associated with progression of the disease.

CONCLUSIONS: Since long term exposure of silica dust lead to non-curable progressive silicosis. ACE can be used as exposure biomarkers to silica dust, providing a better way for early diagnosis and helpful in making strategy for treatment of this deadly disease.

\section{FP 61}

\section{Assessment of Health Status of Youth Using Herbal Tea Through Anthropometric Measurements}

\section{Archana Singh, Tripti Sen}

Department of Biochemistry, Institute of Home

Science, Dr. B.R. Ambedkar University,

Khandari, Agra - 282002 (U.P.) , INDIA

INTRODUCTION:Health-oriented individuals are turning to herbal teas as alternatives to caffeinated beverages such as coffee, tea, and cocoa and for lowcaloric supplements. At the very least, it's a flavorful way of getting enough fluid into your body each day.

OBJECTIVES:The quantity and consumption over a long period of time is of major concern. Thus, the objective of the study to Assessment of Health Status of youth using Herbal Tea through Anthropometric Measurements

METHODOLOGY:Multistage stratified random sampling technique was used for selecting Selecting 100 adolescents of age group 20-35 years in Agra district. An interviewed schedule was developed to collect information regarding socioeconomic profile, dietary pattern, nutritional status etc.

RESULT:The most of herbal tea users were consuming herbal tea for the last one month to reduce weigh. The mean body mass index was more among the users of herbal tea as compared to non-users of herbal tea $(p<0.05)$.Significant effects of herbal tea were observed on the health in youth.

CONCLUSION:Preparation of infusion drinks prepared from plants appears to concentrate biologically active compounds. It is recommended that widespread consumption of herbal infusions should be minimized and varieties of carcinogens, mutagens, and toxicants are made available.

KEY WORDS: Herbal Tea, Youth,BMI, Health

\section{FP 62}

Deciphering the Role of Oxidative Stress and Treatment with Antioxidants In Infected Patients with Salmonella Typhi

Ghizal Fatima ${ }^{1}$, Nar Singh Verma ${ }^{2}$, Farzana Mahdi ${ }^{1}$, Abbas Ali Mahdi ${ }^{3}$, Anthonet N. Ezejiofor ${ }^{4}$

1. Department of Molecular Medicine, Era's Medical College and Hospitals, Lucknow,

2. Department of Physiology, King George's Medical University, Lucknow, India

3. Department of Molecular Medicine, Era's Medical College and Hospitals, Lucknow,

4. Department of Pharmacology, University of Port Harcourt, Nigeria

OBJECTIVE: In the present study we examined the connivance of oxidative and antioxidative parameters in patients with Typhoid fever (TF) and also evaluated its correlation with the severity of its symptoms along with supplement with antioxidants.

INTRODUCTION: Worldwide TF affects nearly 18 million people annually, causing nearly 700,000 deaths. 
Increased oxidative stress results from disparity between products of oxidation and antioxidant defenses. There are several clinical conditions integrated with increased oxidative stress, but novel data suggest a relationship between oxidative stress and TF. Furthermore, there is scant information available in scientific literature about oxidative and antioxidative parameters in TF, and its antioxidant supplement.

METHODS: Oxidative stress was determined by measuring the levels of Lipid Peroxides (LPO), nitric oxide (NO) and Protein carbonyl in plasma and antioxidative parameters like catalase, Glutathione peroxidase (GPx) and Glutathione Reductase (GR) in lysate in 30 patients with TF and 30 healthy volunteers without TF. Clinical parameters of TF were evaluated by Typhoid Fever Questionnaire (TFQ). Antioxidant supplements were given to patients for 4 weeks.

RESULTS: Concentrations of catalase $(\mathrm{p}<0.01)$, GR $(p<0.01)$ and GPx $(p<0.01)$ were significantly lower in patients with TF than in controls, and levels of oxidative stress parameters, LPO $(\mathrm{p}<0.01), \mathrm{NO}(\mathrm{p}<0.01)$ and Protein carbonyl $(\mathrm{p}<0.01)$ were significantly higher in patients than in controls. A significant positive correlation was found between LPO, NO, Protein carbonyl and clinical symptoms of TF among patients group. After 4 weeks of antioxidant supplement to TF patients blood samples were taken for the antioxidant status, it was found higher than the oxidative parameters.

CONCLUSION: The present results indicate that patients with TF are exposed to oxidative stress and this escalated oxidative stress may play a role in the worsening of the infection withSalmonella Typhi. Moreover, our results also show that increased oxidative stress parameters are more strongly amalgamated with TF symptoms. However, supplementation with antioxidants play a major role in perpetuating health of TF Patients.

KEY WORDS: Typhoid fever, Salmonella Typhi, Oxidative stress, Antioxidants.

\section{FP 63}

\section{Iron Status and Pre-Eclampsia}

Beenu $^{1}$, Amrita Chaurasia ${ }^{2}$

1. Department of Biochemistry, M.L.N. Medical College, Allahabad.

2. Department of Obs. \& Gynae., M.L.N. Medical College, Allahabad.

Preeclampsia (PET) is pregnancy induced hypertensive disease. PET is diagnosed clinically with presence of hypertension and proteinuria (with or without oedema) in absence of pre-existing hypertension and renal disease. There is a generalised vasospasm which may result in haemolysis caused by physical destruction of RBC s leading to anaemia and raised serum iron levels. Excess iron is a causative factor of oxidative stress (i.e., in its radical form) involved in the pathogenesis of preeclampsia. Excess iron reacts with free radicals of cell membrane (as it is rich in polyunsaturated fatty acids) and circulating lipoproteins initiating lipid per oxidation both in placenta and vasculature. This causes a change in the serum activities of ferretin, transferrin, ceruloplasmin and total iron binding capacity (TIBC) which may be responsible for hepatic dysfunction, increased vascular resistance and endothelial dysfunction, leading to alterations in biochemical milieu of the body. Thus Pre-eclampsia, vasospasm, hemolysis, raised serum iron levels $\&$ the resulting consequences initiate a vicious circle making Pre-eclampsia a progressive disorder inspite of all the treatments till delivery occurs.

\section{FP 64}

\section{Role of Vitamin D In Ischemic Stroke Patients}

\section{Anand Narayan Singh}

Dept. of Biochemistry, GSVM Medical College Kanpur 208002

BACKGROUND: Stroke is a sudden onset of focal neurological deficit, a major cause of morbidity and 
mortality and the second leading cause of death worldwide. Several mechanisms could make a case for the link between vitamin D deficiency and stroke. First, this knowledgehighlights a possible interaction between vitamin D deficiency and high blood pressure.

AIM: To evaluate of vitamin D in patients of ischemic stroke as compared to normal healthy persons.

METHODS: Total 86 subjects were evaluated, 41 subjects were ischemic stroke cases and 45 subjects healthy persons. We measured serum (25? hydroxyvitamin D [25(OH) D] by chemiluminescence immunoassay method), and LFT, S. creatinine and blood urea, Serum electrolyte $\left(\mathrm{Na}^{+}, \mathrm{K}^{+}, \mathrm{Ca}^{++}\right)$, Blood sugar fasting/post prandial and $\mathrm{HbA} 1 \mathrm{c}$ ) was measured. According to Stroke severity was assessed at admission using the National Institutes of Health Stroke Scale (NIHSS) score.Place of study were indoorpatient was admitted of medicine department at (LLR Hospital, Kanpur, India).

RESULTS:The level of 25(OH) D in stroke patients in the entire group of male/female was $24.58 \mathrm{ng} / \mathrm{ml} / 31.27$ $\mathrm{ng} / \mathrm{ml}$, and without stroke (healthy persons) group of male/female was $21.48 \mathrm{ng} / \mathrm{ml} / 22.4 \mathrm{ng} / \mathrm{ml}$. NO significance difference was found in mean vitamin $\mathrm{D}$ levels between males and females in both groups.

CONCLUSION: Vitamin D as a promising marker for cerebral ischemic vulnerability and to identify stroke patients at high risk for poor outcome. Our results demonstrate that vitamin D deficiency in stroke patients is necessary for vitamin D replenishment will improve the stroke outcome.

KEY-WORD-25-Hydroxyvitamin D, Prognosis, Ischemic strokes, Vitamin D deficiency

\section{FP 65}

\section{Correlation of Vitamin D Levels With Complications of Type 2}

Sukhraj Kaur ${ }^{1}$, Pashaura Singh Sandhu

Department of Biochemistry ${ }^{1}$, Department of Medicine

${ }^{2}$ Government Medical College, Amritsar
INTRODUCTION- Type 2 Diabetes Mellitus is a chronic metabolic disorder of absolute or relative insulin deficiency associated with chronic hyperglycemia. Long term diabetes is associated with development of both micro and macrovascular complications. Vitamin D deficiency appears to be related to the development of type 2 Diabetes Mellitus as it may affect glucose homeostasis. The present study was planned to study the levels of 25-hydroxy vitamin D3 and find a correlation if any between the levels of vitamin D3 and complications of diabetes mellitus.

MATERIAL AND METHODS- 100 known patients of Type 2Diabetes mellitus with complication were recruited for the present study from the OPD and IPD of Medicine Department of GMC ASR. 100 age and sex matched healthy individuals were selected to serve as controls. All the individuals were investigated for baseline parameters and vitamin D3 levels.

RESULTS-The patients were divided depending on the complication and it was observed that levels of vitamin Dwere lower in Type 2 Diabetes $19.26 \pm 0.95 \mathrm{ng} / \mathrm{ml}$ as compared to control $25.49 \pm 1.02 \mathrm{ng} / \mathrm{ml}(\mathrm{P}<0.001)$. Levels of Vitamin D were negatively correlated with Glycated haemoglobin and were positively correlated with Serum Triglyceride levels. This indicating that Vitamin D levels influence glucose homeostasis and also the level of Triglycerides.

CONCLUSION- Management of Type2 Diabetes should include estimation of Vitamin D so as to prevent its deficiency and development of complications.

KEY WORDS- Type 2 DM, Vitamin D, Glycemic Control 


\section{FP 66}

\section{Effect of Mode of Delivery on The Levels of Lipid Profile, Apolipoproteins and Atherogenic Index Determined In Umbilical Cord Blood}

Suman Jain ${ }^{1}$, Sharda Goyal ${ }^{2}$ and U.D. Deshmukh ${ }^{3}$

1. Department of Biochemistry, PIMS, Udaipur, Rajasthan, India.,

2. Department of Obstetrics and Gynecology, GMCH, Udaipur, Rajasthan, India,

3. Department of Biochemistry, PMCH, Udaipur, Rajasthan, India.

BACK GROUND- The data related to fetal cord blood lipid parameters and mode of delivery are measer. The aim of the study was to evaluate the effect on the levels of lipid profile, apolipoproteins and atherogenic index of neonates delivered by normal vaginal delivery and cesarean section.

METHOD- The study was conducted on 640 neonates delivered by normal vaginal (319) and cesarean delivered neonates (321). Lipid profiles, apolipoproteins and atherogenic index were measured in neonatal cord blood.

RESULTS-The mean values of total cholesterol, triglyceride, HDL-C, LDL-C, VLDL-C, Apolipoprotein A-1, Apolipoproetin B and atherogenic index were significantly higher in neonates delivered by normal vaginal delivery than cesarean ones.

CONCLUSION- This study showed that the concentration of lipid parameters changed significantly with mode of delivery.

KEY WORDS: Lipid profile, apolipoprotein, atherogenic, umbilical cord blood

\section{FP 67}

\section{Prognostic Significance of Cystatin C In Acute Coronary Syndrome}

P. Srilakshmi*, M. Vijaya Bhaskar\$, S.Venkatarao**

* Dept. of Biochemistry, Mamata Medical College, Khammam, Telangana State, India.

\$ Dept.of Biochemistry, NIMS, Hyderabad.

**Dept of Biochemistry,Mamata Medical College, Khammam, Telangana State, India.

Email-ID: sri.biochemistry@gmail.com

BACK GROUND: Coronary artery disease (CAD) is a condition in which there is an inadequate supply of blood and oxygen to a portion of the myocardium. The clinical spectrum of CAD is stable angina (SA), unstable angina (UA) and myocardial infarction (MI). ACS includes UA and MI. The frequency of ACS is extremely high among Indians; India has the highest burden of ACS in the world. The rising incidence of ACS in Indians may be associated with changes in the lifestyle, the westernization of the food practices, the growing prevalence of diabetes mellitus and probably genetic factors. In recent years cystatin $\mathrm{C}$ has emerged as a potential marker for cardiovascular risk and predicts the cardiovascular events. Cystatin $\mathrm{C}$ is a naturally occurring protease inhibitor that protects the host tissue from cysteine proteases, which is a proatherogenic factor.

MATERIALS AND METHODS: Study group comprised of 114 patients diagnosed as having ACS based on clinical and bio-chemical criteria. Control group included 66 age and sex matched subjects (non ACS cases) using the above mentioned criteria.

RESULTS: In this study, significant increase of mean serum cystatin C levels was observed in ACS cases than controls. Highest mean cystatin $\mathrm{C}$ values were observed in MI than UA.Highest mean cystatin $\mathrm{C}$ values were observed in ACS cases with risk factors.

CONCLUSION: Cystatin C plays an important role in the development of ACS and serum cystatin $\mathrm{C}$ is a might have a role as a prognostic marker in patients with ACS. 


\section{FP 68}

\section{Analysis of C-Reactive Protein and Homocysteine As Biomarkers For Age-Related Macular Degeneration (AMD)}

Rohit Manyal ${ }^{1}$,P.D. Sarkar ${ }^{2}$, S. Paneri ${ }^{3}$, Rajeev Lohokare ${ }^{4}$, Ravikant Sharma ${ }^{5}$

M.G.M. Medical College, Indore(M.P.)

INTRODUCTION:Age-related macular degeneration (AMD) is a common cause of irreversible visual loss and the disease burden is rising world-wide as the population ages. Inflammation plays a significant role in the incidence and progression of Age-related macular degeneration.

C-reactive protein (CRP) can be markedly elevated in inflammatory conditions.

An elevated homocysteine level is an independent risk factor for the development of vascular diseases.

AIMS \& OBJECTIVES: A study to compare homocysteine and CRP as diagnostic markers for the outcome of AMD in patients attending the tertiary level public health facility of Central India.

MATERIALS \& METHODS: Study was carried out at Deptt of Biochemistry, MGM medical college Indore. (M.P.) after approval from Ethical committee of the institute. During the study period, 30 cases and 30 controls were enrolled.After taking informed consent ,they underwent assay for CRP and Homocysteine (on Fully automated clinical chemistry analyser)

RESULTS: The median value of case in homocysteine group is $26.91 \mu \mathrm{mol} / \mathrm{L}$ and that of control is $12.98(\mathrm{p}$ value $<0.001$ ). The median value of case in CRP group is $6.49 \mathrm{mg} / \mathrm{L}$ and that of control is 1.43 ( $\mathrm{p}$ value $<0.001$ ).

CONCLUSION: Significant statistical difference was observed in Homocysteine and CRP levels of the cases and control ( $\mathrm{p}$ value $<0.05$ ). The present study shows that elevated CRP and Homocysteine levels are associated with AMD, and supports the role of inflammation and atherosclerosis in the pathogenesis of AMD. Further studies are needed to differentiate disease mechanisms of chronic inflammation and atherosclerosis contribute to the pathogenesis of AMD.

KEY WORDS: CRP, homocysteine, AMD

\section{FP 69}

\section{Role of D-Ribose Induced Glycation In Breast Cancer}

Uzma Shahab ${ }^{\mathrm{a}}$, Saheem Ahmad ${ }^{\mathrm{b}}$, Mohammad Waseema, Abbas Ali Mahdia and Mohammed Kaleem Ahmada

\section{a. Department of Biochemistry KGMU Lucknow, \\ b. Department of Biosciences Integral University Lucknow}

INTRODUCTION: Glycation is the non-enzymatic addition of free carbonyl group of reducing sugar to free amino group of protein, DNA and lipid macromolecule. This reaction leads to the formation of advanced glycation end-products (AGEs) through a series of reactions. Due to the Warburg effect and increased oxidative stress, cancer cells frequently accumulate significant amounts of AGEs.D-ribose being a reducing monosaccharide, has the ability to react with proteins and produce glycated derivatives. We investigated the glycating potential of D-ribose with Human Serum Albumin (HSA) and its effect on breast cancer cells.

METHODS:HSA was incubated with D-ribose in PBS under sterile conditions at $370 \mathrm{C}$ for 14 days. AGEs formation and structural changes were characterized using physicochemical techniques. Cultured Human breast adenocarcinoma cellswere treated with D-ribose for MTT assay, wound healing and apoptosis assay.

RESULT:GlycatedHSA samples showed comparatively increased absorption and fluorescence. Its HPLC chromatogram revealed two peaks corresponding to $\mathrm{CML}$ and pentosidine. Moreover, lower doses of D-ribose induced proliferation and migration of breast cancer cells. It also prevented apoptosis in breast cancer cells.

CONCLUSION:Glycation of albumin with D-ribose leads to structural perturbation and generation of AGEs. On the other hand D-ribose promotes breast cancer cells proliferation and migration.

KEYWORDS:AGEs, D-ribose, HSA 


\section{FP 70}

\section{Studies on Methylglyoxal-Modified-IgG Under Normal and High Glucose}

Mohd. Adnan Khan, Khursheed Alam

Department of Biochemistry, Jawaharlal Nehru Medical College, Faculty of Medicine, A.M.U., Aligarh, U.P.

INTRODUCTION: In diabetes mellitus the blood glucose level increases several fold over normal. This may result in profound increase in base-level nonenzymatic glycosylation of potential serum proteins, including IgG. Irrespective of the target protein the nonenzymatic glycosylation is ubiquitously accompanied by free radicalgeneration. During chronic hyperglycaemia in diabetics the formation of methylglyoxal increases manifold andresult in excessive formation of toxic advanced glycation end products (AGEs).In this study, native $\mathrm{IgG}$ has been modified with methylglyoxal (6.67 $\mu \mathrm{M})$ and co-incubated with normal $(5 \mathrm{mM})$ and high glucose $(10 \mathrm{mM})$ for 7 days and characterized by an array of biophysical and biochemicals techniques to understand the effect of glucose crowding on methylglyoxal potential in causing IgG glycosylation.

MATERIALS \& METHODS:Methylglyoxal (MGO) and Protein-A-agarose pre-packed affinity column were purchased from Sigma Chemical Company (St. Louis, MO, USA). D-glucosewas purchased from Qualigens, India.

$\operatorname{IgG}(6.67 \mu \mathrm{M})$ was transferred to assay tubes containing $5 \mathrm{mM} / 10 \mathrm{mM}$ glucose and mixed. To this mixture 6.67 $\mu \mathrm{M}$ MGO was rapidly added and incubated for 7 days at $370 \mathrm{C}$ in capped glass vials. The modification was studied by UV absorbance spectroscopy, fluorescence spectroscopy, far UV and near UV circular dichroism, thioflavin T fluorescence, Congo red binding, SEM, TEM, FTIR spectroscopy and LC-MS.

RESULTS: IgG under high glucose showed more modification by MGO. Furthermore, the extent of modification was found to depend on time and concentrations of both MGO and glucose. Hyperchromicity and increase in fluorescent AGEs was clearly noticed in modified-IgG. MGO-induced change in secondary structure of modified-IgG was revealed by FTIR and CD measurements. MGO modification of IgG also showed aggregation and appearance of amyloid-like structures when probed by thioflavin T, Congo red and SEM and TEM images.

CONCLUSION: IgG modification by MGO under high glucose may severely affect the functionality of $\mathrm{IgG}$ and form aggregates. Since aggregates are known immunogen, their formation in some patients of diabetes mellitus may result in rheumatoid arthritis like features.

KEYWORDS: Hyperglycaemia; Methylglyoxal; AGEs; Crosslink

\section{FP 71}

\section{Delineating the biomarker potential of shelterin complex molecule in multiple myeloma and revealing their therapeutic implications.}

Raman Kumar ${ }^{1}$, Rehan Khan ${ }^{1}$, Nidhi Gupta ${ }^{1}$, Tulika Seth $^{2}$, Alpana Sharma ${ }^{1 *}$

\section{Department of Biochemistry, All India Institute of Medical Sciences (AIIMS),New Delhi, India \\ 2. Department of Hematology, All India Institute of Medical Sciences (AIIMS), New Delhi, India}

BACKGROUND AND OBJECTIVES: Multiple myeloma $(\mathrm{MM})$ is characterized by proliferation of abnormal monoclonal antibody producing plasma cells residing in bone marrow (BM). This disease involves multiple primary and secondary genetic mutations which ultimately generates the phenotype of malignant cells. Role of telomerase activity (RTA) and shelterin complex (TRF1, TRF2, POT1, RAP1, TIN2, TPP1) along with its associated molecules (TANK-1 \& PINX1) has been anticipated in th solid and hematological malignancies. This maiden attempt aims to study expression levels of these molecules in myeloma and revealing therapeutic potential of TanshinoneI (TanI) alone or in combination with lenalidomide by targeting telomerase activity and shelterin molecules.

METHODOLOGY: Newly diagnosed $50 \mathrm{MM}$ patients $\& 20$ controls were recruited for BM aspiration with their 
consent. RTA, mean telomere length, relative mRNA and protein levels were studied in study subjects. Clinical parameters were recorded; correlation was studied between all the studied genes at mRNA levels and between genes and patients' clinical parameters; ROC curves were plotted for RTA, TL, and all studied genes. Anti cancer potential of TanshinoneI and lenalidomide or their combination were studied with different assays on myeloma cells (RPMI8226 \& U266).

RESULTS: Significantly $(\mathrm{p}<0.05)$ high relative mRNA expression were found of all molecules except PINX1 which was significantly lowered in patients compared to controls and was correlating with disease severity and progression. Similarly, higher RTA and lowered mean telomere length was observed in patients. Statistically significant correlation was observed between genes, telomerase activity and with clinical parameters. ROC curves analysis showed very high sensitivity and specificity for TRF2 genes and Telomerase activity. Thus, RTA and shelterin complex along with associated factors plays significant role in causing myeloma and its progression.

TanI caused significant toxicity at low doses in myeloma cells while lenalidomide did not have any toxicity. Cell death was enhanced when combination of both (TanI + lenalidomide) drugs were used as observed in AnnexinV/PI and Tunel assay. TanI and its combination with lenalidomide significantly down regulated protein expression of TANK1, TPP1, and RAP1 in myeloma cells compared to untreated cells.

CONCLUSIONS: In conclusion, present study indicates that increased RTA along with altered mRNA and protein expression of shelterin complex molecules along with its associated partners might play significant role in pathogenesis and progression of MM. Stage-wise significant increased expression and correlation of these molecules with each other and with clinical parameters demonstrated their role as potential bio-marker in MM. Significant down-regulation of RTA and apoptosis by TanI alone or combination with lenalidomide suggests that TanI might have synergizing effect with lenalidomide in causing cytotoxicity in Myeloma.

KEYWORDS: myeloma, shelterin complex, telomere, telomerase, biomarker,
FP 72

\section{Matrix Metalloproteinasesas Biomarkers In Early Diagnosis of Severity Among Pediatric Dengue Infections.}

Debadyuti Sahua, Soundravally Rajendiran ${ }^{a *}$, Rahul Dhodapkarb $^{\mathrm{b}}$ T. Kadhiravan ${ }^{\mathrm{c}}$,

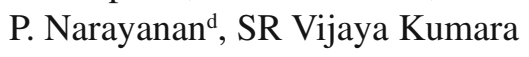

a. Department of Biochemistry,

b. Department of Microbiology,

c. Department of Medicine,

d. Department of Pediatrics, Jawaharlal Institute of Postgraduate Medical Education and Research, Puducherry, India.

INTRODUCTION:Plasma leakage is major pathological hallmark of DHF and DSS, severe forms of Dengue viral (DENV) infection spectrum. MMPsare chiefly secreted by monocytes/macrophages (site of DENV replication) and cause ECM degradation. Balance between MMPs and TIMPs(endogenous MMP inhibitors) may have implication(s) in plasma leakage. Pediatric dengue cases exhibit more severe clinical manifestations.

AIMS AND OBJECTIVE:To estimate serum MMP-9, MMP-13 and TIMP-1 levels during course of DENV infection among pediatric dengue cases and investigate a possible correlation with severity.

MATERIALS AND METHODS: Samplingdone thrice (admission/ febrile stage, toxic stage, during discharge or defervescence) from 19 dengue and 21OFI (control) patients. Dengue patients were classified in accordance with WHO guideline-2009. Serum MMP-9, MMP-13 and TIMP-1 were estimated by ELISA alongside routine hematological and biochemical parameters.

RESULTS: 1) Both the MMPs' serum levels fall during defervescence compared admission. 2) MMP-9 levels are much higher in primary cases with severe clinical manifestations. 3) Upregulation of TIMP-1 towards defervescence (as expected) found among nonsevere cases whereas opposite trend was exhibitedamong severe cases. 4) Rise in TIMP-1 was more pronounced among nonsevere compared to severe cases. 5) Decline in MMP9/TIMP-1and MMP-13/TIMP-1 ratios observed 
fromacute febrile stage towards defervescence; withmore rapidity among nonsevere compared to severe cases.

CONCLUSION: Higher MMP-9, MMP-13 and low TIMP-1 during febrile stage suggest implicationin DENV pathogenesis. Degree of rise in serum TIMP-1 might be determining milder course. Thus MMP-9, MMP-13 and TIMP-1 and their ratio(s) can serve as a potential biomarker for early determination of severity of DENV infection course.

KEYWORDS:Dengue Hemorrhagic Fever (DHF); Dengue Shock Syndrome (DSS); Matrix metalloproteinase (MMP); Tissue inhibitor of metalloproteinases (TIMP); Other febrile illness (OFI).

\section{FP 73}

\section{GC-MS/MS Analysis of Anticancer Sub-Fraction From Tinospora Cordifolia}

Jama $^{1}$ Akhtar Ansari ${ }^{1,2}$, Abbas Ali Mahdi ${ }^{1}$, Mohammad Kaleem Ahmad ${ }^{1}$, Nishat Fatima ${ }^{1,2}$,Abdul Rahman Khan ${ }^{2}$, Satya Narayan ${ }^{3}$, Devendra Kumar Patel $^{3}$

1. Natural Products Research Lab, Department of Biochemistry, King George's Medical University, Lucknow 226 003, U.P., India

2. Department of Chemistry, Integral University, Lucknow 226 026, U.P., India

3. Division of Analytical Chemistry, CSIR-Indian Institute Toxicology Research, Lucknow 226026

INTRODUCTION:Breast cancer is the most common malignancy among women worldwide. The unmanageable side effects caused by conventional chemotherapy drugs counsel for a better and safe drug. Natural products have been recognized as an important source of chemotherapeutic agents with low side effects. Tinospora cordifolia (Willd.) Miers ex Hook. F. \& Thoms. (Menispermaceae ) is an important folk and Ayurvedic plant used for various therapeutical purposes. But there is a scarcity of literature regarding the anticancer activity of $\mathrm{T}$. cordifolia.

MATERIALS AND METHODS:Gas chromatography coupled with triple quadruple mass spectrometer was used to screen out the presence chemical compounds present in sub-fractions. The antiproliferative activity was evaluated against human breast cancer (MDA-MB231 and MCF-7) cells by MTT and colony formation assay. Hoechst 33342 staining was performed to examine apoptosis. H2DCFDA staining was used to determine ROS mediated cell death in breast cancer cell line MDAMB-231 and MCF-7.

RESULTS:The identified compounds were compared with those reported for structurally related molecules. Many new compounds were identified in our work that was not reported from $\mathrm{T}$. cordifolia stem. The anticancer investigation showed that sub-fraction induces apoptosis in human breast MDA-MB-231 and MCF-7 with intracellular ROS generation.

CONCLUSION:In conclusion, we report for the first time the apoptogenic property of sub-fraction obtained from Tinospora cardifolia through induction of ROS generation in breast cancer cells as well as presence of several chemical entities which may be unconditionally attributed to anticancer activity of T. cordifolia.

KEY WORDS:Tinospora cordifolia, antiproliferative activity, Apoptosis, ROS, GC-MS/MS.

\section{FP 74}

\section{Evaluation of Wrightia Tinctoria Non-Polar Fractionfor Antiproliferative Activity And Chemical Characterization}

Nishat Fatima ${ }^{1}$,Abbas Ali Mahdi ${ }^{1}$, Jamal Akhtar Ansari ${ }^{1}$, Mohammad Kaleem Ahmad ${ }^{1}$, Satya Narayan ${ }^{2}$, Devendra Kumar Patel ${ }^{2}$

1. Natural Products Research Lab, Department of Biochemistry, King George's Medical University, Lucknow 226 003, U.P., India

2. Division of Analytical Chemistry, CSIR-Indian Institute Toxicology Research, Lucknow 226 026,

INTRODUCTION: Wrightia tinctoria Roxb. (R.Br.) bark is an important traditional plant commonly known as "Indra Jau". The present work was carried out to evaluate the anticancer potential and phytochemical 
contents of W. tinctoria bark non polar hexane (WTH) fraction.

MATERIALS AND METHODS: The anticancer activity was substantiated by 3-[4,5-dimethylthiazol-2yl]-2,5-diphenyl tetrazolium bromide (MTT) assay and colony formation assay for cell viability, Hoechst staining was performed to examine the apoptosis, and H2DCFDA was used to determine ROS mediated cell death in breast cancer MDA-MB-231 and MCF-7 cancer cells.

RESULTS: Our experimental findings from different in vitro assays showed that the $\mathrm{W}$. tinctoria non polar fractions exhibited significant inhibition of growth and proliferation of breast cancer cells. Fluorescence microscopy with Hoechst and H2DCFDA dye staining showed that the WTH fraction induces ROS mediated apoptosis in MDA-MB-231 and MCF-7 cancer cells. The chromatography fingerprinting demonstrated presence of chemical compounds which belong to different chemical classes.

CONCLUSION: In conclusion, our study revealed an extensive biochemical profile of $\mathrm{W}$. tinctoria bark non polar fraction as well as antiproliferative property. Therefore, further in depth studies are needed to isolate and characterize the active principles.

KEYWORDS: Anticancer, Breast cancer, Wrightia tinctoria, phytochemicals.

\section{FP 75}

Bacopa Monnieri Mode of Actionagainst MPTP Induced Parkinson's Disease Mice Model: An In Silico And In- Vivo Study

Babita Singh ${ }^{1}$, Shivani Pandey ${ }^{1}$, Satyndra Kumar Yadav $^{1}$, Saba Ubaid ${ }^{1}$, Abbas Ali Mahdi ${ }^{2}$

1. Department of Biochemistry, KGMU, Lucknow-226003

2. Era University Sarfazganj, Hardoi road, Lucknow

INTRODUCTION: Parkinson's disease (PD) is a progressive neurodegenerative movement disorder, however itsmanagement is still challenging. Currently studies are being conducted for efficient cure in PD,by using herbal plant. In the present study, an effort has been made to explore neuroprotectivemode of action of herbal plant Bacopa monnieri in MPTP induced neurodegeneration againstmice model of PD through in silico and in vivo study.

MATERIAL AND METHODS:Experimental mice were given $40 \mathrm{mg} / \mathrm{kgbody}$ weight BME treatment orally for one month with prior use of $15 \mathrm{mg} / \mathrm{kg}$ b.w of MPTP for two weeks. After this, neuroprotective effect was studied through neurochemicalanalysis andin silico study.

RESULTS: A significant recovery of spontaneous locomotor activity, antioxidant enzymatic activity was found. The increased levels of Dopamine (DA), 3-4 dihydroxy-phenyl aceticacid (DOPAC), Homovanillic acid (HVA) and decreased levels of lipid peroxides, conjugated dienes were evident as improvement in MPTP+BME group as compared to MPTP treated animals. In-silico study, revealed that BM phytoconstituents mainly bacosides, bacopasides and bacosaponins havecapability to halt the breakdown of MPTP to MPP+ by inhibiting the enzyme Monoamineoxidases B. Further, BM phytochemicals bacosides, bacopasides and bacopasaponins showedinteresting binding affinity with KEAP1 receptor protein and inhibiting the cullin 3 mediatedNuclear factor like 2 (Nrf2) protein ubiquitination process and thereby up regulating theexpression (via Nrf2) of antioxidant enzymes.

CONCLUSION: Both in vivo and in silico studies indicate that BMphytochemical has ability to maintain DA concentrations in PD by virtue of DA synthesisinduction and inhibition of DA degradation. This study may offer a therapeutic approach.

KEYWORDS: Parkinson's disease, MPTP, Bacopa monnieri, Neurodegeneration, Oxidative stress. 


\section{FP 76}

Mir-363-3pidentified As Diagnostic And Prognostic Biomarker In Prostate Cancer Patients

Mohammad Waseem ${ }^{1,2}$, MK Ahmad ${ }^{1}, \mathrm{M}$. Serajuddin ${ }^{2}$, H J khan ${ }^{1}$, S N Sankhwar ${ }^{3}$, S Tiwari ${ }^{1}$, A A Mahdi ${ }^{1}$

1. Department of Biochemistry, King George's Medical University, Lucknow, U. P, India

2. Department of Zoology, Lucknow University, Lucknow, U.P, India

3. Department of Urology, King George's Medical University, Lucknow, U.P, India

BACKGROUND \& OBJECTIVE: Prostate cancer $(\mathrm{PCa})$ is the most common malignant tumor in men in worldwide. Aim of study to profile the differential expression microRNA (miRNA) in prostate cancer tissue to be used as biomarker in PCa patients.

MATERIAL \& METHODS: Human tissue samples for benign prostatic hyperplasia $(\mathrm{BPH})$ and $\mathrm{PCa}$ patients were collected along with the adjacent noncancerous tissue as control and twenty seven tissue samples (control, $\mathrm{n}=8 ; \mathrm{BPH}, \mathrm{n}=8$; $\mathrm{PCa}, \mathrm{n}=11$ were randomly selected for microarray analysis. Raw data was analyzed by TAC software. The miRNAs with more than two-fold change (p-value $<=0.05$ ) in tumor tissues compared with the normal tissues were considered as differentially expressed. We then evaluated microRNA in 50 additional case/control pairs using Reverse transcription (RT)-PCR from advance cDNA synthesis assay kit. The quantitative real time PCR (qRT-PCR) expression analyses of microRNA were done by using the Taqman advance qPCR assay kit. miR-28-3p was taken as endogenous control.

RESULTS: Bioinformatic analysis of our micro array data showed differential expression of miRNAs in $\mathrm{PCa}$ tissue when compared to adjacent noncancerous tissue. Among them miR-1827,miR-363-3p, miR-1282, miR1275, miR-130b-3p,miR-183-5p,miR-4510 was found to be up regulated and miR-711, miR-423-3p, miR-329-5p, miR-486-5p miR-205-5p were found to be down- regulated. In search of novel microRNA we found miR363-3p to be significantly up regulated in PCa tissue as compared to BPH and control $(\mathrm{p}<0.0001)$. Our result depicted that miR-363-3pexpression was increased with advancement of disease which are correlated with high PSA, high Gleason score, Tumor Stage pT3-4, lymph node involvement and metastasis condition. ROC curve analysis of miR-363-3p showed it can distinguish PCa patients from controls $(\mathrm{AUC}=0.0 .953)$ and also distinguish $\mathrm{PCa}$ patients from $\mathrm{BPH}$ patients (AUC $=0.854$ ). Survival analysis pointed out that High miR-363-3pexpression was significantly associated with a poor survival ((P value 0.049$)$.

CONCLUSION: In our study we found up-regulation of miR-363-3p expression increases with progression of disease. Our result also suggested that with high miR363-3pexpression, had significantly shorter progressionfree survival and poor prognosis in patients when compared with patients with low expression of miR-3633p. Therefore, miR-363-3p expression might be helpful in diagnosis and prognosis of PCa patients.

KEY WORDS: MicroRNA, BPH, Prostate cancer, Microarray, qRT-PCR, diagnostic and prognostic marker

\section{FP 77}

Circulating miRNA 223-3p and Mirna 486-5p As Potential Diagnostic Biomarkers For Early Prediction of Type 2 Diabetes Mellitus In North Indians.

Saliha Rizvi ${ }^{1}$, Syed Tasleem Raza ${ }^{1}$, Farzana Mahdi ${ }^{1}$.

1. Department of Biochemistry, Era's Lucknow Medical College and Hospital, Lucknow, India.

INTRODUCTION: Type 2 diabetes mellitus (T2DM) is a major public health problem in India and other developing countries. Diagnostic markers are urgently needed to identify individuals at risk of developing T2DM and greatly improve the care for these individuals and reduce/delay the occurrence and severity of the disease. Altered expression of circulating miRNAs has been documentedin various human diseases including 
T2DMand thus they presentas important sources of noninvasive biomarkers.

AIM: The present study was aimed to determine the role of miRNA 223-3p and miRNA 486-5p as early diagnostic biomarkers for T2DM in North Indians.

MATERIALAND METHODS: Here, we evaluated the expression of miRNA 223-3p and miRNA 486-5p in plasma of three study groups: controls (fasting glucose, 4.8-5.2mmol/L; $\mathrm{n}=20$ ), T2DM-susceptible (fasting glucose, 6.1-6.9mmol/L; $\mathrm{n}=23$ ), and newly diagnosed T2DM (fasting glucose, ?7.0mmol/L; $\mathrm{n}=24$ ) individuals. The miRNA expression analysis and detection was conducted using Taqman ${ }^{\circledR}$ MicroRNA Assay following the standard assay protocol on Applied Biosystems StepOnePlus ${ }^{\mathrm{TM}}$ Real Time PCR systems.

RESULTS:RT-PCR assessment revealed that plasmamiRNA 486-5p levels were significantly higher in T2DM susceptible cases as compared to newly diagnosed diabetics. Also, it was significantly higher in newly diagnosed T2DM cases when compared to controls. The expression of miRNA-223-3p was almost equal in T2DM susceptible and newly diagnosed T2DM cases but they were again higher in our cases as compared to controls. Since the expression of studied miRNA'swascomparatively higher in both newly diagnosed T2DM andT2DM susceptible cases, they may thus act as early diagnostic biomarkers for detection of individuals at risk of developing T2DM in future.

CONCLUSION: The study suggests that detectionof circulating miRNA 223-3p and miRNA 486-5pcan be developed into a noninvasiveand rapid diagnostic tool for the prediction of susceptibleindividuals to developing T2DM

KEYWORDS: Type 2 Diabetes Mellitus, miRNA 2233p,miRNA 486-5p, RT-PCR, North Indians.

\section{FP 78}

\section{Antihyperglyceamic Potential of Aqueous Extract of Different Parts of Butea Monosperma on Alloxan Induced Diabetic Rats: A Comparative Study}

Anthonet Ndidiamaka Ezejiofor ${ }^{1}$, Ghizal Fatima ${ }^{2}$ andFarzana Mahdi ${ }^{2}$

1. Department of Experimental Pharmacology \& Toxicology, Faculty of Pharmaceutical Sciences, University of Port Harcourt Port Harcourt, Rivers State, Nigeria.

2. Department of Molecular Medicine, Era Lucknow Medical College \& Hospital, Sarfarajganj, Hardoi Road, Lucknow 226003 $(U P)$, India

The anti-hyperglyceamic and anti-oxidant potential of the aqueous extract of various parts of B. monosperma was evaluated in alloxan (ALXN)-induced diabetic male adult rats. Experimental animals were divided into eight groups viz., I, II, III, IV, V, VI, VII, and VIII. Diabetes mellitus (DM) was induced in groups II - VIII rats by a single intraperitoneal injection of alloxan $(150 \mathrm{mg} / \mathrm{kg}$ body wt). Group I (control mice) received an equal volume of normal saline. Group III- VIII were further treated with BMF, BMS, BML, BMB and BMR (500 $\mathrm{mg} / \mathrm{kg}$ body wt, p.o.) representing, flower, seed, leave, stem bark and root of BM respectively for a period of 21 days. Body weight, food and water intake and fasting blood glucose (FBG) levels were measured at periodic intervals during the test period. At the end of treatment period, blood was collected by cardiac puncture under mild ether anesthesia and serum was isolated to analyze serum insulin levels. The homogenates of pancreatic was also analyzed for both enzymatic and non-enzymatic antioxidants, such as superoxide dismutase (SOD), catalase (CAT), glutathione peroxidase (GSH-Px), reduced glutathione (GSH), thiobarbituric acid reactive substances (TBARS) and total protein (TP). Alloxan injection resulted in a significantly $(\mathrm{P}<0.05)$ increased concentration of FBG level. The FBG level decreased significantly $(\mathrm{P}<0.05)$ after 21 days treatment of BM parts. 
KEY WORDS:Butea monosperma, anti-hyperglyceamic, anti-oxidant potential

\section{FP- 79}

\section{Evaluation of The Combine Effect of Azadiracta Indicaleaves and Variant Ph on Human Breast Cancer Cell Line}

\section{AparnaMisra $^{1}$, Anchal Trivedi ${ }^{1 *}$}

1. Department of Biochemistry, Era's Lucknow Medical College \& Hospital, Sarfarazganj, Hardoi Road, Lucknow-226003, UP, India.

Cancer is the mostcommon disease and leading cause of death in whole world. After skin cancer, breast cancer is the second most common cancer in women worldwide. Scientists andresearchers have created various drugs for treating cancer including chemotherapy, radiotherapy, surgery and Phytochemical based drug.Phytotherapeutic have no serious side effects, therefore itis a better option forcuring cancer without any side effect. Neem (Azadiractaindica) is fast growing evergreen ancient medicinal plant tree in India. It has many therapeutic properties like-hypoglycemic, antipyretic, antiulcergenic, antifungal and as well as antineoplastic.

Many researchersalso investigated the association between various $\mathrm{pH}$ and its effect observed that the $\mathrm{pHis}$ also effect the cancer cell line. Acidic $\mathrm{pH}$ has increases cancer cell growth and metastasis while in alkaline $\mathrm{pH}$ breast cancer cells shows very different in endocrine resistant. Endocrine study emphasis the major influence of extracellular $\mathrm{pH}$ against of alkalinizing drug therapy. In our research work,ethanolicneemextract and $\mathrm{pH}$ is effective as both individual as well as combined. Itis highly effective on survival and viability of human breast cancer cell line. It was found that a combination of high $\mathrm{pH}$ (8.6) and AI extract 1600 ?g/ml caused significant mortality $(84 \%)$ in the breast cancer cell line (MDAMB- 231).The IC50 value for AI extract at $\mathrm{pH} 8.0$ was found to be $200 ? \mathrm{~g} / \mathrm{ml}$.It was found alkaline $\mathrm{pH}$ is cytotoxic. The cancerous cell can grow in acidic $\mathrm{pH}$ inhibit the growth of cancerous cell line. However, the presence of AI, efficiently alkaline $\mathrm{pH}$ becomes highly cytotoxic and cancer cells more.In future of cancer treatment,either only alkaline $\mathrm{pH}$ or combine with AI can be usedas a potential treatment for the cancer cure.

\section{FP- 80}

\section{Promoter Hypermethylation and Expression of CHD-5 Gene In Pancreatic Adenocarcinoma Patients.}

Dr. N Lakha, Dr. K Krishnamurthy, Dr. A Agarwal, Dr. P Sakhuja, Dr. T. K. Mishra.

\section{Maulana Azad Medical College and Associated Hospitals, New Delhi-110002}

BACKGROUND-Chromodomain helicase DNA binding protein-5 gene (CHD-5) has been identified as tumor suppressor gene. Human chromosome $1 \mathrm{p} 36$ is a region consistently deleted in cancers. CHD-5 is located in this region. CHD-5 is the fifth member of a family of chromatin remodeling proteins. CHD-5 is preferentially expressed in the nervous system and testis. CHD-5 was first identified because of its location in a region of frequent deletion in neuroblastomas. Downregulation of CHD-5 gene expression has been observed in neuroblastoma, breast cancer, colon cancer and ovarian cancer etc. This may be explained by deletions or other mutations. We evaluated CHD-5 gene expression by using quantitative RT-PCR in 20 patients of pancreatic adenocarcinoma (PA). The results were compared with 20 normal healthy controls. We also evaluated whether CHD-5 gene methylation has role in PA by using MSPCR.

MATERIALS AND METHODOLOGY-This study was conducted in the Department of Biochemistry of MAMC, New Delhi. The blood sample of 20 patients of PA were taken along with 20 healthy controls. We evaluated CHD-5 gene expression by using quantitative RT-PCR after cDNA synthesis from RNA extracted from plasma. Promoter hypermethylation was evaluated using MS-PCR after bisulfite treatment of cell-free DNA extracted from plasma samples.

RESULTS-We observed that the expression of CHD-5 gene was reduced in samples of pancreatic adenocarcinoma patients in relation to healthy controls. 
We observed that, out of 20 cases, only three were having promoter hypermethylation whereas none of the control showed promoter hypermethylation of CHD-5 gene. However, it had no significant impact on disease.

CONCLUSION-The CHD-5 gene expression was down-regulated but promoter hypermethylation had no significant impact on presence of pancreatic adenocarcinoma. Also, there was no significant association between CHD-5 gene expression and methylation status.

\section{FP- 81}

\section{Do Vitamin D Deficiency and Renal Dysfunction play a role in the pathogenesis of Fluorotoxic Metabolic Bone Disease (FMBD)?}

Bondu, Joseph Dian*; Selvakumar *;

Seshadri MS **; Fleming, Jude Joseph*

*Department of Clinical Biochemistry, Christian

Medical College, Vellore, Tamil Nadu, India.

** Department of Endocrinology, Christian Medical

College, Vellore, Tamil Nadu, India.

AIM: To study the synergistic role of fluoride exposure and Vitamin D deficiency in the pathogenesis of FMBD.

MATERIALS AND METHODS: Male SpragueDawley rats were divided into 6 groups of six; 3 groups received a Vitamin D deficient diet whereas the other 3 received a Vitamin D adequate diet. Serum 25OHD, calcium, phosphorus, creatinine, ALP, albumin, PTH, Osteocalcin and CTx were measured following exposure to varying levels of fluoride in drinking water $(<1.0 \mathrm{ppm}$, $15 \mathrm{ppm}$ and $50 \mathrm{ppm}$ ). Full body DXA scans were used to examine changes in bone morphology pre and post exposure to fluoride. Renal tubular function was assessed using serum creatinine, urine fluoride and urine Cystatin C. Histopathological examination of sections of bone and kidney tissues were also performed.

RESULTS: Prior to fluoride exposure, DXA scan revealed a significant decrease in BMD and BMC $(p<0.05)$ but a significant increase in fat mass $(p<0.05)$ and fat percentage $(\mathrm{p}<0.01)$ among Vitamin $\mathrm{D}$ deficient rats, with no significant change in biochemical parameters. Following exposure to fluoride, BMD was significantly increased $(\mathrm{p}<0.05)$ in both groups with a corresponding increase in serum ALP, bone fluoride content, Osteocalcin, CTx and urine fluoride with increasing levels of fluoride exposure. Serum creatinine and cystatin $\mathrm{C}$ levels showed no significant changes. Light microscopy examination revealed mild thickening and increased osteoid in $80 \%$ of the Vitamin D deficient rats exposed to high levels of fluoride but renal tubular changes were found only in one experimental and control animal each.

Conclusion: Fluoride deposits in bone affect both osteoblastic and osteoclastic activity. These effects are accentuated in the presence of Vitamin D deficiency.

\section{FP- 82}

Association of Osteoprotegerin level with cardiovascular risk in diabetic subjects is independent of age, body mass index and body composition

Rakchna $\mathbf{M}^{1}$, Nivedita Nanda ${ }^{1}$, Jasmine Manna Rani ${ }^{1}$, Velkumary $\mathrm{S}^{2}$, Jaya Prakash Sahoo ${ }^{3}$

1. Department of Biochemistry,

2. Department of Physiology and

3. Department of Endocrinology Jawaharlal Institute of Postgraduate Medical Education and Research, Puducherry, India

INTRODUCTION: Diabetes mellitus being a major risk factor for cardiovascular diseases (CVD), there is a need to monitor cardiovascular risks (CV) at an early stage. Heart rate variability (HRV) is a non-invasive but sensitive method to assess cardiac autonomic control indicating CV risk. Similarly, osteoprotegerin (OPG) a glycoprotein, correlates with the progression of vascular dysfunction in CVD. Reports on OPG level in insulin resistance (IR) is conflicting in presence of obesity. However, there are no reports regarding association of OPG with autonomic component of $\mathrm{CV}$ risk such as HRV in insulin resistance.

AIM : In the present study, we have evaluated the 
association of OPG level with insulin resistance and HRV in T2DM patients.

MATERIALS \& METHODS: The anthropometry, body fat composition, HRV parameters (RMSSD, SDNN), serum fasting glucose, lipid profile, osteoprotegerin, insulin, HOMA-IR and hsCRP levels, measured in 40 diabetic patients and compared with age, gender and body composition matched non-diabetic controls.

RESULTS \& CONCLUSION: The lipid risk factors calculated from lipid profile suggested an atherogenic profile $(\mathrm{p}<0.05)$ in diabetes group but hsCRP which is a marker for CVD showed no difference. Diabetic subjects had higher OPG $(\mathrm{p}<0.001)$ and HOMA-IR $(\mathrm{p}<0.05)$. The parasympathetic component of HRV (RMSSD, SDNN) were lower $(\mathrm{p}<0.001)$ indicating sympathovagal imbalance which indicates $\mathrm{CV}$ risk. Both OPG and HOMA-IR were associated with RMSSD. Therefore, from the present study we conclude that increased level of OPG in diabetes mellitus is associated with $\mathrm{CV}$ risk and is independent of their age, body composition and hsCRP levels.

\section{FP- 83}

\section{Association of Cord Blood Cholinesterase with Congenital Anomalies and Its Inter-Relation With Fluoride Levels In Cord Blood}

Ghosh A, ${ }^{1}$ Kharb $S,{ }^{1}$ Nanda $\mathrm{S}^{2}$

Department of Biochemistry ${ }^{1}$ and Obstetrics and Gynecology ${ }^{2}$ Pt. B.D.Sharma PGIMS, Rohtak, Haryana

INTRODUCTION : Increase incidence of congenital abnormalities in north-west India suggests the role of some environmental factor as $\mathrm{N}-\mathrm{W}$ India is predominantly an agricultural community and fluoride and pesticides exposure are hypothesized to be one of the causative agents.

AIMS AND OBJECTIVES : Serum cholinesterase levels have been reported to be reduced in acute hepatitis, cirrhosis of liver, organophosphorus poisoning and in some malignant tumours.Levels of serum cholinesterase in newborns with congenital anomalies has not been explored yet. Hence the present study was planned to analyse the levels of cord blood cholinesterase in newborns with congenital anomalies.

MATERIALS AND METHODS : Thirty healthy newborns and thirty newborns with visible congenital anomalies are selected as control and cases respectively. Detailed history of present pregnancy, status of baby, nutritional status of parents, history of pesticides or heavy metal exposure of parents, history of use of any medication were taken and detailed physical examination of babies were done. Five $\mathrm{ml}$ cord blood was collected from placental end of umbilical cord after delivery of baby and serum was separated and serum cholinesterase were estimated enzymatically.

RESULTS AND CONCLUSION : Findings of the present study suggest that low cord blood cholinesterase levels along with high fluoride levels can be caused by various agricultural and industrial pollutant in this region and the interplay between cholinesterase and fluoride might result in congenital anomalies in this region.No such studies are available in Indian context especially in Haryana regarding cord blood cholinesterase levels in newborns with congenital anomalies. This study attempted to collect baseline data so that preventive and diagnostic measures can be planned in environmental health studies for Indian children.

\section{FP84}

\section{Biochemical Profile, Vitamin D Status and Immuno Modulatory Effects In Pulmonary Tuberculosis with and Without Hiv Infection}

\section{Sukhes Mukherjee}

Department of Biochemistry, All India Institute of Medical Sciences, Bhopal

INTRODUCTION: Tuberculosis remains a major global health problem and the second leading cause of death from an infectious disease worldwide. Both drug resistance and HIV worsen the TB disease burden among individuals and are a threat to global TB control and the Indian scenario is not much different with global incidence of tuberculosis of 8.6 million. 
AIM OF THE STUDY: Here in our study we investigated the role of haematological parameters, immunological parameters, biochemical parameters, vitamin D status in patients with pulmonary tuberculosis and with dual infection with HIV.

METHODS: We investigated 100 patients attending RNTCP on short term regimen. The biochemical parameters and haematological were investigated by Cobas c501 model of Roche andD3 levels measured byRoche diagnosis Elesys. Cytokines was measured using Kits.

RESULTS: Our study reveals that mean age of study population was $40.1 \pm 12.9$ and most were between 40 and 49 age group. $30 \%$ of the subjects had a sputum smear grade of $3+$ and $62.5 \%$ showed a CD4 count $<200$. Vitamin D, an immunomodulatory effector, has been proven to play a critical role in inducing anti mycobacterial activity which is accomplished by inhibiting the growth of Mycobacterium tuberculosis (MTB) and up-regulating protective innate host responses.

CONCLUSION: The laboratory markers associated with PTB with or without HIV co infection play a major role, although a nonspecific one, in the diagnosis and prognosis of TB in patients and in future will be an important aspect for clinicians.

KEY WORDS: Immunomodulator, HIV, RNTCP, Tuberculosis, Vitamin D

\section{FP 85}

\section{Hypovitaminosis D and Pulmonary Tuberculosis: A study in a tertiary care institute of Mewat}

Yuthika Agrawal ${ }^{1}$, Vipin Goyal ${ }^{2}$, Nikita Singh ${ }^{1}$, Sangeeta B. Singh ${ }^{1}$

1. Department of Biochemistry, SHKM GMC, Nalhar, Mewat

2. Department of Chest and TB, SHKM GMC, Nalhar, Mewat

INTRODUCTION: Tuberculosis (TB) remains a major challenge to global public health. Vitamin D is an immunoregulatory hormone. Epidemiological evidence also suggests a link between vitamin D deficiency, serum calcium, serum phosphate and TB but the relation is not clear. Our study was aimed to assess the levels of serum Vitamin D, serum calcium and phosphorus in pulmonary tuberculosis (PTB) patients of Mewat.

Material and Methods: Blood samples were collected from 100 patients with newly diagnosed PTB (PTB-0)and PTB cases taking treatment or have completed treatment (PTB-Rx). Age and sex matched controls with same ethnic background, dietary habits and sun exposure were taken. Serum was analysed for vitamin D, calcium and phosphorus.

RESULTS: Serum vitamin D was significantly decreased in both groups of cases (PTB- $0=13.9+5.8 \mathrm{ng}$ / $\mathrm{ml})(\mathrm{PTB}-\mathrm{Rx}=13.6+5.1 \mathrm{ng} / \mathrm{ml})$ as compared to controls $(29.5+6.5 \mathrm{ng} / \mathrm{ml}) .86 \%$ of cases had vitamin $\mathrm{D}$ value $<20 \mathrm{ng} / \mathrm{ml}$. Calcium was comparable in all the groups. Serum phosphorus was significantly increased in newly diagnosed PTB cases $(5.3+1.2 \mathrm{mg} / \mathrm{dl})$ but was within normophosphatemic limits as compared to controls $(4.1+0.4 \mathrm{mg} / \mathrm{dl})$ and was comparable with that of PTB cases on treatment or completed treatment $(5.2+1.7 \mathrm{mg} /$ dl).

CONCLUSION: Hypovitaminosis D was significantly associated with TB infection in our study. Since it has a role in Cell Mediated Immunity it could be one of the causes for the infection. Deficiency was noted in $86 \%$ of the cases, the importance of improving the nutrition and if required even supplementing vitamin $\mathrm{D}$ by food fortification

KEYWORDS:Vitamin D, tuberculosis, Mewat, normocalcemia, hyperphosphatemia

\section{FP86}

\section{Study of bone minerals in women with Systemic lupus Erythematosus}

Siraj Ahmed Khan, D. Shiva Krishna, I. Krishna Mohan, N.N.Sreedevi, M.Vijaya Bhaskar, KSS Saibaba

Nizam's Institute of Medical Sciences 
INTRODUCTION: Systemic lupus erythematosus (SLE) is a chronic inflammatory disease of unknown aetiology predominantly affecting women in their reproductive years. Many patients with SLE have to be treated with systemic glucocorticoids for prolonged periods of time. Premature osteoporosis is one of the long term complications of Systemic Lupus Erythematosis (SLE).

AIMS AND OBJECTIVES: The aim of the present study was to analyze the effect of Lupus on biochemical parameters serum magnesium, calcium, phosphorus, and alkaline Phosphatase.

MATERIAL AND METHODS: 30 Female patients diagnosed with SLE were selected and compared with age matched controls. Serum magnesium, calcium, phosphorus, and alkaline Phosphatase levels were estimated.

RESULT: SLE patients had significantly decreased serum levels of magnesium $(0.51 \pm 0.12$ vs $0.89 \pm 0.2$; $\mathrm{p}<0.0001) \mathrm{mmol} / \mathrm{L}$, decreased levels of calcium $(7.21 \pm 1.23$ vs $8.9 \pm 1.4 ; \mathrm{p}<0.001) \mathrm{mg} / \mathrm{dl}$, increased phosphorus levels $(6.32 \pm 1.73$ vs $4.7 \pm 1.3$; $\mathrm{p}<0.001) \mathrm{mg} /$ $\mathrm{dl}$, and increased levels of alkaline phosphatase (143.3 \pm 54.9 vs $96.6 \pm 41.2 ; \mathrm{p}<0.01) \quad \mathrm{U} / \mathrm{L}$ respectively when compared to controls.

CONCLUSION: SLE patients develop bone loss due to hypothalamo-pituitary gonadal dysfunction, corticosteroid therapy and ovarian dysfunction (SLE specific). This results in variation in serum biochemical parameters like magnesium, calcium, phosphorous and ALP. Our results showed that the patients affected by Lupus showed significant Hypomagnesaemia, Hypocalcaemia, Hyperphosphatemia and high ALP. 


\section{P1}

\section{A Study of Non HDL-C And Ceruloplasmin In Type 2 Diabetes}

\author{
Manzura Rustum Mulani, Veena S. Hatolkar \\ MGM medical college, Aurangabad, Maharastra
}

INTRODUCTION: Cardiovascular disease (CVD) is the primary cause of morbidity and mortality in patients with diabetes and have approximately 2-4 times higher CVD rate than adult without diabetes. A standard traditional lipid profile consists of total cholesterol (TC) , LDL-C \& triglyceride (TG). Although levels of LDL$\mathrm{C}$ are the primary focus of dyslipidemia screening in diabetic people but relying on LDL-C targets alone can be misleading in such patients. Since, they produce highly atherogenic VLDL \& IDL lipoproteins as well as small dense atherogenic LDL particles, inspite of normal LDL$\mathrm{C}$ values. So, residual risk for such events persistently elevated atherogenic particles concentration which has given rise to the debate as to whether non HDL-C \&/or apo B should supplant LDL-C.Non HDL-C might be a useful key standard modified risk factor and better predictor of atherosclerosis in $\mathrm{T} 2$ diabetic patients. Ceruloplasmin $(\mathrm{Cp})$ is a member of inflammation sensitive plasma proteins (ISPs) family that is used in clinical practice to measure the degree of inflammation.

MATERIALS AND METHODS:-All these biochemical parameters estimated on fully auto analyzers (Erba mannheim EM200)

AIMS AND OBJECTIVES:- This study was designed to evaluate the $\mathrm{Cp}$, Non HDL-C, TC and HDL-C as CVD risk markers in T2DM.

RESULTS:-Lipid parameters were significantly different between diabetic and non diabetics subjects. There was increased Non HDL-C, TC \& Cp in DM subjects, HDL-C was significantly decreased in T2DM patients when compared to controls. $(\mathrm{P}<0.05)$

CONCLUSION :- Dyslipidemia and inflammation may prevent the development and progression of atherosclerotic vascular complications in T2DM.

\section{P2}

Exploring Functional Implications of Altered Mitochondrial DNA Copy Number In Pediatric Acute Lymphoblastic Leukemia

Ayushi Jain ${ }^{1}$, Sameer Bakshi², Jayanth Kumar' ${ }^{1}$, Shilpi Chaudhary $^{2}$, Archna Singh ${ }^{1}$

1. Department of Biochemistry, All India Institute of Medical Sciences, New Delhi

2. Department of Medical Oncology, B.R.A.I.R.C.H, All India Institute of Medical Sciences, New Delhi, India.

All India Institute of Medical Sciences, Ansari Nagar, New Delhi.

INTRODUCTION: Changes in mitochondrial DNA (mtDNA) have been identified in various tumors and have been evaluated for prognostic significance. Studies in pediatric ALL have shown that copy number changes might be due to mtDNA mutations or loss of control of mitochondrial replication. The aim of this study was to understand the cause of the change in copy numbers and the resulting effects on the metabolism and activity of mitochondrial electron-chain complexes (ETC).

MATERIALS AND METHODS: Bone marrow aspirates from 60 pediatric ALL subjects were obtained after informed consent. Total cellular DNA extraction and RNA isolation were done on the lymphoblast cell pellets. Real-time PCR using mitochondrial genome specific primers was done to estimate mtDNA copy number and mitochondrial DNA deletion ratio, using beta-actin as the reference gene. Real-time PCR was performed to assess the gene expression of regulators of mitochondrial replication and transcription (TFAM, PolG). Flow cytometry using mitochondrial membrane potential specific dye, JC-1 was done to differentiate between polarized and depolarized membrane potential in patient samples. ELISA plate-well assays were carried out on the cell pellets, to calculate the enzymatic activities of ETC of the mitochondria.

RESULTS: The mean age of subjects was 9.5 years. The median mtDNA copy number (mtDNA copies/copy of beta-actin) was 287.8, which was significantly higher than that in the controls $(90.22)$ ( $p$ value $<0.0001)$. 
TFAM and PolG gene expressions were significantly higher than that in controls ( $\mathrm{p}$ value $=0.0021$ and 0.0157 , respectively). There were variable percentages of polarized and depolarized membrane potentials in the patients. No difference was observed between the enzymatic activities of Complexes I and V between patients and controls.

CONCLUSION: ALL patients show a significant positive correlation between mtDNA copy numbers and gene expression of the regulatory nucleus-encoding genes. This needs to be further validated by analyzing the sequences of mitochondrial D-loop region.

KEY WORDS: Leukemia, mitochondrial DNA copy number, complex activity, metabolism, flow cytometry.

\section{P3}

\section{The Nexus Between C-Myc And Igf2bp1 In B-Acute Lymphoblastic Leukemia}

Gunjan Sharma $^{1}$, Elza Boby ${ }^{1}$, Ayushi Jain ${ }^{1}$, Parthaprasad Chattopadhyay ${ }^{1}$, Anita Chopra ${ }^{2}$, Sameer Bakhshi $^{3}$, Jayanth Kumar Palanichamy ${ }^{1}$

1. Department of Biochemistry,

2. Lab Oncology and

3. Medical Oncology, All India Institute of Medical Sciences, New Delhi, India

INTRODUCTION:Acute lymphoblastic leukemia is the most common childhood cancer.Around $85 \%$ of ALL is of B-cell origin. We have identified IGF2BP1 (Insulin like Growth Factor 2 mRNA Binding Protein 1), an oncofetal protein to be overexpressed in ETV6-RUNX1 translocation positive B-ALL compared to other translocations.IGF2BP1 has been shown to bind to the 3'UTR of oncogenic mRNAs (C-MYC and CD44) in epithelial cancers. We have tried to characterize whether $\mathrm{C}-\mathrm{MYC}$ is an IGF2BP1 target in B-ALL.

AIM AND OBJECTIVES:To decipher the regulation of C-MYC by IGF2BP1in B-ALL.

Comparison of C-MYC expression in ETV6-RUNX1 positive and negative B-ALL patient samples.
Evaluating C-MYC expression after IGF2BP1 overexpression.

MATERIALS AND METHODS:RNA isolation from bone marrow samples done and $1000 \mathrm{ng}$ of DNAse treated RNA used for cDNA synthesis. IGF2BP1 was PCR amplified from ETV6-RUNX1 positive (Reh) cell line, cloned into a pHAGE6 basedexpression plasmid and overexpression confirmed by real time PCR and western blotting. C-MYC expression at different doses of IGF2BP1 vector determined using PPIA and RNA Pol II as reference genes.

RESULTS:C-MYC was significantly downregulated (pvalue $=0.023)$ in ETV6-RUNX1 positive B-ALL compared to translocation negative patients and negatively correlates with IGF2BP1expression. IGF2BP1 overexpression in $293 \mathrm{~T}$ cell line correlated negatively with C-MYC levels in a dose dependent manner.

CONCLUSION:Further work needs to be done to elucidate whether C-MYC is a true target of IGF2BP1 in ETV6-RUNX1 translocation positive B-ALL and if so, does IGF2BP1 have a role to play in regulating C-MYC expression?

KEY WORDS: RNA binding protein, ETV6-RUNX1 translocation, C-MYC, IGF2BP1.

\section{P4}

Evaluation of Secretory Galectin-9 In Uncontrolled Type-2 Diabetes Mellitus

Rakesh Mudaraddi*, Hrudya Perumalreddy*, Praveenkumar Shetty*, Mahesh Bennikal*, Sudheendra Gupta*, Manjunath R*

*SDM College of Medical Sciences \& Hospital, Sattur, Dharwad, Karnataka, India-580009

INTRODUCTION: Glucose is required for the production of energy in all organisms and homeostasis of it is essentially done by glucose transporter (GLUT). GLUT- 2 is expressed by pancreatic $\beta$ cells on its surface as a glucose sensor molecule for appropriate insulin secretion and Galectin-9 is associated with GLUT-2 in 
pancreatic $\beta$ cells and is involved in glucose transport activity.

AIMS AND OBJECTIVES: The main aim of this study is to analyze and compare levels of urinary Galectin-9 in type-2 diabetics with and without nephropathy and in healthy controls.

MATERIALS AND METHODS: In this study, we included 82 subjects and are divided into 3 groups as normal healthy controls, type-2 diabetics without nephropathy and type- 2 diabetics with nephropathy. We have estimated Blood Sugar, Glycosylated Hemoglobin (HbA1C), Serum Urea, Serum Creatinine, Urinary Microalbumin and Galectin-9.

RESULTS \& CONCLUSION: In our study we found Urinary Galectin-9 levels among three groups as 5.63pg/ $\mathrm{ml}, 3.0 \mathrm{pg} / \mathrm{ml}$ and $41.8 \mathrm{pg} / \mathrm{ml}$ respectively. In patients with microabluminuria and nephropathy the urinary albumin excretion was statistically significant. In proper homeostasis of glucose, stable expression of GLUT-2 is required and this is mediated by binding of Galectin-9. Progression of diabetes with renal complication reveals that increase in the urinary excretion of Galectin-9 and albumin through glomerular basement membrane.

In this study we observed, increased urinary Galectin-9 and albumin excretion in type- 2 diabetic nephropathy patients could be related to the micro and macro vascular complications leading to chronic kidney disease.

\section{P5}

\section{Association of Biochemical Parameters and Myeloperoxidase In Chronic Kidney Disease Patients with and Without Diabetes}

Samudrala Sangeeta ${ }^{1}$, Jeevan Ambekar ${ }^{2}$, Tunguthurthi Sudhakar, ${ }^{3}$ Nilima Dongre ${ }^{4}$

1. Ph.D scholar, Department of Biochemistry, BLDE University, Vijayapur,

2. Professor, Department of Biochemistry, BLDE University, Vijayapur,

3. Professor of Biochemistry, CAIMS, Karimnagar,

4. Associate Professor, Department of Biochemistry, BLDE University, Vijayapur.
INTRODUCTION: Chronic kidney disease (CKD) is a worldwide public health problem. It is the common complication of type 2 diabetes mellitus of long duration of more than 5years. It is characterized by the accumulation and/or deficit of various substances in the human body. Patients with CKD are exposed to increased risk of developing oxidative stress due to metabolic disorders, immune deficiency and persistent inflammation. Myeloperoxidase is a heme-containing enzyme found in mammalian neutrophils. MPO may participate as one of the mediators of oxidative modification of biomolecules/tissues and contribute to the development of co-morbidities and complications in patients with CKD.

AIMS AND OBJECTIVES: The present study is undertaken to assess the role of Myeloperoxidase in chronic kidney disease in diabetic patients in and around Karimnagar area.

MATERIALS AND METHODS: A total of 50 healthy normal subjects, 50 Non diabetics with chronic kidney disease and 50 Diabetics with chronic kidney disease were enrolled in the study. Myeloperoxidase, HbA1c, Urea, Creatinine and Albumin Creatinine ratio were estimated in the blood and urine samples.

Thus obtained data was subjected to ANOVA by SPSS statistical package version 20 . ' $p$ ' value of $<0.05$ was considered as statistically significant.

RESULTS: The results of the three groups are expressed as Mean \pm SD. MPO is found to be statistically decreased in CKD patients with type 2 diabetes mellitus compared with other two groups. Myeloperoxidase levels are compared with Urea, Creatinine and eGFR levels in serum. eGFR levels correlated positively with MPO levels.

CONCLUSION: This study found that decreased serum MPO can be used as an indicator for chronic kidney disease in diabetic patients. MPO levels decline steadily as CKD progresses, possibly due to the inhibitory effect of uraemic toxins on the enzyme.

KEYWORDS: Myeloperoxidase, Chronic Kidney Disease, Diabetic Patients, serum creatinine, eGFR. 
P6

Glycosylated Hemoglobin (HbA1c) correlation with severity of Coronary artery disease in stable angina patients : A Hospital based study

Deepak Uppunda $^{1}$, Ranjan Shetty $\mathrm{K}^{1}$, Pragna Rao ${ }^{2}$

Kasturba Medical College, Manipal. Manipal

University, Karnataka -576104

INTRODUCTION: Glycosylated hemoglobin A1C (HbA1c) has been widely recognized as a marker for predicting theseverity of diabetes mellitus (DM) and several cardiovascular diseases. However, whether HbA1c could predict the severity and clinical outcomes in patients with stable coronary artery disease (CAD) remains largely unknown. We determine relationship of HbA1c with severity and outcome in patients with stable CAD.

METHODS: We enrolled 144 patients with stable angina who underwent coronary angiography. The patients were classified into three groups by tertiles of baseline HbA1c level (low group <5.6\%, $\mathrm{n}=53$; intermediate group $5.6-6.2 \%, \mathrm{n}=62$; high group $>6.2 \%$, $\mathrm{n}=29$ ). The relationships between the plasma HbA1c and severity of CAD and early clinical outcomes were evaluated.

RESULTS: The subjects who are prediabetic have 1.77 times more odds of having coronary artery disease than the patiets who are normal OR $(95 \% \mathrm{CI})=3.13$ $(1.20,8.16), P=0.13$. Patients who are diabetic have 3.13 times more odds of havind CAD than the patients who are normal OR $(95 \% \mathrm{CI})=3.13(1.20,8.16)$, $\mathrm{P}=0.02 *$.High HbA1c was associated with higher severity of coronary artery disease.

CONCLUSIONS:From this study we concluded that there is a significant correlation exist between high level of baseline $\mathrm{HbA1c}$ and severity of coronary artery disease in patients with stable angina.appeared and $\mathrm{HbAlc}$ is a somple and reliable measure to predict the severity of CAD.

KEYWORDS: Hemoglobin A1c, Stable angina pectoris, Coronary artery disease, Outcome
P7

\section{Omentin-1 And Il-6 Play Role In Inflammatory Mechanism Of Diabetic Nephropathy In Type 2 Diabetes Mellitus Patients}

$\underline{\text { Md. Yasir }}^{1}$, Senthilkumar G $\mathrm{P}^{1}$, Anithalekshmi M S ${ }^{1}$, Parameswaran $\mathrm{S}^{2}$

1. Department of Biochemistry, Jawaharlal Institute of Postgraduate Medical Education and Research, Puducherry, India

2. Department of Nephrology, Jawaharlal Institute of Postgraduate Medical Education and Research, Puducherry, India

INTRODUCTION: Diabetic nephropathy (DN) is one of the major chronic vascular complication of T2DM and leading cause of end-stage renal disease. Inflammation is one of the proposed pathway which leads to microvascular complications in T2DM but exact mechanism is still unclear. Omentin-1 is an antiin?ammatory adipokine, predominantly expressed in stromal vascular cells of visceral adipose tissue. It promotes insulin signaling. IL-6 is a multifunctional cytokine having role in immune and inflammatory responses. The present study was conducted to elucidate the role of omentin-1 and IL-6 in the pathogenesis of $\mathrm{DN}$ and its association with insulin resistance. We aimed to assess and compare the serum levels of omentin-1 and IL-6 in T2DM patients with and without DN.

MATERIAL AND METHODS: Our study comprised of 2 groups of 41 each. Group A (controls) included T2DM without nephropathy patients and group B (cases) included T2DM nephropathy patients. Parameters studied were serum omentin-1, insulin, IL-6, fasting blood glucose, urea, creatinine, lipid profile, HOMAIR, eGFR and BMI.

RESULTS: Omentin-1 ( $\mathrm{p}=0.03)$ was significantly decreased;Concomitantly, significant increase in levels of insulin ( $\mathrm{p}=0.004)$, IL-6 ( $\mathrm{p}=0.023)$ and HOMA-IR $(\mathrm{p}=0.0004)$ were found in cases compared to controls. Bivariate analysis showedeGFRcorrelating positively with omentin-1 and negatively with insulin and IL-6 in the study population. 
CONCLUSION: Our study suggests omentin- 1 and IL6 having important role in pathogenesis of diabetic nephropathy, throughinflammatory mechanism and insulin resistance, in type 2 diabetes mellitus patients.

KEY WORDS: Diabetic nephropathy;Omentin-1;IL6;Insulin resistance; Inflammation

\section{P 8}

Urinary Albumin Creatinine Ratio and Lipid Profile as A Predictor of Severity of Pre-Eclampsia

Gishu Sweta, R.R.Sinha

Department of Biochemistry,

Nalanda Medical College and Hospital,

Patna, Bihar.

INTRODUCTION: Pre-eclampsia in pregnancy is a common disease. The incidence of pre-eclampsia in India ranges from $5-15 \%$. It is the major cause of low birth weight, fetal growth restriction, preterm delivery of the child and morbidity and mortality both in mother and child. Proteinuria is a major component of pre-eclampsia. Since the collection of urine for 24 hourperiod is cumbersome and delays diagnosis, the albumincreatinine ratio (ACR) is used to measure proteinuria. Many of the postulated pathophysiology of preeclampsia, one of the pathophysiology includes dyslipidemia due to endothelial dysfunction. There is a positive correlation between dyslipidemia and systolic blood pressure in pre-eclampsia cases.

MATERIAL AND METHOD: 50 patients diagnosed as having pre-eclampsia with age between $18-35 \mathrm{yrs}$ and 50 controls with similar age group are studied at Nalanda Medical College, Patna in Bihar after taking consent. Urine samples analysed for microalbumin and creatinine and serum samples are analysed for total cholesterol,triglyceride HDL cholesterol, LDL cholesterol and VLDL.

RESULT: Itis found that there is asignificant increase in thelevel of urinary albumin-creatinine ratio (ACR) in case of pre-eclampsia in comparison to normal pregnant women. Dyslipidemia in the form of significantly increased triglyceride and VLDL concentration and slightly increased total cholesterol and LDL concentration is conspicuously evident in thesubject of pre-eclampsia as compared to normotensive pregnant women.

CONCLUSION:It can be concluded that the spot ACR can be a simple, convenient, accurate and early indicator of significant proteinuria in women with pre-eclampsia. Dyslipidemia is significantly evident in pre-eclampsia and plays an important pathological role.

KEYWORDS: Pre-eclampsia, ACR, lipid profile.

P9

\section{Evaluation of RWbL2-Based Antibody Assays for The Diagnosis And Surveillance of Lymphatic Filariasis In Endemic Pockets}

Suman Kumar Singh, Shivprasad BV, Vishal Khatri, Nitin Amdare, Priyanka Bhoj, Namdev Togre, Kalyan Goswami

Department of Biochemistry and JBTDRC, Mahatma Gandhi Institute of Medical Sciences, Sevagram, Wardha- 442102

INTRODUCTION:The Global Programme to Eliminate Lymphatic Filariasis (GPELF) has emphasized the need for tools that can be used to monitor progress toward programmatic endpointsas well as to conduct surveillance to detect any potential resumption of transmission. The methods involves microscopic examination of thick night blood smears for microfilaria (mf) and immuno-diagnostic tests to detect the circulating filarial antigens or antibodies to assess and monitor the filarial infection.

AIM: To evaluate rWbL2 based IgG4 antibody assays (WbL2-ELISAand WbL2-ICT) in the diagnosis of lymphatic filariasis.

MATERIALS AND METHODS: The recombinant antigens (rWbL2) were prepared using the Escherichia coli (E. coli)-based expression system. The rWbL2 based IgG4 antibody assays (ELISAandICT)were used to screen the active filarial cases. The required samples were 
collectedrandomly from different endemic zones in India.

RESULT AND CONCLUSION:The rWbL2 ELISA showed100\% sensitivity while specificity was found to be $71.99 \%$. Also, when cases were analysed by rWbL2ICT showed $97.87 \%$ sensitivity and $82.81 \%$ specificity. With the help of above results it can be substantiatedthat these both tests can be used as basic tools which may facilitate detection of active filariasis cases.

\section{P10}

\section{Standardization of Solid-Phase Radioassay Using Magnetic Particles for the Detection of Anti-Tpo Autoantibodies}

Chandrakala Gholve*, Savita Kulkarni, Sharmila Banerjee Chandrakal Gholve

Radiation Medicine Centre, BARC, TMH Annexe, Parel, Mumbai- 400012

INTRODUCTION: Three important antigens involved in thyroid autoimmunity are thyroglobulin ( $\mathrm{Tg}$ ), thyroid stimulating hormone receptor (TSH-R) and thyroid peroxidase (TPO). Anti-TPO autoantibodies are found in most of the patients with autoimmune thyroid disorders. Anti-TPO is present in approximately $90 \%$ of Hashimoto's thyroiditis, $75 \%$ of Graves's disease, 10 $20 \%$ of nodular goiter or thyroid carcinoma and $10-15 \%$ of normal individuals. Different methods such as immunofluorescence of tissue-sections, passive hemagglutination, RIA and ELISA have been used for the detection of anti-TPO autoantibodies. At RMC, annually we assay approximately 1500 serum samples for anti-TPO using commercial kits in the patients with thyroid disorders. Our aim was to develop 'in-house' radioassay using magnetic particles coupled to recombinant Protein A, as an import substitute for the commercial anti-TPO kit (Immunotech, France); for the routine measurement of anti-TPO autoantibodies.

MATERIALS AND METHODS:In-house anti-TPO radioassay was optimized by studying the results of varying concentrations of magnetic particles, protein A for coupling, sample volume and 125I-TPO tracer. Various other assay parameters were also optimized and validated. Assay performance was evaluated by studying maximum binding, non-specific binding, reproducibility and analytical sensitivity of the assay.

RESULTS: The standardized in-house anti-TPO radioassay has \%Bmax of $25-30 \%, \mathrm{NSB}<0.4 \%$ and was comparable with the commercial kit. A very significant correlation $(\mathrm{r}=0.71, \mathrm{p}<0.001, \mathrm{n}=114)$ was observed between in-house developed anti-TPO assay and the commercial kit for anti-TPO values in serum samples of patients under study.

CONCLUSIONS: In conclusion, the in-house developed radioassay is cost-effective, sensitive, precise and user-friendly; and hence, can be used routinely for the detection of anti-TPO autoantibodies.

KEY WORDS: Analytical sensitivity, anti-TPO autoantibodies, autoimmune thyroid disorders, radioassay, recombinant Protein A.

\section{P11}

\section{Elevated Serum Ceruloplasmin Level in Patients of Acute Myocardial Infarction (AMI).}

$\underline{\text { Ravi Kant Sharma }}^{1}$, Purnima Dey Sarkar ${ }^{2}$, Sangita Paneri ${ }^{3}$, Rajeev Lohokare ${ }^{4}$, Teena Agrawal ${ }^{5}$, Rohit Manyal. ${ }^{6}$

\section{M.G.M. Medical College \&}

M. Y. Hospital, Indore, M.P.

INTRODUCTION:Myocardial infarction (MI) is still one of the major causes of morbidity and mortality in India. Ceruloplasmin is an important extracellular antioxidant. Ceruloplasmin being an acute phase reactant protein, its level rises immediately after cellular damage in AMI. The increased risk has been attributed to the prooxidant function of ceruloplasmin.

AIMS AND OBJECTIVES: To assess serum ceruloplasmin levels and to determine its role in patients of acute myocardial infarction (AMI).

MATERIALS AND METHODS: The study was carried out at Department of Biochemistry, M.G.M. Medical College, Indore (M.P.) after approval from the Ethical Committee of the institute. The study group 
consists of 50 diagnosed patients of acute myocardial infarction and 50 age and sex matched apparently healthy controls.Samples were analysed on fully automated biochemical analyser. The data expressed in terms of mean $\pm \mathrm{SD}$ and for comparative analysis $\mathrm{p}$ values were calculated.

RESULTS: The mean levels of serum ceruloplasmin were $75.31 \pm 9.61 \mathrm{mg} / \mathrm{dl}$ in cases and $43.2 \pm 8.91 \mathrm{mg} / \mathrm{dl}$ in controls respectively. So in the present study, ceruloplasmin levels were found to be highly significant $(p<0.001)$ in patients of Acute myocardial infarction when compared to controls.

CONCLUSION:Serum Ceruloplasmin levels were significantly higher in patients of AMI than those of control group. Therefore serum Ceruloplasmin may be considered as biochemical risk factor for AMI.

KEY WORDS: Acute Myocardial Infarction, Ceruloplasmin.

\section{P12}

\section{Lead Induces Oxidative Stress and Cognitive Impairment By Interrupting Antioxidant Defense System and Neurotransmitter Level in Children}

\section{Pushkar Singh Rawat ${ }^{1}$, Mohd Zahid ${ }^{1}$, SudhirMehrotra $^{1}$ \\ 1. Department of Biochemistry, Lucknow University, Lucknow}

INTRODUCTION: Lead is one of the oldest element, naturally it occurs from earth's soil and it come in our body through various medium. Potentially Lead induces oxidative stress which causes broad range of physiological, biochemical, and behavioral dysfunctions and damage major organs such as the kidneys and brain. One to six year children are more vulnerable for lead poisoning because they put their hands and toys in their mouth. Lead-based paint is not only source of lead inside homes but also found in common house hold items like pottery, home medical remedies, cosmetics, imported food products and candies, cans with lead-soldered seams, toys, mini-blinds and other vinyl products.
Low lead exposure from childhood causes various adverse effects such as cognitive deficits, neurotoxicity, behavior disorders, and slowed growth, reduced heme synthesis, and hearing impairment.

MATERIAL AND METHODS: In the present study we have done screening in 100 samples on the basis of lead levels. $2 \mathrm{ml}$ venous blood collected in plain and 3 $\mathrm{ml}$ collected in EDTA vials. Oxidative stress was determined by measuring the levels of catalase, superoxide dismutase, oxidized glutathione and reduced glutathione, in lysate and lipid peroxides and dopaminergic neurotransmitters in plasma.

RESULTS: The blood lead levels and antioxidant status in the occupationally exposed workers children in comparison to controls was observed. There was significant variation in the level of ?-aminolevulinic acid dehydratase and the level of zinc protoporphyrin in children compared to controls. These are biomarkers of lead toxicity. Lead might interfere with catecholaminergic and predominantly dopaminergic neurotransmission by interfering the activity associated with $\mathrm{Ca} 2+$-dependent release of acetylcholine, dopamine and amino acid neurotransmitters. Lead exposed children showed a variable changes in the activity of catalase and superoxide dismutase (SOD) compared to controls. Lipid peroxidation (LPO) was monitored by measuring thiobarbituric acid reactive substances (TBARS) that were expressed in terms of malondialdehyde (MDA) equivalents. MDA concentration (plasma) increased in lead exposed children than in controls. Significant changes were also observed in reduced and oxidized glutathione levels.

CONCLUSION: The strong relationship between blood lead levels and oxidative stress markers indicate that oxidative stress should be considered in the pathogenesis of lead toxicity among children with environmental exposure to lead, increase blood lead level resulted in decrease in hemoglobin, anemia in exposed children and alteration in neurotransmitters levels which resulted in cognition impairment in children exposed to lead.

KEY WORDS: $\delta$-aminolevulinic acid dehydratase, zinc protoporphyrin, thiobarbituric acid, cognitive deficits, malondialdehyde. 


\section{P13}

Association of Microalbuminuria And Peripheral Neuropathy In Type 2 Diabetic North Indian Population

Devaansh Syngle $^{1}$, Nidhi Garg ${ }^{2}$, Sudeep Kaur ${ }^{3}$

1. SGRR Institute of Medical \& Health Sciences, Dehra Doon;

2. Chitkara College of Pharmacy, Rajpura;

3. Cardio-Rheuma \& Healing Touch City Clinic, Chandigarh

INTRODUCTION : Diabetes mellitus is characterized by metabolic abnormalities and associated microvascular and macrovascular complications1. Microalbuminuria is a powerful predictor for the future development of vascular complications in diabetes 2 . However, less information is available about the role microalbuminuria in Type 2 diabetes.

We investigated the relationship between microalbuminuria and diabetic neuropathy in Type 2 diabeticNorth Indian population.

MATERIALS AND METHODS : In this crosssectional study, we recruited 45 consecutive type 2 diabetes patients ( 17 males and 28 females) meeting the IDF criteria for diagnosis of diabetes and 30 (6 male, 24 female)age- and sex-matched healthy controls.Detailed clinical history was taken followed by a physical examination that included neurological assessment with the help of Vibration Perception Thresholdmeasured by FDA approved Biothesiometer (Bio-Medical Instrument Company, Newbury, Ohio, UnitedStates)in the study subjects. Micral test,using standard commercial kits, was used for estimation of microalbuminuria.

RESULTS : Among 45 diabetic patients $62 \%$ were males and $38 \%$ females. The mean age was $58 \pm 1.64$ years. The mean duration of diabetes was $9.64 \pm 0.82$ years. The mean $\mathrm{HbA} 1 \mathrm{C}$ was $7.87 \pm 0.23 \%$ and mean microalbuminuria was $49.31 \pm 13.22 \mathrm{mg} / \mathrm{l}$. Type 2 diabetic patients had significantly increased $\mathrm{HbA} 1 \mathrm{c}$ $(7.87 \pm 0.23 \%$ vs. $5.10 \pm 0.18 \%)$, elevated microalbuminuria $(49.31 \pm 13.22 \mathrm{mg} / \mathrm{l}$ vs. $20.45 \pm 7.11$ $\mathrm{mg} / \mathrm{l})$ and impaired VPT $(19.54 \pm 1.66$ vs. $12.20 \pm 1.21$ microns) as compared to healthy controls, respectively $(\mathrm{p}<0.01)$. A positive correlation was found between microalbuminuria and peripheral neuropathy $(\mathrm{r}=0.49$, $\mathrm{p}=0.01$ ).

CONCLUSION : The occurrenceofmicroalbuminuria in type 2 diabetes is significantly associated with peripheral neuropathy which suggests that microalbuminuria is a powerful predictor for the future development and detection of microvascular complications in type 2 diabetes.

KEY WORDS: Microalbuminuria, Peripheral neuropathy, Microvascular complications, Type 2 diabetes.

\section{P14}

Comparative Study of Serum Uric Acid, Serum Calcium and CRP In Preeclamptic Women and Normal Pregnant Women.

Trushna Shah, Drashti Brahmbhatt

Sumandeep Vidhyapeeth, SBKS MI \& RC, Pipariya, Vadodara, Gujarat.

INTRODUCTION:Preeclampsia is one of the major conditions causing maternal morbidity and mortality throughout the world. Hyperuricemia due to oxidative stress is known to be associated with deleterious effects on endothelial function, oxidative metabolism, platelet adhesiveness and aggregation. $\mathrm{C}$-reactive protein (CRP) is an acute phase reactant protein, plays a role in eliciting the inflammatory response characteristic of preeclampsia. Patients with Preeclampsia showed the low levels of serum calcium.

MATERIAL \& METHODS: A case control study conducted on 30 patients with clinically diagnosed preeclampsia and 30 normotensive singleton pregnant women as controls from the general population. $3 \mathrm{ml}$ of venous blood was collected to estimate the Serum Uric Acid, CRP and Serum Calcium levels in each subject. The data was analyzed and expressed in terms of mean \pm SD. The study was done in The Dhiraj General Hospital Pipariya,Vadodara, and Gujarat. 
RESULT: Present study showed statistically significant increase in the levels of serum uric acid, CRP and decreased levels of serum calcium in the Preeclamptic women compared to non Preeclamptic pregnant women.

CONCLUSION:Serum uric acid and CRP levels were significantly high and serum calcium levels were significantly reduced in case. Thus, it can be concluded that serum uric acid, CRP and serum calcium could be considered as a supportive diagnostic tool in preeclampsia along with conventional markers.

KEYWORDS: Preeclampsia; Serum Uric Acid; CRP; Serum Calcium; Oxidative stress; Endothelial injury.

\section{P15}

Role of Homocysteine Metabolism and Genotype Factor V Leiden (FVL) G1691a and Their Correlation With Biochemical Parameters In Patients of Cerebrovascular Stroke

Pramod Sudam Kamble ${ }^{1}$, Mangesh P Bankar², Shreerang P Kulkarni1, P D Zende3w

1. SDM College of Medical Sciences \& Hospital, Sattur, Dharwad, Karnataka, India-580009

2. B J Government Medical College, Pune, Maharashtra, India

3. PSM Institute of Medical Sciences \& Hospital, Islampur, Maharashtra, India

INTRODUCTION:Homocysteine is a nonprotein sulphur containing amino acid formed during methionine metabolism. Elevated total plasma homocysteine levels causes moderate increase in the risk of heart disease and cerebrovascular stroke.Factor Vplaysan important role in the blood coagulation cascade, which take part in the conversion of prothrombin to thrombin with factorXa to formtheprothrombinasecomplex by activated proteinC.Hyper homocysteinemia may trigger single point mutation in FVL which further disturb procoagulant mechanism.

AIMS AND OBJECTIVES: To study the effectof vitamin B12, Folic Acid \& Homocysteine on FVLG1691A genotype among individuals suffering from cerebrovascular stroke.
MATERIAL AND METHODS:The total 50 stroke patients and 50 age and gender matched healthy individuals ervedas controls were selected in the present study. Genotype for FVLG1691A for all above subject sand controls were determined by ARMS-PCR.

RESULTS AND CONCLUSION: Median plasma total homocysteine level in stroke patients was significantly higher $(p<0.0001)$ than controls. The value of serum folic acid in stroke patients was significantly lower $(\mathrm{p}<0.05)$ where as the level of vitamin B12 weredecreased in stroke patients, but not significant when compared with controls. We found that the effect of low dietary intake of vitamin B12\&Folic Acid; genetic factors and hyperhomocysteinemia may be the plausible cause for endothelial cell injury in turn increases risk of cerebrovascular stroke and heart disease.

\section{P16}

\section{Regulation of BRCA1 Mesenger RNA Stability In Sporadic Breast Cancer}

Anil Bapu Bargale ${ }^{1,2}$, Jayaram Shetty $\mathrm{K}^{2}$, PraveenKumar Shetty ${ }^{1}$, Suchetha Kumari N ${ }^{2}$, R D Kulkarni ${ }^{1}$

1. SDM College of Medical Sciences \& Hospital, Sattur, Dharwad, Karnataka, India-580009

2. K S Hegde Medical Acadamy, NITTE University, Deralakatte, Mangalore, Karnataka, India-575018

INTRODUCTION: BRCA1 is a nuclear phosphoprotein involved in genome integrity by regulating cell cycle checkpoints, DNA repairand apoptosis. BRCA1 down regulation occurs in sporadic breast cancer (BC). Post-transcriptional regulation of gene expression hasevolved as a means of fine-tuning of protein levels. Regulation of the dynamics of mRNA molecules is a wide spread phenomenon and there are many tumor suppressor genes regulated by this manner. There are several post-transcriptionalregulatory motifs, including CU-rich, U-richand AU-rich elements, which are usually located in the 5' and 3' UTR's. Posttranscriptional regulation ofBRCA1 is poorly characterized and under appreciated. 
AIMS AND OBJECTIVES:To elucidate the molecular mechanism of AnxA2 mediated post-transcriptional regulation of BRCA1 in sporadic breast cancer.

MATERIAL AND METHODS: DRB chase \& RNA immuno precipitation experiments were conducted to study the BRCA1 mRNA stability in MDAMB-231 (AnxA2 overexpressing) \& MCF-7 (AnxA2 low expression) BC cell line models.

RESULTS AND CONCLUSION: In order to investigate BRCA1 mRNA levels in MDAMB231\&MCF-7 cells, we conducted DRB chase experiment. The BRCA1 mRNA destabilization is significantly increased upon AnxA2 induction in MCF7 cells. Further we knocked down AnxA2 in MDAMB231 cells we found, BRCA1 mRNA stabilization which proves AnxA2 destabilizes BRCA1 mRNA. In RIP-CHIP experiment we pulled BRCA1 mRNA by AnxA2 specific monoclonal antibody;mRNA was extracted and amplified by RT-qPCR and resolved on agarose gel; we found BRCA1 amplification band. This supports our finding that AnxA2 has role in the regulation of BRCA1 at mRNA level.In the present study, we demonstrated that AnxA2 has a role in post-transcriptional regulation of BRCA1 expression.

KEY WORDS:BRCA1, breast cancer, AnxA2, MDAMB-231, MCF-7

\section{P 17}

Relationship Between Vitamin D Insufficiency, Bone Mineral Density, Bone Metabolism In Indian Women

Seema Kadam, Sonam Hatkar, Anushree Patil, Suchitra Surve, Beena Joshi, M.I. Khatkhatay, Meena P. Desai

National Institute for Research in Reproductive Health (ICMR),J. M. Street, Parel, Mumbai 400012

INTRODUCTION: Vitamin D insufficiency is known to have adverse effects on bone metabolism and is an important risk factor for low bone mass.

AIMS AND OBJECTIVES: The aim of the study was to determine the relationship between Vitamin D insufficiency with bone mineral density, bone turnover markers in healthy Indian women.

MATERIALS AND METHODS: We determined serum levels of 25H-hydroxy vitamin D [25(OH)], estradiol (E2), bone turnover markers (BTO) Osteocalcin(OC) and C-terminal Telopeptide (CTX) by commercial ELISA kits in 200 apparently healthy Indian women aged (18-65years) who underwent BMD measurements by DXA. Calcium(Ca),Phosphorus( $\mathrm{Ph})$ were estimated on an Autoanalyzer.

RESULTS: The prevalence of Vitamin D insufficiency [25(OH)D :16-29ng/ml] was observed in $31 \%$ women $(\mathrm{n}=62,23 \pm 0.49 \mathrm{ng} / \mathrm{ml})$ while sufficient levels $(>30 \mathrm{ng} /$ $\mathrm{ml})$ were seen in $23 \%$ women $(\mathrm{n}=47,40.52 \pm 3.19 \mathrm{ng} /$ $\mathrm{ml})$. $\operatorname{VDD}(<15 \mathrm{ng} / \mathrm{ml})$ was prevalent in $46 \%$ of women with $t$ score of-3.385 \pm 0.02 . In age group 18-29 years $\operatorname{vit}[25(\mathrm{OH}) \mathrm{D}: 11.87 \pm 6.60 \mathrm{ng} / \mathrm{ml} \mathrm{p}<0.0001)$ levels were low with normal BMD,OC and CTX levels and $\mathrm{Ca}$ and $\mathrm{Ph}$ levels in the normal range. Insufficient Vit $\mathrm{D}$ levels $25(\mathrm{OH}) \mathrm{D}: 16-29 \mathrm{ng} / \mathrm{ml}$ in the normal, osteopenic and osteoporotic women $(23.10 \pm 0.96 \mathrm{ng} / \mathrm{ml}$ vs $21.59 \pm 0.79$ $\mathrm{ng} / \mathrm{ml}$ vs $18.14 \pm 0.69 \mathrm{ng} / \mathrm{ml}$; with $\mathrm{t}$ scores ranging from $0.022 \pm 0.15,-1.589 \pm 0.067$ and $-2.38 \pm 0.126: p<0.001$ resp) were observed. OC and CTX also showed an inverse relation with $\mathrm{BMD}$ and $\mathrm{E} 2$ levels in the osteoporotic postmenopausal women who showed signs of hypovitaminosis with vit[25(OH)D] :16.35 $\pm 1.79 \mathrm{ng} /$ $\mathrm{ml}$.

CONCLUSIONS: Our study shows that Vit D insufficiency in an otherwise healthy population is a common risk factor for osteoporosis associated with increased bone remodeling and low bone mass.

KEY WORDS: VitD, Bone Mineral Density, Bone Turnover Markers (BTO), Osteoporosis.

\section{P18}

Evaluation of Thr92ala Single Nucleotide Polymorphism (Snp) In Patients Having Diabetes Mellitus And Thyroid Disorders.

$\underline{\text { Smita Gawandi }}^{1}$ *, J. Kumarasamy ${ }^{1}$, S. Cherian ${ }^{2}$, 
Sharmila Banerjee ${ }^{1}$, Savita Kulkarni ${ }^{1}$

1. Radiation Medicine Centre, BARC, Mumbai, 2BARC Hospital Mumbai

INTRODUCTION: Type 2 deiodinase (DIO2) enzyme plays a key role in the peripheral T4 to T3 conversion. The SNP (rs225014), at 92 codon of the DIO2 gene i.e. Thr92Ala lowers DIO2 activity in tissues and affect cellular T3 levels. It is a predisposing factor for coexistence of thyroid disorders (TD) in Diabetes Mellitus (DM). The determination of this SNP in DM may identify the susceptible patients to develop TD. Similarly, this SNP is associated with hypothyroidism at tissue level and linked with insulin resistance (IR). Thyroid patients having IR are more prone to develop cardiovascular disease (CVD), dyslipidaemia \& DM. The Screening for DIO2 SNP in TD may help physicians to implement personalised treatment in thyroid patients.

MATERIALS AND METHODS: The study includedDiabetes Mellitus patients $(n=20)$, hypothyroid patients taking Eltroxin supplementation i.e. Hypo-Rx $(n=29)$ and thyroid cancer patients taking Eltroxin replacement therapy i.e. Thyro-Rx $(n=18)$. Thyroid function testsi.e. T4, T3, TSH and AMA were estimated by RIA/IRMA. Genomic DNA of all the subjects were screened for the Thr92Ala SNP by PCR technique.

RESULTS AND CONCLUSION: The percentage of homozygous Ala/Alamutant genotype was highest in the DM group (35\%) when compared to Hypo-Rx (17\%) and Thyro-Rx (24\%) groups. The Ala/Ala genotypes from all the patients' groups showed higher AMA positivity and lower T3/T4 ratio as compared to heterozygous genotypes. These results were suggestive of the association of this SNP with thyroid function abnormalities. It will be worthwhile to determine the relevance of DIO2 SNP with thyroid dysfunction as well as with co-existence of TD in DM in larger ethnic population.

KEY WORDS:Thr92Ala SNP, Diabetes mellitus, Thyroid disorders

\section{P19}

Hepatoprotective activity of ethanolic extract of tuber of Actinoscirpus grossus (L.f.) Goetgh. \& D.A. Simpson against paracetamol induced hepatotoxicity in Wistar albino rats.

Savin Chanthala Ganapathi ${ }^{1 *}$, Rajendra Holla ${ }^{2}$, Shivaraja Shankara ${ }^{3}$

1. *Dept of Pharmacology, KVG Medical College \& Hospital Sullia D.K, Karnataka, India, 574327, emailid-savincg@gmail.com

2. Dept of Pharmacology,KS Hegde Medical Academy, NITTE University, Deralakatte, Mangalore, Karnataka, India 575018

3. Dept of Biochemistry, KVG Medical College \& Hospital Sullia D.K, Karnataka, India, 574327

INTRODUCTION-Actinoscirpus grossus (L.f.) Goetgh.\&D.A.Simpson commonly known as Kasheruk in Indian system of Medicine, belongs to Cyperaceae family.A. grossushas been indicated for different ailments such as anti diarrheal, anti emetic. The root is slightly sweet in taste and indicated for cooling, laxative and liver disorders.

AIMS \& OBJECTIVES: Aim of this study is to evaluate the hepatoprotective activity of Actinoscirpus grossus (L.f.)Goetgh \& D.A Simpson against paracetamol induced hepatotoxicity in Wistar albino rats.

MATERIALS AND METHODS: Hepatotoxicity was induced by giving high dose of paracetamol $3 \mathrm{~g} / \mathrm{kg}$ bodyweight followed by A. grossus( $200 \mathrm{mg} / \mathrm{kg}, 400 \mathrm{mg} /$ $\mathrm{kg}$ ), and reference standard drugs (Silymarin $50 \mathrm{mg} / \mathrm{kg}$, liv52 5ml/kg). Test and reference standard drugs were administered orally for 15 consecutive days.On 15th day blood was collected in the tubes and biochemical investigation was done. All the animals were sacrificed. Liver is dissected out, cleaned to remove extraneous tissues, volume and weight of liver is noted and piece of liver tissue was preserved in $10 \%$ formalin for histopathological processing

RESULTS\&CONCLUSION: High dose of paracetamol $3 \mathrm{~g} / \mathrm{kg}$ manifested by statistically significant increase in liver marker enzymes such as serum SGOT. 
SGPT, Alkaline phosphatase, and bilirubin level. After administration of A. grossus $200 \mathrm{mg} / \mathrm{kg}$ and $400 \mathrm{mg} / \mathrm{kg}$ and reference standard drugs lowered activities of liver enzyme activities. Results obtained from histopathological studies supported the hepatoprotective activity of A. grossus against paracetamol induced hepatotoxicity. Analysis of the data generated during the study indicates paracetamol produced significant liver injury at the dose injected. Injurious effects were reversed by both reference standard and test drug.

KEY WORDS: Hepatotoxicity, A. grossus, hepatoprotective activity

\section{P20}

\section{Thyroid Dysfunction And Thyroglobulin Level} In Iodine Deficient Children of Udayapur, Nepal

$\underline{\text { Saroj Kunwar }}^{1}$, Basanta Gelal ${ }^{1}$, Kisun Deo Mehta ${ }^{1}$, Gaurishankar Shah ${ }^{2}$, Nirmal Baral ${ }^{1}$, Madhab Lamsal ${ }^{1}$

1. Department of Biochemistry, BPKIHS, Dharan

2. Department of Pediatrics and Adolescent Medicine, BPKIHS, Dharan

INTRODUCTION: Iodine deficiency is a major global public health problem in the developing countryaffecting young children. Deficiency during childhood reduces somatic growth, cognitive and motor function. Nepal lies in the endemic area of iodine deficiency due to geographical situation with $19.4 \%$ prevalence in school age children.

OBJECTIVES: This study aimed to investigate thyroid dysfunction and thyroglobulin level in iodine deficient children and to establish the relation between these factors.

MATERIAL AND METHODS: A community-based cross sectional study was conducted in schools of hilly and plain region of Udayapur district by enrolling of 1012 School age children(SAC)aged 6-14 years for assessment of urinary iodine concentration (UIC) by APDM method.Serum thyroglobulin ( $\mathrm{Tg}$ ), thyroid stimulating hormone (TSH), free triiodothyronine (fT3) and free thyroxine (fT4) were measured in iodine deficient children by collecting 83 blood samplesby commercial
ELISA kit.Data were expressed in frequency, percentage, mean \pm SD, median (IQR) and chi-square test, independent T test, Mann Whitney Test,Kruakal Wallis test were applied considering $\mathrm{p}$ value $? 0.05$ is statistically significant at $95 \%$ confident interval.

RESULTS : The prevalence of iodine deficiency in schoolchildrenwas $23.25 \%$ in hilly and $8.57 \%$ in plain area. The median UIC of hilly and plain area were 212 $\mu \mathrm{g} / \mathrm{L}$ and $244 \mu \mathrm{g} / \mathrm{L}$ respectively $(\mathrm{p}=0.029)$. The mean fT3, fT4 and TSH of iodine deficient children were $22.45 \pm 14.05 \mathrm{pg} / \mathrm{mL}, 0.96 \pm 0.28 \mathrm{ng} / \mathrm{dL}$ and $3.60 \pm 1.44$ $\mathrm{mIU} / \mathrm{L}$ respectively. The Median (IQR) serum thyroglobulin was $17.5(12,29.4) \mathrm{ng} / \mathrm{mL}$. We found $6 \%$ cases of hypothyroidism and $3.6 \%$ cases of subclinical hypothyroidism in iodine deficient children. No significant correlation was observed between serum $\mathrm{Tg}$ with fT4 $(r=0.012, p=0.916)$ and $\operatorname{TSH}(r=0.056$, $\mathrm{p}=0.615)$. There is significant positive correlation between thyroglobulin and fT3(r=0.273, $\mathrm{p}=0.013)$.

CONCLUSION : Iodine deficiency with thyroid dysfunction remains a public health problem in Udayapur district of eastern Nepal.

KEY WORDS: School Age Children, Thyroglobulin, Urinary Iodine Concentration

\section{P21}

\section{Study of Serum Ferritin In Newly Diagnosed Cases of Hypothyroidism}

Salma Ahmed, Rashmi Rajkakoti

Department of Biochemistry, Assam Medical College \& Hospital, Dibrugarh, Assam

INTRODUCTION: Ferritin is an iron storage protein found in almost all of the body tissues. Serum ferritin levels also have been reported to be altered in patients with thyroid disease. Thus changes in the serum concentration of ferritin reflect thyroid function.

AIMS \& OBJECTIVES: To study the serum levels of ferritin in newly diagnosed cases of hypothyroidism and compare with those of healthy controls. \& to assess any correlation between thyroid profile and ferritin in 
hypothyroidism.

MATERIALS \& METHODS: Ferritin levels were estimated in 30 newly diagnosed cases of hypothyroidism using immunoradiometric method. T3, T4 and free T4 were estimated using radioimmunoassay and TSH by immunoradiometric method. These were then compared with age and sex matched healthy controls. Results were correlated statistically.

RESULTS:Serum ferritin levels were found to be significantly reduced in patients with hypothyroidism compared to normal subjects $(\mathrm{p}<0.001)$.

CONCLUSION: Hypothyroidism is associated with low serum ferritin levels. The estimation of serum ferritin may help in understanding the etiopathogenesis and monitoring of hypothyroid patients.

KEYWORDS: Immunoradiometric, Ferritin, Hypothyroid

\section{P22}

\section{To Evaluate Serum Amylase Levels In Metabolic Syndrome}

Sumit Dokwal, Veena Singh Gahlaut, Manish Raj Kulshrestha, Piyush Bansal.

Pt. B D Sharma PGIMS, Rohtak.

INTRODUCTION: Few recent studies have reported low serum amylase levels to be a risk factor of metabolic syndrome (MetS) associated with worse lipid profile parameters, suggesting an endocrine-exocrine relationship in pancreas. However they also reported increasing prevalence of stroke and requirement for drug treatment for hypercholesterolemia with higher amylase levels. Other studies have reported elevated amylase levels in diabetes.

AIMS AND OBJECTIVE : To evaluate serum amylase levels in metabolic syndrome.

MATERIALAND METHOD: Study group comprised of 200 subjects (25-75 yrs) with MetS. Persons with any other chronic and acute illness, renal dysfunction and drug intake (OCP, steroids, aspirin etc.) known to influence amylase levels, persons with high urea levels and those with amylase $<30 \mathrm{IU} / \mathrm{L} \&>200 \mathrm{IU} / \mathrm{L}$ were also excluded. Control group comprised of 50 healthy controls.

RESULTS AND CONCLUSION: Amylase levels was significantly higher than in control group (71.80 $\pm 29.06 v s 59.9 \pm 21.40 \mathrm{IU} / \mathrm{L}, \mathrm{p}=0.010)$. Amylase levels positively correlated with serum triglycerides $(\mathrm{r}=0.210, \mathrm{p}=0.032)$, ALT $(\mathrm{r}=0.223, \mathrm{p}=0.023)$ and urea $(\mathrm{r}=0.209, \mathrm{p}=0.039)$ and were negatively correlated with HDL levels $(r=0.267, p=0.006)$ in the study group. The study found higher amylase levels in MetS which were correlated significantly with higher TG and lower HDL levels. Hyperinsulinemia a feature of MetS is known to increase amylase secretion. Pancreatic tissue in Type 2 DM has been reported to have inflammatory hypercellularity, loss of cell adhesion and paracrine communication, apoptosis, ECM remodeling, fibrosis in both islet (endocrine) and acinar(exocrine) tissues. This may be leading to increase release of amylase as a nonfunctional plasma protein, reflecting the slow continuous pancreatic inflammation and damage.

KEY WORDS: serum amylase, inflammation, fibrosis, HDL, acinar

\section{P23}

\section{Antinuclear antibodies status and related risk factors in non pregnant adult Indian women}

Barun Kumar Chakrabarty $^{1}$, Mukul Bajpai ${ }^{1}$, Sougat Ray $^{2}$, Manu Chopra ${ }^{3}$

\section{51 Base Hospital1, SSO Health, HQWNC \\ 2. Army Hospital R\& $R$ \\ 3. Military Hospital Namkum, Army Medical corps}

INTRODUCTION:Antinuclear antibodies (ANA) are the most commonly evaluated non specific autoantibodies in clinical practice. Previous studies showed ANAs are frequently detected in healthy individuals and expression of ANAs are more common in women and older age groups. To explain the reason of sex differences differential chromosomal, hormonal and reproductive factors are postulated, but till now the 
association is not clearly understood. There is paucity of Indian data of ANA status amongst women. In this study we examined the relationship of ANA status with socio demographic and reproductive factors in a representative sample of non pregnant adult Indian women.

AIMS AND OBJECTIVES: Estimation of ANA status prevalence in non pregnant Indian women by enzyme linked immunosorbent assay (ELISA) technique.

Effects of socio demographic and reproductive factors with ANA prevalence.

MATERIAL AND METHODS:This study was conducted in the zonal level hospital. Non pregnant adult women who had ever menstruated and voluntarily completed the socio demographic and reproductive history questionnaire were included in the study. Serum ANA screening test were performed for the detection of IgG class antibodies to ANA in humanserum by ELISA.

RESULTS: We analyzed data on socio demographic and reproductive factors in relation to serum ANA status in adult non pregnant females using SPSS statistical software. The values thus obtained statistically similarwith those obtained in developed countries.

CONCLUSION:These findings highlight the importance of considering sociodemographic and reproductive history in studies of autoimmunity in women in Indian set up.

KEYWORDS:Antinuclear antibodies (ANA), non pregnant adult women, risk factors,ELISA

\section{P24}

Fabrication of Antibody-Chip on Track-Etched Membranes For Serum Thyroglobulin Estimation.

Bharti Jain, J. Kumarasamy, C. Gholve, M.G.R. Rajan, Savita Kulkarni

Radiation Medicine Centre, BARC, TMH Annexe, Parel, Mumbai- 400012

INTRODUCTION - Antibody-chip fabrication is an important aspect of microarray immunoassay (MI) development that influences its performance with regard to sensitivity, specificity and reproducibility. We have fabricated antibody-chip on polycarbonate track-etched membranes (PC-TEM) as a novel solid support and optimized it with regard to spot homogeneity, morphology, non-specific interactions and performance of antibodies on chips. Thyroglobulin (Tg) which is primarily used as a tumor marker for residual or recurrent differentiated thyroid cancers was selected as a target of interest.

AIMS AND OBJECTIVES : Immobilization of antibodies on PC-TEM to fabricate high performance antibody-chip.

Development of non-competitive MI for human $\mathrm{Tg}$

MATERIALS AND METHODS- Antibody-chip was fabricated on PC-TEM using polyclonal antibodies raised in camel. Glutaraldehyde activated hydrophobic and hydrophilic PC-TEMs were immobilized with $0.3 \mu \mathrm{l}$ $(1 \mathrm{mg} / \mathrm{ml})$ of antibodies in both dry and humid chamber. MI was optimized using I25I-anti-Tg monoclonal antibodies. TEMs were imaged using phosphorimager and spot intensity was quantified using ImageJ analysis software. Tg MI was validated using several parameters viz. sensitivity, working range, precision and correlation to IRMA assays.

RESULTS AND CONCLUSION - Hydrophobic PCTEM immobilized with antibodies in humid chamber produced homogenous spot with good morphology and reproducibility and MI results correlated well with IRMA. MI was sensitive $(0.05 \mathrm{ng} / \mathrm{ml})$, reproducible (intra-and inter assay CV <20\%) with clinically useful working range $(0.05-250 \mathrm{ng} / \mathrm{ml})$ and showed excellent correlation to IRMA $(r=0.97, p<0.001, n=140)$. MI for $\mathrm{Tg}$ can be used for serum $\mathrm{Tg}$ measurement in a cost effective and sample economical manner. Although current work addresses thyroglobulin, the technique can be applied to estimate several analytes, especially TSH and Tg autoantibodies.

KEY WORDS: Antibody-chip, PC-TEM, Thyroglobulin 


\section{P25}

\section{Comparison Between Rapid Screening Test and Elisa for the Detection of Anti HCV Antibody}

Jay Prakash Sah, Pawan Joshi, Rudra Karki, Aasif Karim

National Path Lab and Research Centre, Butwal, Nepal

INTRODUCTION : WHO reported that approximately 170 million people are infected with HCV worldwide.

AIMS AND OBJECTIVES : To compare the performance rapid test (ICT) with 3rd generation ELISA (Gold standard) for the detection of anti-HCV antibody.

MATERIALS AND METHODS : The study comprised of 130 bloods samples, which were tested by ELISA kits for HCV. All Samples were retested by rapid test kits. The results of sample by ELISA were compared with the rapid tests. Statistical analysis was performed by using SPSS software package version 16.0. The Chisquare test was used to find the difference between two variables of the study.

RESULTS \& CONCLUSION : 100 samples were nonreactive and 30 samples were reactive by ELISA. All samples were retested by RDT, 1 sample showed reactive out of 100 and similarly, 1 sample showed non-reactive out of 30. By using ELISA technique for HCV infection our results showed $99 \%$ specificity and $96.66 \%$ sensitivity of ICT technique. Our study shows ICT results for screening were similar to ELISA due to its comparable sensitivity and specificity with ELISA. It can be recommended that rapid test (ICT) may be allowed to be used for initial screening of HCV especially, in remote areas or where cost is an issue. Further research with larger sample size and higher techniques are required to found the credibility of rapid test for their sensitivity and specificity.

KEY WORDS: ICT, ELISA, HCV.
P26

Gestational Impaired Glucose Tolerance (Gigt) Induced Suppression of Fetal Thyroid SecretionEffect on Fetal Outcome

Suganya Shanmugam ${ }^{1}$, Pooja Dhiman $^{1}$, Soundravally Rajendiren ${ }^{1}$, Archana Nimesh ${ }^{1}$, Dilip Kumar Maurya ${ }^{2}$.

1. Department of Biochemistry,

2. Department of Obstetrics \& Gynaecology, JIPMER, Puducherry-06.

INTRODUCTION: Gestational impaired glucose tolerance (GIGT), is a milder form of Gestational diabetes mellitus (GDM), which is often poorly managed. Although, GDM is known to be associated with increased incidence of thyroid dysfunction, no study has been done to study the effect of GIGT on thyroid status and its effect on fetal outcome. Here, we carried out a study to assess thyroid function and glycemic status in both maternal and cord blood of the subjects with GIGT, and to find their association with the fetal outcome

MATERIAL \& METHOD: Women who came to the hospital for safe confinement during 37-40th weeks of gestation were recruited in the study. Based on the 2 hour post prandial blood glucose levels with 75 grams OGTT, done at 24-28 weeks of gestation, all the subjects were stratified into two groups: 1) Cases or GIGT group women with blood glucose levels between120-140mg/ $\mathrm{dl}$ and 2) Controls - women with blood glucose levels of less than $120 \mathrm{mg} / \mathrm{dl}$. Three $\mathrm{ml}$ of venous blood was collected from mothers and $3 \mathrm{ml}$ of cord blood was collected during delivery. New-borns were assessed for birth weight, head circumference, abdominal circumference, thigh circumference and crown heel length.Glycated haemoglobin was carried out using immunoturbidimetry (DiaSys Diagnostic Systems $\mathrm{GmBh}$, Germany) and fructosamine was estimated using dye binding method (Biosystems, Spainn).Estimation of Total T3 (TT3), free T3 (FT3), Total T4 (TT4), free T4(FT4) and TSH was done by chemiluminescence in Siemens Advia Centaur CP, using competitive immunoassay.

RESULTS: Although within the normal reference range, GIGT Mothers had higher concentration of free and total 
T4 than controls. Cord fructosamine levels were significantly higher in babies of GIGT mothers than controls, indicating the reflection of maternal hyperglcemia. There was a positive correlation between the maternal glycated haemoglobin and cord blood fructosamine in the GIGT group. Statistically significant lower levels of total T3 and T4 with high TSH levels were found in babies with GIGT mothers, indicating the suppressive effect of maternal hyperglycemia on fetal thyroid function. Birth weight, head circumference and thigh circumference were significantly higher in babies born to mothers with GIGT, which may be a combined effect of maternal hyperglycemia and fetal thyroid suppression.

CONCLUSION: Maternal hyperglycemia, even in milder form of GIGT may cause suppression of fetal thyroid function. Both these factors may predispose to change in fetal anthropometry, leading to a large baby. Therefore, it is recommended to evaluate maternal and cord thyroid function for timely management strategies.

KEY WORDS: glucose; intolerance; pregnancy; fetal; outcome.

\section{P27}

CSF Lactate Level: A Useful Diagnostic Test To Differentiate Scrub Typhus Meningitis From Aseptic, Bacterial And Tuberculous Meningitis

\section{P. Yesudas Sudhakar}

\section{Deparment of Clinical Biochemistry, CMC, Vellore}

INTRODUCTION: Scrub typhus is an acute zoonotic febrile illness caused by the obligate intracellular bacteria Orientia tsutsugamushi. Acute meningitis is a lifethreatening infectious disease, and requires a prompt etiological diagnosis to start appropriate therapy since delays in therapy can lead to disastrous neurological sequel and death. The differentiation of scrub meningitis from other forms of meningitis (bacterial, tuberculous and viral) may be challenging as most clinical and laboratory features are often overlapping. A characteristic eschar is present only in a minority of patients. Rapid techniques such as Gram stain, CSF cell counts, CSF glucose and protein lack the sensitivity and specificity to establish an accurate diagnosis. Conclusive investigations like bacterial culture, viral PCR etc require several days to return results, rendering them futile in guiding immediate management decisions. Rapidly available biomarkers like cerebrospinal fluid (CSF) lactate have been evaluated to distinguish the various forms of meningitis before culture and PCR results are available.

The reliability of CSF lactate for differentiation of bacterial meningitis from aseptic meningitis has been established in numerous studies have been reported to be independent predictors of mortality. However, to the best of our knowledge, no data exists in the literature to examine the predictive ability of CSF lactate for scrub typhus meningitis.

AIM:To evaluate if CSF lactate is useful in differentiating scrub typhus meningitis from aseptic bacterial and tuberculous meningitis.

MATERIALS AND METHODS: A cross-sectional study was carried out at Christian Medical College Vellore.Patients were recruited for the study from August, 2014 to 2016. Patients above the age of 15 years presenting to the emergency department or General Medicine OPD with an acute febrile illness and clinically suspected to have meningitis were enrolled in the study. Thirty six cases were initially recruited for each of the scrub typhus meningitis and aseptic meningitis, but some had an alternate diagnosis and somedid not meet the diagnostic criteria were excluded.CSF specimens obtained from lumbar puncture were collected in $\mathrm{NaF}$ Vacutainer tubes and transported on ice to the lab. The lactate assay was performed within 24 hours by kinetic enzymatic test on the Beckman Coulter analyser.

RESULT: The age-specific reference range is set at 1.2$2.1 \mathrm{mmol} / \mathrm{L}$ for this study (Leen WG et al, PLoS ONE 2012). The values were lowest in aseptic meningitis (median 2.4; range 1.2-4.5 mmol/L), followed by scrub typhus (median 3.0; range 1.5-10.6 $\mathrm{mmol} / \mathrm{L}$ ), TB meningitis (median 5.7; range 1.6-13.7 $\mathrm{mmol} / \mathrm{L}$ ) and bacterial meningitis (median 6.9; range 2.1-26.5 mmol/ L). Figure 10 shows the median $( \pm \mathrm{IQR})$ lactate values for each diagnosis. The difference in CSF lactate between scrub typhus and bacterial meningitis is 
significant.

CONCLUSION: The current diagnosis of scrub typhus meningitis relies on the co-existence of clinical and laboratory features of scrub typhus infection. This prospectivestudy of patientswith acute meningitis has shown a good diagnostic accuracy of CSF lactic acid levels in differentiating scrub typhus from aseptic, bacterial and tuberculous infections. The inclusion of this rapid, reliable and inexpensive modality in the initial evaluation will provide clinically useful information to differentiate between various clinical entities. This may lead to increased confidence in diagnosing aseptic meningitis, which is likely to translate into reduced use of empirical antimicrobial therapy, shorter hospital stays and reduced morbidity/mortality in patients with scrub typhus meningitis.

KEY WORDS:Scrub typhus, Orientia tsutsugamushi, CSF, tuberculous

\section{P28}

\section{Serum Calcium and Magnesium Level In Preeclampsia}

Chaudhari RK ${ }^{1}$, Niraula $\mathrm{A}^{1}$, Bataju $\mathrm{M}^{1}$, Khan $\mathrm{SA}^{1}$, Bhatta $\mathrm{R}^{2}$, Chhetri $\mathrm{S}^{2}$, Lamsal $\mathrm{M}^{1}$

\section{Department of Biochemistry, \\ B.P. Koirala Institute of Health Sciences, Dharan, Nepal \\ 2. Department of Obstetrics and Gynecology, B.P. Koirala Institute of Health Sciences, Dharan, Nepal}

INTRODUCTION: Preeclampsia (PE) is a disorder of pregnancy characterized by hypertension with proteinuria after 20 weeks of pregnancy in a previously normotensive and non-proteinuria patients which may progress to eclampsia.Studies have documented that deficiency of minerals like calcium, magnesium and zinc may have a role in etio-pathogenesis of preeclampsia.

AIMS AND OBJECTIVES:The objective ofthis study was to assess, and compare serum total calcium, magnesium and uric acid level in preeclampsia and normotensive pregnancy.

MATERIALS AND METHODS: Ahospital based comparative cross-sectional study conducted at BPKIHS. A total number of 72 women (36 diagnosed PE and 36 healthy pregnant women) were recruited by purposive sampling technique. Serum total calcium, magnesium, creatinine and uric acid was estimated by Cobas c311 autoanalyzer. Data were analyzed using SPSS version 16.0.

RESULTS AND CONCLUSION: Mean serum calcium $(\mathrm{mg} / \mathrm{dl})$ and magnesium level $(\mathrm{mg} / \mathrm{dl})$ in PE $(8.69 \pm 1.59$ and $0.79 \pm 0.15$ )was significantly lower as compared to normotensive pregnant women $(10.13 \pm 0.66$ and $0.85 \pm$ 0.05 ; $\mathrm{p}$ value $<0.001)$. Serum uric acid $(\mathrm{mg} / \mathrm{dl})$ and creatinine $(\mathrm{mg} / \mathrm{dl})$ was raised in PE $(4.40 \pm 2.14$ and 0.49 $\pm 0.24)$ compared to the control women $(2.70 \pm 0.80$ and $0.30 \pm 0.07 ; \mathrm{p}$ value $<0.001)$

This study depicts lower serum calcium and magnesium levels in PE compared to normal pregnant women. Thus, the assessment of serum calcium and magnesium levels helps in the assessment of severity in preeclampsia, thereby preventing the progression of preeclampsia to eclampsia.

KEYWORDS: Preeclampsia, Creatinine, Uric Acid

\section{P29}

Status of Serum Calcium, Phosphorus And Magnesium In Patients With Thyroid Dysfunction

Das BKL ${ }^{1}$, Joshi BR ${ }^{1}$, Mishra $\mathrm{B}^{1}$, Sapkota $\mathrm{S}^{1}$, Maskey $\mathrm{R}^{2}$, Chaudhari $\mathrm{RK}^{1}$, Lamsal $\mathrm{M}^{1}$

1. Department of Biochemistry,

2. Department of Internal medicine

INTRODUCTION: Thyroid dysfunction comprises one of the most common endocrine abnormalities. Mineral metabolism like calcium, magnesium and phosphorus is frequently disturbed in thyroid dysfunction.

OBJECTIVES: The present study was intended to assess and correlate the serum calcium, phosphorus and magnesium level with thyroid dysfunction. 
MATERIALAND METHODS: This is a hospital based comparative cross sectional study conducted from $\mathrm{Feb}$ 2016-Aug 2016 after obtaining the ethical approval from Institutional Review Committee (IRC). Forty diagnosed case of hyperthyroid, 40 hypothyroid and 40 healthy subjects were enrolled in the study. Serum calcium, phosphorus and magnesium was analysed in cobas c311 autoanalyzer. Statistical analysis was done using ANOVA. P value $<0.05$ was considered as significant.

RESULTS: Mean age group in the study was $40.91 \pm 11.22$ years. Mean value of serum calcium was $10.85 \pm 0.6 \mathrm{mg} / \mathrm{dl}, 10.17 \pm 0.93 \mathrm{mg} / \mathrm{dl}$ and $10.44 \pm 0.28 \mathrm{mg} /$ dl respectively for hyperthyroid, hypothyroid and euthyroid subjects. Similarly, Mean value of serum phosphorus and magnesium was $4.08 \pm 0.67 \mathrm{mg} / \mathrm{dl}$, $3.83 \pm 0.66 \mathrm{mg} / \mathrm{dl}$ and $3.82 \pm 0.73 \mathrm{mg} / \mathrm{dl}$ and $2.11 \pm 0.22 \mathrm{mg} /$ $\mathrm{dl}, 2.6 \pm 0.5 \mathrm{mg} / \mathrm{dl}$ and $2.26 \pm 0.19 \mathrm{mg} / \mathrm{dl}$ respectively for hyperthyroid, hypothyroid and euthyroid subjects. There was significant difference in calcium and magnesium level in hyperthyroid, hypothyroid and euthyroid subjects $(\mathrm{P}<0.001)$. There was no significant difference in phosphorus level in hyperthyroid, hypothyroid and euthyroid subjects $(\mathrm{P}=0.17)$.

CONCLUSION: This study concludes that serum calcium was high in hyperthyroidism compared to euthyroid and serum magnesium was high in hypothyroidism when compared to normalsubjects. A regular follow up of these serum minerals should be done in thyroid hormone deficiency which would be of great helpin its management.

KEY WORDS: Calcium, Phosphorous, Magnesium, hyperthyroid, hypothyroid, euthyroid

\section{P30}

\section{Iron Homeostasis and Its Related Biochemical Complications In Preeclampsia}

Nila SG, Zachariah Bobby, Sajini Elizabeth Jacob, Gowri Dorairajan.

\section{Department of Biochemistry, JIPMER, Puducherry}

OBJECTIVE: To compare indices of iron homeostasis, low grade inflammation, oxidative stress and endothelial dysfunction between healthy pregnant women(HC) and Preeclampsia patients(PE).

MATERIALS \& METHODS: In a cross-sectional study Preeclampsia patients $(n=40)$ and healthy pregnant women $(n=40)$ between 30 to 38 weeks of gestation both on Iron supplementation were recruited from OG department of JIPMER, Puducherry. Blood samples were collected from them and serum was stored for further analysis. Serum levels of Hepcidin, Transferrin, Ferritin, Iron, Hscrp, ADMA were measured by Elisa and MDA,TAS by Manual Methods.

RESULTS : The serum levels of Iron (PE:703 \pm 484 $\mathrm{HC}: 393 \pm 180$ in $\mu \mathrm{g} / \mathrm{dl} ; \mathrm{p}<0.000$ ), Ferritin (PE:47 \pm 36 $\mathrm{HC}: 24 \pm 14$ in $\mu \mathrm{g} / \mathrm{L} ; \mathrm{p}<0.000$ ), Hepcidin (PE :76 \pm 31 HC: $62 \pm 20$ in $n g / m l ; p<0.016)$ Tsat(PE: $89 \pm 65$ HC:43 \pm 26 in \%; $<<0.000)$ and $\mathrm{MDA}(\mathrm{PE}: 21.5 \pm 4.5 \mathrm{HC}: 6.3 \pm 0.81$ in $\mu \mathrm{mol} / \mathrm{L} ; \mathrm{p}<0.000)$ were significantly higher in PE compared to HC. Serum Transferrin $(622 \pm 30$ and $742 \pm 228$ in $\mathrm{mg} / \mathrm{dl} ; \mathrm{p}<0.013$ ). and TAS (PE :407.8 \pm 110 $\mathrm{HC}: 634.86 \pm 217.07$ in $\mu \mathrm{mol} / \mathrm{L} ; \mathrm{p}<0.000)$ were significantly lower in PE. ADMA and Hscrp levels were higher in PE but was notsignificant.

Conclusion: In Preeclampsia patients serum, storage and circulating levels of Iron were elevated significantly which in turn elevated the levels of the regulatory protein Hepcidin and induced oxidative stress in them. The significant Hepcidin elevation points more towards the ongoing Iron overload rather than the disease. The alarming increase in oxidative stress warns about the deleterious effect of Iron therapy in Preeclampsia. There was no significant evidence of endothelial injury and low grade inflammation which reinforces the current treatment regimen in Preeclampsia.

KEY WORDS: Iron homeostasis, Preeclampsia, Hepcidin, Transferrin, Ferritin

\section{P31}

\section{Antimullarian Hormone and Insulin Levels In Polycystic Ovarian Syndrome}

Shakti Suslade, Sangeet Paneri, Jayshree Sridhar

School of Biochemistry, D.A.V.V, Indore 
INTRODUCTION:Polycystic ovarian syndrome (PCOS) is a common metabolic dysfunction and hetrogeneous endocrine disorder in women of reproductive age, also called Hyperandrogenic anovulation or stein-leventhal syndrome.

AIM:The aim of the study to analyze serum Antimullerianhormone (AMH) and Insulin level in PCOS Patients and its correlation with Healthy control subject.

MATERIAL AND METHODS:Present study was carried out in M.Y. Hospital and K. R. G's Blessed mom Centre during June 2016 to Jan 2017. Study comprised total 80 Subjects divided in to two groups control \& cases. 40 Healthy women aged between 20 to 40 year taken as control \& 40 PCOS patients aged between 20 to 40 year taken as cases. Fasting sample form each subject was collected and analyzed for Antimullerian Hormone and insulin.

RESULT:The result revealed that serum AMH and insulin levels were significantly higher in PCOS patient than in healthy control subjects.

CONCLUSION: Our finding indicate that increased $\mathrm{AMH}$ and insulin level might be contribute to the pathogenesis of PCOS. Early estimation of AMH \& Insulin level in women may decrease the risk of PCOS and associated disorders, although further investigation is necessary to elucidate the detailed mechanism.

KEY WORDS:PCOS, Antimullerianhormone, Insulin

\section{P32}

\section{Study of $\boldsymbol{\beta}$-Hcg and Dyslipidemia in Pregnancy Induced Hypertension During Second Trimester of Pregnancy}

Neha Jaiswal, Ranjana Mathur and Jairam Rawtani.

Department of Biochemistry, Dr. S.N. Medical College, Jodhpur.

INTRODUCTION: Pregnancy induced hypertension (PIH) is defined as hypertension that develops for the first time in pregnancy after 20 weeks of gestation.
Pregnancy associated hypertensive disorders are common complications responsible for fetal, neonatal and maternal morbidity. WHO estimates that one woman die every minute due to the complications of Hypertensive Disorders of Pregnancy (HDP).

OBJECTIVE: To evaluate ?-HCG and lipid profile in second trimester of pregnancy and compare the results with healthy control.

MATERIALS AND METHODS: 25 pregnant women with singleton pregnancy between 14 and 26 weeks of gestation attending antenatal clinic of Department of OBG, MDM Hospital, Jodhpur and 25 healthy control female subjects of similar age groups were studied. Serum ?-HCG was estimated by ELFA technique. Serum lipid profile was evaluated by Enzymatic method on Beckman Coulter.

RESULTS: Statistically significantly elevated values of serum Cholesterol, Triglycerides and decreased values of HDL Cholesterol was observed in pregnant females during second trimester showing dyslipidemia as compared to healthy controls. Total Cholesterol : HDL Cholesterol ratio and Triglycerides : HDL cholesterol ratio is also increased in PIH. Similarly the values of Serum ?-HCG were also significantly high during second trimester. There was a positive correlation between serum cholesterol and serum triglycerides with ?-HCG and negative correlation between HDL and $\beta$-HCG.

CONCLUSION: Elevated serum $\beta-\mathrm{HCG}$ and Dyslipedemia in second trimester can be considered as predictors of subsequent PIH/ Pre-eclampsia. Thus, Dyslipidemia and $\beta$-HCG associated with increased risk of PIH. Therefore, proper timely regulation and management of these parameters can reduce the risk of complications during pregnancy.

KEY WORDS: $\beta$-HCG, PIH, Dyslipedemia, Preeclampsia

\section{P33}

Association of Vitamin D, C-Reactive Protein , And Polyunsaturated Fatty Acids Among Diagnosed Breast Cancer Women. 
Preethika A, Suchetha Kumari, Sukanya Shetty, Shipra S, VijethShetty, JayaramShetty.

K S Hegde Medical Aacademy, NITTE University, Deralakatte.

INTRODUCTION: Mechanism of data suggests that different types of fatty acids play a role in carcinogenesis and the vitamin D and C-reactive protein(CRP) may modulate the relationship but epidemiologic evidence is lacking. Our aim was to investigate the association between serum poly unsaturated fatty acids and overall and breast cancer risk and to evaluate the effect of vitamin $\mathrm{D}$ and C-reactive protein on these relationships.

MATERIALS AND METHOD: In this descriptive comparative study twenty breast cancer women and equal numbers of age matched healthy women wererecruited. Serum fatty acids were quantified using gas chromatography. Vitamin D and CRP levels were measured using commercially available ELISA kits.

RESULTS: Omega 3 and omega 6 fatty acids ratio were inversed in breast cancer women compared to control women. Significant difference in vitamin D $(\mathrm{p}<0.0001)$ and CRP levels were observed between breast cancer and control women. In stratified analysis,for both the case and control groups put together significant associations were observed between omega 3: omega 6 and vitamin $\mathrm{D}$ level $(\mathrm{r}=0.4, \mathrm{p}<0.05)$ and no significant association were observed between omega3: omega 6 and CRP level.

CONCLUSION: Polyunsaturated fatty acids were differentially associated with cancer risk. This study has provide evidence that vitamin D deficiency and high levels of CRP has been very prevalent in breast cancer patients more than comparable matched control population. Risk of breast cancer has increased with inversed omega fatty acids ratio,decreased vitamin D levels and increased inflammation, suggesting the need for high quality studies that look at the social and dietary associations in breast cancer women.

KEYWORDS: Breast cancer, polyunsaturated fatty acids, vitamin D, C-reactive protein.

\section{P34}

\section{Serum Ceruloplasmin Albumin Ratio as a Biochemical Marker To Assist The Prognosis of Pulmonary Tuberculosis Patients.}

Teena Agrawal ${ }^{1}$, P.D. Sarkar ${ }^{2}$, S. Paneri ${ }^{3}$, R. Lohokare ${ }^{4}$, Ravikant Sharma ${ }^{5}$, Rohit Manyal ${ }^{6}$, Bhavna Tiwari ${ }^{7}$

M. G.M. Medical College \& M. Y. Hospital, Indore, M.P.

INTRODUCTION: Pulmonary tuberculosis (PTB) is a global disease affecting about one third of the world's population. It is caused by mycobacterium tuberculosis. India has the highest number of TB cases in the world and it is leading cause of death with its high attendant mortality and morbidity. Reason lies in the emergence of multidrug resistant TB strains and HIV infection which reactivate latent TB making it lethal. . In tuberculosis, the albumin content decreases while the globulin like Ceruloplasmin increases that leads to high ceruloplasmin albumin ratio.

AIM \& OBJECTIVE:To estimate and to compare the serum level of ceruloplasmin, albumin (C/A) and its ratio in diagnosis and prognosis of pulmonary tuberculosis patients.

MATERIALS \& METHODS: The study was carried out in department of Biochemistry, M. G.M. Medical College after ethical clearance. In this prospective cohort study 50 newly diagnosed pulmonary tuberculosis patients attending to pulmonary medicine OPD of M.Y.Hospital. The control population comprised of age and sex matched 50 healthy volunteers. Serum ceruloplasmin was estimated by tubidimetric immunoassay and serum albumin was estimated by BCG method on fully automated analyzer.

RESULT: The study revealed that MEAN $\pm \mathrm{SD}$ for serum ceruloplasmin, serum albumin and ceruloplasmin albumin ratio in newly diagnosed pulmonary tuberculosis and after completion of treatment $84 \pm 12$, $3122 \pm 299,0.031 \pm 0.005$ and $42 \pm 5,4566 \pm 478$, $0.02 \pm 0.003$ respectively. $\mathrm{P}$ value $<0.05$ was considered statistically significant. 
CONCLUSION: Serum Ceruloplasmin albumin ratio can therefore be incorporated as surrogate marker to assist in diagnosis as well as prognosis of pulmonary tuberculosis.

KEY WORDS: pulmonary tuberculosis, ceruloplasmin, albumin

\section{P35}

\section{Early Diagnostic Markers In Diabetic Nephropathy Patients}

Abdulrahman Hefdhallah Amer ${ }^{1}$, N. Haridas ${ }^{2}$

1. Medical Lab. Technology,

Pramukhswami Medical College, Karamsad.

Sardar Patel University, V. V. Nagar Anand,

Gujarat, India Pin Code- 388325.

Thamar University-Yemen

2. Biochemistry Pramukhswami Medical College,

Karamsad Anand, Gujarat, Pin Code: 388325.

INTRODUCTION: Diabetes mellitus is one of the most important causes of chronic complications of kidney diseases. Cystatin $\mathrm{C}(\mathrm{CysC})$ is a better marker in early diagnoses in kidney diseases.In our studywas done to estimate clinical advantage of serum Cystatin C levels for predicting renal impairment in patients with type 2 diabetes in Gujarat, India. Also, in order to evaluate the association between serum creatinine and Cystatin C.

MATERIAL AND METHODS: A total of 170 participants were involved and classified into two groups; Diabetic nephropathy and Diabetics without nephropathy.Serum samples were collected to measure Cystatin $\mathrm{C}$ using immunoturbidimetric assay (On TURBODYNE TM SC). Glucose, Urea, Creatinine, HbA1c, Cholesterol, Triglycerides, and HDLCholesterolusing Automated Analyzer (SiemensDimension-RXL-and X pand). The LDL-Cholesterol was calculated using Friedewald formula. The cross-sectional study was approved by the Human Research Ethics Committee (HREC) in Pramukhswami Medical College (PSMC), Karamsad. The period for samples collection was from September 2015 to April 2017.
RESULTS:The results showed that the mean Cystatin $\mathrm{C}$ level was higher in diabetic with nephropathy patients versus diabetics without nephropathy, was significant (1.87 \pm 0.51 vs. $1.025 \pm 0.30 \mathrm{mg} / \mathrm{L}$, respectively, with Pvalue $<0.001)$. Personal correlation results showed positive correlation between Cystatin $\mathrm{C}$ and Age, Creatinine, Urea and $\mathrm{HbA} 1 \mathrm{c}$ were (R-value 0.214 and $\mathrm{P}$-value 0.005), (R-value 0.55 and $\mathrm{P}$-value $<0.001$ ), (Rvalue 0.66 and $P$-value $<0.001$ ), (R-value 0.164 and $P$ value 0.033 ), respectively.No significant correlation with gender (R-value 0.075 and $\mathrm{P}$-value 0.33 ). The results also showed that the serum Cystatin $\mathrm{C}$ level was increased in some patients which have the normal level of Creatinine), 28 cases out of 85 total cases. But serum Cystatin C level was abnormal in all patients that have the increase abnormality of the serum creatinine level, with $(\mathrm{P}=0.001)$.

CONCLUSION: The results of this study suggest that Cystatin $\mathrm{C}$ measurement in serum is useful for early detection of renal impairment in the course of diabetes.

KEY WORDS : Cystatin-C, Creatinine, diabetic without nephropathy, Diabetic Nephropathy.

\section{P36}

\section{A Study of Correlation Between Electrophoretic Pattern of Serum Proteins and Serum Crp In Psoriasis}

Isha Sharma*, A.K. Bhargava**, Deepti Gautam**, Ranjana Mathur*, Jairam Rawtani*

Department of Biochemistry, *Dr. S.N. Medical College, Jodhpur and

**Jhalawar Medical College, Jhalawar

INTRODUCTION- Psoriasis is a chronic inflammatory skin disease and a major health problem(2\% in World and $0.4-2.8 \%$ in India) on an individual basis; it has negative impact on patient's quality of life. The mean age of onset of first occurrence between 25-30 years, with the second peak at 55-60 years. Psoriasis is characterized by sharply demarcated, erythematous and scaly plaques of different sizes,distributed mostly on scalp,extensor 
aspects of elbows and knees,lumbosacral region and umbilicus.Alterations in serum CRP levels and electrophoretic patten of serum proteins are seen because of impairment in catabolic or anabolic metabolism in liver.

AIMS AND OBJECTIVES- The aim of the study was to investigate the correlation between the electrophoretic pattern of serum proteins and level of serum CRP in psoriasis, to assess if these biochemical parameters emerged as a predictive tools in prognosis, diagnosis and better management of psoriasis.

MATERIALS AND METHODS- 15(10M,5F) normal healthy controls without any clinical symptoms of disease and $25(15 \mathrm{M}, 10 \mathrm{~F})$ psoriatic patients between age of 1870years attending OPD of Dermatology,S.R.G. Hospital, Jhalawar (Raj.) were included.The subjects having risk factors that may affect the level of serum proteins and serum CRP were excluded. The separation of serum proteins was done by Cellulose Acetate Membrane Electrophoresis and Quantitative Estimation of Serum CRP was done by Immunoturbidimetric method. Unpaired and Paired- t test, Chi square test, and Correlation coefficient(r value) was used in data analysis.

RESULTS- Electrophoretically in psoriatic patients statistically highly significant $(\mathrm{p}<0.0001)$ decrease in serum Albumin, highly significant $(\mathrm{p}<0.0001)$ increase in alpha- 1 band and significant ( $p$ value $=0.012$ ) increase in alpha-2band,significant $(\mathrm{p}$ value $=0.032)$ decrease in beta band and highly significant ( $p$ value $<0.0001$ ) decrease in gamma band was observed as compared to healthy controls. Serum CRP was also highly significantly ( $p<0.0001)$ increase in serum CRP level was observed as compared to healthy controls.

Highly significant negative correlation $(\mathrm{r}$ value $=-0.729)$ between CRP and albumin, highly significant positive correlation ( $\mathrm{r}$ value $=0.620$ ) between $\mathrm{CRP}$ and alpha- 1 band as well as alpha- 2 band( $\mathrm{r}$ value $=0.589$ ), significant negative correlation $(r$ value $=-0.389)$ between CRP and beta band as well as gamma band(r value $=-0.705)$ was observed in psoriasis pateints.

CONCLUSION- From above results it is concluded that CRP is a useful inflammatory marker for diagnosis of psoriasis and also there was principle significant changes in serum proteins such as decrease in albumin and an increase in alpha-1 and alpha-2globulin and decrease in beta and gamma globulin. So evaluation of these biochemical parameters emerged as predictive tools in prognosis and diagnosis of psoriasis with reference to therapeutic significance.

KEY WORDS:Psoriasis, erythematous, CRP, electrophoretic pattern

\section{P37}

Serum Interleukin-6 Concentration in Schizophrenia: Elevation Associated with Duration of Illness

Bhavana Singhal ${ }^{1}$, Shakuntala Saini ${ }^{2}$, Suresh gupta ${ }^{3}$

1,2. Department of Biochemistry, SMS Medical College \& Attached hospitals Jaipur, Rajasthan.

3. Department of Psychiatry, SMS Medical College \& Attached hospitals Jaipur, Rajasthan.

INTRODUCTION: Schizophrenia may be accompanied by an activation of inflammatory response system. Interleukin -6 (IL-6) which can function as both a pro- and anti-inflammatory cytokine has been reported to be elevated in schizophrenic patients.

AIMS AND OBJECTIVES: The aim of this study is to investigate an association between the serum IL-6 concentration and total duration of illness (TDI) in patients with schizophrenia.

MATERIALS AND METHODS: IL-6 levels were determined in schizophrenic $(\mathrm{n}=80)$ patients and were compared with healthy controls $(n=50)$ by using enzyme linked immune sorbent assay(ELISA). Patients were interviewed and diagnosed by the psychiatrist as per ICD10 ( International classification of mental disorders) criteria of mental disorders. The severity of illness was assessed by positive and Negative Syndrome Scale (PANSS).

RESULTS: Mean serum IL-6 level was significantly higher in the schizophrenic patients as compared with the control subjects. Serum IL-6 was significantly 
correlated with total duration of illness (TDI).

CONCLUSION: The elevated serum IL-6 levels in schizophrenia may play a role in the pathophysiology of schizophrenia and develop during illness and may be related to treatment or to disease progression.

KEYWORDS: Interleukin-6, Positive and negative syndrome scale, total duration of illness.

\section{P38}

\section{Human Adipose Derived Stem Cell Obesity Bioinformatics In Adipose Tissue Engineering: Obesity Regulating Stem Cell Surface Proteomics (ORS-CSP)}

\section{Rakesh Sharma}

Innovations and Solutions Incorporated USA, Bioproteomics Lab, Florida State University, Woodwards Avenue, Tallahassee, FL 32304

$\mathrm{A}$ dipose or fat tissues as waste from liposuction at obesity centers may be used to isolate mesenchymal stem cells. The biochemical action of lipase enzymes in presence of phospholipids degrades the adipose or fat tissues suitable to preparemesenchymal stem cells. The purity of stem cells is characterized by protein expression on cell surface in specific cell phenotype. These cells differentiate in presence of specific lipogenic inducer in cell cultures. In vitro stem cell culture is a technique to develop controlled cell proliferation and maintain specific phenotype. In adipose stem cell cultures, phenoltyping and controlled cell differentiation both are determined by stem cell surface marker CD105 proteins. Moreover, stem cells display multi-differentiation potential and serve as 'Obesity molecular bioinformatics tool' or molecular differentiation protein fingerprints. The database of cell differentiation proteins is very significant in decision making of obesity treatment and drug development. The concept is based on the fact that stem cell surface proteins involved in cell differentiation might be adipogenic or drug targets. In this paper, adipose tissue engineered stem cell isolation and characterization by different stem cell surface proteins as bioinformatic tool to search adipogenesis inhibitors and differentiation blockers is described retrieved from MMDB (Molecular Modeling Database) and PDB (Protein Data Bank). Different polymeric scaffold materials serve as potential breast and thorax extracellular matrices suitable for human use under clinical practice regulations. In conclusion, adipose stem cell isolation from fat tissue, stem cell surface protein markers as bioinformatics tools with current practices and future perspectives are addressed in the light of adipose tissue engineering matrices.

KEY WORDS: Mesenchymal stem cells, Enzyme digestion, Obesity, Adipose stem cells, Adipose Tissue Engineering

\section{P39}

\section{Pseudoesterase Activity of Human Serum Albumin: Analysis By Docking And Molecular Dynamics Stimulation}

Deepak Kumar ${ }^{1}$, Meenu Narwal ${ }^{2}$, Rajasri Bhattacharyya ${ }^{1}$, Dibyajyoti Banerjee ${ }^{1 *}$

Department of Experimental Medicine and Biotechnology, Post Graduate Institute of Medical Education and Research, Chandigarh, India. Department of Biotechnology, Maharishi Markandeshwar University, Mullana, Ambala, India

INTRODUCTION: There is a considerable current interest in developing newer methods for albumin estimation because satisfactory microalbuminuria detection is not a reality. It is now well known that human serum albumin (HSA) exhibits slow turnover enzyme activity towards several ester substrates which is popularly recognized as pseudoesterase activity. Recently, it is getting explored for estimation of albumin and even a US patent in the consult subject is registered that a sensitive probe has been designed (US9340821 B2).However, a systematic study, to the best of our knowledge, is lacking for comparative evaluation of the ester substrates for albumin. It is in that context we have analysed the potential of various ester substrates as candidates to estimate pseudoesterase activity of HSA. 
AIMS AND OBJECTIVES: To identify the ester substrates which are candidates for pseudoesterase activity of HSA and to understand them in relation to their hydrolysis potential through true esterases.

MATERIAL AND METHODS: Some available ester substrates are downloaded from PubChem, ChemSpider database and docking is done with the site of HSA known for pseudoesterase activity. Similar exercise is done with some true esterases like Butyrylcholinesterase (BChE). The results were verified by Molecular dynamics stimulations.

RESULTS AND CONCLUSION: It is observed that p-Nitrophenylacetate (PNPA) binds with HSA with more favourable binding energy in comparison to $\mathrm{BChE}$. The binding also shows conformations after molecular dynamics stimulation runs. PNPA may be used as a substrate for assaying pseudoesterase activity of HSA.

KEYWORDS: Pseudoesterase, In-silico, Human serum albumin.

\section{P40}

\section{Serum Uric Acid Levels In Cardiovascular Diseases In Bundelkhand Region}

Ayush Agrawal, Prabhakar Singh Bais, Neeraj Sharma, Hari Madhav Sharan

Deptt. of Biochemistry, MLB Medical College, Jhansi (U.P.)

OBJECTIVE -The aim of the study was to evaluate the serum uric acid level in cardiovascular diseases in a tertiary care centre of Bundelkhand region.

INTRODUCTION - Serum uric acid is important in the diagnosis, prognosis and medical management of cardiovascular diseases. Uric acid is an important member of antioxidants \& therefore plays an important role in scavenging the liberation of free radicals. If the level of antioxidants is low then the defense mechanism of the body is decreased $\&$ we suffer from a large number of diseases such as cardiovascular diseases. The uric acid is a waste product normally present in the blood as a result of breakdown of purines. The role of uric acid as an independent marker of cardiovascular disorder has been controversial for decades.

MATERIALS \& METHODS - We have studied uric acid level in 50 cardiovascular diseases patient and 50 normal healthy controls both male and female of different age groups. Uric acid estimation was done by commercial kit method supplied by Transasia Biochemicals Ltd. Solan (H.P.).

RESULTS - In our study the level of uric acid varies from $5-12 \mathrm{mg} / \mathrm{dl}$ with mean + S.D. $(8.7+3.14)$ in patients suffering from cardiovascular diseases. In chest pain serum uric acid mean value was $8.9 \mathrm{mg} / \mathrm{dl}$ with S.D. 3.49 as compared with normal healthy person, the value was significantly higher $(\mathrm{P}<0.0001)$, in case of hypertension mean + S.D. $(7.52+2.47)(\mathrm{P}<0.014)$, In coronary syndrome mean + S.D. $(9.5+3.65)(\mathrm{P}<0.001)$, In myocardial infarction mean + S.D. $(8.8+3.15)$ $(\mathrm{P}<0.0003)$ and in ventricle failure mean serum uric acid was 7.7 with S.D. $3.59(\mathrm{P}<0.0037)$.

CONCLUSION - Our study was attempt to evaluate uric acid is an important cardiovascular risk factor. By this study we could confirm that the uric acid levels are increased in cardiovascular disease patients and it can be utilized in evaluating the prognosis of the disease.

KEYWORDS:- Cardiovascular Dieases, Uric Acid, Myocardial Infarction, Antioxidants, Free Radicals.

\section{P41}

\section{Study of Serum Adenosine Deaminase and Uric Acid In Rheumatoid Arthritis}

Chanchal Garg, Neelima Singh, Kiran Saxena, VedikaRathore, Roshan Kumar Mahat, Mritunjay Kumar Mishra

Chirayu Medical College \& Hospital, Bhopal, (M.P.)

INTRODUCTION:Rheumatoid arthritis (RA) is a chronic disease, characterized by extracellularmatrix degradation, destruction of joint cartilage and loss of function. During inflammatoryreactions, the enzyme adenosine deaminase (ADA) is released into theextra cellular fluid. Uric acid is an aqueous, endogenous 
antioxidant with higher concentration in plasma than vitamin Cand vitamin E. It contributes 2/3rd of free radical scavenging capacity in plasma. Hence, the present study attempt to estimate the levels of ADA and uric acid that represent the oxidant-antioxidant status in RA patients and verify its diagnostic potential in the rural area of Bhopal.

MATERIALS AND METHODS: The present study included 100 subjects of which 50 were RA patients and 50 were healthy controls. In both the groups, ESR, ADA, CRP, RF and uric acid levels were analyzed.The comparison of parameters between the groups was carried out using student $t$ test.

RESULT: All rheumatoid arthritis patients included in the present study had positive RF. ESR, CRP and ADA levels were found to be significantly increased in rheumatoid arthritis patients as compared to normal healthy control individuals where as there was no significant difference in the level of uric acid between RA patients and normal healthy individuals.

CONCLUSION: The increased level of ADA in rheumatoid arthritis patients indicates inflammation. Owing to the simplicity and also the cost effectiveness of ADA assay, ADA may be recommended as a marker of inflammation in patients with RA. However, further studies are needed to accept the concept.

KEY WORDS: Rheumatoid arthritis, Adenosine deaminase, CRP

\section{P42}

Prevalence of Phosphides and Phosphorous Poisoning Detected At Manipal Poison Detection Centre (MPDC), Manipal

Gagana H, Vinutha R Bhat

Department of Biochemistry, Kasturba Medical College Manipal, Manipal University,

Manipal 576104.

INTRODUCTION: Phosphorous is a highly reactive, poisonous element, occurring in solid state. Red phosphorus is non-volatile and unabsorbable. Yellow phosphorus is a local and systemic toxin. The dangers of phosphorous poisoning now are much more from its organic compounds which are used as insecticides and rodenticides.

AIM: To study prevalence and demographic profile of Phosphides and Phosphorous Poisoning cases received at MPDC.

METHODOLOGY: Qualitative test (using silver nitrite solution and aqueous lead acetate) for phosphides and phosphorus poisoning was performed with stomach wash contents of patients and reported positive only when the silver nitrite paper was blackened.

RESULTS: Among 74 samples of suspected phosphides and phosphorous poisoning aged about 1 to 78 years in both males and females of which 42 were detected positive and 32 were negative. Over which phosphides and phosphorous poisoning was detected positive in $52.3 \%$ in males $(n=22)$ and $47.6 \%$ in females $(n=20)$. Around $57.1 \%$ of phosphides and phosphorus consumers were between the age group of 25 to 55 years.

DISCUSSION: Phosphorous is a general protoplasmic poison causing cardiac, hepatic, renal and multi organ failure. Cardiac toxicity includes hypotension, tachycardia, cardiogenic shock. Liver being susceptible, transplantation is recommended in few cases. Phosphorus poisoning lacks specific antidote. Treatment is directed at removal of the poison and supportive therapy with possible survival benefit of early intravenous $\mathrm{N}$-acetyl cysteine administration.

CONCLUSION: This study contributes to determination of percentage of phosphides and phosphorous poisoning among different age groups. As this doesn't have a specific antidote,further research can be conducted.

KEY WORDS:Yellow phosphorus, insecticides, rodenticides

\section{P43}

Role of Oxidative Stress For Prediction of Cardiovascular Disease In Prediabetic Subjects 
Roshan Kumar Mahat, Neelima Singh,

Vedika Rathore

Department of Biochemistry, GR Medical College, Gwalior

INTRODUCTION:Prediabetes is a condition in which the blood glucose level is above normal but below the diagnostic threshold for diabetes mellitus. The association between oxidative stress and risk for cardiovascular disease in prediabetes in poorly defined. Hence, the present study intended to explore the risk of cardiovascular disease in prediabetes by assessing the markers of oxidative stress i.e. 8-hydroxy-2-deoxyguanosine $(8 \mathrm{OHdG})$, malondialdehyde (MDA) and reduced glutathione (GSH).

MATERIALS AND METHODS:This was a crosssectional case control study, which included 80 cases(prediabetic subjects)and 80 healthy control subjects. In all the cases and controls, oxidative stress markers 8-OHdG, MDA and GSH were estimated. The comparison of parameters between the groups was carried out using student $t$ test.

RESULTS:There were significant increase in the levels of $8-\mathrm{OHdG}$ and MDA in prediabetic subjects as compared to control subjects where as the level of reduced glutathione (GSH) was significantly decreased in prediabetes compared to that of controls.

CONCLUSION:In conclusion, the alterations in the levels of oxidative stress markers in prediabetic subjects indicate their higher risk for the development of atherosclerosis and cardiovascular diseases in future.

KEYWORDS: Oxidative stress, prediabetes, 8-OHdG, MDA, GSH

\section{P44}

\section{The Analysis of 16s rRNA For Predicting Antibiotic Resistance}

Vandana Singh ${ }^{1}$, Kunal Maniar ${ }^{2}$, Rajasri Bhattacharayya ${ }^{1}$, Dibyajyoti Banerjee ${ }^{1}$

1. Department of Experimental Medicine \&
Biotechnology, Post Graduate Institute of Medical Education \& Research, Sector 12, Chandigarh, India.

2. Department of Pharmacology, Post Graduate Institute of Medical Education \& Research, Sector 12, Chandigarh, India.

INTRODUCTION: Antibiotic resistance is a global concern which is recently complicated further by the emergence of multiple drug resistance strains of pathogens. Indiscriminant use of antibiotics is undisputedly one of the major culprit but however far from control. Therefore, newer strategies are warranted for monitoring antibiotic resistance. Recently, after the advent of Next Generation Sequencing (NGS), metagenomic data are reposited in public databases. 16s ribosomal data is also available from a wide range of environment and clinical sample. We have analyze sequences, exploring In-silico tools to understand signatures of antibiotic resistance in the reposited $16 \mathrm{~s}$ rRNA.

MATERIALS AND METHODS: We have considered 16S rRNA databases.Streptomycin, tetracycline, spectinomycin are considered which are known to bind with 16s rRNA. Mutant sequences responsible for antibiotic resistance were identified through literature study. Using PERL script the antibiotic sensitive/resistant binding region were identified and analyzed.

RESULTS: we have identified antibiotic sensitive and resistant 16s rRNA from Greengene database. There is a difference in thermodynamic profile of wild type $16 \mathrm{~s}$ rRNA with respect to the mutant. Moreover it is observed that, predicting antibiotic resistance is not possible because the responsible fragment for sensitive or resistance is not amplified. However, in Grenegene database available freely at internet is properly sequenced and can be used for analysis of antibiotic resistance.

CONCLUSION:A protocol is developed that can be used for identifying antibiotic sensitive or resistance against the above mentioned three antibiotics from $16 \mathrm{~s}$ rRNA.

KEYWORDS: 16s r RNA; NGS; Antibiotic resistance 


\section{P45}

\section{Validation of A Prototype Kit For Osteocalcin In The Diagnosis and Management of Osteoporosis}

Sonam Hatkar, Seema Kadam, Khatkhatay MI, Meena Desai

National Institute for Research in Reproductive Health (ICMR), J. M. Street, Parel, Mumbai- 400012

INTRODUCTION: Osteocalcin (OC) is a sensitive biomarker for evaluating bone turnover in humans. Expensive commercial OC kits prohibit their use in routine screening and management of osteoporosis.

AIM: To assess the diagnostic potential of Osteocalcin (OC) prototype kit previously developedin the laboratory in screening, diagnosis and management of osteoporosis.

MATERIALS AND METHODS: Developed OC assay was validated with 290 samples.Bone mineral density (BMD) measurements at the hip and spine by DXA were available in 260 samples and the study was carried out at the Institute.The study included 163 premenopausal age: $21-45$ years and 97 postmenopausal women age:4665 years classified in three groups as normal,osteopenic and osteoporotic based on their T-scoreand clinical/ abnormalsamples $(n=30)$. OCassay was validated for diagnostic sensitivity, specificity by correlating with BMD measurements and commercial ELISA kit from Ray Biotech, USA ( $\mathrm{n}=260)$ and clinical/abnormal samples $(\mathrm{n}=30)$.

RESULTS: Mean serum OC levels by developed assay were significantly different in three classified groups $(11.74 \pm 0.810 \mathrm{v} / \mathrm{s} 14.51 \pm 0.672 \mathrm{v} / \mathrm{s} 15.45 \pm 1.66 \mathrm{ng} / \mathrm{ml}$, $\mathrm{p}<0.001)$. OC levels significantly correlated with the commercial kit (Pearson $\mathrm{r}=0.424, \mathrm{p}<0.001$ ). ROC analysis determined a cut off value of $\mathrm{OC}>19.60 \mathrm{ng} / \mathrm{ml}$ with diagnostic sensitivity of $20.5 \%$ and specificity $77.9 \%$ whileOC value of $>17.3 \mathrm{ng} / \mathrm{ml}$ by commercial kit gave diagnostic sensitivity of $46.5 \%$ and specificity $80.6 \%$ in the osteoporotic group indicating a high bone turnover and a negative correlation with BMD.

CONCLUSION:The developed cost effective prototype kit for OC can be used as a diagnostic bone turnover marker for screening and management of osteoporosis.
KEY WORDS: Osteocalcin, ELISA, Diagnostic, bone turnover marker, Osteoporosis

\section{P46}

\section{Suppression of In Vitro And In Vivo Growth of Metastatic Melanoma Cells Upon Inhibition of Bmp Signalling Pathway}

Bhuvanesh Sukhla ${ }^{1}$ Kalal ${ }^{1,2}$, Vinitha Ramanath Pai ${ }^{3}$, Dinesh Upadhya ${ }^{4}$

1. Department of Biochemistry, Yenepoya Medical College, Yenepoya University, Mangaluru India,

2. Yenepoya Research Centre, Yenepoya University, Mangaluru, India.

3. Department of Biochemistry, Yenepoya Medical College, Yenepoya University, Mangaluru, India

4. Department of Anatomy, Kasturba Medical College, Manipal University, Manipal, India

INTRODUCTION: Studies have shown that bone morphogenetic protein (BMP) signaling pathways are deregulated in melanoma. Activation of BMP pathway is known to cause growth, migration and invasion of melanoma cells.

AIM: The aim of this study was to evaluate the effect of inhibition of BMP signaling pathway using a cell permeable small molecule inhibitor LDN193189 on the growth of melanoma cells under in vitro and in vivo conditions.

MATERIALS AND METHODS: The MTT [3-(4,5dimethylthiazol-2-yl)-2,5-diphenyl tetrazolium bromide] assay was done to test cytotoxicity of LDN-193189 (a potent inhibitor of BMP pathway) on human melanoma cell line, A375 and mouse melanoma cell line, B16F10. For in vivo study, 6-8 week old female C57BL/6 mice were inoculated subcutaneously with $1 \times 105$ B16F10 cells. Visible tumors were developed within next 5-10 days. The tumor size was measured using a vernier caliper on alternate days. The tumor volume was calculated by the formula: volume $(\mathrm{mm} 3)=(0.52) \mathrm{X}$ (length) $\mathrm{X}$ (width) $\mathrm{X}$ (height) (in $\mathrm{mm}$ ). When the tumor reached a size of $100 \mathrm{~mm} 3$, mice were randomly divided into two groups of 5 mice each. One group was injected 
with normal saline (control group) while another group was intraperitoneally injected with LDN-193189 at a dose of $3 \mathrm{mg} / \mathrm{kg}$ body weight twice daily for five consecutive days. Tumor size was measured on alternate days in both groups. Animal health was monitored and autopsy was performed after termination when either the tumor in control group reached $3000 \mathrm{~mm} 3$ or 30 days post tumor implantation.

RESULTS AND CONCLUSION: MTT assay revealed that, LDN-193189 inhibited the proliferation of A375 and B16F10 melanoma cells in a dose dependent manner with the IC50 value of 1.7 and $1.8 \mu \mathrm{M}$ respectively. The animal experiment showed that in comparison to control group tumors, twice daily injection of the LDN-193189 at $3 \mathrm{mg} / \mathrm{kg}$ body weight caused a significant reduction in tumor size, indicating that inhibition of BMP signaling pathway blocks melanoma growth.

KEY WORDS: BMPsignalling, Metastatic Melanoma, Tumor growth.

\section{P47}

Hypovitaminosis D and Pulmonary Tuberculosis: A Study In A Tertiary Care Institute of Mewat

Yuthika Agrawal $^{1}$, Vipin Goyal ${ }^{2}$, Nikita Singh ${ }^{1}$, Sangeeta B. Singh ${ }^{1}$

1. Department of Biochemistry, SHKM GMC, Nalhar, Mewat

2. Department of Chest and TB, SHKM GMC, Nalhar, Mewat

INTRODUCTION: Tuberculosis (TB) remains a major challenge to global public health. Vitamin D is an immunoregulatory hormone. Epidemiological evidence also suggests a link between vitamin D deficiency, serum calcium, serum phosphate and TB but the relation is not clear. Our study was aimed to assess the levels of serum Vitamin D, serum calcium and phosphorus in pulmonary tuberculosis (PTB) patients of Mewat.

MATERIAL AND METHODS: Blood samples were collected from 100 patients with newly diagnosed PTB (PTB-0)and PTB cases taking treatment or have completed treatment (PTB-Rx). Age and sex matched controls with same ethnic background, dietary habits and sun exposure were taken. Serum was analysed for vitamin $\mathrm{D}$, calcium and phosphorus.

RESULTS: Serum vitamin D was significantly decreased in both groups of cases (PTB- $0=13.9+5.8 \mathrm{ng} /$ $\mathrm{ml})(\mathrm{PTB}-\mathrm{Rx}=13.6+5.1 \mathrm{ng} / \mathrm{ml})$ as compared to controls $(29.5+6.5 \mathrm{ng} / \mathrm{ml}) .86 \%$ of cases had vitamin $\mathrm{D}$ value $<20 \mathrm{ng} / \mathrm{ml}$. Calcium was comparable in all the groups. Serum phosphorus was significantly increased in newly diagnosed PTB cases $(5.3+1.2 \mathrm{mg} / \mathrm{dl})$ but was within normophosphatemic limits as compared to controls $(4.1+0.4 \mathrm{mg} / \mathrm{dl})$ and was comparable with that of PTB cases on treatment or completed treatment $(5.2+1.7 \mathrm{mg} /$ dl).

CONCLUSION: Hypovitaminosis D was significantly associated with TB infection in our study. Since it has a role in Cell Mediated Immunity it could be one of the causes for the infection. Deficiency was noted in $86 \%$ of the cases, the importance of improving the nutrition and if required even supplementing vitamin D by food fortification

KEYWORDS:Vitamin D, tuberculosis, Mewat, normocalcemia, hyperphosphatemia

\section{P48}

Genetic Polymorphism of tumor necrosis factor alpha (TNF- $\alpha$ ) and tumor necrosis factor beta (TNF- $\beta$ ) genes and risk of oral pre cancer and cancer.

Om Prakash Gupta*1, Shainda Laeeq ${ }^{2}$, Shalini Gupta ${ }^{3}$

1. Associate Professor, Dept. of surgery, SUHMMH Medical College, Saharanpur, India.

2. Senior Research Scholar, Dept. of Oral Pathology \& Microbiology, King George's Medical University, Lucknow, India.

3. Professor, Dept. of Oral Pathology \& Microbiology, King George's Medical University, Lucknow, India.

$\mathrm{O}$ ral cancer is one of the eight most common cancers in the world and occurs more often in males in developing countries than developed countries. Oral 
precancereous lesions, a benign morphologically altered tissue that has a greater than normal risk of malignant transformation, such as leukoplakia etc., is also very common. Leukoplakia and submucous fibrosis are early indicators of damage to the oral mucosa with a transformation rate of $2-12 \%$ to frank malignancies. ?any environmental factors and genetic factors are implicated in the development of oral cancer . Several factors related to angiogenesis, inflammation and thrombosis have also been associated with oral oncogenesis. Such factors previously implicated in cancer, inflammation and thrombotic events are tumor necrosis factor alpha (TNF?) and beta (TNF-?), which are respectively encoded by TNF-?and TNF-?genes.

TNF-?-238G/A is a proinflammatory multifunctional cytokine produced by macrophages. It plays an important role in the regulation of immune response since its increase after traumatic injury generates a cytokine cascade resulting in activation, proliferation and hypertrophy of mononuclear and phagocytic cells. TNF?-238G/A has been implicated in the pathogenesis and progression of various malignancies. The biological activities of TNF-?-238G/A and the fact that its gene is located within the major histocompatibility complex have suggested that polymorphisms in this locus may be associated with autoimmune, infectious and neoplastic disorders. In light of the recently found contribution of inflammation-related factors to oral cancer, the possible correlation of tumor necrosis factor alpha and beta genes (TNF-? and TNF-?) with risk of oral cancer was investigated.A study was done in King George Medical University, Luck now ,India in whicha total 250 patients with Oral pre cancer \& cancer and 250 healthy volunteers were genotypes for the TNF-? (-238) G/A andTNF-?(252) A/G gene polymorphism. Genotypes were identified by polymerase chain reaction (PCR) restriction fragment length polymorphism(RFLP). Genotype frequencies were evaluated by Chi-square test and Odds ratio (OR) relative risk.

TNF-? (-238) G/A, TNF-?(-252) A/G polymorphism were significantly associated with Oral pre cancer \& cancer patients as compared to healthy volunteers.(GA, $\mathrm{p}=0.0050 *$, AG, $\mathrm{p}=0.497 *$ )

We found that the TNF-? (-238) G/A, TNF-?(-252) A/G polymorphism were significantly associated with Oral pre cancer \& cancer.

\section{P49}

\section{Association of Vitamin D Deficiency With Parathyroid Hormone Levels and Relevance To Renal Function and Insulin Resistance}

K.S.S. Sai Baba, Iyyapu Krishna Mohan, Siraj Ahmed Khan, Rachel Jacob.

Department of Biochemistry, Nizam's Institute of Medical Sciences, Panjagutta, Hyderabad-500082, Telangana.

INTRODUCTION: The rationale of the current study was to investigate whether the inverse association between vitamin $\mathrm{D}$ deficiency and parathyroid hormone levels is age-dependent and whether it has any role in modulating renal function and insulin resistance.

MATERIALS AND METHODS: To test this hypothesis, we have carried out ahospital based study on 848 subjects ( 558 men and 290 women) with the mean age of $50.9 \pm 15.9 \mathrm{y}$. IntactPTH and $25-\mathrm{OHD}$ levels were measured using commercially available kits (Simens Healthcare Diagnostics Inc., U.S.A) on ADVIA Centaur ${ }^{\circledR}$ XP Immunoassay system, which utilize sandwich and competitive immunoassays, respectively based on chemiluminometric technology.

RESULTS: Vitamin D deficiency was predominant irrespective of age group $(p=0.21)$ and gender $(p=0.12)$. An inverse association between vitamin D and PTH was observed $(r=-0.24)$ in middle age subjects $(\mathrm{p}=0.02)$. The data segregation based on plasma vitamin $\mathrm{D}$ levels which were $<20 \mathrm{ng} / \mathrm{ml}, 20.1-30 \mathrm{ng} / \mathrm{ml}$ and $>30 \mathrm{ng} / \mathrm{ml}$ confirmed the inverse association between vitamin D and PTH levels (ptrend: 0.007). Subjects with low plasma vitamin D and increased PTH exhibited elevated blood urea, serum creatinine and blood glucose. Subjects with Vitamin D deficiency showed a 3.03-folds (95\% CI: 2.264.07) and 2.09-fold (1.41-3.10) increased risk for diabetes and renal disease, respectively.

CONCLUSION: Based on the results of the present study, it is suggested that in those with vitamin D deficiency need to be evaluated for possible presence of 
renal dysfunction and insulin resistance in addition to assessing their PTH status.

KEY WORDS: Vitamin D, Parathyroid hormone, hyperparathyroidism, insulin resistance, diabetes.

\section{P50}

\section{Vitamin D Deficiency- Relationship with Hypothyroidisim}

Divya Shukla, Ashish Shukla

Department of Biochemistry, Subharti Medical College, Meerut, U.P., India.

INTRODUCTION: Vitamin D deficiency a common problem in the general population, and its role as an immune modulator has been recently emphasized. Low levels of vitamin $D$ have also been associated with thyroid diseases.However, the research of role of vitamin D in autoimmune and thyroid dysfunctions is not conclusive. In view of these we planned to conduct the hospital based study to find out the relationship between thyroid dysfunctions and vitamin D.

MATERIALS AND METHODS:Present study was conducted in the Department of Biochemistry, Chatrapati Shivaji Hospital, Subharti Medical College Meerut, U.P. India.Serum vitamin D (25-OH) levels were measured in 50 referred patients from Rural (24) \& Urban(26) aged 18- 55 years and compared with age sex matched 40( rural-20 and urban-20) were taken as control from the same area health centre of our college. Thyroid hormones (TSH, and fT4) levels were evaluated in all participants.

RESULT:The present study patient's concentration of vitamin D was significantly lower in hypothyroid patients $(12.36 \pm 3.29)$ than in controls $(57.59 \pm 14.48)$. Whereas fT4 levels were significantly lower $(6.01 \pm 2.54)$ and TSH levels were significantly high $(13.89 \pm 2.3)$ as compared to healthy controls (fT4 level 13.87 \pm 4.01 and TSH level $3.52 \pm 1.12$ ). Furthermore study highlights that vitamin $\mathrm{D}$ deficiency levels were significantly lower in females than their male counterpart.

CONCLUSIONS:Study highlights that patients with hypothyroidism suffer from hypovitaminosis D which is significantly associated with the degree and severity of the hypothyroidism. It is suggested that vitamin D deficiency is one of the potential factor in pathogenesis of autoimmune thyroid disorders.

KEY WORDS: Autoimmune thyroid disorders, Hypothyroidism, Vitamin D deficiency.

\section{P51}

\section{A Cross-Sectional Study of Insulin Resistance Between Urban and Rural Population In Western Uttar Pradesh India}

Yogesh Kumar Rai, Ashish Shukla

Department of Biochemistry, Subharti Medical College, Meerut, U.P., India.

INTRODUCTION:Insulin resistance is defined as decreased sensitivity in responsive metabolic actions of insulin to stimulate glucose utilization and inhibition of hepatic glucose production in diabetic patients.Type 2 diabetesis rapidly increasing in India causing nearly 5.8 million deaths per year. Primary reasons of insulin resistance in India are nutrition and lifestyle transitions.

MATERIAL AND METHOD:A community-based cross-sectional study was conducted in Chatrapati Shivaji Hospital, Subharti Medical College Meerut, U.P. India. A total of 78 subjects of type 2 diabetes ((42 Urban and 36 Rural subjects) having an age group of 40-65 years were participated from urban and rural area of Meerut District for determination of HOMA-estimated insulin resistance (HOMA-IR).

RESULT: The mean serum fasting glucose, fasting insulin, HOMA-IR values of type 2 diabetics in urban subjects were $182.16 \pm 17.15,9.37 \pm 2.13,4.21 \pm 0.09$ and in rural subjects were $173.87 \pm 15.54,7.34 \pm 1.63,3.15$ \pm 0.06 respectively. We found urban subjects had significantly higher fasting Glucose, fasting insulin and HOMA-IR values than rural subjects $(\mathrm{p}<0.001)$.

CONCLUSIONS:Urbanization influence insulin resistance to a greater extent in western uttar pradesh region. Thus it is concluded that insulin resistance in urban areas was associated with sedentary life style 
associated with higher dietary fat intake and lower occupational physical activity.

KEY WORDS:HOMA- IR, Insulin resistance, Type 2 diabetes.

\section{P52}

\section{Prevalence of Complications In Type 2 Diabetics of Jammu Region and Correlation With Duration of Diabetes}

Usha Kumari Gupta, Mridula Mahajan,

Sukhraj Kaur, Anjali Tikoo, R P Kudyar.

GMCAmritsar, Acharya Shri Chander college of Medical Sciences and Hospital (ASCOMS) Sidhra Jammu.

INTRODUCTION:Diabetes is the most challenging health problem of 21 st century. It is one of the most common non communicable diseases, preventable and treatable. It is an important cause of mortality and morbidity because of insidious and silent course of the disease and is increasing in numbers dramatically in low and middle income countries (WHO 2016). Prolonged hyperglycemia seen in uncontrolled diabetes mellitus is responsible for various macrovascular complications like (CAD, PVD and Stroke) and microvascular complications (like retinopathy, nephropathy and neuropathy). It has been reported that prevalence of diabetic complications is , neuropathy $24.6 \%$, CAD $23.6 \%$, nephropathy $21.1 \%$, retinopathy $16.6 \%$ and PAD $5.5 \%$ (Seema Abhiijeet Kaveshwar and Jon Cornwall, Australasian Medical Journal 2014; 7(1):45 48.)

AIMS AND OBJECTIVES: Keeping in view the prevalence of diabetic complications present study was planned to investigate the prevalence of complications in type 2 diabetics of Jammu region and relation to duration of diabetes.

MATERIAL AND METHODS: Study was conducted in department of biochemistry in collaboration with department of medicine ASCOMS Jammu. Individuals suffering from type 2 diabetes were included and investigated for various biochemical parameters. All the individuals were divided into groups depending on duration of diabetes and type of complications.
RESULTS AND CONCLUSION: It was observed, as the duration of diabetes increased the prevalence of micro and macrovascular complications increased from $1 \%$ to $21 \%$ (both micro and macrovascular complications) 2$15 \%$ microvascular complications and $2-14 \%$ macrovascular complications. Increase in duration of diabetes leads to increase in complications.

KEY WORDS: Type 2 diabetes, complications, microvascular, macrovascular and duration of diabetes.

\section{P 53}

Implication of Therapeutic Intervention on Putative Oxidative stress markers in Cervical Cancer

Suman Gautam ${ }^{1}$, A.A Mahdi ${ }^{1}$ M.L.B Bhatt ${ }^{3}$, Seema Mehrotra ${ }^{2}$, Ranjana Singh ${ }^{1}$, Komal S Pasi ${ }^{3}$, B Anjum ${ }^{1}$

1. Department of Biochemistry, King George's Medical University, Lucknow, India.

2. Department of Obstetrics \&Gynecology, King George's Medical University, Lucknow, India.

3. Department of Radiotherapy, King George's Medical University, Lucknow, India.

BACKGROUND: The present study was undertaken in patients of carcinoma cervix to evaluate the Implication of chemo radiotherapy on status of oxidative stress biomarker such as protein carbonyl, lipid hydro peroxide and antioxidants defence mechanism of melatonin and total thiol levels. Methodology: Patients were delivered radiotherapy by external beam radiotherapy (EBRT) followed by brachytherapy. All patients were given chemotherapy in the form of injection cisplatin. Blood samples were collected from patients as well as control before treatment and within 24 hours and six weeks after chemo radiotherapy. Newly diagnosed women with cervical cancer [ $\mathrm{N}=192], 30-65$ years of age and age- matched clinically healthy women $[\mathrm{N}=192]$ were included in this study. Result \&conclusion: The mean $\mathrm{LOOH}$ and PC levels in all three groups of cases were comparatively higher than controls. Further, the mean $\mathrm{LOOH}$ and PC levels increased in cases after the chemo-radiotherapy as compared to pre chemo- 
radiotherapy., $\mathrm{P}<0.001)$.Study also found that the mean Melatonin level and total thiol level in all three groups of cases lowered comparatively than controls. Further, the mean Melatonin and total thiol in cases decreased after the chemo-radiotherapy as compared to pre chemoradiotherapy. We suggest that plasma $\mathrm{PC}, \mathrm{LOOH}, \mathrm{PC}$, Melatonin and T-SH may serve as biomarkers for oxidative stress in patients with gynecological malignancy. A highly structured study with a larger sample size is required to establish the precise role of oxidative stress in pathobiology of cancer. Such oxidative biomarker can be used for diagnosis and prognosis of diseases in future.

KEYWORDS: chemotherapy, oxidative stress, radiotherapy, cervical cancer

\section{P54}

Variation In Level of Lipid Profiles In Patients of Hypothyroidism

Ashish Shukla ${ }^{1}$, Yogesh Kumar Rai ${ }^{2}$, Gautam Sarkar ${ }^{3}$

Department of Biochemistry, Subharti Medical

College, Meerut, U.P. India

INTRODUCTION:Thyroid hormones play an important role in regulating lipid metabolism, and thyroid dysfunction can result in lipid abnormalities which increase the risk of cardiovascular disease. The aim of our study was to assess the association between hypothyroidism and variation in serum lipid profile levels.

MATERIALANDMETHODS:The present study was conducted on 100hypothyroidism patients (study subjects) and 50 healthy normal (controls) in the Department of Biochemistry, Subharti Medical College, Meerut. Serum concentrations of thyroid hormones FT3, FT4 and TSH were measured by automated immunoassay system. Total cholesterol, Triglycerides and HDL-C were measured by fully automated system. Serum LDL-C and VLDL-C were calculated according to the Friedwald formula.Results of this prospective study wereanalyzed by SPSS 14.0.
RESULT:Study assessed 100 subjects including 43\% males and $57 \%$ females, with a mean age of $42.36 \pm 8.3$ years (ranged 30 - 60 years). Total cholesterol, triglycerides, LDL-C and VLDL-C level was significantly increased with decresed level of thyroid hormones (FT3 and FT4)in study group as compared with controls. Mean TC, TG and LDL-C and VLDL-C concentrations weresignificantly increased with increased level of serum TSH level. TSH level was associated negatively with, HDL-C and it increased with decresed level of HDL-C in study group.

CONCLUSION:Our study found an association between hypothyroidism and dyslipidemia. It is recommended that patient of dyslipidemia should get investigate for hypothyroidism.

KEYWORDS: Cardiovascular disease, Hypothyroidism, Lipid Profile, Thyroid Hormones

\section{P55}

Correlation Between Insulin Resistance and Leptin In Pre-Diabetics and Type 2 Diabetes Mellitus

\section{Parama Banerjee, Praveen Sharma}

Department of Biochemistry, All India Institute of Medical Sciences, Jodhpur

INTRODUCTION: The main pathology underlying Diabetes Mellitus is defective insulin secretion and function. Insulin resistance precedes development of diabetes. Leptin - an adipocytokine which regulates body weight has been found to play a role in insulin resistance.

AIM AND OBJECTIVE:The correlation between insulin resistance, beta cell function, obesity and altered leptin levels was studied.

MATERIALS AND METHODS: Participants of this cross-sectional study included 25 newly diagnosed Diabetics and 25 pre-diabeticswithout any comorbidities. They were age and sex matched with 25 clinically healthy controls. Study subjects of both sexes were aged between 18-65 years. Serum fasting insulin and leptin levels were estimated by ELISA. Other serum 
analytes including glucose, liver function test and renal function test parameters were estimated by autoanalyzer. Height, weight, waist circumference and hip circumference were noted. Body mass index, insulin resistance and beta cell function (using HOMA) were calculated.

RESULTS:Leptin levels correlated with insulin resistance values. Mean insulin resistance values were $6.61 \pm 3.54,4.19 \pm 2.09$, and $1.84 \pm 0.86$ for diabetics, pre-diabetics, and controls, respectively. Beta cell function values were $44.88 \pm 36.94,121.6 \pm 55.84$, and $129.4 \pm 64.07$ for diabetics, pre-diabetics, and controls, respectively. Leptin levels were highest among prediabetics, at $26.09 \pm 21.44 \mathrm{ng} / \mathrm{ml}$.

CONCLUSION: Correlations were observed between insulin resistance values and leptin levels in all three groups (diabetics, pre-diabetics, and controls). Additional investigations in larger study populations will be necessary to further explore this association.

KEYWORDS: Insulin resistance, leptin, diabetes, beta cell, HOMA

\section{P56}

FTO Gene Polymorphism and It's Association With Type 2 Diabetes Mellitus In North Indian Populations

$\underline{\text { Kahkashan Naaz }}^{*}$, Ipsita Chaudhary, Anil Kumar

Rama Medical College, Kanpur

AIM: The present study investigated the association of variant-rs9940128 (in intron 1) of the fat mass and obesity-associated (FTO) gene with type 2 diabetes mellitus (T2DM) in North Indian population.

METHODS: Unrelated study subjects (n-110 normal glucose-tolerant [NGT] controls and 110 cases [T2DM]) were randomly selected. Genotyping was done by the polymerase chain reaction-restriction fragment length polymorphism method and samples were sequenced to validate the genotypes obtained.

RESULTS: The polymorphism rs9940128 A/G of the FTO gene was found to be highly significant and thus was associated with T2DM .

CONCLUSION: Among North Indians, the rs9940128 A/G variants of the FTO gene was associated with T2DM.

KEYWORDS: FTO gene, Polymorphism, T2DM

\section{P57}

\section{Beneficial Effects on Health of Various Components of Nyctanthesarbor-Tristis}

$\underline{\text { Rajender Pal Singh }}{ }^{1}$, Subendu Sarkar

Department of Experimental Medicine and

Biotechnology, Post Graduate Institute of Medical

Education and Research, Chandigarh, India.

INTRODUCTION: Diabetes mellitus, hyperlipidaemia, hypertension, metabolic syndrome, etc. have received considerable current consideration because these conditions are widely prevalent around the globe and far from control.It has been recently highlighted from various laboratories that extracts of Nyctanthesarbortristis has beneficial effect on metabolic status particularly on lipid profile of experimental animals as well as human subjects.

AIMS AND OBJECTIVES: To derive a mechanism of action of components of Nyctanthesarbor-tristis on lipid profile of a human subject.

MATERIALAND METHODS: Relevant literature has been searched and a logical correlation has been made to hypothesize the mechanism of the hypolipedimic action of components of Nyctanthesarbor-tristis.

RESULTS AND CONCLUSION: It has been observed that various compounds present in the plant have the potential to inhibit lipid biosynthesis in human host and it is felt that extracts of the plant should be urgently considered for translational research.

KEYWORDS: Pharmacognosy, Hyperlipidemia, Diabetes mellitus. 


\section{P58}

Analysis of Antibody Response (Igm, Igg, Igg3) To Chikungunya Virus Using A Panel of Peptides Derived From Envelope Protein For Serodiagnosis

Priyanka Verma, D.N. Rao Alpana Sharma

Department of Biochemistry, All India Institute of

Medical Sciences, New Delhi-110029

BACKGROUND: Many epidemic outbreaks of Chikungunya fever (CHIKF) have been reported throughout the world including India after its reemergence in 2005. The immuno protective role of envelope proteins during Chikungunya virus (CHIKV) infection has been reported. With the aim of identifying the immunodominant epitopes within the envelope protein we investigated the detailed analysis of fine specificity of antibody response in different individuals during CHIKV infection.

METHODS: The peptides corresponding to the full length of E1, E2 and E3 proteins of S27 strain of CHIKV were synthesized and their seroreactivity with CHIKV positive patients' sera collected from different epidemic regions of India was determined using indirect ELISA.

RESULTS: The data analysis reveals many potent epitopes throughout the length of envelope E2 protein thus displaying it as the most promising antigen for diagnostic purpose. We found that the main IgG isotype response to envelope protein was predominantly of subclass IgG3. Interestingly, most of the epitopes were found to be conserved for detecting $\operatorname{IgM}, \mathrm{IgG}$ and $\mathrm{IgG} 3$ antibody response.

CONCLUSIONS: Peptides E2P3, E2P7, E2P16 and E2P17 were revealed as the most immunodominant peptides that together can form the basis for designing an accurate, economical and easy to synthesize a peptidebased immunodiagnostic for CHIKV. This study provides new and important insight into the humoral response generated by CHIKV S27 strain during the early phase of infection.

KEY WORDS: Chikungunya fever, E2P3, E2P7, E2P16 and $\mathrm{E} 2 \mathrm{P} 17$
P59

Secreted Frizzled Related Protein 4: A Novel Marker of Pancreatic Beta Cell Dysfunction In Prediabetic Obese Subjects.

$\underline{\text { Kumar Mukesh }}{ }^{1}$, Singh Neelima ${ }^{2}$, Sharma Ashish Kumar ${ }^{1}$

Department of Biochemistry, G.R. Medical College \& J.A.Group of Hospitals, Gwalior (M.P.)

INTRODUCTION: Secreted frizzled-related protein 4 (SFRP4) is a proinflammatory cytokine and a potent regulator of $\mathrm{Wnt} / \beta$ catenin signaling pathway. It causes apoptosis of $\beta$ cells and decreases expression of $\mathrm{Ca}^{+}$ channels thereby its concentration is increased in the obesity and impaired glucose tolerance.

AIMS AND OBJECTIVES:The objective of the present study was to investigate whether the SFRP4 concentration in serum is altered in obesity and impaired glucose tolerance.

MATERIALS AND METHODS: The present study includes 30 prediabetic obese subjects (IGT group) and 30 normal healthy individuals as controls. In all the cases and controls, Oral glucose tolerance tests were conducted and Serum SFRP4 levels were measured by ELISA.

RESULTS AND CONCLUSION: Levels of serum SFRP4 in the IGT group (cases) were significantly higher than those in the Control group $(\mathrm{P}<0.001)$. The serum SFRP4 levels were positively correlated with BMI, IGT and HOMA-IR and were negatively correlated with HDL-C. Our study provides evidence that the concentrations of serum SFRP4 inprediabetic obese subjects were increased and correlated closely with pancreatic beta cell dysfunction and obesity (chronic lowgrade inflammation). Hence, SFRP4 may participate in the development of pancreatic beta cell dysfunction in prediabetic obese subjects.

KEYWORDS: SFRP4, prediabetes, obesity, Wnt signaling, IGT, HOMA-IR 


\section{P60}

\section{Effect of Risperidone on Markers of Synaptic Plasticity and Inflammation In Schizophrenia Spectrum of Disorder}

$\underline{\text { Raghavi C, Hanumanthappa Nandeesha }}{ }^{1}$, Shivanand Kattimani $^{2}$

Department of Biochemistry ${ }^{1}$ and Psychiatry ${ }^{2}$, Jawaharlal Institute of Postgraduate Medical Education and Research, Puducherry, India

BACKGROUND:Schizophrenia is a neurodevelopmental disorder that affects around $1 \%$ of the population worldwide.Even though its pathophysiology remains elusive several studies have hypothesized that it could be the result of deregulation of synaptic plasticity and alterations of inflammatory processes.Neurotropin-3and Matrix metalloproteinase- 9 which play a role in the growth and differentiation of neurons are known to be associated with aberrant synaptic plasticity.Risperidone is an atypical antipsychotic drug used in the treatment of schizophrenia. Till date there are limited, but inconclusive data available in the literature about the effects of Risperidone on Matrix metalloproteinase9(MMP-9), Neurotropin-3(NT-3), Interleukin-10 (IL-10) and Interleukin-17(IL-17) levels in schizophrenia

AIM OF THE STUDY:The present study was designed to assess the effect of Risperidone on IL-10, IL-17,NT3,MMP-9 in patients with Schizophrenia spectrum of disorder.

METHODS:We conducted a longitudinal follow-up study on 70patients who are diagnosed as Schizophrenia spectrum of disorder as per DSM-V, aged 18 to 45 years, and to be subsequently treated with $4 \mathrm{mg}$ of Risperidone once daily.Serum IL-10,IL-17,NT-3,MMP-9 were compared at baseline and after 6 weeks following Risperidone treatment.

RESULTS: We found a significant increase in circulatory levels of NT-3 $(\mathrm{p}=<0.05)$ and a significant decrease in MMP-9 levels $(\mathrm{p}<0.05)$ in patients receiving Risperidone.There was no significant change in the circulatory levels of inflammatory markers before and after treatment. PANSS Score was also found to be significantly decreased $(\mathrm{p}<0.001)$ after 6 weeks of treatment with Risperidone

CONCLUSION: Risperidone reduces MMP-9 and increases NT-3 levels in patients with schizophrenia spectrum of disorder thereby reducing the disease severity.

KEY WORDS: Risperidone, MMP-9, NT-3, Schizophrenia

\section{P61}

Urinary Protein Excretion In Smoker and Non-Smoker Young Indian Males In NonSedentary Professions

Indu Saxena ${ }^{1}$, Monish Gupta ${ }^{2}$

All India Institute of Medical Sciences, Jodhpur-342005, Rajasthan

INTRODUCTION: Smoking causes renal damage and proteinuria in persons suffering from diabetes mellitus, cardiovascular diseases, and renal diseases, although its immediate effect on glomerular filtration rate in healthy non-smokers and smokers is debatable. Since the effects of BMI, age and physical activity levels have not been considered in many of the previous studies, this study was undertaken to determine if urinary protein excretion is different in young Indian smokers compared to nonsmokers in non-sedentary professions, and can the difference in protein excretion be determined using protein creatinine ratio from random spot urine samples to detect deterioration of renal status of healthy smokers.

METHODS: We obtained random spot urine from 103 healthy Indian men, engaged in occupations requiring physical activity, thus ensuring physical work of at least 8h per day (51 smokers, smoking 10-19 cigarettes/day for the past 5-10y) aged 20-30 y. Protein creatinine ratio, $\mathrm{PCR}$, was calculated as: $\mathrm{PCR}=$ urinary protein $(\mathrm{mg} / \mathrm{dl}) /$ urinary creatinine $(\mathrm{mg} / \mathrm{dl})$

RESULTS: There was no significant difference in BMI. Resting BP was higher in smokers. Our results indicate that a 5-10 years period of smoking was sufficient to produce elevation of blood pressure in 20-30 year old 
individuals. Protein excretion was higher in case of smokers indicating that even moderate smoking status of 5-10 years was sufficient to produce proteinuria in young individuals.

CONCLUSIONS: The comparison of basal parameters of smokers and non-smokers revealed slightly lower BMI in smokers, but the difference was not significant. The BMI is reported to decrease in new smokers, and the smoker population of this group had been smoking for an average of 7.2 years, therefore this effect was not visible. Urinary protein creatinine ratio was significantly higher in smokers, indicating that moderate smoking of less than 10 years duration causes increased protein excretion in healthy Indian male subjects of comparable age, BMI, and levels of physical activity. Thus proteinuria should be checked at regular intervals in chronic smokers for the early detection of renal damage.

This work was the part of ICMR-sts project.

KEY WORDS:Proteinuria, protein-creatinine ratio, smokers

\section{P62}

Assessment of Adiponectin and Hs-Crp In Patients of Coronary Artery Disease with and Without Type 2 Diabetes Mellitus

Mritunjay Kumar Mishra, Roshan Kumar Mahat

Department of Biochemistry, MGM's Medical College \& Hospital, Kamothe, Navi Mumbai, (M.H.), India

INTRODUCTION: Atherosclerosis of the coronary arteries cause coronary artery disease (CAD) and is the leading cause of mortality and morbidity among patients with type 2 diabetes mellitus. Secretion of various bioactive substances from adipose tissue, conceptualized as adipocytokines, has been widely recognized to play a contributory role in insulin resistance, diabetes and cardiovascular disease. In contrast to circulating inflammatory factors, adiponectin has anti-diabetic, antiatherogenic and anti-inflammatory properties. Lower adiponectin levels were found to be associated with obesity, type 2diabetes and CAD.
MATERIALS AND METHODS: A total of one hundred twenty (120) subjects (age group 25-70 years) of either sex were enrolled in this study. The study subjects were categorized into four groups i.e. Group I (30 normal healthy individuals), Group II (30 patients with diabetes mellitus having coronary artery disease), Group III (30 non-diabetic patients with coronary artery disease) and group IV (30 patients with diabetes mellitus without coronary artery disease). In all the groups, serum adiponectin and hs-CRP were estimated by quantitative sandwich enzyme immunoassay technique and immuneturbidimetric method respectively.

RESULTS: The mean serum level of adiponectin was decreased in all study groups where as there was increase in mean serum level of inflammatory marker i.e. hs-CRP in all study groups compared to controls and were statistically highly significant.

CONCLUSION:The results of present study indicate that there occurs alteration in the levels of inflammatory markers in CAD. Hence, early assessment of such markers can help to identify high risk patients.

KEY WORDS: Coronary artery disease, Adiponectin, hs-CRP, Diabetes Mellitus.

\section{P63}

\section{Status of Liver Enzymes In Prediabetic Young Adult Subjects}

Sharma Ashish Kumar, Singh Neelima, Kumar Mukesh

Department of Biochemistry, G.R. Medical College, Gwalior.

INTRODUCTION:The majority of the metabolic processes occur in the liver. An alteration in metabolic processes may results in development of many diseases like prediabetes. The liver enzymes are predominantly take part in the metabolic reactions of the carbohydrates, lipids and proteins. So, liver enzymes are the most important concern for development of prediabetes.

AIMS AND OBJECTIVES: To estimate the liver enzymes in prediabetic young adult subjects. The major 
objective of the study was the screening of prediabetes in general population

MATERIALS AND METHODS:The present study includes 50 prediabetic young adult subjects having age 18-38 and 50 age and gender matched healthy control subjects. The Impaired Glucose tolerance (IGT) and the liver enzymes (AST, ALT, ALP and GGT) were determined by fully automated Mindray BS-400 analyzer using GOD-POD end point method and kinetic method respectively.

RESULTS AND CONCLUSION:The serum level of ALT and GGT were statistically highly significant and serum ALP was statistically significant while serum AST was statistically non-significant in prediabetic young adult subjects as compared to age and gender matched healthy control subjects. The deranged liver enzymes (ALT, GGT and ALP) are mainly concern with the development of prediabetes. These liver enzymes can be used as a surrogate biomarker for prediabetes and related risk assessment in prediabetic young adult subjects.

KEYWORDS: liver enzymes, prediabetes, IGT.

\section{P64}

\section{PCSK9 Gene Variants In Patients with Coronary Artery Disease - A Pilot Study}

Ankita Bane $^{1}$, C.K. Ponde ${ }^{2}$, Rajesh Rajani ${ }^{2}$,

Sudhir Pillai ${ }^{2}$, Tester F. Ashavaid $1^{3}$

\section{Research Laboratories,}

2. Department of Cardiology,

3. Department of Biochemistry,

P.D. Hinduja Hospital and

Medical Research Centre, Mumbai

INTRODUCTION:Proprotein convertase subtilisin/ kexin type 9 (PCSK9) is a circulating protein involvedin lipid homeostasis. Gain-of-function mutations in PCSK9leadto higher plasma LDL-C concentrations and an increased risk of cardiovascular disease. Recently, PCSK9 has been projected as an emerging target for treatment of hypercholesterolemia and atherosclerosis.Here, we investigated the presence of
PCSK9 variants in angiographically proven coronary artery disease $(\mathrm{CAD})$ positive and $\mathrm{CAD}$ negative patients.

MATERIALS AND METHODS:Altogether, 60 patients (including 30 age and gender matched controls)qualified for this study. GenomicDNA was isolated from whole blood samples bya manual saltingout procedure. PCR products (150-300bp) were synthesized by amplifyingexons 1-5 of PCSK9, which were screened viathe PCR-SSCP technique(currently being standardised in-house to investigate the presenceof variants). PCR-SSCP results were further validated by Sanger DNA sequencing.

RESULTS: Mobility shifts were observed in exon 2 of 2 patients after SSCP analysis. DNA sequencing revealed a $\mathrm{C} / \mathrm{G}$ heterozygous transition at codon 86 of exon 2 in both patients. The presence of these variants in 2 patients within a small subset of 30 cases, while analysing limited exonic regions, hints at the possibility of mutations occurring at a higher frequency in a larger sample size.

CONCLUSION:These preliminary findingswarrant the need to replicate this study in a larger cohort using genotype analysis to determine the prevalence of PCSK9gene variants. In a highly heterogeneous Indian population consisting of diverse ethnic groups, mutational heterogeneity of PCSK9 is likely to exist.

KEY WORDS: PCSK9, LDL-cholesterol, coronary artery disease, hypercholesterolemia, SSCP

\section{P65}

\section{Correlation of Cardiac Markers With C-Reactive Protein In Patients of Acute Myocardial Infarction}

Vedika Rathore, Neelima Singh, Roshan Kumar Mahat, Chanchal Garg, Rakesh Kumar Shah

Department of Biochemistry, Gajra Raja Medical College, Gwalior

INTRODUCTION: Acute myocardial infarction (AMI) is a significant cause of morbidity and mortality worldwide, which results from occlusion of coronary artery. Various cardiac markers are used for the diagnosis 
of AMI. Recently, a new cardiac marker i.e. glycogen phosphorylase BB (GPBB) has been invented as an early marker of AMI. AMI is associated with profound systemic inflammatory response and inflammatory marker C-reactive protein (CRP) has been found to increase after AMI. Hence, the present study attempt to investigate the correlation between inflammatory marker i.e. C-reactive protein (CRP) and cardiac markers i.e. glycogen phosphorylase BB (GPBB), Myoglobin and CKMB in AMI patients.

MATERIALS AND METHODS: The present study includes 100 AMI patients and 100 normal healthy individuals as controls. In all the cases and controls, GPBB, myoglobin, CKMB and CRP were estimated. Myoglobin and GPBB were measured by ELISA where as CKMB and CRP were measured by diagnostic kits supplied by ERBA.

RESULTS: The mean levels of GPBB, Myoglobin, CKMB and CRPwere significantly increased in AMI cases as compared to controls. Also, there were significant positive correlations between all the studied cardiac markers and CRP in AMI cases.

CONCLUSION: The positive correlation between CRP and cardiac markers in AMI indicates that there occurs inflammation in acute myocardial infarction patients. Hence, it is concluded that CRP is a risk marker of AMI and can be used for diagnostic purpose in patients of AMI.

KEYWORDS: Acute myocardial infarction, Glycogen phosphorylase BB, Myoglobin, CKMB, C-reactive protein

\section{P66}

\section{Biochemical Parameters In Neuroblastoma}

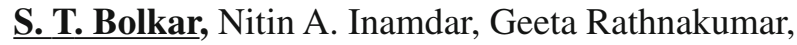
Mukta Ramadwar, B.J. Shinde, N. D. Kamble

Dept of Biochemistry, Tata Memorial Hospital, Parel

INTRODUCTION:The most common types of solid tumors in children include brain tumors, neuroblastoma, rhabdomyosarcoma, wilms tumor, and osteosarcoma.
Neuroblastoma is the second most common solid tumour in childhood and it makes up $8 \%$ of the total number of children's cancers. Most children who get this cancer are younger than five years old. Apart from the biochemical parameters, routinely used parameter like Vanillyl Mandellic Acid (VMA) for the patients with neuroblastoma along with neuron specific enolase(NSE), ferritin (FER), lactate dehydrogenase (LDH), and gamma glutamyl transferase (GGT) were also studied to assess the utility in diagnosing the patients with neuroblastoma.

AIM OF THE STUDY:The present study was undertaken to evaluate the utility of urinary VMA and other markers like NSE, Ferritin, LDH and GGT to assist in the diagnosis of neuroblastoma.

MATERIALAND METHODS: The study involved 40 healthy controls and 91 untreated cases of histologically proved neuroblastoma referred to the Tata Memorial Hospital for further management and treatment.

RESULTS AND CONCLUSION: The urinary VMA levelsand the serum levels of NSE, LDH had increased significantly, $\mathrm{p}<0.0001$ whereas the GGT did not show significance at $\mathrm{p}<0.0001$, as compared to the normal healthy group. Serum NSE, FER, LDH along with urinary VMA could be of help in diagnosing the patients with neuroblastoma.

KEY WORDS: neuroblastoma, NSE, Ferritin, LDH, GGT

\section{P67}

Association Between Serum 25 (OH) D Levels, Interleukin-6 And Risk of Metabolic Syndrome Among Women In A Tertiary Care Hospital

Deepa Gupta $^{1}$, Aditi Gupta ${ }^{1}$, Amit Kumar Shrivastav ${ }^{1}$, Kamini Vinayak $^{1}$, Arun Raizada ${ }^{1}$, Neelima Singh ${ }^{2}$

1. Department of Biochemistry, Medanta-The Medicity, Gurgaon, Department of Biochemistry, G.R. Medical College, Gwalior (M.P.)

INTRODUCTION:25(OH) D plays an important role in bone metabolism and seems to have an anti- 
inflammatory and immune-modulating properties. The immune-modulatory effects of $25(\mathrm{OH}) \mathrm{D}$ and its inverse link with inflammation draw more attention towards metabolic syndrome (MS). Therefore, the importance of $25(\mathrm{OH})$ D levels and pro-inflammatory marker interleukin-6 (IL-6), as a risk factor for the occurrence of MS among women has been focused in the present study.

AIMS AND OBJECTIVES: To find out the association between serum $25(\mathrm{OH})$ D levels, IL-6 and risk of MS among women in a tertiary care hospital

MATERIALS AND METHODS: A total of 332 women who were enrolled for executive health checkup at Medanta-The Medicity, Gurgaon were consented voluntarily and included in the study. Demographic information was achieved by some questionnaires. Anthropometric measurements were recorded by standard methods and tools. Serum $25(\mathrm{OH})$ D was assessed by chemiluminescence and IL- 6 was estimated by ELISA method. Biochemical parameters were analyzed by fully automated vitros- 56002355 analyzer. Vitamin D deficiency was defined by international endocrine society and MS was defined according to NCEP-ATP III for Asian Indian.

RESULTS AND CONCLUSION: Vitamin D deficiency were highly prevalent in women with MS as compared with women without MS. Statistically significant inverse association were found between low level of serum $25(\mathrm{OH})$ D levels and IL-6 $(\mathrm{p}<0.01)$. Hypovitaminosis D was associated with increased level of IL6 and risk of developing MS. By improving Vitamin D status would be useful in improving the risk of having MS among women.

\section{P68}

\section{The Study of Prognostic Factors in Multiple Myeloma}

N. D. Kamble, Nitin A. Inamdar, Geeta Rathnakumar, Mukta Ramadwar, B.J. Shinde, S.T. Bolkar

Dept of Biochemistry, Tata Memorial Hospital, Parel

INTRODUCTION:Multiple Myeloma (MM) is a cancer of plasma cells. The simplest measure of prognosis in $\mathrm{MM}$ is based on Serum $\mathrm{M}$ protein concentration on Protein Electrophoresis, beta-2microglobulin (?2M), lactate dehydrogenase (LDH) and albumin.

AIM OF THE STUDY: To determine the role of prognostic factors in newly diagnosed multiple myeloma patients.

MATERIALS AND METHOD: The material for the study comprised of 87 normal healthy controls and 90 newly diagnosed multiple myeloma in Tata Memorial Hospital, Parel with no prior treatment. Analysis of serum creatinine, calcium, phosphorus, LDH, Total proteins, Albumin, and B2M was done by photometry on Beckman coulter AU 640 autoanalyzer. Immunoglobulines IgG, IgA, IgM, Kappa light chain and Lambda light chains were estimated by nephelometry on Beckman coulter Immage 800 Immunoassay analyzer. Protein electrophoresis and Immunofixation was done using agarose gel on Interlab G26 is a fully automated clinical electrophoresis system.

RESULTS: In a multivariate analysis, serum beta 2 microglobulin levels and stage were the most significant prognostic factor even though serum creatinine, albumin and LDH levels were found significant at a $p<0.05$. We conclude that beta 2 microglobulin level is one of the most useful prognostic factors in patients with multiple myeloma.

CONCLUSION: Levels of $\beta 2 \mathrm{~m}$, creatinine, $\mathrm{LDH}$ and $\mathrm{M}$ band concentration declined during the treatment (chemotherapy), hence we conclude that these results will help the clinicians to tailor-made the chemotherapy doses for betterment of patients and improve survival rate in MM patients.

KEY WORDS: Multiple myeloma, prognostics, albumin, beta 2 microglobulin

\section{P69}

Comparison of Beta-2-Microglobulin- B2M and CD56 Expression In Multiple Myeloma

Geeta Rathnakumar, Nitin A. Inamdar, 
Mukta Ramadwar, B.J. Shinde, N.D. Kamble,

S.T. Bolkar

Tata Memorial Hospital, Parel, Mumbai.

INTRODUCTION: Multiple myeloma (MM) is a type of blood cancer where uncontrolled cell division of plasma cell takes place, producing monoclonal antibodies in greater concentration. Serum Protein Electrophoresis is commonly used to identify patients with multiple myeloma and other disorders of serum protein. Electrophoresis separates proteins based on their physical properties, and the subsets of these proteins are used in interpreting the results.

The simplest measure of prognosis in MM is based on $\beta 2 \mathrm{~m}$ and albumin. Higher levels of $\beta 2 \mathrm{~m}$ and lower levels of albumin are associated with a poorer prognosis.

Expression of CD56 is commonly used to identify abnormal plasma cells, since plasma cells from the bone marrow of healthy individual are negative for the CD56 antigen.

AIM AND OBJECTIVE: To compare $\beta 2 \mathrm{~m}$ and CD 56 expression with other parameters and to correlate these markers to improve the diagnostic utilities of them.

MATERIALS AND METHOD:50 (18 female and 32 male with age group 35-74 years) newly diagnosed and untreated patients with MM were selected. ?2m was estimated using turbidimetry method and CD56 expression using flow cytometer. Serum protein electrophoresis (SPE) was done on fully automated electrophoresis analyzer Interlab G26. Quantitation of Immunoglobulin's were done with nephelometry method on Immage 800 analyzer.

CONCLUSION: $\beta 2 \mathrm{~m}$ with and CD56 expression may be a useful additional tool for precise and early diagnosis, with Serum Protein Elctrophoresis. As good correlation exists between $\beta 2 \mathrm{~m}$ \& CD56 expression. with $90 \%$ specificity it may help in prognosis of MM.

KEYWORDS:Multiple myeloma, CD56, $\beta 2 \mathrm{~m}$, albumin

\section{P70}

\section{Thyroid Profile and Risk of Spontaneous Abortion}

Manohar A, Gahlawat P*, Singh A, Nanda S,*

Kharb S

Department of Biochemistry, Obstetrics and

Gynecology

*, Pt. B.D. Sharma PGIMS, Rohtak, Department of

Biochemistry, Pt. B.D. Sharma University of Health

Sciences, Rohtak

BACKGROUND: Pregnancy is associated with significant but reversible changes in thyroid function. Abnormal thyroid hormone levels could give rise to malplacentation that underlies the association between maternal thyroid dysfunction and adverse obstetric outcome such as fetal brain damage, preterm births and fetal death.

OBJECTIVE OF THE STUDY: Present study was planned to predict the risk of abortion in pregnancy by studying the relation between thyroid profile and rate of abortion.

MATERIALS AND METHODS: This prospective observational study was carried out on 100 pregnant women with singleton pregnancy of 6-12 weeks of gestation and they were divided into three groups namely, group 1 ( $n=30$,control group) normal pregnant women with no history of abortion; group $2(n=35)$ included pregnant females with history of previous abortion; and group $3(n=35)$ comprised of pregnant women coming with chief complaint of bleeding per vaginum. Five $\mathrm{ml}$ blood was taken and serum was separated using centrifugation. T3 (triiodothyronine), T4 (thyroxine) and TSH (thyroid stimulating hormone) levels were estimated by chemiluminiscence.

RESULTS: Thyroid stimulating hormone levels were significantly higher in first trimester in women who had spontaneous abortion as compared to pregnant women who had successful continuation of pregnancy. TSH was strongly associated with abortion in first trimester in all the three groups.

CONCLUSION: Screening of thyroid disorders in early 
pregnancy has clinical significance and adequate thyroxine replacement therapy if given in cases of hypothyroidism would help to reduce the risk of miscarriage in these women.

KEY WORDS: Thyroid, gestational age, pregnancy, abortion

\section{P71}

\section{Biochemical Analysis of Employees Attending Cardiac Camp of Supreme Court of India}

\author{
Rajni Saijpaul*, Shyama Gupta**, Manoj Saini*** \\ *Senior Specialist, Department of Biochemistry, \\ VMMC \&Safdarjung Hospital, New Delhi \\ ** CMO I/C, Supreme Court of India \\ *** Senior Medical Officer, Supreme court of India
}

INTRODUCTION:Lifestyle diseases like Hypertension, Diabetes Mellitus and Atherosclerotic disease is the biggest killer of mankind in the present day. India is undergoing a rapid health transition with rising burden of coronary heart disease (CHD). The aim of the Camp was to screen the employees of Supreme Court of India clinically and by biochemical analysis pertinent to lifestyle related disorders and to present the prevalence of lifestyle diseases among the Staff and treatment needed

MATERIAL \& METHODS: The program was aimed with an intention to make all employees not only become aware of their health status but also understand and realize the importance of need for regular preventive check-ups and taking early corrective measures to impede the bigger health problems.

The Cardiac Health Check-up Camp was held from 13th December 2014 to 21st January 2015 (between 08: 00 hrs to $17: 00$ hrs with prior online Registration for the Camp.

The employees were screened for Fasting Blood Sugar,HBA1c, Lipid Profile, LFT, KFT

RESULTS: A total number of employees were 544 during the screening program. Out of 544 patients screened a staggering no225 patients had abnormal glucose metabolism (41.3\%). Out of these 84 were diabetics (15.4\%) and remaining 141(25.9\%) had Impaired Fasting Glucose levels. Males were are at more risk of having abnormal glucose metabolism $(\mathrm{N}=189$, $84 \%)$. Out of 544 people screened 160 (29.4\%) had high Total cholesterol levels, 125(22.9\%) had increased Serum Triglyceride levels, and $306(56.2 \%)$ had reduced serum HDL cholesterol levels.

\section{CONCLUSION:}

1. $41.3 \%$ of screened employees had an abnormal glucose metabolism

2. More male employees had an abnormal glucose metabolism $(84 \%)$

3. Nearly $1 / 3$ rd of the employees had an increased Serum Total cholesterol

4. Nearly $1 / 4$ th of the employees had an increased Serum Triglyceride levels

5. More than $50 \%$ of the employees had a reduced Serum HDL levels

KEYWORDS: Lifestyle diseases, Glucose metabolism, Lipid Profile, HDL-cholesterol, Triglycerides

\section{P72}

Comparison of Cardiovascular Risk Between Diabetic \& Non-Diabetic End Stage Renal Disease (ESRD) Patients Undergoing Haemodialysis

Priya. K, Sangeeta. K

Teerthanker Mahaveer Medical College \& Research Centre, Moradabad, Uttar Pradesh

INTRODUCTION: This study proposes to see whether end stage renal disease (ESRD) patients undergoing haemodialysis with additional diabetes mellitus exhibit increased cardiovascular risk markers in comparison to end stage renal disease (ESRD) patients undergoing haemodialysis without diabetes mellitus.

OBJECTIVES: In this study,an attempt has been made to compare the Lipid profile and fibrinogen as 
inflammatory markers for assessing cardiovascular risk between diabetic and non-diabetic end stage renal disease (ESRD) patients undergoing maintenance haemodialysis.

MATERIAL AND METHODS: We enrolled 32 patients of both sex with diabetes mellitus end stage renal disease undergoing haemodialysis and 32 non- diabetic end stage renal disease undergoing haemodialysis for this study.

RESULTS:Our data came outwith a significant increase in the parameters like plasma fibrinogen, plasma glucose concentration, atherogenic index (TC/HDLc), serum lipid profile (TC, LDLc, TG, VLDLc) and decrease in serum HDLc in diabetic ESRD patients in comparison to non diabetic ESRD patients undergoing maintenance haemodialysis.

CONCLUSION: Our study conclude that elevation in plasma fibrinogen level, serum lipid profile and atherogenic index (TC/HDLc) in diabetic ESRD patients undergoing maintenance haemodialysis which indicates higher risk of adverse cardiac events and atherosclerosis as a major cause of coronary heart disease in diabetic ESRD patients.

KEY WORDS: Plasma Fibrinogen, Haemodialysis, Diabetes

\section{P73}

Correlation Between The Insulin Resistance and Treatment with Either Metformin or Sulfonylurea In Type 2 Diabetes Mellitus :- A Prospective Observational Study

Pragati Srivastava $^{1}$, Ashok Shenoy $\mathrm{K}^{2}$, Mukta. N. Chowta $^{3}$, Caron. S.M. D'Silva ${ }^{4}$, Bhuvaneshwari Sudershan ${ }^{5}$

\section{Post Graduate Department of Pharmacology,} Kasturba Medical College Mangalore, Manipal University, Manipal,

2. Department of Pharmacology, Kasturba Medical College Mangalore, Manipal University, Manipal,

3. Department of Pharmacology, Kasturba Medical College Mangalore, Manipal University, Manipal 4.Medical Pharmacology, Biostatistics and
Epidemiology, St. Matthew's University, Grand Cayman

4. Post Graduate Department of Pharmacology, Kasturba Medical College Mangalore, Manipal University, Manipal

INTRODUCTION: Chronic hyperglycemia is due to inadequate production of insulin and/or "resistance" to the glucose lowering action of insulin. The main aim of this study is to determine the correlation between the insulin resistance and treatment with either metformin or sulfonylurea in newly diagnosed type 2 diabetes mellitus. The study also compared the degree of insulin resistance before and after treatment with either metformin or sulfonylureas in newly diagnosed type 2 diabetes mellitus

MATERIALS AND METHODS: It was a prospective observational study done in patients of newly diagnosed cases of Type 2 Diabetes Mellitus. A total of 60 patients were included in two groups of 30 patients each to receive either metformin or sulfonylurea as a monotherapy for a period of 3 months. Routine blood investigation and HOMA-IR were done at baseline and at the end of 3 months.

RESULTS: In the metformin group statistically significant decrease in the blood glucose level in both fasting and post prandial state $(p$ value $<0.05$ ) was observed whereas insignificant difference was observed for the other parameters: $\mathrm{HbAlc}$, serum insulin and HOMA-IR. Correlation between the baseline insulin resistance and glycemic control was also seen to be significant in each group ( $\mathrm{p}$ value $<0.05$ ).

CONCLUSION: Metformin as a monotherapy has proven to have a significant response in lowering the blood glucose levels in both fasting and postprandial state as compared to sulfonylurea as a monotherapy. The effect of both the drugs in lowering the insulin resistance is comparable when given as a monotherapy and showed positive correlation.

KEY WORDS:Insulin resistance, HOMA-IR, metformin, sulfonylurea 


\section{P74}

\section{Dyslipidemia And Reduced Estrogen Level As Predictors of Cardiovascular Disease In Post Menopausal Hypertensive Women}

Rakesh Kumar Shah, Neelima Singh, Roshan Kumar Mahat, Vedika Rathore, Prerna Vyas Gokhale

Department of Biochemistry, GR Medical College, Gwalior

INTRODUCTION: Menopause is the permanent cessation of menstruation and prevalence of hypertension increases after menopause. Menopause leads to changes in lipid profile due to hormonal changes related to menopause and may increase the development of cardiovascular disease (CVD). Hence, the present study attempts to compare the levels of lipid parameters and estrogen between pre and post-menopausal hypertensive women.

MATERIALS AND METHODS: 50 post-menopausal hypertensive women and 50 pre-menopausal hypertensive women were recruited for the study. In both pre and post-menopausal hypertensive women, serum estrogen and serum lipid profile [total cholesterol (TC), triglyceride (TG), HDL Cholesterol, LDL-Cholesterol and VLDL-Cholesterol] were measured. The comparison of parameters between both pre and post-menopausal hypertensive women was carried out using student $t$ test.

RESULT: There was significant increase in serum Total Cholesterol (TC), Triglycerides (TG), LDL-cholesterol and VLDL-cholesterol levels in post-menopausal hypertensive women as compared to pre-menopausal hypertensive women. HDL-cholesterol and estrogen levels were significantly decreased in post-menopausal hypertensive women compared to pre-menopausal hypertensive women.

CONCLUSION: The alteration of lipid profile in post menopausal hypertensive women indicate their higher risk for the development of cardiovascular disease in future. However, further studies are needed to accept the concept.
KEY WORDS: Post-menopausal women, Lipid profile, estrogen, cardiovascular disease

\section{P75}

Relevance of MTHFR Gene Mutations In Hyperhomocysteinemia Thrombotic Patients - A Study From Western India

\section{Minal Paradkar ${ }^{1}$, Balkrishna Padate ${ }^{2}$ and}

Tester Ashavaid ${ }^{1}$

\section{Department of Laboratory Medicine and Biochemistry section,}

\section{Hemat-Oncology and Bone Marrow transplant}

INTRODUCTION: Hyperhomocysteinemia (HCA) known to be associated with increased thrombotic tendency has been considered as a risk factor for coronary artery disease and atherosclerosis. HCA is known to be due to genetic factors like mutation in methylene tetrahydrofolate reductase (MTHFR) genes. Association of MTHFR C677T and A1298C genes with cardiovascular diseases have been reported however, the frequency distribution of both these mutations shows ethnic heterogeneity.

AIMS AND OBJECTIVES: Considering the implication of MTHFR gene in complex disorders coupled with its high heterogeneity and ethnic differences, the present study was undertaken to determine MTHFR genetic variants in thrombosis patients from Western India.

MATERIALS AND METHODS: 124 thrombosis patients were included in the study and DNA was extracted by modified Miller etal protocol and C677T and A1298C mutations were detected by ARMS PCR.

RESULTS AND CONCLUSION: The minor allele frequency (MAF) for C677T allele was $16.1 \%$ and for A1298C allele was $37.5 \%$. In case of C677T mutation the homocysteine (Hcys) levels were significantly higher in TT alleles (64.65) as compared to CC alleles (32.43) and CT alleles (30.54). In case of A1298C mutations the Hcys levels did not show significant differences. Similar trend was also observed in patient groups with DVT and brain thrombosis. The haplotype analysis also showed 
significantly higher levels of Hcys in T677T/A1298A as compared other haplotypes. The Hcys levels did not showed significant differences in compound heterozygous C677T/A1298C haplotypes. Thus, in this study amongst various MTHFR genetic variants studied only homozygous TT alleles showed significance with HCA.

KEY WORDS: Hyperhomocysteinemia, MTHFR, thrombosis

\section{P76}

\section{Evaluation of Serum Lipid Profile In Ovarian Cancer Patients At A Tertiary Care Centre}

Priti Rani, Richa Madhawi, Santosh Kumar, Rekha Kumari, AnandS Saran, Uday Kumar

Department of Biochemistry, I.G.I.M.S, Patna, Bihar,India

OBJECTIVE: The aim of this study was to compare serum lipid profiles: total cholesterol (TC), triglyceride (TG), high density lipoprotein cholesterol (HDL-c), low density lipoprotein cholesterol (LDL-c) and very low density lipoprotein cholesterol (VLDL-c) and lipid ratio among ovarian cancer (OC) patients and healthy controls.

MATERIALS AND METHODS: Total 100 subjects were enrolled in this study, out of which 50 were clinically and histologically provencases of ovarian carcinoma and 50 healthy women were taken as control group. Plasma levels of Total Cholesterol, Triglyceride High Density Lipoproteins and Low Density Lipoproteins were determined using a fully automated analyzer. VLDL was calculated by Friedewald's formula.

RESULTS: In our study, it was found that the mean serum cholesterol, TGs, LDL-c and VLDL levels in Ovarian cancer patients was higher as compared to the controls. The HDL level is higher in controls as compared to study group. TGs, HDL-c VLDL were found highly significant in Ovarian Cancer patients as compared to controls. The Ratio of TC/HDL, LDL/HDL, HDL/LDL was also found significantly higher in Ovarian Cancer patients as compared to controls.
CONCLUSIONS: The results of the present study shows that serum lipid levels, except HDL-cholesterol, are increased in Ovarian Cancer patients. As there is an alteration in the serum lipid profile during ovarian cancers, it may be helpful for prognosis of the disease.

KEY WORDS: lipid profiles, total cholesterol, ovarian cancer, VLDL

P77

\section{Evaluating The Effect of Hypoxia on Differentiation Capacity of Human Fetal Neural Stem Cells In Vitro}

Dey $\mathbf{D}^{1 *}$, Singhal $\mathrm{C}^{2}$, Manhas J, Sharma JB, Kumar J, Chattopadhyay P, Seth $\mathrm{P}^{2}$, Sen $\mathrm{S}^{1}$

1. Department of Biochemistry, All India Institute of Medical Sciences, New Delhi

2. Department of Molecular and Cellular

Neuroscience, National Brain Research Centre,

Manesar, Haryana.

INTRODUCTION: Neural stem cells (NSCs) can differentiate into neuronal and glial lineages. Studies indicate that "physiological hypoxic conditions" strongly influence their growth and differentiation. We wanted to evaluate the effect of hypoxia on the differentiation capacity of fetal neural stem cells (FNSCs).

METHOD: Human fetal brains were obtained from aborted fetuses of mothers undergoing medical termination of pregnancy between 12-20 weeks of gestation $(n=3)$ after Institutional Ethics Committee clearance and informed consent. FNSCs were isolated and grown in NSC growth media. These cells were then characterized by immunocytochemistry using Nestin and Sox2. FNSCs were exposed to various grades of hypoxia $(20 \%, 6 \%, 2 \%$ and $0.2 \%)$ mimicking in vivo oxygen concentrations, for 48 hours, and their respective RNA were isolated. Exposure to hypoxia was confirmed by appearance of hypoxia markers (CA9, VEGF AND PGK1) with the help of Q-PCR. After exposing the FNSCs to hypoxia, their commitment towards neuronal or glial lineages were evaluated with specific markers, using Q-PCR. 
RESULT: Nestin and Sox2 expression confirmed the identity of FNSCs. Appearance of hypoxia markers (CA9, VEGF AND PGK1) were observed. On exposure to hypoxia, expression of Nestin and PAX6 (stem cell markers) were maintained in the FNSCs. Appearance of neuronal commitment markers (DCX and ACSL1) were observed at lower oxygen concentrations. Interestingly, glial lineage marker, NG2 was not expressed, but Olig-1 and Olig-2 expression were observed. GFAP expression could not be detected.

DISCUSSION: Stem cell markers (Nestin and PAX6) were maintained when FNSCs were exposed to moderate hypoxia (2\%). However, when FNSCs were exposed to increased hypoxia $(0.2 \%)$, these cells initiated a commitment towards the neuronal lineage, evident by increased expression DCX and ACSL1. Our findings suggest that moderate hypoxia is important to maintain the undifferentiated neural stem cell character of FNSCs. However, increase in hypoxia can modulate FNSCs to undergo neuronal differentiation.

KEY WORDS: Neural stem cells, hypoxia, fetal neural stem cells, CA9, VEGF AND PGK1

\section{P78}

\section{Assessment of Parathyroid Hormone, C-Reactive Protein And Interleukin-6 Levels In Patients With Urolithiasis.}

Senthil Kumar GP, Kalyani $C^{1}$, Manikandan $\mathrm{R}^{2}$

1. Department of Biochemistry, Jawaharlal Institute of Postgraduate Medical Education and Research (JIPMER), Puducherry-605 006, India.1

2. Department of Urology, JIPMER, Puducherry.

INTRODUCTION:Vitamin D is important in maintaining calcium homeostasis, inflammatory marker,parathyroid hormone its effect on stone formation are still not clear.

OBJECTIVES: To assess the serum levels of PTH, vitamin D and IL-6, urinary and serum calcium and phosphorus levels in patients with renal calculi and to compare to control group.
MATERIAL AND METHODS: 41 renal calculi diagnosed patients were selected as cases and compare to health control. Routine biochemical parameters such as Serum levels of vitamin D, Parathyroid hormone, IL-6 were estimated.

RESULTS: There was a statistical significant difference in the serum levels of vitamin D, Parathyroid hormone, interleukin-6 serum calcium and urinary calcium and phosphorous levels between the cases and the control. There was negative correlation between the levels of Parathyroid hormone and vitamin D.

CONCLUSION:There was vitamin D insufficiency increased levels of PTH and IL-6 abnormalities in patients presenting with renal calculi.

KEYWORDS: Vitamin D, Parathyroid hormone, IL-6

\section{P79}

Study of Red Cell Distribution Width Levels and Its Association with Glycated Hemoglobin In Type 2 Diabetes Mellitus Patients.

Somnath Bag*, Renuka. P.

Department of Biochemistry, SRM Medical College

Hospital \& Research Centre, Kattankulathur.

INTRODUCTION: Diabetes mellitus is one of the most common and complicated metabolic disorders. Incidence of type 2 diabetes mellitus is continuously increasing due to overweight and inactive life style. Hyperglycemia can decrease the RBC lifespan. Red cell distribution width (RDW) is the measurement of red cell variability and it can alter the RBC lifespan. This study aims to establish a relation between glycated hemoglobin and RDW in type 2 diabetes mellitus.

MATERIALS AND METHODS: HbA1c, FPG and RDW values from 100 type 2 diabetic patients and 100 healthy controls were collected retrospectively from Master Health Checkup database of SRM Medical College Hospital \& Research Centre, Kattankulathur. A Pearson correlation coefficient was computed to assess the relationship between $\mathrm{HbA1c} \&$ RDW. 
RESULTS: Red cell distribution width (RDW) was significantly higher in diabetic patients than in controls subjects $(\mathrm{p}<0.05)$. It was also higher in patients with uncontrolled glycemia $(\mathrm{HbA} 1 \mathrm{c}>7 \%)$ than those with good control (HbA1c ? 7\%; $\mathrm{P}<0.05)$.

CONCLUSION: Elevated RDW in diabetic subjects reflects the presence of anisocytosis which could be due to impaired erythropoiesis and RBC destruction caused as a result of oxidative stress. Hyperglycemia alters the $\mathrm{RBC}$ life span which is indicated by RDW.

KEYWORDS: Red cell distribution width, Type 2 Diabetes mellitus, HbA1c.

\section{P80}

Effect of Methotrexate Monotherapy on T-Cell Sub-Sets In Peripheral Circulation In Psoriasis

M Priyadarssini, Medha Rajappa*,

Laxmisha Chandrashekar, DM Thappa

Department of Biochemistry and Department of Dermatology, Jawaharlal Institute of Postgraduate Medical Education and Research, Puducherry, India

INTRODUCTION: Psoriasis is a T-helper (Th)-1/Th17mediated chronic inflammatory disease. There is an increased population of T-helper cells in the skin lesions as well as in the peripheral circulation in psoriasis. Systemic methotrexate is one of the effective treatment modalities for severe psoriasis. However, the effect of methotrexate on different T-cell subsets is understudied.

OBJECTIVES: To study the effect of methotrexate on psoriatic T-cell profile.

MATERIALS AND METHODS: It is a follow-up study involving 50 patients with severe psoriasis on systemic methotrexate treatment for 12 weeks. $5 \mathrm{ml}$ of blood was collected from the patients, PBMCs were isolated and T cell phenotyping was done by flow cytometry.

RESULTS: With methotrexate, we observed an increase in the percentages of Th2/T-reg cells, and a relative decrease in the percentages of Th1/Th17 cells, along with a significant reduction in the median PASI score.
CONCLUSION: Methotrexate helps in the restoration of immune balance by decreasing Th1 and Th17 cells and increasing the Th2 and Treg cells, thus causing significant reduction in disease severity. Methotrexate converts the pro-inflammatory $\mathrm{T}$ cell phenotype to a protective anti-inflammatory phenotype, thus significantly suppressing the inflammation in psoriasis

KEY WORDS: psoriasis, methotrexate, T cell phenotype, immune mediated inflammation

\section{P81}

\section{Association of Serum Magnesium with Obesity In Postmenopausal Women}

\author{
Alka Yadav, Madhuri Gupta, R.C.Gupta \\ Department of Biochemistry, \\ National Institute of Medical Sciences and \\ Research Jaipur, Rajasthan.
}

INTRODUCTION:Obesity is a complex, multifactorial condition in which excess body weight may put a female at risk of serious health problems such as dyslipidemia, hypertension, diabetes mellitus and cardiovascular diseases. Magnesium is a vital divalent metal ion and plays an important role in so many enzymatic reactions occurring in the body. At the cellular level, magnesium also serves as a cofactor for many intracellular enzymes that generate energy via hydrolysis of ATP and are involved in the metabolism of carbohydrates, fats and proteins. It also assists the action of insulin. The effects of magnesium on obesity are crucial to women's health, since women are more likely to be obese than men. Therefore, the present study was planned to estimate and compare the serum magnesium level in obese and nonobese postmenopausal women and to find out the relationship between serum magnesium levels and degree of obesity.

MATERIAL AND METHODS: Twenty five obese postmenopausal women having $(\mathrm{BMI} \geq 30)$ with their final menstrual period at least one year prior to the study were taken as the study group and 25 non-obese (BMI $\geq$ 22.9) post-menopausal women were taken as control group. All subjects were asked to give detailed dietary 
history using Food Frequency Questionnaire (FFQ).Venous blood sample was collected after an overnight fast for estimation of serum total magnesium in all subjects. Serum magnesium was measured colorimetrically using ready to use kit (Xylidyl blue method).

RESULTS: Obese postmenopausal women had significantly higher weight $(78.36 \pm 0.064 \mathrm{~kg})$ and BMI $(32.68 \pm 1.7 \mathrm{~kg} / \mathrm{mt} 2)$ compared to non-obese postmenopausal women (wt. $54.72 \pm 4.80 \mathrm{~kg}$ and BMI $21.75 \pm 1.68 \mathrm{~kg} / \mathrm{mt} 2$ ). The mean $\pm \mathrm{SD}$ serum magnesium concentration found in the obese postmenopausal women was $1.40 \pm 0.45 \mathrm{mg} / \mathrm{dl} \mathrm{Vs} 2.03 \pm 0.49 \mathrm{mg} / \mathrm{dl}$ in the nonobese group. Pearson's correlation analysis showed a significant $(\mathrm{r}=-0.9)$ negative correlation of BMI with serum magnesium in obese postmenopausal women.

CONCLUSION: The present study shows a lower level of serum magnesium in the obese postmenopausal women compared to the non-obese postmenopausal women. This decrease correlates with increasing degree of obesity. Proper body weight regulation with magnesium supplementation may play a role in the management of health related issues after menopause.

KEY WORDS: Obesity, Magnesium, postmenopausal, BMI

\section{P82}

Preparation of A Human Blood with Desired Measures of Blood Components and Its Stabilization

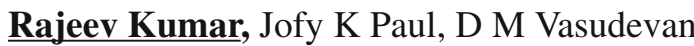

Agappe Diagnostics Pvt. Ltd., Pattimattom,

Dist. Ernakulum, Kerala 683562 (India)

INTRODUCTION: We are developing hematologycontrols and calibrators which shall be used to calibrate the blood analyzers in the diagnostic laboratories. Running the blood controls will verify the calibration of the blood analyzers. There are 3 levels of controls available (Low, Normal and High); each level has a defined range of values for the blood components. We have developed a new technology by which one can set components of the blood as per the desired values, then we add stabilizer to corresponding controls. After the maturation period, the product is ready to perform on the hematology analyzers.

AIMS AND OBJECTIVES: Development of a method to set blood components with desired values which will be used as a hematology control for blood analyzers and stabilizing it for six months at $2-8^{\circ} \mathrm{C}$

MATERIALS AND METHODS: Whole blood stabilization: Gentamycin and ampicillin with Agappe blood stabilizer TriFog-60 was addedstepwise in the blood.

COMPONENT SEPARATION:Centrifugation technique was used to isolate the plasma, WBC, platelets and RBCsatat 2200rcf(relative centrifugal field) for 10 minutes at $4{ }^{\circ} \mathrm{C}$ by using Thermo Scientific SorvallCentrifuge.

REMOVAL OF PLATELETS FROM THE PLASMA: Controls were centrifuged at $500 \mathrm{rcf}$ for 10 minutes at $4{ }^{\circ} \mathrm{C}$ and then turbid plasma which contains platelets and few WBCs was replaced with platelet free plasma.

HEMATOLOGY ANALYZER: Mindray BC 3000 Plus was used in all experiments.

RESULT AND CONCLUSION: Newly developed technology to set blood parameter is functional and open vial stability observed to be more than 20 days, whereas closed vial stability observed to be 4 months so far.

KEY WORDS: Calibrators, Gentamycin, Ampicillin, Mindray BC 3000 Plus

\section{P83}

A Comparative Study of Liver Function Tests Between Pregnant and Non Pregnant Women In Different Trimesters At Rims, Ranchi.

Shrishti Dhar Prasd, Anand Kumar,

RIMS, Ranchi

INTRODUCTION:The pregnant women experiences physiological changes to support fetal growth and 
development.During pregnancy liver functions are adapted to physiological need.Serum estrogen and progesterone level increase progressively and reach a maximum during third trimester.These sex steroid have effect on metabolic, synthetic an excretory function of liver. Understanding of these adaptations requires evaluation during different trimesters for avoiding any risks in outcome of pregnancy.Liver disease that occur during pregnancy can present a challenge for health care providers.

MATERIALS AND METHOD: A total of 200 women were screened.Among 150 healthy pregnant women ,50 from each trimester and 50 non pregnant women formed controls.Samples are collected from department of obstetrics and gynecology attending for ANC at RIMS, Ranchi.

Venous sample about $5 \mathrm{ml}$ were collected from pregnant and non pregnant women.Labroatory data were estimated by full automated analyzer.

RESULTS: Mean ALT level in patients of our socioeconomic environment is $10+2.4 \mathrm{IU} / \mathrm{L}$ for non pregnant,and for pregnant women of 1 st 2 nd,and $3 \mathrm{rd}$, trimester are $12.6+4.3,17.3+5.4$, and $25.7+12.8 \mathrm{IU} / \mathrm{L}$ respectively.Mean AST level in pt of our socioeconomic is $22.3+4.8 \mathrm{IU} / \mathrm{L}$ for non pregnant and for pregnant women of $1 \mathrm{st}, 2 \mathrm{nd}$, and $3 \mathrm{rd}$, trimester are 19.3+5.2,26.8+7,2 and 32.6+9.8 IU/L respectively.Mean ALP level in pt of our socioeconomics is $52.7+21.6 \mathrm{IU} /$ Lfor non pregnant and for pregnant women of 1st, 2nd, and $3 \mathrm{rd}$, trimester are $50.6+20.7,70.4+25.3$, and 210.5+79.3 IU/L respectively.

CONCULSION: Among these enzymes, serum ALP level raises more and especially during third trimester. Establishing upper normal level will be of much help for obstetrician in mangament of pregnancy related problem and differentaiating physiological changes from pathological conditions such as hyperemesis gravidarum,intrahepatic cholestasis of pregnancy,acute fatty liver of pregnancy,pre-eclampsia,eclampsia and HELLP syndrome etc.

KEYWORDS: Aspartate transaminase, Alkaline phosphatase, Alanine transaminase, Hemolysis, elevated liver enzymes and low platelets.

\section{P84}

Correlative Study of Soluble Vascular Cell Adhesion Molecule-1 And Estrogen In Knee Osteoarthritis Among Postmenopausal Women

$\underline{\text { Shrivastava Reetika }}^{1}$, Singh Neelima ${ }^{2}$, Dhakad RKS ${ }^{3}$

1,2. Department of Biochemistry,

3. Department of Orthopaedic, Gajra Raja Medical College, Gwalior

INTRODUCTION: Knee osteoarthritis is a leading cause of pain and functional disability among elderly female population. The high incidence of osteoarthritis in women just after menopause has suggested that estrogen deficieny plays an important in the onset and progression of disease. Osteoarthritis is associated with a local inflammatory process. VCAM-1 is an inducible cell surface glycoprotein and mediates heterotypic cellular aggregation. Therefore, the aim of this study is to assess the role of soluble VCAM-1and its relation with estrogen in postmenopausal women having clinical symptoms of knee osteoarthritis.

MATERIALS AND METHODS: The present study includes 80 postmenopausal women of age 50 years or above with clinical symptoms of knee osteoarthritis as cases and 80 normal healthy female age matched individuals as controls. In all the cases and controls, serum VCAM-1 concentrations and estrogen were estimatedby ELISA method. Statistical analysis was made by student independent sample t-test. Correlation was determined by using spearman's rank correlation coefficient.

RESULT: Serum level of soluble VCAM-1 was found statistically highly significantly increased and estrogen was found highly significantly decreased $(\mathrm{p}<0.001)$ in postmenopausal women with symptoms of knee osteoarthritis as compared to control healthy subjects. A significant negative correlation was observed between VCAM-1 and estrogen.

CONCLUSION: These findings show that in postmenopausal women lack of estrogen hormone induces soluble VCAM-1 production. VCAM-1 mediates the interaction of chondrocytes with immune cells and 
contributes to immune-mediated cartilage damage. Hence, serum level ofsoluble VCAM-1 could be used as an early biomarker for inflammatory response and cartilage damage in osteoarthritis.

KEYWORDS: Vascular cell adhesion molecule1(VCAM-1), Knee osteoarthritis, Estrogen

\section{P85}

\section{Evaluation of Serum Vitamin B12 Level In Primary Hypothyroidism}

Pritam Prakash ${ }^{1}$, RajeevRanjan Sinha ${ }^{2}$, Rekha Kumari ${ }^{3}$, Uday Kumar ${ }^{4}$, Anand Sharan ${ }^{5}$

Department of Biochemistry, Indira Gandhi Institute of Medical Science, Patna

OBJECTIVE:The objective of my study is to evaluate the level of serum vitamin B12 in patient of primary hypothyroidism. The vitamin B12 level was determined in patients of primary hypothyroidismand compared with normal subjects.

MATERIAL AND METHOD-Fifty five patients of known hypothyroidism and fifty normal subjects were taken for study. Serum sample were collected and level of vitamin B12 was measured by Backmen Coulter Access 2.

RESULT-The result showed serum vitamin B12 level of primary hypothyroid patients were significantly lower as compared to normal subjects(145.5 \pm 65.71 vs $396.4 \pm 197.6) \mathrm{p}<0.0001$.

CONCLUSION- There is very high prevalence of vitamin B12 deficiency in hypothyroid patients. So screening of serum vitamin B12 level should be undertaken in all hypothyroid patients in early course of disease when it is in potentially reversible condition and we will prevent vitamin B12 related complicationin hypothyroid patients.

KEY WORDS- vitamin B12, hypothyroidism.
P86

TNF-Alpha G-308A Promoter Gene Polymorphism Is Associated with Coronary Artery Disease Phenotypes In Indian Population: An Explorative Study

$\underline{\text { Reena Kumari }}^{1,4}$, M Kaleem Ahmad ${ }^{1}$, Sandeep Kumar $^{3}$, Rajeev Singh ${ }^{1}$, Sharad Chandra ${ }^{2}$, Akshayaya Pradhan $^{2}$, Sudhir Kumar ${ }^{4}$.

1. Department of Biochemistry,

2. Department of Cardiology, KGMU, Lucknow,

3. Department of Clinical Immunology, SGPGIMS, Lucknow,

4. Department of Zoology, University of Lucknow, Lucknow

INTRODUCTION: The C-reactive protein (CRP) and the tumor necrosis factor-alpha (TNF-?) are considered markers of inflammation and have been shown to predict the risk of incident of coronary artery disease (CAD). The promoter region polymorphism at position $-308 \mathrm{G} /$ A of TNF-? has been shown to increase transcription of the gene. Aim of this study is to investigate in an Indian population the impact of the G-308A TNF-? variant on various components of the $\mathrm{CAD}$ and to study its relation with circulating TNF-? and hsCRP levels.

METHODS: The G- 308A TNF-? polymorphism has been studied in 301 diagnosed CAD subjects (Age

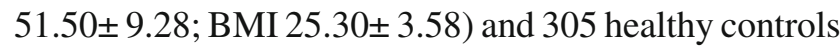
(Age 51.57 \pm 9.50; BMI $24.06 \pm 7.26$ ). The G-308A variant was detected by real time PCR by using Taqman SNP genotyping assay. Furthermore serum TNF-alpha and hsCRP levels were also measured in both the groups.

RESULTS: Homozygous variant genotype (AA) (GG v/s GA+AA) ( $\mathrm{p}=0.030: \mathrm{OR}=1.61: 95 \% \mathrm{CI}=1.06-2.44)$ and variant allele $(\mathrm{A})(\mathrm{p}=0.006$ : $\mathrm{OR}=1.71: 95 \% \mathrm{CI}=1.17$ 2.51) of the G-308A TNF- $\alpha$ polymorphism was observed significantly high in the CAD population as compared to controls group. Furthermore, on dividing the subjects into two groups according to the presence or absence of the variant allele $\mathrm{A}$, significantly high levels were observed in TC, LDL, TC/HDL, LDL/HDL, TNF- $\alpha$ and hsCRP however present study also showed significantly lower level of HDL/LDL in variant genotype group 
(GA+AA) as compare to wild type genotype (GG).

CONCLUSIONS: The findings of present study suggest that the G-308A mutation of the TNF- $\alpha$ gene is likely to play an important role in the development of coronary artery disease and metabolic abnormalities.

KEY WORDS: C-reactive protein, polymorphism, hsCRP, HDL/LDL

\section{P87}

Assessment of Serum ?n-Carboxymethyllysine And Soluble Receptor of Advance Glycation End Product Levels Among Type 2 Diabetes Mellitus Patients With And Without Acute Coronary Syndrome

S. Shanija ${ }^{a}, \underline{\text { P.S. Adole }}^{\mathrm{a}}$, K.V. Vinod ${ }^{\mathrm{b}}$, R.P. Saya ${ }^{\mathrm{c}}$

a. Department of Biochemistrya and Medicine

b. Jawaharlal Institute of Postgraduate Medical

Education and Research, Puducherry, India;

Department of Medicine

c. Kanachur Institute of Medical Sciences \&

Research Center, Natekal, Mangalore, India.

INTRODUCTION: One of the most predominant macro-vascular complications of type 2 diabetes mellitus (T2DM) is acute coronary syndrome (ACS). Advance glycation end products (AGEs) play an important role in development and progression of ACS in T2DM patients. Soluble receptor of AGEs (sRAGE) decoys the effect of AGEs by binding with them.

AIM AND OBJECTIVES:The present study was conducted to determine the association between serum ?N-carboxymethyllysine (CML), an important AGE and sRAGE level with risk of ACS in T2DM patients.

MATERIALS AND METHODS: Thirty seven T2DM patients with ACS were selected as cases and 37 T2DM as controls. Routine biochemical parameters were carried out on Auto-analyzer and serum CML and sRAGE levels were estimated by ELISA. The data were recorded and analyzed on SPSS system and were compared by student t-test or Mann-Whitney test.
RESULTS \&CONCLUSION: Serum CML level and CML/sRAGE ratio were significantly increased in the cases as compared to control. Correlation analyses showed serum CML concentration and serum CML/ sRAGE ratio were positively correlated with weight, waist circumference, body mass index and serum urea and very low density lipoprotein-cholesterol. Multivariate regression (Binary logistics) analysis after adjusted for waist circumference, weight, BMI and systolic blood pressure showed that serum CML was significantly associated with ACS risk among T2DM patients. Receiver Operating Characteristic curve depicted that high CML level (>31.83 ng/ml) and CML/ sRAGE ratio $(>15.48)$ is associated with greatest risk of ACS among type 2 DM patients. Hence, T2DM patients with high serum CML concentration and CML/ sRAGE ratio were at risk of ACS independent of other cardiovascular risks.

KEY WORDS: Type 2 diabetes, cardiovascular, sRAGE, acute coronary syndrome

\section{P88}

\section{Assessment of Kidney Function Tests In Organophosphorous Poisoning Cases}

Brijesh. S. Naik, Priyanka shridharan, Vinutha.R. Bhat, E.G Padmanabha Udupa.

\section{Department of Biochemistry, Kasturba Medical College Manipal University,Manipal. 576104}

INTRODUCTION: Organophosphorous compounds are one of the most common cause of insecticide poisoning worldwide. Organophosphorous pesticides are the derivative of phosphorous acid which is extensively used in agriculture sector. In acute organophosphorous poisoning acetylcholinestrase is accumulated in synaptic junctions. This leads to cholinergic crisis and intermediate symptoms which may lead to acute renal failure and severe metabolic acidosis. The study is based on analysis of renal function test with patients who are exposed to organophosphorous pesticides.

AIM AND OBJECTIVE: To study the changes in serum levels of urea and creatinine in patients with 
organophosphorous compound poisoning

MATERIALS AND METHOD: Blood samples of 15 patients who were exposed to organophosphorous poisoning were taken and anonymized. Urea was estimated using urease or GLDH method. Creatinine was estimated by modified Jaffe's method.

RESULTS AND DISCUSSION: Dysfunction of kidney is assessed by organ function test. In the present study there was statistical significant increase in urea $(p<0.001)$ when compared to control and no significant difference were observed in creatinine level. Organophosphate intoxication is a very infrequent cause of acute deterioration of renal functions. It is suspected that due to organophosphorous poisoning direct damage occurs at renal tubules. Due to this urea is not excreted and there is increase in blood urea. The levels of creatinine is used as an indicator of glomerular filtration rate. Increase in urea level indicates that there could be stress on kidney due to organophosphorous toxicity.

CONCLUSION: The op poisoning particularly at high dosage has a toxic effect on kidney.

\section{P89}

\section{Blood Lead Levels and Anemia In School Going Children of Western Rajasthan}

Shailja Sharma, Praveen Sharma

Department of Biochemistry, All India Institute of Medical Sciences, Jodhpur, India

INTRODUCTION:Lead exposure is estimated to account for $0.6 \%$ of the global burden of disease, with the highest burden in developing regions of the world. Childhood lead exposure is estimated to contribute to about 600,000 new cases of children with intellectual disabilities every year. Anemia in children is also a substantial problem in India and leads to increased morbidity and mortality. The aim was to find out the blood lead level and its association with haemoglobin levels in school going children.

MATERIAL \& METHODS: Students belonging to Jodhpur (India) from various schools from government as well as private sector were selected randomly and written informed consent was obtained from the parents/ guardians of participants. Personal details, complaints, dietary habits, history of pica, symptoms suggestive of altered behaviour, blood transfusions, history of pain abdomen, constipation, delirium and convulsions were noted in questionnaire .Venous blood sample in EDTA vial was collected and blood lead level (BLL) was estimated the very same day after ensuring quality control. BLL was measured using Lead Care II analyser by standardized anodic stripping voltametry technique ensuring quality control. Samples were measured in duplicates. Hemoglobin was estimated using cyanmethemoglobin method.

RESULTS: Total 426 children (5-12yrs) participated in the study. Their mean BLL was 5.21 $\pm 3.32 \mathrm{ug} / \mathrm{dL}(<3.3$ $22.6 \mathrm{ug} / \mathrm{dL}$ ). Children with increased BLL ( $>5 \mathrm{ug} / \mathrm{dL})$ were found to be $18.87 \%$ which is considerably high in comparison to western counterparts. Children residing in urban region were found to have BLL $>5 \mathrm{ug} / \mathrm{dl}$ (22.18\%) in comparison to rural inhabitants $(9.77 \%)$. The students of government school were found to have BLL $>5 \mathrm{ug} / \mathrm{dL}$ in $23.98 \%$ of cases in comparison to private school students which were $13.17 \%$ in this category. No correlation was observed between BLL and haemoglobin $\%$. Anemia is a significant problem in school going children [12yr (19.23\%)and 5-11yr(15.24\%)] with possible underlying cause being nutritional rather than lead exposure.

CONCLUSION: Significant number of Indian children have increased BLL. According to CDC guidelines no safe BLL in children has been identified. Even low levels of lead in blood have shown to affect IQ, ability to pay attention, academic achievement and effects of lead exposure cannot be corrected. Anemia is a significant problem in school going children with possible underlying cause being nutritional rather than lead exposure in the selected population.

KEY WORDS: Lead exposure, Blood lead level, BLL, Children, haemoglobin, anemia 


\section{P90}

\section{Evaluation of Liver Enzymes In Organophosphorous Poisoning Cases}

Priyanka Shridharan, Brijesh S.Naik, Vinutha R.Bhat, E.G. Padmanabha Udupa

Department of Biochemistry, Kasturba Medical College Manipal, Manipal University,

Manipal. 576104

INTRODUCTION: Organophosphorous poisoning is one of the most common poisoning seen in developing countries like India. organophosphorous compounds are quickly absorbed through respiratory system, gastrointestinal tract etc. which inhibits acetyl cholinesterase at synapses. This causes stimulation of nicotine receptor which leads to several symptoms like sweating, brachycardia etc. Severe symptoms include acidosis, acute renal failure, and death. The purpose of the study is to investigate the variation in liver enzyme in patient who are exposed to organophosphorous poisoning.

AIMAND OBJECTIVE: To study the changes in serum levels of liver enzymes in patients with organophosphorous compound poisoning.

MATERIALS AND METHODS: Blood samples of 15 patients who were exposed to organophosphorous poisoning were taken and anonymized. Liver enzymes such as AST and ALT were analysed using modified IFCC (international federation of clinical chemistry) method.

RESULTS AND DISCUSSION: In the present study AST level was markedly elevated in organophosphorous poisoning patients, whereas there was significant increase in ALT in organophosphorous poisoned patients with $(p<0.01)$ and their increase depends on type of compound consumed. Estimation of liver enzymes is used as a diagnostic tool to monitor the treatment of liver damages and it includes measurement of ALT and AST. Liver accepts and metabolizes organophosphates their oxidation and sulfate or glucuronate conjugation and may undergo oxidative damage in case of organophosphorous poisoning. Significant increase in ALT indicating muscular functional impairment is due to organophosphorous poisoning.

CONCLUSION: The findings of this study reflect the usefulness of serum AST and ALT in the management of organophosphorous pesticide poisoning and a good prognostic indicator of survival.

KEY WORDS:Organophosphorous, acetyl cholinesterase, AST, brachycardia

\section{P91}

\section{Comparison Between Serum Holotranscobalamin And Total Vitamin B12 As Indicators of Vitamin B12 Status}

Sanjay Kumar, Pratik Poladia, Babu G Pillai, Umakant Gavhane, Ulka gosavi, ManikchandraTiwari, Rajani Mohite, Swati wayker, Preeti Chavan, Vivek Bhat, Hemant Vira

TATA Memorial Centre ACTREC, Kharghar, Navi Mumbai

AIM:This study was undertaken to compare the diagnostic sensitivity and specificity of total vitamin B12 analyses to active B12 (holoTC) analyses in a patient sample

INTRODUCTION:- Current assays measure total serum vitamin B-12 as cobalamin. However, new assays measuring holotranscobalamin (active vitamin B-12) are said to reflect actual vitamin B-12 status in body. In this study levels of these two assays were measured and analysed for deciphering the correlation between the two.

METHOD AND MATERIAL:Serum samples were selected from 83patients at ACTREC-TMC who had been referred to Biochemistry Laboratory for assessment of vitamin-B12 status.For each patient serum total vitamin-B12 level and active vitamin-B12 level were determined by chemiluminescent microparticle immunoassay on Architect1000i analyzer (Abbott).

RESULTS:R score by pearson correlation analysis is 0.652 with a high statistical significance $(\mathrm{P}<0.0001)$. There is positive correlation, which means there is a 
tendency for active vitamin B-12 concentrations to increase with increase in total vitamin B-12 concentrations.

CONCLUSION: Thus our study concludes that measurement of total vitamin B-12 as well as active vitamin B-12 serum concentrations are equally effective in diagnosing the status of vitamin B-12 in patients and both methods can be used interchangeably.

KEY WORDS: holoTC, vitamin B-12, cobalamin

\section{P92}

\section{Graphene Nanoparticle Enhanced Elisa For High Sensitive Malaria Diagnosis}

Ajai Kumar S, D M Vasudevan

Agappe Diagnostic Limited, Agappe Hills, Pattimattom, Cochin, Kerala, India

INTRODUCTION: Even though microscopy is the gold standard for malaria diagnosis, more advanced techniques like ELISA, PCR \& Immunochromatography are widely used for precise detection of malaria antigens. A new approach, Graphene nanoparticle enhanced ELISA can provide more sensitive and accurate diagnosis for malaria. In this study, Plasmodium HRP II detection by Graphene sandwich ELISA have been comparedwith conventional sandwich ELISA. Results shows that graphene ELISA is more sensitive (2-3 times) than the conventional ELISA. Nanoparticle sized graphene with its inert nature can provide tremendous surface area for the capture antibodies which in turn can provide more sensitivity and can increase the lower detection limit of the antigen.

AIM\& OBJECTIVES: Development of GrapheneELISA for high sensitive Malaria diagnosis.

\section{MATERIALS \& METHODS:}

$\sqrt{ }$ Graphene nanoparticle activated with APTES silanization: $\mathrm{KOH}$ treated microtiter plates will be functionalized with graphene nanoparticles dispersed in aminopropyltriethoxysilane (APTES).

$\sqrt{ }$ Capture antibody activation with EDC-NHS: Under optimized conditions silanized graphene particles can make strong amide bonds with the ethyldimethyl aminopropyl carbodiimide - N Hydroxysuccinimide (EDC-NHS) activated capture antibodies.

$\sqrt{ }$ Standardization of Graphene ELISA: Conditions has to be optimized with capture as well as detector antibody to obtain the maximum sensitivity.

RESULT \& CONCLUSION: Advanced Malaria diagnosis Graphene-ELISA kit for Plasmodium falciparum detection with high sensitivity than the conventional ELISA kits.

The graphene enhanced ELISA technology can be further used for development of diagnostic platforms which necessitate more sensitivity and early detection.

KEY WORDS: malaria, Immunochromatography, Graphene nanoparticle, APTES silanization

\section{P93}

\section{Analysis of Laboratory Sample Rejections In The Pre-Analytical Stage At Anoncology Center}

AshwadeepKarmore, P. Chavan,V. Bhat, P. Poladia, U.Gavhane, M.Tiwari, S.Pal, B.Pillai, U.Gosavi, R. Mohite, S. Vaykar, A. Kadam, P.Dalvi

Tata Memorial Centre, Advanced Centre For Treatment Research \& Education in Cancer.

INTRODUCTION: Clinical laboratories play a crucial role in the diagnosis and management of patients. These are some of the key indicators of errors that can help \& identify potential improvements in patient safety during pre-analytical phase in clinical laboratories.Clot was found to be the major cause of rejection of samples, followed by insufficient sample volume, patients clinical history was not provided, improperlylabeledsamples, samples collected in expired vacutainers,samples received without requisition, unlabelled samples \& haemolysed samples.Errors in clinical laboratories have a great impact on safety and care of patients. The preanalytical phase is responsible for about $70 \%$ of errors. Quality indicators in the clinical laboratory provide a useful tool for continuous improvement of laboratory 
services. In this study, we aimed to evaluate the sample rejection ratios according to the types of pre-analytical errors.

MATERIALS AND METHODS : A retrospective, intervention and prospective analysis of the samples rejected from the total samples received in our laboratories, during a period from January 2015 to June 2017 was undertaken.

RESULTS: Out of 216631 samples received during January 2015 - June 2017, 318 samples ( $0.15 \%$ ) were rejected. The most common reasons for rejection is clotted blood samples (57.14\%),followed by improperly labelled samples(14.28\%), haemolysed samples (11.42\%), insufficient sample volume (8.57\%), Samples without requisition $(5.71 \%)$ \& Samples in expired vacutainers $(2.85 \%)$,

CONCLUSION : This study has shown that the most frequent causes of pre-analytical errors are clottedsamples, improperly labeled samples.haemolysed samples \& samples with insufficientvolume.

KEY WORDS : Quality indicators,pre-analytical, sample rejection.

\section{P94}

\section{The Study of Cancer Patients with CK-MB Value Higher Than Total CK}

Babu Pillai, Sanjay kumar, Aswadeep Karmore, Pratik Poladia, Umakant Gavhane, Manik Tiwari, Ulka Gosavi, Swati Vaykar, Vivek Bhatt, Preeti Chavan

Tata Memorial Hospital, ACTREC, Kharghar, Navi Mumbai

INTRODUCTION:The measurement of Creatinine Kinase enzymes and its proportion to the MB fraction,is a major diagnostic test for acute myocardial infarction. The MB fraction is $<5 \%$ of the total ck under normal conditions. In case of myocardial infarction this fraction is elevated.However,some times in laboratory practice one may come across conditions where creatinine Kinase $(\mathrm{CK}) \mathrm{MB}$ levels are greater than total $\mathrm{CK}$ value.This poses a challenge to the laboratory reporting the values and to the clinician treating the patients.

MATERIAL AND METHODS :We analysed three patients, the first one is $36 \mathrm{y}$ female having primary myelofibrosis, second one is 53y male having Myelodysplastic syndrome(MDS) and the last patient have 43y male having Squamous Carcinoma.Serum samples for all the patients were sent to the laboratory for estimation of cardiac markers.serum CK-MB and CKI were measured by RXL Max fully automatic Biochemistry Analyzer.

RESULTS : There are reported cases in literature, where CK-MB activity greater than total CK is reported in association with Macro-Creatinine Kinase syndrome in patients of Cancer.

CONCLUSION : Our cases corroborate the fact that whenever CK-MB activity greater than total CK is encountered,the history and diagnosis of malignancy should be kept in mind.

KEY WORDS : Myocardial infarction, CK-MB, Creatinine kinase

\section{P95}

\section{A Comparative Evaluation of Hba1c Measurement In Two Different Anticoagulant Tubes}

JananiIswarya, Geethanjali Arulappan

Department of Clinical Biochemistry, Christian Medical College, Vellore.

INTRODUCTION: Diabetesis classified as a worldwide epidemic.Assessing glycemic control in diabetics is crucial for the management of the disease and for the prevention of long-term complications. HbA1C is currently the most widely used index of average glycemia and its importance in diabetes has increased manifold in recent years. Most of the commercial kits for HbA1Cestimation requires sample to be collected in EDTA tube and this requires additional sample collection for the patient. Hence this study was carried out to check the variation between two different 
anticoagulant tubes and to check its stability when stored at $2-8^{\circ} \mathrm{c}$ for 7 days.

AIM: To compare the performance of K2-EDTA and sodium Fluoride- Na2-EDTA vacutainer tubes in the measurement of $\mathrm{HbA} 1 \mathrm{C}$ and to check its stability during storage.

MATERIALS: This study was conducted at Christian Medical College,vellore, where samples for study were obtained from patients during routine hospital visit. 100 samples were recruited for this study.

METHODS: HbA1C was measured in both K2-EDTA and sodium fluoride Na2-EDTA vacutainer tubes by Ion Exchange HPLC method(Variant II turbo Biorad Analyzer).

RESULT: There were no significant differences noted in the mean values of Hbalc measured between the two different anticoagulant tubes on day 1 , day 3 , day 5 and day 7 of storage.

CONCLUSION: The findings from this study concludes that need for separate EDTA sample for HbA1C estimationis not necessary as the values obtained from sodium Fluoride vacutainer tube showed no significant variationswith the values obtained from k3 EDTA anticoagulant tube.

KEY WORDS: Diabetes, HbA1C, glycemia,

\section{P96}

To Study The Expression of Nitric Oxide and Inducible Nitric Oxide Synthase (INOS) And Their Correlation With Inos Gene Polymorphic Variants In Tuberculosis Patients And Controls

\author{
Ambrish Tiwari ${ }^{1}$, Sudhasini Panda1, \\ Kalpana Luthra ${ }^{1}$, Archana Singh ${ }^{1}$ \\ 1. Department of Biochemistry, All India Institute of \\ Medical Sciences, New Delhi-110029
}

INTRODUCTION: Nitric oxide act as free radical and help in killing mycobacterium tuberculosis.

OBJECTIVE: To study expression of nitric oxide synthase and serum levels of nitric oxide and their corelation with iNOS gene polymorphism in patients with Tuberculosis and controls.

METHODS: In this cross sectional study, we had done ARMS-PCR for genetic analysis, qPCR for expression of iNOS ,ELISA for iNOS protein level and colorimetric assay for NO level in Active tuberculosis patient and correlated with values with control.

RESULTS: Analysis of (T/C) showed TT (wild homozygous) to be present in $24.45 \%$ of patients as compared to $32.43 \%$ of household contacts and $38.71 \%$ of healthy controls. The TC (variant heterozygous) genotype was seen in $61.22 \%$ of active TB patients as compared to $54.05 \%$ of household contacts and $54.83 \%$ healthy controls. CC (Variant homozygous) genotype was present in $14.2 \%$ of active TB patients as compared to $13.51 \%$ of household contacts and $6.45 \%$ of healthy controls. Analysis revealed that active TB group, household contacts and healthy controls had significant difference in amounts of iNOS mRNAs with p-value of 0.005. Analysis revealed that there was no significant difference in the serum levels of iNOS among all the three groups with a p-value of 0.07 . The difference in levels of nitric oxides among all three groups was found to be non-significant with a $p$ - value of $>0.05$.

CONCLUSION: Expression iNOS mRNA and protein as well as NO were found to be significantly increased in samples with TT genotype (wild type) compared to $\mathrm{TC}$ and $\mathrm{CC}$ (variant) genotype suggesting the association of iNOS genotype with the production of iNOS mRNA and protein. Though iNOS expression at mRNA level was decreased in Active Tb patient, iNOS protein and NO level was higher in Active TB group as compared to controls. This may be due to counteracting actions of various bacterial and host factors for its survival and defense respectively leading to unrelated expression of iNOS mRNA in respect to serum iNOS protein and nitric oxide levels in active TB group.

KEY WORDS: iNOS, gene polymorphism, TB 


\section{P97}

\section{Evaluation of Vitamin D Level In Patients of Epilepsy At Rims Ranchi Jharkhand}

Anand Kumar, Shrishti Dhar Prasad

Department of Biochemistry, RIMS, Ranchi.

INTRODUCTION: Epilepsy is a second most leading neuronal disorder received much less attention. We review evidence supporting a link between vitamin D and epilepsy including those coming at RIMS medicine OPD as well as ecological studies. Vitamin D and its receptor have important regulatory effects during brain development such as cell differentiation and apoptosis. It has been shown that vitamin D deficiency poses a risk for neurological diseases such as epilepsy etc. The synthesis of nerve growth factor is regulated by 1, 25( $\mathrm{OH}) 2 \mathrm{D} 3$. It was shown at molecular levels that rat with low vitamin D3 have persistently low level of NGF. In the literature, several studies and hypothesis suggested an association between low vitamin d level and seizures;-Vitamin D may positively modulates brain neuromodulators and neuroreceptors viaGABA-A receptors. -vitamin d may affect seizures via acting through VDR to induce certain genes in the brain encoding cytokines and enzymes which involve in metabolism of neurotransmitter.

MATERIAL AND METHOD: We conducted an observational study of epilepsy patient treated by clinician at RIMS ranchi. Vitamin D level were catogarised as low ( $<20 \mathrm{ng} / \mathrm{ml})$, borderline( $20-30 \mathrm{ng}$ / $\mathrm{ml}$ ), or normal ( $>30 \mathrm{ng} / \mathrm{ml}$ ).

RESULTS: Vitamin D level obtained on 60 patient with epilepsy. Mean age was $45 \mathrm{yrs}$. The mean vitamin $\mathrm{D}$ level was 24 ( SD 11.8 range 7 to 46 ), and $56 \%$ had level < 20. Vitamin D deficiency was present in $58 \%$ of enzymeinducing and $26 \%$ of non enzyme inducing antiepileptic drug group.

CONCLUSION:Vitamin D deficiency is common in epileptic patients which were on antiepileptic therapy. Vitamin D assessment should be a routine measurement for epileptic patients.

KEY WORDS : Epilepsy, vitamin D and drugs.

P98

Incidence of Paraquat Poisoning detected at
Manipal Poison Detection Centre (MPDC),
Manipal.

Thejesh S, Vinutha R Bhat.

Department of Biochemistry, Kasturba Medical College Manipal,Manipal University,

Manipal 576104.

INTRODUCTION: Paraquat is classified under viologen, itis one of the most common herbicides, which is quick acting. Ingestion leads to acute respiratory distress syndrome. Although it lacks specific antidotes, activated charcoal, haemodialysis or haemoperfusion is an effective treatment if given in time.

AIM: To study Incidence of paraquat poisoning detected at MPDC.

METHODOLOGY: Sodium dithionite test on urine was tested positive as a blue/black colour, indicates the presence of Paraquat. Related herbicide, diquat gives a yellow green colour, but interference is insignificant in the presence of Paraquat.If the colour fades on continued agitation in air, Paraquat or diquat is confirmed.

RESULTS: Among 48 suspected paraquat poisoning samples aged about 14 to 74 years in both males and females, 10 were detected positive and 38 were negative.Over which paraquat poisoning was detected positive in $60 \%$ in males $(n=6)$ and $40 \%$ in females $(n=4)$. Around $60 \%(n=6)$ of paraquat consumers were seen in the age group of 14 to 28 years, where about $67 \%(n=4)$ were teenagers.

DISCUSSION: Mild intoxication of paraquat leads to lung injury,pulmonary fibrosis and acute renal failure, where survival chances are considerably high. High dose of paraquat leads to multiorgan dysfunction, liver being the foremost victim, death occurs within 3 days.N-Acetyl cysteine(antioxidant) is given as primary antidote which reduces production of reactive oxygen species. 
CONCLUSION: This study determines the percentage of paraquat poisoning among different age groups.Study shows that younger generations, especially teenagers show maximum suicidal attempts which has to be addressed by counselling and moral programme.

KEY WORDS: Paraquat, viologen, herbicide, MPDC

\section{P99}

\section{Assessment of Beta 2 Microglobulin In Patients of Diffuse Large B Cell Lymphoma As A Prognostic Marker.}

Garima Gupta*, Veena Singh Ghalaut\#, V

Lokanathan\#, Praveen Sharma*

* Department of Biochemistry, All India Institute of Medical Sciences, Jodhpur, \# Department of Biochemistry, PGIMS, Rohtak

INTRODUCTION : Lymphoma is the proliferation of lymphoid cells, which arise as discrete tissue masses. It has been broadly divided into non-Hodgkin's lymphoma (NHL) and Hodgkin's lymphoma (HL). NHL contributes to about $85 \%$ of all malignant lymphomas. Diffuse large B-cell lymphoma (DLBCL) is the most common subtype of non-Hodgkin lymphoma, a heterogeneous disease with a variety of molecular aberrations and diverse clinical outcomes. Beta-2 microglobulin(?2M) is synthesized in all nucleated cells and forms the light chain subunit of the MHC class I.

AIM: To determine the role of ?2M in the prognosis of patients with DLBCL so as to validate its role as a convenient biomarker.

METHOD : 30 diagnosed patients with DLBCL and 30 age and sex matched healthy controls were taken. ?2M was estimated in newly diagnosed patients before initiating treatment and also in controls by ELISA. DLBCL patients were given chemotherapy following the CHOP Regimen (cyclophosphamide, hydroxydaunomyicin, oncovin, and prednisolone). Six chemotherapeutic cycles were given. Serum ?2M was repeated in cases after completion of chemotherapy.

RESULTS: The levels of serum ?2M levels were found to be significantly higher in patients with DLBCL $(\mathrm{P}<0.01)$. The levels were also higher significantly in patients with in advanced stages (stage III and IV) $(6.12 \pm 0.32 ? \mathrm{~g} / \mathrm{ml})$ than those with in early stages (stage I + II $)(\mathrm{P}<0.01)(3.08 \pm .065 ? \mathrm{~g} / \mathrm{ml})$. There was also a significant decrease in serum ?2M levels after therapy, in patients who achieved remission.

CONCLUSION : ?2M can be considered as a significant prognostic tool as the levels were significantly different between the pre and post - treatment groups and also declined significantly only in patients achieving remission.

KEY WORDS: Lymphoma, lymphoid cells, Beta-2 microglobulin

\section{P100}

\section{THE Role of Oral Pyridoxine In Renal Stones}

Mugadha Kowli, $*$ Rohini Bhadre, **Nilay

Chakarabarti***

\section{K.J. Somaiya Medical College, Mumbai, Maharashtra}

AIMS AND OBJECTIVES: To Study The Role Of Oral Pyridoxine (Vitamin B6)Supplementation In Addition To Standard Dietary Advice, Compared Against Only Dietary Advice, In The Reduction Of Urinary Oxalate Levels In Patients With Urinary Calculi Along With Proven Hyperoxaluria.

MATERIALS \& METHOD:74 Subjects with Hyperoxaluria and Calcium OxalateCalculi were Enrolled and Randomly Divided into Interventional Group(N=38) who Received Oral Pyridoxine 40mg/day with Dietary Restriction ofOxalate Rich Food and Control Group $(\mathrm{N}=36)$ who received only DietaryRestriction of Oxalate Rich Foods. Both Groups were evaluated and

Statistically Compared at the end of 3 Months. Unpaired $\mathrm{T}$ Test and MannWhitney Test were applied to compare the Results.

RESULT AND DISCUSSION: In Our Study, in the 24 Hour UrinaryOxalate Levels was reduced significantly $(P=0.0001)$ this reduction was Higher in the 
Interventional Group as Compared to the Control Group. Also, TheMean Change in 24 Hour Urinary Oxalate Levels, showed asignificant difference $(\mathrm{P}=0.0001)$ when comparison was done between Interventional Groupand Control Group..Hyperoxaluria Is an Important factor for Urinary Stone formation.Pyridoxine (Vitamin B6) is acofactor in the Alanine-Glyoxalate-Transaminase Pathway; which converts Glyoxalate To Glycine and reduces Oxalate production by inducing the Enzyme.

CONCLUSION: Oral Pyridoxine Supplementation in the dose of $40 \mathrm{mg} /$ Day inaddition to Dietary restriction of Oxalate Rich Foods results instatistically significant reduction of Urinary Oxalate Excretion,by decreasing the precipitation of Calcium Oxalateand thereby reducing the chances of recurrent Calcium Oxalate Stone formation. It can be used as asimple, cheap and easy to implement prophylactic measure for prevention of recurrence Of Calcium Oxalate Urolithiasis.

\section{P101}

\section{Serum Osteocalcin Levels In Type 2 Diabetes Melitus}

Avinanda Mahanta, Kailash Bhattacharyya,

Gauhati Medical College and Hospital, Guwahati-32, Assam

INTRODUCTION: Type 2 Diabetes Mellitus is the predominant form of diabetes worldwide, accounting for $90 \%$ of cases globally. Osteocalcin (OC)is an osteoblast derived non collagenous bone matrix protein. In several studies done in the last decade it has been found that though osteocalcin is a marker for bone metabolism it has a possible role in glucose metabolism. There is a possibility of existence of a feedback loop among bone, pancreas and adipose tissue. This study seeks to evaluate the relationship of osteocalcin in patients with type 2 diabetes mellitus compared to that with healthy controls in a tertiary care hospital of North-East India.

MATERIALS AND METHODS:The study was conducted in two broad groups. Case group contained 45 newly diagnosed cases of Type 2 Diabetes mellitus and control group contained 45 age and sex-matched subjects without Type 2 Diabetes mellitus. Demographic data (age, sex, weight, height) were determined by appropriate method, medical records and proper history taking. Clinical examination was done. Blood samples were collected by proper methods. Plasma glucose and HbA1c were determined by using auto analyzer and serum osteocalcin levels by elisa technique.

RESULTS: The results showed that the mean level of osteocalcin was significantly lower $(p<0.001)$ in case group containing newly diagnosed diabetics compared to healthy control group. There was significant negative correlation of serum Osteocalcin level with the glycemic status of the patient.

CONCLUSION: It was concluded from the study that apart from the known role of Osteocalcin in bone metabolism there may be a role of Osteocalcin in relation to energy metabolism in type 2 diabetes.

KEYWORDS: *Diabetes, *Osteocalcin

\section{P102}

\section{Prevalence of Vitamin D Deficiency In Orthopedic Patients: A Hospital Based Study}

$\underline{\text { Rekha Kumari }}^{1}$, Ritesh Runu², Anand Saran ${ }^{3}$, Uday Kumar ${ }^{4}$

\section{1, 3, 4, Department of Biochemistry,}

2. Department of Orthopedics, Indira Gandhi Institute of Medical Sciences Patna

INTRODUCTION : Vitamin D is considered essential for bone and muscle health and some studies have demonstrated the positive effects of vitamin D on metabolic diseases and cancer.But vitamin D deficiency is a global health problem.Lack or less availability of sunlight in some regions of the world, winter season, purdasystem,less intake of vitamin D containing food and unfortified food, illiteracy are other causes of high prevalence of vitamin D deficiency. Reasons of vitamin $\mathrm{D}$ deficiency are different in different regions in the world.In western countries, food products arefortified with vitamin D, but in developing countries like India, food fortification with Vitamin D is in a nascent stage. 
AIMS AND OBJECTIVES: This study was done to find the prevalence of vitamin D deficiency in orthopedic patients.

MATERIALS AND METHODS: Fifty patients over 20 years of age and both sexes having orthopedic problem were included in the study. $25(\mathrm{OH}) \mathrm{D}$ estimation was done with venous blood sample of the patients by chemiluminescence immunoassay method. Vitamin D deficiency was defined as serum levels of $25(\mathrm{OH}) \mathrm{D}<20$ $\mathrm{ng} / \mathrm{mL}$, insufficiency $20-29 \mathrm{ng} / \mathrm{mL}$ and normal level as $=$ or $>30 \mathrm{ng} / \mathrm{mL}$.

RESULTS: $37 \%$ patients were found to have deficient level, $8 \%$ insufficient level and only $5 \%$ patients were having normal level of vitamin D.

CONCLUSION: This result indicates vitamin D deficiency as a major health problem which needs further study with large number of orthopedic patients with stratification of patients in different groups.

KEY WORDS :Vitamin D, Deficiency, Orthopedic Patients,

\section{P103}

\section{Thyroid Profile In Diabetes With or Without Complication}

Anmol Kohli ${ }^{1}$, Sukhraj Kaur ${ }^{1}$, Mridula Mahajan ${ }^{1}$ and Pashaura Singh Sandhu

Department of Biochemistry ${ }^{l}$, Department of Medicine ${ }^{2}$ Government Medical College, Amritsar

INTRODUCTION- Diabetes Mellitus is a result of social influence and changing lifestyle. It is characterized by anbsolute or relative insulin deficiency in insulin secretion and/or insulin associated with chronic hyperglycemia. Diabetes and thyroid disorders have been shown to mutually influence each other and associated between both conditions have been long reported. Patients with Type2 DM are more prone to thyroid disorders. Hypothyroidism is them leads to an aggravation of microvascular complications (nepropathy, retinopathy, neuropathy). Diabetic patients with hypothyroidism are at an increased risk of cardiovascular
disease.Free and total T3 and T4 concentrations are usually normal or low in patients with nephropathy. Insulin an anabolic hormone enhances the levels of fT4 while it suppresses the levels of $\mathrm{T} 3$ by inhibiting hepatic conversion of $\mathrm{T} 4$ to $\mathrm{T} 3$.

MATERIAL AND METHODS-The present study is being conducted in Department of Biochemisrty in association with Department of Medicine GMC ASR. 100 patients suffering from type 2 Diabetes with complications were recruited for the past study and 100 age and sex matched individuals viewed as controls.

RESULTS-It was observed that mean \pm S.E levels of free T3 were $2.3 \pm 0.19, \mathrm{~T} 41.6 \pm 0.06$ and TSH is $2.3 \pm$ 0.44. Level of fT3 and fT4 were significantly reduced compared to controls. Further the study group was classified depending on the type of complications ie microvascular and macrovascular and it was observed that in patients with macrovascular complications levels of TSH were more whereas in patients with microvascular complications levels of fT3 and fT4 were less.

CONCLUSIONS- Patients with Type 2 DM with increased levels of TSH are more prone to diabetic complication.

KEY WORDS: Diabetes Mellitus, nepropathy, retinopathy, neuropathy, TSH

\section{P104}

\section{Evaluation of Calcium, Phosphorus and Vitamin D Level In Different Stages of Pregnancy In East Indian Population}

Ravi Kumar, Rekha Kumari, Priyanka Prasad, Swarnima Singh, Vibha Sushilendu

Department of Biochemistry, Indira Gandhi Institute of Medical Sciences, Patna, Bihar, India

INTRODUCTION: Vitamin D, serum calcium $(\mathrm{Ca})$ and inorganic phosphorus deficiency during pregnancy is a significant public health problem in many parts of the world. It is associated with an increased risk of preeclampsia, gestational diabetes, and preterm births. 
Approximately, $99 \%$ of the $\mathrm{Ca}$ and magnesium in the human body is located in the skeleton. $\mathrm{Ca}$ is a essential nutrient and mineral consumed in food and supplements. The mineral is stored bones where it is used to promote strength and support changes and growth throughout life. There is also free $\mathrm{Ca}$ in blood used by cells during the normal cellular function. The objective of this study was to assess the role of $\mathrm{Ca}$, phosphorous and vitamin $\mathrm{D}$ level in pregnant women at different age groups compare to nonpregnant women in East Indian population.

MATERIALS AND METHODS: This cross-sectional study was conducted on 180 normal women between 22 and 45 years of age in their first 24-28 weeks of pregnancy and 50 normal nonpregnant women. After 3 months of follow-up, serum levels of vitamin $\mathrm{D}$ and $\mathrm{Ca}$ and inorganic phosphorous were measured. Written informed consent was obtained from the subjects.

RESULTS: The mean age of women in the study group was $25.34 \pm 5.40$ years; while that in the control group was $25.80 \pm 5.90$ years. The age of study and control groups was matched. The result shows that serum $\mathrm{Ca}$ and inorganic phosphorous in pregnant women lower significantly $(\mathrm{P}<0.001)$, especially in the third trimester as compared to controls. However, vitamin-D levels in pregnant women lower significantly $(\mathrm{P}<0.001)$ at all trimester as compared to controls.

CONCLUSION: Vitamin D deficiency coupled with Ca and inorganic phosphorous deficiency may found to be highly prevalent in pregnant East Indian women. The decrease in serum vitamin-D, Ca and inorganic phosphorous levels parallels increase in gestational age. This may result from mineral transfer from mother to developing fetus.

KEY WORDS: Calcium, inorganic phosphorous, pregnancy, vitamin D

\section{P105}

Incidence of Tetrahydro Cannabinol Toxicity Detected At Manipal Poison Detection Centre, Manipal

Nayak Deeksha Dayanand, Vinutha R Bhat,
Padmanabha Udupa

Department of Biochemistry, Department of Forensic Medicine and Toxicology Kasturba Medical College Manipal, Manipal University, Manipal 576104

INTRODUCTION: Tetrahydro Cannabinol (THC) refers to a psychotropic cannabinoid and is the principal psychoactive constituent of cannabis. THC is considered one of the most commonly used illicit drugs in the world. The action of THC result from its partial agonist activity at the cannabinoid receptor CB1, located mainly in the central nervoussystem and $\mathrm{CB} 2$ receptor mainly expressed in the cells of the immune system. The psychoactive effects of THC are primarily mediated by the activation of cannabinoid receptors, which result in decrease in the concentration of the second messenger molecule cAMP through inhibition of adenylate cyclase.

AIM: To evaluate the incidence of tetrahydro cannabinol toxicity

MATERIALS AND METHODS: The reports of suspected poisoning cases came for evaluation to Manipal poisoning detection centre from 2016 to 2017 $(n=73)$ were obtained from laboratory information system after proper anonimization by laboratory incharge.

RESULT: Among 73 patients of suspected poisoning, aged about 17 to 70 years, of which 18 were detected positive for THC. Among 18 positive cases, 55\% were males and $45 \%$ were females.

DISCUSSION: THC toxicity is highly influenced between the age grouped from 17 to 26 years. Since exposure to THC in younger generation is more, creating awareness among them is necessary to avoid THC poisoning. Delta $9 \mathrm{THC}$ is the most psychoactive cannabinoid producing euphoria, relaxation, perceptual alterations, diminished pain and difficulties with memory and concentration. Repeated use over days to weeks induces considerable tolerance to the behavioural and psychological effects of cannabis.

KEY WORDS: Tetrahydro Cannabinol, psychotropic cannabinoid, adenylate cyclase. 


\section{P106}

\section{Yolk Sac Tumor with Omental Metastasis}

Mamta Saraf, Shilpa Asegawker

United Ciigma Hospital, Aurangabad, Maharashtra

INTRODUCTION : Extra-Ovarian yolk sac tumor arising in the omentum is extremely rare.As yolk sac tumor originated from the omentum has been rarely reported, its clinical information is very limited.

MATERIALAND METHODS : A Female of 22 years admitted to our hospital with complaintof abdominal pain and abdominal distension. She was diagnosed with yolk sac tumor with omental meastasis.She received 6 cycles of chemotherapy.But she died after twomonths due to lack of follow up and not taking chemotherapy on regular intervals.

CONCLUSION : Diagnosing yolk sac tumor may be difficult because the normal mensturalcycle and puberty can cause pain and pelvic symptoms. Yolk sac tumors have the worst prognosis of all ovarian germ cell tumors. They grow quickly and recur easily and are not easily treatable once they have recurred.

KEY WORDS : yolk sac tumor, omental metastasis, chemotherapy.

\section{P107}

\section{Urine and Serum Fetuin- A Levels In Patients With Urolithiasis}

S. Vanitha, Rajat Arora, Victoria Job, F.S. Geethanjali Department of Clinical Biochemistry, CMC Vellore.

INTRODUCTION:Identifying molecules and metabolic disturbances which affect the pathway of stone formation provides opportunities to modulate the process to prevent or decrease the rates of stone formation. Fetuin-A is a 59kd glycoprotein secreted by liver and it has been identified to inhibit extra osseous mineralization via formation of protein mineral complexes known as calciprotein particles (CPPs). CPPs inhibit calcium phosphate precipitation by facilitating mineral solubilization and clearance from the circulation and thus preventing ectopic calcification. Fetuin-A has been proven to decrease vascular calcification and its low serum levels are associated with increased vascular stiffness.

AIM: The objective of this study was to compare the 24 hour urine and serum fetuin-A levels of patients with and without urolithiasis.

METHODS: This was a case control study conducted from april 2014 to march 2016. Serum and 24 hour urine fetuin-A levels were measured in 41 patients with bilateral, multiple or recurrent urinary tract calculi (Group A) and 41 matched controls with no calculi (Group B). Fetuin levels were measured by enzyme linked immunosorbent assay (ELISA). Serum and urine fetuin-A levels in the two groups were compared.

RESULTS: The median (range) 24 hour urine fetuin-A value in Group A was 11.9 (1.12-221) $\mathrm{mg} /$ day and in Group B was 37.7 (1.28-125) mg/day. This difference was statistically significant (Man-Whitney test, $\mathrm{P}=$ 0.0169). The median (range) serum fetuin-A in Group A was $0.67(0.05-2.68) \mathrm{gm} / \mathrm{L}$ and in Group B it was 0.99 $(0.01-5.5) \mathrm{gm} / \mathrm{L}$. The difference between serum values in the two arms was not statistically significant (ManWhitney test, $\mathrm{p}=0.1817$ ) but serum creatinine adjusted mean log serum fetuin and urine fetuin were significantly different in the two arms $(\mathrm{p}=0.003)$. The mean \pm SD (range) serum creatinine in Group A was 0.98 \pm 0.25 (0.56$1.58) \mathrm{mg} \%$ and in Group B it was $0.83 \pm 0.16(0.58-1.18)$ $\mathrm{mg} \%$, (two sample $\mathrm{t}$ test, $\mathrm{p}=0.0031$ ).

CONCLUSIONS: Patients with urolithiasis have lower urine fetuin-A and creatinine adjusted serum fetuin -A levels.

KEYWORDS: fetuin-A, urolithiasis, kidney, ELISA

\section{P108}

Establishment of Reference Interval For Lipid Profile Parameters In Apparently Healthy Nepalese Adult Population.

$\underline{\text { RV Mahato }}{ }^{1}$, SK Sah, ${ }^{2}$ PP Rout ${ }^{3}$ M Lamsal $^{3}$ 
1. Tribhuvan University, Institute of Medicine, Mahrajgunj Medical Campus, Department of Biochemistry, Kathmandu, Nepal

2. Purbanchal University, Asian College of Advanced Studies, Department of Biochemistry, Lalitpur, Nepal.

3. BP Koirala Institute of Health Science, Department of Biochemistry, Dharan, Nepal

4. Civil Service Hospital, Department of Pathology, Kathmandu, Nepal

INTRODUCTION: Cardiovascular disease (CVD) is one of the leading causes of morbidity and mortality in the adult population in Nepal. During last decades, the incidences of CVD in Nepal increases drastically throughout the country. Lipid profile tests are the basis for the prediction of CVD. The number of medical laboratory is growing day by day in every part of the country but all most all of the laboratory are using the reference values supplied in the reagents kits which is mainly for either American or European population. Therefore, the interpretation of laboratory values with kit values is not the proper way for the prediction of CVD for the Nepalese population. Every lab should have their own reference values for each test in the contest of their locality. For this reason, this study has been designed to establish the reference range for the lipid profile test for healthy adult Nepalese population.

METHODS: A total617 apparently healthy individuals were recruited nationwide from five different regions of the country. Blood samples were collected and sera were separated and stored at?800 C. All the samples were measured collectively by auto-analyzer AU480 (Beckman-Coulter).Test results were standardized by measuring the value-assigned panel of sera which was provided by IFCC committee on Reference Intervals and Decision Limits (C-RIDL). With application of the latent abnormal values exclusion (LAVE) method, reference intervals (RIs) were derived by both parametric and nonparametric method by use of Reference Master Software provided by C-RIDL.

RESULTS: The reference interval (RI) values (lower limit-upper limit) for total cholesterol, triglyceride, HDLcholesterol and LDL-cholesterol obtained were (2.536.14), (0.42-3.32),(0.28-1.46) and (1.05-4.0) $\mathrm{mmol} / \mathrm{L}$ respectively. However, gender wise analysis demonstrated higher range for Cholesterol, triglyceride and LDL-cholesterol in men than female while HDLcholesterol has higher range in female than male.

CONCLUSION: It can be suggested that the reference interval values for lipid profile parameters obtained by this study can be used for the interpretation of laboratory values in the diagnosis, care and treatment of cardiovascular disease patients in context of Nepalese population.

KEY WORDS: Cardiovascular disease, HDLcholesterol, LDL-cholesterol

\section{P109}

\section{Cooling of Blood Sample: An Effective Alternative For Sodium Fluoride As A Preservative For Blood Glucose Samples}

\author{
Ketki K* $*$ Shailesh Kumar \\ *Department of Biochemistry, Heritage Institute of \\ Medical Sciences, Varanasi \\ Department of Forensic Medicine, Heritage Institute \\ of Medical Sciences, Varanasi
}

INTRODUCTION: Even with the use of fluoride it takes approximately three to four hours for these glycolytic inhibitors to become fully effective and stabilize glucose levels. If we avoid sodium fluoride as a preservative then the same samples can be used for other assays in cases when sample size must be restricted.

MATERIALS\& METHODS: Six samples in duplicate one with fluoride and other without fluoride are taken from diabetic patients attending our out-patient department of Heritage institute of medical sciences,varanasi.

RESULTS: Fluoride containing sample showed 112 $\mathrm{mg} \%$ at zero and $109 \mathrm{mg} \%$ at three hrs respectively (decrease of $3 \mathrm{mg}$ ) and refrigerated samples without fluoride, showed $108 \mathrm{mg} \%$ and $95 \mathrm{mg} \%$ respectively (decrease of $13 \mathrm{mg}$ ) However the decrease was not statistically significant between zero hr and $3 \mathrm{rd}$ hr values. 
CONCLUSION: Our results show that effective cooling of blood samples immediately after withdrawal can be an effective preservative than the fluoride and also we feel it should be recommended as it avoids taking two different samples if the patient wants some other tests and in cases where samples should be restricted.

KEYWORDS: Blood sugar, fluoride containing vacutainers /refrigerated samples in plain vacutainers

\section{P110}

Use of Serum Cancer Biomarkers and Prevalence of Cancer In Central Nepal: A Tertiary Care Hospital Based Study

\section{$\underline{\text { Sanjaya Kumar Shah }}{ }^{1}, \mathrm{RV}$ Mahato ${ }^{2}$}

1. Department of Medical Laboratory Technology, Asian College for Advance studies, Purbanchal University, Nepal

2. Department of Biochemistry, Institute of Medicine, Tribhuwan University, Nepal

INTRODUCTION: Cancer is one of the major problems worldwide. In Nepal, some 30,000 new cases of cancer are diagnosed every year in the country. The burden of cancer increases in Nepal. Due to the unavailability of a population based cancer registry it is difficult to precisely predict of incidence rates. However, using hospital-based study to predict the cancer incidence in Nepal. Biomarker is a biological molecule found in blood, other body fluids, or tissues that is a sign of a normal or abnormal process, or of a condition or disease. Large numbers of cancer biomarkers are used worldwide but limited number of serum cancer biomarkers are being used in Nepal. Aim of this study to find out the commonly used cancer biomarkers and prevalence of cancer in central Nepal.

AIM: Aim of this study to find out the commonly used cancer biomarkers and prevalence of cancer in central Nepal.

METHODS: A hospital based retrospective study was conducted. Study was carried out using data retrieved from the register maintained in the Department of Laboratory service of Bhaktapur Cancer Hospital, a tertiary care hospital, Bhaktapur, Nepal between $14 \mathrm{~h}$ April, 2015, and 15 April 2016. Total 821 samples were included. SerumCancer Biomarkers were analysed in Fully Automated mini vidas (biomerieux) - CLIA Method

RESULTS: 275 samples were analyzed for Cancer Biomarkers, CA 15.3: 89\% normal and 31\% Breast Cancer. 219 samples were analyzed for CEA: 71.8\% normal and $28.3 \%$ Colon cancer, $(\mathrm{p}<0.05) .106$ samples were analyzed for beta HCG: $72.6 \%$ normal and $27.4 \%$ ovarian germ cell tumor. 104 samples were analyzed for CA 19.9: $64.4 \%$ normal and $35.6 \%$ colorectal cancer. 61 samples were analyzed for PSA: $60.7 \%$ normal and $39.3 \%$ prostate cancer. 196 samples were analyzed for CA125. $61.7 \%$ normal and $38.3 \%$ ovarian cancer $(\mathrm{p}<0.05)$ There was a significant positive correlation with age above 60 years and ovarian cancer. 61 samples were analyzed for AFP. 80.3\% normal and $19.7 \%$ benign liver disease $(\mathrm{p}<0.05)$.

CONCLUSION : This study revealed that Commonly used cancer biomarker are CA 15.3,CEA, beta HCG, CA19.9, PSA, CA125 and AFP. Prevalence of Breast cancer, Colon cancer, ovarian cancer and benign liver disease relatively significant in number and Above 60 years female are more prevalent to the ovarian cancer. Continual future research and large sample size is warranted to study more significant result.

KEY WORDS:Tumour markers,Breast cancer, Colon cancer, ovarian cancer and benign liver .

\section{P111}

\section{A Study of Biochemical And Hematological Abnormalities In Dengue and Chikungunya Viral Fever}

Jyoti Shukla, Sumita Sharma, Pankaj Sharma, Nita Garg

Department of Biochemistry, Shri Guru Ram Rai Institute of Medical \& Health Sciences, Dehradun

INTRODUCTION:- Emerging viral infections have become a serious problem in recent years. Emergence or reemergence of severe arboviral hemorrhagic fevers caused by mosquito borne viruses, such as Dengue virus 
and Chikungunya (CHIK) virus, have been frequently reported in the Indian subcontinent in the past few years. From the clinical perspective, these infections have similar clinical manifestations and are difficult to distinguish from one another.

OBJECTIVE:-To compare the laboratory features of confirmed cases of Chikungunya(CHIK) and Dengue fever(DF).

MATERIAL \& METHOD :-Data has been compared between 27 ( $9 \mathrm{M}+18 \mathrm{~F}$ ) Chikungunya (RT-PCR Positive) patients group and 24 ( $16 \mathrm{M}+8 \mathrm{~F}$ ) dengue (NS1 antigen positive) patients group for thrombocyte count, hepatic function markers(SGOT and SGPT) and renal function (blood urea and serum creatinine) was compiled and studied.

RESULTS:- Females were found to be more affected in Chikungunya while in Dengue fever males were more affected. Chikungunya patients had significantly higher platelet count $(193.5 \times 10 ? / \mathrm{L} \pm 84.25 \times 10$ ?/L) than dengue fever(101.79X10?/L \pm 42.51X10?/L). Urea(CHIK$33 \mathrm{mg} / \mathrm{L} \pm 12.6 \mathrm{mg} / \mathrm{dl}$, DF- $34.04 \pm 18.08 \mathrm{mg} / \mathrm{dl})$ and creatinine (CHIK- $0.9 \pm 0.24 \mathrm{mg} / \mathrm{dl}$, DF- $0.85 \mathrm{mg} / \mathrm{dl} \pm$ $0.28 \mathrm{mg} / \mathrm{dl}$ ) levels were comparable in both the groups, indicating that there was no renal dysfunction. Transaminases were found to be higher in dengue fever( SGOT- 158.25 U/L $\pm 77.14 \mathrm{U} / \mathrm{L}$, SGPT- $89.87 \mathrm{U} / \mathrm{L} \pm$ $32.5 \mathrm{U} / \mathrm{L}$ ) than Chikungunya [SGOT- $45.37 \mathrm{U} / \mathrm{L} \pm 18.82$ U/L , SGPT- $42.63 \mathrm{U} / \mathrm{L} \pm 18.22 \mathrm{U} / \mathrm{L}]$

CONCLUSION:-Dengue and Chikungunya infections continue to co-exist in many tropical countries. Our study has shown that it is possible for clinicians to use simple laboratory tests to predict these infections for appropriate management even if there is substantial overlap in clinical presentation.

KEY WORDS: Dengue virus, Chikungunya, Transaminases, creatinine

\section{P112}

Assessment of Serum Bile Acids and Hepatic Enzymes In Suspected Cases of Intrahepatic Cholestasis of Pregnancy
Sumita Sharma, Kiran Bhat, Rajeev Singh Kushwaha, Tariq Masood, R.K Singh*

Department of Biochemistry, Shri Guru Ram Rai Institute of Medical and Health Sciences, Dehradun (UK), *T.S. Mishra Medical College \& Hospital, Amausi, Lucknow (UP)

INTRODUCTION: Intrahepatic cholestasis of pregnancy (ICP) or obstetric cholestasis is the most common pregnancy related liver disorder characterized by maternal pruritus in the third trimester withelevated serum bile acidsand increased rates of adverse fetal outcomes. The etiology of ICP is multifactorial with environmental, hormonal, and genetic contributions. It results from the cholestatic effects of reproductive hormones and their metabolites in genetically predisposed women. The present study was conducted to assess the levels of serum bile acids and activities of various hepatic enzymes in ICP.

MATERIAL AND METHODS:The present study included 54 suspected cases of ICP, who presented in the OPD/IPD of Shri MahantIndiresh Hospital attached to Shri Guru Ram Rai Institute of Medical and Health Sciences,Dehradun. Serum bile acids andhepatic enzymes were investigated inthe above patients.

RESULTS: On statistical analysis, it was found that the serum alanine transaminase showed better correlation $(\mathrm{r}=0.347, \mathrm{p}=0.01)$ with serum bile acid levels as compared to aspartate transaminase $(\mathrm{r}=0.303, \mathrm{p}=0.02)$. Since alkaline phosphatase is elevated in pregnancy due to production by the placenta, so it cannot be used as a reliable marker of ICP, although it also showed significant correlation with bile acid levels. No significant correlation was found between gamma glutamyltranspeptidase and bile acid levels.

CONCLUSION:It can be concluded that serum alanine transaminase can be used as a marker in the follow up of patients diagnosed with ICP for better management and prognosis.

KEY WORDS: Intrahepatic cholestasis of pregnancy, serum bile acids, alanine transaminase, aspartate transaminase. 


\section{P113}

\section{Role of Natural Antioxidants In The Treatment Of Osteoporosis - A Global Epidemic}

\author{
Faiza Ismail*, Naiyer Asif**, Abbas Ali Mahdi***, \\ Najmul Islam*\# \\ *Department of Biochemistry, \\ **Department of Orthopaedic Surgery, \\ *\# Department of Biochemistry, Faculty of Medicine, \\ J. N. Medical College, Aligarh Muslim University, \\ Aligarh, 202002, Uttar Pradesh, India, \\ *** Department of Biochemistry, \\ King George Medical University, Lucknow, \\ Uttar Pradesh, India.
}

INTRODUCTION: Osteoporosis is a progressive and chronic disease of the skeleton characterized by bone fragility, due to reduced bone mass. The imbalance between the generation of reactive oxygen species (ROS) and the activity of antioxidant defence system leads to oxidative stress by providing a more oxidized bone microenvironment. There is a synergistic action of ROS, TNF- $\alpha$ and IL- $1 \alpha$ levels on each other, leading to an increase in bone markers and increased osteoclasts activity and hence osteoporosis. In the present work an attempt was made to regulate ROS production in vivo by natural antioxidants like punicalagin from pomegranate and resveratrol from grapes having potent antioxidant as well anticancer activities.

MATERIALS AND METHODS: Effects of antioxidants were evaluated in the treatment of osteoporosis by employing cell culture study, ELISA, GPx activity determinationand GSH assay.

RESULTS: Results have shown that there is a suppressed GPx activity in osteoporosis patients $(n=30 ; p<0.001)$ indicating impairment of $\mathrm{H} \alpha \mathrm{O} \alpha$ neutralizing mechanism. Intramonocyte GSH levels were also observed to be decreased, again an indication of weak antioxidant system. Levels of by-product of lipid peroxidation i.e. malondialdehyde (MDA) was high in monocytes culture of osteoporosis patients, pointing to the increased oxidative stress. Punicalagin and resveratrol $(0-20 \mu \mathrm{g} / \mathrm{ml})$ increased GSH levels and GPx activity dose dependently to almost normal values. MDA levels were decreased dose dependently by these antioxidants $(0-25 \mu \mathrm{g} / \mathrm{ml})$. TNF- $\alpha$ and IL- $1 \alpha$ levels in 24 hours monocytes culture supernatants were down regulated dose dependently $(0-$ $100 \mu \mathrm{g} / \mathrm{ml}$ ) in patients.

CONCLUSION:Antioxidants thus have an important role in curbing the free radicals the main culprit of osteoporosis and thus helpful in the management of osteoporosis.

KEY WORDS: Glutathione peroxidase (GPx) activity, GSH, TNF- $\alpha$, natural antioxidant.

\section{P114}

\section{Ischemia Modified Albumin In Prediabetes And Diabetes Mellitus}

Nadeem Khan G, Vijetha Shenoy Belle

Department of Biochemistry, Kasturba Medical College Manipal, Manipal University, Manipal. 576104

INTRODUCTION: Ischemia modified albumin is a novel marker for oxidative stress. The serum levelof ischemia modified albumin has been increased in many oxidative stress condition like Diabetes mellitus, coronary artery diseases, hypothyroidism, myocardial infarction etc. Thus this study is conducted to measure ischemia modified albumin in diabetics and correlate IMA with glycated hemoglobin.

Objectives: To estimate \& compare Ischemia modified albumin in Normal, Prediabetes and Diabetes mellitus.

Meterials and Methods: Institutional ethics committee permission was obtained prior to the study, total 57 serum sample was obtained (17 normal subject, 20 prediabetic and 20 diabetic), IMA level were estimated by spectrophotometric method. Glycated hemoglobin was measured by immunoturbidimetric method. Statistical analysis was done using ANOVA and Pearson's correlation. $\mathrm{P}$ value $<0.05$ was considered to be significant.

Results: Ischemia modified albumin is increased in Diabetes $(0.50 \pm 0.10)$ and Prediabetes $(0.51 \pm 0.10)$ compared to Normal $(0.47 \pm 0.12)$ but statistically not 
significant.

Conclusion: No changes observed in present study, IMA levels in Diabetes and Prediabetes conditions. Since sample size is small, large sample size is required to obtain reliable results.

Key words:Ischemia, albumin, oxidative stress, pre diabetic, diabetic

\section{P115}

Evaluation of Serum Copper And Zinc with Tsh Level In Subclinical Hypo And Hyper Thyroid Patients of Coastal Karnataka Region.

Thriveni G.K, Krithishree. S, Shobha. U. Kamath

Department of Biochemistry, Kasturba Medical

College Manipal, Manipal University,

Manipal. 576104

INTRODUCTION: Thyroid stimulating hormone (TSH) is one of a family of glycoprotein hormones including $\mathrm{LH}$ and FSH. Thyroid diseases are the commonest endocrine disorders of the world. Population study estimated that 108 million people in India are suffering from endocrine and metabolic diseases of which thyroid abnormalities contribute 42 million. Hyperthyroidism may result from the increased concentration of thyroid hormone. Hypothyroidism may result from decreased concentration of thyroid hormone. Copper and Zinc are important for healthy thyroid function. Zinc is responsible for converting inactive T4 form of thyroid hormone, thyroxin into active T3 form. Copper stimulates the thyroid and protect the body against too much thyroxin in the blood. Thus, this study was conducted to measure serum zinc and copper levels in thyroid patients and to correlate it with TSH levels.

MATERIALS AND METHOD: Blood samples of 60 patients were collected out of which 20 weresubclinical hyperthyroidism, 20 were subclinical hypothyroidism and 20 control. Serum copper and zinc levels are estimated by kit method using colorimetric method.

RESULTS: The mean serumzinc $(\mathrm{P}=0.04)$ is significantly increased in subclinical hyperthyroid patients $(567.5 \pm 51.91)$ compared to subclinical hypothyroid patients $(310.2 \pm 124.7)$. Copper $(\mathrm{p}=0.5)$ is decreased in subclinical hyperthyroid patients (120.5 \pm 62.97$)$ but was not statistically significant.

CONCLUSION: Significant relationship between zinc, copper and TSH with subclinical hypo and hyper thyroid patients were detected. There is a positive correlation between TSH and zinc in subclinical hypothyroid patients. Zinc has definite role in the metabolism of thyroid hormones.

KEY WORDS: Thyroid stimulating hormone, LH, FSH, zinc, copper

\section{P116}

Sialic Acid Levels In Subclinical Hypothyroid And Subclinical Hyperthyroid Patients Ofcoastal Karnataka Region.

Krithishree S, Thriveni G.K, Shobha U Kamath.

Department of Biochemistry, Kasturba Medical

College Manipal, ManipalUniversity,

Manipal,576104.

INTRODUCTION: Sialic acid (SA) is a N- or Osubstituted derivative of neuraminic acid, and it is found as a terminal component of glycolipids and glycoproteins. Excessive ROS can target oligosaccharides in protein structuresand change their biological functions by breaking SAs located in the terminal position. Additionally, excessive levels of TSH, which can be seen in subclinical hypothyroidism, was shown to cause increased production of ROS which may further result in breaking of SA located in terminal position. Thus, this study is conducted to measure sialic acid level in patients with subclinical hypothyroidism and subclinical hyperthyroidism.

OBJECTIVES: To estimate and compare sialic acid in normal, subclinical hypothyroidism and hyperthyroidism patients.

MATERIALS AND METHODS:Total 60 serum samples were obtained and was divided into 3 groups(20 normal controls, 20 subclinical hypothyroidism and 20 
subclinical hyperthyroidism). Sialic acid level was estimated by spectrophotometric method at 530nm. Statistical analysis was done using ANOVA and Pearson's correlation.

RESULTS: MeanTSA was found to be highest in the patient group with subclinical hypothyroidism (87.2 \pm 29.4$)$ compared with the control group (77.5 \pm 23.9$)$ and patients with subclinical hyperthyroidism (61.2 \pm 15.7$)$, but statistically were not significant. Further a larger sample size may be required to obtain reliable results.

CONCLUSION: Increased sialic acid in the serum indicates oxidative stress. The patients with subclinical hypothyroidism have the highest serum sialic acid levels, indicating greater oxidative stress in this group as compared to normal controls and subclinical hyperthyroidism patients.

KEY WORDS: Sialic acid, glycolipids, glycoproteins, TSH

\section{P117}

Effect of Different Fractions of methanolic Extract of Cinnamomumzeylanicumon Sheep Kidney Angiotensin Converting Enzyme: An In Vitro Study

Padmanabha Udupa E G, Ranjini H S and Vinutha R Bhat

Department of Biochemistry, Kasturba Medical College, Manipal, Manipal University, Manipal, India- 576104

INTRODUCTION: Angiotensin converting enzyme (ACE, EC 3.4.15.1), is a zinc metallopeptidase that cleaves Angiotensin I to Angiotensin II (a potent vasoconstrictor). It is most commonly associated with the regulation of blood pressure. Captopril, a known ACE inhibitor isused for treating hypertension, which is well tolerated with certain side effects. Medicinal plant extracts are in use to treat many diseases and can be a good alternative for therapeutic outcomes because of their wellness, effectiveness and quality.
AIM: The present study was aimed to estimate the effect of angiotensin converting enzyme (ACE) activity in presence of different fractions of methanolic extract of Cinnamomumzeylanicumon kidney ACE.

METHOD: ACE activity of kidney tissue was measured with Hippuryl-Histidyl-Leucine (HHL) as substrate and the hippuric acid released was measured spectrophotometrically at $228 \mathrm{~nm}$. Similarly, ACE activity was measured in the presence of captopril and withdifferent fractions (Petroleum ether, ethyl acetate and aqueous) of methanolic extract of Cinnamomum zeylanicumin the enzyme assay.

RESULTS:The linearity of ACE activity in kidney was established with HHL as substrate for an incubation period of $30 \mathrm{~min}$ at $37^{\circ} \mathrm{C}$. ACE activity was confirmed with specific ACE inhibitor Captopril.ACE activity was determined in the presence of petroleum ether, ethyl acetate and aqueous fractions of methanolic extract of C.zeylanicum. Ethyl acetate fraction has significantly inhibited ACE activity by $17.98 \%$ whereaspetroleum etherand aqueousfractionsincreased ACE activity by $11.7 \%$ and $4.9 \%$ respectively.

CONCLUSION: Ethyl acetate fraction of methanolic extract contained ACE inhibitor, whereas petroleumether and aqueous fractions contained ACE stimulants. Further isolation \&identification of the active componentsand in vivo studiesof these fractionsmay beuseful in the treatment of hypertension and hypotension or related disorders.

KEY WORDS: Angiotensin converting enzyme, Hippuryl-Histidyl-Leucine, Cinnamomum zeylanicumin,

\section{P118}

\section{Status of Serum Vitamin D In Healthy Persons}

Mohammad Shahrukh, Rajeev Singh Kushwaha, Tariq Masood, Sumita Sharma.

Department of Biochemistry*, Shri Guru Ram Rai Institute of Medical and Health Sciences and Shri Mahant Indiresh Hospital, SGRR University, Dehradun, Uttrakhand. 
INTRODUCTION:Vitamin D insufficiency affects almost $50 \%$ of the population worldwide. An estimated 1 billion peoples worldwide, across all ethnicities and age groups. The high prevalence of vitamin D insufficiency is a particularly important public health issue because hypovitaminosis $\mathrm{D}$ is an independent risk factor for total mortality in the general population. Children and young- and middle-aged adults are at equally high risk for VDD and insufficiency worldwide. VDD is common in Australia, the Middle East, India, Africa, and South America. Pregnant and lactating women who take a prenatal vitamin and a calcium supplement with vitamin D remain at high risk for VDD.

AIM \& OBJECTIVE: To assess of Vitamin D levels in healthy Indians.

MATERIAL \& METHODS: This study was conducted in the Department of Biochemistry, at SMI Hospital Patel Nagar, Dehradun (UK). We took 50 healthy individuals of serum Vitamin D who attended OPD, where as 20 male patients and 30 female patients. The data were compared using suitable statistical methods. Serum Vitamin D was measured by Immunoassay method with the help of VITROS ECi/ ECiQ Immunodiagnostic systems.

RESULT: The mean value of Serum Vitamin D was found to $16.37 \pm 10.35$ in male as compared to $17.39 \pm 12.20$ in female patients, where as the age mean value $51 \pm 19.44$ of male patients as compared to $44.70 \pm 14.70$ of female patients. The serum Vitamin D level was slightly lower in males as compared to females.

CONCLUSION: It is suggested to measure the serum 25-hydroxyvitamin $\mathrm{D}$ level as the initial diagnostic test in patients as well as in healthy person, who at risk for deficiency. Treatment with either vitamin D2 or vitamin D3 is recommended for the deficient patients. This conclusion can't be drawn as the study in as progress.

KEY WORDS: Vitamin D, 25-hydroxyvitamin D, hypovitaminosis D

\section{P119}

\section{Study of Serum CA125 In Patients With Massive Exeudative Pleural Effusion}

Monit Singh*, Rajeev Singh Kushwaha*, Tariq Massod*, Sumita Sharma*, Dev Singh Jangpangi** and R K Singh***.

Department of Biochemistry and Pulmonary Medicine, Shri Guru Ram Rai Institute of Medical and Health Sciences and Shri Mahant Indiresh Hosspital, SGRR University, Dehradun, Uttrakhand. Department of Biochemistry, TS Mishra Medical College and Hospital, Amausai, Lucknow.

INTRODUCTION: Primary Infection and Malignancies are inflammatory causes of fluid accumulation in the pleural space. TB is one of the infective causes of pleural effusion and is similar to Malignancies, although their management is extremely different. CA 125 is a glycoprotein tumor marker with Molecular weight of $200 \mathrm{KD}$, which is found on the surface of ovarian and some normal and inflammatory cells. In both Malignancy and Tuberculosis, this tumor Marker increases in serum and consequently in pleural fluid. This study was conducted to evaluate and compare CA-125 tumor marker in Exeudative pleural effusion resulting from Malignancies and tuberculosis.

MATERIALS AND METHODS: The Study was conducted in Department of pulmonary medicine and biochemistry of Shri Guru Ram Rai Institute of Medical and Health Sciences and Shri Indiresh Hospital, Dehradun in the period from 2016 to 2017. A total number of 33 blood samples were taken in which $21 \mathrm{~TB}$ patients ( 15 men and 6 women), with the mean $( \pm$ SD) age of $52.1 \pm 16.4$ years and 12 patients was affected by malignant tumors ( 4 men and 8 women ) with the mean $( \pm \mathrm{SD})$ age of $60.4 \pm 12.6$ years were evaluated during 2016 to 2017. In Malignant cases, diagnosis was made through microscopic inspection of the biopsy samples and cytology of pleural fluid. For recognition of tuberculosis, culture and biopsy of pleura as well as ADA marker was used. CA-125 and ADA was measured by Immunoassay method with the help of VITROS ECi/ ECiQ Immunodiagnostic systems.

RESULTS: The mean $( \pm \mathrm{SD})$ level of CA-125 in Serum was $82.508 \pm 44.191$, and $147.69 \pm 136.04 \mathrm{U} / \mathrm{ml}$, while ADA mean levels are 73.22 \pm 54.36 , and $47.52 \pm 39.3 \mathrm{IU} /$ $\mathrm{L}$ in tuberculosis and Malignancies respectively. The Malignancy was confirmed by the help of Biopsy which 
shows 12 patients positive and 21 patients Negative.

CONCLUSION: CA-125 marker levels in Pleural Effusion may be used as a diagnostic biomarker for differentiation of TB, Pneumonic, and Malignancy induced pleural effusion.

KEYWORDS: CA-125, Exeudative pleural effusion, Tuberculosis, Malignancy.

\section{P120}

\section{Body Weight Changes In Streptozotocin Induced Diabetic Rats Treated With Bixaorellana}

Vinutha R Bhat ${ }^{1}$, Padmanabha Udupa ${ }^{1}$, Samreen

Sheikh $^{1}$, Anushree U Salian ${ }^{2}$, Yousef Razeiah ${ }^{1}$

1. Department of Biochemistry, Manipal University,

Manipal,

2. Department of nuclear medicine, Manipal

University, Manipal

OBJECTIVE: The objective of present study was to evaluate the effects of the aqueous extract of BixaOrellana on diabetes mellitus induced experimental adult Wistar rats by Streptozotocin and comparison of changes in body weight between normal and diabetic rats. Intra-venous injection of $60 \mathrm{mg} / \mathrm{kg}$ dose of Streptozotocin in adult wistar rats, makes pancreas swell and at last causes degeneration in Langerhans islet beta cells and induces experimental diabetes mellitus in the 2-4 days.

METHODS: Diabetes was induced by administering streptozotocin dissolved in saline while the normal control group was given propylene glycol. Animals were randomly assigned into different 4 groups. Group 1(Normal Control, NC), treated with vehicle (ethylene glycol) n=6, Group 2(Diabetic Control, DC), treated with vehicle (ethylene glycol) n=6, Group 3(Insulin treated-- ie: 6 units/kg body weight ) n=6, Group 4(Test, T), treated with aqueous extract of B. orellana $(200 \mathrm{mg} / \mathrm{kg}$ body weight orally) $n=14$. The body weight was measured in day 1, 15 and day 30 in both control and experimental group.

RESULTS:The treatment with the aqueous extract (200 $\mathrm{mg} / \mathrm{kg}$ body weight) showed no statistical significance with respect tobody weight,when it is compared among the group 2 and 3 on day $1(\mathrm{p}<0.127)$,day $15(\mathrm{p}<0.519)$ and day $30(\mathrm{p}<0.127)$. The alteration in body weight of group 3 was noticeable in day $30(257.11 \pm 33.03) \mathrm{g}$ when compared with group $2(226.16 \pm 40.3)$ gas well as diabetic control group in day $1(226.33 \pm 22.65) g$.

CONCLUSION: The employed dose of the extract showed considerable increase in weight in day 30 that might be significant clinically and indicates a long term usage of aqueous extract of BixaOrellanamay have beneficial effect on diabetes.

KEY WORDS: Bixa Orellana, Streptozotocin, Langerhans islet beta cells, B. orellana

\section{P121}

To Evaluate The Effect of Levothyroxine Replacement Therapy on Lipid Profile Parameters In Patients With Subclinical Hypothyroidism In Tertiary Care Centre of Kumaon Region

$\underline{\text { Seema Gupta }}{ }^{1}$, Sangeeta Singh ${ }^{2}$, Paramjeet Singh ${ }^{3}$, Basant Joshi ${ }^{4}$

Department of Biochemistry1, 2, 4, Department of Medicine, Government Medical College, Haldwani, Uttarakhand

INTRODUCTION: Subclinical hypothyroidism (SCH) represents a condition of mild to moderate thyroid failure characterized by normal levels of thyroxine (T4) and triiodothyronine (T3), with mildly elevated concentration of thyroid stimulating hormone (TSH) with or without clinical symptoms. Various studies have shown inconsistent results concerning the derangements of lipid profile parameters in $\mathrm{SCH}$,also there is no consensus on whether substitution of levothyroxine (L-t4) has beneficial effects on serum lipid profile.This study was therefore planed to evaluate the effects of hypothyroidism on lipid profile and to study the effects of L-t4 treatment on thyroid and lipid parameters in area around kumaon region. 
MATERIAL \& METHODS: This study included a group of 100 newly diagnosed $\mathrm{SCH}$ patients and 75 age $\&$ sex matched euthyroid controls. Thyroidfunction tests (T3, T4 ,TSH) and lipid profile parameters (Total cholesterol (TC) ,Low density lipoprotein cholesterol (LDLc), Triglyceride, High density lipoprotein cholesterol (HDLc)were estimated in all subjects after an overnight fast of 12 hours.Out of $100 \mathrm{SCH}$ patients 58 selected patients were given L-t 4 treatment and were followed up after 3 months with repeat thyroid and lipid profile tests.

RESULTS: our resultsshowedsignificantly raised levels of TC, LDLc ,TG in SCH patients when compared with euthyroid controls. HDLc levels were also raised in $\mathrm{SCH}$ patients but insignificant statistically. After thyroxine replacement therapy for 3 months, there was a significant decrease in levels of TC and LDLc. The mean levels of TG and HDLc were also reduced after L-t4 therapy but insignificant statistically.

CONCLUSION:Our study demonstrated the subclinical hypothyroidism has adverse effects on lipid profile parameters. The restoration of euthyroidism with Lthyroxine therapy effectively reduces atherogenic lipids and thus may reduces the risk of cardiovascular complications.

KEY WORDS: hypothyroidism, levothyroxine, euthyroid, atherogenic

\section{$\mathbf{P 1 2 2}$}

\section{Rays Induced Oxidative Stress In Cerebral Tissue of Albino Wistar Rats}

Sudha K, Reshma K, Gaya PR, Vinitha Dsouza, Charu Yadav

Department of Biochemistry, Kasturba Medical College Mangaluru, Manipal University, Manipal, India

INTRODUCTION: Radiotherapy has been increasingly used as an effective tool in the treatment of cancer. Radiations might produce toxic effects to the surrounding normal cells mediated by free radicals. The study of normal tissue response to $\mathrm{x}$ rays is of great importance in cancer patients who undergo radiotherapy. Brain is the logical target of free radical injury due to high lipid content and low antioxidants. Hence, this study was undertaken to study the toxic effects of radiation on brain.

MATERIALS AND METHODS :Albino rats exposed to whole body radiation of 6 Gy per minute formed test group and rats that were not irradiated formed control group. Each group had eight animals each. Malondialdehyde, SOD, catalase, glutathione and acetyl choline esterase were estimated in brain homogenates by spectrophotometric methods.

RESULTS: Brain antioxidant enzymes viz., SOD and catalase decreased significantly in rats exposed to radiations compared to normal rats. Acetyl cholinesterase a marker of low grade inflammation increased significantly in $\mathrm{x}$ ray treated rats. The increase in lipid peroxidation was also statistically significant in these rats compared to control group.

CONCLUSION: On the whole, it can be concluded that free radical toxicity may be one of the major factors contributing to radiation pathology in rats.

KEY WORDS: Antioxidant enzymes, Acetyl cholinesterase, Lipid peroxidation

\section{P123}

Study of Chronic Effects of Varying Dosage of X-Rays on Hepatotoxicity And Nephrotoxicity In Wistar Albino Rats

\author{
Reshma K* , Sudha K*, Debjith C* , Chiranth*, Rahul* \\ Department of Biochemistry, Kasturba Medical \\ College, Mangalore,Manipal University
}

INTRODUCTION:Ionising radiations are reported to cause damage to living systems. This study focuses on the chronic effects of various doses of radiation in the liver and kidneys of albino wistar rats subjected to irradiation using X-rays.

MATERIALS AND METHODS: In the present study, albino wistar rats were exposed to various doses of X- 
rays (4gy,6gy and $6.6 \mathrm{gy}$ ) and the animals were observed for a period of 30 days,following which they were sacrificed and liver and kidney tissues, which are the targets of radiation induced damage, were analysed for the oxidative stress markers namely reduced glutathione, SOD, catalase and lipid peroxidation indicater,MDA. Histopathological studies of the liver and kidneys were also performed.

RESULTS: Indicated that there was a significant increase in GSH and SOD levels in the animals exposed to radiation compared to controls $(\mathrm{p}<0.05)$. A decrease in these values was observed at 6.6 gy compared to $6 \mathrm{gy}$ which was non significant.. However there was a significant and consistent decrease in catalase and a similar increase in MDA with increased doses of $\mathrm{X}$ rays $(\mathrm{p}<0.05)$. Damage in hepatic structural architecture with disruption of central vein as well as necrosis of the epithelial cells of PCT and DCT with lymphocyte infiltration, correlated positively with increased doses of $\mathrm{x}$ rays.

CONCLUSION: Our study strongly supports the hypothesis of the involvement of free radicals in radiation induced damage to living systems .

KEY WORDS: Radiation, oxidative stress, hepatotoxicity, nephrotoxicity

\section{$\mathbf{P 1 2 4}$}

\section{Assessment of Plasma B-Type Natriuretic Peptide (BNP) And High Sensitive C-Reactive Protein Among Diagnosed Patients of Diabetes Mellitus With And Without Cardiovascular Disease.}

$\underline{\text { Smriti Rai }}{ }^{1}$, Mingma L. Sherpa ${ }^{2}$, Sameer Bhandari ${ }^{3}$, Bidita Khandelwal ${ }^{4}$.

Sikkim Manipal Institute of Medical Sciences, Central Referral Hospital,5th Mile Tadong, East Sikkim. 737102.

INTRODUCTION: Role of plasma B-type natriuretic peptide (BNP) and hsCRP as a marker for diagnosing cardiovascular events among DM is being studied separately and together in different populations. Our study is designed to assess the levels of BNP and hsCRP among patients with DM attending the Central Referral Hospital, Gangtok, Sikkim.

AIMS AND OBJECTIVE: To assess the levels of plasma B-type natriureticpeptide (BNP) and hs-CRP among diabetics in Sikkim and tocompare their levelbetween diabetics with and without cardiovascular events.

METHODOLOGY: 75 diagnosed patients of diabetes mellitus were included in the study out of which 37 had associated cardiovascular diseases. Blood sugar (GODPOD method), glycated haemoglobin (immunoturbidimetric test), plasma B-type natriuretic peptide (BNP) and high sensitive $\mathrm{C}$-reactive protein (hsCRP) values were measured from participants' serum.

RESULTS: Significant increase in the level of BNP $(<0.001)$ and hsCRP $(0.001)$ was found in patients of diabetes with cardiovascular disease compared to diabetics without cardiovascular disease. Sensitivity of the test (BNP) was $>97 \%$ with specificity of $<36 \%$ and the Sensitivity of the test (hsCRP) was $90 \%$ and the specificity was $65 \%$ only.

CONCLUSION: Our study findings suggest the potential capability of BNP and hsCRP in diagnosing and predicting CVD in diabetic patients.

KEY WORDS: Diabetes mellitus, cardiovascular disease, B-type natriuretic peptide, high sensitive CRP.

\section{P125}

\section{High Prevalence Vitamin D Receptor (VDR) Gene Polymorphism Causes Hypovitaminosis D In School Going Females of Central India}

\author{
Roji Khan, S.K Goel, Rashmi Chowdhary \\ Department of Biochemistry, All India Institute of \\ Medical Sciences (AIIMS) Bhopal
}

INTRODUCTION: Vitamin-D plays an important role in health promotion during adolescence. Vitamin-D deficiency and insufficiency are common in adolescents worldwide. Few data on vitamin-D status and risk factors for hypovitaminosis D in Indian adolescents are currently available. Studies done in northern India have found a 
very high prevalence of vitamin-D deficiency among pregnant women and adolescent girls apart from having abundant sunlight in the country.

AIMS AND OBJECTIVES:The aim of the study is to find out the status of vitamin-D and their relationship with vitamin D receptor (VRD) gene polymorphism in school girls (age 12-17 years) of Bhopal (Madhya Pradesh) to find out any genetic or environmental factors association for the reason of hypovitaminosis D.

MATERIALS AND METHODS: Vitamin-D (25hydroxyvitamin D), parathyroid hormone (PTH), Serum Calcium, Phosphate, Alkaline Phosphate levels were evaluated by auto analyzers and VDR gene polymorphism (Fok1,Apa1,Taq1,Bsm1) was done by using PCR-Restriction Fragment Length Polymorphism (RFLP) in Bhopal from four school's hundred healthy adolescents (12-17 years) from two different socioeconomic strata.

RESULTS AND CONCLUSION: Enrolled adolescents are $>90 \%$ having hypovitaminosis D associated with high values of PTH.Logistic regression showed increased risk of hypovitaminosis D and these results when compared with VDR gene polymorphism, Bsm1 showing 36\%, Apa1 23\%, Taq1 7.48\% and Fok1 6.48\% polymorphism respectively comparable to other biochemical parameters. Our results suggest that high prevalence of hypovitaminosis $\mathrm{D}$ in adolescents (12-17 years) of central India in which 60-70\% correlates to VDR gene polymorphism rather than malnutrition or lack of sun exposure.

KEY WORDS: Vitamin-D, VDR gene polymorphism

\section{P126}

Study of Serum Adenosine Deaminase and Zinc In Diagnosis of Extrapulmonary Tuberculosis And Pulmonary Smear Negative Tuberculosis

Vijay K Shah, Z G Badade, Kavita More

Department of Biochemistry, MGM Medical College, Navi Mumbai

INTRODUCTION: Tuberculosis is a highly prevalent chronic infectious disease caused by Mycobacterium tuberculosis.India has highest burden of both TB and MDR TB based on estimates reported in Global TB Report 2016. ADA primary function in humans is the development and maintenance of the immune system but full biological role of ADA is not yet completely understood. Zinc deficiency can increase susceptibility to TB because the cell-mediated immunity plays a major role in the disease.Zinc is the only cofactor necessary for activity, which is located in the deepest recess of the ADA active site, which may cause low level of serum Zinc. Even it may likely due to the redistribution of zinc from plasma to other tissues, decrease of zinc-carrier protein ?2-macroglobulin and rise of Metallothionin a protein that transports zinc to the liver.

AIMS AND OBJECTIVES: To evaluate, compare\& correlate the level of serum ADA \& Zinc in patients with Pulmonary TB, Extra Pulmonary TB and Smear Negative TB.

MATERIALS AND METHODS: Total 120 volunteers were enrolled in 3 groups.Group I includes 40 healthy Individuals, Group II 40 patients diagnosed with EPTB and Group III 40 patients diagnosed with SNPTB. Blood sample were collected for the estimation of serum ADA and Zinc levels.

RESULTS AND CONCLUSION: In the present study serum zinc levels of patients with Extra Pulmonary, Pulmonary Smear negative TB was found low as compared to healthy individual but vise-versa of ADA levels i.e. Strong Negative correlation. Which means Zinc supplement during TB treatment can play vital role in faster recovery. It can be included in routine diagnosis and prognosis of tuberculosis.

KEY WORDS: TB, MDR TB, Zinc, Pulmonary Smear, SNPTB

\section{P127}

Role of Bulk Elements and Glucose In Depression- A Pilot Study

Preeti Gupta $^{1}$, P.B. Desai ${ }^{2}$

1. Department of Biochemistry, L.N. Medical 
College, Bhopal (M.P.),

2. Department of Biochemistry, DM Wayanad

Institute of Medical Sciences WAYANAD 673577

Kerala, India

INTRODUCTION: Depression is a universal problem with the worldwide frequency of approximately $15-20 \%$ of total population. Studies in India have reported prevalence rates of depression that vary from $21-83 \%$ in primary care practices. Recent years have witnessed an explosion of knowledge in field of minerals and their effects on different organs. In this instance, we are trying to establish some important medical and diagnostic links between these bulk elements concentration, blood sugar and depression.

OBJECTIVE : Some studies have suggested that the mineral metabolism are associated with mood disorders. In this instance, we are trying to establish some important medical and diagnostic links between these bulk elements concentration, blood sugar and depression.

METHOD: The study sample consisted of 15 mild and 15 severely depressive subjects who were repeatedly admitted to the psychiatric inpatients services and 30 age and sex matched healthy controls. The fasting serum sodium, potassium, calcium and fasting blood sugar levels were measured in the study subjects.

RESULTS: Subjects with depression had decreased levels of sodium, potassium and calcium compared to healthy controls and ' $p$ ' value of sodium, potassium is less than 0.00 where as for calcium ' $p$ ' value is less than 0.01 all of which are statistically significant.

CONCLUSIONS \& INTERPRETATION:This study serves as pilot project for more detailed retrospective and prospective studies in the future.

KEY WORDS:Depression, sodium, potassium \& calcium.

\section{P128}

Ischemia Modified Albumin And Paraoxonase-1 Levels In Serum of Patients With Malignancy Undergoing Intravenous Contrast Enhanced Computed Tomography As Biochemical Markers
Shobha U Kamath ${ }^{1 *}$,Chanda Jha ${ }^{1}$, Sambit Dash ${ }^{2}$, Ravindra Prabhu A ${ }^{3}$, L Ramachandra ${ }^{4}$, Rajgopal Shenoy $\mathrm{K}^{4}$

\section{Department of Biochemistry, Kasturba Medical College, Manipal University, Manipal, India}

2. Department of Biochemistry, Melaka Manipal Medical College, Manipal University, Manipal, India,

3. Department of Nephrology, Kasturba Hospital, Manipal, India,

4. Department of Surgery, Kasturba Hospital, Manipal, India

INTRODUCTION: Followingcontrast enhanced computed tomography (CECT)adverse effects including contrast induced nephropathy (CIN) is a risk in patients with renal insufficiency, diabetes and malignancy. Administration of contrast media (CM) in CECT, puts patients with malignancyat further risk of oxidative stress and damage. Estimating serum Ischemia modified albumin (IMA) as an early marker for acute ischemia and oxidative stress and then correlating it with paraoxonase-1 (PON-1) levels in this group of patients before and after CECT may add to information regarding oxidative status of the patient.

OBJECTIVES: To estimate serum levels of IMA and PON-1 in patients with malignancy before and after an intravenous CECT procedure

MATERIALS AND METHODS: 22 adult in-patients with malignancy regardless of type of malignancy and body region were included in this prospective study. Venousblood samples were obtained 12-24 hours before and 12-24 hours after contrast media administration in CECT. Serum IMA and PON-1 levels were estimated using methods described by Bar-Or et al., and Dantoine et.al., respectively.

RESULTS: The mean IMA levels in post-CECT $(0.83 \pm 0.40)$ patients was higher compared to the preCECT patients $(0.71 \pm 0.45)$. In post-CECT samples, PON-1 levels $(47.3 \pm 18.6)$ were lower compared to preCECT patients $(54.0 \pm 20.4)$

CONCLUSION: Our study shows that IMA increases and PON-1 decreases post CECT. Such altered levels could be associated with ischemia/oxidative activity following $\mathrm{CM}$ administration. A combined estimation 
of these parameters may identify patients at risk for adverse effects of CECT including CIN.

KEY WORDS: Contrast induced nephropathy, paraoxonase-1, Serum IMA, PON-1

\section{P129}

To Study The Levels of Serum Cholesterol And Hscrp Level In Children With Nephrotic Syndrome.

$\underline{\text { Seema Patel }}{ }^{1}$, Manoj Kumar ${ }^{2}$, Shobha Sharma ${ }^{3}$, B.C. $\mathrm{Kabi}^{4}$

1. Department of Biochemistry, SGT university, Gurugram;

2. Department of Biochemistry, Sharda University, Greater Noida, UP;

3. Department of Pediatrics, VMMC and SJH, New Delhi. Department of Biochemistry, $V M M C \& S J H$.

INTRODUCTION: Nephrotic syndrome(NS)is characterized by proteinuria, edema, hypoalbuminemia and hypercholesterolemia. The latter is a risk factor for atherosclerosis suggesting a higher risk for cardiovascular disease in children suffering from NS. This change is proposed to be due to vascular endothelial dysfunction, reflecting the inflammatory response to tissue damage. One potential inflammation marker high sensitive C Reactive Protein (hsCRP) was aimed to be evaluated in these children.

OBJECTIVES: Hence the study was designed to measure the levels of serum hsCRP and Cholesterol level.

METHODS: It is a cross sectional study conducted in tertiary-care hospital. Forty children with first episode nephrotic syndrome attending the Pediatric Outpatient Department, Vardhaman Mahavir Medical College \& Safdarjung Hospital within 14 years of age and equal no of age and sex matched healthy control children were included in the study.Serum hsCRP was measured by Enzyme linked immunosorbent assay technique and Cholesterol level by enzymatic method using cholesterol esterase and cholesterol oxidase conversion in Autoanalyser.
RESULT: Serum hsCRP level was elevated in children with NS compared to healthy controls. A positive correlation between serum hsCRP and cholesterol level in cases $(\mathrm{r}=0.53, \mathrm{p}<0.05)$ was observed.

CONCLUSION: Children suffering from Nephrotic syndrome are shown with raised hsCRP and positively correlated with hypercholesterolemia. Literature says hypercholesterolemia persists in such patients even after long period remission. It is proposed that regular monitoring serum hsCRP level in such patients may help in understanding role of inflammatory mediated atherosclerosis changes of risk of coronary artery disease in later part of life.

KEYWORDS: High sensitive C Reactive Protein, Cholesterol, Nephrotic syndrome

\section{P130}

\section{Study of Polymorphism of Genes Associated In Lipid Metabolism Basis For Dyslipidemia In Unselected Population of Madhya Pradesh}

Neha Masarkar*, Dr. Rashmi Chowdhary, Dr. Sagar Khadanga

Department of Biochemistry, All India Institute of Medical Sciences \&, Bhopal and Department of Biotechnology, Barkatullah University*,Bhopal.

INTRODUCTION: Dyslipidemia is a multifactorial disease in which lipoproteins plays an important role. The plasma concentrations of lipoproteins and their metabolic fates are modulated by apolipoproteins. Studies identify that polymorphism in Apolipoprotein C3 (APOC3) and Fatty acid-binding protein 2 (FABP2) genes predispose individuals to Dyslipidemia. FABP2 is responsible for transport of free fatty acids in the intestinal endothelium cells. APOC 3 modulates triglyceride metabolism through inhibition of lipoprotein lipase.

AIMS: The aim of this study was to investigate the association between FABP2 Ala54Thr and APOC3 rs5128 genetic polymorphism and Dyslipidemia in 
population of Madhya Pradesh, and its association with occurrence of Coronary Heart Diseases (CHD).

METHODOLOGY: Total 60 subjects; 20 Diabetic dyslipidaemias (Group 1), 20 Non-Diabetic dyslipidaemias (Group 2) and 20 apparently healthy case controls (Group 3) were studied. Biochemical analyses, genomic DNA isolations were done. Genotyping was done by PCR-RFLP (PCR-Restriction Fragment Length Polymorphism). Statistical analysis using SPSS was done.

RESULTS \& CONCLUSION: For APOC3 gene maximum polymorphism- $35 \%$ was seen in group 2 . No polymorphism observed in group 1 and 3 . For FABP2 gene, $50 \%$ polymorphism observed in group 2, 35\% in group 1 and $30 \%$ in group 3 . These observations were used to identifyrisk groups to predict the probability of developing atherosclerosis. Individuals of group 2 were homozygous for both the genes, thus riskof developing CHD was high. Individuals of group 1 and group 3 had mixed genotypes and were at intermediate risk.Our data indicates that APOC3 and FABP2 polymorphisms are associated with Dyslipidemia in individuals with polymorphic genes particularly in group 2. In group3 insulin intolerance and lifestyle factors were responsible for Dyslipidemia. Group 3 individuals were carriers. Analysis of risk groups suggests group 2 individuals for immediate lifestyle changes to avoid the risk of developing CHD.

KEYWORDS: Dyslipidemia, APOC3, FABP2, Polymorphism, CHD.

\section{P131}

\section{Tobacco Exposure by Various Modes Modulate Pro-(Il-12) And Anti-(Il-10) Gene Expression And Influence The Median Survival of Prostate Carcinoma Patients}

Shailendra Dwivedi $^{1 \#}$, Apul Goel ${ }^{3}$, Sanjay Khattri², Anil Mandhani ${ }^{4}$, Praveen Sharma ${ }^{1}$, Sanjeev Misra ${ }^{5}$, Kamlesh Kumar Pant ${ }^{2}$.

1. Department of Biochemistry, All India Institute of Medical Sciences (AIIMS), Jodhpur, Rajasthan, India-342005
2. Department of Pharmacology and Therapeutics, King George Medical University (KGMU), Lucknow, Uttar Pradesh, India -226003,

3. Department of Urology, King George Medical University (KGMU), Lucknow, Uttar Pradesh, India-226003

4. Department of Urology, Sanjay Gandhi Post Graduate Institute of Medical Sciences, Lucknow Uttar Pradesh, India-226010

5. Department of Surgical Oncology, All India Institute of Medical Sciences (AIIMS), Jodhpur, Rajasthan, India-342005

INTRODUCTION: Inflammation is an important hallmark of all cancers and net inflammatory response is determined by a delicate balance between pro- and anti-inflammatory cytokines, which may be affected by various environment factors including tobacco exposure.

OBJECTIVE: The present study was designed to explore the effect of various modes of tobacco exposure on Interleukin-12 (IL-12) and Interleukin-10 (IL-10) inflammatory cytokine levels and survival in prostate carcinoma $(\mathrm{PCa})$ patients.

MATERIALS AND METHODS: 5 mlblood samples of 285 cancer patients and 285 controls with $94 \mathrm{BPH}$ (Benign Prostatic Hyperplasia) were recruited after taking ethical clearance and their consent ; baseline levels of serum IL-12 and IL-10 were measured by ELISA kits and mRNA expression of both genes were quantified by (Sybergreen chemistry) real time PCR in various tobacco exposed groups.IL-18 and IL-10 levels were analyzed by ANOVA (one-way and two-way). mRNA expression was analyzed by appropriate statistical tool. Relative inflammatory mRNA levels of both were calculated using [(1/2)?Ct], while fold change expressions were evaluated using the equation $2-? ? \mathrm{Ct}$ (Livak and Schmittgen, 2001). Five year survivals of patients were analyzed by Log-rank (Mantel-Cox) test (graph pad version 5).

RESULTS: The serum pro-inflammatory (IL-12) among tobacco smokers groups showed unique trend i.e. the levels were highest in bidi's smokers, followed by chillum, cigarette, hookah (bidi's smokers > chillum $>$ cigarette smokers $>$ Hookah). Moreover pro-(IL12) and anti-inflammatory (IL-10) were in highest in 
smoker, chewer and alcohol users (combined users) have shown significantly higher levels $(\mathrm{p}<0.001)$ with significant low median survivals (27.1 months, standard error=2.86 and $95 \%$ CI: 21.4-32.62;) than non users. Stage (III and IV) of tobacco addicted patients have also shown significantly increased levels of IL-12 and IL-10.

CONCLUSIONS: IL-12 and IL-10 seems to be influenced by various modes of tobacco exposure and inflammation also affects median survival of cancer patients.

KEY WORDS: IL-12, IL-10, prostate carcinoma

\section{P132}

\section{Serum Hepcidin Level As An Early Predictor or Marker of Iron Deficiency In Young Female.}

Shaveta $^{1 *}$, S K Bansal ${ }^{2}$, Seema Patel ${ }^{3}$, B.Karunanand ${ }^{4}$

$1 *$ Department of Biochemistry,

2. Professor, Department of Biochemistry,

3. Department of Biochemistry,

4. Department of Biochemistry, SGT Medical College, Hospital \& Research Institute, Gurugram.

INTRODUCTION: Most common nutritional disorder in world is iron deficiency. Maintenance of body iron status is important in young female of reproductive age group as they will be child bearers of future generation and early detection would call for early intervention. Ideal screening test would be capable of identifying iron deficiency in absence of anemia. Therefore, the present study was aimedto determine the utility of serum hepcidin in iron deficiency and to access the baseline value of hepcidin in young female, which would implicate the status of iron in the body.

OBJECTIVE: Hence the study was designed to measure the level of serum hepcidin-25 in young female.

METHODS: This is a cross-sectional study performed on two hundred students in SGT University, Gurugram (18-25 years) non pregnant female $(\mathrm{Hb}>12 \mathrm{mg} / \mathrm{dl})$ with normal RBC indices were included in this study.Serum from 200 healthy volunteer students were analysed for hepcidin-25 by ELISA kits.Estimation of iron status parameter was done.Statistical evaluation was done by SPSS.

RESULT: Serum Hepcidin-25 was significantly declined in patient of iron deficiency. Serum hepcidin levels showed positive correlation with serum ferritin and significantly negative correlation with soluble transferrin receptor level.

CONCLUSION: Therefore, estimation of serum hepcidin-25 may be considered as a valuable tool in assessing iron status in young healthy female population. Serum hepcidinhas significant association with traditional parameter of iron status and can prove to be useful indicator of iron deficiency.

KEYWORDS: serum hepcidin, serum ferritin, Iron deficiency, Hemoglobin, Iron status.

\section{P133}

Role of An Isolated Compound From The Leaves of Astilberivularisin Restraint Stress Induced Gastric Ulceration and Anti-Oxidant Defense Indices In Rats

P. Mitra ${ }^{1}$, T. Ghosh ${ }^{2}$, P. Sharma ${ }^{1}$, P. K. Mitra ${ }^{2}$

1. Department of Biochemistry, All India Institute of Medical Sciences, Jodhpur, Rajasthan, India,

2. Department of Medical Biotechnology, Sikkim Manipal University, SMIMS, Sikkim, India.

INTRODUCTION: Astilberivularis (A. rivularis), family - Saxifragaceae, is one of the medicinal plants of Sikkim Himalaya. Ethnic use of A.rivularis, as reported in literature, is in peptic ulcer. Root juice of the plant, two teaspoonful thrice a day, is given to patients suffering from peptic ulcer. We also found anti peptic ulcer activity of A. rivularisleaves in experimental animals. Recently we have isolated a compound from the leaves ofA. rivularis. In this study, we evaluated the role of the compound in restraint induced gastric ulceration and anti-oxidant defense indices in rats.

METHODS: Wister strain albino rats were assigned by 
weight into four groups (six rats per group, average body weight, $184.20 \pm 6.3$ ). Healthy rats receiving de-ionized water were served as control. Restraint induced gastric ulcer was developed in rats by the method of Brodie and Hanson with slight modification. Rats were fasted for $24 \mathrm{~h}$ when no food but de-ionized water was supplied ad libitum. Each rat was then placed in single cage. Limbs of the rat were put together and tightened with adhesive tapes so that the animal could not move. Under this condition rats were kept for 24 hours. Compound isolated from A. rivularis was given in the dose of $50 \mathrm{mg} / \mathrm{kg}$ (orally) in another group of rats 30 minutes prior to apply restraint stress. Ranitidine $(50 \mathrm{mg} / \mathrm{kg}$ ), given orally 30 minutes prior to administer restraint stress in another set of experiment, was used for comparison. After 24 hours of restraint stress, animals were euthanized by decapitation. Stomachs were taken out and examined for ulcers to evaluate ulcer index. Antioxidant indices like superoxide dismutase, catalase, glutathione, and glutathione peroxidase were measured in gastric mucosa.

RESULTS:Compound isolated from Astilberivularis L leaves significantly $(\mathrm{P}<0.001)$ reduced ulcer index in rats induced by restraint stress. Ulcer index came down to $12.27 \pm 0.82$ from $30.42 \pm 1.1$ in restraint stress group. Inhibition (59.66\%) was comparable to that of ranitidine group where ulcer inhibition came $61.24 \%$, Anti-oxidant indices (superoxide dismutase, catalase, glutathione, and glutathione peroxidase) decreased during ulceration, were found enhanced by the compound. Results were comparable to that of ranitidine group.

CONCLUSION: Compound isolated from A. rivularis leaves had anti gastric ulcer activity in restraint induced gastric ulceration in rats and the activity was mediated through anti-oxidant defense mechanism.

KEY WORDS: Astilberivularis, Saxifragaceae, Ranitidine,

\section{P134}

\section{Seasonal Variation In Hepatoprotective Activity of Ageratumconyzoideslinn Leaves on Antitubercular Drugs Induced Hepatotoxicity In Rats}

T. Ghosh ${ }^{1}$, P. Mitra ${ }^{2}$, P. Sharma ${ }^{2}$, P. K. Mitra ${ }^{1}$ Presenting Author:- T. Ghosh

1. Department of Medical Biotechnology, Sikkim Manipal University, SMIMS, Sikkim, India.

2. Department of Biochemistry, All India Institute of Medical Sciences, Jodhpur, Rajasthan, India,

INTRODUCTION: Ageratum conyzoides L. (Family, Asteraceae) is a medicinal plant. Leaves, root, stem and flower of A. conyzoides L. are widely utilized in traditional medicine.The plant is specially known for its antibiotic efficacy, analgesic, anti-oxidative and hepato protective effects as well as blood booster. In this study we evaluated seasonal variation in hepatoprotective activity of A.conyzoidesL. leaves on antitubercular drugs induced hepatotoxicity in rats

METHODS: A.conyzoides L.leaves were collected from the Medicinal Plants Garden of the University of North Bengal, Dist. Darjeeling, West Bengal, India in January - February, March- April, May - June, July - August, September - October, November - December. Wister strain albino rats(average body weight, $168.86 \pm 1.24$ ) were divided into eight groups of 6 each.First group of animals (control) received normal diet and de-ionized water while animals of the second group, in addition to normal diet and de-ionized water, took isoniazid $(27 \mathrm{mg} /$ $\mathrm{kg} /$ day $)+$ rifampicin $(54 \mathrm{mg} / \mathrm{kg} /$ day $)+$ pyrazinamide (135 mg/kg/day) suspension orally for one month fordevelopment of hepatotoxicity in rats. Other groups of rats received powdered leaves of A.conyzoides L. of different seasons in addition to hepatotoxicity inducing drugs. On 30th day blood samples were collected from the rats by cardiac puncture under ether anesthesia. Serum total protein, serum bilirubin and serum alanine aminotransferase (ALT) were estimated.

RESULTS: Results showed that antitubercular drugs could induce hepatotoxicity in rats by increasing serum bilirubin and ALT. Serum bilirubin and ALT levels were significantly increasedto $2.56 \pm 0.11 \mathrm{mg} / \mathrm{dl}$ and 199.67 $\pm 5.12 \mathrm{U} / \mathrm{ml}$ respectively (Normal values, bilirubin: 0.98 $\pm 0.05 \mathrm{mg} / \mathrm{dl}$, ALT : $39.98 \pm 4.24 \mathrm{U} / \mathrm{ml}$ ). Administration of A.conyzoides L. leaves of all seasons could protect rats from antitubercular drugs induce hepatotoxicity but maximum effect was found with the leaves of May and June. 
CONCLUSION:A.conyzoides L. leaves of the period May and June had maximum hepatoprotective activity on anti-tubercular drugs induced hepatotoxicity in rats.

KEY WORDS:Ageratum conyzoides, antitubercular drugs, hepatotoxicity

\section{P135}

Comparison of Fasting Blood Glucose, Glycated Hemoglobin and Triglycerides Levels In Patients with Atherosclerosis

Shaik allabhakshu, Priyanka Shridharan, Krishnanada Prabhu RV

Department of Biochemistry, Kasturba Medical College Manipal, Manipal University, Manipal

INTRODUCTION: Measurement of Fasting blood glucose (FBG) level and glycated haemoglobin (HbA1c) is widely used for long term management of diabetes and its complications. Dyslipidemia, an abnormal level of lipids in theblood, is frequently associated with diabetes as a strong risk factor for developing cardiovascular disease, and its control can prevent cardiovascular complications. In this study we compared the fasting blood glucose,HbA1c and triglycerides in atherosclerosis patients with and without diabetes mellitus. .

AIM AND OBJECTIVE: comparison of the FBG,HbA1c and TG in atherosclerosis patients with and without diabetes mellitus.

METHODS AND MATERIALS:This is a crosssectional study using data collected from the diabetic patients. A total of consenting 100 patients of type 2 diabetes mellitus were enrolled over a period of 6 months. For statistical analysis, SPSS Version 17.0 was used and Chi-square and Pearson's correlation coefficient was used to find the association between TG and $\mathrm{HbA} 1 \mathrm{c}$ and fasting glucose.

RESULTS:FBG and TG are significantly increased in diabetic patients ( $\mathrm{p}=0.007, \mathrm{p}=0.005)$ compared to normal. The above parameters also showed significant increase in diabetic patients $(\mathrm{p}=0.013, \mathrm{p}=0.002)$ compared to pre- diabetic patients.

DISCUSSION AND CONCLUSION:In the present study it has been highlighted the relationship between FBG,HbA1c, and TG.Diabetes is multifactorial having a wide range of lipid abnormalities. This study evaluated the correlation between the glycated haemoglobin glucose and triglycerides.

KEYWORDS: Fasting blood glucose,Glycated Hemoglobin, Triacylglycerol, Diabetes, Atherosclerosis

\section{P136}

\section{Pseudohyperamylasemiainpatients With Renal Dysfunction}

Tapasyapreeti Mukhopadhyay,

Sudhanshu Shekhar, Sudip Kumar Datta

Department of Laboratory Medicine, All India Institute of Medical Sciences, New Delhi

INTRODUCTION:Serum total ?-amylase activity isderived mainly of pancreatic(p-AMY) and salivary glands(s-AMY). Increased serum levelsare used in diagnosis of several clinical conditions including pancreatitis, parotitis, intestinal obstruction,etc.About $20 \%$ of circulating amylase is eliminated through kidneys and hence kidney dysfunction has been reported to be associated with higher serum amylase levels.However, not much study has gone into the correlation of this pseudohyperamylesmia with the degree of renal impairment.

AIM AND OBJECTIVES:To compare the serum amylase levels in patients withdifferent stages of chronic kidney disease $(\mathrm{CKD}) \mathrm{v} / \mathrm{shealthy}$ controls and to correlate the same with estimated glomerular filtration rate (eGFR) if any.

METHOD:In the present cross-sectional study,180 serum samplesreceived in the Clinical Biochemistry laboratory from patients of CKD (e-GFR $<60 \mathrm{~mL} / \mathrm{min}$ per $1.73 \mathrm{~m} 2$ ) without any known causes for hyperamy lasemiawere estimated for serum total amylase using IFCC recommended enzymatic colorimetric method on Hitachi Modular P800 biochemistry autoanalyzer (Roche 
Diagnostics, USA).e-GFRwas calculated using 4 parameter MDRD formula from serum creatinine values obtained by rate blanked Kinetic Jaffe's method. 47 age and sex matched healthy controls were also included in the study. Results were compared using ANOVA with post hoc Bonferroni test and Pearson's correlation.

RESULT:Out of 180 cases, 117 (65\%)belonged to stage 5 (e-GFR $<15 \mathrm{~mL} / \mathrm{min}$ per $1.73 \mathrm{~m} 2), 42(23.3 \%)$ in stage 4 and $21(11.6 \%)$ in stage 3CKD. The study showed that the serum totalamylase level was higher in CKD patients(165.15 $\pm 122.30 \mathrm{U} / \mathrm{L})$ as compared to controls $(69.5 \pm 21.92 \mathrm{U} / \mathrm{L}),(\mathrm{p}<0.05)$. Also highest levels were observed in stage $5(176.75 \pm 132.88 \mathrm{U} / \mathrm{L})$ followed by in stage $4(156.64 \pm 103.23 \mathrm{U} / \mathrm{L})$ and stage $3(135.76 \pm$ 93.28 U/L). $(\mathrm{p}<0.05)$. However, no significantcorrelation $(r=-0.103)$ was observed for amylase levels with eGFR.

CONCLUSION: In patients of chronic kidney dysfunction, hyperamylasemia must be interpreted with caution.

KEY WORDS:Chronic Kidney Disease (CKD), e-GFR, Hyperamylasemia, Pseudohyperamylasemia

\section{P137}

\section{Serum Total Acid Phosphataselevels In Patients With Renal Dysfunction}

Sudhanshu Shekhar, Tapasyapreeti Mukhopadhyay, Sudip Kumar Datta

Department of Laboratory Medicine, All India Institute of Medical Sciences, New Delhi

INTRODUCTION:Almost all patients of prostate cancer have an elevated total acid phosphatase (ACP) level, however currently it is used mostly for staging of patients. Not much is known about the metabolism and route of elimination of ACP. The present study was designed to assess whether renal function plays a role in determining ACP levels.

AIM AND OBJECTIVE: To compare the serum total ACP levels in patients with kidney dysfunction without any known benign or malignant prostate disease v/s healthy controls.
METHOD: In the present cross-sectional study, 63 serum samples received in the Clinical Biochemistry laboratory from patients of renal dysfunction (eGFR $<60 \mathrm{~mL} / \mathrm{min}$ per $1.73 \mathrm{~m} 2$ ) without any known or obvious causes of benign or malignant pathology of prostate were estimated for serum total ACP using enzymatic colorimetric method described by Hillman on Hitachi Modular P800 biochemistry auto-analyzer (Roche Diagnostics, USA). e-GFR was calculated using 4 parameter MDRD formula from serum Creatinine values obtained by Kinetic Jaffe's method. 46 age and sex matched normal healthy controls were included in the study. Results were analysed using ANOVA.

RESULT: Out of 63 cases, $41(65.07 \%)$ belonged to stage 5 (e-GFR $<15 \mathrm{~mL} / \mathrm{min}$ per $1.73 \mathrm{~m} 2), 17(26.98 \%)$ in stage 4 (e-GFR 15 to $<30 \mathrm{~mL} / \mathrm{min}$ per $1.73 \mathrm{~m} 2$ ), and 5 $(7.9 \%$ ) in stage $3 \mathrm{CKD}$ (e-GFR 30 to $<60 \mathrm{~mL} / \mathrm{min}$ per $1.73 \mathrm{~m} 2) .41$ patients $(65.07 \%)$ had values more than upper reference limits (URL); (URL: <6.6 U/L). Overall, serum total ACP level was higher in CKD patients (7.66 $\pm 2.50 \mathrm{IU} / \mathrm{L})$ as compared to controls $(4.74 \pm 0.9 \mathrm{U} / \mathrm{L})$, $(\mathrm{p}<0.05)$. Interestingly, highest levels were observed in stage $4(9.47 \pm 2.82 \mathrm{U} / \mathrm{L})$ followed by in stage $5(7.33 \pm$ $1.85 \mathrm{U} / \mathrm{L})$ and stage $3(4.1 \pm 1.90 \mathrm{U} / \mathrm{L})$.

CONCLUSION: Renal function could be an important determinant for ACP levels and hence in patients with renal dysfunction, ACP levels should be evaluated with caution.

KEY WORDS: Acid phosphatise, benign, malignant, MDRD formula

\section{P138}

\section{Association of Vitamin D Level And Gene Polymorphism (Taq1 And Fok1) In Occupationally lead Exposed Workers}

$\underline{\text { Himani }}^{1}$, Sudip Kumar Datta ${ }^{2}$, Jamal Akhtar Ansari, Abbas Ali Mahdi, Dilutpal Sharma, Rajiv Ranjan³, Busi Karunanand ${ }^{1}$

1. Department of Biochemistry, SGT College Gurugram,

2. Department of Lab Medicine, All India Instituteof Medical Sciences, New Delhi, 
3. Department of Biochemistry, Hindu Rao Hospital, New Delhi, 3 Department of Biochemistry, King George's Medical University, Lucknow

INTRODUCTION: Vitamin D and its metabolites enhance intestinal absorption and renal reabsorption of calcium and phosphate, increasing body calcium and phosphate. These actions are mainly mediated by its binding to a nuclear receptor, vitamin D receptor (VDR). Lead may share with calcium the same metabolic pathways for its toxicokinetics

AIMS AND OBJECTIVES: To study the association of vitaminD levels and vitaminD receptor gene polymorphism (Taq1 and Fok1) on lead in the occupationally exposed lead battery workers.

MATERIAL AND METHODS: Fifty occupationally exposed lead battery workers and fifty controls were recruited for the study with their ethical consent.Ten milliliter of blood was collected in respective vials. Serum calcium and phosphorous levels were measured using Modular P biochemistry auto-analyzer (Roche diagnostics, USA). Serum Vitamin D was estimated by competitive ELISA. Genomic DNA was extracted from EDTA blood by using commercially available Qiagen (Hilden, Germany) kits. Vitamin D receptor gene (Fok1 \& Taq1) polymorphism was studied using PCR-RFLP method. Blood lead levels were determined using Inductively coupled plasmamass spectrometry (ICP-MS) from whole blood. Data was analyzed statistically using the graphpad prism 5.0 software.

\section{RESULTS AND CONCLUSION: Results} demonstrated that there was significant increased in blood lead level in the occupationally exposed lead battery workers compared to the healthy controls. In contrary to the above results, healthy controls demonstrated significantly higher vitamin D levels in the serum in comparison to the battery workers while there was insignificant change in the calcium and phosphorous levels in the recruited study subjects. Further, in the polymorphism study, lead exposed battery workers had significantly lower frequency of FokI (TT,TC) whereas no significant difference was observed in the TaqI. Additionally, subjects negative for FokI SNP had significantly higher blood lead levels than subjects having FokI SNP. Finally, vitamin D levels in lead exposed battery workers positive for FokI had significantly higher serum levels than the subjects negative for FokI. In conclusion, the present study suggests an involvement of the blood lead levels and vitamin $\mathrm{D}$ receptor gene polymorphism in occupationally lead exposed battery workers. Further, FokI polymorphism does play significant involvement in the maintenance of the blood lead levels in the body.

KEYWORDS: Blood Lead, Vitamin D, Vitamin D receptor (FokI, TaqI) Occupationally exposed lead battery workers.

\section{P139}

\section{Relation of Insulin Resistance (IR) With Viral Etiology and Cytokines Il-6, Il-2 In Patients with Chronic Liver Disease}

\author{
M. Irshad, Asif Iqbal, M.A. Ansari, \\ Raghavendra L. \\ Clinical Biochemistry Division, Department of Lab \\ Medicine, All India Institute of Medical Sciences \\ (AIIMS), New Delhi-110029, India
}

INTRODUCTION : Insulin resistance (IR) leading to Diabetes mellitus (DM) is frequently observed in patients with chronic liver diseases in India. Hepatitis viruses' induced chronic liver diseases are also on the rise in the country. Although IR levels depend on viral etiology, a little information is available on IR in liver diseases caused by hepatitis viral infections. In this study, we investigated the occurrence of level of IR in patients with different liver disease and relate its level to viral etiology and cytokines IL-6 and IL-2 levels to understand the underlying mechanism.

MATERIALS AND METHODS: A total number of 79 patients including 35 patients (agerange 23-49 years) with chronic viral hepatitis $(\mathrm{CVH}), 33$ patients (age range 3458 years) with cirrhosis (CIR) and11 patients (age range 43-54) with hepatocellularcarcinoma (HCC) of both gender and in adult age groupwere included in this study plan.IR level was calculated using HOMA-IR formula. Bloodanalysis for insulin, C-peptide, IL-6, IL-2 and hepatitis serology were done using EIAprocedure. Data 
were analyzed using appropriate statistical tests.

RESULTS: IR levels were raised in all the disease groups than healthy controls. IR was significantly high in $\mathrm{CVH}$ patients $(\mathrm{p}<0.01)$. Accordingly, C-peptide also showed a strong tendency to be elevated in $\mathrm{CVH}$ patients $(\mathrm{p}=0.06)$. However, analyzing IR and C-peptide levels across Hepatitis $\mathrm{B}$ and $\mathrm{C}$ viruses revealed that $\mathrm{HCV}$ and HBV had comparable effect in rising IR in CVH and $\mathrm{HCC}$ patients. In cirrhosis patients, however, $\mathrm{HCV}$ markedly increased IR levels while IR levels in HBV infected patients were comparable to controls $(\mathrm{p}<0.01)$. Serum IL-2 was undetectable in almost all of the patients except for few patients in CIR group. Serum IL-6 was remarkably increased in all the disease groups compared to healthy controls $(\mathrm{p}<0.05)$. Within disease groups, IL6 level were highest in HCC patients followed by CIR and CVH. Further, IL-6 level was remarkably elevated in $\mathrm{HCC}$ patients infected with $\mathrm{HCV}$ as compared to HBV $(p<0.001)$ only. No difference in IL-6 levels between viral etiologies was observed in $\mathrm{CVH}$ and CIR patients.

CONCLUSION: Raised IR and IL-6 in chronic liver disease is not associated with viral etiology.

KEYWORDS: Chronic liver diseases, Insulin resistance, Hepatitis viruses, IL-6, IL-2

\section{P140}

Evaluation of Antioxidant, Lipid Peroxidation And Toxic Effects After Apple Intake In Healthy Human Volunteers

\author{
Faizal.P1 $^{1}$, R. Manikkumar ${ }^{2}$, K.T Augusti ${ }^{1}$ \\ Dept. of Medical Biochemistry, \\ School of Health Sciences, Kannur University \\ Thalassery Campus, Palayad, Kerala, 670661 \\ Sankars Health Care Scans and Diagnostics, \\ Vandanam, Alleppey, Kerala, 688005
}

INTRODUCTION: Several studies have demonstrated the amelioration of enzymic and nonenzymic antioxidants as well as decrease of lipid peroxidation status after the intake of apple without any observable toxicity in animal models. Human trials are comparatively less studied on these aspects. Many a literatures shows that the extensive damage to the cells caused by free radicals appears to be a major contributor in ageing and other debilitating diseases such as cancer, cardiovascular diseases, cataracts, compromised immune system, rheumatoid arthritis and brain dysfunction. As per several literatures reviewed the saying "An Apple a day keeps the doctor away" is true with regard to apple. As per the reviews the fruit apple possess antioxidant, anti-peroxidative and antitoxic effects.

AIMAND OBJECTIVE: The Primary objective of this study was to evaluate the circulatory levels of selected antioxidant parameters and lipid peroxidation marker in healthy human volunteers before and after the intake of apple for a period of 0-90 days. As the secondary objective of the study we also assessed the toxic effects of apple supplementation by determining Hepatobiliary and Renal function tests.

MATERIALS AND METHODS:The work has been carried out at the Dept. of Medical Biochemistry, School of Health Sciences, Kannur University. Ethical committee clearance has been obtained for the conduct of this study. An informed consent was collected from each participant prior to the study. A clinical proforma was given to each participant to collect data such as weight, diet pattern and previous history of illness. A total number of 25 healthy human volunteers (age group of 18 - 65Yrs) as adjudged from their clinical features were selected for the study. They were divided into three groups as Group Ia - Healthy human volunteers before apple intake, Group Ib - Healthy Human volunteers after 45 days of apple intake and Group Ic - healthy human volunteers after 90 days of apple intake. Participants suffering from any diseases/disorders that may interefere with the study were excluded. $5 \mathrm{~mL}$ of blood sample was taken for the analysis. Blood samples were collected before the intake, 45 days after the intake and 90 days after the intake of fresh apple fruit at a dosage of $200 \mathrm{~g} /$ day. Serum/Plasma was separated and estimated using the standard methods.

RESULT AND CONCLUSION: The final result showed that majority of the study population showed a significant increase in enzymic and non enzymic antioxidants and a decrease in oxidative stress after the intake of apple without any observable toxicity in Liver 
function and renal function. The significant healthy variations were more after the intake of apple for a period of 90 days than that of 45 days. Various nutraceuticals present in the fruit apple may be responsible in increasing antioxidant activity and decreasing the oxidative stress.

KEYWORDS: Free radical, Nutraceuticals, Oxidative stress

\section{P141}

A Study on The Evaluation of Antioxidant, Lipid Peroxidation And Toxic Effects of Orange Intake In Healthy Human Volunteers

\section{$\underline{\text { R. Manikkumar }}^{1}$, Faizal.P ${ }^{2}$, K.T Augusti ${ }^{2}$}

1. Sankars Health Care Scans and Diagnostics, Vandanam, Alleppey, Kerala, 688005

Dept. of Medical Biochemistry, School of Health

Sciences, Kannur University Thalassery Campus, Palayad, Kerala, 670661

INTRODUCTION: Extensive epidemiologic studies have shown significant inverse relationship between the incidence of various diseases and dietary intake of fruits and vegetables. There is a substantial evidence that excessive production of free radicals can cause or exacerbate many human diseases. Orange, the fruit is been used by peoples owing to its taste and potential health benefits. The various phytochemicals present in this fruit are potent antioxidants and anti-inflammatory agents, thereby counter acting oxidative damage and inflammation which is responsible for the pathogenesis of various diseases. As there is only limited, but promising human data, the present study was aimed to further strengthen support for the unique antioxidant and antiperoxidative properties of the fruit orange.

AIM AND OBJECTIVE: The Primary objective of this study was to evaluate the blood levels of selected antioxidant parameters and lipid peroxidation marker in healthy human volunteers before and after the intake of orange for a period of 0-90 days. As the secondary objective of the study we also assessed the toxic effects if any of orange supplementation by determining Hepatobiliary and Renal function tests.
MATERIALS AND METHODS:The work has been carried out at the Dept. of Medical Biochemistry, School of Health Sciences, Kannur University. Ethical committee clearance has been obtained for the conduct of this study. An informed consent was collected from each participant prior to the study. A clinical proforma was given to each participant to collect data such as weight, diet pattern and previous history of illness. A total number of 25 healthy human volunteers (age group of 18 - 65Yrs) as adjudged from their clinical features were selected for the study. They were divided into three groups as Group Ia - Healthy human volunteers before apple intake, Group Ib - Healthy Human volunteers after 45 days of orange intake and Group Ic - healthy human volunteers after 90 days of orange intake. Participants suffering from any diseases/disorders that may interefere with the study were excluded. $5 \mathrm{~mL}$ of blood sample was taken for the analysis. Blood samples were collected before the intake, 45 days after the intake and 90 days after the intake of fresh orange fruit at a dosage of $200 \mathrm{~g} / \mathrm{day}$. Serum/Plasma was separated and estimated using the standard methods.

RESULT AND CONCLUSION: The final result showed that majority of the study population showed a significant increase in enzymic and non enzymic antioxidants and a decrease in oxidative stress after the intake of orange without any observable toxicity in hepatobiliary function and renal function. The significant healthy variations were more after the intake of orange for a period of 90 days than that of 45 days.

KEYWORDS: Phytochemicals, Oxidative stress, Antioxidant

\section{P142}

\section{Role of Platelets In The Pathogenesis of Antiphospholipid Syndrome}

N K Singh, D Dash, ${ }^{*}$ D Bandyopadhyay, Shorya Taliyan, B K Gupta, Haren Baruah, Dilutpal Sharma

Institute of Medical Sciences (IMS), Banaras Hindu University (BHU), Varanasi, India 
INTRODUCTION:Pathogenesis of Antiphospholipid Syndrome (APS) is an ongoing area of research and studying the role of platelets will be helpful in developing newer diagnostic and therapeutic strategies.

MATERIALS AND METHODS: Forty patients with APS, diagnosed as per modified 2006 Sapporo's Criteria and who were not on aspirin or any other antiplatelet drug, were included. The same number of age- and sexmatched healthy controls was also recruited for comparison. The following platelet function studies were performed using the blood samples collected from APS patients as well as healthy controls: platelet aggregation studies, platelet secretion of dense granules (a. total degranulation b. platelet secretion of granules in relation to time c. visualization of platelet degranulation), clot retraction studies, and western blot studies on clot retracted samples for demonstration of activated proteomes.

RESULTS:A significant increase $(\mathrm{P}<0.001)$ in the platelet aggregation in APS patients as compared to healthy controls was noted. The subjects also showed a significant increase $(\mathrm{P}<0.05)$ in the platelet granule release as well as more degra $\mathrm{n} \mathrm{l}$ at i o $\mathrm{n}(\mathrm{P}<0.001)$ i n re 1 at i o $n$ to $t \mathrm{i} m$ e at stored condition, which were well-visualized under phase-contrast microscope. Sixtyfive percent of APS patients showed lesser as well as delayed clot retraction as compared to healthy controls, signifying that the platelet clots are less retractile in APS patients.

CONCLUSION:The study clearly demonstrates the hyperactivity of platelets in APS patients in each step of their activation as compared to the controls. This indicates the major role played by platelets in APS pathogenesis.

KEYWORDS: Antiphospholipid Syndrome, Sapporo's Criteria, Platelet

\section{P143}

Study of Prevalence of Hypothyroidism In Type 2 Diabetes Mellitus Patients of Lucknow And Surrounding Areas
Gazala Afrin, Aparna Misra, Brijesh Rathore, Vishnu Kumar, Kanchan Singh, Vaibhav Shukla*

Department of Biochemistry \& Department of Medicine*. Era's Lucknow Medical College and Hospital, Lucknow, Uttar Pradesh, India.

INTRODUCTION:Diabetes mellitus and thyroid hormone dysfunction are two common endocrinopathies seen in adult population. Insulin and thyroid hormones are intimately involved in cellular metabolism, so excess or deficit of any of them can derange the function of another.

AIM OF THE STUDY: To see the prevalence of hypothyroidism in type 2 Diabetes Mellitus patients.

MATERIALS AND METHODS: A total of 182 patients with diagnosed Type 2 Diabetes Mellitus of either sex, aged $>20$ years were included in the study. Analysis of serum TSH by ELISA was done in all patients of the study.

RESULT: A total of 182(88 males and 94 females) Type 2 Diabetes Mellitus patients were included in the study. Hypothyroidism was seen in $35(19.23 \%)$ patients. Hyperthyroidism was seen in $6(3.29 \%)$ patients. Euthyroid patients were 141(77.47\%).Out of 94 females $26(27.65 \%)$ had hypothyroidism whereas out of 88 males $19(21.59 \%)$ had hypothyroidism.

CONCLUSION: We conclude that prevalence of hypothyroidism was $19.23 \%$ and seen more in female Type 2 Diabetic patients as compared with male patients.

\section{P144}

Evaluation of Antiproliferative Active Phytochemicals From Chloroform Fraction of Solanum Nigrum L. Berries Using GC-Ms/Ms Analysis

Homa Jilani Khan ${ }^{1,2}$, Mohammad Kaleem Ahmad ${ }^{2}$, Mohd Waseem², Abdul Rahman Khan ${ }^{1}$, Abbas Ali Mahdi

1. Department of Chemistry, Integral University, Lucknow-226 026, India

2. Molecular Cell Biology Lab, 
Department of Biochemistry,

King George's Medical University,

Lucknow-226 003, India,

INTRODUCTION:Solanum nigrum is a traditional Indian plant acclaimed for its medicinal properties since antiquity. Among all plant parts fruit berries have shown to be most pharmacologically active part.

MATERIAL AND METHODS:In the present investigation, we tried to characterize the bioactive principles of chloroform fraction of S. nigrum (CFSn) fruit berries using GC-MS analysis. Our phytochemical investigation of CFSn was well supported by its total phenolic content and antioxidant activity which we evaluated subsequently.Further, we investigated the anticancer activity against breast cancer cell lines (MDAMB-231 and MCF-7) and human normal transformed (HaCaT) cells as well.

RESULT:We identified 29 compounds belonging to different chemical classes viz. alkaloids, flavonoid, carbohydrates, glycosides, phytosterols, proteins, phenolic compounds, and saponins. More specifically, we found two novel phenolic compounds, Benzoiisovanillin and Syringic acid (4-hydroxy-3, 5dimethoxybenzoic acid), which may be responsible for its pharmacological properties. Our in vitro results indicated that CFSn exhibited significant antiproliferative activity against both these cell lines and due induction of cancer cell death through apoptosis.

CONCLUSION: Our study emphasizes the need for isolation and characterization of specific bioactive compounds of CFSn and determination of their mechanism of action responsible for its anticancer activity in breast cancer cells.

KEYWORDS: Antiproliferative, Black nightshade, Breast cancer, Phenols, Tri-terpenoids

\section{P145}

GAPDH Knock-Out Induces Stem Cell Phenotype And Arsenic Resistance In Human Cells
Imran Ahmad $^{1,2,}$ Sabita Chaurasia ${ }^{1,2}$, Dinesh Kumar Singh 1,2

1. Immunotoxicology Division, Systems Toxicology and Health Risk Assessment Group, CSIR-Indian Institute of Toxicology Research (CSIR-IITR), Vishvigyan Bhavan, 31, Mahatma Gandhi Marg, Lucknow, 226 001, Uttar Pradesh, (India).

2. Academy of Scientific and Innovative Research (AcSIR), CSIR-IITR Campus, Vishvigyan Bhavan, 31, Mahatma Gandhi Marg, Lucknow, 226001 , Uttar Pradesh, (India).

INTRODUCTION: Glyceraldehyde-3-phosphate dehydrogenase (GAPDH) is an essential glycolytic enzyme that has recently been shown to participate in various other cellular physiological processes like tRNA export, DNA replication, DNA repair, endocytosis, cytoskeletal organization, iron metabolism, and cell death. However, the effect of GAPDH knockout (absence of GAPDH) on cellular physiology is not yet known.

METHODS: We knocked out GAPDH in human cervical cancer cell line (HeLa) using genome engineering/editing tool CRISPR (Clustered Regularly Interspaced Short Palindromic Repeats) and CRISPRassociated gene 9 (Cas9) and treated them with arsenate, a potent environmental toxicant.

RESULTS: We observed that the cancer cells were able to survive even in absence of GAPDH. Morphologically, the knockout cells exhibited features of induced pluoripotent stem cells (iPSCs). Stem cell specific gene transcription is also observed. Interestingly, they also showed resistance to arsenic toxicity.

CONCLUSION: Overall, we conclude that GAPDH knockout induces stem cell like properties and arsenic resistance in human cancer cells.

KEY WORDS- GAPDH, CRISPR Cas, Knock out, arsenic, stem cell

\section{P146}

Study of Vitamin D [25-(OH)D] Levels In New Sputum Positive Pulmonary Tuberculosis Cases \& Its Correlation With Treatment Outcome. 
Jasmine Sinha, Anu Chandra, Kanchan Singh , Manisha Singh, Abhishek Agarwal*

Department of Biochemistry \& Department of

Pulmonary Medicine*.

Era's Lucknow Medical College and Hospital, Lucknow, Uttar Pradesh, India.

INTRODUCTION:Pulmonary tuberculosis is a widespread, infectious disease caused by various strains of mycobacteria, usually Mycobacterium tuberculosis. Susceptibility to disease after infection by Mycobacterium tuberculosis is influenced by multiple environmental and host factors. Vitamin D modulates monocyte-macrophage activity in the body and plays a role in human innate immunity to certain infectious agents. This role may be important in the body's defense against tuberculosis, in which action of macrophages is a key step, in pathogenesis.

MATERIALS AND METHODS: In this study 80 cases of new sputum positive pulmonary tuberculosis patients of either sex, aged between 18-60years enrolled for DOTS in category-1were examined for serum vitamin D levels and routine follow ups for DOTS treatment were done to check treatment outcome.

RESULTS: The prevalence of hypovitaminosis D in patients with new sputum positive pulmonary tuberculosis came out to be $55 \%$ and the Vitamin D deficiency was found to be higher among women than male $(p=0.003)$. No significant correlation was seen with vitamin D level with DOTS treatment outcome.

CONCLUSION: We conclude that prevalence of hypovitaminosis $\mathrm{D}$ is $55 \%$ and seen more in female patients as compared to male patients suffering from pulmonary tuberculosis. No significant correlation was seen with role of vitamin D on DOTS treatment outcome.

\section{P147}

An Optimized Method To Genotype Rs671 Snp Through PCR-RFLP For Candidate Gene Association Studies

Pilla VSN Kiran Kumar, Prasenjit Mitra,
Shailja Sharma, Praveen Sharma

Department of Biochemistry,

All India Institute of Medical Sciences, Jodhpur, Rajasthan, India.

INTRODUCTION: Asingle base substitution $\left(\mathrm{G}^{\prime} \mathrm{A}\right)$ is the basis for rs671 single nucleotide polymorphism (SNP) of ALDH2 gene, in which the 'A' allele (formerly known as $\mathrm{ALDH} 2 * 2$ ) encodes a non-functional or inactive enzyme, whereas the 'G' (ALDH2*1) allele codes for a functionally active enzyme. This SNP has been found to be associated with various disorders including alcohol dependence, diabetes, cancer, lead toxicity in various populations. But apart from real time PCR, the reported PCR-RFLP or PCR-CTPP methods are either not easy or cost effective and often provides inconclusive results. The objective of this study was to design a costeffective and easy method of genotyping rs671.

METHODS: Peripheral venous blood was obtained. Genomic DNA was extracted through commercially available kits and quantified. Primers to genotype rs671 were designed and a polymerase chain reaction protocol was optimized for proper amplification of the region of ALDH2 gene inclusive of the SNP. Following amplification, search for restriction enzymes able to cut the restriction site was performed and AcuI was selected for restriction digestion. Post PCR-RFLP, results were observed via agarose gel electrophoresis.

RESULTS:The optimized technique provided good amplification and appreciable results after restriction digestion. Post amplification, a 502 bp band was obtained. After restriction digestion, GG genotype yielded two bands of 297 bp and 205 bp, whereas GA genotype yielded three bands of 502 bp, 297 bp and 205 bp.Bands were appreciably visualized through agarose gel electrophoresis.

CONCLUSION: We report a modified PCR-RFLP technique for successful rs671 genotyping. This method is relatively easy and cost-effective. Thus, it may be used in centers which do not have facilities for genotyping through Real-time PCR.

KEYWORDS: SNP, PCR-RFLP, rs671 


\section{P148}

10,000 Fold Effect: Intracarotid Sodium Nitroprusside on 5th Post Ischemic Stroke Day In Middle Cerebral Artery Occlusion (MCAO) Rat Model

Lori Tewari*, RK Singh*, Lavanya*, M K Mishra*, R Singh*, H Afreen*, V K Tewari**, H K D Gupta**, V Bhosle***, R Shukla ***, Sheeba***

* Department of Biochemistry, TSM Medical College, Amausi, Lucknow, UP, India

** Department of Neurosurgery, Advance Neuro and General Hospital, Lucknow, UP, India, 226010.

***Department of Clinical Trials and Department of Experimental Pharmacology, CSIR-CDRI, Lucknow, UP

INTRODUCTION: Ischemic-stroke at later stages $(>4.5$ hrs) have very few treatment options-left. SodiumNitroprusside(SNP), a NO-donor was tested earlier in Rat middle-cerebral-artery-occlusion(MCAO)model in early-stages (5-60mints) and found-useful but in delayedstroke-cases (60-120 minutes), found-useless. This was due to local INDUCIBLE-NITRIC-OXIDESYNTHASE (iNOS) ENZYME and SUPEROXIDE (causes destructive effects) formation which was skipped.

AIM: To evaluate the effect of INTRACAROTIDSODIUM-NITROPRUSSIDE(ICSNP) in MCAO-ratmodel of ischemic-stroke-(I/R model) post-5th-strokeday.

MATERIALS/METHODS: Sprague-Dawley-rats-24, weighing 250-280g, at CDRI-Lucknow were used. Study was approved by Institutional-animal-ethical-committee.

Rats were divided in three groups.

Group"A"(n=4) were taken as sham with standard procedure but without any injection on 5th day.Group"B" $(\mathrm{n}=8)$ as control with injection of saline on 5th-day and group"C" $(\mathrm{n}=12)$ received SNP at dose of $3 \mathrm{mcg} / \mathrm{kg} / \mathrm{min}$ given directly in internal-carotid-artery via external-carotid-artery with a modified-intraluminalstump-technique as ischemia/reperfusion(I/R) in ipsilateral-MCAO-intracarotid-artery region as a single- dose-therapy on 5th-day and then wound closed. Wait for full recovery for $2 \mathrm{hrs}$, neurobehavioral assessment done.Thereafter the brains were quickly removed and sliced at $2 \mathrm{~mm}$ intervals. Animals showing no sign of neurological deficit were excluded from the study.Tested animals were compared with control animals for neurological-deficit, percentage-of-infarction by 5triphenyltetrazolium-chloride(TTC) staining and nNOSexpression and scores were summed-up.

The statistical-analysis was done by Newman-Keuls-test, Graph-Pad-prism(version-5) and $\mathrm{p}<0.05$ was considered statistically-significant.

RESULTS: ICSNP(group C) showed a good reduction in the cerebral-infarction of $53.42 \%$ as compared to control(group B). Group-A mean-change in NewmanKeuls test and Graph-Pad-prism(version-5) was increase of 1.44 -points $/ 6.55 \%$, compared to Group-B decrease of 0.7 -points. $60 \%$ decrease in ischemic-zone noted in Group-A.

CONCLUSIONS: The use of single-dose-intracarotidsodium-nitroprusside is beneficial $(53.42 \%)$ in 5 th-poststroke-day.

KEYWORDS: 10,000 Fold Effect; Post 5th Day Ischemic Stroke Management; Intracarotid Sodium Nitroprusside

\section{P149}

\section{Emerging Epidemic of Type 2 Diabetes Mellitus In Indian Youth}

\section{Manorama Swaim}

$\mathrm{T}$ ype 2 diabetes mellitus (DM) is becoming an increasingly prevalent disorder among young person's between the age group of 18-39 years. Life style factors leading to increased obesity due to lack of physical activity, genetic and familial factors leading to insulin resistance appear to be crucial in the pathogenesis. In the Indian context; the main types include maturity onset diabetes of young (MODY) malnutrition modulated diabetes mellitus (MMDM), fibrocalcinous pancreatic 
diabetes (FCPD) and latent autoimmune diabetes in the adult (LADA).

MODY can result from at least 6 different genetic mutations, that lead to beta cell dysfunction (MODY, $1-6)$

Treatment Options for Type 2 Diabetes in Adolescents and Youth (TODAY) study have found that these youths develop early and rapidly progressing signs of kidney and cardiovascular diseases. With this clinical scenario in mind we tried to find out the incidence of type 2 diabetes in young adults of our locality. The study group comprises of first year MBBS and BDS students admitted to our medical college. The diagnosed cases will be followed throughout their medical career till they leave the institution. Fasting blood sugar, plasma insulin and lipid profile will be estimated to measure HOMA-IR and dyslipidemia in both cases of controls. In the diagnosed cases early treatment modalities in form of life style modification and pharmaco-therapeutic approaches can prevent the future complications.

\section{P150}

To Estimate Serum Calcium, Serum Phosphorus And 25 (OH) Vitamin D2 Levels In Sedentary And Less Exposed To Sun And Heavy Working Females Exposed To Sun

\author{
Muzamil Bashir ${ }^{1}$, B.K. Gupta ${ }^{2}$, Niren Singh Koch $^{3}$ \\ Dept of Biochemistry. Subharti Medical College, \\ Meerut
}

INTRODUCTION: Vitamin D is a steroid hormone involved in mineral ion homeostasis, it promotes calcium absorption in the gut and maintains adequate calcium and phosphate concentration for bone mineralization by osteoblasts and osteoclasts. Vitamin D levels are influenced by many factors such as; diet, geographical locations, physical activity and exposure to sunlight. With this background in mind the present study has been aimed to compare the values of $25(\mathrm{OH})$ vitamin $\mathrm{D} 2$, serum calcium and phosphorus levels in sedentary less exposed to sun and heavy working females exposed to sun.

MATERIALS AND METHODS:The study is being conducted in the Department of Biochemistry, Subharti Medical College, Meerut. 100 apparently Healthy females of age group 21-50 years are included in the present study, out of which 50 are sedentary workers not exposed to sun(Case) and the rest 50 are labour class working 6-8 hours daily exposed to the sun (Control). $5 \mathrm{ml}$ of Venous Blood is taken in plain vials under all aseptic conditions from all individuals participated in the study. The samples are processed by technical laboratory methods and in related automatic machines (Vidas and Vitros 250).

RESULT: The mean value of serum vitamin D ( 20.68 5.34), calcium (8.51 0.65) and phosphorous (4.03 0.33) in control groups are higher than the mean value of vitamin D (7.56 1.83) calcium (6.17 0.90$)$ and phosphorous ( 3.360 .48 ) in the case group and the mean difference between two groups is statistically significant ( $\mathrm{p}$ 0.001)

CONCLUSION: From this study it can be concluded that along with sun exposure one should take balanced diet,vitamin supplements and regular physical exersice to maintain adequate bone mineralization. The study is still in progress.

\section{P151}

\section{Ast \& Alt Levels In Adenocarcinoma Breast Patients Being Treated With Cyclophosphamide.}

Navanil Roy, Mrinal Gupta

Shri Shankaracharya Institute of Medical Sciencesl, Bhilai, Chhattisgarh, Babasaheb Ambedkar Medical College, 2New Delhi .

INTRODUCTION: Adenocarcinoma of the breast is a form of breast cancer originating in the milk ducts or lobules. Presently it is seen that breast cancer has surpassed cervical cancer as the most common cancer among Indian women.

The drug used for treatment was cyclophosphamide. Cyclophosphamide also k/a cytophosphane is a anti cancer drug used in various cancers.

AIMS \& OBJECTIVE: To analyze the serum levels of AST \& ALT in adenocarcinoma breast patients being 
treated with cyclophosphamide attending a tertiary care hospital in Durg-Bhilai region.

MATERIALS \& METHODS: 50 diagnosed adenocarcinoma breast cases were selected as the cases. 50 healthy females were taken as control. Fasting blood samples were collected in plain vacutainers. Serum AST \& ALT were measured by automated analyzer Cobas 360 .

RESULTS: AST levels in the serum was increased in cases (76 IU/L \pm 8.05$)$ than the control (32 IU/L \pm 2.56 ) which was statistically significant ( $\mathrm{p}$ value $<0.001)$. ALT levels were also increased in the cases $(80 \mathrm{IU} / \mathrm{L} \pm 6.42)$ than the controls $(36 \mathrm{IU} / \mathrm{L} \pm 1.24)$ which were also statistically significant ( $\mathrm{p}$ value $<0.001$ ).

CONCLUSION: The objective of the study is to enable the oncologists that they can ensure proper dose maintenance of cyclophosphamide as the drug is highly hepatotoxic.

KEY WORDS: Adenocarcinoma, Cyclophosphamide, Hepatotoxic.

\section{P152}

Acceptance of Medical Faculty For E-Learning Modules - A Pilot Study

Ritu Singh, Parijat Gogoi, Veenu Razdaan

Lady Harding Medical College, New Delhi.

INTRODUCTION : E-learning in medical education is the use of internet resources and teaching methodologies for teaching learning experience. This has gained focus over the last few years with the ever increasing time paucity for the students and faculty in the light of their routine work load and patient care. The various e-learning technologies provide a forum to regulate pace of learning, control over content and time and possibility to discuss topics across distances.

AIMS AND OBJECTIVES : To sensitize faculty to elearning as a modern tool of facilitating online small group discussions with other faculty and post graduate students.
To assess the acceptance of faculty for this method of learning

Materials and methods: Participants included interested Faculty (13) from seven medical colleges and postgraduate students (10). After the discussion of 6 topics via e-learning for a duration of $2 \frac{1}{2}$ months, detailed feedback questionnaires and open ended answers were taken.

RESULTS AND CONCLUSION: This study showed that both the faculty and students benefitted from the e-learning experience. The faculty is finding the concept interesting and is realizing that it must be updated with the tools of e-learning to be able to teach the new generation with global knowledge and would like to add it to postgraduate education to a significant extent. The students on the other hand seem to have a rosier picture of e-learning, possibly because they are comfortable with internet tools. It highlights the fact that e-learning is a concept whose time has come.

KEY WORDS: E-learning, medical education

\section{P153}

\section{Study of Serum Sodium and Serum Potassium Level In Chronic Renal Failure}

Preeti Singh, Prasenjit Mitra, Shailja Sharma, Praveen Sharma

Department of Biochemistry, All India Institute of Medical Sciences, Jodhpur, India

INTRODUCTION: Chronic renal failure is a progressive loss in renal function over a period of months or years. It is a long term condition caused by damage to both kidneys. There is no single cause and damage is usually irreversible and can lead to ill health. It is associated with significant morbidity and mortality.

MATERIALS AND METHODS: The present study included 50 chronic renal failure patients of both sexes and 50 healthy controls of both sexes. Special care were taken to ensure that females were neither on contraceptive pills and nor pregnant. Healthy controls were screened to exclude the presence of malnutrition, metabolic 
diseases and other diseases like CRF with Hypertension, cardiovascular disease, and diabetes.All patients were subjected to a thorough history taking with special emphasis to exclude the presence of other diseases which are known to alter the sodium and potassium levels in serum except hypertension, cardiovascular disease and DM.

RESULT: The comparison of serum sodium and serum potassium levels between control cases and CRF cases were found to be statically significant $(\mathrm{p}<.005)$.

CONCLUSION: Serum sodium level decrease and serum potassium level were found elevated in CRF cases as compare to control cases. In renal disease with CRF shows low serum sodium level and high serum potassium level as compare to control. This is due to malfunctioning of aldosterone and rennin-angiotensin system, glomerular filtration and decreased reabsorption. So these patients sometime need replacement therapy.

KEYWORDS: CRF (Chronic renal failure), DM (Diabetes mellitus)

\section{P154}

\section{Role of Pleural Fluid, Serum Protein Carbonyl Gradient In Differentiating Exudative And Transudative Effusions}

$\underline{\text { Raghumoy Ghosh }}^{1}$, Debojyoti Bhattacharjee*2, Purvi Purohit $^{1}$, Prasenjit Mitra ${ }^{1}$, Shailja Sharma ${ }^{1}$, Praveen Sharma $^{1}$

\section{All India Institute of Medical Sciences, Jodhpur, Basni, Jodhpur-342005, Rajasthan, India \\ 2. Calcutta National Medical College, 32 Gorachand Road, Kolkata-700014, WB, India}

INTRODUCTION: Pleural effusions often cause a diagnostic dilemma. The first step in the evaluation of pleural effusions is the distinction between exudates and transudates. In this background, a study was designed to distinguish these two broad subgroups of pleural effusions, based on the protein carbonyl gradient between pleural fluid and serum.

AIMS AND OBJECTIVES: The study aims to realize if transudative and exudative type of effusions can be differentiated by comparing the protein carbonyl levels of the pleural fluids

MATERIALS AND METHODS:After obtaining ethical clearance from the IEC, forty four exudative and thirty six transudative pleural effusion cases based on clinical findings and evaluation by Light's criteria were selected. Protein carbonyl was measured by established photometric method using dinitrophenylhydrazine (DNPH) method in pleural fluid and serum of all the cases.

RESULTS:Oxidative stress, estimated by the protein carbonyl gradient between pleural fluid and serum, was found to be higher in exudates when compared to transudates $(\mathrm{p}<0.001)$. No significant difference was existent between exudates of different etiology. However, the gradient was higher in exudates from males compared to females $(\mathrm{p}<0.05)$. No such difference was found among transudates.

CONCLUSIONS:Pleural fluid to serum protein carbonyl gradient may serve as an useful tool for differentiating pleural effusions.

KEYWORDS : Oxidative stress, Pleural fluid,Protein Carbonyl gradient, Exudate, Transudate

\section{$\mathbf{P 1 5 5}$}

Can Impaired Diaphyseal Fracture Healing Predicted Early? : A Cohort Study of Biomarkers

$\underline{\text { Sabir Ali }}{ }^{1}$, Ajai Singh ${ }^{1}$, Abbas Ali Mahdi ${ }^{2}$

1. Department of Orthopaedic Surgery and

2. Department of Biochemistry, King George's Medical University, Lucknow, India.

INTRODUCTION: Currently, clinico-radialogical method was used to analyse the healing progression of fractures globally, but even they are also unable to presume the impaired healing early. So till date no reliable method is available to predict impaired healing early, so that it can be interventionally managed as required within time. In behalf of that we planned the 
present study with a Question/Purpose that whether the Osteocalcin (OC) and Osteopontin(OPN) expression have any correlation to fracture healing progression?

METHODOLOGY:In this study total 121 adult fractured patients and 108 healthy controls were analysed. Peripheral blood sample were taken from controls (at once) and fractured casesfollow-ups to quantify the OC and OPN mRNA and protein expression along with clinico-radiological follow-up examinations.

RESULTS: As per the clinico-radiological status at 24th week, fracture patients were divided into normal healing $(\mathrm{n}=102)$ and impaired healing $(\mathrm{n}=19)$ groups. Mean RUST score between normal healing and impaired healing group showed significant statistical difference at each follow-up. In both groups, expressions of OC (mRNA \& protein) gradually up-regulated from the baseline to end of follow-up, whereas OPN mRNA as well as protein gradually up-regulated from the baseline to a peak value at 10th day, then declined. Significant correlation was found between the mRNA expressions of OC and OPN with the RUST score at most of the follow-ups, however the protein expressions were not showed any significant correlation.

CONCLUSION: The OC and OPN expression provide an early prediction of the healing outcomes of tibial fractures.

\section{P156}

\section{Gold Nanoparticles Synthesized From Nelumbo Nucifera Are Antiadipogenic}

Sabita Chourasia $^{1,2}$, Imran Ahmad1 ${ }^{2}$, Jai Shankar ${ }^{3}$, Neeraj Satija ${ }^{4}$, Nidhi Arjaria ${ }^{3}$, Prem Narayan Saxena ${ }^{3}$, Raja Gopal ${ }^{3}$, Somendu Kumar Roy ${ }^{6}$, Mohan Kamthan ${ }^{7}$, Dinesh Kumar Singh ${ }^{\dagger 1,2}$

1. Immunotoxicology Division, Systems Toxicology and Health Risk Assessment Group, CSIR-Indian Institute of Toxicology Research (CSIR-IITR), Vishvigyan Bhavan, 31, Mahatma Gandhi Marg, Lucknow, 226 001, Uttar Pradesh, (India).

2. Academy of Scientific and Innovative Research (AcSIR), CSIR-IITR Campus,
3. Electron Microscopy Laboratory, Analytical Toxicology Group, CSIR-IITR.

4. Developmental Toxicology Division, CSIR-IITR.

5. Nanotherapeutics and Nanomaterial Toxicology Group, CSIR-IITR.

6. Analytical Toxicology Laboratory, Regulatory Toxicology Group, CSIR-IITR.

7. Environmental Microbiology Laboratory, CSIR-IITR.

INTRODUCTION : Gold nanoparticles (AuNPs) have recently been synthesized using biological materials from various sources like bacteria, fungi, flowers, leaves, roots etc. However, very few studies have demonstrated the physiological use of the same as nanomedicine.

METHODS: In the current study, we synthesized gold nanoparticles using different parts of sacred lotus (Nelumbo nucifera) flower and chacterized physicochemically. We used mesenchymal stem cells and NIH3T3L1 preadipocytes for the study of anti-adipogenic properties of AuNP.

RESULTS: Our results demonstrate that biologically synthesized gold nanoparticles are biocompatible and non-toxic. Additionally, they inhibit adipocyte differentiation from mesenchymal stem cells and NIH3T3L1 preadipocytes. The mechanism of action for anti-adipogenic activity of these AuNPs has also been deciphered.

CONCLUSION: These AuNPs can potentially be used as nanomedicine to check obesity.

KEYWORDS: gold, nanoparticles, nanomedicine, adipogenesis, obesity.

\section{P157}

\section{Metallothioneins: Novel Therapeutic Target In Osteosarcoma}

$\underline{\text { Salma. }}^{1}$, Dilutpal Sharma ${ }^{2}$, Ajai Singh ${ }^{1}$, Abbas Ali Mahdi ${ }^{2}$, Sabir Ali ${ }^{1}$

1. Department of Orthopedic Surgery, King George Medical University, Lucknow, Uttar Pradesh, INDIA 
2. Department of Biochemistry,

King George Medical University, Lucknow, Uttar Pradesh, INDIA

$\mathrm{O}$ steosarcoma is the third most common cancer in adolescence and second causal agent of death associated with cancer.Instead of amputation, present treatment is a combination of chemotherapy and surgery which has greatly improved survival rate.However, unfortunately this survival rate has remained unchanged in certain metastatic stages. To a certain extent, the reason behind this may be due tochemoresistance to anti OS therapy. In clinical applications, till now there is no specific diagnostic/prognostic marker. Metallothioneins (MTs) are intracellular proteins containing the highest amount of thiol groups which binds to severalcytotoxic agents such as alkylating agents and platinum compounds. The involvement of MT in many physiological and pathophysiological processes such as apoptosis, proliferation, angiogenesis, and detoxification of heavy metals suggestive of vital role of MT in carcinogenesis as well as chemotherapy.MT in tumor cell plays an important role in defense mechanism against the effect of radiation by inhibiting the processes that lead to apoptosis. In bone tumors, MT levels were found to be increased in sera from patients with osteosarcoma compared with healthy patients and in tumor biopsies compared with non-malignant bone biopsies. Increased MT2A contributes to chemotherapy resistance in osteosarcoma. Therefore, increased MT level in serum might be a novel potential diagnostic/prognostic biomarker or also will be the therapeutic target for the treatment of osteosarcoma patients.Increased MT levels could provide a zinc cofactor reserve that increases the cell's reparative potential when faced by DNA damage by cytotoxic agents. Although the physiologic role of MT in resistance to the cytotoxic effects of anticancer drugs remains unclear, a better understanding of the interaction between MT and chemotherapeutic agents is important and may open new horizon in the treatment of cancer.

\section{P158}

\section{Role of IGF2BP3 In Migration And Invasion of Epithelial Cancer Cell Lines}

Sanjeev Goswami, Gunjan Sharma, Elza Boby, Parthaprasad Chattopadhyay, Jayanth Kumar Palanichamy

Department of Biochemistry, All India Institute of Medical Sciences, New Delhi, India

INTRODUCTION: RNA Binding Proteins (RBPs) play crucial role in biogenesis, stability and functioning of RNAs. Insulin likegrowth factor binding protein 3 (IGF2BP3) is an oncogene correlated with poor prognosis and metastasis in cancers but themechanism of oncogenesis and metastasis is not known. We try to address these lacunae.

AIM AND OBJECTIVES:To study the role of IGF2BP3 in migration and invasion of epithelial cancer cell lines by correlating the expression of IGF2BP3 and its targetwith the migration and invasion.

MATERIALS AND METHODS:RNA isolation and cDNA synthesis followed by real time PCR for IGF2BP3 ant its target expression in epithelial cell lines done.Migration and invasion assays were also done. The experiments were repeated after IGF2BP3 knockdown by specific siRNAs.

RESULTS: IGF2BP3and its targets (CD44, MALAT1) are highly expressed in $\mathrm{HeLa}$ and $\mathrm{SiHa}$ in comparison to MCF7.Knockdown of IGF2BP3 leads to a decrease in the expression level of CD44, MALAT1 and decrease in the migratory and invasive potential of $\mathrm{HeLa}$ and $\mathrm{SiHa}$.

CONCLUSION: High IGF2BP3 expression correlates with high migration and invasion potential of HeLa and SiHa compared to MCF7.Decrease in expression levelof targetsafter IGF2BP3 knockdown implies their stabilisation by IGF2BP3.Decrease in migration and invasion of HeLa cells after IGF2BP3 knockdown implies its role in stabilizing pro-migratory transcripts.

KEY WORDS: RNA binding protein, IGF2BP3, CD44, MALAT1.

\section{P159}

To Study Serum Biomarkers In Cases of Fever Withmalaria And Controls. 


\section{$\underline{\text { Shiva Vasudev }}^{1}$}

1. Dept. of Biochemistry, S.M.S Medical College Jaipur

INTRODUCTION: The infection of liver cells by thePlasmodialsporozoites form of the malarial parasite can cause cellular inflammation leading to the leakage of parenchymal (transaminases) and membranous (alkaline phosphatase) enzymes of the liver and erythrocytes (Lactate dehydrogenase and acid phosphatase) to the circulation.Hence the assessment of acid phosphatase (ACP), LDH (lactate dehydrogenase) and liver enzymes like AST (Aspartate transaminases), ALT (Alanine transaminases) ALP (Alkaline phosphatase) and albumin in malaria patients could represent additional and useful parameters in determining the clinical and prognostic aspects of the disease.

AIMS \& OBJECTIVES: To assess the diagnostic value of serum LDH, AST, ALT, ALP activity and albumin (liver function biomarkers), LDH, ACP activity(RBC function biomarkers) in malaria cases and controls.

MATERIALS \& METHODOLOGY: 30 cases and control were taken for the study. Patient selection was done by simple random sampling with history of fever, chills \& rigor after applying inclusion and exclusion criteria.

RESULTS AND CONCLUSION: Estimating serum enzyme levels as biomarkers in malaria; we observed that enzyme AST, ALT, ALP, ACPincreases significantly ( $\mathrm{p}<$ $0.05)$. LDH activity increases very significantly $(\mathrm{p}<$ $0.001)$ and albumin decreases significantly $(\mathrm{p}<0.05)$ in patients as compared to control subjects. Hence there is a rationale in using these biochemical markers in malaria.

\section{P160}

Antioxidant and Scavenging Activities of Trianthema Portulacastrum

Uttam Das, Tanmay Saha and Subir Kumar Das

Department of Biochemistry, College of Medicine \& JNM Hospital, WBUHS, Kalyani, Nadia 741235, West Bengal
INTRODUCTION: The global demand for herbal medicine is increasing. India is one of the blessed nations produced large amount of medicinal herbs. Trianthema portulacastrum Linn. (Aizoaceae) also acts as good source of organic substance in diet. Different parts are used traditionally to treat alcohol poisoning, liver ailments, bronchitis, heart diseases, asthma, ascites, anemia, beri-beri, dropsy, corneal ulcers, edema, inflammation, migraine, rheumatism, piles and night blindness. An ethanolic extract of T. portulacastrum exhibit a potent early hepatoprotective activity in acute and chronic liver damage.

AIM OF THIS STUDY:The objective of the present study was to assess the antioxidant activity and scavenging property of different parts of $\mathrm{T}$. portulacastrum.

MATERIALS AND METHODS: T. portulacastrum collected from fields in kalyani and were authenticated from the Department of Botany, University of Kalyani, Kalyani, Nadia. Different parts of the plant such as leaf, stem, and root were separated. Different biochemical parameters, such as protein, carbohydrate, total polyphenols, flavonoids and ascorbic acid content were measured. Antioxidant properties were estimated enzymatically and non-enzymatically, which include reduced glutathione content and activities of ascorbic acid oxidase (AAO), Poly phenol oxidase (PPO), catalase, peroxidise, Glutathione s-transferase (GST)and superoxide dismutase(SOD).Scavenging properties were measured using DPPH, hydrogen peroxide, hydroxyl radical,nitric oxide and superoxide anion radical utilising methanolic extracts, and overall anti oxidant activity was monitored from total antioxidant status.

RESULTS: Leaf extract of T. portulacastrum showed highest antioxidant activity like catalase $(2.83 \pm 0.15$ $\mathrm{mg} \mathrm{H} 2 \mathrm{O} 2$ decomposed $/ \mathrm{min} / \mathrm{g}$ ), reduced glutathione $(4.03 \pm 0.25 \mathrm{mg} / \mathrm{g}$ fresh wt), ascorbic acid content $(0.21$ $\pm 0.03 \mathrm{mg} / \mathrm{g}$ of fresh weight) and protein content (33.93 $\pm 1.79 \mathrm{mg} / \mathrm{g}$ of fresh weight $)$ and SOD $(1.6 \pm 0.02 \mathrm{U} / \mathrm{g}$ tissue) and AAO (11.73 $\pm 0.25 \mathrm{mg}$ ascorbic acid decomposed/g tissue/min) in stem extract, Peroxidase $(6.37 \pm 0.02 \mathrm{U} / \mathrm{min})$ and GST $(3.59 \pm 0.05 \mathrm{nmole} / \mathrm{mg}$ tissue $/ \mathrm{min}$ ) in whole plant extract, while carbohydrate content $(378.67 \pm 37.42 \mathrm{mg} / \mathrm{g}$ of fresh weight $)$ was found higher in in root extract, and polyphenol oxidase activity 
$(0.02 \pm 0.001 \mathrm{U} / \mathrm{min} / \mathrm{ml})$ in root and whole plant extract. Among leaf, Stem and whole plant extract leaf extract showed maximum scavenging property compared to stem and whole plant extract.

CONCLUSION: The results indicate that hepatoprotective activity of T. portulacastrum is due to its antioxidant and scavenging property.

\section{P161}

\section{Dried Blood Spot Technology Coupled With Tandem Mass Spectrometry For The Screening of X-Linked Adrenoleukodystrophy}

Archana Natarajan $^{1}$, Rita Christopher ${ }^{1}$,

Netravathi.M ${ }^{2}$, Maya Bhat ${ }^{3}$

\section{Metabolic Laboratory, Department of}

Neurochemistry,

2. Department of Neurology,

3. Department of Neuroimaging and Interventional

Radiology, National Institute of Mental Health and

Neuro Sciences (NIMHANS), Bengaluru, Karnataka

INTRODUCTION: $X$-linked Adrenoleukodystrophy (X-ALD) with an incidence of 1:17,000 is one of the most common peroxisomal disorder and inherited neurodegenerative diseases which cause demyelination of the central nervous system and adrenal dysfunction. It is caused due to the mutation of the $\mathrm{ABCD} 1$ gene which impairs the peroxisomal Very Long Chain Fatty Acid (VLCFA) degradation resulting in accumulation of VLCFA in the cytosol. Accumulation of one of the VLCFAs- C26:0 VLCFA, has shown to result in accumulation of C26:0-lysophosphatidylcholine (C26:0LPC) in X-ALD patients. Estimation of plasma VLCFA by Gas Chromatography Mass Spectrometry (GC-MS) is cumbersome, expensive and invasive. The advantage of using dried blood spots (DBS) for newborn screening has been well established but alternate non-invasive, less expensive and simple method for VLCFA estimation for screening of X-ALD has not been developed in India so far.

AIMS AND OBJECTIVES: To develop a simple, noninvasive and less expensive DBS based tandem mass spectrometry (LC-MS/MS) method for estimating VLCFA-LPCs.

MATERIALS AND METHODS: $3 \mathrm{~mm}$ dried blood spots were punched out from the filter paper and a panel of 4 VLCFA-LPCs (C26:0, C24:0, C22:0 and C20:0) were measured using a lab standardised and validated LC-MS/MS method.

RESULTS: Out of 48 suspected cases with clinical and radiological features of leukodystrophy we identified 16 positive cases by the LC-MS/MS method developed by us. Plasma VLCFA concentration was also elevated for these cases.

CONCLUSION: We developed a simple LC-MS/MS method for the screening of X-ALD using dried blood spots for the first time in India.

\section{P162}

\section{A Study on Relationship of Serum Vitamin D3 With Anthropometry And Metabolic Markers Intype Ii Diabetes Mellitus}

Taru Goyal, Prasenjit Mitra, Shailja Sharma, Praveen Sharma

Department of Biochemistry, All India Institute of Medical Sciences, Jodhpur, India

INTRODUCTION:Type II diabetes is associated with serious morbidity and increased mortality.From a worldwide perspective, the total number of people with diabetes is expected to rise from 171 million in 2000 to 366 million by 2030 . Low serum 25 hydroxyvitamin D3 (vitamin D3) is known to agitate cellular function in many tissues, including the endocrine pancreas, which are involved in obesity and type 2 diabetes mellitus (T2DM).Vitamin D may play a vital role in modifying risk of diabetes. The aim of this study was to evaluate the relationship of serum hydroxy vitamin $\mathrm{D}[25(\mathrm{OH}) \mathrm{D}]$ level with various clinical and metabolic parameters in newly diagnosed patients of T2DM.

MATERIALS AND METHODS: The study comprised of 100 newly diagnosed T2DM patients and 50 age and 
gender matched healthy controls. Patients with type 1 diabetes, or other endocrine disorders, chronic kidney diseases or receiving vitamin D supplementation were excluded from the study. Blood samples were analyzed using standard techniques for fasting \& post prandial blood glucose,glycated hemoglobin (HbA1c), serum 25(OH)D and lipid profile. Anthropometric indices like height, waist circumference, weight of the subjects was also noted. Differences in various parameters between the two groups were analyzed for significance using the student's t-test with a significance level set at $P$ value $<0.05$.

RESULT: The vitamin D levels were not significantly different in between the two groups, although a decreasing trend was observed in T2DM patients. $28 \%$ of T2DM patients were found to be vitamin $\mathrm{D}$ insufficient $(15-30 \mathrm{ng} / \mathrm{ml})$ and $71 \%$ of the T2DM patients were vitamin $\mathrm{D}$ deficient $(<15 \mathrm{ng} / \mathrm{ml})$. In addition to this, Vitamin D3 levels had modest inverse associationswith HbA1c\%,LDL,BMI, Blood Pressure and a direct association with HDL. No association was observed with TG level.

CONCLUSION: The results of the present study show that majority of the patients with T2DM are vitamin D deficient and the serum $25(\mathrm{OH}) \mathrm{D}$ level in the patients with T2DM is related to anthropometric, lipid and glucose parameters. Further studies are required to evaluate the relationship of vitamin $D$ with serum triglycerides and other related clinical or metabolic parameters.

KEYWORDS: Vitamin D, Diabetes mellitus

\section{P163}

\section{Association of Fasting Blood Glucose Level And Dyslipidemia In Pre Diabetic Individuals}

*Kalpana Singh, *Neha Srivastava, **Bhawana

Mahajan, *Abbas Ali Mahdi

* Department of Biochemistry, King George's

Medical University, Uttar Pradesh, Lucknow

**Department of Biochemistry, G. B. Pant Institute of

Post Graduate Medical Education \& Research, New

Delhi
INTRODUCTION:Pre-diabetes is a condition in which blood glucose level is above normal but below the diagnostic threshold for diabetes mellitus.Impaired lipid profile i.e. dyslipidemia is well known to be associated withcardiovascular disease (CVD) in type 2 diabetic patients and can also occur in pre-diabetic individuals. This study was aimed to compare the lipid profile level among pre-diabetic individuals having normal fasting glucose with the individuals having impaired fasting glucose (IFG).

MATERIAL AND METHODS: 50 pre-diabetes individuals were enrolled in our study having $\mathrm{HbA} 1 \mathrm{c}$ between $5.7 \%-6.4 \%$ and not on lipid lowering drugs. Depending on fasting blood sugar (FBS) level, patients were categorized into two groups i.e., group 1: normal (FBS ? $110 \mathrm{mg} / \mathrm{dl}$ ) and group 2: impaired fasting glucose (FBS: 110 - $125 \mathrm{mg} / \mathrm{dl}$ ). Out of 50 patients enrolled, 37 constituted groups 1 and 13 belonged to group 2. Sample for FBS and lipid profile were collected from all the patients and estimated by Dry Chemistry Analyzer.

RESULTS: No differences were observed in the level of total cholesterol, triglyceride, HDL-c, VLDL, LDL, ratio of TC/HDL and LDL/HDL amongpre-diabetic individuals having normal and impaired fasting glucose level.

CONCLUSION: Association of pre-diabetes and dyslipidemia is still in question. Studies with largersample sizeare required to assess the correlation between pre-diabetes and dyslipidemia in order to ascertain whether early interventions can prevent cardiovascular diseases.

KEYWORDS: Diabetes, Dyslipidemia, Fasting blood glucose, Impaired Fasting blood glucose

\section{P164}

GC-MS Analysis of Dental Filling Prepared From Artocarpus Heterophyllus (Jackfruit) Latex- An Innovative Biocomposite Material

\footnotetext{
*Jitendra Rao, *Tuba Afsheen, **Kalpana Singh, ***Prashant Gupta, ****O. P. Sidhu

* Department of Prosthodontics, ** Department
} 
of Biochemistry, ***Department of Microbiology,

King George's Medical University, Lucknow

****Department of Phytochemistry, CSIR-National

Botanical Research Institute, Lucknow

\section{INTRODUCTION: Jackfruit (Artocarpus} heterophyllus) is one of the important members of family Moraceae which is widely distributed lactifer tree with traditional and medicinal uses. The white milky latex comprises of different compounds, one of them is resin. The present study was aimed to isolate and extract jackfruit resin and to elucidate its potential benefits as an alternative substitute in dental restoration.

MATERIAL AND METHODS: Latex was collected by using demineralised water which caused it to coagulate. The coagulated latex was washed, dried and dissolved in acetone. The solution was filtered and allowed to dry completely under vacuum to obtain slightly sticky mass called resin, which was confirmed by UV absorption method. Resin was modified to prepare a dental material before filling it in the cavity of artificial tooth by the addition of certain fillers and modifiers. Both extracted resin and dental filling material were studied for the presence of metabolites by GC-MS (Gas Chromatography- Mass Spectrometry) analysis.

RESULTS: During the drying of latex, its color changed from white on day 1 st to off-white on 7 th day. The presence of resin was confirmed at $272 \mathrm{~nm}$. The modified resin was filled in completely dried artificial tooth.GCMS profiling of resin and dental filling material demonstrated presence of four and eight metabolites respectively. Modified dental filling material was found better than resin for dental restoration.

CONCLUSION: Jackfruit latex may serve as a potential herbal biocomposite material which can be used as an inexpensive alternative to currently available costly procedures and materials in dental restoration. Also, synthetic composite materials available commercially contain BIS-GMA resin which has been reported carcinogenic.

KEYWORDS:Dental restoration, Jackfruit latex, GCMS, Dental filling material
P165

\section{Potential of Selected Vegetable Peels As Natural Antioxidant Source And Their Antimicrobial Efficacy Against Different Test Pathogens}

\author{
*Rini Tiwari, *Rakesh Kumar Yadav, \\ **Kalpana Singh \\ * Department of Conservative Dentistry and \\ Endodontic, King George's Medical University, \\ Lucknow \\ ** Department of Biochemistry, King George's \\ Medical University, Lucknow
}

INTRODUCTION: Vegetable peel, usually a waste product which is thrown into the environment, has a very good antioxidantandantimicrobial potentiality. The present study was aimed to investigate the antimicrobial and antioxidant properties of four different vegetable peels.

MATERIAL AND METHODS:The ethanolic and methanolic extracts of bottle gourd (Lagenaria siceraria) peels, french beans (Phaseolus vulgaris) peels, garden pea (Pisum sativum) peels, water chestnut (Trapa natans) peels were evaluated for their antimicrobial activity against six microorganisms (Aspergillus fumigates, Bacillus subtilis, Staphylococcus aureus, Helicobacter pylori, Klebsiella pneumoniae, Pseudomonas aeroginosa) by agar well diffusion method. Several potential antioxidant activities including reducing power assay, superoxide anion scavenging activity, and total antioxidant capacity were evaluated using colorimetric methods. Furthermore phenolic contents including, total flavonoid content and total phenolic content was also performed using colorimeter.

RESULTS: Trapa natans showed highest result in TPC and TFC with $0.193 \mathrm{mg} \mathrm{GAE} / \mathrm{g}$ and $0.00081 \mathrm{mg} \mathrm{QE} / \mathrm{g}$ respectively and other peel extract showed moderate results. The methanolic extracts of all the vegetable peels showed moderate to high activity against all the investigated microbial strains. Ethanolic extract of Trapa natans and methanolic extract of Lagenaria siceraria was most effective against all microorganisms $(29 \mathrm{~mm}$ and $34 \mathrm{~mm}$ inhibition zone against B. subtilis). Overall, ethanolic extracts were more effective than the 
methanolic extracts.

CONCLUSION: The present study showed that among the tested peels, T. natans exhibited strong antioxidant and antibacterial property. These results suggest that vegetable peels could be exploited as a potential source of natural antioxidant and antimicrobial agent.

KEYWORDS: Antioxidant activity, Antimicrobial activity, Vegetable peels

\section{P166}

\section{Role of Interleukin-10 (A1082g) Gene Polymorphism Is Associated With Risk For Oral Squamous Cell Carcinoma}

$\underline{\text { Syed Rizwan Hussain }}^{1}$, Mohammad Kaleem Ahmad ${ }^{2}$, Shalini Gupta ${ }^{3}$, Nitu Nigam ${ }^{4}$, Pritivi kumar Singh ${ }^{4}$ and Abbas Ali Mahdi ${ }^{2}$

1. Molecular Biology Unit CFAR,

2. Department of Biochemistry,

3. Oral Pathology \& Microbiology and

4. Cytogenetic Unit CFAR, King George's Medical

University, Lucknow, Uttar Pradesh, India- 226003.

INTRODUCTION: The functional polymorphism IL$10(\mathrm{~A} 1082 \mathrm{G})$ has influence on the gene expression and is also associated with risk for Oral squamous cell carcinoma (OSCC). The present case - control study was carried out to evaluate the possible association between IL-10 (A1082G) gene polymorphisms and OSCC in a north Indian population.

METHODS: Analysis of IL-10 (A1082G) genotype in 232 OSCC cases and 221 healthy controls of comparable age, gender, smokers, tobacco chewing and alcohol consumptionwas carried out. IL-10 (A1082G) gene polymorphism in the cases and controls were evaluated by polymerase chain reaction and restriction fragment length polymorphism (PCR-RFLP).

RESULTS: The frequency ofIL-10 (A1082G) gene polymorphism AA, AG, GG genotype were $29.74 \%$, $68.10 \%, 2.15 \%$ in OSCC cases and $57.46 \%$, $42.08 \%, 0.45 \%$ in healthy controls, respectively. The average frequency of $\mathrm{G}$ mutant allele was $36.20 \%$ in OSCC cases compared to $21.50 \%$ among the controls and this allele was associated with increased risk for OSCC cases. Heterozygous AG genotype was found statistically significant in OSCC cases than in controls $[\mathrm{OR}=1.6,95 \% \mathrm{CI}=1.1-2.2, \mathrm{p}=0.003]$, whereas homozygous mutant GG genotype was not found significant $[\mathrm{OR}=4.7,95 \% \mathrm{CI}=0.55-41.1, \mathrm{p}=0.2]$. Moreover, we found that $\mathrm{G}$ allele was significant in OSCC cases of tobacco chewing.

CONCLUSION: The results of present study indicated that the frequency of IL-10 (A1082G) gene polymorphism $\mathrm{G}$ allele and $\mathrm{AG}$ genotype is associated with OSCC cases as compared with controls; this may be due to smoking and tobacco chewing.Our findings showed that in IL-10 (A1082G) gene polymorphism AG genotype and $\mathrm{G}$ allele may participate in the progression of OSCC.

KEYWORDS: Polymorphism, IL-10 (A1082G) gene. PCR-RFLP, Oral squamous cell carcinoma.

\section{P167}

Apoptotic Inhibition of Methyl-4-Phenyl-1,2,3,6Tetrahydropyridine (MPTP) Induced Dopaminergic Neuronal Cell Death By Ethanolic Extract Bacopa Monnieri

Shivani Pandey ${ }^{1}$, Babita Singh ${ }^{1}$, Satyndra Kumar Yadav ${ }^{1}$, Abbas Ali Mahdi

1. Department of Biochemistry, KGMU, Lucknow-226003

2. Era University Sarfazganj, Hardoi road, Lucknow

INTRODUCTION: Parkinson's disease (PD) is a neurodegenerative disease which causes rigidity, resting tremor and postural instability.Baccopa monnieri (BM), a herb traditionally recognized as brain tonic by the Indian system of medicine, Ayurveda.The present study investigates the mechanisms of neuroprotection elicited by ethanolic extract of BM in MPTP mice model system

MATERIAL AND METHODS:Male Swiss albino mice weighing 30-40 gram were used in experiment. Experimental mice were given $15 \mathrm{mg} / \mathrm{kg}$ b.w of MPTP 
for two weeks and then subsequently treatment of BM $40 \mathrm{mg} / \mathrm{kg}$ bodyweight orally for one month. Inhibition of apoptotic pathways was studied via behavioral, biochemical, Immunohistochemical and expression analysis.

RESULTS:An ethanolic extract of BM was co-treated with the MPTP induced mouse model of PD and was shown to signi? cantly rescue the motor behaviour (Rotarod, Grip Strength and Foot Printing test). Furthermor, on biochemical parameters BM significantly showed marked improvement and the levels of Dopamine, DOPAC and HVA were enhanced significantly. There was a significant reduction in tyrosine hydroxylase (TH) immunoreactivity in the substantia nigra ( $\mathrm{SN}$ ) in the MPTP treated group, which was considerably restored by the use of BM extract.BM also facilitated neuroprotection by creating an antiapoptotic environment indicated by reduced apoptotic (Bax and caspase-3) and increased levels of antiapoptotic $(\mathrm{Bcl} 2)$ protein expression, respectively.

CONCLUSION: Present study suggests that BM treatment provides nigrostriatal dopaminergic neuroprotection against MPTP induced Parkinsonism by the modulation of oxidative stress and apoptotic machinery possibly accounting for the behavioural effects.

KEYWORDS: neurodegenerative; antioxidant; Baccopa monnerri;substantia nigra; caspase-3; apoptotic.

\section{P168}

The Association Oftnf- $\alpha$ (-G238A And -G308A) Gene Polymorphism With Risk of Oral Cancer

Prithvi Kumar Singh ${ }^{1}$, Mohammad Kaleem Ahmad ${ }^{2}$, Nitu Nigam ${ }^{1}$, Syed Rizwan Hussain ${ }^{1}$, Kumar $^{3}$, Abbas Ali Mahdi², Vijay Kumar ${ }^{3}$, Girish Chandra ${ }^{4}$

1Department of Centre for Advance Research (Cytogenetics Unit), 2Department of Biochemistry, 3Department of Surgical Oncology,4Department of Anaesthesiology, King George Medical University UP, Lucknow-India
BACKGROUND:Cancer is a multifactorial disease, resulting from complex interactions between genetic and environmental factors. Tumor necrosis factor alpha (TNF-?) is a pro-inflammatory cytokine, which plays a pivotal role in the pathogenesis of a variety of infectious and inflammatory diseases. Tumor necrosis factor alpha (TNF-?) gene polymorphism has been a potential determinant of susceptibility to various types of cancer.

OBJECTIVE: To explore the association of TNF-? promoter (-G238) and (-G308A) gene polymorphisms with the susceptibility oforal cancer.

METHODS:A total 300 patients with oral cancer and 300 healthy volunteers were genotyped for the TNF-?- 238G/A and-308G/A polymorphism. Polymorphism variants were examined by polymerase chain reactionrestriction fragment length polymorphism (PCR-RFLP). Genotype frequencies were evaluated by Chi-square test strength of associations by odds ratio with $95 \%$ confidence intervals.

RESULTS:The genotypes of TNF-? (-G308A) were not significant differences in thegenotype and allele frequencies of the TNF-? (-G308A) gene polymorphism between oralcancer patients and control groups ( $\mathrm{p}$ : $>0.05)$. The genotypes of TNF-? (-G238A) gene polymorphism were significantdifferences in the between oral cancer patients and control groups(OR: 23.15, CI: 1.32-7.53, p: 0.01). Moreover the allele $G$ and $A$ frequencies was alsosignificantly deference in between case controls (OR: 3.07, CI: 1.29-7.28, p: <0.007).

CONCLUSIONS:Our results suggest that the TNF-? (G238A) polymorphism may be significantly associated with risk of oral cancer and TNF-? (-G308A) polymorphism may not be associated with increased risk of oral cancer.

KEYWORDS: Cytokines, Cytokines, Tumor necrosis factor- alpha, Inflammation, Genotype

\section{P169}

Publication Trends In The Indian Journal of Clinical Biochemistry

$\underline{\text { Shorya Taliyan }}^{1}$, Debapriya Bandyopadhyay, Haren 
Baruah, Dilutpal Sharma, Bharat Kumar Gupta.

Department of Biochemistry, Subharti Medical College,Swami Vivekanand Subharti University, Subhartipuram, Delhi-Haridwar Bypass Road (N.H. 58),Meerut - 250005, Uttar Pradesh, India

INTRODUCTION: The Indian Journal of Clinical Biochemistry (IJCB) is the official journal of the Association of Clinical Biochemists of India (ACBI). It publishes papers reporting original research work, critical up-to-date topics of current interest and brief communications on all aspects of clinical biochemistry. There is a constant effort put in by most leading journals in trying to evaluate the quality of research work they publish, the range of topics covered, their authorship, citation and readership to assess the impact of their dissemination of scientific knowledge in the subject. No assessment has been done till date of the nature of articles published in IJCB.

METHODS: This work reviews the publications in this journal over a 4-year period (2011-2014). It assess the types of articles published, the coverage of various subspecialties of clinical biochemistry in the journal, and explores the authorship patterns in these publications.

RESULTS: In the six volumes reviewed, the IJCB has published peer reviewed articles that contributed significantly to the existing knowledge of clinical biochemistry. This journal particularly dealt with the applications of biochemistry to the diagnosis, treatment, monitoring and prevention of human diseases. Most of the publications were from teaching institutes. The highest numbers of publications in all the volumes were based on "antioxidants and free radicals" followed by those on "diabetes mellitus".

CONCLUSION: IJCB has delivered broad-based, balanced coverage of human metabolism in health and disease, biomarkers and recent advances between 2011 and 2014, with contributions from all over India, as well as abroad.

KEY WORDS: Antioxidants and free radicals, Diabetes mellitus, Publications .

\section{Post Phototherapy Changes In Serum Calcium And Magnesium Levels In Neonatal Hyperbilirubinemia Cases In A Tertiary Care Hospital of Ne India}

Mousumi Goswami, Kailash Bhattacharyya

Gauhati Medical College and Hospital, Gauhati

INTRODUCTION- Neonatal hyperbilirubinemia is the most common abnormal clinical finding during the first week of life andobserved in $\sim 60 \%$ of term neonates and $80 \%$ of preterm infants. It is the most common cause for readmission during the early neonatal period and a matter of concern for the parents as well as paediatricians. Phototherapy plays a significant role in the treatment and prevention of hyperbilirubinemia in neonates.Serum electrolyte levels (magnesium and calcium) in neonates should however be assessed as significant changes are seen following phototherapy.

OBJECTIVE- -To evaluate the changes seen in serum calcium and magnesium levels in neonates receiving phototherapy.

MATERIALS AND METHODS- A hospital based prospective study was conducted in a tertiary care hospital of North East India from January 2017-March 2017. 50 eligible neonates admitted in a neonatal intensive care unit for neonatal hyperbilirubinemia were enrolled in our present study. Serum Calcium and Magnesium levels of each neonates were estimated before and after phototherapy using semi auto analyser.

RESULTS- The study included 50 neonates receiving phototherapy for neonatal hyperbilirubinemia. Male female ratio being 1.17:1. Incidence of low birth weight babies was $32 \%$ and preterm babies was $32 \%$ compared to term babies being $68 \%$.Incidence of hypocalcemia was high $(75 \%)$ in low birth weight babies compared to normal babies (41\%). There was highly statistically significant decrease of the serum magnesium levels after phototherapy,more in low birth weight babies(81\%) compared to normal babies(61\%) suggesting phototherapy induced hypocalcemia and hypomagnesemia in neonatal hyperbilirubinemia more commonly in low birth weight babies. 
CONCLUSION- Neonates undergoing phototherapy are at a higher risk of hypocalcemia and hypomagnesemia, which is seen commonly amongst low birth weight babies. The study highlights the importance of monitoring electrolytes changes to prevent further complications and reduce economic and morbidity burden of neonates in tertiary care hospitals.

KEYWORDS- Neonatal Hyperbilirubinemia , Serum Calcium, Magnesium, Phototherapy

\section{P171}

\section{Cost Effective Screening Tests For The Diagnosis of Inborn Errors of Metabolism}

Nazmin Fatima, Gyanendra Kr. Sonkar, Sangeeta Singh, Abbas Ali Mahdi

\section{Department of Biochemistry, King George's Medical University, Lucknow}

INTRODUCTION:Inborn Errors of Metabolism (IEM) are caused by defect in the gene that are responsible for the production of an enzyme. These defective gene causes reduced or no formation of enzymes that are necessary for the normal functioning of various metabolic pathways, in such a way that the toxic substance starts accumulating in an abnormal amount on different organ and cause to damage various systems in the body in one form or the other.IEM are heterogeneous and inherited as autosomal recessive traits. The term IEM was coined by Dr. A.E. Garrod in 1908. Individual IEM are rare disorder having an incidence of less than 1 per 1, 00,000 birth. However collectively the incidence may approach to 1 in 800 to 2500 births. In India the overall prevalence of IEM is one in 2497 newborns and in Andhra Pradesh, one in 1000 newborns. More than 3500 IEMs are believed to exist and nearly 700 IEMs have been identified till date.

AIMS \& OBJECTIVES: Our present study aimed to screen IEM suspected children using a cost effective and simple biochemical testsand confirmed by high performance liquid chromatography (HPLC).

METHODS: Cross sectional study was done in the Department of Biochemistry in collaboration with the
Department of Pediatrics, King George's Medical University (KGMU) Lucknow. This study was conducted on 150 children suspected for IEM, aged below 15 years having history of lethargy, convulsion, consanguinity, regression of milestone, coarse facial, mental retardation, skeletal deformities, sibling death with similar illness, etc. was included in the study. Various biochemical test and high performance liquid chromatography was performed in urine and blood samples.

RESULTS: A total of 150 subjects were enrolled in our study, out of which 57 (38\%) tested positive for a specific metabolic disorders. HPLC result revealed, twelve (8\%) cases were found to be positive for amino acid disorders i.e. two $(1.3 \%)$ cases for phenylketonuria, five (3.3\%) cases each for tyrosinemia and five (3.3\%)cases for MSUD in plasma sample of suspected cases.

CONCLUSION: Early screening for genetic disorders, along with accurate diagnosis and intervention, can save lives and result in improved long-term health for affected newborns.Most IEM cases remain undiagnosed the possible reasons being poverty, lack of facilities, ignorance or due to misdiagnosis by clinicians. There is a need to start a national program in India for routine screening of all newborns as in other countries worldwide, in order to decrease the morbidity and mortality due to IEM in future generation and may be useful for reducing the burden of disease in India.

KEY WORDS: Inborn errors of Metabolism, mental retardation, newborns, tyrosinemia, MSUD

\section{P172}

\section{Emerging Diagnosis of Acute Myocardial Infaction Via Cardiac Biomarker : A Review}

Mohammad Amil Rahman, Roshan Alam, Dhananjay Tiwari, Priyanka Thapa Mangar

Department of Biochemistry, Integral Institute of Medical Science \& Research

$\mathrm{V}$ arious studies have shown that people of Indian origin have an increased risk of ischemic heart disease (IHD) compared with most other ethnic groups the early diagnosis of cardiac ischemia is fundamental 
for the effective management of AMI patients. Inadequate diagnosis of patients with chest pain often leads to inadequate admission of patients without acute myocardial infaction (AMI) and vice versa Cardiac markers are used in the diagnosis and risk stratification of patients with chest pain and suspected acute coronary syndrome (ACS).The troponins are regulatory proteins found in skeletal and cardiac muscle. Three subunits have been identified: troponin I (TnI), troponin $\mathrm{T}(\mathrm{TnT})$, and troponin $\mathrm{C}(\mathrm{TnC})$. The genes that encode for the skeletal and cardiac isoforms of $\mathrm{TnC}$ are identical; thus, no structural difference exists between them. However, the skeletal and cardiac subforms for TnI and troponin TnT are distinct, and immunoassays have been designed to differentiate between them.According to European Society of Cardiology (ESC)/American College of Cardiology Foundation (ACCF)/American Heart Association (AHA)/ World Heart Federation (WHF) guidelines, MI refers specifically to myocardial necrosis due to myocardial ischemia. However, although elevations in the serum levels of TnI, TnT, and CK-MB indicate the presence of injury-associated necrosis of myocardial cells, such elevations do not point to the underlying mechanism of the necrosis. While myocardial necrosis occurs in MI, it can also be a product of predominantly nonischemic myocardial injury, as occurs in association with heart failure, arrhythmia, myocarditis, renal failure, pulmonary embolism, and percutaneous or surgical coronary procedures. The National Academy of Clinical Biochemistry (NACB) working with the ACC/ ESC guidelines has recommended adoption of the 99th percentile upper reference limit as the recommended cutoff for a positive troponin result. Ideally, the precision of the assay at this cutoff level should be measured by a $\mathrm{CV}$ that is less than $10 \%$. Copeptin may be useful to rule out MI rapidly when used along with troponin.

KEY WORDS: Acute myocardial Infaction, Cardiac Troponins, CK-MB.

\section{P173}

\section{Lipid Peroxide Levels And Antioxidant Status In Healthy And Alcoholic Hepatitis Patients}

$\underline{\text { Sanjay Bhatt }}{ }^{1}$, Shivani Pandey ${ }^{2}$, Bindu Sati ${ }^{2}$, Singh RK ${ }^{3}$

1. Department of Biochemistry, S.R.M.S I.M.S , Bareilly

2. Department of Biochemistry KGMU, Lucknow GBPIHED, Srinagar Garhwal,

3. TSM Medical College and hospital, Lucknow

BACKGROUND: Alcohol remains the single most significant cause of liver disease throughout the Western world, responsible for between 40 and $80 \%$ of cases of cirrhosis in different countries.

MATERIAL \& METHODS: The study was conducted in Department of Biochemistry, Santosh medical college \& Hospital, Ghaziabad and Veer Chandra Singh Garhwali Govt. Institute of Medical Sciences \& Research 164 alcoholic hepatitis patients were subjected to detailed clinical examination and laboratory investigations and the results were compared with 82 controls. Blood samples were collected for oxidative stress parameters. It was observed that there was a significant increase in activities of Catalase, SOD, MDA, GPX and GR activity in patients with alcoholic hepatitis when compared to controls.

RESULTS: Results of our study show higher oxygen free radical production, evidenced by elevated levels of MDA and decreased levels of Catalase, SOD, GPx, GR, and TAS activity, supporting the evidence of oxidative stress in alcoholic hepatitis patients. Decreased concentrations of antioxidant support the hypothesis that alcoholic hepatitis is an important causative factor in pathogenesis of lipid peroxidation.

CONCLUSION: The antioxidant defense mechanisms might be impaired in patients with alcoholic hepatitis. These findings also provide a theoretical basis for development of novel therapeutic strategies, such as antioxidant supplementation.

KEY WORDS: Catalase, Superoxide dismutase (SOD), Malondialdehyde (MDA), Glutathione Peroxidase (GPx), GlutathioneReductase (GR), Total antioxidant status (TAS), Alcoholic Hepatitis (AH). 


\section{P174}

\section{Antibacterial and Antifungal Activity of The Seeds of Hyoscyamus Niger L Against Microbes of Urinary Tract Infection}

M.M. Abid Ali Khan, *Murtaza Abid, Sabiha Kazmi, S.H.A Kazmi, Amber Hasan

Department of Botany,

Shia P.G. College, Lucknow, U.P., India

*Department of Biochemistry, King George's Medical

University, Lucknow, U.P., India

INTRODUCTION: The methanol extracts obtained from the seeds Hyoscyamus niger L. (Solanaceae) were investigated for their antimicrobial activities against the pathogens causing complicated urine tract infections.

MATERIALS AND METHODS: The seeds of plant were extracted with aqueous $60 \%$ methanol. The extract was screened against urinary tract pathogens (Enterococcus faecalis, Escherichia coli, Klebsiella pneumonia, Pseudomonas aeruginosa, Proteus mirabilis and Candida albicans) by disc diffusion method and microdilution method. Some antibacterial and antifungal antibiotics were used as a positive effect reference standard to determine the sensitivity of the stains.

RESULTS: The extracts showed strong antimicrobial activity against Enterococcus faecalis, Klebsiella pneumonia and Candida albicans with inhibition zones of 26.0, 19.0 and $16.0 \mathrm{~nm}$ with MIC's and MBC's or MFC's of 4.0 (8.0), 32 (64) and 64 (128) $\mu \mathrm{g} / \mathrm{ml}$, respectively. Also, the extracts exhibited moderate activity against the other test microorganism.

CONCLUSION: Our findings support the use of Hyoscyamus niger L. in traditional medicine for the treatment against the urine tract pathogens.

KEYWORDS: Urinary tract infection (UTI), Hyoscyamus niger, Antimicrobial activity and Antibacterial activity.

\section{P175}

\section{Predicting Serious Cardiac Outcomes Following Acute Coronary Syndromes Using Cardiac Biomarkers}

Mayank Bhasin*,JavinBishnu Gogoi**

Department of Biochemistry, VCSG Govt. Institute of

Medical Science \& Research, Srinagar, Garhwal, Uttarakhand, India, Pin-246174, Email-

drjbgogoi@gmail.com

INTRODUCTION:Acute coronary syndrome (ACS) is one of the largest contributors to global mortality worldwide of which most of the burden is borne by developing countries. Post ACS complications are the major contributors to death even in those patients who receive early medical attention. The goal of preventing reinfarction and death after recovery from Myocardial infarction (MI) has led to an extensive search for strategies to evaluate the risk of occurrence of these complications after initial infarction. Use of cardiac biomarkers (measured for diagnosing MI) for such evaluation will not only be less cumbersome but will give us a very early prediction for the adverse future outcomes and help to identify the high risk patients.

OBJECTIVES: The present study is undertaken with an objective to assess the sensitivity, specificity, positive and negative predictive value of cardiac biomarkers for the prediction of subsequent development of complicatingcardiac outcomes (upto72hrs) following an attack of ACS. As well as the patients with acute chest pain are to be screened and diagnosed for ACS by using the cardiac biomarkers.

MATERIAL AND METHODS: A longitudinal observational study was conducted in 120 patients with symptoms of ACS attending the Emergency Department and Medicine Department of HNB Base Hospital, Srinagar, Garhwal, Uttarakhand, India. History was taken and detailed clinical examination of the study participants was carried out. A panel of biochemical tests including enhanced cardiac troponin-I(en-cTnI), NT-pro BNP (Brain Natriuretic Peptide), CK-MB(Creatine kinase$\mathrm{MB}$ ) and hs-CRP (high sensitive C-Reactive Protein) was employed at the time of admission using Cardiac Marker 
Analyser (AQT90 Flex, Radiometer). Patients were followed upto 72 hours following the initial episode for the development of any serious cardiac outcome.

RESULTS:17 (14.2\%) participants were found to have at least one of the serious cardiac outcomes within 72 hours of the episode of acute coronary syndrome. Various serious cardiac outcomes observed during the study were heart failure (7 patients), arrthymias (5 patients), recurrent angina ( 3 patients) and cardiogenic shock (2 patients). ROC (receiver operating characteristics)curves were plotted for these biomarkers and sensitivity and specificity of the prediction of these outcomes using encTnI was $76.4 \%(95 \% \mathrm{CI})$ and $90.3 \%(95 \% \mathrm{CI})$ respectively. NT-pro BNP yielded sensitivity of $58.82 \%$ (33.43\%-80.57) and specificity $73.78 \%$. CK-MB and hsCRP showed sensitivity of $52.94 \%$ and $35.3 \%$ respectively. This prediction was statistically significant for en-cTnI(pvalue <0.0001) and NT-pro BNP (pvalue $=0.0013$ ) by Wilcoxon Mann Whitney test. Obesity especially truncal obesity, association with diabetes and hypertension and smoking or alcoholism were found to be additional risk factors for these outcomes.

CONCLUSION: The presentstudyconcludesthatencTnIandNT-pro BNPcan be usedas potentialpredictors of serious cardiacoutcomes likerecurrentangina, congestive heart failure (CHF), arrhythmias, etcfollowingan episodeof ACS.CK-MB andhs-CRPdo not prove to be promising biomarkers for this prediction.

KEY WORDS:Acute coronary syndrome,cardiac biomarkers, predictors, en-cTnI,NT-pro BNP.

(Acknowledgement- We are thankful to ICMR, New Delhi for funding this project.)

\section{P176}

\section{Thyroid Dysfunction In Patients With Type 1} Diabetes: A Longitudinal Study

Manisha Hoon ${ }^{1}$, SuryakantNagtilak

Department of Biochemistry, Subharti Medical College,Swami Vivekanand Subharti University, Subhartipuram,Delhi-Haridwar Bypass
Road (N.H. 58), Meerut - 250005,Uttar Pradesh, India

INTRODUCTION:Cross-sectional studies have reported that the risk of thyroid dysfunction in patients with type 1 diabetes is two- to threefold higher than in the general population. However, longitudinal studies to determine the natural history of thyroid dysfunction in patients with type 1 diabetes are lacking.

MATERIAL AND METHODS:We analyzed the incidence of thyroid dysfunction over time in a cohort of 50 patients ( 22 men and 28 women) enrolled in the Rural and Urban health centres of Subharti Medical College, Subharti University, Meerut (Uttar Pradesh) and prospectively followed for 2 years. Patients underwent measurement of thyroid function tests (thyroidstimulating hormone [TSH], thyroxine, and triiodothyronine) every six months for 2-years.

RESULTS:A total of 7 patients had hypothyroidism, and 1 patient experienced transient hyperthyroidism. The mean age of diagnosis was $12+/$ - 5years for type 1 diabetes and $11+/-5$ years for hypothyroidism. Hypothyroidism was more common in female $(21.4 \%)$ than in male $(4.5 \%)$ subjects. There were no differences in lipid profile, and $\mathrm{HbA}(1 \mathrm{c})$ between patients with and without thyroid dysfunction.

CONCLUSIONS:This longitudinal study confirms the association between autoimmune thyroid dysfunction and type 1 diabetes. Our results indicate that all subjects with type 1 diabetes should undergo annual screening by serum TSH measurement to detect asymptomatic thyroid dysfunction.

KEYWORDS: Type 1diabetes, Hypothyroidism

\section{P177}

\section{Thyroid Dysfunction In Patients With Type 1 Diabetes: A Longitudinal Study}

Manisha Hoon', SuryakantNagtilak

Department of Biochemistry, Subharti Medical

College,Swami Vivekanand Subharti University, Subhartipuram,Delhi-Haridwar Bypass Road (N.H.

58),Meerut - 250005,Uttar Pradesh, India 
INTRODUCTION:Cross-sectional studies have reported that the risk of thyroid dysfunction in patients with type 1 diabetes is two- to threefold higher than in the general population. However, longitudinal studies to determine the natural history of thyroid dysfunction in patients with type 1 diabetes are lacking.

MATERIAL AND METHODS:We analyzed the incidence of thyroid dysfunction over time in a cohort of 50 patients ( 22 men and 28 women) enrolled in the Rural and Urban health centres of Subharti Medical College, Subharti University, Meerut(Uttar Pradesh) and prospectively followed for 2 years. Patients underwent measurement of thyroid function tests (thyroidstimulating hormone [TSH], thyroxine, and triiodothyronine) every six months for 2-years.

RESULTS: A total of 7 patients had hypothyroidism, and 1 patient experienced transient hyperthyroidism. The mean age of diagnosis was $12+/$ - 5years for type 1 diabetes and $11+/-5$ years for hypothyroidism. Hypothyroidism was more common in female (21.4\%) than in male $(4.5 \%)$ subjects. There were no differences in lipid profile, and $\mathrm{HbA}(1 \mathrm{c})$ between patients with and without thyroid dysfunction.

CONCLUSIONS:This longitudinal study confirms the association between autoimmune thyroid dysfunction and type 1 diabetes. Our results indicate that all subjects with type 1 diabetes should undergo annual screening by serum TSH measurement to detect asymptomatic thyroid dysfunction.

KEYWORDS: Type 1diabetes, Hypothyroidism

\section{P178}

\section{Hematological Malignancy Development Due To Aberration By The Bunch of Regulatory Protein Involved In Regulation of Hematopoietic Stem Cell}

Vivek Singh, Ranjana Singh,Abbas Ali Mahdi

Department of Biochemistry, King George's Medical University, Lucknow, U. P, India
$\mathrm{H}$ ematopoiesis is the process by which all lineages of blood cells are generated in a stepwise manner from immature cells present in the bone marrow and subsequently released into circulating blood peripheral organs for further maturation steps and effector function. At the apex of this hierarchy are hematopoietic stem cells (HSCs), which are the only self-renewing cells capable of life-long production of all lineages of blood cells. In response to differentiation cues, HSCs give rise to nonself renewing multipotent progenitors (MPPs), which then progressively differentiate into an array of more lineagecommitted progenitors and ultimately produce all of the highly specialized and differentiated into fully-grown mature blood cells. A large body of work has shown an essential role for two major categories of molecular networks in the maintenance of HSCs function, cellintrinsic process and cell extrinsic development pathways. As essential components of these regulatory networks are often deregulated in cancer, it is not surprising that improper control of HSCs maintenance is intimately tied to the development of hematological malignancies. Hematological malignancies are heterogeneous group of diseases that can globally be viewed as aberrant hematopoietic (stem cells) process initiated by a population of leukemic stem cells (LSCs) that are able, like normal HSCs, for both self-renewal and differentiation in an aberrant fashion thereby propagating themselves and giving rise to differentiated progeny that represent the bulk of cells found in the tumor. Knowledge of this would help researchers and clinicians better chance of understanding the pathogenesis of relevant blood diseases and to develop new strategies for therapeutic manipulations of HSCs.

KEY WORDS: stem cell, leukemia, hematopoietic stem cells (HSCs).

\section{P179}

In Vitro Evaluation of Apoptotic Potential And Metabolic Profiling of Tridex procumbens

Meenakshi Shukla ${ }^{\mathbf{1}, 2}$, HomaJilani Khan, ${ }^{1 \mathrm{~A}}$. A Mahdi ${ }^{1}$, Ranjana Singh ${ }^{1}$, MK Ahmad ${ }^{1}$

\section{Department of Biochemistry, King George's}


Medical University, Lucknow, U. P, India

2. Department of Biotechnology,

Dr.A.P.J Abdul Kalam Technical University, Lucknow, U.P, India

BACKGROUND \& OBJECTIVE: Breast cancer is the most common malignant tumor andsecond leading cause of death in womenworldwide. It is a heterogeneous multifactorial disease caused due to genetic, reproductive, environmental, and dietary and lifestyle related risk factors.Phytochemicals present in medicinal plants are frequently used in the treatment of cancer. The medicinal values of many plants have been explored but a large number of them are unexplored,T.procumbens is one of them. The aim of this studyis to explore the antiproliferative activity of aerial parts of T.Procumbensagainst human breast cancer (MDA-MB231 \& MCF-7) Cell lines.

MATERIAL \& METHODS:Breast Cell lines (MDAMB-231 \&MCF-7) along with the human normal transformed (HaCaT) cells were selected for MTT analysis. Flower part of T.procumbens dissolve in hexane solvent (i.e. hexane extract) showing most significant IC50value (p-value ? 0.05)could be considered as a potent anti-proliferative agent. We then further evaluated the anti- metastatic property using wound healing assay.

RESULTS: Our results revealed that hexane extract of T.procumbens flower exhibit highly significant antiproliferative and anti-metastatic activity against both the cell lines (MDA-MB-231, MCF-7).

CONCLUSION: Based on our finding and available literature there is need for isolation and characterization of specific bioactive compounds of T.procumbensand determination of their mechanism of action responsible for anticancer activity in breast cancer cells.

KEYWORDS: Malignant, Antiproliferative, Breast cancer, Wound healing, Metastasis

\section{P180}

Hepatoprotective Potential of Tridax Procumbens Against Isoniazid And RifampicinInduced Hepatotoxicity In Albino Rats $\underline{\text { Rida Sagheer }}^{1,2}$, R. Singh ${ }^{2}$, M. Nasibullah ${ }^{1}$, M. Shukla ${ }^{2}$. M. Sibghatullah ${ }^{1}$, H. Firoj ${ }^{1}$ A.A. Mahdi², A. R. Khan ${ }^{1}$

1. Department of Chemistry, Integral University Lucknow- 226026, India,

2. Department of Biochemistry, King George's Medical University, Lucknow - 226003, India.

BACKGROUND AND OBJECTIVE:Tridax procumbens belongs to Asteraceae family has been demonstrated to be hepatoprotective by Ayurvedic practitioner but it has not been scientifically explored so for. Therefore the present study was carried out for the assessment of hepatoprotective role of T. procumbens against anti-tuberculosis drug - induced hepatotoxicity in male Wistar rat.

MATERIALS AND METHODS: Male Wistar albino rats weighing 100-150g divided into 7 groups of 6 animals each. Group-1 (control) normal saline $1 \mathrm{ml} / \mathrm{Kg}$ p.o. Group-2 (positive) silymarine- suspension $200 \mathrm{mg} /$ Kg p. o. for 14 days. Group-3 (toxicant) - INH (Isoniazid) \& RMP (Rifampicin) $(50 \mathrm{mg} / \mathrm{Kg}, 100 \mathrm{mg} / \mathrm{kg})$ i. p. on day 14th. Group-4 silymarine same as group 2 for 14 days and toxin on day 14 thGroup-5 (treatment) MTPL (methanolic extracts of T.procumbens leaves) 300mg/ $\mathrm{Kg} \mathrm{p}$. o. for 14 days and toxin as same dose in group 4 Group-6 MTPS (methanolic extracts of T. procumbens stem) same as group 5 Group-7 MTPF (methanolic extracts of T. procumbens flower) same as in group 5 . Blood was collected from orbital plexus and liver was dissected out. LFT, Creatinine and cholesterol were measured and liver was subjected for histopathological examination. The consequences of MTPL, MTPS and MTPF on antioxidant enzymes SOD (superoxide dismutase) and CAT (catalase) were monitored to assess antioxidant activity.

RESULTS: SGOT, SGPT ALP and total bilirubin were significantly elevated in INH \& RMP treated group and displayed considerable decrease in SOD, CAT and TP in a dose dependent manner. Whereas MTPL (300mg/ $\mathrm{kg} \mathrm{p}$. o.) showed more significant reduction of in these parameters and increasing the SOD, CAT and TP levels. MTPL was found to have hepatoprotective potential as compare to MTPS and MTPF. 
CONCLUSION: It may be due to numerous reasons like antioxidant and free radicals scavenge property, ability to stabilizing the hepatic cells and reducing neutrophil infiltration into the hepatocytes.

KEY WORDS: MTPL, isoniazid, rifampicin, SOD, CAT, total protein, hepatoprotection

\section{P181}

\section{The Role of Micro-RNA Expression In Cancer Biology}

\section{$\underline{\text { Ashish Chaturvedi }}$}

Experimental and Public Health Laboratory, Department of Zoology, University of Lucknow, Lucknow-226007, U.P., India.

$\mathrm{C}$ ancer has a major impact on society in both developed and developing countries. In 2016, an estimated 1,685,210 new cases of cancer were diagnosed in the United States and 595,690 people died from cancer. MicroRNAs (miRNAs) are approximately 22 nucleotides in length, non coding RNA speciesand play an important role in regulation of many aspects of cancer biology including proliferation, differentiation, metabolism and apoptosis. The miRNAs predominantly bind to $3^{\prime}-$ untranslated regions ( $3^{\prime}$-UTR) and sometimes to $5^{\prime}$ UTR, codons of the transcribed mRNAs through specific seed sequences. This binding eventually results in either translation repression of target mRNAs to protein or induces their exonuclease-mediated cleavage, thereby regulating the expression of target genes. Moreover, their deregulated expression is also linked to the onset and progression of many diseases including cancer. Aberrant expressions of many miRNAs have been associated with cancer progression, metastasis as well as chemoresistance and are therefore classified as tumor suppressor miRNAs or oncogeneic miRNAs (OncomiRs). The our data suggested that targeting miRNAs in cancer can be a novel approach in area predictive biomarker which may be used as diagnostic, prognostic and therapeutic outcome.

KEYWORD: Cancer, Diagnostic, Prognostic and Therapy.

\section{P182}

\section{Alkaloids Contents of Hyoscyamus Niger L. And Their Pharmacological Action}

S. Rais Haider, Amber Hasan, *Murtaza Abid, S. Arshad Hasan Rizvi, S. Nazeer Haider Zaidi

Department of Botany, Shia P.G. College, Lucknow, U.P., India *Department of Biochemistry, King George's Medical University, Lucknow, U.P., India

INTRODUCTION: Medicinal plants produce a wide variety of secondary metabolites, such metabolites include alkaloids which are very prominent class of compounds exhibits novel pharmacological action. Hyoscyamus niger L. is an important medicinal plant belongs to family solanaceae. This plant is rich source of medicinal substances including tropane alkaloids.

MATERIALS AND METHODS: Seeds of H. niger were extracted for isolation of alkaloids, $5 \mathrm{ml}$ ammonia (25\%) and methanol was added over dried seeds powder to extract alkaloids in alcoholic medium, then methanol was evaporated and residue dry matter was washed with petroleum ether and finally lower phase was collected now added $25 \%$ ammonia and alkaline solution was collected washed with chloroform and dehydrated with anhydrous sodium sulphate and filtered with Whatman filter paper now ethanol is added and extract analyzed by TLC plates and HPLC, Hyoscyamine and scopolamine were obtained.

RESULTS:Results showed that on performing TLC scopolamine was major alkaloids then hyoscyamine in seed and on HPLC analysis scopolamine and hyoscyamine retention times obtained 9 and 11 minutes respectively, these alkaloids showed antispasmodic effect on gastrointestinal and genitourinary actions.

KEYWORDS: Hyoscyamus niger L., Solanaceae, Pharmacological actions, Tropane Alkaloids, Hyoscyamine and Scopolamine, HPLC and TLC.

\section{P183}

Saliva : A Non Invasive Diagnostic Fluid In The Next Mellinium 
*Mona Saxena, **Manvendra Pratap Singh, **Raja Roy, \#C.S. Saimbi, \#\#R.C. Saxena

* Department of Biochemistry, Saraswati Medical College, Unnao

**Sanjay Gandhi Post Graduate Institute of Medical Sciences and Hospital, Lucknow

\# Career Post Graduate Institute of Dental Sciences \& Hospital, Lucknow

\#\#King George's Medical University, Lucknow

$\mathrm{H}^{2}$ ealth is wealth, hence, yoga has been popularised as a preventive measure from diseases and to maintain health. If still wrong happens, it must be detected at the earliest where noninvasive methodology is the method of choice. Besides blood there are many secretions like sweat, saliva, urine etc where saliva is the easiest to be obtained. Saliva is secreted from the cells of the salivary glands filtering many biomolecules which may be altered in disease process as in inflammation which alters the physicochemical characteristics of the cellular biomembrane. For detection of these biomolecules there are several methods but nuclear magnetic resonance is one of the best technique based on absorption of electromagnetic radiations of the radiofrequency region (4 Mega Hertz) by nucleus of atom where protons absorb EM radiations. This is most powerful tool for elucidating number of protons/ hydrogen in a compound or fluid containing biomolecules.

The study has been supported by our observations measuring 99 metabolites by proton NMR spectroscopy of saliva which has shown a clear distinction between control and diseased cases of periodontitis where we have observed an elevation in the level of some major and new metabolites which may serve as early biomarker in detection of diseased state. After identifying specific \& selective culprit biomarker biomolecule amongst large number of metabolites by NMR, the specific molecule can be subjected to be evaluated by developing quick, easy and simpler biochemical tests.

Saliva samples are readily available, its collection is also non invasive and it could be developed as a diagnostic tool to identify various types of diseases in clinical practice by metabolite profiling in such diseases.

\section{P184}

\section{Menopause, (Estrogen Level) Associated With Cardiovascular Disease In Women}

Saiyad Shakiru Islam, Roshan Alam, Dhananjay

Tiwari, Priyanka Thapa

Department of Biochemistry, IIMS\&R, Integral

University, Lucknow

$\mathrm{C}$ ardiovascular disease (CVD) is the leading cause of death in women, and knowledge of the clinical consequences of atherosclerosis and CVD in women has grown tremendously over the past 20 years. Research efforts have increased and many reports on various aspects of ischaemic heart disease (IHD) in women have been published highlighting sex differences in pathophysiology, presentation, and treatment of IHD . Women largely share similar (CVF) cardiovascular risk factors for IHD with men; however, women with suspected or confirmed IHD have less coronary atherosclerosis than men, even though they are older and have more cardiovascular risk factors than men.In young women who have undergone early or surgical menopause, who do not take estrogen, their risk for heart disease is also higher. This is because during menstrual cycle the estrogen level maintain the integrity of the pericardium. Hence after menopause the estrogen level falls down and thereafter integrity of the pericardium is no longer maintained by the estrogen. Thereafter, the risk of CVD increases after menopause. And this is how till menopause women are not that susceptible to CVD, but after menopause share the same risk factors as that for men.

KEYWORDS: Estrogen, Cardiovascular Disease, Menopause.

\section{P185}

\section{Microvascular Complications And Vitamin-D Deficiency In Type 2 Diabetes}

Mohammad Khalid, Roshan Alam, Dhananjay

Tiwari, Priyanka Thapa 
Department of Biochemistry, IIMS\&R, Integral

University, Lucknow

$\mathrm{D}$ iabetes is accompanied by many short terms and long terms complications. Vitamin D deficiency is a global health problem which has been shown to affect both insulin secretion and action. Epidemological evidence also suggests a potential association with vitamin D insufficiency and metabolic dearrangements including diabetes.

Growing evidences suggests that metabolic syndrome causes microvascular complications in the subjects with diabetes. Researches showed that improvement of vitamin D status of subjects with type 2 diabetes leads to significant amelioration of glycemic status. Researches also showed that there exists the inverse relationship between body fat mass and vitamin $\mathrm{D}$ status.

The vitamin D plays role in the development of both metabolic syndrome and cardiovascular disease in nondiabetic subjects. The hypothesis was made that vitamin D status could be determinant of such late diabetic complications mostly by affecting the risk of metabolic syndrome in the individuals with type 2 diabetes.

Vitamin D deficiency remains the most worldwide underdiagnosed and undertreated nutritional deficiency. Deleterious and long term skeletal effects of vitamin D deficiency are proposed by numerous biochemical and epidemiological study to be associated with many chronic diseases, as cardiovascular diseases,diabetes mellitus.

KEYWORDS: Vitamin D, cardiovascular diseases, type 2 diabetes mellitus.

\section{P186}

\section{Clonal Origin of Bladder Cancer}

Mohammad Zaid Kidwai, Roshan Alam, Dhananjay Tiwari , Priyanka Thapa

\section{Department of Biochemistry, IIMS\&R, Integral}

University, Lucknow

$\mathrm{P}$ atients with cancer of the urinary bladder often present with multiple tumors, appearing at different times and at different sites in the bladder .
Researches shows that a number of tumors of bladder can arise from single transformed cell . Every tumor in a given patient has inactivation of same $\mathrm{X}$-chromosome , suggesting that all the tumors arose from the same progenital cell . By contrast, in patients who inherit a predisposition to tumors of bowel and parathyroid, the examination of multiple tumors reveals according to the researches variations in the pattern of $\mathrm{X}$-chromosome inactivation. Independent somatic alterations are apparently responsible for the initiation of neoplasia in these tumor types .

Monoclonality is a fundamental characteristic of neoplasia. One transformed cell gives rise to daughter cells. The accumulation of further genetic changes in subsequent daughter cells, each providing an additional growth advantage, has been well documented in each human cancer . This concept of clonal origin and expansion poses a difficulty for the understanding of neoplasia in organs where several metachronous tumors occurs .In patients with bladder cancer, for example , several tumor scattered over the bladder epithelium are often discovered at cytoscopy .

However, only one transforming event occurs and the progeny of a single transformed cell spread through the bladder,giving rise to genetically related tumors.

KEYWORDS : Multiple tumors, neoplasia , Xchromosome, metachronous tumors .

\section{P187}

\section{Evaluation of Serum Total And Ionized Calcium In CKD Patients}

Mayank Kumar Singh, Arya Desh Deepak, Monica Kakkar, Shahbaj Ahmad

Swami Rama Himalayan University (SRHU), Dehradun

INTRODUCTION:Chronic kidney disease (CKD) is emerging to be an important chronic disease globally, mostly due to increase in the incidence of Diabetes Mellitus (DM) and hypertension. Abnormalities in calcium, phosphorus,calcitriol and parathyroid hormone $(\mathrm{PTH})$ are associated with $\mathrm{CKD}$. Patients of 
CKD have much higher chances of vascular and soft tissue calcification if calcium is over-supplemented.

AIM: The aim of this study was to evaluate serum ionized and total calcium in CKD patients and to predict if ionized calcium estimation is a better predictor of calcium levels in the serum.

MATERIALS AND METHODS: For this study, we evaluated 60 newly diagnosed patients of CKD who were not on any calcium supplement. Levels of creatinine, albumin, phosphorus, calcitriol, PTH, total calcium and ionized calcium were analyzed.Statistical analysis was done between ionized calcium with various parameters using Pearson two tailed correlation coefficient for association.

RESULT:We found strong association of ionized calcium with creatinine, total calcium and phosphorus while no significant correlation was observed with albumin, albumin corrected calcium, PTH and calcitriol levels. Ionized calcium proved to be a better predictor of serum calcium level than total calcium level and albumin corrected calcium.

CONCLUSION: Ionized calcium is physiological/ functional component of serum calcium. We propose that ionized calcium should be estimated routinely instead of total calcium as it will help in proper supplementation of calcium and prevent metastatic calcification of various organs which occurs due to over-supplementation of calcium based on hypocalcemia predicted by serum total calcium level and albumin corrected calcium estimation.

\section{P188}

\section{Role of Isolated Natural Plant Products In Treatment of Microbial Infections}

$\underline{\text { Sabiha Kazmi, }}$ *Murtaza Abid, S. Nazeer Haider Zaidi, S.H.A. Kazmi, S. Rais Haider

Department of Botany, Shia P.G. College, Lucknow, U.P., India *Department of Biochemistry, K.G.M.U., Lucknow, U.P., India

INTRODUCTION:The use of and search for drugs and dietary supplements derived from plants have accelerated in recent years. Ethnopharmacologists, botanists, microbiologists, and natural-products chemists are combing the Earth for phytochemicals and "leads" which could be developed for treatment of infectious diseases. While 25 to $50 \%$ of current pharmaceuticals are derived from plants, none are used as antimicrobials. Traditional healers have long used plants to prevent or cure infectious conditions; Western medicine is trying to duplicate their successes.

MATERIAL AND METHODS:The natural products in pure form were isolated using organic solvents, column chromatographics on polyamide, amberlite, DEAEcellulose, Cm-Celulose and Sepadex-gel filteration, further identification was carried out by HPLC, SDSpolyacrylamide gel electrophoresis and UVspectrophotometry on spectronic-2000 etc.

RESULTS: Plants are rich in a wide variety of secondary metabolites, such as proteins, glycoproteins, tannins, terpenoids, alkaloids, and flavonoids, which have been found in vitro to have antimicrobial properties. This review attempts to summarize the current status of botanical screening efforts, as well as in vivo studies of their effectiveness and toxicity. The structure and antimicrobial properties of phytochemicals are also addressed. Since many of these compounds are currently available as unregulated botanical preparations and their use by the public is increasing rapidly, clinicians need to consider the consequences of patients self-medicating with these preparations.

KEYWORDS: Natural Plant Products, Microbial Infections, Microbiologists, Antimicrobial Agents and Ethnopharmacologists.

\section{P189}

\section{Nigella Sativa (Black Seed): "Seed of Blessings"}

Syed Uzma Jalil ${ }^{1}$, Mohammad Israil Ansari*2 ${ }^{* 2}$,

S. Arshad Hasan Razvi ${ }^{3}$,

Amity Institute of Biotechnology, Amity University Uttar Pradesh, Lucknow Campus, Lucknow-226 028, Indial. Department of Botany, University of Lucknow, Lucknow-226 007, India2. 


\section{Department of Botany, Shia PG College, Lucknow}

$\mathrm{T}$ The medicinal plant Nigella sativaL. is well known for its wide spectrum of therapeutic potential throughout the world. It is very popular from ancient times by using in various traditional systems of medicine like Unani and Tibb, and Ayurveda. Nigellasativa seed and its oil are known to contain many active components to include thymoquinone (30\%-48\%), thymohydroquinone, dithymoquinone, p-cymene (7\%-15\%), carvacrol (6\%-12\%), 4-terpineol (2\%-7\%), t-anethol $(1 \%-4 \%)$, sesquiterpenelongifolene (1\%-8\%) ?-pinene and thymol etc. Seeds contain two different types of alkaloids; i.e. isoquinoline alkaloids e.g. nigellicimine and nigellicimine-N-oxide, and pyrazol alkaloids or indazole ring bearing alkaloids which include nigellidine and nigellicine. Moreover, Nigellasativa seeds also contain alpha-hederin, a water-soluble pentacyclictriterpene, and saponin. Bioactive components are reported to possess very useful pharmacological effects to include analgesic (painkilling), anti-bacterial, anticonvulsant, anti-inflammatory, antidepressant, antianxiety, antimalarial, anti-ulcer,anticancer,anti-cholinergic, anti-fungal, anti-hypertensive, antioxidant, antispasmodic, antiviral, bronchodilator, gluconeogenesis inhibitor (anti-diabetic), antipyretic, antipakinsonism, hepatoprotective (liver protecting), hypotensive, insulin sensitizing, interferon inducer, leukotriene antagonist, renoprotective (kidney protecting), tumor necrosis factor alpha inhibitor, and neuroprotective. Most of the therapeutic properties of this plant are due to the presence of thymoquinone, which is a major active chemical component of the essential oil. Because of various pharmacological activities of Nigella sativaseeds it is considered as one of the greatest forms of healing medicine and it is also believed that the Islamic prophet Mohammed stated that it is "a remedy for all diseases except death."

\section{P190}

Evaluation of Different Formula To Estimate LDL-C In Nepalese Populations

P. Padmavathi*, V.Sathiyapriya, G.Brindha, Pregna B Dolia, Samiya Begam
Department of Biochemistry, ACS medical college and hospital, Chennai, India

INTRODUCTION: According to NCEP ATP III guideline the primary target of therapy in cardiovascular disease is based on Low Density Lipoprotein-cholesterol (LDL-c) concentration. Estimating LDL-c by direct homogenous assay is expensive especially in developing countries. Hence in clinical practice most of the clinical laboratory uses calculation method for estimating LDLc concentration. There are different formulas been used to calculate LDL-c concentration,the most frequently used is Friedewald formula.In recent studies have shown that among them novel 180-c method showed the best agreement with direct LDL-c method when compared with other formulasin Asian population. The aim of the study was to (i) Evaluate different formulas for estimation of LDL-c in Nepalese population. (ii)And to identify which formula shows best agreement with novel 180-c method.(iii) And to determine whether it is really in the need to substitute the Friedewald formula.

MATERIALS AND METHODS: This prospective study conducted atUniversal College of Medical Sciences and Teaching Hospital (UCMSTH), Bhairahawa, Nepal.We measured Lipid profile $(n=1000)$ of In and Outpatients samples which were sent to the clinical biochemistry laboratory,from January to December 2013. Total sevenformulas were used to estimate LDL-c mg/ dl. Those are novel 180-cell method and equations suggested by Friedewald et al, Hattori et al, Anandaraja et al, , Cordova et al, Ahmadi et al, \& Rao et al.Twostatistical concepts were utilised to compare the accuracy of different equations to estimate LDL-c by using 180cell method as reference value. The intra class correlation coefficient ICC was calculated to compare the degrees of agreement between the formulas.Good agreement ICC $>0.75$, moderate agreement ICC $<0.5$ or $<0.75$. Calculated weighted kappa $(\mathrm{k})$ index to distinguish the most accurate equation to estimate LDL-c according to NCEP ATPIII classification guideline.

RESULTS: The intraclass correlation coefficient for Friedewald equations (ICC: 0.991), Hattori et al.,(ICC: 0.969), Anandaraja et al.,(ICC:0.979), Cordova et al.,(ICC:0.965), Ahmadi et al.,(ICC:0.623), Rao et al., (ICC:0.989) $(\mathrm{p}<0.001)$. Among these Friedewalde- 
quationshowed the highest agreement in terms of ICC and also highest weighted kappa index (k index: 97.49) which indicating the best agreement with novel 180 cell measurement.

DISCUSSION/CONCLUSION:Studies have highlighted Novel 180 cell method with high accuracy to use in clinical practice which uses adjustable factor for TG:VLDL-c ratio using stratification approach according to TG and non HDL-c levels(TC-HDL-c = non HDL-c) But in routine clinical practice calculating LDL-c by novel 180 cell method for each patient where there is manual entry of reports seems to be burden to the laboratory technologist.We used 180-c method as a reference value for LDL-c estimation with previously reportedformulas as the first external validation in Nepalese population. The most commonly used Friedwald equationappeared to be more accurate than the newly derived formulas. This suggests that we still can use Friedwald formula in Nepalese population.

KEYWORDS: LDL-c, Evaluation, Different formulas

\section{P191}

Synergistic Involvement of Il-17 And Haematological Indices As Surrogate Marker For Monitoring of Azathioprine Therapy In Inflammatory Bowel Disease Patients.

\author{
U.Tiwari , Priyatma , M. Saroj, S. Prakash \\ Department of Laboratory Medicine AIIMS New Delhi \\ Email:Umesh_tiwari1987@yahoo.co.in
}

BACKGROUND: Anaemia and leucopoenia are frequent dose related complications by azathioprine therapy in patient with IBD. Interleukin (IL)-17 is known proinflammatory cytokine with robust effect on many blood cells that enhances the MCV and hematocrit in patient with IBD after the Azathioprine therapy.

OBJECTIVES: The aim of study was to evaluate the haematological indices and in patients with IBD with and without azathioprine drug to assess the potential impact of inflammatory cytokines.

MATERIALS AND METHODS: Blood samples were collected from 30 patients with IBD with azathioprine therapy and 15 patients without azathioprine based on simple sampling. The mean age $40.86 \pm 10.25$, male/ female ratio $(1.12 ; 1)$ were matched with the subjects and all subjects were recruited from the department of Gastroenterology and Human Nutrition, AIIMS, New Delhi. Clinical history and colonoscopy proven IBD cases were taken with and without Azathioprine therapy. The patient were categorised on the basis of haemoglobin levels in terms of healthy, mild and moderate anemia as per WHO cut-off. The levels of TNF- , IL-17, vitamin B12, Folate, Ferritin and haematological indices were done in all subjects.

RESULTS: The mean of IL-17 and TNF- was significantly higher in patients with IBD compared with disease control $(0.33 \pm 0.06$ vs. $0.42 \pm 0.14$, $)(\mathrm{P}=0.02)$, but their vitamin $\mathrm{B} 12$ levels was lower $(12.53 \pm 8.41$ vs. $54.94 \pm 17.95$, $)(P=0.05)$. However, the Ferritin levels was similar in both groups $(5.34 \pm 2.40$ vs. $8.07 \pm 3.28$, $(\mathrm{P}>0.05)$ and there was not any significant difference between the noted hematologic indices among patients with different stages of anemia except MCV and hematocrit of patients whose on Azathioprine therapy.

CONCLUSIONS: IL-17 as a proinflammatory cytokine enhances macrocytosis in patient with IBD after the Azathioprine therapy. Decreased Vitamin B12 level and Macrocytosis in IBD with azathioprine drug indicating cytokine related damage in $\mathrm{RBC}$ and drug related haematological changes cannot be ruled out. Thus IL-17 synergistically enhances the haematological indices in IBD patients and could a surrogate marker for monitoring of patients after the Azathioprine therapy

\section{P192}

An Assessment of Outbreak of Acute HBV with High Fatality Due To Unsafe Injection Practices, Associated With Hbv Mutants In Sabrakantha Gujarat, India

\section{Priyatma, Shyam Prakash}

Department of Laboratory Medicine, AIIMS, New Delhi

INTRODUCTION: The majority of outbreaks have 
been associated with unsafe injection practices, which include reuse of contaminated syringes, needles, acupuncture needle, finger stick devices and multidose vials. The HBV infection has diverse clinical manifestations ranging from subclinical infection and acute viral hepatitis (AVH) to acute liver failure (ALF), chronic hepatitis, cirrhosis and hepato-cellular carcinoma.

OBJECTIVE: To assess the unsafe practices associated with HBV mutant and their quantification in outbreak fatality among acute HBV infection cases.

MATERIAL AND METHODS : The blood samples were collected from suspected cases of acute viral hepatitis and acute liver failure. The serology of hepatitis panel was tested and HBV DNA Quantitation was done by QPCR. The genotyping, basal core promoter (BCP)/ Precore (PC) mutant's was done by RFLP and genotyping, $\mathrm{BCP} / \mathrm{PC}$ mutant's pattern was identified by the restriction patterns of DNA fragments.

RESULT: HBV DNA viral load was higher in ALF $(6.6 \pm 2.2 \log$ copies $/ \mathrm{ml})$ cases than AVH $(5.1 \pm 1.8 \log$ copies $/ \mathrm{ml})$ cases $(\mathrm{p}=0.9)$. Overall genotype $\mathrm{D}$ was the commonest genotype $(n=89,90.2 \%)$ and there was no significant difference between AVH $(n=70,88.6 \%)$ and $\operatorname{ALF}(n=19,100 \%)$ regarding the genotype distribution. The commonest virus was of wild type (70.8\%) followed by virus with dual basal-core mutations (BCP) and precore mutations (PC) (20.4\%). Notably, all cases with ALF had infection with mutant virus: either BCP mutations combined with PC mutations $(89.4 \%)$ or PC mutations alone $(10.6 \%)$ whereas majority of AVH cases had infection with wild type of virus $(n=69,87.3 \%)$ $(\mathrm{p}=<0.0001)$.

CONCLUSION : The HBV infection was raised due to unsafe injection practices by physicians in Gujarat. The fatal cases had mutation in basal core and pre-core region of HBV genome. HBV DNA viral load was also higher in them.

\section{P193}

Development of An Inexpensive Method For Detection of HCV Rna By Loop Mediated Isothermal Amplification (Lamp)
Manish Saroj, Priyatma, Shyam Prakash

Department of Laboratory Medicine, All India

Institute of Medical Science, New Delhi-110029

INTRODUCTION: Since HCV RNA is the major cause of liver disease so both diagnosis and therapeutics need to assess the HCV RNA. QPCR is the only available method for quantitation of HCV RNA and required expertise, sophisticated equipments and infrastructure. There is a need to develop an alternate method which is inexpensive, rapid and fast detection of HCV RNA.

OBJECTIVE: To develop quantification method for detection of HCV RNA by loop mediated isothermal amplification in HCV infected patients.

MATERIAL \& METHODS: 913 samples were collected from Liver clinic and blood collection centre, of AIIMS, New Delhi. Only 86 samples were positive for HCV antibody. The primer of LAMP was designed by LAMP designer software. $25 \mu$ reaction mixture was done at $60^{\circ} \mathrm{Cincluding}$ forward inner primer and backward inner primer, forward and backward primers, Betaine, Tris - $\mathrm{HCl}, \mathrm{MgSo} 4$ and Bst DNA polymerase for amplification of target molecules. The reaction mixture was quantified at $645 \mathrm{~nm}$ using HNB.

RESULT: The sensitivity of LAMP method was found up to $90 \%$, specificity $100 \%$, positive predictive value $96.1 \%$ and negative predictive was $100 \%$ respectively. The amplification range of LAMP method was found $20 \mathrm{U}$ to $107 \mathrm{U} / \mathrm{ml}$.

CONCLUSION: LAMP is inexpensive, sensitive and alternate technique for HCV RNA levels. Thus, thistechnology can be transferred to other centre where the expertise and necessary infrastructure are unavailable.

\section{P194}

Effects of Short-Term And Long-Term Use of Lithium on Thyroid Function Tests In Patients With Bipolar Disorder

\section{Rajesh Kumar Gupta $^{1 *}$}

Email:hirajesh_iom@yahoo.com 
BACKGROUND: Lithium has been used by most psychiatrists as a long term effective therapy for the treatment of bipolar disorder as well as reducing the risk of suicide and short term mortality in patients. However, some studies have also reported varying degrees of thyroid abnormalities in lithium treated patients, but it is unclear whether there is significant association with duration of therapy. We aimed to determine the effect of long term use of lithium on thyroid function tests and possible prevalence of hypothyroidism in women and men with bipolar disorder.

METHODS: This cross-sectional study was conducted in 75 bipolar disorder patients ( 24 males, 51 females) treated with lithium and equal number of controls. Diagnosis of bipolar disorder was made by psychiatrist according to ICD-10-DCR guidelines and DSM-IV criteria. Serum fT3, fT4 and TSH were measured by enhanced chemiluminescence immunoassay. Statistical analysis was performed using SPSS 20.0 version.

RESULTS: The prevalence of primary hypothyroidism and subclinical hypothyroidism were found significantly increased in lithium treated group (12\% and $17 \%$ respectively) which were further increased with duration of treatment, showing no significant difference of subclinical hypothyroidism in sex $(17.6 \%$ female vs. $16.6 \%$ male), but primary hypothyroidism cases were only observed in female (17.6\%) not in male. The mean fT3 level of lithium treated group was decreased as compared to control group $(5.61 \pm 1.35$ vs. $6.02 \pm 1.1, \mathrm{p}=$ $0.051)$, also showing decreased level of fT4 (17.57 \pm 6.35 vs. $19.71 \pm 4.56, \mathrm{p}=0.019)$. But mean TSH level was found significantly $(\mathrm{P}<0.001)$ higher in lithium treated group than that of control $(9.67 \pm 12.47$ vs. $3.41 \pm 3.69)$.

CONCLUSION: Our findings indicate that use of lithium therapy is associated with higher degree of primary hypothyroidism and subclinical hypothyroidism which is being increased with duration of lithium therapy. These results also show slightly higher prevalence of hypothyroidism in female but statistically not significant.

KEYWORDS: Bipolar Disorder, Lithium, Hypothyroidism

\section{P195}

\section{Role of Oxidative Stress After Routine Iron Supplementation In Normal And Anemic Women During Pregnancy: Central Indian Scenario}

\author{
$\underline{\text { Ajai Kumar }}^{1}$, Kalbe Jawad ${ }^{2}$, Abbas Ali Mahdi ${ }^{3}$ \\ 1. Department of Biochemistry, King George \\ Medical College, Lucknow (U.P)-India \\ 2. Department of Biochemistry, Uttar Pradesh \\ University of Medical Science, Saifai, Etawah(U.P)- \\ India \\ 3. Era University, Sarfazganj, Hardoi road,Lucknow.
}

INTRODUCTION: Iron deficiency anemia (IDA) is one of the most common nutritional disorders worldwide, affecting people of all ages in developed and developing countries. Oxidative stress causes dimunition of various antioxidant enzymes.

OBJECTIVE: The objective of the study was to detect impact of iron supplementation in anaemic \& nonanaemic pregnant subjects on oxidative stress.

MATERIAL AND METHODS: Patients are divided into two groups, Control groups (60 non-anemic pregnant women) \& Study groups (60 anemic pregnant women). The $5 \mathrm{ml}$ blood sample was collected from different groups of subjects.Biochemical markers estimation was carried out by standard protocols.

RESULTS: In controls groups, a singnificant decrease were found in catalase, superoxide dismutase, glutathione peroxidase and glutathione reductase while level of lipid peroxidase was found to have increased significantly after iron therapy. In study group, and increment in all markers except lipid peroxidase was seen and the level of lipid peroxidase was decreased following iron supplementation which was statistically significant $(\mathrm{p}<0.001)$.

CONCLUSION: It may be concluded that iron deficiency anaemia is associated with free radical generation and peroxidation of vital body molecules which implies increased risk for pregnant women

KEYWORDS: Anemia, Oxidative stress, Iron supplementation, pregnancy. 


\section{P196}

\section{A Comparative Study of Toxic Heavy Metals In Seminal Plasma and Whole Blood In Infertile Men}

Vimmi Kishore $^{1}$, Kalbe Jawad ${ }^{1}$, Tariq Mahmood ${ }^{1}$, Jamal Akhtar Ansari², Mohammad Kaleem Ahmad², Abbas Ali Mahdi ${ }^{2}$, Suruchi Mathur ${ }^{1}$

1. Department of Biochemistry, Uttar Pradesh University of Medical Sciences, Saifai, Etawah, U.P.

2. Department of Biochemistry, King George's Medical University, Lucknow, U.P.

INTRODUCTION: Male infertilityis a significant social and medical health issue with a high prevalence worldwide. Heavy metals such as lead $(\mathrm{Pb})$, Cadmium (Cd) and Arsenic (As) play important role in causing infertility in men. The aim of the current study was to compare the level of 3 toxic heavy metals lead $(\mathrm{Pb})$, Cadmium (Cd) and Arsenic (As) in seminal plasma and whole blood of male partners of infertile couples.

METHODS:A total of 50 samples of blood and semen were collected from 25 men attending the infertility clinic of UPUMS Saifai out of which 10 were normospermic and 15 were oligospermic. The concentrations of $\mathrm{Pb}, \mathrm{Cd}$ and As were determined by Inductively coupled plasma optical emission spectrometry.

RESULTS: When the $\mathrm{Pb}$ and $\mathrm{Cd}$ level were compared in the seminal plasma of the study subject suffering from oligospermia as against the normal control, the levels were found significantly increased. Moreover, in case of blood samples the level of $\mathrm{Pb}$ and $\mathrm{Cd}$ were also found significantly increased. However, the concentration of arsenic was found absent in both cases.

CONCLUSION: The result of present study suggests that $\mathrm{Pb}$ and $\mathrm{Cd}$ may be related to a moderate alteration of seminal parameters which leads to male infertility.

KEYWORDS: Male infertility, Pd, Cd, As,ICP-OES.

\section{P197}

\section{Effect of Manganese Nanoparticles On Neuroblastoma Cells}

Murtaza Abid, *Arshiya Parveen, **Syed Husain Mustafa Rizvi and Abbas Ali Mahdi

Department of Biochemistry, King George's Medical University, Lucknow, U.P.

*Department of Molecular Medicine and

Biotechnology, Sanjay Gandhi Postgraduate Institute of Medical Sciences (SGPGI), Lucknow, India

**College of Nanoscale Science and Engineering, State University of New York, Albany, NY, USA

INTRODUCTION: Nanoparticles (NPs) are particles with a diameter typically smaller than $100 \mathrm{~nm}$ that differ from their bulk form (diameter $<1000 \mathrm{~nm}$ ) and usually have unique properties. Although nanoparticles have some benefits and used in pharmaceutical filed to develop substances for drug delivery (De Jong and Borm, 2008) and for development of anticancer, antibacterial and antiviral drugs, they also have severe negative effect on biological system such as inflammation and brain cancer.

Man made nanoparticles and materials are being rapidly produced in large quantities throughout the world. With the increased probability of exposure from large yield industry production, the issue of toxicity has led to new investigations of dosing and exposure to elemental metals. Although a wide and growing number of applications for nano materials exist, there is a serious lack of information concerning the human health and environmental implications of these manufactured nano materials.

Materials and Methods are carried out under following subheads: Synthesis of Manganese Oxide (MnO2) nanoparticles, Transmission electron microscopy of synthesized Manganese nanoparticles NPs, Hydrodynamic size of MNNPs (DLS), XRD analysis of MnNPS, FTIR analysis of MNNPS, Cell Culture, MTT Assay, LDH Assay, Determination of ROS, Statistical Analysis.

RESULTS: Studies on human have indicated that elevated levels of Mn may put humans at risk of 
Parkinsonism (Olanow, 2004). This suggests that there are may be significant pathological consequences and risks to the central nervous system (CNS) when manufacturing nano scale metals. Larger amount of manganese metal or powder applications are typically found in industries involving steel and non steel alloy production, battery manufacture, colorants, pigments, ferrites, welding fluxes, fuel, additives, catalyst, and metal coatings. Due to the recent development of nanomaterials, macro sized manganese particles are likely to be replaced by manganese nano particles. The applications of Mn nano materials are currently being pursued for catalysis and battery technology (Han et al., 2005). So occupational and consumers exposure to MnNPs are likely to vary accordingly. Risk of occupational and environmental exposure to MnNPs has obviously increased and exposure of MnNPs and its associated risk to humans at occupational as well as domestic exposure should be deeply investigated.

The results presented here demonstrate that MnNPs adversely affect SH-SY5Y human neuroblastoma cell and induce significant cytotoxicity and oxidative stress with a clear dose dependent manner. This findings is of special interest as the particular role of neurons in several neurodegenerative diseases has been intensely discussed, and it seems likely that this cell type is being initially during pathogens. The high vulnerability of SH-SY5Y human neuroblastoma cell toward MnNPs exposure, as demonstrated here.

KEYWORDS: Manganese Oxide Nanoparticles, Neuroblastoma Cells, Cytotoxicity and Oxidative Stress.

\section{P198}

\section{To Estimate Prevalence of Mineral Bone Disease In Chronic Kidney Disease Patients Using Biochemical Markers}

R Choudhary $^{1}$, D Gupta $^{1}$, A Gupta $^{1}$, A Srivastava $^{1}$, A Harith $^{1}$, S Bansal ${ }^{2}$, B Bansal ${ }^{3}$, A Raizada ${ }^{1}$

1. Department of Biochemistry, Medanta-The Medicity, Gurgaon

2. Department of Nephrology, Medanta-The Medicity, Gurgaon.
3. Department of Endocrinology, Medanta-The Medicity, Gurgaon.

INTRODUCTION: Chronic kidney disease (CKD) is a condition characterized by a gradual loss of renal function over time. Mineral Bone Disease (MBD) is a systemic disorder of mineral and bone metabolism leading to bony abnormalities, cardiovascular and other soft tissue calcifications. There is direct effect of diabetes on bone demineralization and cardiovascular calcification.

AIMS \& OBJECTIVES: To study the prevalence of MBD in CKD patients with the help of biochemical markers i.e. Calcium (Ca), Phosphorus (P), Alkaline Phosphatase (ALP) and intact Parathyroid hormone (iPTH). Also to study the levels of these parameters in diabetic CKD-MBD patients.

MATERIALS \& METHODS : This is a cross sectional observational study, in which 250 patients attending the Nephrology OPD of Medanta-The Medicity, Gurgaon were evaluated for serum levels of $\mathrm{Ca}$, P, ALP using Colorimetric Method and iPTH using Chemiluminescent microparticle immunoassay method on fully automated Vitros 5600 and Architect analyzer.

RESULTS: Prevalence of various biochemical abnormalities in CKD-MBD i.e. hypo-calcemia, hyperphosphatemia, raised ALP and hyper-parathyroidism was $37.2 \%, 49.2 \%, 42.8 \%$ and $83.6 \%$ respectively. In subgroup analysis serum levels of $\mathrm{Ca}(\mathrm{p}<0.04)$ and ALP $(\mathrm{p}<0.03)$ were significantly higher in diabetic as compared to non-diabetic CKD-MBD patients. Although not statistically significant, we found increased levels of $\mathrm{P}$ and $\mathrm{iPTH}$ in the same studied groups.

CONCLUSION: High prevalence of MBD in Indian CKD patients was demonstrated. We also found that there was progression of MBD with advancing stages of CKD. Disordered mineral metabolism begins earlier and was more severe among diabetic CKD patients as compared with non-diabetics.

KEY-WORDS: CKD-MBD, hypo-calcemia, hyperphosphatemia, hyper-parathyroidism. 


\section{P199}

\section{Circulatory Levels of IGF-1 \& Osteocalcin And Their Relationship With Bone Mineral Density Inthe Postmenopausal Women}

Israr Ahmad ${ }^{\mathbf{a}, \mathbf{b}}$, Tabrez Jafar, Mohammad Waseem, c, Farzana Mahdib, Md. Arshad', Siddharth Kumar Dasd, Abbas Ali Mahdi"a

a. Department of Biochemistry, King George's Medical University, Lucknow-226003, India.

b. Department of Biochemistry, Era's Lucknow Medical College \& Hospital, Lucknow-226003, India.

c. Department of Zoology, Lucknow University, Lucknow-226007, India

d. Department of Rheumatology, King George's

Medical University, Lucknow-226003, India

INTRODUCTION:In India osteoporosis is an important health problem due to the higher prevalence of vitamin D deficiency across all ages, low level of awareness and higher risk of complications. This disease is characterized by decreased bone mass, decreased bone strength and higher risk of bone fracture. The aims of the present study to compared the relationship betweenbone mineral density (BMD) with insulin-like growth factor-1(IGF1) and osteocalcin in postmenopausal women with and without osteoporosis.

METHODOLGY: 254 osteoporotic women (age 55.82 \pm 6.91 ) and 254 postmenopausal non-osteoporotic women (age 54.76 \pm 6.26) were included in the study. BMD were assessed by dual energy X-ray absorptiometry (DXA) at the lumbar spine (L1-L4), hip, forearm and femoral neck.Insulin-like growth factor-1(IGF-1) and serum osteocalcin were measured by enzyme-linked immunosorbent assay (ELISA).

RESULT: Bone mineral density was significantly lower inpostmenopausal women with osteoporosis at all skeletal sites. Circulatory levels of IGF-1were significantly decreased in osteoporotic women as compared to those in non-osteoporotic women while osteocalcin levels were significantly increased in osteoporotic women as compared to those in nonosteoporotic women. There was a significantcorrelation between IGF-1 and BMD and a significant negative correlation between osteocalcin with BMD.

CONCLUSION: Measurement of circulatory levels of IGF-1 and osteocalcin with BMD in postmenopausal women may help in the identification of those at risk for developing lowbone mass and osteoporosis.

KEYWORDS: Osteoporosis, Bone Mineral Density,Insulin-like growth factor-1,Dual Energy X-ray Absorptiometry

\section{P200}

\section{Association of Col4a1 (RS 605143 and RS565470) Gene Polymorphism With Coronary Artery Diseases (CAD)}

Apoorv Pankaj ${ }^{1 *}$, Anu Chandra ${ }^{2}$

Department of Biochemistry, Era's Lucknow Medical College, Lucknow, India

BACKGROUND: CAD is one of the biggest cause of death around the world, with the number of people affected continually increasing. Increasing Incidence of CAD is a major concern not only in India but across the world. Genetic Polymorphism of many genes is associated with increased risk of various diseases in a particular ethnic group.

METHODS: This case control study was carried out to investigate the association of COL4A1 gene polymorphism with CAD. The study includes 25 angiographically proven CAD cases and 25 controls. Blood was collected in EDTA tube. Genomic DNA was extracted using phenol-chloroform extraction method (Sambrook J, 1989). Polymerase Chain Reaction(PCR) was done on extracted DNA. PCR product was digested with $5 \mathrm{U}$ of restriction enzyme (Fermentas, UK). Statistical analysis was performed using SPSS 20.0 version.

RESULTS: For SNP-1 rs605143 Frequency of COL4A1 AG, GG, AA genotype in CAD samples were $52 \%, 40 \%$, $8 \%$ and in Control samples were $64 \%, 32 \%, 4 \%$ respectively. Frequencies of COL4A1 A and G alleles were $34 \%$ and $66 \%$ in CAD samples and $36 \%$ and $64 \%$ 
in control Samples. For SNP-2 rs 565470 Frequency of COL4A1 CT, CC, TT genotype in CAD samples were $60 \%, 12 \%, 28 \%$ and in Control samples were 52\%, 16\%, $32 \%$ respectively. Frequencies of COL4A1 C and T alleles were $42 \%$ and $58 \%$ in CAD samples and $42 \%$ and $58 \%$ in control Samples.

CONCLUSION: Polymorphism of COL4A1 gene (rs605143, rs565470) was not associated with the risk of CAD (Statistically). For rs605143, frequency of A allele was significantly higher in Control subjects than in CAD patients which indicates that A allele of rs605143 is a protective factor for CAD while the frequency of $G$ allele was significantly higher in CAD cases than controls suggesting it to be a risk factor for CAD. For rs565470, no significant difference was found between the allelic ratio of the case and the control samples.

KEYWORDS: Coronary Artery Disease, COL4A1, Gene Polymorphism

\section{P201}

Correlation of Carotid Intima Media Thickness And Lp-Pla2 In Patients With Cardiovascular Disease Risk

Brijesh Rathore*, Almas Qureshi, Devendra Kumar and Jalees Fatima

Department of *Biochemistry and Medicine, Era's Lucknow Medical College, Era University, Sarfarajganj, Hardoi Road, Lucknow-226003

INTRODUCTION: Cardiovascular disease (CVD) is a wide array of disorders including diseases of cardiac muscle and vascular system of heart, brain and other vital organs. There are various factors involved for rapid increase in CVD patients. According to WHO, 17.3 million CVD deaths occurred in 2008 and the toll may increase upto 23.3 million by the year 2030. There are two emerging risk markers i.e, Carotid Intima Media Thickness(CIMT) and LpPLA2 for CVD risk patients. Lipoprotein phospholipase A2 (Lp-PLA2) is a member of phospholipase-A2 family, produced by monocytes, macrophages, mast cells etc. Lp-PLA2 activity has been correlated with CVD patients, while Carotid Intima
Media Thickness (CIMT) represents a preclinical atherosclerosis marker. Our study is aimed to compare LpPLA2 and CIMT in CVD risk patients.

OBJECTIVE: To compare CIMT and Lp-PLA2 in patients with risk of cardiovascular disease.

MATERIAL AND METHODS: Study was conducted in the Department of Biochemistry and Medicine, Era's Lucknow Medical College and Hospital, Lucknow. Blood of 140 patients was collected and analyzed for lipid profile, HbA1c and LpPLA2 activity. Ultrasonographic scanning of the carotid arteries was performed.

RESULTS: In our study, the ratio of male to female was 45:25 for cases and 47:23 in control. Significant changes were recorded insystolic and diastolic blood pressure, Fasting blood sugar, Postprandial blood sugar in cases as compared to controls. LpPLA2 activity was found significantly elevated in cases. Univariate analysis revealed CIMT-RT and CIMT-LT non-significant correlation with LpPLA2 activity.

CONCLUSION: Present study indicates that patients with CVD risk reflected the changes in CIMT and LpPLA2 activity. ROC analysis revealed that CIMT can serve as a good diagnostic indicator for future cardiovascular event in risky patients.

\section{P202}

\section{Deciphering The Antibacterial Potency of Different Extracts of Butea Monosperma Flower}

Raza Abbas $^{1}$, Ghizal Fatima ${ }^{2}$,

Anthonet N. Ezejiofor ${ }^{3}$, Farzana Mahdi ${ }^{4}$, Priyanka Shukla

Era's Medical College and Hospital1,2,4,5 Medical School of Nigeria3

OBJECTIVE: To investigate the antibacterial activity, using extraction procedures with solvents like, ethanol, methanol and distilled water to validate medicinal uses of Butea monosperma (B. monosperma) in controlling infections. 
METHODS: The antibacterial activity of flowerextracts (ethanol, methanol and distilled water) was evaluated on the blood agar and Mac-Conkey agar plating method against clinically isolated Gram-positive and negative pathogenic bacteria in vitro.

RESULTS: Pathogenic bacteria used were Citrobacter freundii, Escherichia coli, Klebsiella pneumoniae, Pseudomonas aeruginosa, Salmonella typhi, Shigella sp., Enterococcus sp., Staphylococcus aureus (S. aureus), along with standard bacterial strains. After overnight incubation no visible growth was seen in all the culture plates. These bacteria had been recorded to have significant inhibitions by flower extracts, obtained by extraction procedures with different solvents.

CONCLUSIONS: Flower-extracts with and ethanol had shown significant antibacterial activity against all bacteria. B. monosperma flower-extract could be used in treating infectious diseases, caused by the range of tested bacteria, as complementary and alternate medicine.

KEYWORDS: Butea monosperma, Gram-positive bacteria, Gram-negative bacteria, Antibacterial activity

\section{P203}

\section{Antihyperglyceamic Potential of Aqueous Extract of Different Parts of Butea Monosperma on Alloxan Induced Diabetic Rats: A Comparative Study}

\section{Anthonet Ndidiamaka Ezejiofor ${ }^{1}$, Ghizal Fatima ${ }^{2}$} and Farzana Mahdi ${ }^{2}$

\section{Department of Experimental Pharmacology \&} Toxicology, Faculty of Pharmaceutical Sciences, University of Port Harcourt Port Harcourt, Rivers State, Nigeria.

2. Department of Molecular Medicine, Era Lucknow Medical College \& Hospital, Sarfarajganj, Hardoi Road, Lucknow 226003 (UP), India

$\mathrm{T}$ he anti-hyperglyceamic and anti-oxidant potential of the aqueous extract of various parts of B. monosperma was evaluated in alloxan (ALXN)-induced diabetic male adult rats. Experimental animals were divided into eight groups viz., I, II, III, IV, V, VI, VII, and VIII. Diabetes mellitus (DM) was induced in groups II - VIII rats by a single intraperitoneal injection of alloxan (150 mg/kg body wt). Group I (control mice) received an equal volume of normal saline. Group IIIVIII were further treated with BMF, BMS, BML, BMB and BMR (500 mg/kg body wt, p.o.) representing, flower, seed, leave, stem bark and root of BM respectively for a period of 21 days. Body weight, food and water intake and fasting blood glucose (FBG) levels were measured at periodic intervals during the test period. At the end of treatment period, blood was collected by cardiac puncture under mild ether anesthesia and serum was isolated to analyze serum insulin levels. The homogenates of pancreatic was also analyzed for both enzymatic and nonenzymatic antioxidants, such as superoxide dismutase (SOD), catalase (CAT), glutathione peroxidase (GSH$\mathrm{Px})$, reduced glutathione (GSH), thiobarbituric acid reactive substances (TBARS) and total protein (TP). Alloxan injection resulted in a significantly $(\mathrm{P}<0.05)$ increased concentration of FBG level. The FBG level decreased significantly $(\mathrm{P}<0.05)$ after 21 days treatment of BM parts.

KEY WORDS:Butea monosperma, antihyperglyceamic, anti-oxidant potential

\section{P204}

\section{PD-L1 Expression In Colorectal Adenocarcinoma: An Immuno-Histochemical Study In Tissue Microarray.}

Radhieka Misra ${ }^{1 *}$, Nuzhat Husain ${ }^{2}$, Saumya Shukla²*, Aparna Misra ${ }^{2 *}$

Department of Biochemistry, Era's Lucknow Medical College, Lucknow, Uttar Pradesh.

BACKGROUND:Colorectal cancer (CRC) is the third most common cancer inmen and the second most common cancer in women, accountingfor about 700,000 deaths per year.Colorectal carcinogenesis is driven by sequential genetic and epigenetic alterations of tumour cells and is also influenced by tumour-host interactions.It has been shown already that T-cell-mediated immunity 
plays a major role in repressing tumour progression. Programmed cell death ligand 1 (PD-L1) is a key immunoregulatory molecule that, upon interacting with its receptor, $\mathrm{PD}-1$, suppresses the CD8 cytotoxic immune response in both physiologic and pathologic pathways. 1 PD-L1 and PD-1 have come under close scrutiny in recent years as potential targets for cancer therapy. The prognostic value of PD-L1 expression in different cancers is still controversial. Thus, in this study we aimed to assess the expression of PD-L1 gene in tumour cells in colorectal cancer cases.

METHODS:The study included 25 cases of CRC.Adequate clinical details were documented including the age, sex, presenting clinical features, type of resection and pertinent radiological findings.

Evaluation and assessment of PD-L1 IHC using tissue microarray, staining in cytoplasm and/or membranous staining in both the tumour cells as well as the normal intestinal tissue was done. The data was collected in an excel format and PDL-1 expression was correlated with the different parameters- age, sex, site, size, tumour grade, pathological $\mathrm{T}$ stage, pathological $\mathrm{N}$ stage, lymphovascular invasion, perineural invasion, number of nodes absent and present and number of positive lymphnodes was analyzed statistically using Fisher's Exact Test.

RESULTS:In this study only 3 cases (9\%) had PDL-1 positive in the tumour cells. The different parameters displayed no significant statistical result as the $\mathrm{p}$ value was $>0.05$.

CONCLUSION:PD-L1 cannot be used as a new immune marker in colorectal cancer that has both predictive and prognostic impact since the research conducted showed no significant statistical observation and result.

\section{P205}

Oxidative Stress Indices During Pregnancy-A Comparative Study

\section{Soobia Karim Ansari}

Dept of Biochemistry, GMC Jalaun,Orai
BACKGROUND-Pregnancy is a physiological state accompanied by a high energy demand and increased oxygen requirement.It is a stressful condition in which many physiological and metabolic functions are altered to a considerable extent.Because of the mitochondria rich placenta,pregnancy favours oxidative stress.

AIM-The present study was undertaken to compare the markers of oxidative stress i.e.lipid peroxidation and antioxidant enzymes such as Superoxide dismutase (SOD) and catalase (CAT) and Total antioxidant capacity (TAC) in Healthy non pregnant and Normal pregnant women during pregnancy(I,IIand III trimester).

METHODS-The study was conducted on 90 normal pregnant women and 50 healthy non pregnant women in SRN and Kamla Nehru Memorial Hospital ,associated with MLN Medical college,Allahabad.Estimation of Lipid peroxidation i.e.Malondialdehyde by reaction with thiobarbituric acid,SOD by Marklund and Marklund,CAT by method of Beutler and Total antioxidant capacity (TAC) by FRAP assay.

RESULT-Significant increase in the levels of Lipid peroxidation and significant decrease in the levels of SOD and CAT were found in I,II and III trimester of healthy pregnant women, when compared with Healthy non pregnant women.TAC levels was also increased in the healthy pregnant womens.

CONCLUSION-Present study observed a progressive increase in oxidative stress as pregnancy advances ,being maximum in the third trimester of pregnancy.Activities of SOD and CAT decreased gradually with the progression of pregnancy.Total antioxidant capacity also increased significantly but was not proportionate to the increase in MDA levels thereby indicating the stressed state of the pregnant women.

KEY WORDS: Lipid peroxidation, Superoxide dismutase, Catalase, Total antioxidant capacity .

\section{P206}

Dietary Manipulations of Folate And Vitamin B12 Levels During Pregnancy Resulted In Epigenetic Alteration of Imprinting Genes In Maternal And Fetal Tissues 
Aatish Mahajan, Divika Sapehia, Jyotdeep Kaur

Department of Biochemistry, Postgraduate Institute of Medical Education and Research, Chandigarh, India.

BACKGROUND:Thealtered ratio of folic acid and vitamin B12 in maternal dietduring pregnancy influences the DNA methylation patterns of offspring.

OBJECTIVE: The present study was planned to study the effect of dietary folate and B12 during pregnancy on expression of imprinting genes in maternal and fetal tissues.

MATERIALAND METHODS:Four week old C57BL/ 6 female mice (F0 generation) were randomized into nine groups NBNF, NBFO, NBFD, BDNF, BDFD, BDFO, $\mathrm{BONF}, \mathrm{BOFO}$ and BOFDand were fed diet for six to eight weeks while malemice were fed normal diet.On day 20 of gestation, half of the pregnant mice were sacrificed, fetal and maternal tissues were isolated whereas rest of the pregnant mice werecontinued togivebirthtothelitter(F1 generation). Both males and females fetuses in F1 generation were given same diet as fed to their mothers for 6 weeks. Mating was performed within same groups and fetuses born were denoted as F2 generation. Folate, homocysteine and vitamin B12 levels were analysed in serum. The mRNA expression of some imprinting genes Igf2, KCNQIOT1, H19, Igf2Rand folate transporters RFC, PCFT, FR? were analyzed by Real-Time PCR. Global DNA methylation levels were analyzed by using ELISA kit.

RESULTS:Folate and Vitamin B12 levels were significantly decreased and homocysteine levelsincreased in folate and B12 deficient groups. In B12 deficiency,abnormal growth was observed in fetuses born in F2 generation. In maternal and fetal tissues overexpression of H19, a negative regulator of growth,was observed in case of folic acid and B12 deficiency conditions in maternal as well as fetal tissuesand loss of expression of IGF2, (a growth promoting gene)was observed under folate deficient conditions and in all groups of B12 deficiency.Igf2R was down-regulated in both maternal as well as fetal tissues in case of deficient conditions of folate and/or B12. PCFT, RFC and FOLR1 expression were increased in both deficient groups in maternal and fetal tissues.In maternal tissues, a decreased global methylation was observed in brain, liver \& kidney whereas in placenta it was increased. Fetal tissues behaved similar to placenta in BDNF, BDFD \& BOFD groups

CONCLUSION:Maternal folate and B12 levels during pregnancy influence fetal growth by altering the expression of imprinting genes.

ABBREVIATIONS: NB: Normal B12, NF: Normal folate, FD: Folate deficient, FO: Folate over supplementation, BD: B12 deficient, BO: B12 over supplementation

\section{P207}

\section{Advanced Trends In Blood Vessel Cardiovascular} Diseases And Their Remedies

\author{
K. P. Mishra*,Ashish Shukla**, Vivek Srivastava*** \\ *Professor, Department of Biochemistry, L.L.R.M \\ Medical College, Meerut \\ **Assistant Professor, Department of Biotechnology, \\ RBSETC, Bichpuri, Agra \\ ***Associate Professor, Department of Biotechnology, \\ RBSETC, Bichpuri, Agra
}

$\mathrm{W}$ hen the blood pressure in the arteries is persistently elevated at a constant rate then the condition of Hypertension arises very commonly nowadays. This is also called as called as high blood pressure or high vital sign or blood vessel cardiovascular disease. Two major categories of cardiovascular diseases commonly occur, primary disease and the secondary disease. Approximately $90-95 \%$ of cases are of primary type and the foremost reason behind this is the ruinous condition they follow, includes excess consumption of alcohol, Table salt, body weight etc. Persisting 5-10\% of people suffer this due to narrowing of kidney arteries, chronic kidney diseases and endocrine disorder. Assessment of cardiovascular disease primarily includes: Confirmation of hypertension, Risk factors, Underlying causes, organ injury \& Indications and contraindications for medication medicine. Hypertension is directly responsible for $57 \%$ of all stroke deaths and $24 \%$ of all coronary heart disease deaths in India. The American 
Heart Association estimated the direct and indirect costs of hypertension in 2010 were $\$ 76.6$ billion. In India, onethird of urban adult Indians and close to one fourth of rural adult Indians are hypertensive. Regional differences exist in rural areas of India for prevalence of HTN. Urban areas of India show no significant differences in HTN prevalence. Only quarters of rural Indians suffering from HTN is aware of and are being treated for HTN. Fortytwo percent of urban Indian hypertensive patients are aware of their hypertensive status. Thirty-eight percent of urban Indians are being treated for their HTN. Only one-tenth of rural Indians and one-fifth of urban Indians suffering from BP have their BP under control. Globally cardiovascular disease accounts for approximately 17 million deaths a year, nearly one third of the total population. Out of these, complications of hypertension account for 9.4 million deaths worldwide every year.

Treating high blood pressure can take a multi-pronged approach including diet changes, medication, and exercise. Hypertension, or high blood pressure, is dangerous because it can lead to strokes, heart attacks, heart failure, or kidney disease. The goal of hypertension treatment is to lower high blood pressure and protect important organs, like the brain, heart, and kidneys from damage. Many advanced treatment approaches and medications have been established for the treatment and management of hypertension such as Biomarkers. Biomarkers are biological measures that are in the causal pathway of disease or have utility for risk stratification. Consequently, biomarkers are key to implementation of at least 3 aspects of personalized medicine: detection and diagnosis of disease, risk assessment and prognosis, and prediction of responses to therapeutic or preventative interventions.

Serving as a model for the application of this recommendation to hypertension, the GENetics of Hypertension Associated Treatment (GenHAT) study is determining whether variants in hypertension susceptibility genes interact with antihypertensive medication to modify coronary heart disease risk in hypertensives.

\section{P208}

\section{Role of Serum FLC Measurement, Ratio of Total To Free Light Chains And Bjp In Diagnosis of Multiple Myeloma.}

\author{
B.J. Shinde, Nitin A. Inamdar, Geeta Rathnakumar, \\ Mukta Ramadwar, N. D. Kamble
}

INTRODUCTION: Multiple myeloma (MM) is the 2nd most common disease of hematological malignancies. Male to Female ratio of incidences in India is 13:8 per million. Diagnosis and monitoring of MM and related conditions are usually carried out by means of serum and urine protein electrophoresis and immunofixation. Immunoglobulin free light chains (FLCs) are by-products of immunoglobulin synthesis and in normal subjects are released into the circulation in small quantities.Several reports have demonstrated the usefulness of the free light chains assay for the diagnosis and monitoring of MM.

AIM OF THIS STUDY: To evaluate the role of serum FLC measurement in differential diagnosis of MM and clinical significance of total light chain \& free light chain ratio and bence jones proteins in the diagnosis of mulitple myeloma.

METHODS AND MATERIAL: The material for the study comprised of 120 newly diagnosed (with serum protein electrophoresis) multiple myeloma cases in Tata Memorial Hospital, Parel. Analysis of serum Free light chain Kappa and Lambda, Total Protein, and B2M was done by photometry on Beckman coulter AU 2700 autoanalyzer. Total Kappa light chain, Lambda light chains and urinary Bence Jones Protein were estimated by nephelometry on Beckman coulter Immage 800 Immunoassay analyzer.

RESULTS AND CONCLUSION: Our data depicts the highest sensitivity and specificity for BJP when compared with Ratio of total light chain to free light chain assay and can be considered reliable for the diagnosis, monitoring, and prognosis of different plasma cell disorders. 


\section{P209}

\section{Circulatory Level of Aldose Reductase In Diabetic And Diabetic Nephropathy Patients}

\author{
Sanchit Tiwari $^{1,2}$, Mohd. Waseem ${ }^{1}$, I Ahmad ${ }^{1}$, \\ R. Alam ${ }^{2}$, S.K. Sonkar ${ }^{3}$, M.K. Ahmad ${ }^{1}$ \\ 1. Department of Biochemistry, KGMU Lucknow. \\ 2. Department of Biochemistry, IIMSR, IU Lucknow. \\ 3. Department of Medicine, KGMU Lucknow.
}

INTRODUCTION: In diabetic nephropathy complications, hyperglycemia may cause damage at a cellular level in both glomerular and tubular locations, often preceding overt dysfunction. Aldose reductase 2 (ALR2), a key enzyme in the polyol pathway, catalyzes nicotinamide adenosine dinucleotide phosphatedependent reduction of glucose to sorbitol, leading to excessive accumulation of intracellular reactive oxygen species (ROS) in various tissues of DM including the kidney, eyes, neurons and heart.

METHOD: Serum level of Aldose Reductase 2 done by Enzyme Linked immunosorbent Assay (ELISA)and molecular studies including the detection of AR C(106) $\mathrm{T}$ gene polymorphisms using the polymerase chain reaction (PCR)/ restriction fragment length polymorphism technique.

RESULT: The circulatory level of ALR2 in healthy control was $7.07 \pm 2.11 \mathrm{ng} / \mathrm{ml}$ and in Type-2 Diabetes patients was $14.44 \pm 3.11 \mathrm{ng} / \mathrm{ml}$ and Diabetic Nephropathy patients was $16.11 \pm 4.16 \mathrm{ng} / \mathrm{ml}$. ALR2 levels were increased by $104.24 \%$ and $127.86 \%$ in Type-2 Diabetes and Diabetic Nephropathy patients as compared with healthy control. The genotype distribution and allele frequency of AR C(-106) T showed no statistical significance also the genotypes were not associated with any of the different studied parameters

CONCLUSION: We observed circulatory level of ALR2 is highly significant association with T2DM and DN when compare to Healthy Controls. The C(-106) T polymorphism in the AR gene is not involved in the pathogenesis of Diabetic Nephropathy in type 2 diabetes.

\section{P210}

\section{Does Smoking Alter MicroRNA-21 Expression In Bladder Cancer Patients?}

Zainab Siddiqui ${ }^{1}$, Anand Narain Srivastava ${ }^{2}$, Satya Narain Sankhwar ${ }^{3}$, Syed Tasleem $\mathrm{Raza}^{4}$, Osman Musa $^{5}$, Abdul Naeem ${ }^{6}$, Kainat Fatima ${ }^{6}$, Shahnawaz Ahmed $^{6}$, Nishat Fatima ${ }^{7}$.

1. Department of Pathology, Era's Lucknow Medical College and Hospital(ELMC\&H), Lucknow, India;

2. Director Research, ELMC\&H, Lucknow, India;

3. Department of Urology, King George's Medical University(KGMU), Lucknow, India;

4. Department of Biochemistry, ELMC\&H, Lucknow, India;

5. Department of Surgery, ELMC\&H, Lucknow, India;

6. Research Metabolic Unit, ELMC\&H, Lucknow, India;

6. Research Metabolic Unit, ELMC\&H, Lucknow, India;

6. Research Metabolic Unit, ELMC\&H, Lucknow, India;

7. Department of Biochemistry, ELMC\&H, Lucknow, India;

6. Research Metabolic Unit, ELMC\&H, Lucknow, India;

7. Department of Biochemistry, (KGMU), Lucknow, India.

BACKGROUND- Bladder cancer (BC) represents 4.7\% of all new cancer cases in the U.S and it ranks ninth amongst them. It is an outcome of multi-factorial process that includes genetic (dysregulated microRNA, mutations in CCDN1, FGF3, KRAS genes etc.) and/or non-genetic risk factors (occupational exposure, smoking etc.), thus influencing the progression, angiogenesis, metastasis and invasion of the bladder cancer cells. Recent researchers have found that both genetic and non-genetic risk factors have cumulative effect in the occurrence of $\mathrm{BC}$ like polymorphism in glutathione $\mathrm{S}$-transferase and $\mathrm{N}$-acetyl transferase genein cigarette smokers modulates BC risk. Therefore we want to study microRNA-21(miR-21) gene-expression in $\mathrm{BC}$ smokers. 
MATERIAL AND METHODS- miR-21 expression analysis (Taqman Assay) was performed using commercial kits by qRT-PCR and miR-21expression was calculated using the 2-??ct method.

RESULTS- In our study smokers had higher expression of miR-21than non-smokers. $(\mathrm{p}<0.05)$.

CONCLUSION- We conclude from our study that smoking may modulate the expression of miR-21 in BC patients.

KEYWORDS- Bladder cancer, smoking, microRNA21.

\section{P211}

Prevalence of Hyperhomocysteinemia In Patients With Deep Venous Thrombosis In Haryana

Sinha L, Singla A*, Vashist MG*, Bala J, Goel K, Aparna, Kharb S, Sachdeva A

Department of Surgery

* and Biochemistry, Pt. B.D. Sharma Post Graduate

Institute of Medical Sciences Rohtak, Haryana, India.

BACKGROUND: Deep vein thrombosis (DVT) affects a significant proportion of population and data in Indian population on serum homocysteine levels is lacking.

OBJECTIVE OF THE STUDY: The present study was undertaken to analyze the levels of serum homocysteine in patients with DVT in Haryana region of India.

MATERIALS AND METHODS: A total of 100 patients were selected for study and divided into two groups. Group A: 50 patients having clinical features suggestive of DVT (study group) and group B contained 50 patients which did not have clinical features suggestive of any venous disorder (controls). Homocysteine was estimated by ADVIA Centaur CP Immunoassay System.

RESULTS: In group A, 11 out of 18 (61.11\%) patients were hyperhomocysteinemia in the age group 21-30 years. The prevalence of hyperhomocysteinemia was $60.46 \%$ (26 out of 43) in patients with age less than 60 years as compared to $42.86 \%$ (3 out of 7) in patients aged more than 60 years $(\mathrm{p}>0.05) .60 .71 \%$ males with
DVT patients had hyperhomocysteinemia as compared to $54.55 \%$ female DVT patients ( $\mathrm{p}>0.05$ ). $35.71 \%$ with DVT below knee region and $66.67 \%$ with DVT extending above knee region had hyperhomocysteinemia $(\mathrm{p}<0.05)$.

CONCLUSION: Serum homocysteine levels should be measured in all cases of DVT especially in cases where no predisposing risk factor (idiopathic DVT) was present and in the patients of DVT in youngerpopulation. Homocysteine can prove to be good, early diagnostic marker in idiopathic DVT.

KEY WORDS: homocysteine, deep vein thrombosis, diagnosis, Color Doppler.

\section{P212}

Salivary Bone Alkaline Phosphatase - An Alternative Tool for Growth Assessment

\section{Dr Hana Khan}

Department of Orthodontics and Dentofacial

Orthopaedics, Sardar Patel Post Graduate Institute of

Dental and Medical Sciences, Lucknow, Uttar

Pradesh.

BACKGROUND : The use of clinical and radiographic guidelines to predict a patient's skeletal maturation is a routine practice for healthcare practitioners. These findings help in assessing the pubertal or adolescent growth spurt and also define whether it is imminent, present or complete. Such information is useful for treatment planning and evaluation of treatment progress in patients with skeletal and growth related disorders. As chronologic age is not reliable for evaluation of growth status, the biologic age needs to be determined. The use of radiographs to assess skeletal and dental maturation and evaluation of biomarkers for osteoblastic activityare of help in this regard. These Biomarkersare measureable in blood and other body fluids like saliva, gingival crevicular fluid and urine. As radiographic methods are highly subjective and involve radiation exposure and blood assay are invasive requiring patient co operation, saliva can be used as a diagnostic medium. It harbours a wide spectrum of proteins/peptides and 
nucleic acids and reflects the body's health and wellbeing. Its collection is non invasive and cost effective, also it can be repeated at short time interval without fear of any harm. The purpose of this study was to associate salivary Bone Alkaline Phosphatase (BAP) with MP3 staging (which is an established skeletal maturity indicator) to find whether BAP levels in saliva can be used as clinical tools to determine the growth status of the subject.

METHOD : A cross-sectional study was conducted on a sample of 120 healthy subjects in the age range of 7 30 years with equal distribution of males and females in each group. Each group was in turn divided in to $4 \mathrm{sub}$ groups of 15 each depending on their chronological age. Oral prophylaxis was done for all subject 15 days prior to sample collection. Unstimulated saliva was collected by passive drool method and Radiovisiography (RVG) was used to capture the image of the Middle Phalanx of the 3rd finger (MP3). BAP was estimated using ELISA and MP3 was graded using Hagg and Taranger staging. The results were subjected to statistical evaluation. BAP levels in saliva were then co related with MP3 staging to determine if Salivary BAP levels can be used as clinical tools to determine the growth status of the subject.

RESULTS : A strong co relation was seen between salivary BAP levels and MP3 staging in both males and females. A gradual increase in salivary BAP was seen from stage $\mathrm{F}$ to $\mathrm{FG}$. The peak levels was seen in $\mathrm{G}$ stage which was followed by a sudden decline in BAP levels from $\mathrm{H}$ stage to I stage.

CONCLUSION : This study concluded that salivary BAP levels can be used as biomarkers for evaluation of skeletal maturation status in subjects.

KEYWORDS: Biomarkers,Bone Alkaline Phosphatase, MP3 Staging.

\section{P213}

\section{Contamination of mobile phones used by medical students and health care workers in a tertiary care hospital, North India}

Bushra Khalid*, Priyanka Shukla, Farzana Mahdi, Mastan Singh
BACKGROUND : Mobile phones are popular communication devices; a potential vector for bacteria trans-mission which can be contaminated through human skin, pockets, environment and food particle. The constant handling and heat generated by the phones creates a prime breeding ground for all sorts of microbes.

METHODS : This study was conducted in Department of Microbiology, Era Lucknow Medical College \& Hospital, Lucknow including health care workers, Medical and Para Medical students. A total of $40 \mathrm{swab}$ samples from mobile phones were collected after taking informed written consent.

METHODOLOGY : A sterile cotton swab(moistened with Peptone water) was taken for specimen collection and bacterial culture. Swab was rotated on keyboard screen, back and sides of mobile phones . After collection of sample, tube containing Peptone water was incubated overnight, plating was done on next day on Blood Agar, Mac Conkey agar. Alcohol sanitizer was used to sanitize hands of sample collectors before swabbing mobile phone. Incubation was done at 37?C for 24-48 hours. Visible growth from each plate was identified by Gram's staining and biochemicals.

RESULTS : All mobile phones showed evidence of bacterial contamination. A total of 40 bacterial isolates were obtained of which, Bacillus(28), Micrococcus(4), Staphyloccus aureus,(1) CONS(5) and Klebsiella.(2)

CONCLUSION : Training programs should be conducted to increase awareness about decontamination of mobiles. Regular hand washing can reduce the contamination of phones. Decontamination of mobiles with alcohol disinfectant wipes or with $70 \%$ isopropyl alcohol can be effective.

KEYWORDS : Healthcare workers, Mobile phones

\section{P214}

Adenosine Deaminase Activity In Subjects With Altered And Normal Liver Function Profile Test.

Seraj Ahmad Khan ${ }^{1}$, Lamsal M², Chaudhari RK ${ }^{1}$, Tamang $\mathrm{B}^{3}$, Lamichane $\mathrm{S}^{3}$ 
Department of Biochemistry, BPKIHS, Dharan, Nepal

INTRODUCTION: Adenosine Deaminase (ADA) is a nonspecific marker for cellular immunity, is raised in diseases eliciting a cell-mediated immune response. High serum ADA activities were observed in patients with different liver diseases. In this study we aim to determine the association of serum ADA and transaminases levels in liver disease patients and normal control. Methodology: In this comparative cross sectional study 80 subjects, 40 with liver disease and 40 without liver disease were enrolled. Blood samples were drawn and analysis was done. Liver function profile was done by Autoanalyzer (cobas 311, Germany) and ADA activity was measured by Giusti and Gallanti method. Independent $t$ test and Mann Whitney $U$ test for association; Pearson's correlation and Spearman's Rho for correlation study. Multiple linear regression model use for independent association of the variable with ADA. $\mathrm{P}<0.05$ set as statistically significant.

RESULTS: Based on liver function profile subjects were divided into Group 1 (normal liver function) and Group 2 (altered liver function) with 40 individual in each group and mean age of $40.0 \pm 16.5$ years and $42.4 \pm 16.3$ years respectively. Gender were uniformly distributed. Total bilirubin, direct bilirubin, aspartate transaminase (AST), alanine transaminase (ALT), alkaline phosphatase (ALP) and adenosine deaminase was higher in group 2 and was statistically significant. ADA was significantly correlated with albumin, AST, ALT and ALP in subjects with normal liver function whereas it was significantly correlated only with direct bilirubin in subjects with altered liver function. Conclusion: Serum ADA activity is significantly raised in subjects with altered liver function profile.

KEYWORDS: Adenosine Deaminase, Aspartate Transaminase, Alanine Transaminase, Alkaline Phosphatase.

\section{P215}

Cross Sectional Study of Variation of C-Reactive Protein (CRP) Levels In Diabetic And Non Diabetic Subjects With Respect To Their Fasting Blood Sugar (Fbs) Value
Shivaraja Shankara Y $\mathbf{M}^{* 1}$, Sujith $S^{2}$

$1 *$ Dept of Biochemistry, KVG Medical College Hospital Sullia D.K, Karnataka, India, 574327,

2. Dept of Biochemistry, KVG Medical College \& Hospital Sullia D.K, Karnataka, India, 574327,

INTRODUCTION: According to WHO, 422 million adults are living with diabetes mellitus globally. The number of people with diabetes has risen to 422 million in 2014. The increase in rates of diabetes mellitus in developing countries follows the trend of urbanization and lifestyle changes, increasingly sedentary life styles and global nutrition transition.Diabetes mellitus is known to have an inflammatory component with respect its etiopathogenesis.

AIMS AND OBJECTIVES: The present study has made an attempt to compare the levels of serum Creactive Protein (CRP) between type 2 diabetic subjects and healthy controls. The study also aimed to find the correlation between plasma glucose and CRP levels, and the variations in the CRP levels.

MATERIALS AND METHODS: The study subjects were of two categories namely, type 2 diabetic patients $(n=56)$, and age- and sex-matched healthy controls $(n=56)$. In both the groups of subjects, Fasting plasma glucose was estimated by glucose oxidase-peroxidase method, and serum CRP was assayed by nephelometry.

RESULTS AND CONCLUSIONS: Serum CRP level was significantly higher in diabetic patients $(137.5 \mathrm{mg} /$ L) when compared to healthy controls $(12.4 \mathrm{mg} / \mathrm{L})$; $\mathrm{P}$ $<0.001$. There was significant correlation between serum $\mathrm{CRP}$ level and fasting/post prandial plasma glucose $(\mathrm{r}=$ $0.889 ; \mathrm{P}=0.005)$. Among the diabetic subjects, $100 \%$ had CRP levels above the reference range while among the healthy controls, $1.3 \%$ CRP levels above the reference range.The role of inflammation the pathogenesis of diabetes mellitus and its complication is evident from the findings of the study.

KEY WORDS: CRP, nephlometry, diabetes, fasting blood sugar 


\section{P216}

Objective Structured Practical Examination (OSPE) As An Assessment Method of Laboratory Practical Skill Sessions In First MBBS Students Of Biochemistry: A Research Study In Saims, Regional Centre of Medical Education, Indore

\author{
Bindu Sharma $^{1}$, Deepti Mandsorwale ${ }^{2}$ \\ Department of Biochemistry, VAMC \& RH, Banthra, \\ Shahjahanpurl;Department of Biochemistry, Govt. \\ Medical College, Kannauj2
}

INTRODUCTION:OSPE act as a good tool to evaluate skill competency in Biochemistry subject.

MATERIAL AND METHODS:The first MBBS students admitted for 2014-16 batches of SAIMS, Indore were the subjects for the study. After successfully completing the syllabus, OSPE notification was announced 10 days in advance.A total of 122 students were assessed. Each student was assessed by attending 4 procedure and 8 question stations. Coefficient of reliability of questions administered was done by calculating Cronbach's alpha. Among 122 students are present \& took this OSPE exercise.

RESULTS:15 students failed to achieve an average of $50 \%$ or above in the assessment. However, 39 students on an average achieved $>75 \%, 41$ students achieved between 65 to $75 \%$ and 27 students scored between 50 to $65 \%$. Cronbach's alpha of the questions administered showed to be having high internal consistency with a 0.80 score. $99 \%$ of students believed that OSPE helps them to improve and $82 \%$ perceived in both learning and evaluation tool. $100 \%$ of the faculty agreed or strongly agreed that such assessment tested objectivity, $87.5 \%$ felt that is measured practical skills better and $62.5 \%$ felt eliminated examiner biasto a greater extent.

CONCLUSION:OSPE was more objective, measured practical skills better and eliminated examiner bias. Studentfeedback reflects that such assessment helps them to improve practical skills \& it is effective tool both for teaching and evaluation of MBBS students of Biochemistry.
KEYWORDS: OSPE (objective structured practical examination)/ OSCE(objective structured clinical examination), $\mathrm{MCQ}$ (multiple choice question), cognitive domain.

\section{P217}

\section{Confirming Monoclonality In Multiple Myeloma With Serum Immunoglobulins}

Nitin A. Inamdar, B.J. Shinde, Geeta Rathnakumar, Mukta Ramadwar, N. D. Kamble

Department of Biochemistry, Tata Memorial Hospital, Parel, Mumbai

Q erum Protein electrophoresis (SPE) and Immunofixation (IFE) is considered Gold standards in Multiple Myeloma (MM) Patients till date. In suspected MM patients SPE is initially asked and in M band is found than IFE is asked to confirm the same. Haemolysed and unclotted blood sample give rise to false positive band. This leads to follow expensive IFE tests. If serum Immunoglobulins $\operatorname{IgA}, \operatorname{IgM}$ and $\operatorname{IgG}$ along with serum total Kappa and Lambda light chain quantification is done and these results are interpreted properly, it can find out monoclonality in more than $95 \%$ of patients.

\section{P218}

\section{Lactoferrin and Its Mechanistical Insights Into The Anticancer Activity In Breast Cancer}

Mohit Adhikary $^{1,2,5}$, Arun B Chand ${ }^{3,4,5}$

1. Research Content Management, SPS Pvt. Ltd. (Wiley), G N Chetty Road, Chennai, Tamil Nadu

2. Department of Biochemistry, J N Medical College, Nehrunagar, Belgaum, Karnataka

3. Life Trust Medi Diagnostic Pvt Ltd, Aanandanagar Marg, Kathmandu Nepal 24442, Nepal

4. Department of Medical Microbiology, Shi-Gan International College of Science \& Technology, Narayan Gopal Chowk, 


\section{Chakrapath Kathmandu}

5. College of Paramedical Sciences, SGRR Institute of Medical and Health Sciences, Patelnagar, Dehradun, Uttarakhand

INTRODUCTION: Lactoferrin, a glycoprotein and a regulator of free iron in the body fluid of mammals is majorly found on external secretions such as milk. For the development of cancer the proliferating cells need iron, and the iron binding capacity of lactoferrin provides it the anticancer activity, it scavenges the free iron present in the plasma iron.

METHODS: We reviewed the mechanism with which lactoferrin brings about the anticancer activity in breast cell lines in various studies as the exact mechanism is still unclear. Two mechanisms in different studies have been proposed, where lactoferrin either activated the immune cells to release tumor cytotoxic effectors or it is internalized by the cancer cells which in turn triggers apoptosis as evidence of anticancer activity in metastatic breast cell lines.

RESULTS:Basis the analyses, we observe a strong evidence of lactoferrin inhibiting metastatic breast cancer by triggering apoptosis through its association with a differentialextracellular acidification rate and $\mathrm{V}-\mathrm{H}+-$ ATPase localization. It induces intracellular acidification and inhibits both protonpumping and hydrolytic activities of V-H+-ATPasein highly metastaticbreast cancer cells.

Conclusion: Studies that would provide an insight into the mechanism and the interactions of the lactoferrin with the V-H+-ATPase at a molecular level would assist in examining the hypothesis of long-term and short term effects in inhibiting metastatic breast cancer and its application as an anticancer agent.

KEYWORDS: Lactoferrin, anticancer, breast cancer, V-H+-ATPase

\section{P219}

\section{Hepcidin-Ferritin Ratio Is Decreased In Diabetes Mellitus}

Padmanabhan V, JoeVarghese,JithuJames, JasminePrasad, Molly Jacob

\section{Department of Biochemistry, Christian Medical}

College, Vellore 632002, Tamil Nadu, India

INTRODUCTION: Increases in body iron stores have been reported to be associated with diabetes mellitus (DM). The mechanisms that link these two conditions are unclear. Hepcidin is the central regulator of iron homeostasis. It is, therefore, conceivable that changes in hepcidin may underlie the association between these conditions. This study was designed to determine if this was so.

METHODS: Adult males, not previously known to be diabetic, who were referred for medically-indicated estimations of fasting and post-prandial blood glucose levels, were recruited after obtaining informed consent. Anthropometric measurements were also donefor each subject. The results of the fasting plasma glucose level were used to categorize subjectsas diabetics (diagnosed for the first time) and non-diabeticsbased on criteria recommended bythe American Diabetic Association. The blood sample obtained was used to estimate haematological parameters, glucose, insulin, C-reactive protein (CRP), ferritin, iron, total iron-binding capacity (TIBC) and hepcidin. Homeostatic model assessment (insulin resistance [HOMA-IR]), an indirect measure of insulin resistance, and HOMA-B (an index of pancreatic beta cell function) were calculated (using values for fasting glucose and insulin). Appropriate testswere used for statistical analyses, depending on the distribution of the data obtained.

RESULTS: A preliminary analysis was done for 42 subjects in whom biochemical estimations have been completed. The diabetic $(\mathrm{n}=21)$ and non-diabeticsubjects $(n=21)$ were similar in age and anthropometric measurements. Fasting plasma glucose levels, postprandial plasma glucose andHOMA-IR values were significantly higher and HOMA-B values significantly lower in the diabetic group. Serum ferritin levels were significantly higher in those with DM(median 147 [IQR 114-251.5] ng/ml) than in non-diabetics (median 79 [IQR 46.65-155.5] ng/ml). Serum hepcidin levels were similar in the 2 groups (controls: median 12.28 [IQR 9.86 15.37] vs diabetics: 13.15 (IQR 8.4 1-25.54]). Hepcidinferritin ratio was significantly lower in those with DM (0.15 [IQR 0.08 - 0.24])thanin control subjects( 0.08 (IQR 
0.06-0.12). Other parameters measured (haemoglobin, serum iron, TIBC, transferrin saturation, CRP and insulin levels)were similar in the two groups.

On univariate analysis, serum ferritin showed known significant correlations with other parameters of iron metabolism. Fasting plasma glucose levels correlated significantly and positively with serum ferritin and negatively with hepcidin-ferritin ratios. Multivariate analyses showed correlations between serum ferritin and other iron-related parameters (as mentioned above) and a trend towards a negative correlation between fasting plasma glucose levels and hepcidin-ferritin ratio $(\mathrm{p}=$ $0.07)$.

CONCLUSION: Serum ferritin, often used as a marker of body iron stores, was significantly higher in subjects with diabetes mellitus, but hepcidin levels were not. Lower hepcidin-ferritin ratios seen in the diabetics suggest that serum hepcidin levels were inappropriately low for the increased iron stores in these subjects, as indicated by elevated serum ferritin levels. The significance of these observations requires elucidation. This study is ongoing with analyses pending on more patients and also in those with pre-diabetes.

KEY WORDS: Diabetes mellitus, iron, hepcidin

\section{P220}

Fluoride Induced Alteration In Hypothalamic Testicular Axis Hormones And Deterioration In Antioxidants Status In Fluorotic Patients

Dushyant Singh Chauhan, Sudhanshu Mishra, Sandeep Tripathi

Department of Advanced Sciences\&Technology, Nims Institute of Engineering \& Technology,Nims University Rajasthan, Jaipur, 303121

INTRODUCTION:Endemic fluorosis is the major environmental born public health problem in India. Fluoride ubiquitously found in the water or Rajasthan, Karnataka, Punjab and as well as in the other parts of the country and worldwide and responsible for fluorosis. Fluoride has its effect on various organ systems, including the spermatogenesis. Excessive fluoride has been associated with the reduced quality of the sperm but mechanism of action still unknown.High endemic areas of fluorosis found to be responsible for hypothalamic-testicular toxicity.

MATERIALAND METHODS:In this study we attempt to investigate the early markers of Fluoride $(F)$ induced reproductive toxicity in 100 human males.

RESULTS:Semen morphological parameters, hypothalamic- testicular axis hormones namely LH, FSH, prolactin, testosterone and the oxidative stress markers was examined in Fluoride exposed population. We found that $\mathrm{LH}, \mathrm{FSH}$, testosterone and prolactin values was significantly $(\mathrm{p}<0.05)$ alters in fluoride exposed population. Increased lipid peroxidation and Protein carbonyl content and decreased antioxidant status i.e., SOD, CAT, GPx and GSH was observed. Sperm count, motility and viability was delineated in exposed population.

CONCLUSION:On the basis of results it may conclude that the environmental exposure of fluoride alters the hypothalamic testicular axis hormones in human male. This study suggests that hypothalamic testicular axis hormones and oxidative stress parameters can be useful as early markers for determination of disease fluorosis in population those residing in high fluoride regions. Moreover, further study may require for the depth knowledge of fluoride toxicity.

KEY WORDS: Fluoride; Hypothalamus; testicular axis; oxidative stress

\section{P221}

\section{Neurolipofuscinogenesis In Alumnum Induced Neurotoxicity}

Sandeep Tripathi ${ }^{1}$, Manisha Choudhary ${ }^{1}$, Devesh Kumar Joshi ${ }^{1}$, Sudhanshu Mishra ${ }^{1}$, Abbas Ali Mahdi ${ }^{2}$

1. Department of Advanced Science, Nims Institute of Engineering \& Technology, NIMS University Rajasthan, Jaipur India

2. Department of Biochemistry, King George's Medical University, Lucknow, India 
BACKGROUND:Aluminum (Al) intake might increase risk of Alzheimer disease. The brain aluminum content increases with age. Neurolipofuscin is considered a reliable biomarker of aging and it is distorts the protein building machinery of the cell and interferes with the important autophagic process. The aim of the study was to evaluate effect of $\mathrm{Al}$ on mitochondrial aging associated with Autophagy-lysosome-neurolipofuscin pathway.

METHODS: To achieve these objectives we administered $\mathrm{AlCl} 3$ (100 mg / kg b.w.) to young and aged rats for 90 days. Thereafter, animals were sacrificed for biochemical and ultrastructural studies in the hippocampus.

RESULTS:Increased lysosomal-macroautophagy and lipofuscinogenesis activity discernible in the hippocampal region. Moreover, caspase- 3 and bax were increased in proportion of the ROS, lipid peroxidation and LDH. The biochemical and morphological changes in Al treated young rats were comparable to those of untreated old control rats. The ability of mature neurons in the brain to export accumulating metabolic waste by lipofuscin exocytosis is a significant adaptation.

CONCLUSION:Together these findings indicate widespread neuropathology and focused axonal neurodegeneration in $\mathrm{Al}$ treated groups in association with autophagy-lysosome-neurolipofuscin pathway, provide the basis for future mechanistic assessment for developing $\mathrm{Al}$ induced Alzheimer's like disease.

\section{P222}

Surface Stem Cell Marker CD133 Expression And Its Clinicopathological Significance In Gallbladder Cancer: An Immunohistochemical Study.

Naseem Fatima $^{1}$, A.N Srivastava ${ }^{1}$, Zainab Siddiqui, Vijay Kumar².

1. Department of Pathology, Era's Lucknow Medical College \& Hospital, Lko. U.P-India.

2. Department of Surgical Oncology, King George's Medical University, Lko, U.P-India
BACKGROUND: Gallbladder cancer (GBC)is an aggressive, third most common type of malignancy in north India. Survival rate is very low due to poor prognosis. CD133 is considered the most robust surface marker, at present very few research reports suggest the significance of CD133 expression with progression of GBC. The present work aimed at a comparative study of CD133 expression between GBC, benign lesions, and gallbladder epithelium with chronic cholecystitis.

MATERIAL AND METHODS: Immunohistochemistry (Polyclonal, Proteintech, USA) was performed to evaluate CD133 expression in 40 fresh tissues of GBC (radical/biopsy), 15 cases of adenomatous hyperplasia GB, and 10 cases of chronic cholecystitis (histopathological proven). Results:It was found that CD133 was highly expressed in GBC tissues as compared to benign gallbladder tissues. Well differentiated tumor was reported in $70 \%$ (28/40), lymphatic invasion in $77.5 \%(31 / 40)$ and Liver metastasis in $32.5 \%$ (13/40). Higher expression of CD133 was identified in 65\% (26/ 40) GBC, low expression was observed in $22.5 \%$ (9/ 40). Weak staining was observed in $40 \%(6 / 15)$ adenomatous hyperplasia of gallbladder. However chronic cholecystitis cases showed negative staining. The higher expression of CD133 was significantly associated with degree of differentiation $(\mathrm{p}=0.003)$, and liver metastasis $(\mathrm{p}=0.001)$.

CONCLUSION:Strong expression of CD133 in gallbladder cancer tissue indicates that CD133 is closely related to higher stage of disease and metastasis.

KEY WORDS:Gallbladder cancer, premalignant gallbladder, CD133, Immunohistochemistry.

\section{P223}

Evaluation of Oxidative Stress and Antioxidant Enzymes In Type 2 Diabetic Patients

Shruti Priya $^{1}$, Vishnu Kumar ${ }^{2}$, Mrinal Ranjan Srivastava ${ }^{3}$
1. Department of Biochemistry, Hind Institute of Medical Sciences, Safedabad, Lucknow .
2. Department of Biochemistry and
2. Department of Community Medicine, Era's 
Lucknow Medical College \& Hospital,

Lucknow-226 003

$\mathrm{D}$ iabetes mellitus is a group of metabolic disorder characterized by hyperglycemia with glycosuria and it is well documented that increased level of blood glucose is a marker of disorder of carbohydrate metabolism and is associated with the initiation of diabetic dyslipoproteinemia and other complications. Prolonged free radical mediated lipotoxicity and abnormal glucose tolerance are involved in the pathogenesis of diabetes mellitus. Diabetic dyslipoproteinemia is characterized by the increased level of cholesterol, reduced HDL and high triglyceride (TG) levels.The present study was carried out to explore the status ofoxidative stress and antioxidant enzymes in type2 diabetic patients. This study was conducted on type 2 diabetic patients attending the diabetes OPD, Hind Institute of Medical Sciences, Safedabad, Lucknow. All biochemical assays were carried out by the standard kit methods. A marked increase in plasma levels of lipid peroxide following decrease in antioxidant enzymes and reduced glutathione were noted in type 2 diabetic patients compared to healthy controls. Type 2 diabetic patients are consistently associated with disorder of metabolism and disturb redox status.

KEY WORDS: Lipid profile-Lipoprotein profileOxidative stress-Antioxidants-LCAT.

\section{P224}

The Predictive Ability of Maternal Serum Hif$1 \alpha$ In Pre-Eclampsia And Gestational Hypertension

Jenu Maria Thomas ${ }^{1}$, Revathi P Shenoy ${ }^{1}$, Parvati V Bhat $^{2}$, Asha Kamath ${ }^{3}$, Shashikiran Umakanth ${ }^{4}$, Angel Treasa Alex ${ }^{5}$, Krishnananda Prabhu ${ }^{1}$

1. Department of Biochemistry,

4. Department of Community Medicine, Kasturba Medical College, Manipal,

2. Department of $O B G$,

4. Department of General Medicine, Dr. TMA Pai Hospital, Udupi,
5. Department of Pharmaceutical Biotechnology, Manipal College of Pharmaceutical Sciences, Manipal.

INTRODUCTION: Pre-eclampsia (PE) is a multi system disorder of unknown etiology characterizedby the development of hypertension to the extent of 140/90 $\mathrm{mmHg}$ or more with edema or proteinuria or both, induced by pregnancy after the 20th week of gestation. Gestational hypertension( $\mathrm{GH}$ ) is often considered as a transitional state developed wherein the majority of the women who develop the condition between 24-35 weeks of gestation progress to pre-eclampsia. PE and GH are the primary cause of maternal and fetal/neonatal mortality and morbidity across the globe. HIF-1? is a transcription factor responsible for the regulation of angiogenesis and thus oxygen and nutrient supply. It controls the action of oxygen on trophoblastic invasion. It is also known to induce the transcription factor coded for VEGF and thus promotes tumor angiogenesis This study trying to find the predictive ability of maternal serum HIF-1? in PE and GH.

MATERIALS AND METHODS : After the approval by the Institutional Ethics Committee (IEC290/2013) at Kasturba Medical College, This study was conducted with a cohort design and duration of three and half years and was carried out in the Department of Biochemistry, KMC, Manipal, and Department of OBG, Dr. TMA Pai Hospital, Udupi. Pregnant women attending outpatient department at Dr. TMA Pai Hospital, Udupi in both the first trimester (from the diagnosis of pregnancy confirmed by ultrasound till $12+$ weeks) and second trimester (from 13th week till 28 weeks of gestation) fulfilling all the criteria were included in the study and Serum HIF-1? levels were measured during both trimesters. They were followed up in the third trimester till the delivery. Based on the pregnancy outcome they were categorized as 1)Controls- those who have had a healthy pregnancy; 2)Cases- those who developed GH/ PE.

RESULTS AND DISCUSSION: We observed the HIF1 ? levels were to be significantlyelevated in bothtrimesters of pregnancy among PE/GH groups as compared to controls. Limited studies were found measuring the first trimester values and following up to 
check the presence of hypertensive disorder in pregnancy. Through, statistical analysis, the cut off value was also deduced for both trimesters to differentiate the incidence of PE/GH. However, there are no established data available on HIF- $1 \alpha$ levels in pregnancy to support our study.

CONCLUSION: Even though significant difference observed between cases and controls, furtherfollow up studies required to evaluate the HIF $-1 \alpha$ levels and to consider HIF $-1 \alpha$ as the predictive marker for PE and $\mathrm{GH}$

\section{P225}

\section{Association of Gstm1, Gstt1 and Gstp1 Polymorphisms in Predisposition to Vitiligo}

Apurva Shrivastava, Shivam Choudhary, *Dinesh Asati, Kotnis Ashwin

* Department of Dermatology, All India Institute of Medical Sciences Bhopal, MP, India. Department of Biochemistry, All India Institute of Medical Sciences Bhopal, MP, India

INTRODUCTION: Vitiligo is a common, noncontagious, skin depigmentation disorder characterized by milky white patches on skin. Vitiligo is associated with deep social stigma in India and the patients have poor quality of life due to long duration of treatment without definite outcome. Vitiligo occurs due to melanocyte death however exact cause of melanocyte death is unknown. Patients with vitiligo exhibit high levels of free radicals in vitiligo lesions and are benefitted by antioxidant therapy supporting oxidative stress in aetiopathology of Vitiligo. GSTs present in the skins are crucial antioxidants and their aberrant function may genetically predispose to vitiligo.

AIMS AND OBJECTIVES: The aim of the present hospital based case control study was to analyse the association of null genotype of GSTM1, GSTT1 and genotypes of GSTP1 (Ala113Val rs1138272; Gly169Asp rs41462048, Ile104Val rs1695) with occurrence to Vitiligo in central India. Correlation of genotype with demographic factors; type and duration of vitiligo was also analysed.

MATERIALS AND METHODS:Genomic DNA from whole blood was isolated from 260 vitiligo patients and 350 healthy unaffected controlsfrom a tertiary care hospital after obtaining informed consent. The protocol is approved from IHEC. Genomic DNA was PCR amplified using specific primers to analyse GSTM1 and GSTT1 null genotype and PCR-RFLP to analyse genotype of GSTP1 (Ala113Val rs1138272; Gly169Asp rs41462048, Ile104Val rs1695).

RESULTS AND CONCLUSION:In the present studyvitiligo was observed in both men and women at all ages.25\% patients showed family history of vitiligo and acrofacial and active vitiligo were the most common type. GSTM1 null genotype $(\mathrm{p}=0.025 ; \mathrm{OR}=0.646$; $95 \% \mathrm{CI}=0.437-0.953)$ and GSTP1Ile104Val rs 1695 $(\mathrm{p}=0.027 ; \mathrm{OR}=0.415 ; 95 \% \mathrm{CI}=0.171-0.973)$ showed significant association withvitiligo. There was no association of habit with Vitiligo. Association of genotype was observed with kobernisation. Correlation of genotype with GST levels in the patients and disease activity is warranted. 